LA-UR -93-4375

TITLE: WORKSHOP ON ADVANCES IN SMOOTH PARTICLE HYDRODYNAMICS

\author{
AUTHOR(S): Charles A. Wingate \\ Warner A. Miller
}

\author{
SUBMITTED TO: WORKSHOP PROCEEDINGS \\ LOS ALAMOS NATIONAL LABORATORY \\ SEPTEMBER 21-23, 1993
}

\title{
DISCLAIMER
}

\begin{abstract}
This report was prepared as an accouni of work sponsored by an agency of the United States Government. Neither the United States Government nor any agency thereof, nor any of their employees, makes any warranty, express or implied, or assumes any legal liability or responsibility for the accuracy, completeness, or usefulness of any information, apparatus, product, or process disclosed, or represents that its use would not infringe privately owned rights. Reference berein to any specific commercial product, process, or service by trade name, trademark, manufacturer, or otherwise does not necessarily constitute or imply its endorsement, recommendation, or favoring by the United States Government or any agency thereof. The views and opinions of authors expressed herein do not necessarily state or reflect those of the United States Government or any agency thereof.
\end{abstract}




\section{WORKSHOP ON ADVANCES IN SMOOTH PARTICLE HYDRODYNAMICS}

Los Alamos National Laboratory,

September 21-23, 1993

\section{WORKSHOP PROCEEDINGS}

Organizing Committee:

Chuck Wingate

MS F645

Los Alamos National Laboratory

Los Alamos, NM 87545

Warner Miller

MS B288

Los Alamos National Laboratory

Los Alamos, NM 87545 email: caw Qlanl.gov

phone: 505-667-8954

fax: 505-665-3389

email: wam @regge.lanl.gov

phune: 505-667-3747

fax: 505-665-4055 


\section{PREFACE}

This groceedings contains copies of the viewgraphs presented at the September 1993 Workshop on Advances in Smooth Paricle Hydrodynamics held at Los Alamos National Laboratory, Los Alamos, New Mexico. This was the second conference of this series. The first conference in this series was held at Phillips Lab in Albuquerque, New Mexico in January 1993 and was organized jointly by Phillips Lab and Sandia National Laborkiory. Proceedings from the first conference, including a videotape of the meeting, can be obtained from Steve Attaway at Sandia.

We arc now in the process of planning the third SPH meeting. If anyone has any suggestions for the next meeting please contact Chuck Wingate or Wamer Miller at Los Alamos or Steve Attaway at Sandia.

We are indebted to Ms. Jan Muir who worked very diligently to assemble these proceedings, and to the Theoretical Division Office for their administrative assistance. We wish to acknowledge support for this conference from NASA under the High Performance Computing and Communications Program.

Chuck Wingate (X-1, MS F645)

Wamer Miller (T-6, MS B288)

Los Alamos National Laboratory

Los Alamos, NM 87545

December, 1993 


\section{TABLE OF CONTENTS}

Agenda

\section{F. A. Allahdadi}

L. Baker

P. Shapiro

H. Martell

D. L. Hicks

J. W. Swegle

SPH: Instabilities, Wall Heating, and

S. W. Attaway 
W. Benz

Tensor SPH

M. Davies

W. Benz

Tidal Disruption of Stars

287

M. Fullbright

M. Warren

Breaking the $10,000,000$ Particle Limit in SPH

P. J. Mann

Modelling Relativistic Collapse:

SPH vs FEM

P. Laguna SPH without $\mathrm{H}$

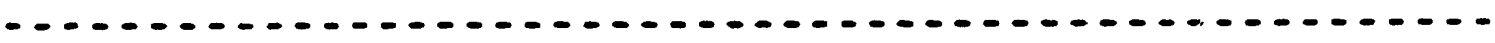

A. Kheyfets Relativistic KSPH Avoidance of Velocity--

W. A. Miller

Biased Kernels

W. H. Zurek

H. Sponłolz

Tidal Compression and Disruption of Stars near a Supermassive Rotation Black Hole

J. J. Monagan

Relativistic SPH Viscosity and Energy

387

L. Brewin

A. Lun

List of Attendees and Others

by Company/Institution

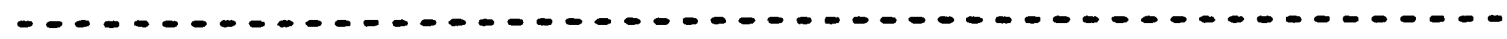

Alphabetical Listing of Attendees 


\section{WORKSHOP ON ADVANCES IN SMOOTH PARTICLE HYDRODYNAMICS}

Los Alamos National Laboratory, September 21-22 and September 23, 1993

At the Study Center (top floor of the library)

\section{AGENDA}

Tuesday, September 21, 1993

8:30 Welcome and administrative announcements

8:35 Bob Stellingwerf What is SPH?

9:15 Joe Monaghan SPH Masters Negative Stress

9:45 Break

10:15 Larry Libersky Cylindrical SPH / SPH with energetic reactions

10:55 Dave Amdahl Topics in SPH

11:30 Lunch

1:30 Charles Luehr Progress on Interface Problems in SPH

2:00 Lou Baker Boundaries and Interfaces in SPH

2:30 Break

3:00 Mike Owen Methodulogy and Tests of Adaptive SPH

3:30 Mike Fisher SPH Energy Conservation for Viscous Flows

5:30 Reception at the University House (next to the Study Center) 
Wednesday, September 22, 1993

8:30 Administrative Announcements

8:35 Gordon Johnson Linked Penetration Calculations

9:00 Chuck Wingate Topics in SPH

9:30 Marv Alme SPHIX Simulations of Oblique Impact of Steel Fragments onto Steel Armor Plate (with Nick Ferriter)

10:00 Break

10:30 Jeff Swegle Stability and Consistency of the SPH Method

11:00 Dave Amdahl, Mike Fisher, Chuck Wingate and Bob Stellingwerf Graphics Demos

11:30 Lunch

1:30 Darrell Hicks SPH: Instabilities, Wall Heating and Conservative Smoothing

2:00 Willy Benz Tensor $\mathrm{H}$ and Fragmentation

2:30 Break

3:00 Bob Stellingwerf Workshop on Numerical problems in SPH 
Thursday, September 23, 1993

9:00 Wojciech Zurek Opening Remarks

9:15 Willy Benz Tidal Distuptions and Elliptic Kernels

9:55 Mike Warren Breaking the 1.e07 Particle Limit in SPH

10:35 Break

11:00 Patrick Mann SPH vs. FEM: Relativistic Spherical Collapse

11:40 Lunch

1:00 Pablo Laguna SPH without $H$

1:40 Warner Miller Avoidance of Velocity-Biased Kernels

2:30 Break

3:00 Hanno Sponholz Tidal Disruptions and Accretion Disks

3:40 Joe Monaghan Energy and Viscosity in Relativistic SPH 
$-4-$ 


\section{WHAT IS SPH ?}

\section{Overview, Comments, Trieste}

\section{Smooth Particle Hydrodynamics Workshop}

September, 21, 1993, LANL

Bob Stellingwerf

Los Alamos National Laboratory

Applied Theoretical Division

Los Alamos 


\section{SPH is a MONTE CARLO Techniquel}

- First proposed as such by Lucy in 1977.

- Many improvements have been made to SPH since then, but it still is based on a Monte Carlo integration step.

- Basic Monte Carlo theorem, uniform sampling, $\mathrm{i}=1, \mathrm{~N}$, over volume $\mathrm{V}$ :

$$
\int f(x) d V=\frac{V}{N} \Sigma f_{j}=\Sigma f_{j} \Delta V_{j}
$$

- For random sampling with probability density $p d V$ (Lucy):

$$
p(r)=\frac{M}{N} \sum w\left(r \cdots r_{j}\right)=\sum w\left(r-r_{j}\right) \Delta m_{j}
$$

where $w(r-r)$ is a kernel that provides a local, compact support.

- Then for any specific quantity, $Q$ (something/gm), we can compute the corresponding "density" $q=p Q$ (something/cc) via:

$$
q(r)=\sum Q_{j} w\left(r-r_{j}\right) \Delta m_{j}
$$

Los Alamos 


\section{SPH is a MONTE CARLO Technique (continued)}

- Now, since $Q_{j}$ is a constant, and all of the local variation of $Q(r)$ has been transferred to the $w(r-r)$, we have:

$$
\nabla q(r)=\sum Q_{j} \nabla w\left(r-r_{j}\right) \Delta m_{j}
$$

- Now, to do physics we interpret the $\Delta m_{j}$ as the mass associated with "particle" $j$, and the interpolated velocity" as the mean velocity of that mass, and presto, we have a (real) Lagrangian scheme!

- Note, however, that the "particles" are still actually Monte Carlo sampling points, the kernels overlap, and SPH is actually not a particle scheme at all, much less a Lagrangian hydro scheme...

- Much progress since Lucy can be attributed to Monaghan, who noted:

1. Since an interpolation is involved to apply the integral theorem, a considerable body of interpolation theory can be applied.

2. By applying some algebraic tricks, the SPH equations can be made to conserve mass, momentum, angular momentum and (optionally) total energy.

3. The sampling error can be reduced by starting with a regular grid of points. 


\section{SPH Volume Element}

- A handy form of the Monte Carlo integral for SPH is:

$$
(f(r))=\int f\left(r^{\prime}\right) w\left(r-r^{\prime}\right) d V=\sum f_{j} w\left(r-r_{j}\right) \Delta V_{j}
$$

- Where $<.>$ indicates the interpolated function, and the usual choice for $\Delta V_{j}$ is $\left(m_{j} / p_{j}\right)$.

- Other possibilities can be considered: Lucy used $\left\langle f_{i}\right\rangle=\frac{\sum f_{j} \omega\left(r_{i}-r_{j}\right) m_{j}}{p_{i}}$ or we could consider $\left.\quad f_{i}\right)=\left(\frac{m_{i}}{p_{i}}\right) \sum f_{j} w\left(r_{i}-r_{j}\right)$.

- Another possible choice might be

$$
\left\langle f_{i}\right\rangle=\frac{\sum f_{j} w\left(r_{i}-r_{j}\right)}{\sum w\left(r_{i}-r_{j}\right)}
$$

- this has the advantage that neither the mass nor the density enters the interpolation, and edge effects are eliminated (functions are constant to the edge of the kernel), but the implied boundary condition at the edge of an object is $\Delta P=0$, rather than $P=0$.

- Another problem with all of these alternatives is that symmetry (required for momentum and energy conservation) is usually lost, but not all possibilities have been explored. 


\section{SPH Problem Areas}

- Adequate coverage of $w\left(x-r^{\prime}\right)$ is needed to approximate the integral. This means lots of neighbors, and preferrably a non-uniform spacing. Interpolation theory, on the other hand, requires regular spacing and gives best results with narrow kernels. A balance is needed.

- Spherical particles impose a maximum 1D strain that can be tolerated, and imply an elasticity limit that can cause premature fracture (tennis ball problem). Widening the kernel fixes this in most cases.

- Instabilities related to the "cell-centered" averaging are sometimes seen. These are most often encountered in quiescent regions under tension, although the analogous compressional instability has never been seen.

- Interfaces in which some quantities (such as mean molecular weight) vary discontinuously can cause large fluctuations when perturbed.

- Density given by the sum of masses formula cannot be constant at the edge of an object. This is incompatible with a solid equation of state.

- Densities given by integrating a continuity equation may rot be consistent with the masses used in the integral sums. 


\section{SPH- Some Comments}

- Tennis Ball Problem: extra viscosity, different kernel, or simply increasing the smoothing length (more neighbors) fixes this. The optimum fix may be to allow non-spherical kernels.

- Fracture: very high strain/high fracture experiments are modeled very well for both ductile and brittle materials, even without a fracture model. Benz/Asphaug model shows promise of modeling fracture accurately on all scales.

- Continuity Equation: seems to work, but no consistent way of setting the masses, or verifying that mass is conserved has been proposed.

- Interfaces: for individual problems a workable solution can often be found by carefully matching masses, sizes and placement of particles. No general treatment is known. Probably the most serious problem now facing the technique.

- Cell-centered instability: usually seen at very late time in relaxing problems such as crater formation. Varying the pressure average sometimes helps (Libersky). Not normally a serious problem for most applications. 
Masters Negative STtess

J.J. Monaghan Monash University

J.A. Morris Honoush University

$-11-$ 
Program

- Why use particle methods

- Different forms of SPH

- Lessons from MHD - stability

- Stability when

$$
P_{a}=\rho_{0} c^{2}\left[\frac{\rho_{a}}{\rho_{0}}-1+\mathbb{R}\right]
$$

- Adjusting Kernels for the momentum conserving form

- Stability of Pressure difference form.

- Conclusions

$-12-$ 


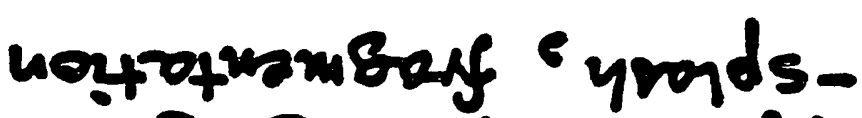

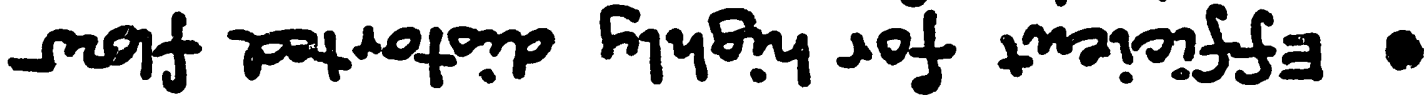

poppro rquos s sisfind payosildius

\section{poo6 noitonsasnos}

pinif

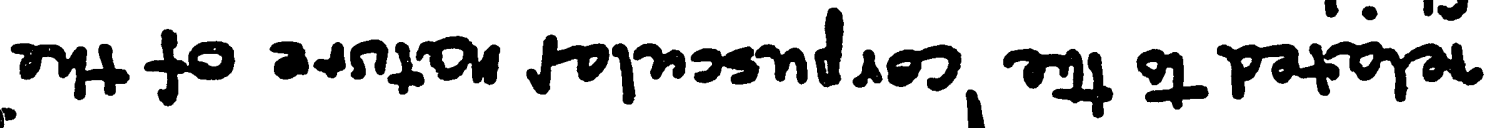
bytropp op poutam aloptrod ont ormosag

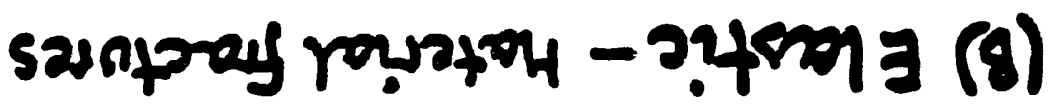

\section{$7 \cdot 0$}

mon satamprod

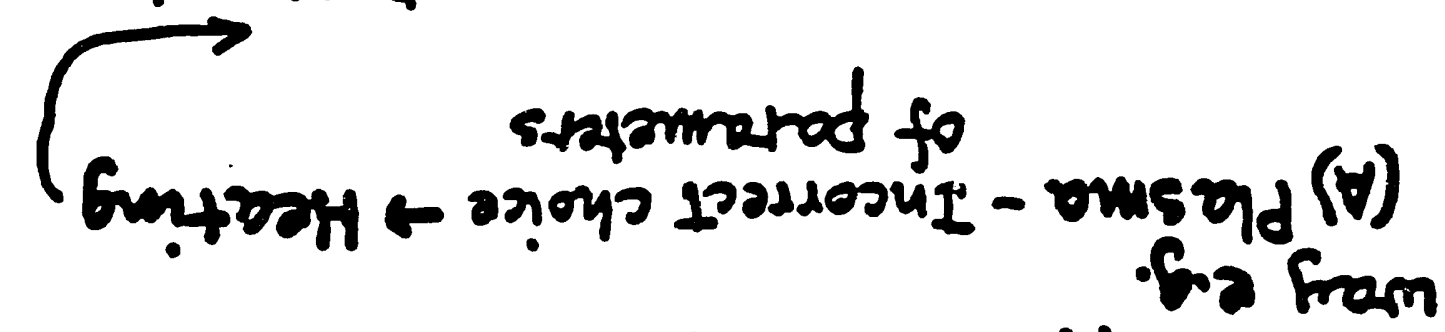

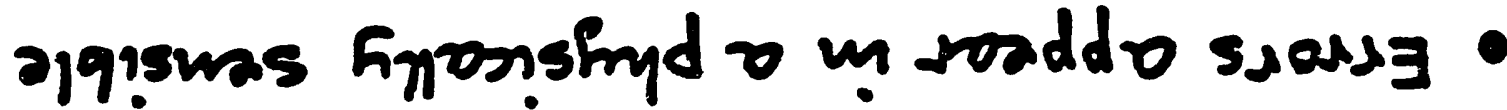

mascheramo

rom = varg $+\frac{7 \varepsilon}{V e}$

snba pinif m smsat P!joqusd fit ryt ampes of fran paintou oyt apuend say 
S.P.H.

- Particle method with out a grid

- Properties from Interpolation from Particles

Mutant Forms

- Different Kernels

- Different arrangement of terms

- Different time stepping

- Different $h$ variations

- Different viscosity -other dissipation

-14- 
M. H.D. Problems

Mom

$\frac{d \vec{v}}{d t}=-\frac{\overrightarrow{\nabla P}}{\rho}+\frac{\vec{J} \times \vec{b}}{\rho}$

ideal gas

or

$$
\frac{d v_{i}}{d t}=-\frac{1}{\rho} \frac{\partial P_{i}}{\partial x^{i}}+\frac{1}{\rho} \frac{\partial M_{i j}}{\partial x^{j}}
$$

where

$$
H_{i j}=\frac{1}{\mu_{0}}\left(B_{i} B_{j}-\frac{1}{2} B^{2} \delta_{i j}\right)
$$

G.Phillips, J.J.Monaghan M.N.R.A.S. 216, 883 ,(1985)

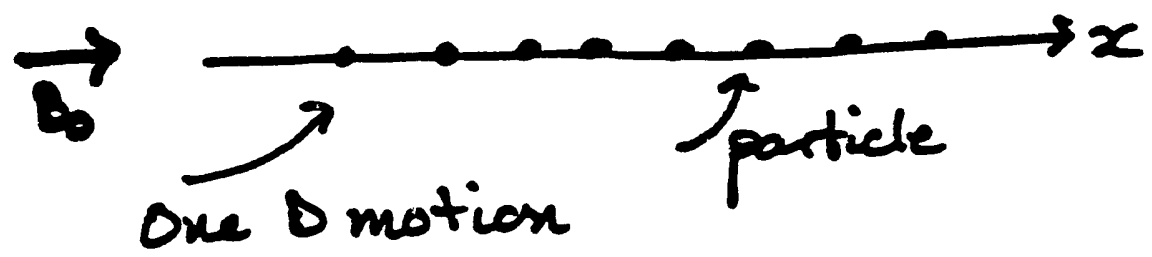

$$
\begin{aligned}
& \frac{d v_{a}}{d t}=-\sum_{b} m_{b}[\frac{P_{b}}{\rho b^{2}}+\frac{P_{a}}{\rho a^{2}}-\frac{1}{2 e_{b}} \frac{B_{a}^{2}}{\rho^{2}} \underbrace{\vec{\nabla} W_{a b}} \\
& P_{\text {erturbations }}
\end{aligned}
$$

1 theseparticles

Potential

Botential

Wele

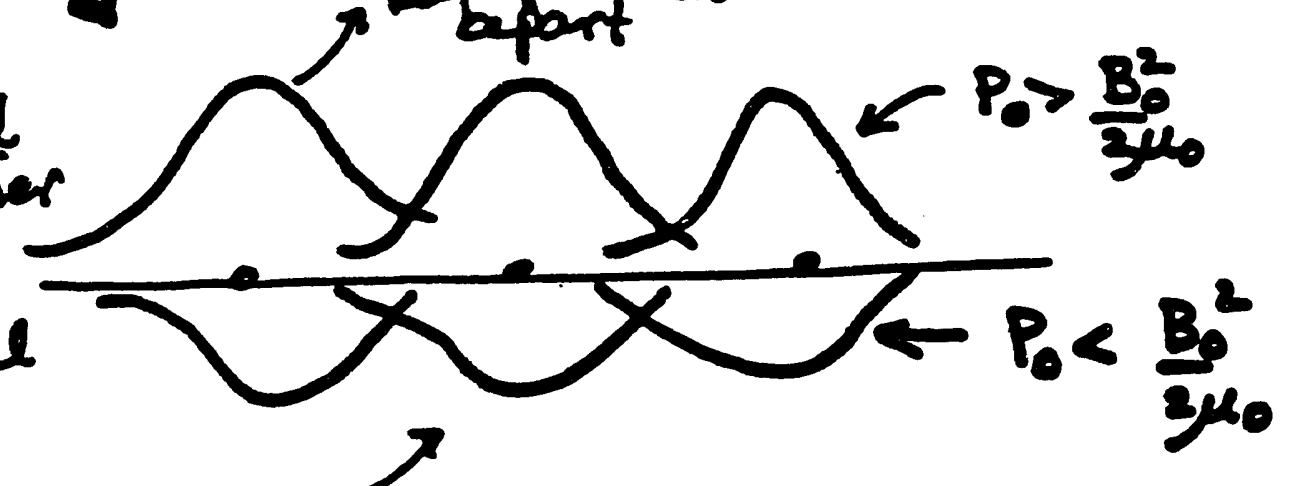

Fries topult particlestogether

$-15-$ 
How The Instability

Was Removed

Pressure term

Magueticterm

either

calculate $\vec{J}, \vec{B}$ then $\vec{J} \times \vec{B}$

or

Make $M_{i j}<0$ by subtracting a constant.

$-16-$ 
Analysis

Jeff Swegle tothers. (Jerry Brackbill ......) Joe Morris, Willy Bens

$$
P_{a}=\rho_{0} c^{2}\left[\frac{\rho_{a}}{\rho_{0}}-1+\mathbb{R}_{0}\right]
$$

$R=1 \rightarrow$ isothermal gas

$R=0 \rightarrow$ like metal

$R<0 \rightarrow$ unstable cases

$R=i-\frac{B_{0}^{2}}{2 \mu} \rightarrow$ mag. case.

ID gas

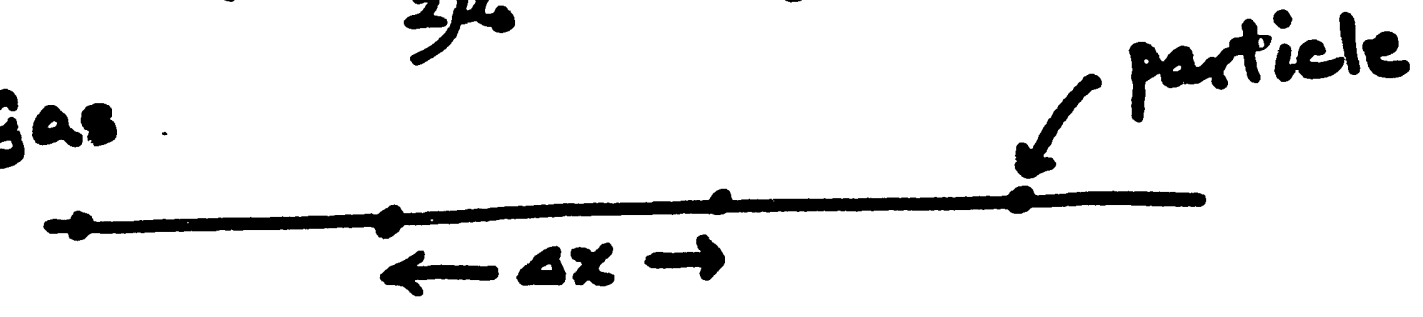

Small $\int$ Pert $\alpha e^{i(k(a \Delta x)-\omega t)}$

oscillation $\rho a=\rho \circ$ background

$-17-$ 


$$
\begin{aligned}
& 99^{9} q_{m} \frac{9}{2}=2 \delta \\
& \operatorname{sim} \nu_{\Delta}\left(-\frac{q d}{q d}+\frac{q d}{d}\right) a_{m} \dot{z}^{q}=\frac{\nabla \delta}{d \Delta} \\
& \left.\left[\%+1-\frac{\delta}{\delta}\right], 2 D_{\delta}=x_{d}\right\} \\
& \text { ग00न }
\end{aligned}
$$

$$
\begin{aligned}
& 0>9 \text { 5? 0> }
\end{aligned}
$$

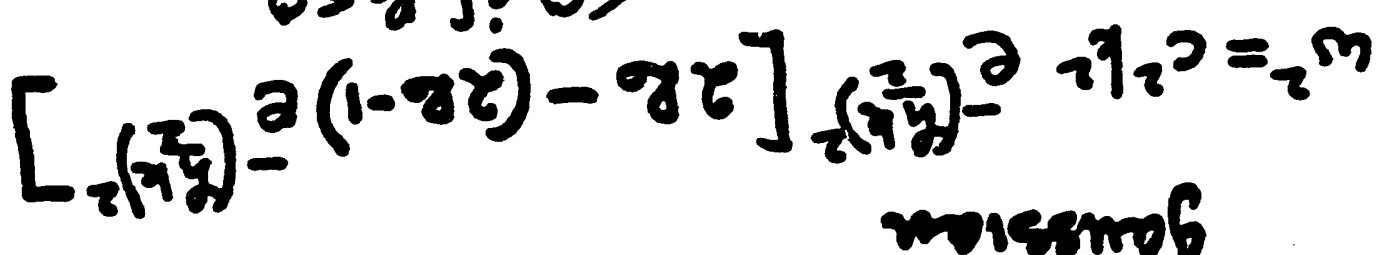

$$
\begin{aligned}
& \left(y^{\prime} x_{2} n(1-y z)-\left(y^{\prime} x\right) n \partial z\right] z^{\prime} y_{z} z=z_{2}
\end{aligned}
$$

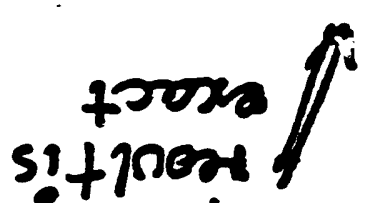

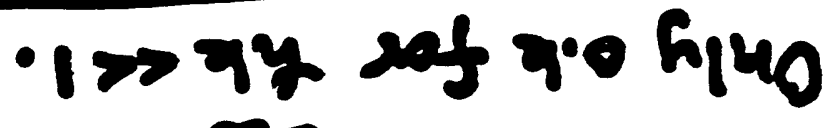

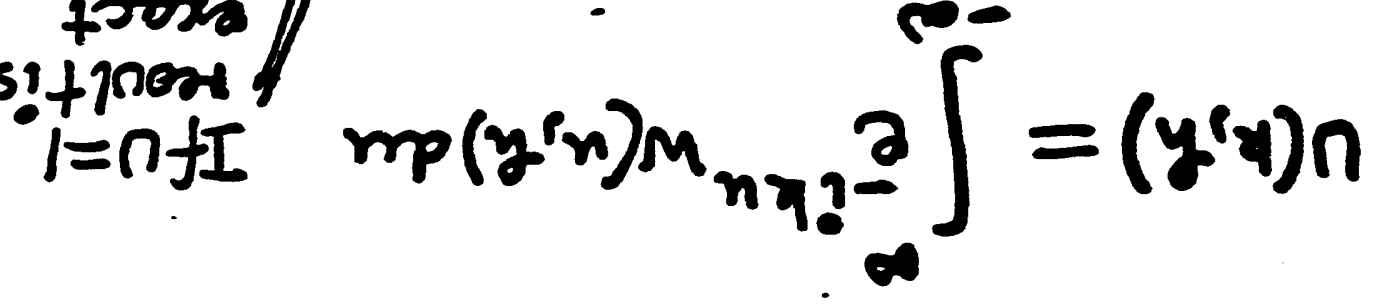

$$
\begin{aligned}
& \operatorname{xp}(x) f \int^{\infty} \sim f^{\infty} \bar{z}
\end{aligned}
$$

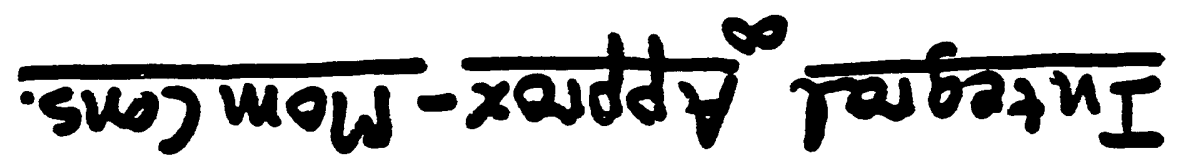


Poisson Summation

$\sum_{n=-\infty}^{\infty} f_{n}=\sum_{l=-\infty}^{\infty} \int_{-\infty}^{\infty} f(x) e^{-2 \pi i l x} d x$

Gives useful expressions

Direct Summation

$$
\begin{aligned}
w^{2}= & 2 c^{2} R_{0} \sum_{j} \Delta x(1-\cos (j k \Delta x))\left(\frac{\partial^{2} w}{\partial x^{2}}\right) j \\
& +c^{2}(1-2 R)\left\{\sum_{j} \Delta x \cdot \sin (j k \Delta x)\left(\frac{\partial w}{\partial x}\right)_{j}\right\}^{2}
\end{aligned}
$$

$-19-$ 


\section{Wo hernel}

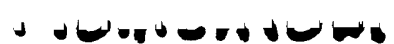
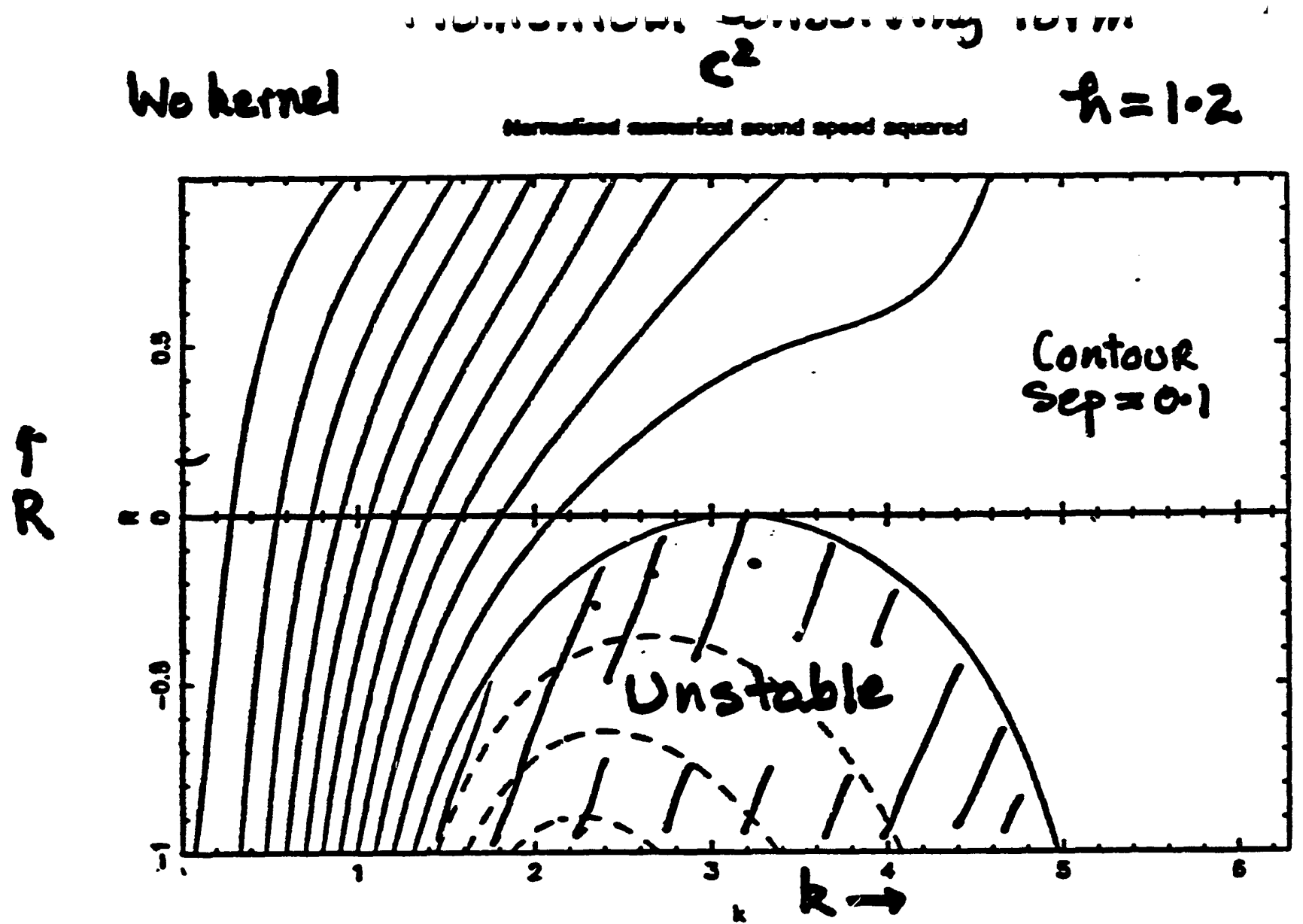

C 8 Normotioed mumbitool sound epeed anvered

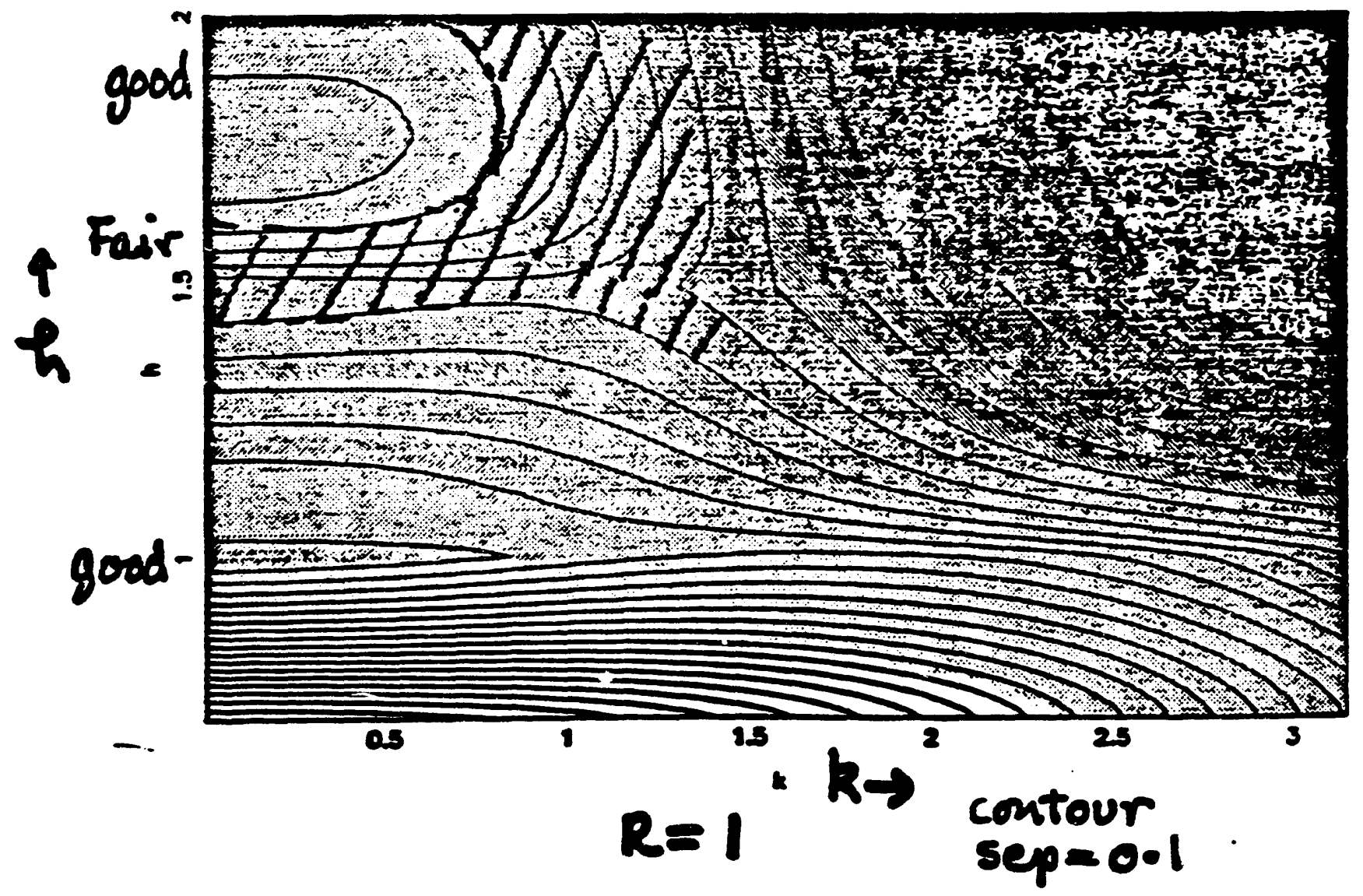




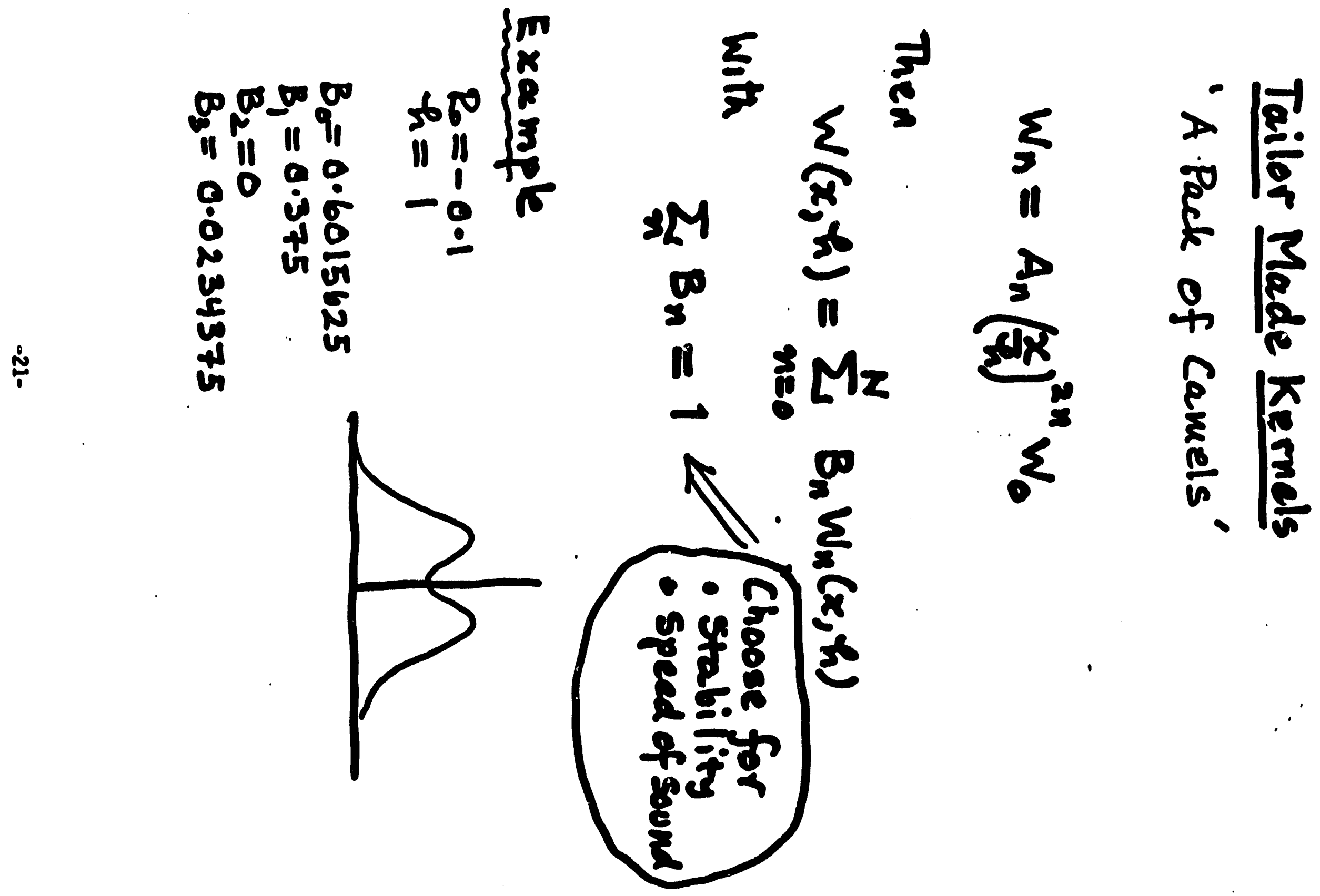



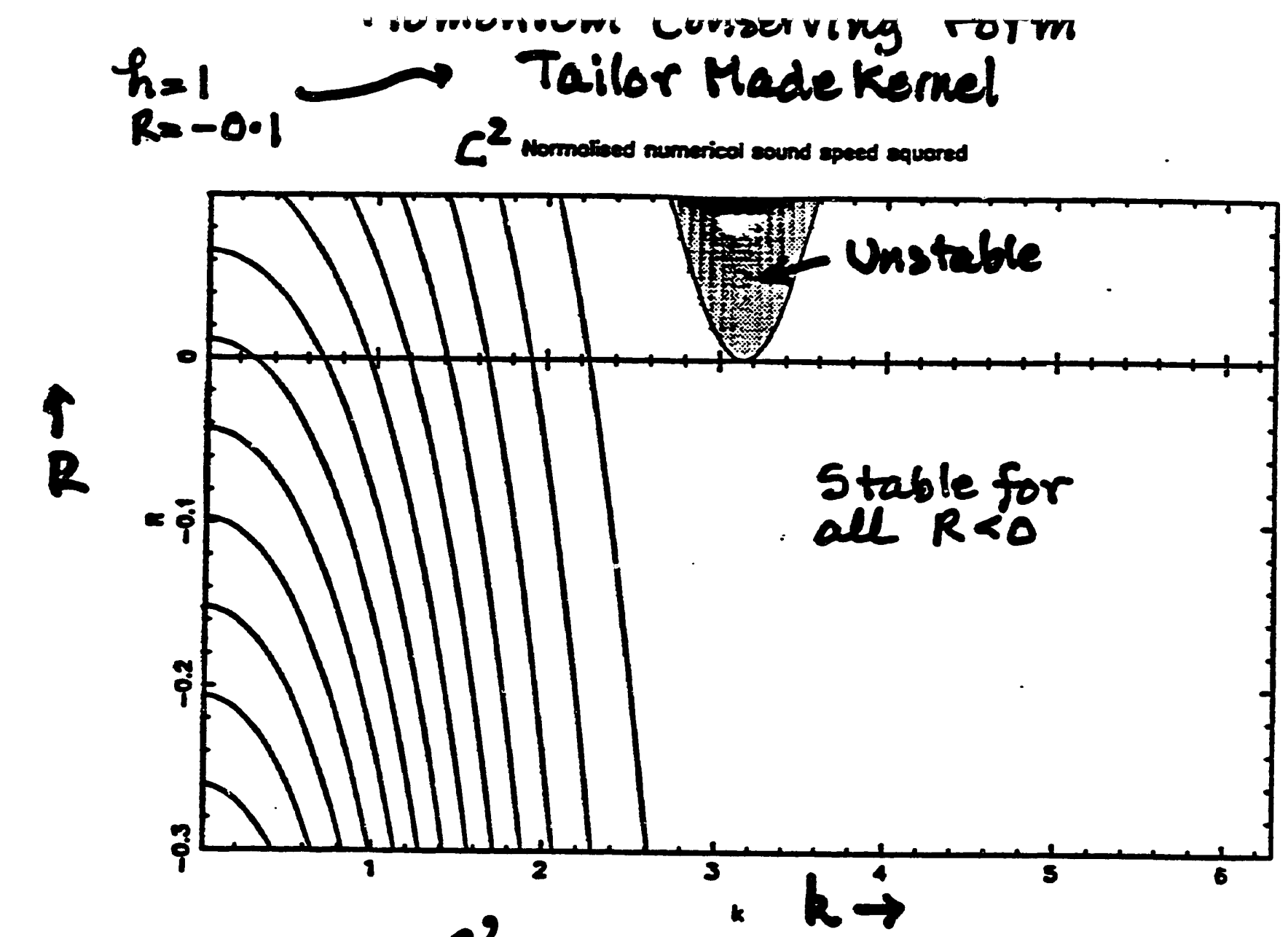

$\mathrm{C}^{2}$ Normoliaed mumericol sound spead squared

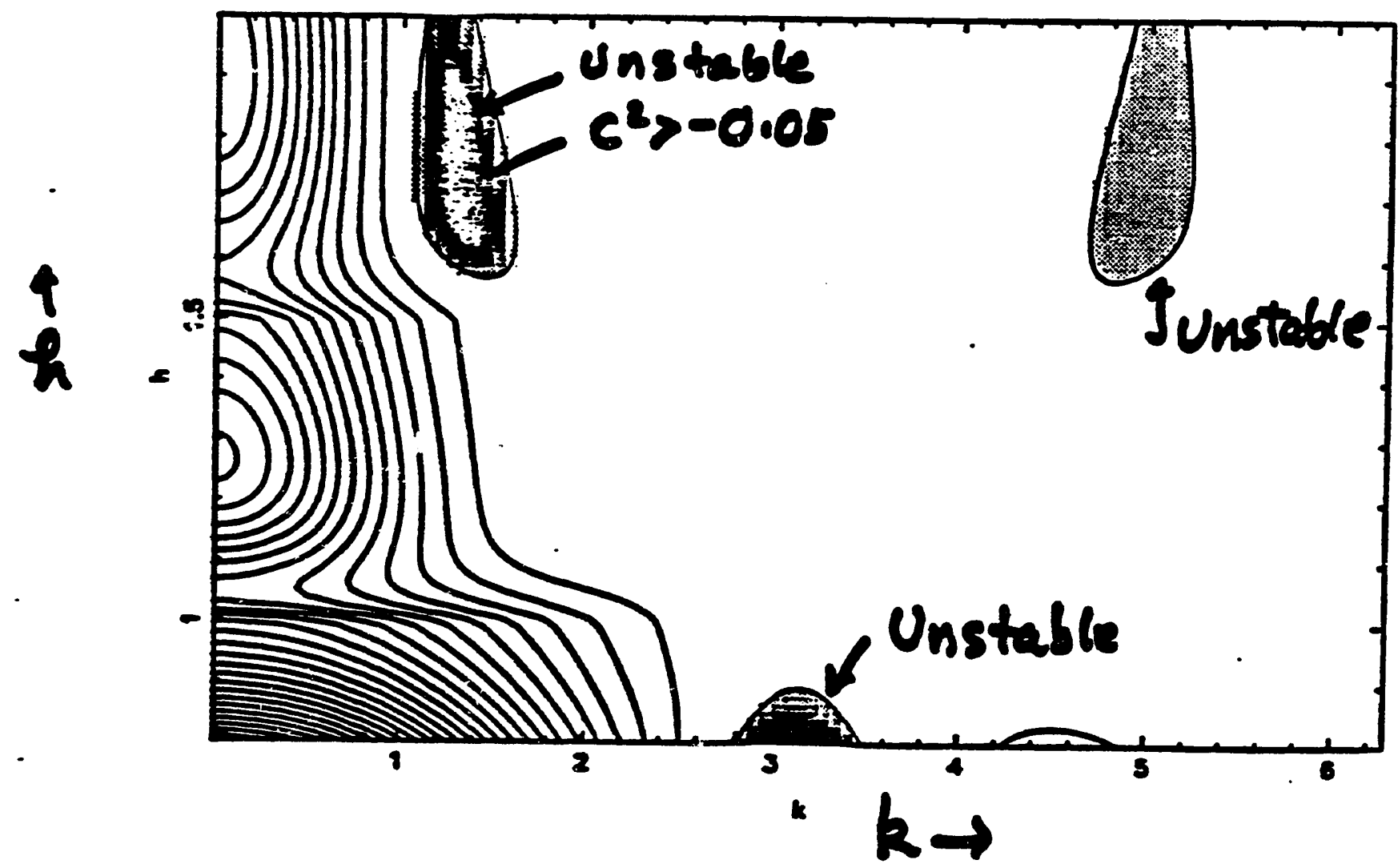



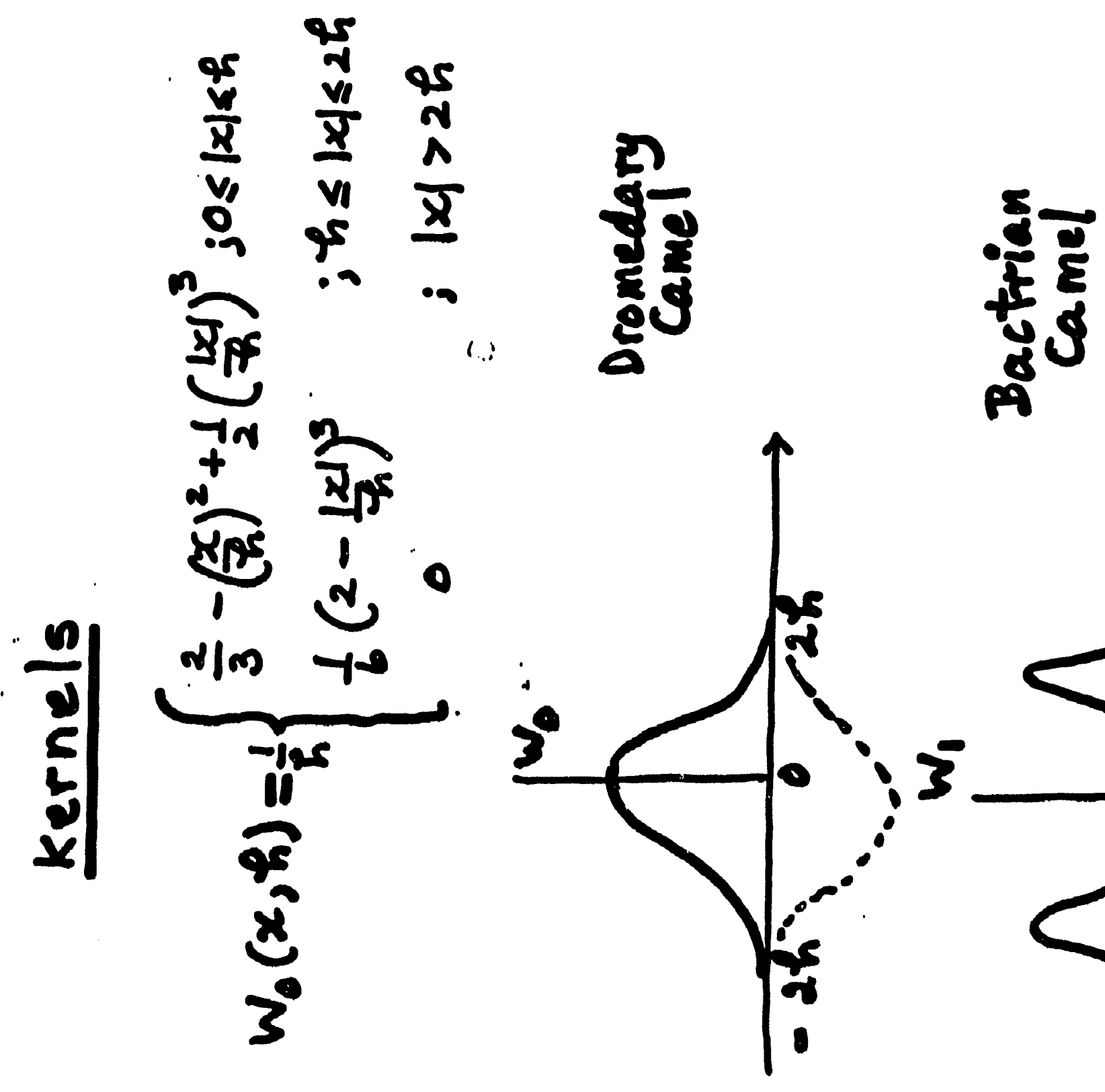

A

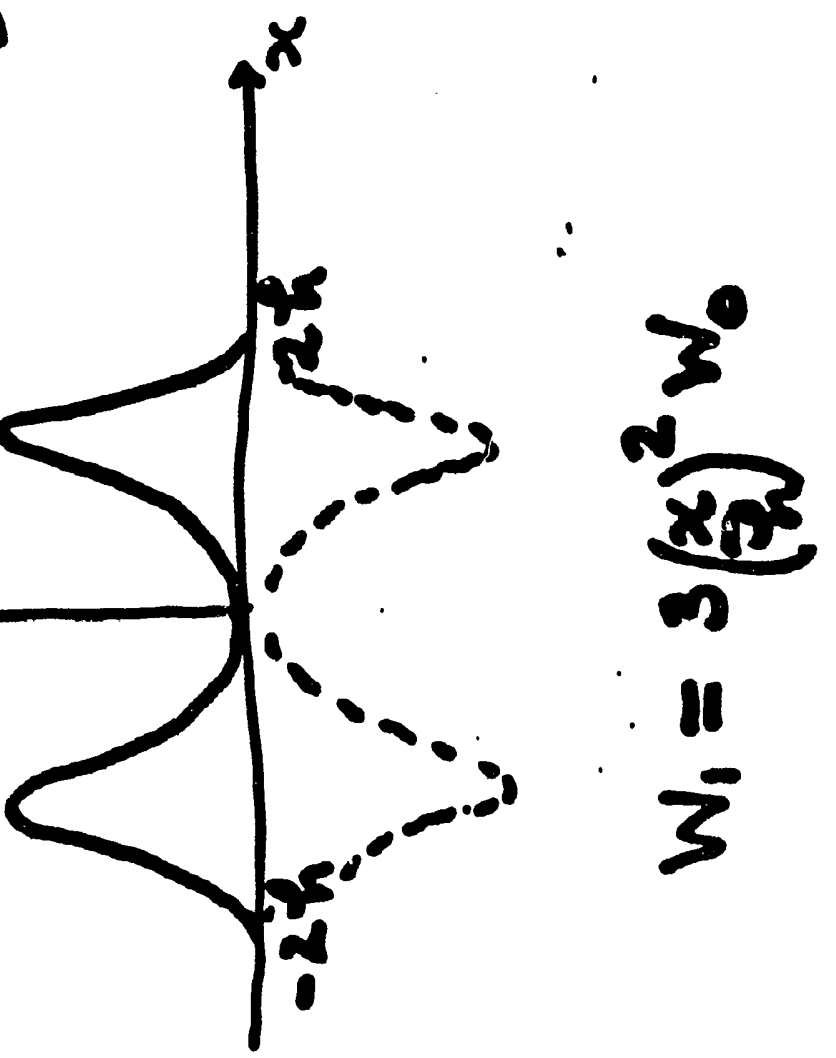


Pressure Difference VP

$$
\left(\frac{\nabla P}{\rho}\right)_{a}=\sum_{b} m_{b} \frac{\left(P_{b}-P_{a}\right)}{\rho_{a} \rho^{b}} \nabla_{a} W_{a b}
$$

Stability for ID

$$
\begin{aligned}
& \omega^{2}=\left(\frac{m c}{\rho_{0}}\right)^{2}\left[\sum_{-a}^{\infty} \sin (k \Delta x j) \frac{\partial w_{j}}{\partial x}\right]^{2} \\
& \text { ALWAYS STABLE } \\
& \text { Excellent } C
\end{aligned}
$$

Thermal Energy Equ. (Not Needed Here).

$$
\frac{d u_{a}}{d t}=-\frac{P_{a}}{\rho_{a}} \sum_{b} u_{b} \frac{\left(\overrightarrow{v_{b}}-\overrightarrow{u_{b}}\right)}{\rho_{b}} \cdot \nabla_{a} u_{a b}
$$

$-24-$ 


\section{$c^{2}$ For Pressure Difference Form}

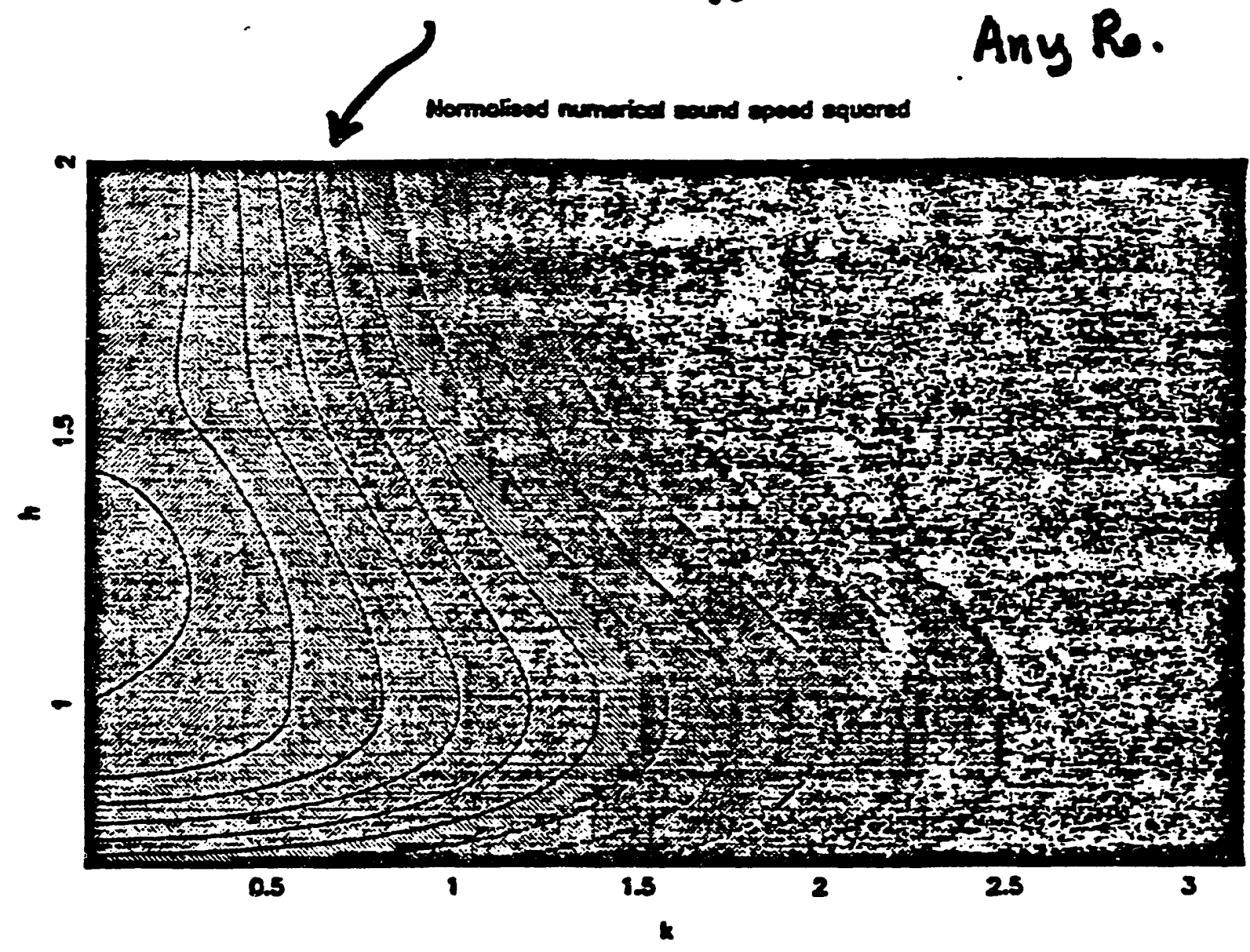

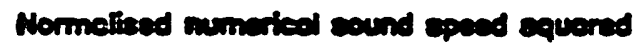

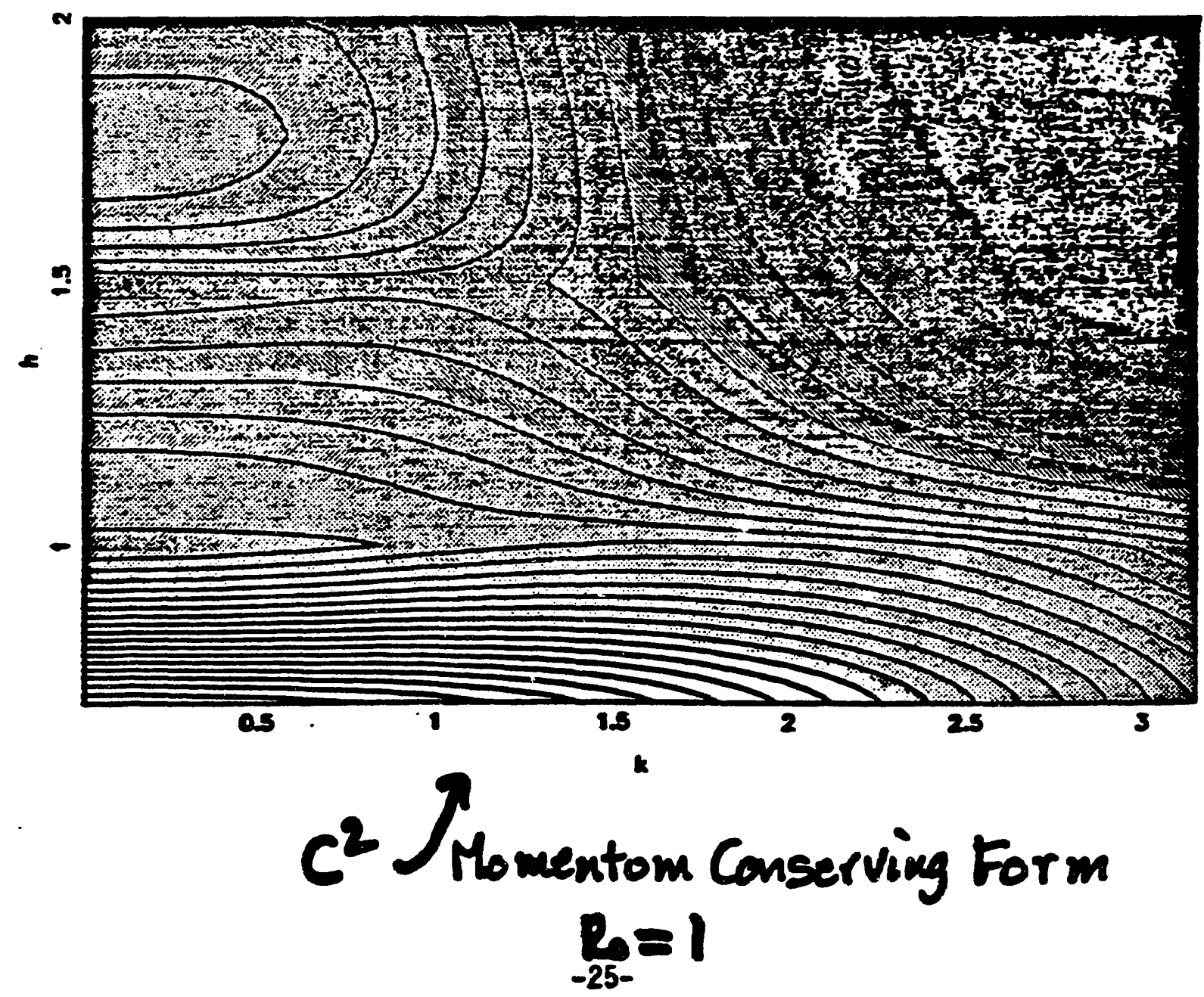


SHOCK PROFILES
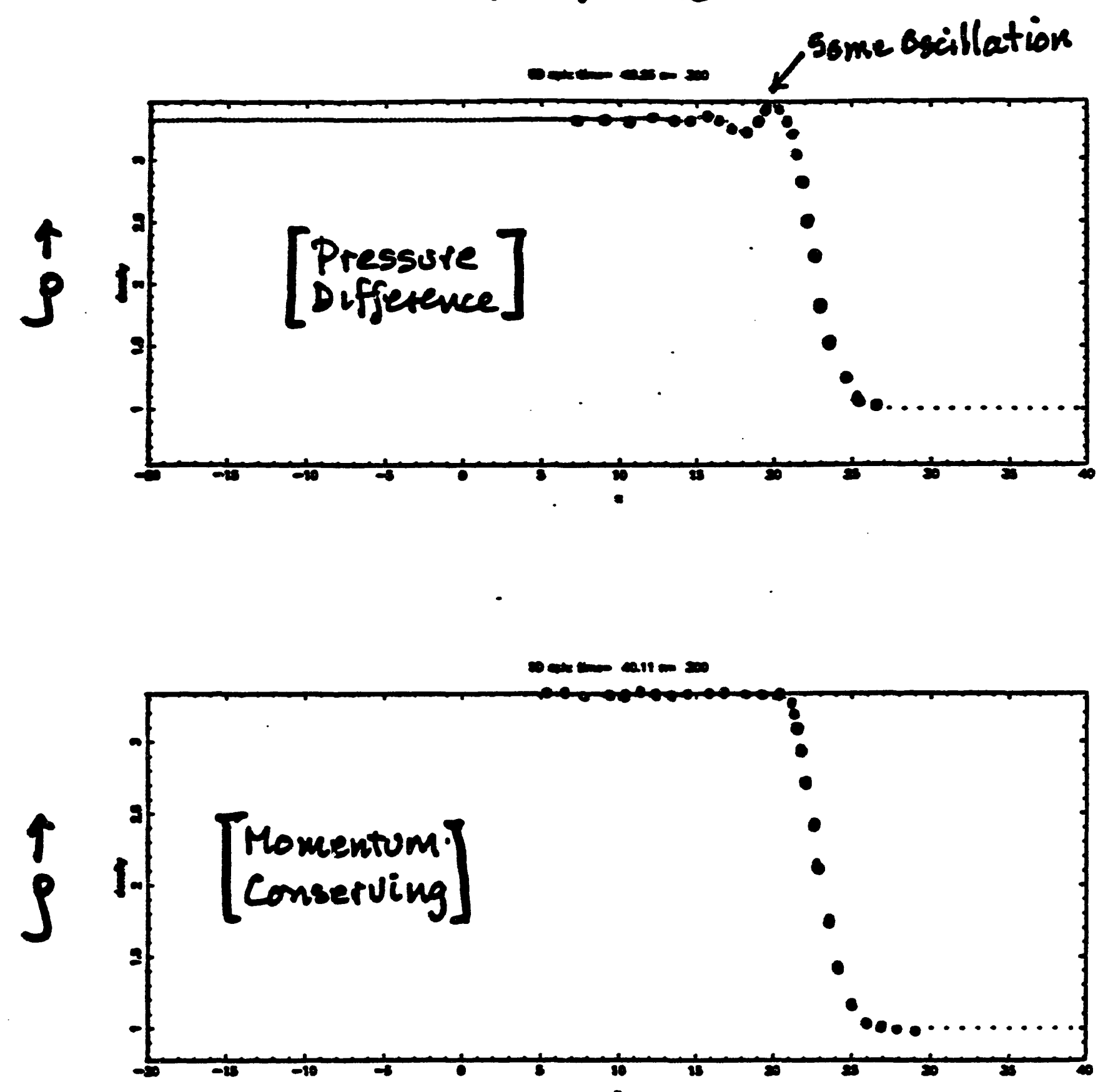

P. Diff

- 'Go Good C

- Conserve mom with Lagrange Milt.

$-26-$ 
Take Home Message

- If $P \geqslant 0$ use Mom Conserving form

- If $P<0$ use Press. Diff form Tailor made Kernels

-

$$
\begin{aligned}
& \stackrel{\lambda \geqslant 0}{P} \delta_{i j}+\dot{M}_{i j} \\
& \frac{d \vec{v}_{i}(a)}{d t}=-\sum_{b} m_{b}\left(\frac{P_{b}}{\rho_{a}^{2}}+\frac{P_{b}}{j b^{2}}\right) \nabla_{a} W_{a b} \\
& +\sum_{b} m_{b}\left[\frac{M_{i j}(b)-M_{i j}(a)}{\rho a j b}\right] \nabla_{a, j} W_{a b}
\end{aligned}
$$

$-27-$ 
ซ 


\title{
CALCULATION OF REACTIV FLOW USTNG SMOOTEID PARTICLEEDRODYNAMICS
}

\author{
LD. Libersty AG. Pewehek Per-Anders Persion \\ Esergetic Materials Reserrah Texing Center (EMRTC) and \\ Deparument of Physies \\ New Mecico Instivite of Mining and Technology \\ Socorre, NM 87801
}

Reactive flow is incorporated within the frumetwork of Smoothed Particle Hydrodynamics and a mixure nule is formulated which allows for treamem of large grained explosives and propellants. The method is tested using lengsh scales fine enough to resolve the reacrion sone. The simulution seems 20 reproduce ided detonarion theory quine well for planer steady-state detomation in Composition-B.

\section{ETIRODUCTION}

Sonoothed Pariele Hydrodynumies (SPF) is a relativety new compurational tectrique that seems well arised to simulation of renctive flow. The method uses Laprongina fluid elements (paricles) thut are nor tied to any undertying spatial grid. The gridless nerure of the rectrique mnikes it possible to compune highly distorted fow in a gure Lagrengien frame. The method has other rice femires ach as the relutive eace of adding bew physices and of exrending calculations to three dimencions. Application of SPH to the enlavincien of reactive fow has the potential therefore, for improved searrey and excended applicability. The main puppose of this paper is to describe the inclusion of renctive flow wishin the SPH frumework. In so doing we inuoduce sew ways of treaving the tempersure in which (a) thend equitibrim is sot a mixure nule and (b) the tempersure referesce enve is determined antyrienlly. A simulation of ploner ready-ante detonstion in Composivion-B is shown to reproduce ided deconmion theory guive well.

\section{T:150RY}

The SPH representuion of the fluid conservation equations are given below (eee [1] for derivations).

$$
\frac{d \rho_{1}}{d t}=\rho_{1} \sum \frac{m_{j}}{\rho_{j}}\left(U_{i}-U_{j}\right) \frac{\partial T_{y}}{\partial t}
$$

$$
\begin{aligned}
& \frac{d U_{1}^{c}}{d}=-\sum_{j} m_{j}\left(\frac{P_{1}}{\rho_{1}^{2}}+\frac{P_{1}}{P_{j}^{2}}+\Pi_{v}\right) \frac{\partial W_{v}}{\partial P_{1}} \\
& \frac{d E_{1}}{d t}=\sum_{j} m_{j}\left(U_{i}^{*}-U_{j}\right)\left(\frac{P_{i}}{\rho_{1}^{2}}+\frac{1}{2} \Pi_{j}\right) \frac{\delta W_{t}}{\alpha_{l}}
\end{aligned}
$$

Varibles are coordinutes $(x)$, time $(1)$, density $(p)$, velocity $(U)$, specific imternd energy (E), prescure $(P)$ and arificial viscosiny (II). The subseripts refer to : given paricle (I) and its naighbors (j). Internecions between paricles are waighted by the imerpolation kemel $W$ called the smoothing finction. Iypically, $W$ is a-spline whose width is mensured by the parmeter $h$, the suoothing length. Notice thrt only pradients of $W$ sppent in these equations. Becruse of this no undertying spativ grid is required. The formulution can be extended to inchude reactive flow by adding a consinutive relation and a rate equation for the unburned frection (2) of reacting muterial. From this poin on we itop the particle index in order to use gubseript notution for other purposes.

$$
\begin{aligned}
& P=P(0, E, \lambda) \\
& \frac{d \Omega}{d}=f(P, P, \lambda)
\end{aligned}
$$


Rescing muerial is pariniosed into unbuned solid (s) and rencted gas (g) phases sccording to conservation luws for mass and exersy.

$$
\begin{aligned}
& V=\lambda V_{2}+(1-\lambda) V_{2} \\
& E=\lambda E_{8}+(1-\lambda) E_{8}
\end{aligned}
$$

Here, $V=1 / p$ is the specific volume. In order to close the symen of egurtions (1-5) two additional constrints are required. For high dencisy mitiony exploxives, it hes

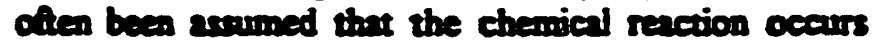
bomogenecusty, therefore one chooses preasure and tempereure eqpitiorim berween the solid and gas pheses of the burning muterial. Fowever, for composite reacting meterials brving grain sizes typical of many explosives and propellants the asumption of rempesume equitionium is not vatid. A more realintic aseumption for large ganined materials would be that nepligible bea tronfer occurs berween the swo phases, i.e., the unrescted solid behnves isentropically. We therefore cloce the system of equations (1-6) by imposing the consurints of pressure equilitoriun and an adiabric solid.

$$
\begin{gathered}
P_{s}=P_{s} \\
\frac{E_{s}}{\partial_{s}}=-P_{s}
\end{gathered}
$$

In order to use (9) we must bnow which icentrope the reacting muerial is on. For ideal stendy rate detonxions this is the isentrope paesing through the vonNewman point. For transitions to deconation a fumily of adinbats defined by the solid Flugoniot are encoumered by the reacing material. A progmoxic equarion for the exuropy is added for this pupose.

$$
\frac{d S}{d}=\frac{d Q}{T}
$$

The bea added (dQ) is the arificial viscous work (II verus in equation 3). Equrtion (10) sleo introduces the temperenure $T$ which we compurte by linear excrapolation form the priscipal adiaber.

$$
T=I_{s}+\left(E-E_{s}\right) / C_{r}
$$

URing the Fins Lw

$$
d E=T A S-P d V \text {. }
$$

the following rebrions can be derived

$$
\left.\left.\left.\frac{\partial T}{\partial T}\right)_{s}=\frac{\partial P}{\partial S}\right)_{V} \quad \frac{\partial E}{\partial S}\right)_{V}=T
$$

Assuming a Grumeisen equation of sate

$$
P(V, E)=P_{n}(V)+\frac{\gamma}{V}\left[E-E_{g}(V)\right]
$$

the presure derivaive in (13) en be writuen as

$$
\left.\left.\left.\left.\frac{\partial P}{\delta}\right)_{V}=\frac{\partial T}{\partial V}\right)_{s}=\frac{E P}{\partial E}\right)_{V} \frac{\partial E}{\partial S}\right)_{V}=\frac{Y}{V} T
$$

An equation for the solid adiabut sempereture is, therefore,

$$
\left.\frac{\partial T}{\partial V}\right)_{s}=\frac{\gamma}{V} T
$$

which for $y / V$ econst integrutes to

$$
T^{3} e^{\left(r_{0} m_{0}\right) t}=T_{0} e^{T_{0}}
$$

We obrain the adiabat energy $E_{S}$ by integration.

$$
\begin{gathered}
E_{s}(V)=-\int_{V}^{V} P d V= \\
\int_{0}^{y}\left[P_{n}+\frac{\gamma}{V}\left(E-E_{n}\right)\right] d V
\end{gathered}
$$

where the Ifugonion presure and exergy are given by

$$
\begin{aligned}
& P_{m}=\frac{C^{0}\left(V_{0}-V\right)}{\left[V_{0}-S\left(V_{0}-V\right)\right]^{2}} \\
& E_{m}=\frac{1}{2} P_{m}\left(V_{0}-V\right)
\end{aligned}
$$

Here, $C$ and $S$ are coefficiens in the linear shock speed particle epped relutionship $U_{s}=C+S U_{p}$ and $V_{0}$ is the normal volume. Choosing yrha to be constum, and leting $F=E \exp [a V]$ we have

$$
F=-\int_{V_{0}}^{\nu} e^{N}\left[P_{H}-\frac{\gamma}{V} E_{M}\right] d V
$$

which intequates 20

$$
\begin{aligned}
& F(V)=\frac{1}{2 S^{2}}\left\{-C^{2}\left(3 S-a V_{0}\right) v_{0}+\left[1+\frac{\left(2-a V_{0} / S\right)}{\left(S V I V_{0}-S+1\right)}\right]\right\}(22) \\
& \left.+\frac{C\left(2 S^{2}-4 a V_{0}+\omega^{2} V^{2}\right)}{2 S^{2}}\right)
\end{aligned}
$$


where Ei is the exponential incegral. The adiabut energy is therefiore.

$$
E_{s}(V)=F(V) e^{-\frac{\pi}{V_{0}}}
$$

Equmions (11), (17) and (23) are used to colaulate the rempermure of the solid required in (10). Methods using fits to the Inugoniot tempernure for the reference curve [2] hove cansed us dififailies, apperently becane we require temperanues cutside the region of validity of the fil. The antycic sure of our method eliminntes such problems.

\section{EQUATIONS OF STATL}

For the unreacted solid mnerial the Conmeinen equnion of stre $(14,19)$ is used. A. HOM representation of the BKW [2] equarion of strie is ured for the gaseous detonicion products.

$$
\begin{aligned}
& P=P_{1}+\frac{f}{t}\left(E-E_{1}\right) / V \\
& m\left(P_{A}\right)=A+B(\operatorname{m} V)+C(\operatorname{m} V)^{2}+D(\operatorname{m} V)^{3}+E(\ln V)^{4} \\
& h\left(E_{A}\right)=K+L(\ln P)+M(\ln P)^{2}+N(\ln P)^{3}+O(m P)^{4}( \\
& -1 / \beta=R+S(\ln V)+T(\min )^{2}+U(\min )^{3}
\end{aligned}
$$

Here, the subseript "A" represents the adiabu passing through the CJ poin. The coefficients are compuned using the "BKW" code [3].

For a parially reacted paricie $(0<\lambda<1)$ we apply the mivoure rules $(8,9)$ in the following way. Firs we identify which adiaba the parricle is on by couparing the particle encropy with the entropy on the solid Ilugosiot. The funily of adiabats is chuncterized by the solid In ingoniot arve for the urreacted materinl. Secood, upper limits on the vohume (V/Z) and enery (E/A) of the solid are provided by $(6,7)$ to enaure poxitive $V_{g}$ and $E$. Near, we identify the bower limin for the colid volume as $V(5 / \lambda)$ on the adivbet. Inving in seceptable volume range for the partinlly reacted solid, we pick the aribumetic mean is the waring guess for $V_{s}$ and compute $V_{g}$ using (8). The staring solid exery is $E_{S}\left(V_{g}\right)$ with (9) providing $E_{\text {. }}$. A Newron-Rupheon iteration is thea med towerd preasure equilibrium berween the solid and gas phases, constraining the solid to move slong its atinbut.

\section{Carcumatron}

A condimentiond simulation wh performed in orter to exnmine pinner stendy-strte deconmion in Compoxition-B exploxive using the sew formentism within the frumework of Smoothed Particle Fiydrodynamies. For this estentrion we rook the smoothing length $(h)$ to be $1 \mu \mathrm{m}$. Burring was initiated by impacting the explosive with equivilent iner mnerial. The shock pressure inpur to the eample was kept just below $\mathrm{PCI}_{\mathrm{Cl}}(290 \mathrm{~Kb})$ so as to minimize the run disunce to detonition. A Forest-Fire rute [4] was used to describe the decomposition.

$$
\frac{d \lambda}{d}=\lambda \sum_{n=1}^{n} C_{1} P^{-1}
$$

The coefficients in (27) were obsained uring the ATFiRE code [S] and are listed in Tablel. Fits for the Comp-B isentrope $(25,26,27)$ are given in Table 2. Solid parmeters used were: $V_{0}=0.583 \mathrm{co} / \mathrm{s}, C=0.21 \mathrm{~cm} / \mathrm{ks}$ $S=1.5$ and $r=2.0$.

\begin{tabular}{|c|c|c|c|}
\hline 1 & $C_{i}$ & 1 & $C_{i}$ \\
\hline 1 & $-1.035458043 E+01$ & 8 & $8.206591093 E+09$ \\
\hline 2 & $4.734274495 E+02$ & 9 & $-1.298662700 E+10$ \\
\hline 3 & $-1.675370422 E+04$ & 10 & $1.618379369 E+11$ \\
\hline 4 & $.475674643 E+05$ & 11 & $-1.260581243 E+11$ \\
\hline 5 & $-8.493147154 E+06$ & 12 & $-7.728984899 E+11$ \\
\hline 6 & $1.153593435 E+08$ & 13 & $7.437676727 E+11$ \\
\hline 7 & $-1.140236515 E+09$ & 14 & $3.616777570 E+11$ \\
\hline
\end{tabular}

Table 1. Forear-Fire Rate Coefs for Comp-B

\begin{tabular}{|c|c|c|}
\hline$A-E$ & $K-0$ & $Q U$ \\
\hline$-3.525848 E+00$ & $-1.560376 E+00$ & $7.502720 E+00$ \\
\hline$-2.334291 E+00$ & $5.331214 E-01$ & $-.412090 E-01$ \\
\hline $3.972673 E-01$ & $8.063108 E-02$ & $1.512926 E-01$ \\
\hline $3.045104 E-03$ & $3.338168 E-03$ & $6.776332 E-02$ \\
\hline$-1.752264 E-01$ & $-6.8439992 E-01$ & $-2.424033 E-02$ \\
\hline
\end{tabular}

Table 2. HOM fin 10 BSWW EOS for Comp-B

Figures 1,2 and 3 show differens appects of the SPH compured rendy-rrate detonution in Composition-B explosive. Odly fully or pertinlly reacted particles are plotted for charity. Inest "flyer plate" paricies and purticles sot yat shocked are omitued. Figure 1 shows the preasure profile. The penk presure at the shock front is 450 ITb which is the von-Nermam spike. The renction is $100 \%$ complete $400 \mathrm{\mu m}$ behind the spike at 290 KW, the Chrpmo-Jougue (C) strte. The reaction is $75 \%$ complete $80 \mathrm{um}$ behind the shock from. These aumbers compire wall with experimentlly measured values for Composition-B.

In Figure 2 the prescure is plotted on the ordinne and the resction extent on the sbscisen. The vonNenmann spike is seen sear $\lambda=1$ and the CJ poim is seen near $\lambda=0$. Notice the large mumber of particles $(-100)$ comprising the renction zone. Notice also that adequate resolution of the thoek from is achieved in the sease thrt so subsumial reaction rikes place until the vonNemmen epitse is renched. In Figure 3 the presaure is 
agnin plowed on the ordinute but now the specific volume is used for the sbecisen. This representuion clearty shows the Rxyleigh line excending from the vonNerman spike to the CJ point. Thx compression to the von-Neamano point is not abrupt, and that the path to this point is curved, are both due to the arificin viscouiny spreading out the shock In addition to the results presemed here we have also modeled cases using lowe flyerplate speeds (reduced shock inpur) and observe boild-up to ready-stute detonnion consirem with wodge test results. We expect to presem this date orilly at the conference.

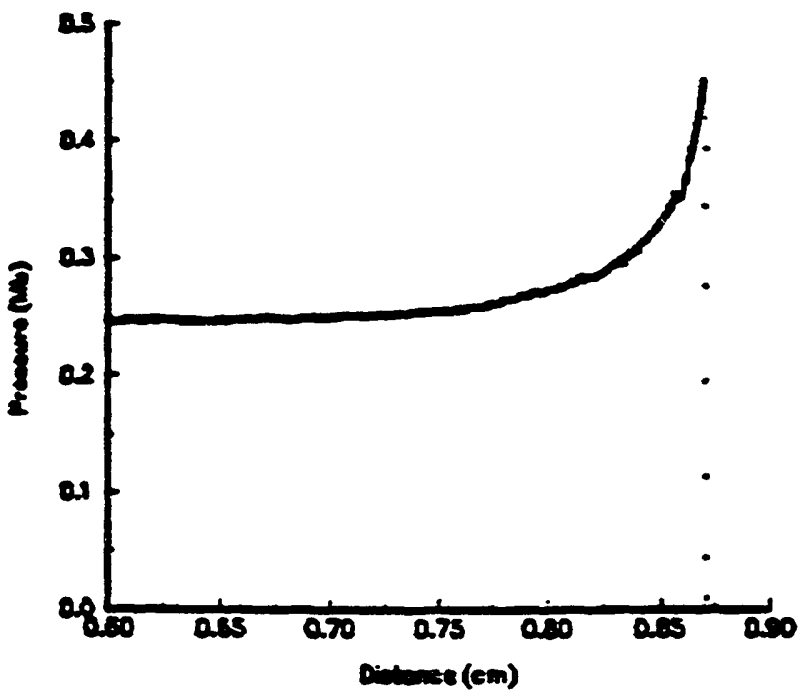

Figere 1. SFH competed presure prefile for grendyane plant detonmon in Composition-B explosive.

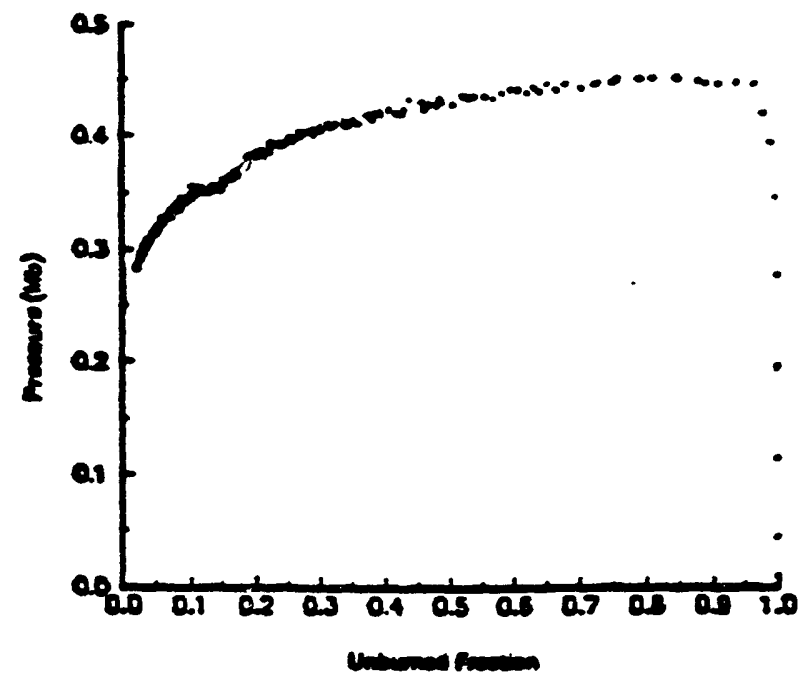

Figure $2.8 \mathrm{~V} 2 \mathrm{~d}$ shom approdimmety 100 pertides couprising the receican zoce.

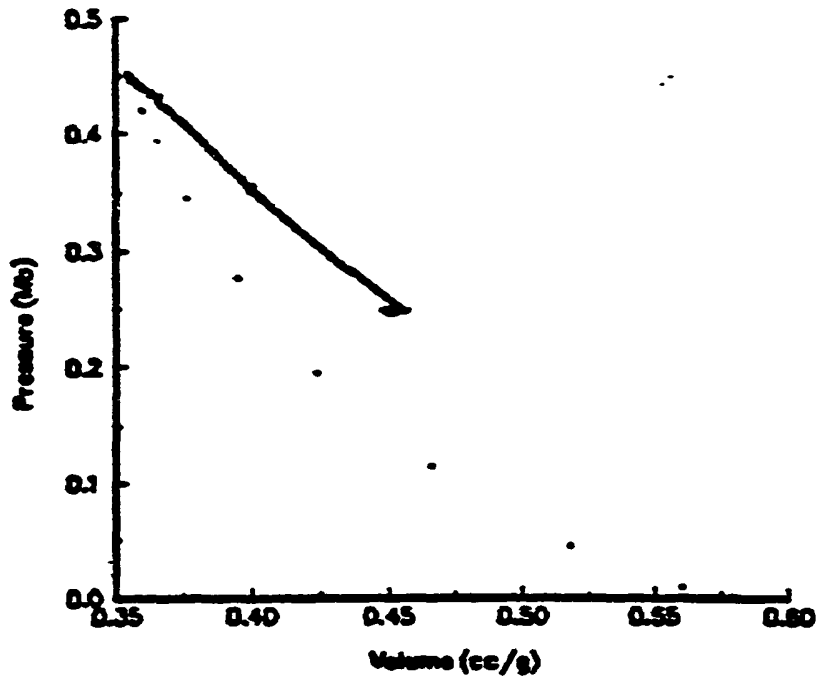

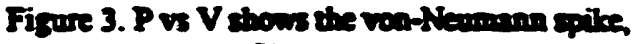
Ryleigh line and $C \mathrm{Coinh}$

\section{CONCLUSIONS}

We have derived a new formalism for treating reactive flow and have incorporated it within the frumework of Smoothed Particle Hydrodynamics. The mixture rules extend the applienbility of reactive flow calculations to large-grined mrerials and the SPH compuring technique allows for eary excension to threedimensions and improved scouracy. We hrve tested the approsch by caloulating planur ready-srae detonation in Composition-B. Results show ideal detonntion theory so be reproduced quite well.

\section{RATRRNAXS}

1. Libersky, LD.; Pescebek, A G.; Camey, T.C.; Hipp, JR; Allahdadi, F.A.; J. Compur. Phys., aceepted for pubtiention., Feb., 1993.

2. Mader, CI., Numeried Modeling of Detonntions. Univ. of Crif. Prees, Los Angeles, CA,1979, p.327.

3. ATBKW Computer Program Los Alamos Code No. LP-181 Los Almos Nationd Lab., NM, 1986.

4. Mader, CL.; Forest C.A., Two-Dimensional Homogeneous and Heterogeneous Detonation Wave Propagation, LA -6259, Los Alamos Nationd Lab., NM., 1796.

5. ATFIRE Computer Progrem, Mader Consultam Co., Fonobulu, III, 1986. 


\section{Topics in SPH}

\section{Capt David Amdahl}

$\dot{\psi}$

21 September 1993 SPH Meeting Los Alamos NM 


\section{Outline}

- Aero-SPH

- Review

- Revisit the Previous Work

- Variable $\mathrm{H}$

- Wall Heating

- Supersonic Airfoil 


\section{Review}

- SPH Requires No Grid

Saves Time and Opens Problem Domain of Traditional CFD Methods

- Validate SPH

- Boundary Conditions

Solid Boundary, In-Flow, and Out-Flow

- Inviscid, Compressible Flow Supersonic with Local Sub-Sonic Regions 


\section{Ramp Problem}

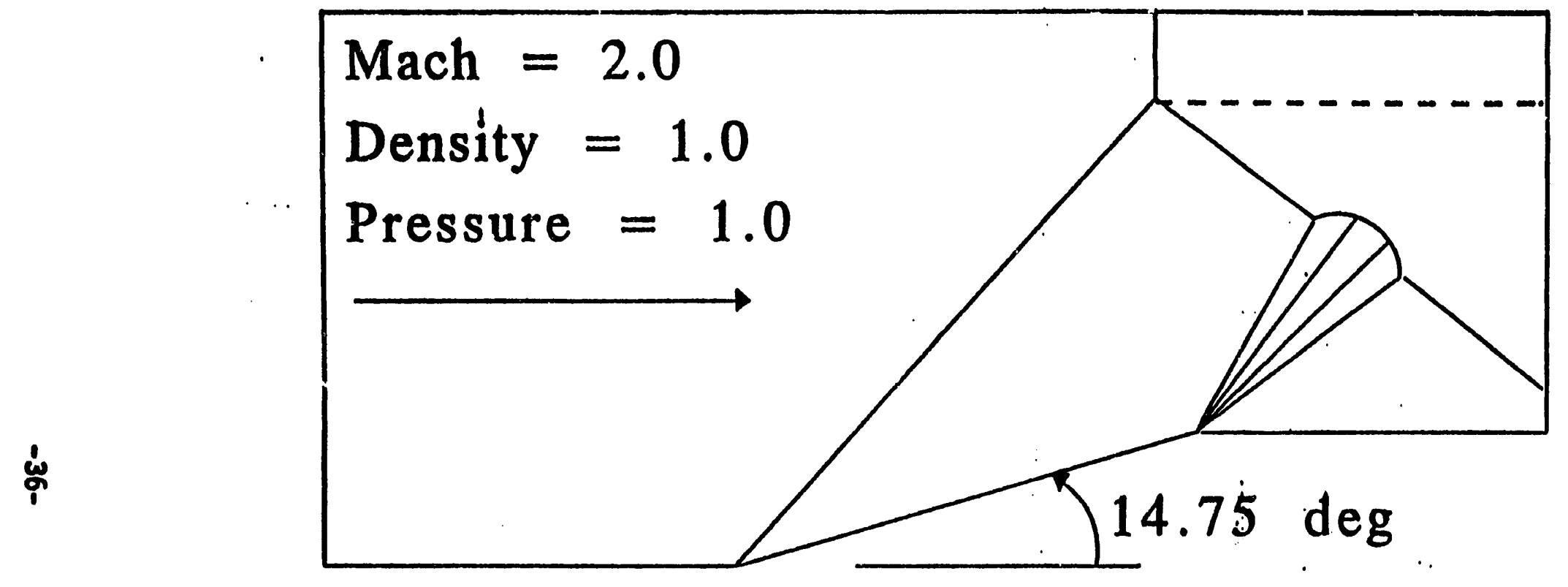




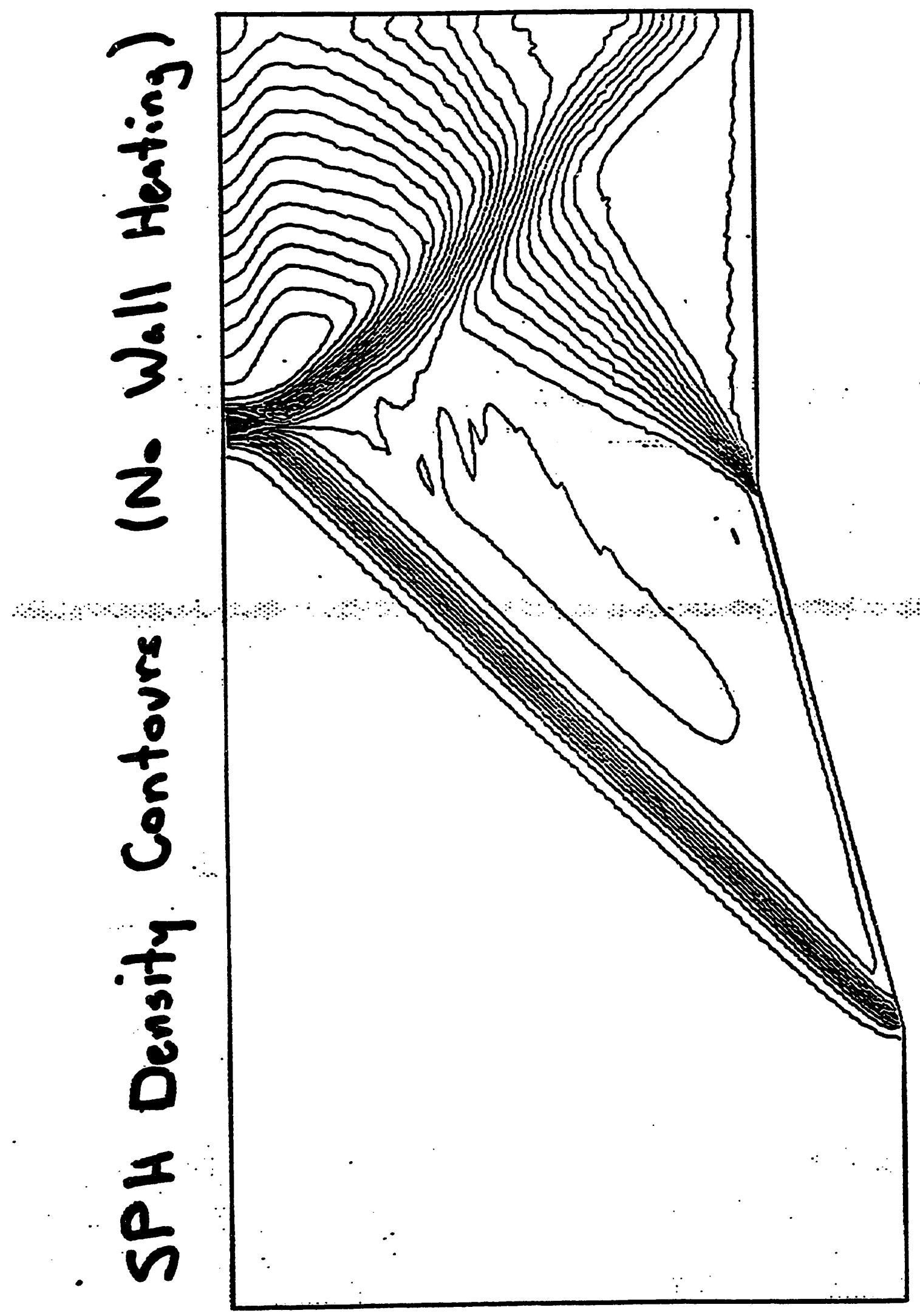




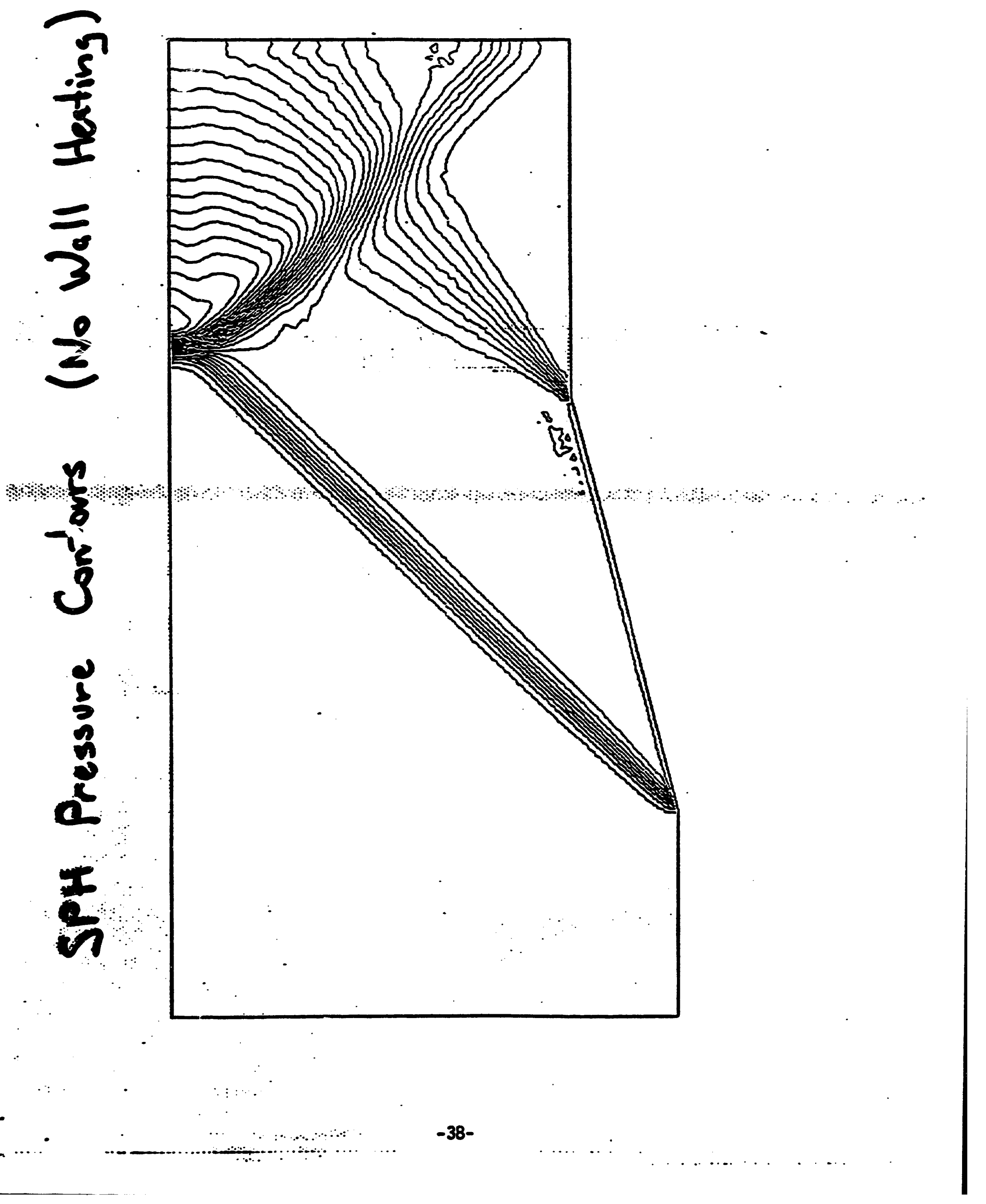




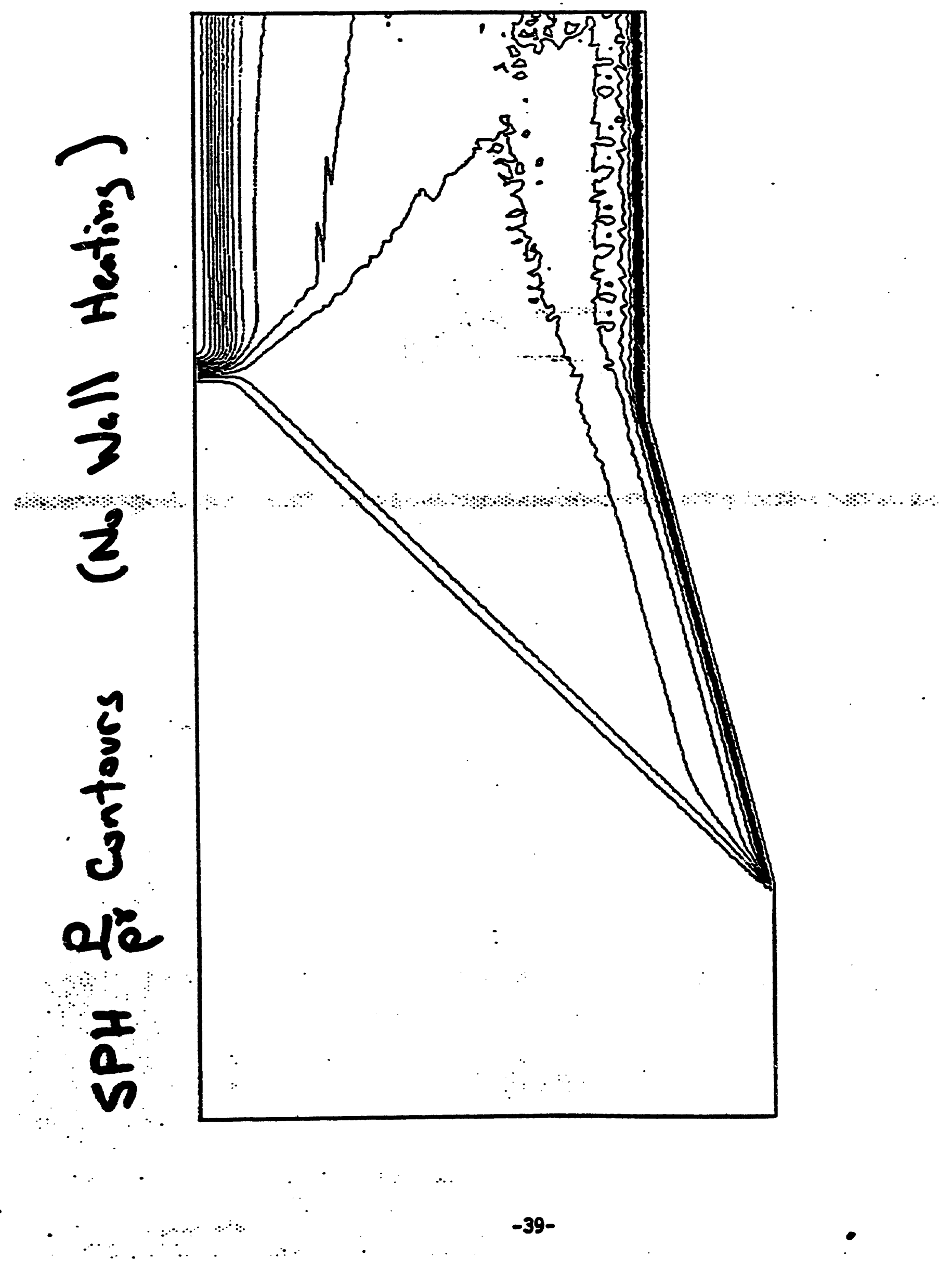


SPH Density Contours (Wall Heationg O.S. 1.0)

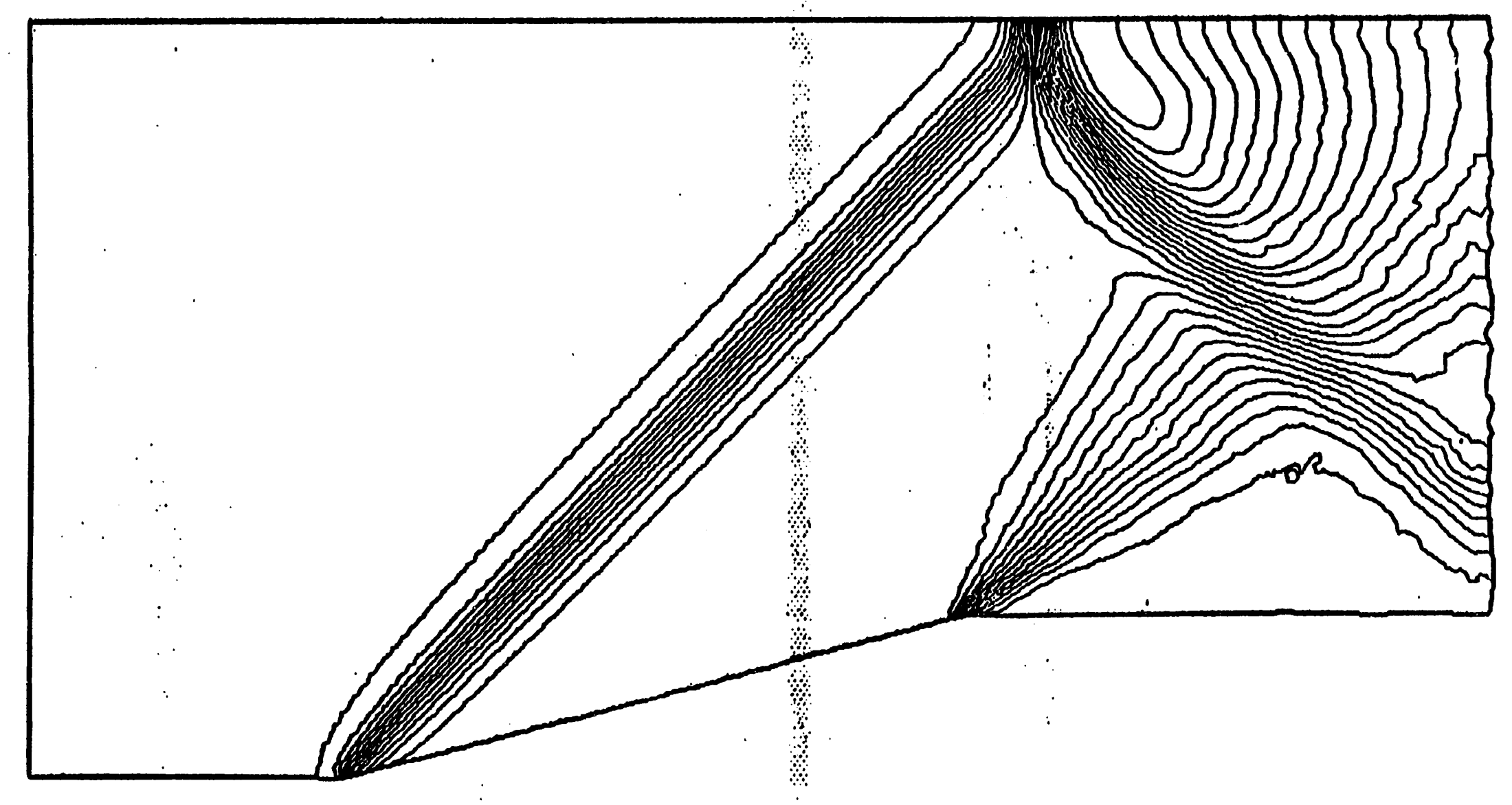




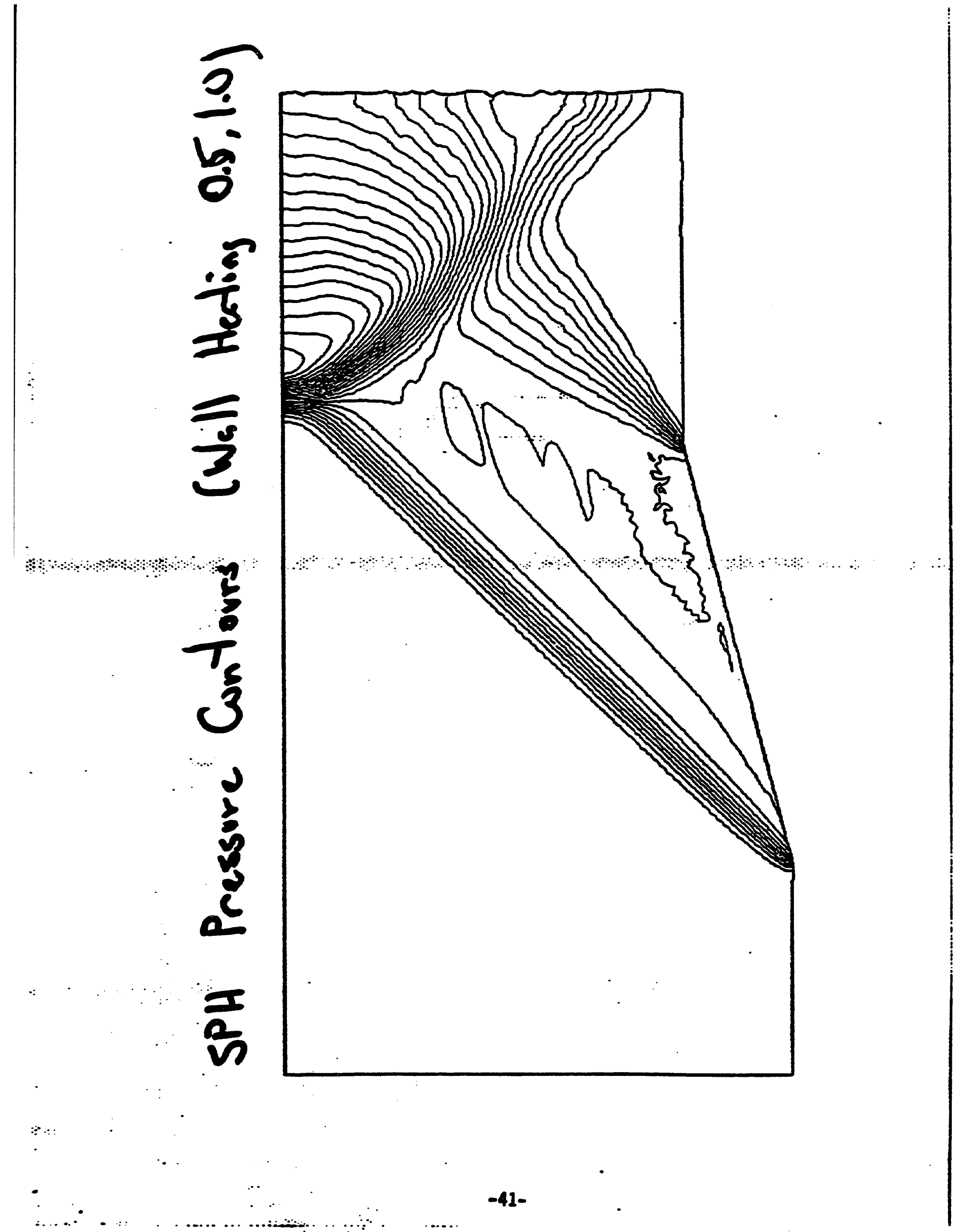




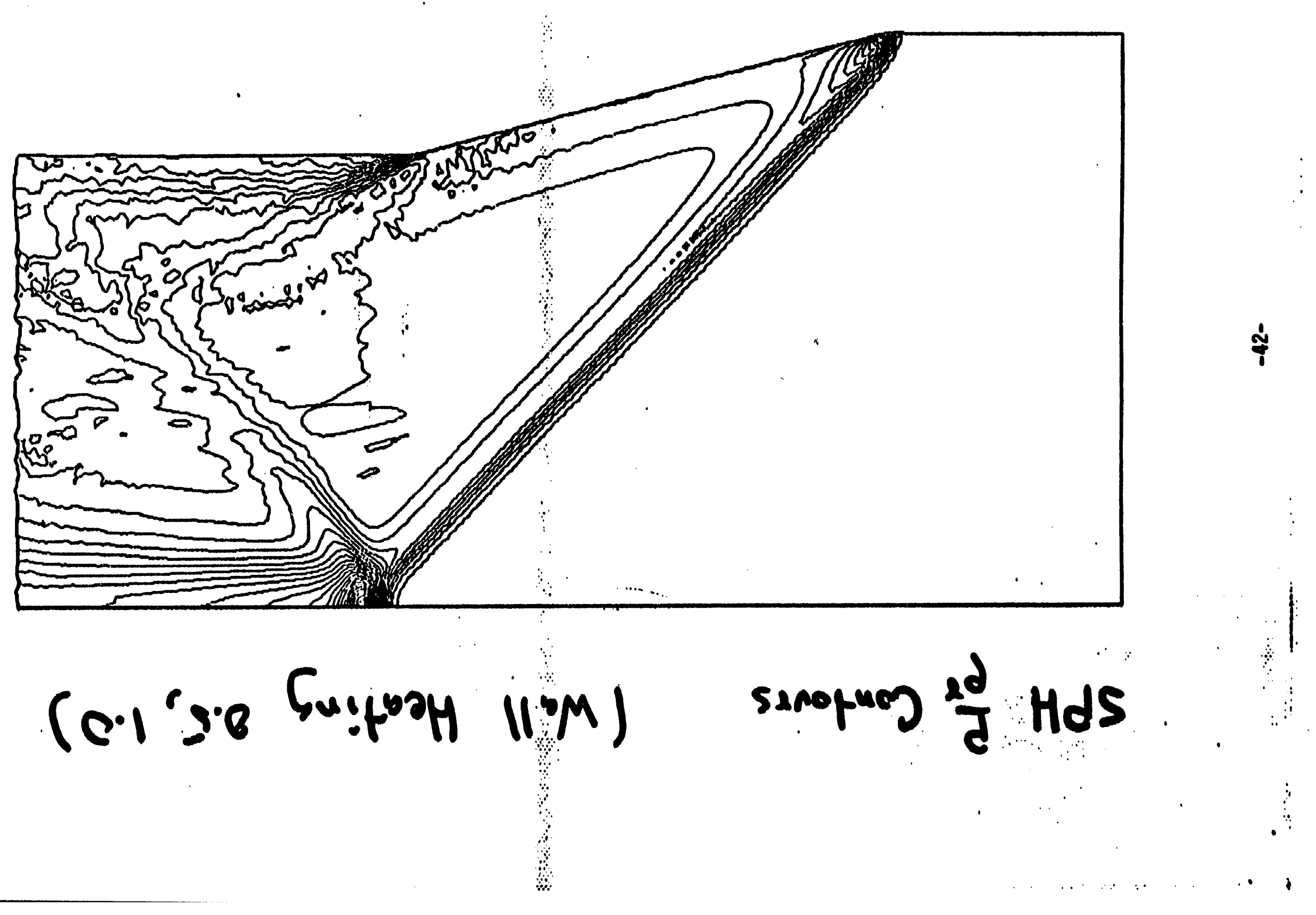




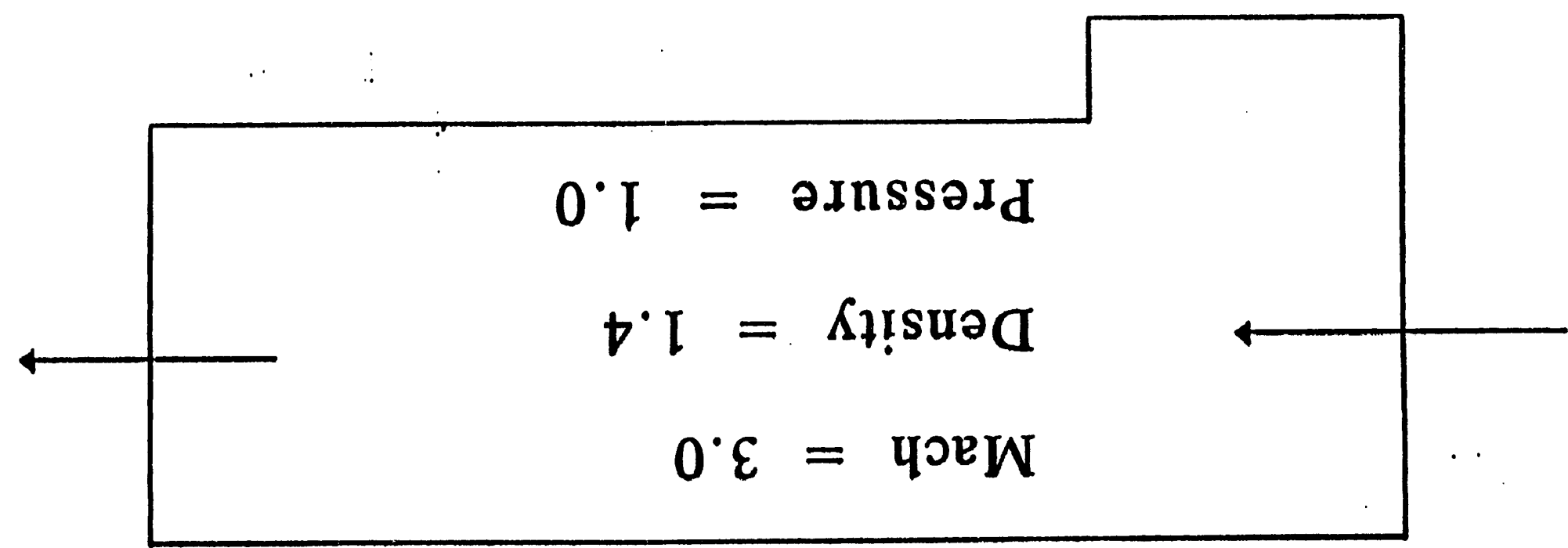



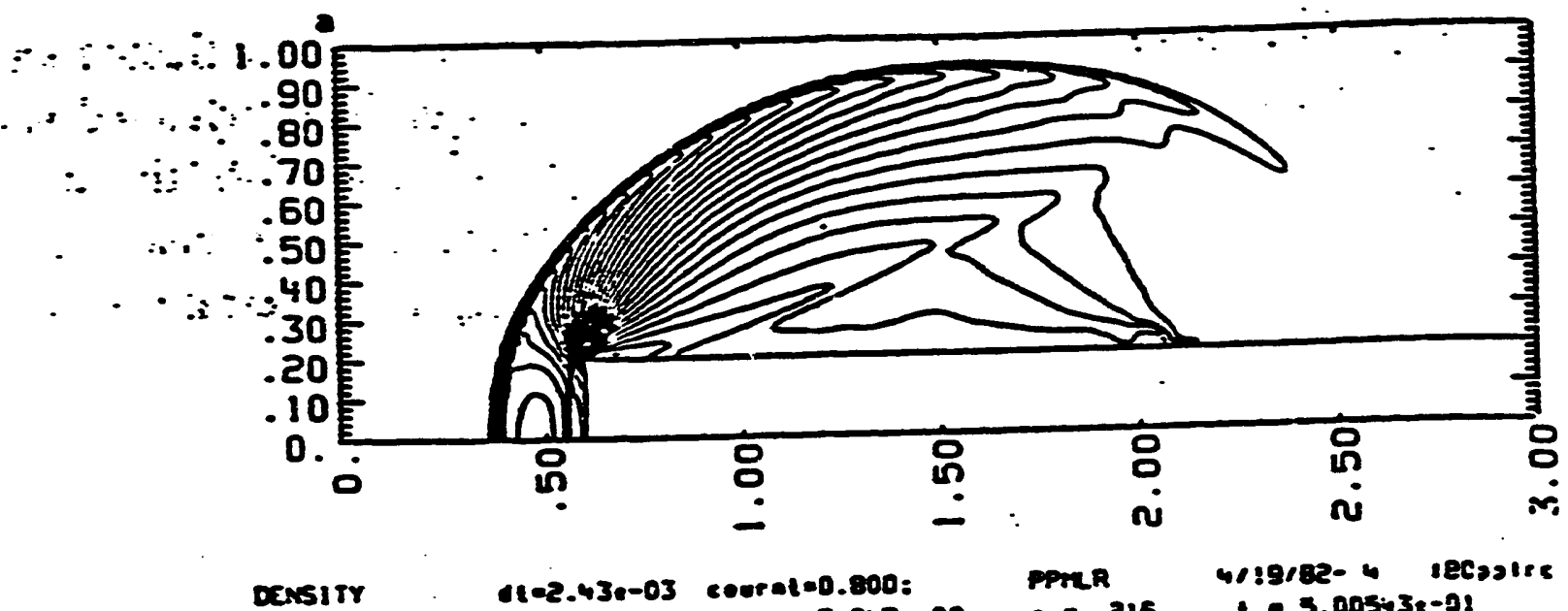

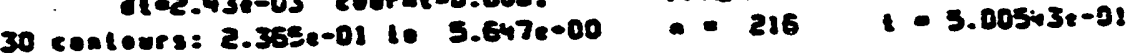
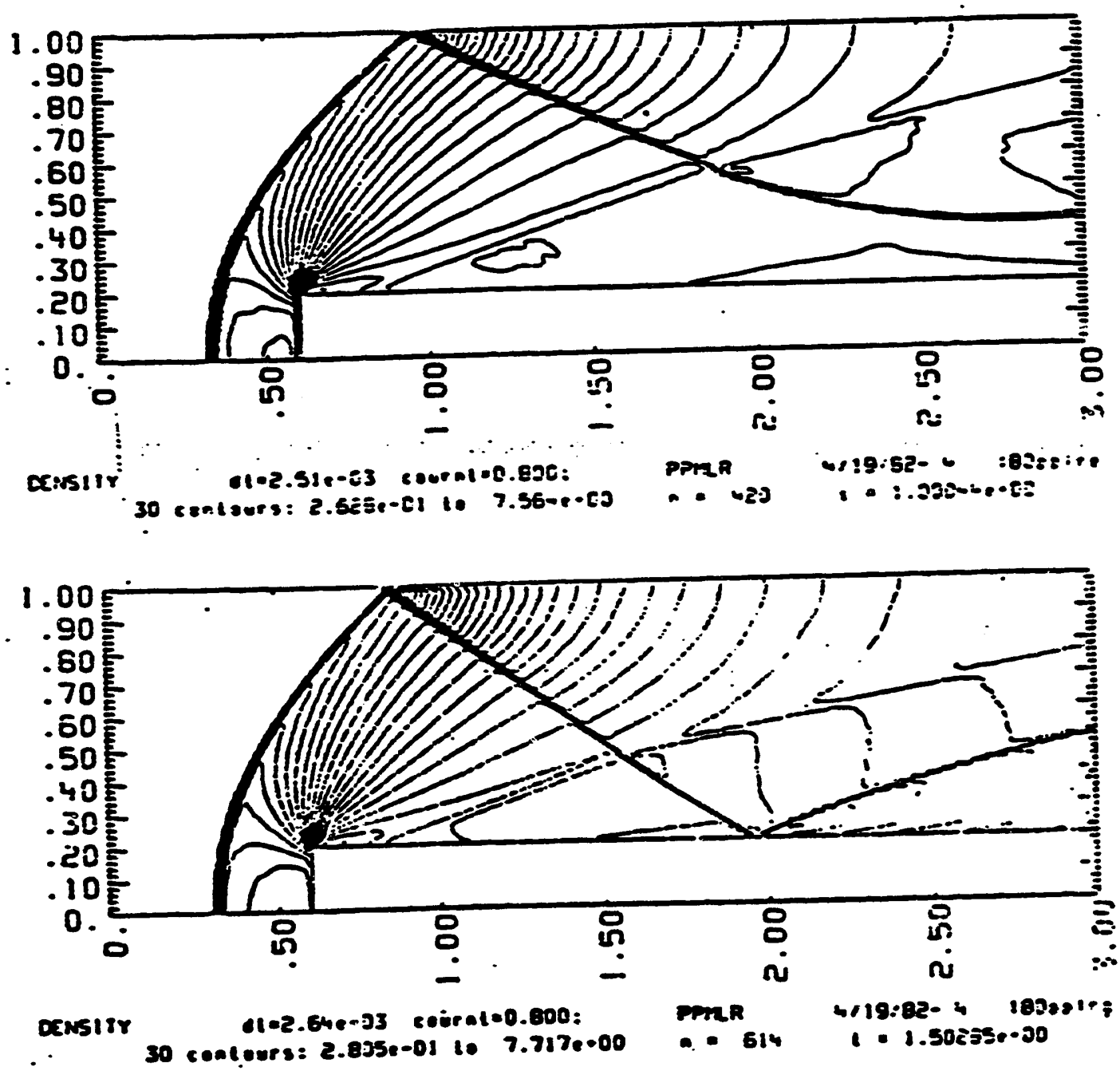

Fig. 3. The time evolution of the Mach 3 wind tunnel problem discussed in Section IVb. The resules vere obtained with the PPMLR scheme using a uniform grid with $\Delta x=\Delta y=1 / 80$. The contours of jensity are shown at time intervals of 0.5 up to time 3. At time 4 , the contours of density, pressure. $t=p / p^{y}, v_{y}, v_{x}$, and $\left(v_{x} / c\right)-1$ are also plotted. In each plot 30 equally spaced contours are shown with the contour for the lowest level or for any. negative level drawn as a doited line. 


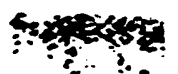

$\cdots$

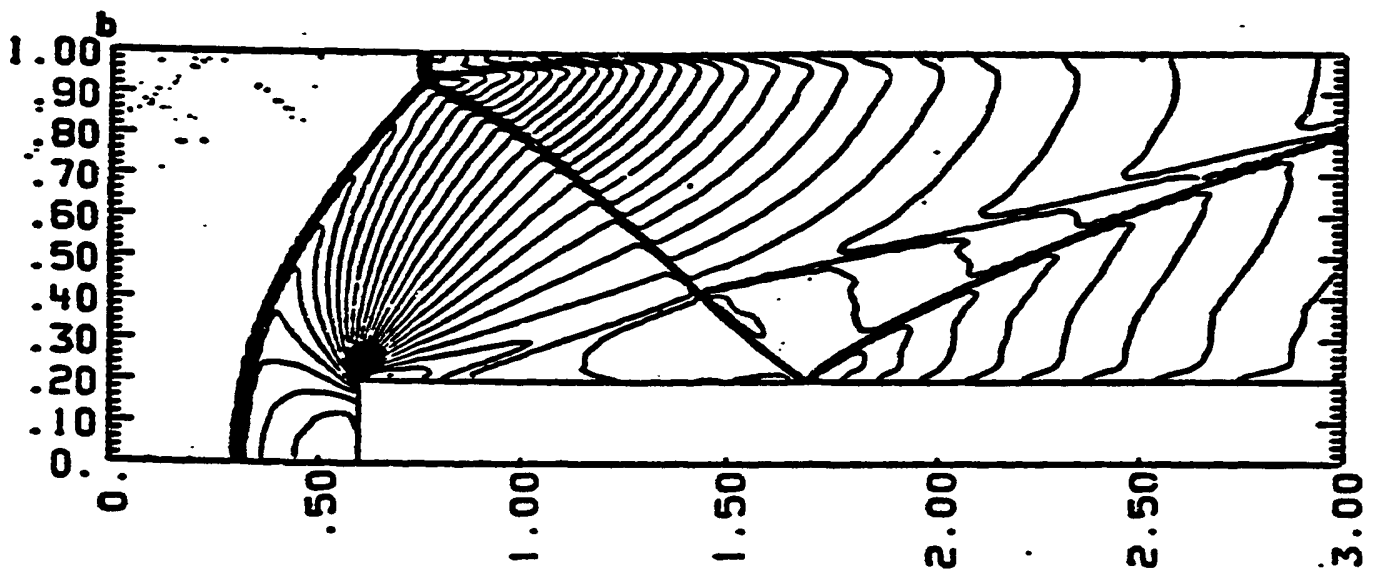

densitr

$01-2.69 e-03$ courat=0.000:

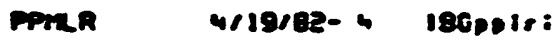
30 centours: 2.659e-01 $106.650 e+00 \backsim 002 \quad 1 \cdot 2.00455 e \cdot 00$

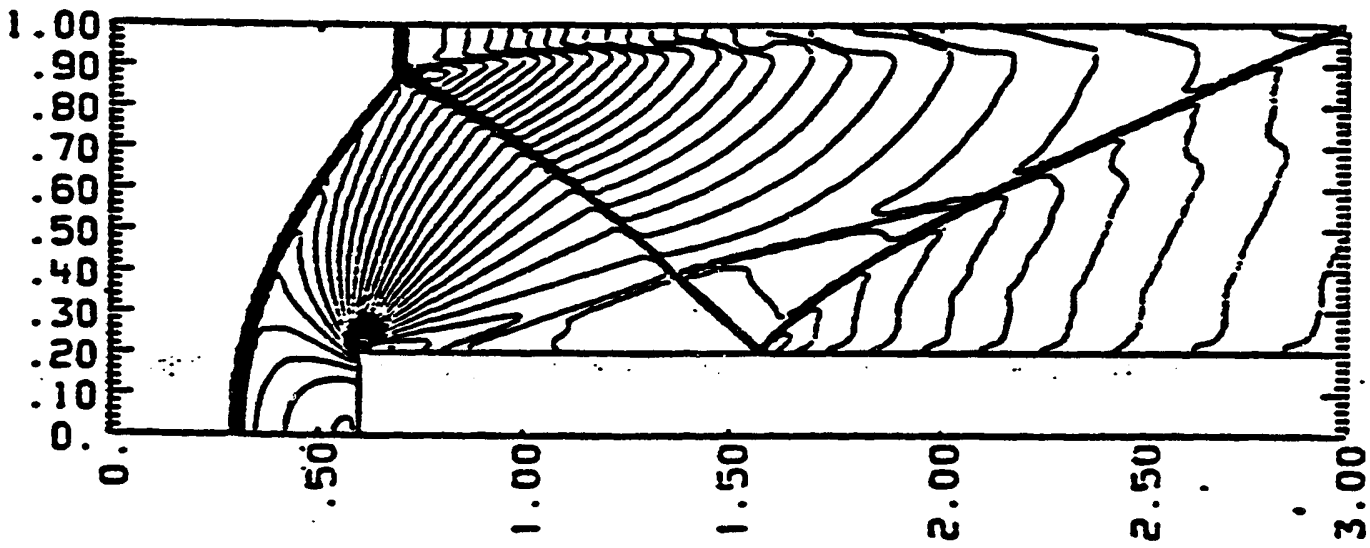

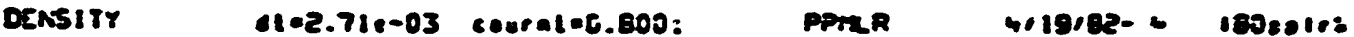

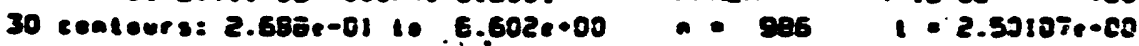

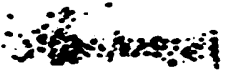

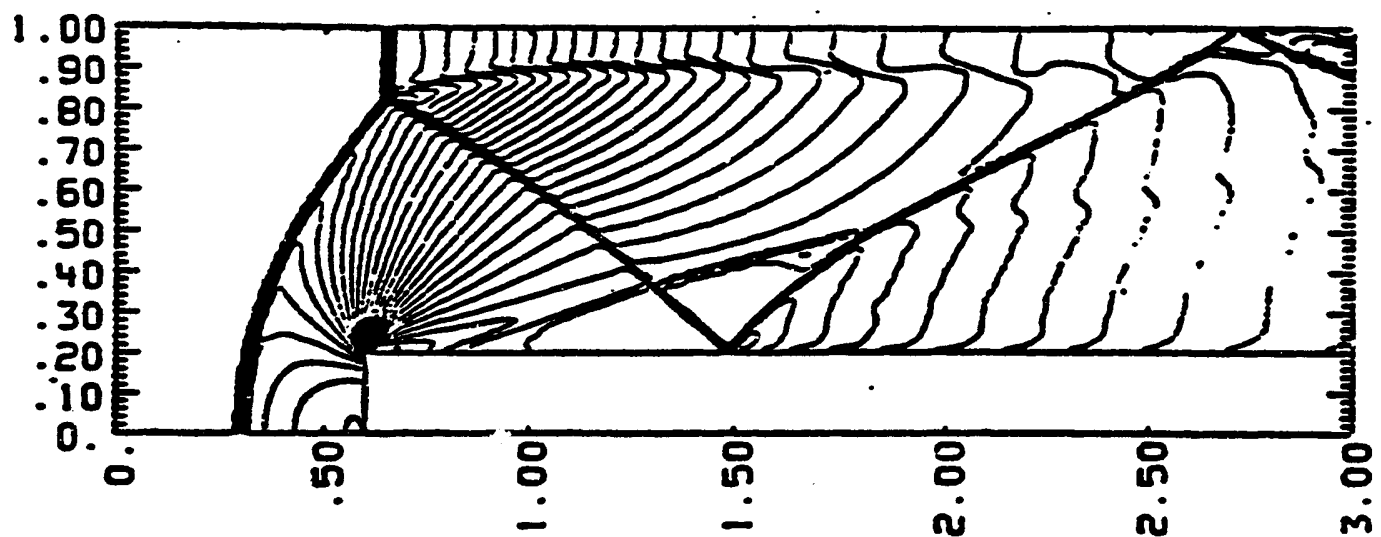

oznstit

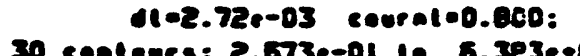

Pren

$410185-428002160$

$\therefore \quad \quad \quad$

Fig. 3-Continued. 


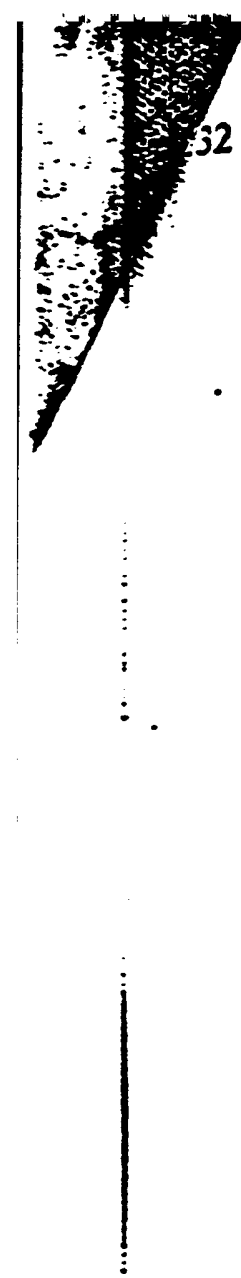

WOODWARD AND COLELLA
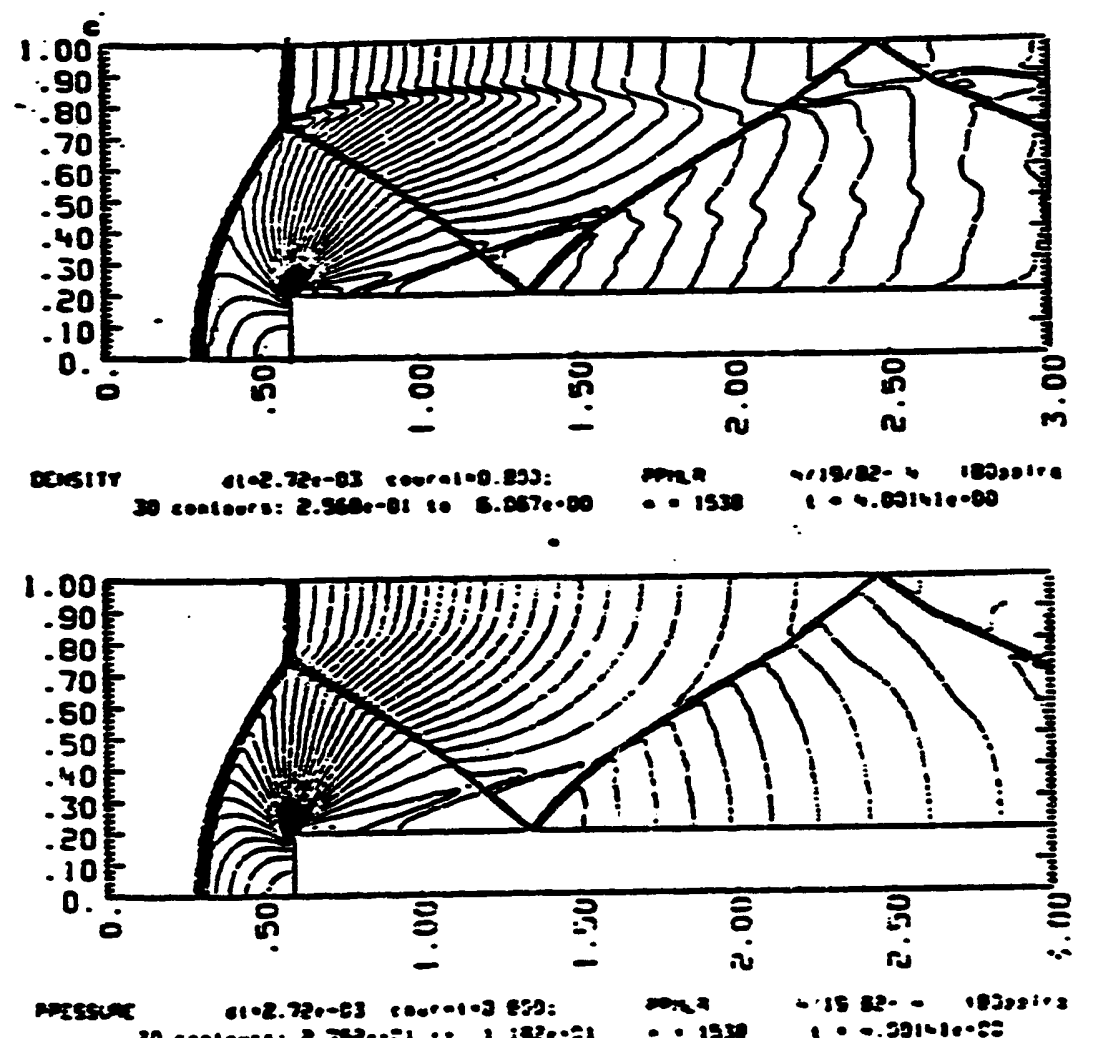

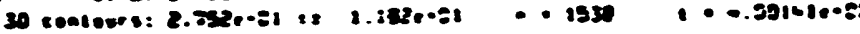

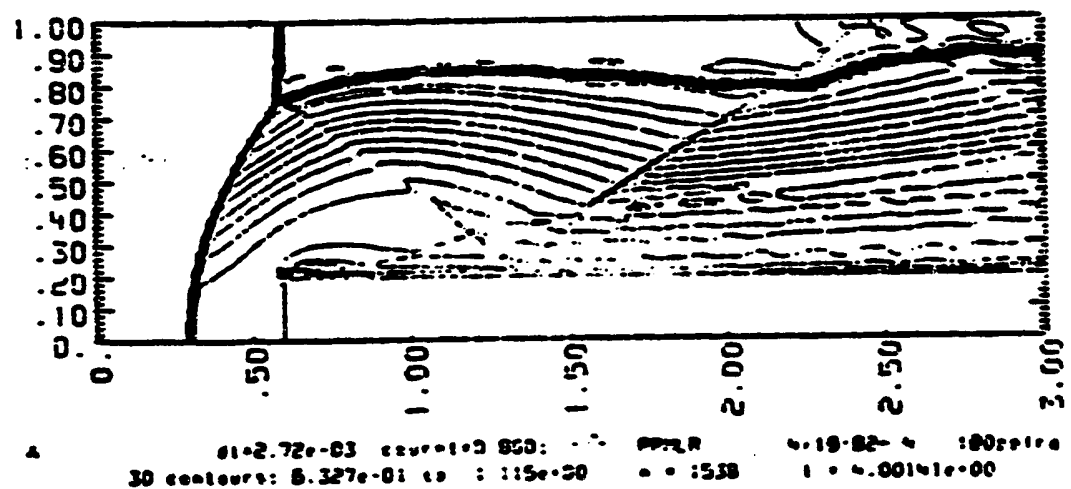

FiG. 3-Continued.

zone- used to set the entropy. This condition is based on the assumption of a nearly steady flow in the region near the corner. It is clearly inappiopriate at the very outset of the calculation. These conditions remove the grosseis errors generated near the corner, but of course large errors in the flow direction there are bound to remain These errors may be the cause of an overexpansion observed at the corner in all the runs, although similar effects occur in wind tunnel experiments of this type using resl. viscous air.

The time evolution, up to time 4 , of the density distribution in the wind tunnel is displayed in Fig. 3. The flow at time 4 is still unsteady. A steady flow develops by time 12 and is st: vill focus here Fig. 3 were obta ${ }^{4}$ $80 \times 240$ zones. converged to its : : vearly correct. $1 ! 1$ pansion at the ce. 1 panded flow finat. 10 change in pos: 


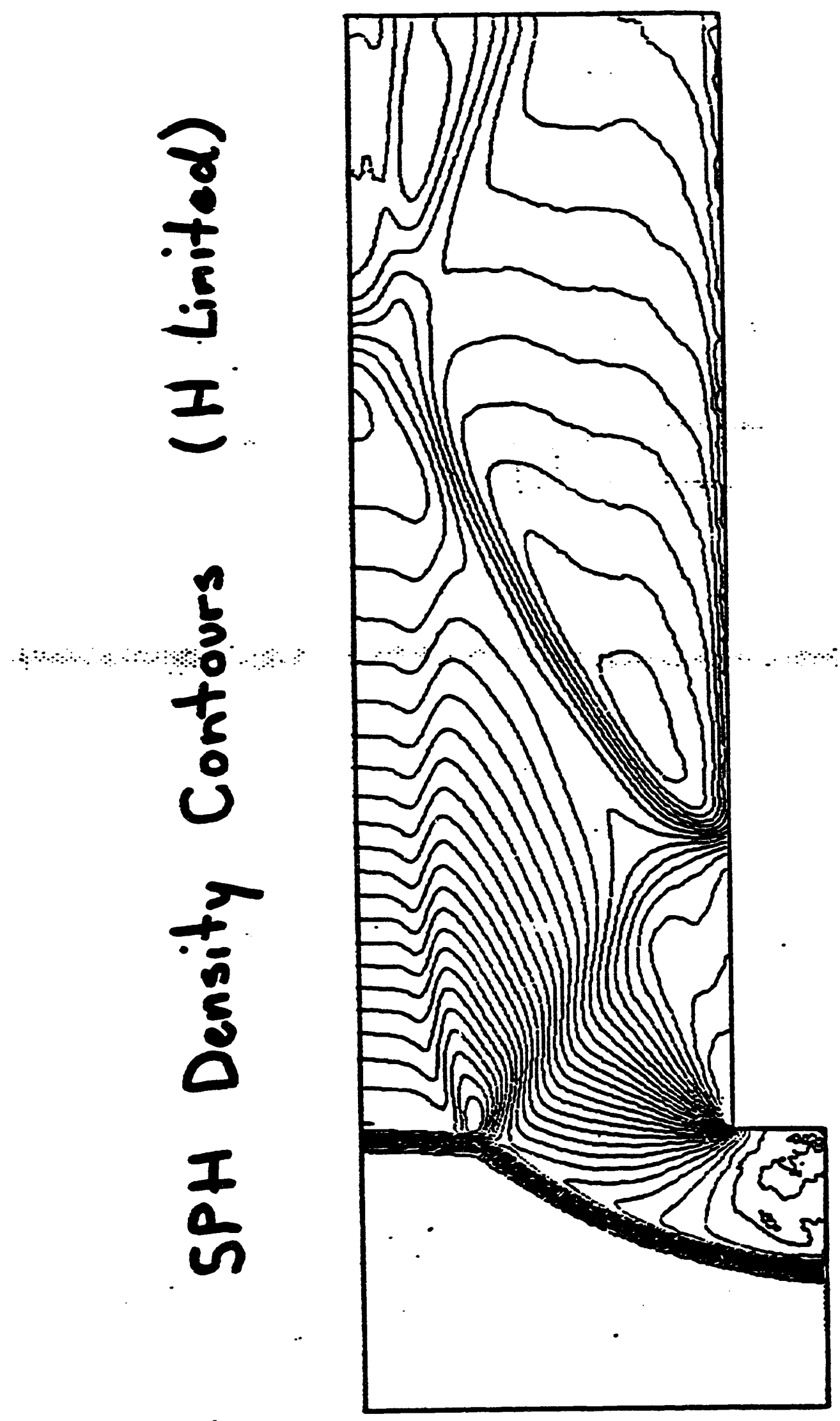




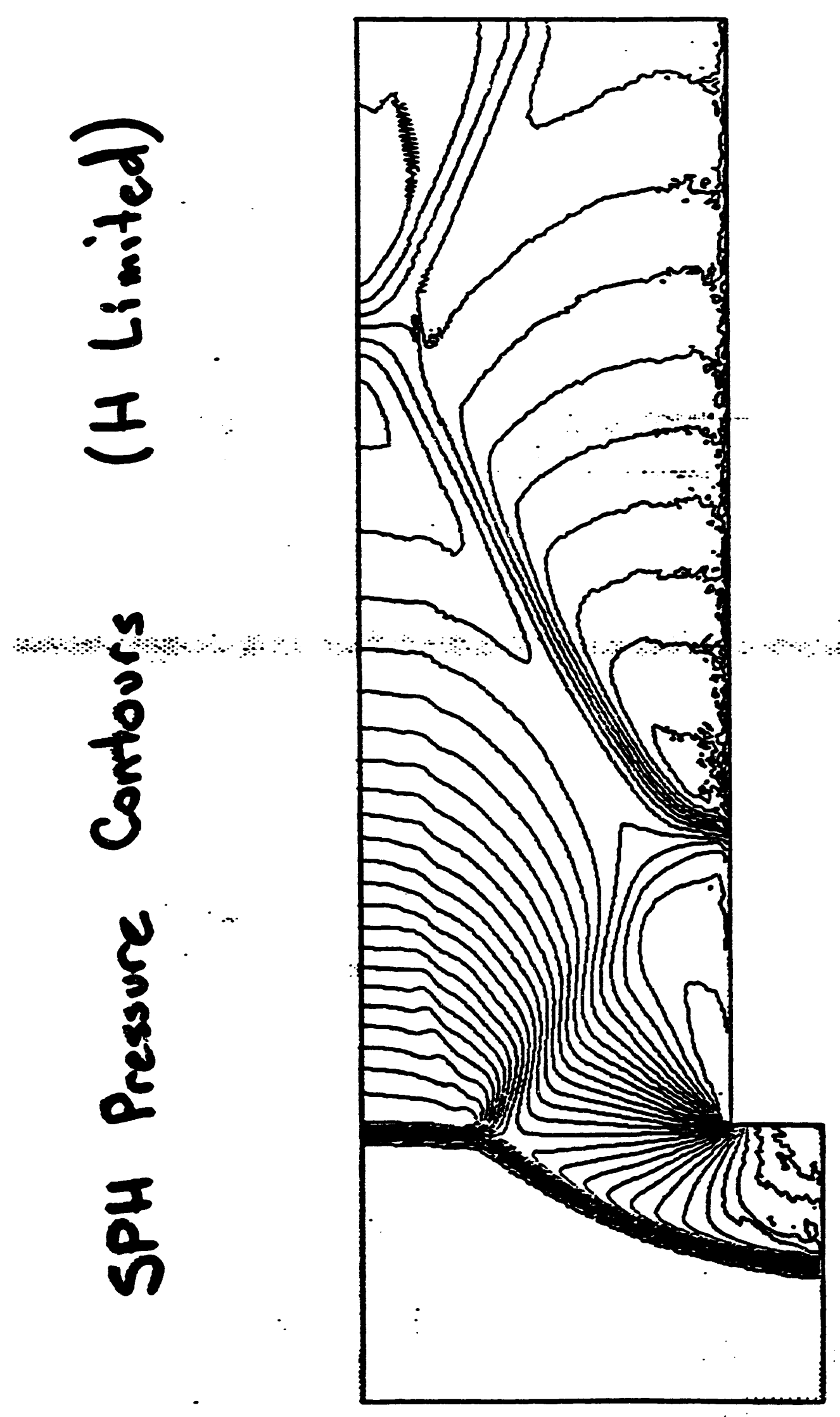




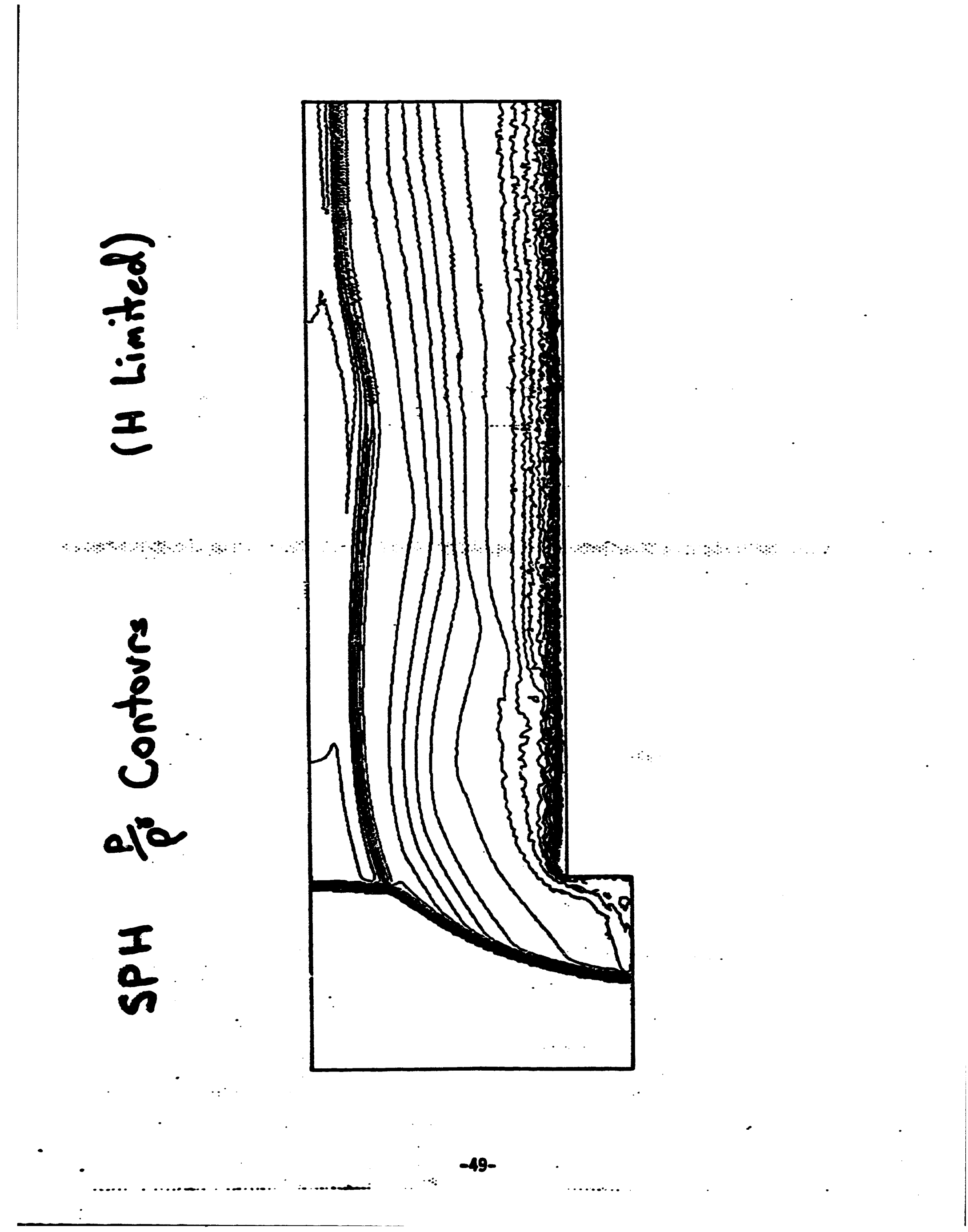




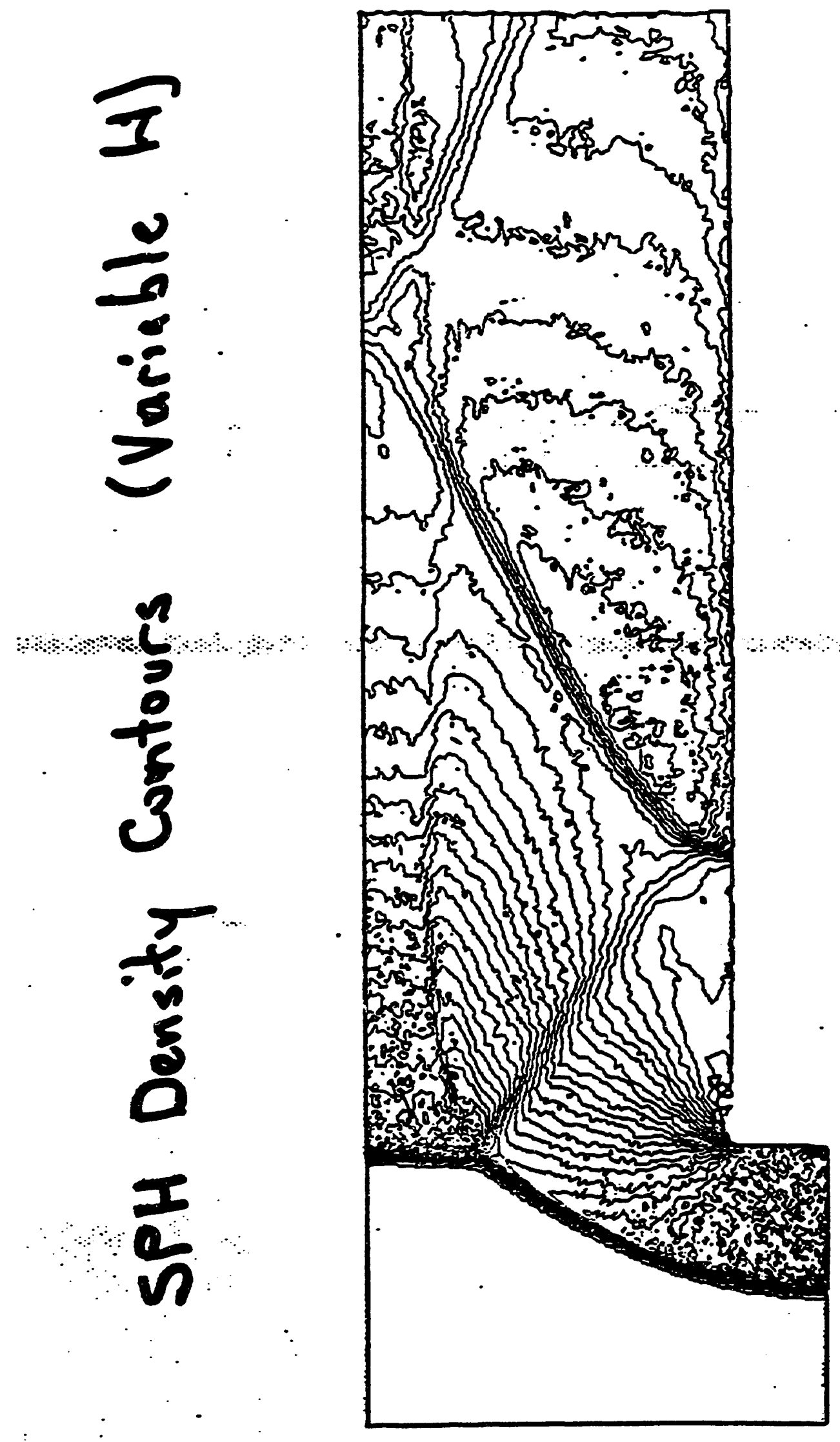


SPH Pressure Contours (Variable H)

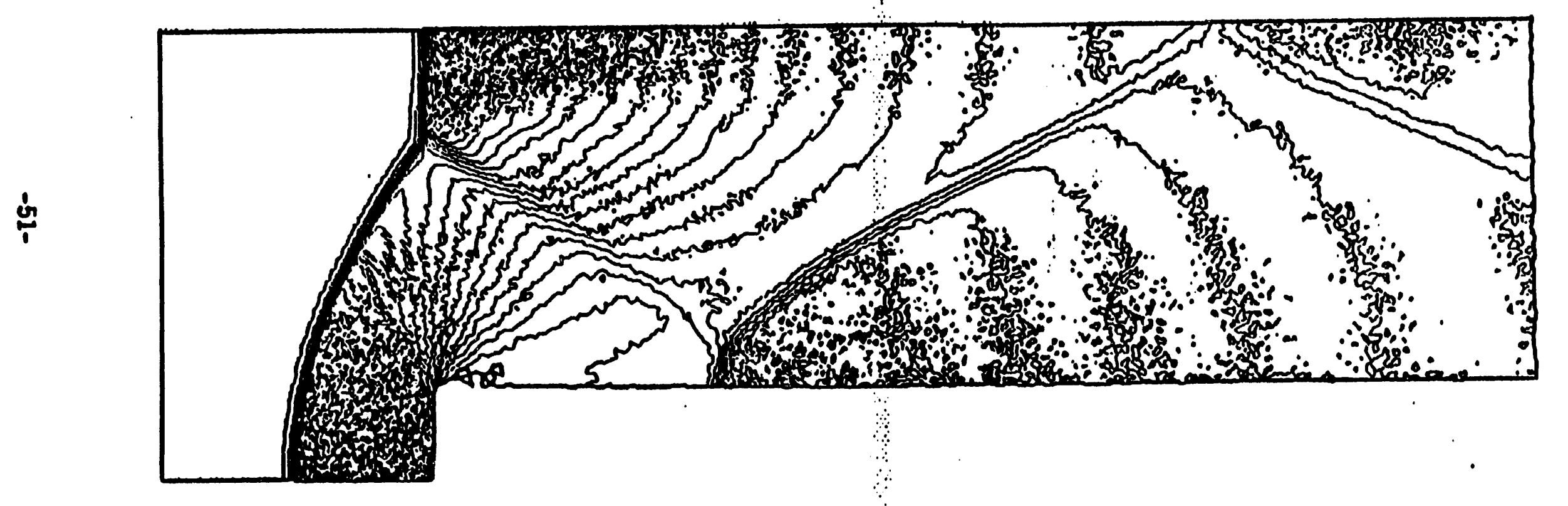




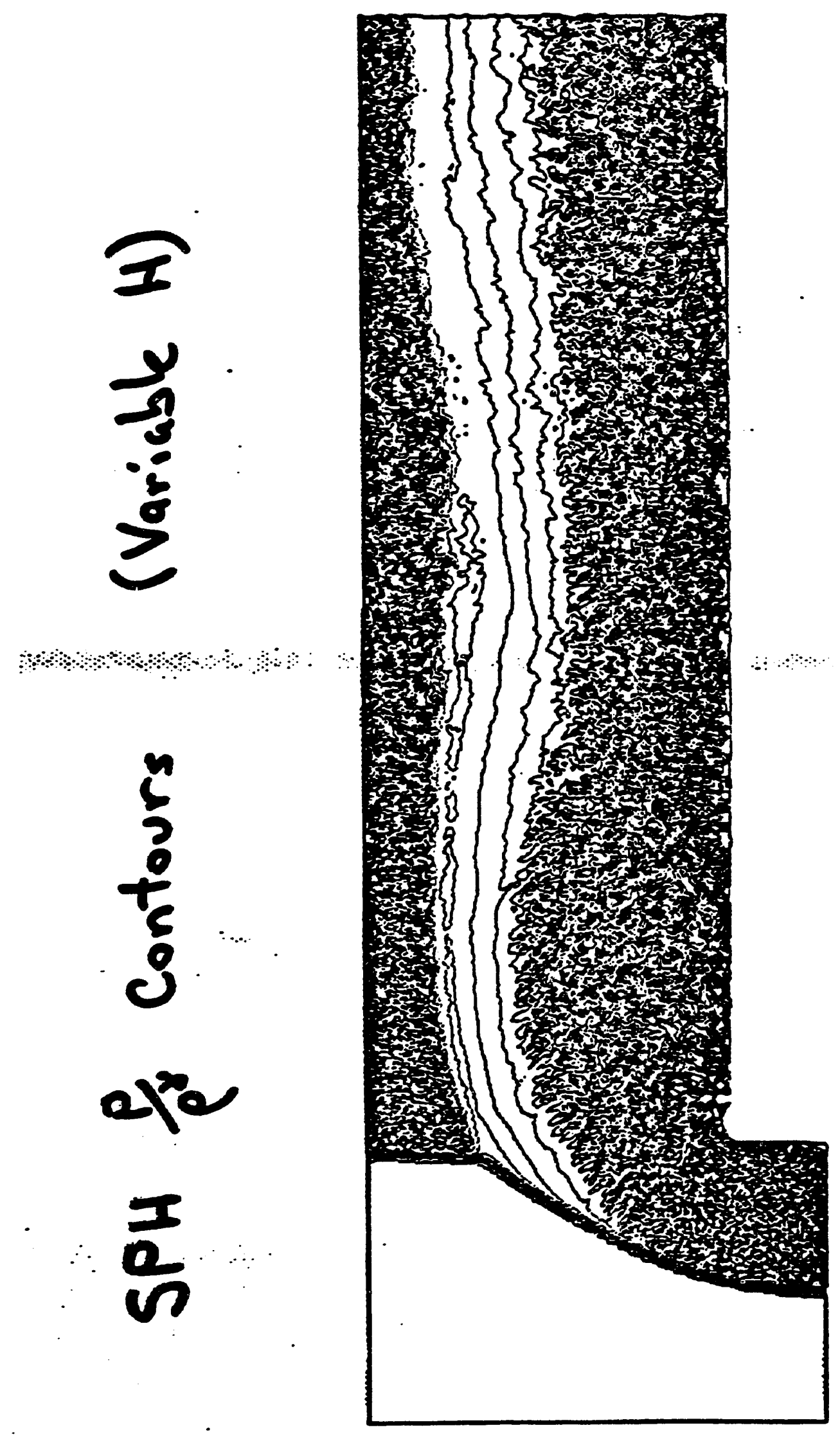




\section{Supersonic Airfoil in a Tunnel}
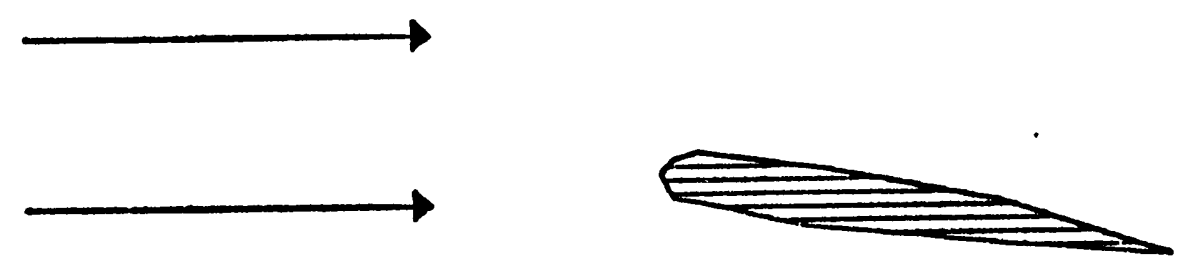

NACA 0012 Airfoil

$\underset{\mathfrak{u}}{\mathfrak{u}}$

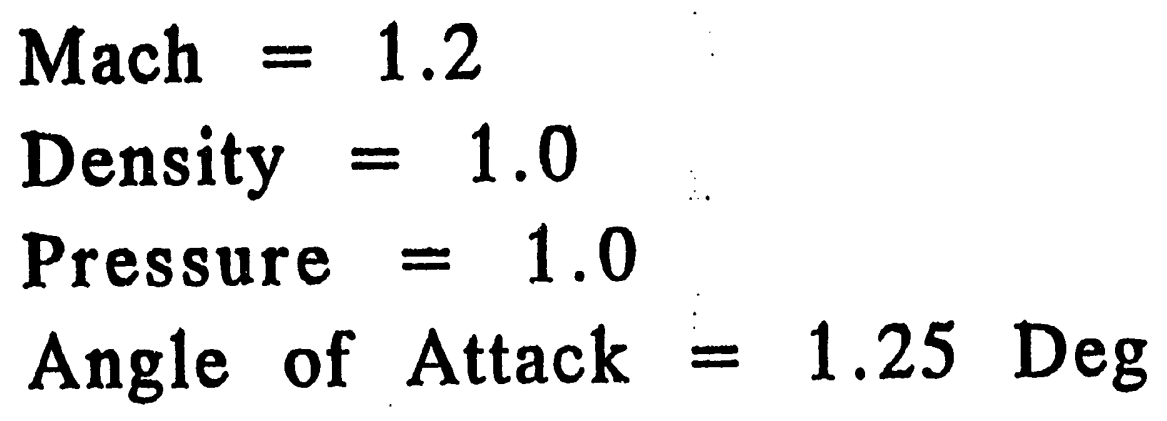




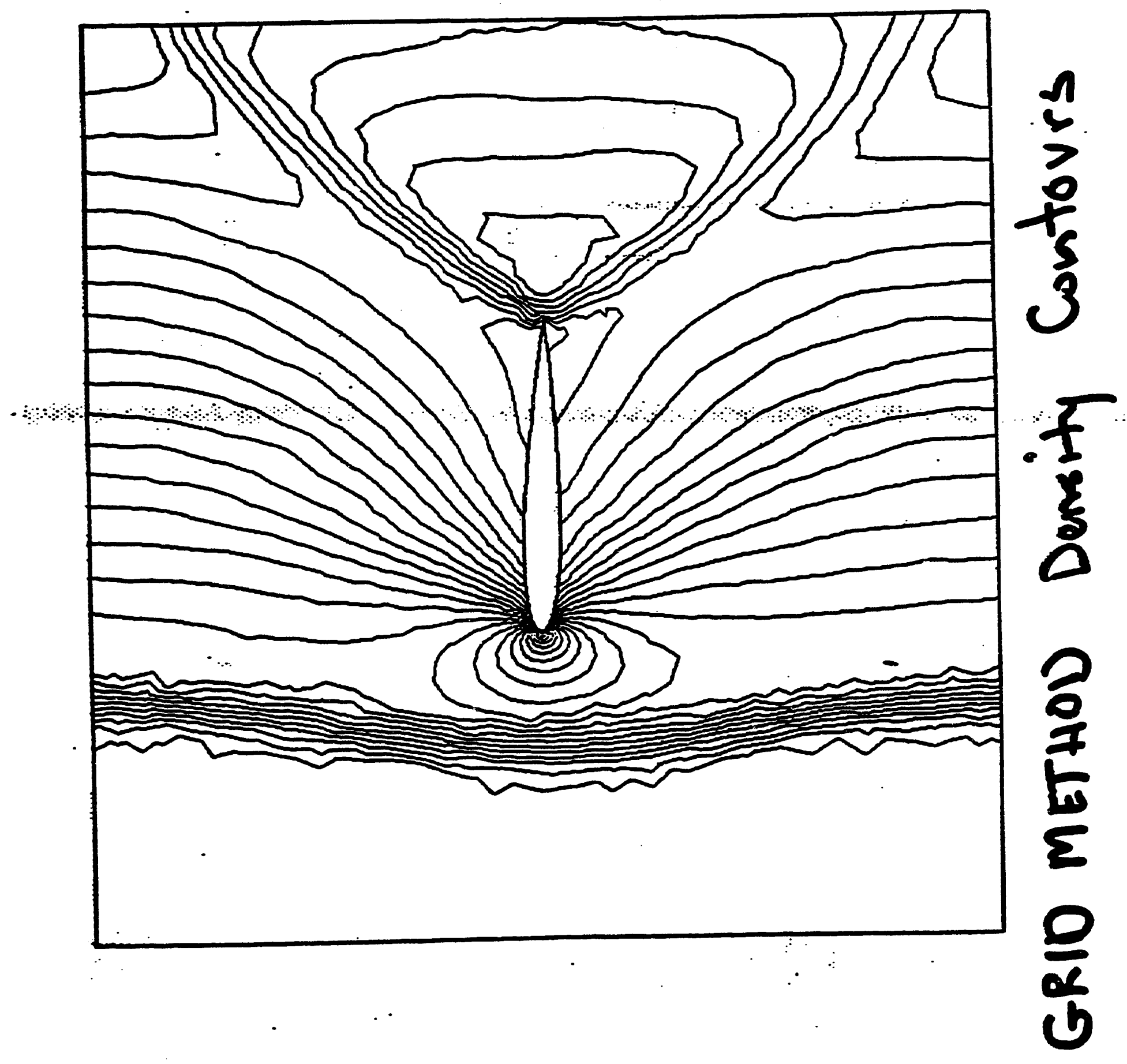




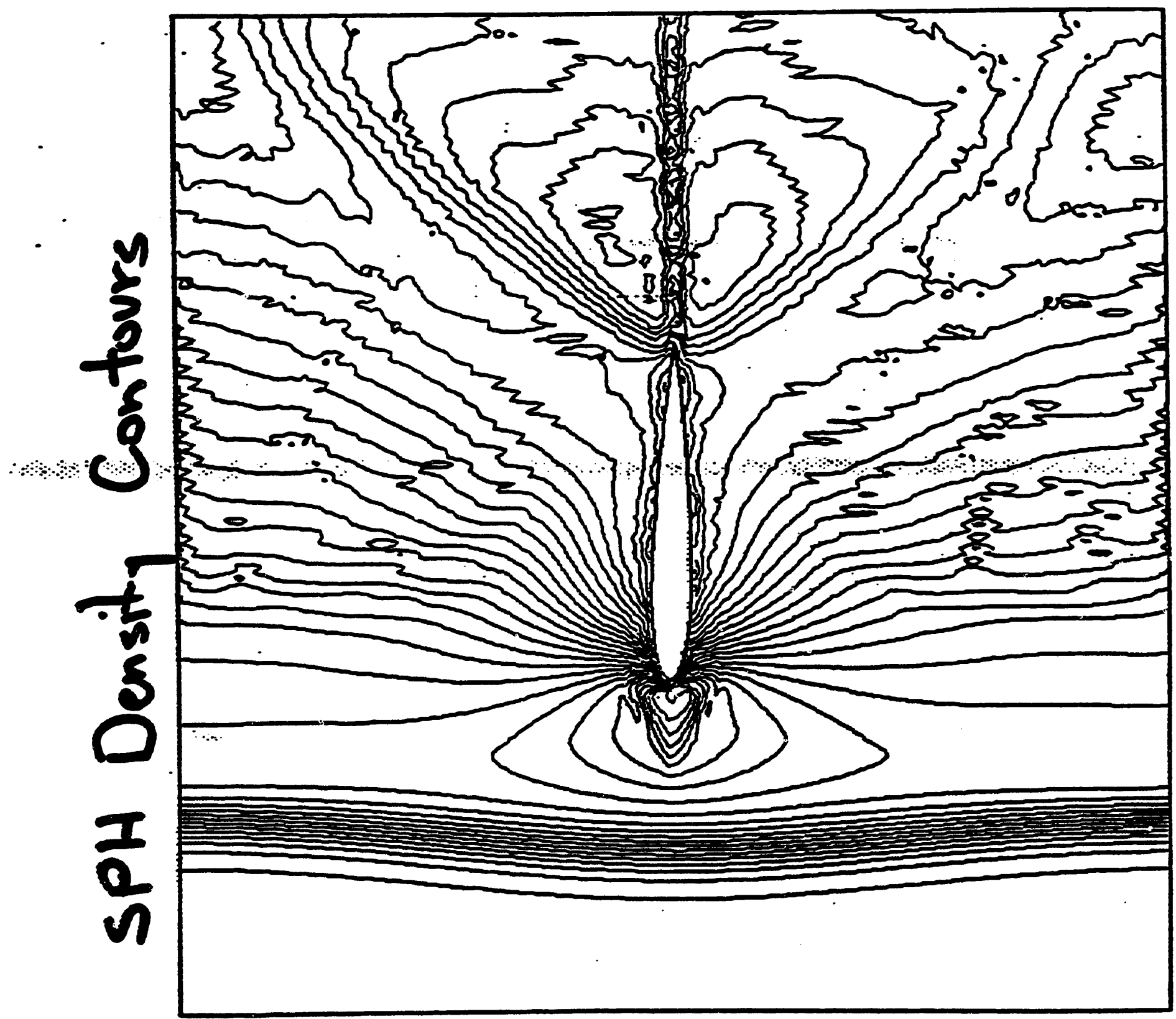




$$
-
$$




\section{Conclusion}

- Boundary Conditions !!!

- Noise Reduction, Sharpen Discontinuities

- Overall, Improved Quality of Solution 
$-58-$ 


\section{PROGRESS ON INTERFACE PROBLEMS IN SPH}

Charles P. Luehr \& Firooz A. Allahdadi USAF Phillips Laboratory, PL/WSSD

\section{OUTLINE:}

1. Cutoff Method for Separated Impact

2. Density Deviation Cancellation

3. Effective Relative Velocity Scheme

4. Velocity Divergence Based on Estimates Used in Artificial Viscosity Theory 


\section{CUTOFF METHOD FOR SEPARATED IMPACT}

Smoothing function cutoff with shutoff:

Diff. kinds interact by cutoff method Same kinds interact as usual.

Example (see diagram):

Dim. is 1 . Cutoff dist., $d=2 h / 3$.

No. of particles per $h$ is $3 / 2$.

Procedure:

1. Find active particles (i.e., particles within dist. $d$ from any particle of the other kind).

2. For all $i$ and $j$ of diff. kinds:

a. If neither is active, they do not interact.

b. If either is active, they interact as usual. 

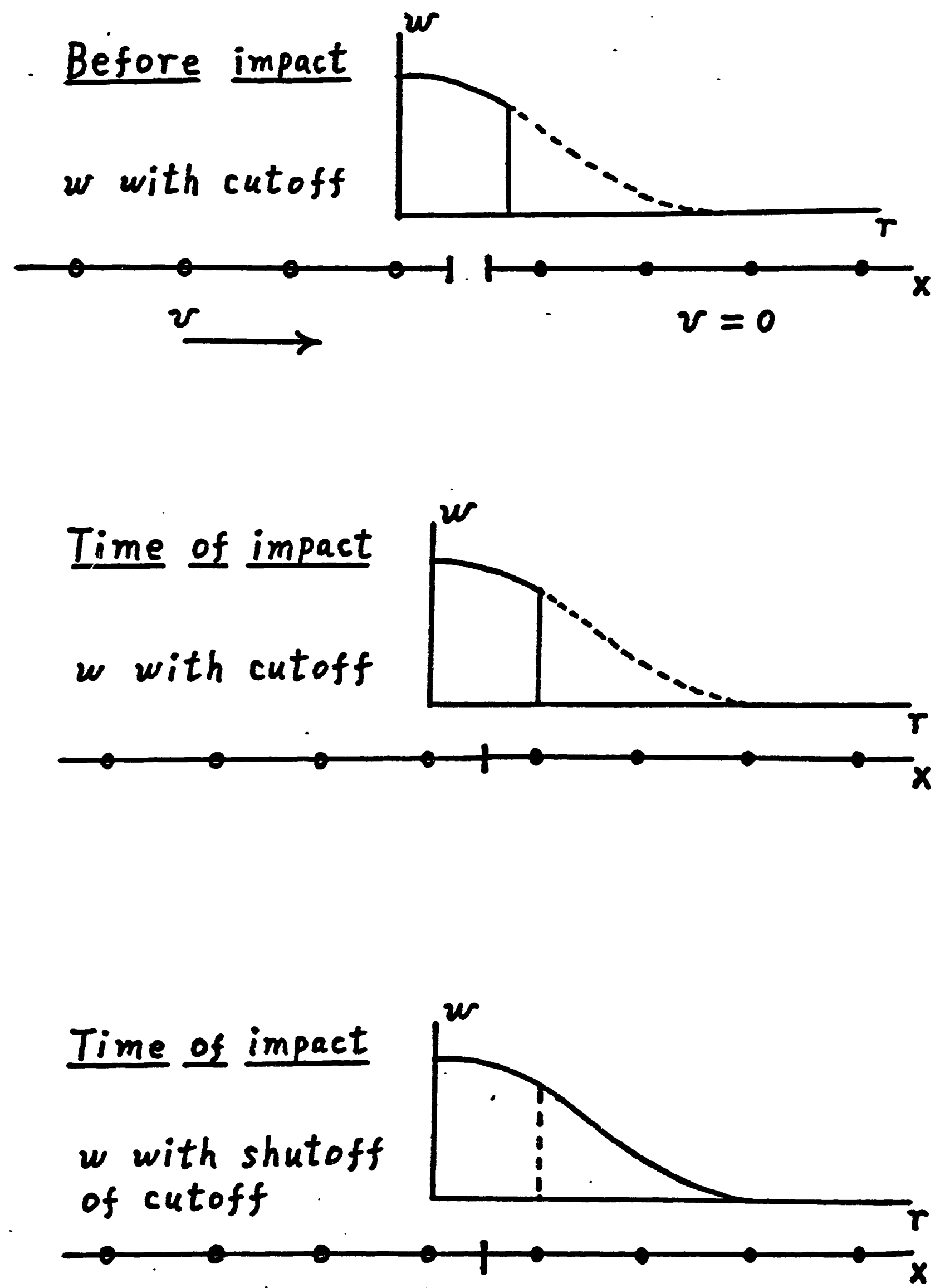
$\operatorname{run} 62$

$$
\begin{aligned}
& 0,0 \\
& 0.0,0.0,3 \\
& 5000,1 \\
& 2.5,2.5,0.1 \\
& 0.50,1.00 \\
& 50 \\
& 0.3,3.0 \\
& -1.0,3.0 \\
& \text { Istrt. } 000000 \\
& 2 \\
& \text { aluminum } \\
& 0.0,1.0 \\
& 2.71,1.0 \mathrm{E}-08,0.01 \\
& 0.01,1.5 \\
& \text { aluminum } \\
& 1.02,2.02 \\
& 2.71,1.02-08,0.0 \\
& 0.01,1.5 \\
& 2.0 \\
& \text { ratrt }
\end{aligned}
$$

[ IHOD ( 0-Original, 1=Mode1, 2=Mode2), IVARH (0=Fixed, 1=Var)]

[H (ignored), NPH (Ignored), IEOS (1=PerGas, 2=Grun, 3=Mie-Grun)]

[Number of Iterations, Density ( $0=$ Sumo, $1=$ Cont) ]

[Alpha, Beta, Epsilon, (Artificial Viscosity)]

[G1, G2 (Wall Heating)]

[Interval]

[CFI Yo.., Stop Time]

[Yin, X max (Problem Domain)]

[Restart $(0=\mathrm{NO}, 1=$ Yes $)$ ]

[Number of Lines]

[Material]

[Start and End]

[Density, Internal Energy, and Velocity] [H, MPH]

[Material]

[Start and End]

[Density, Internal Energy, and Velocity] [H, MPH]

[Number of Time Dump Intervals]

$$
\begin{aligned}
& \frac{\text { runG 2 }}{\text { Original method }} \\
& \text { Separated impact } \\
& \text { Al on Al at } 0.1 \mathrm{~km} / \mathrm{sec}
\end{aligned}
$$

1 user after impact

$-62-$. 


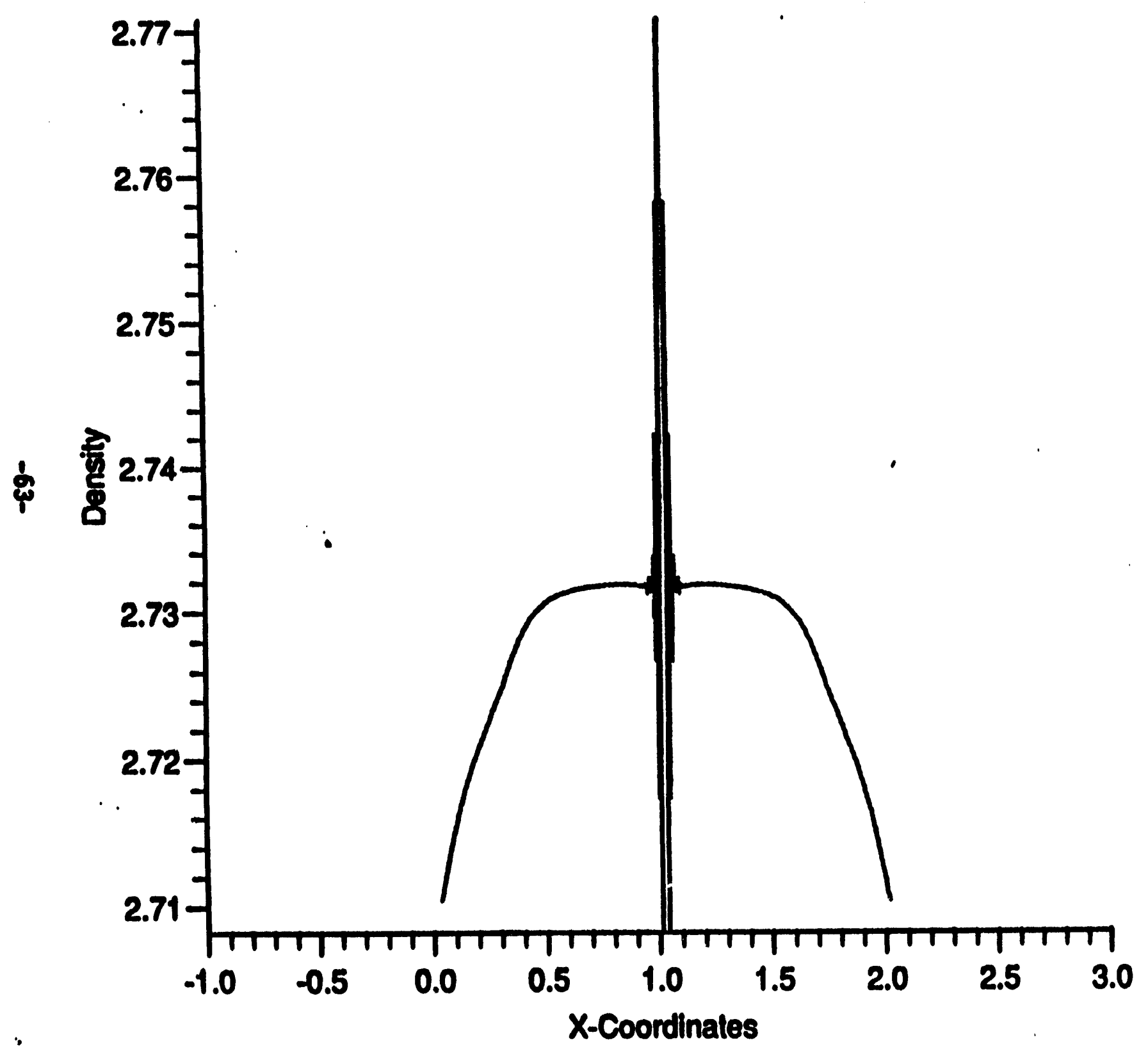
Max Density $=2.770667$
Min Density $=2.708231$

Alpha $=\mathbf{2 . 5 0 0 0}$

Beta $=2.5000$

Eps $=0.1000$

G1 $=0.5000$

$G 2=1.0000$

$C F L=0.3000$

Calculation

29 December 1992 
10

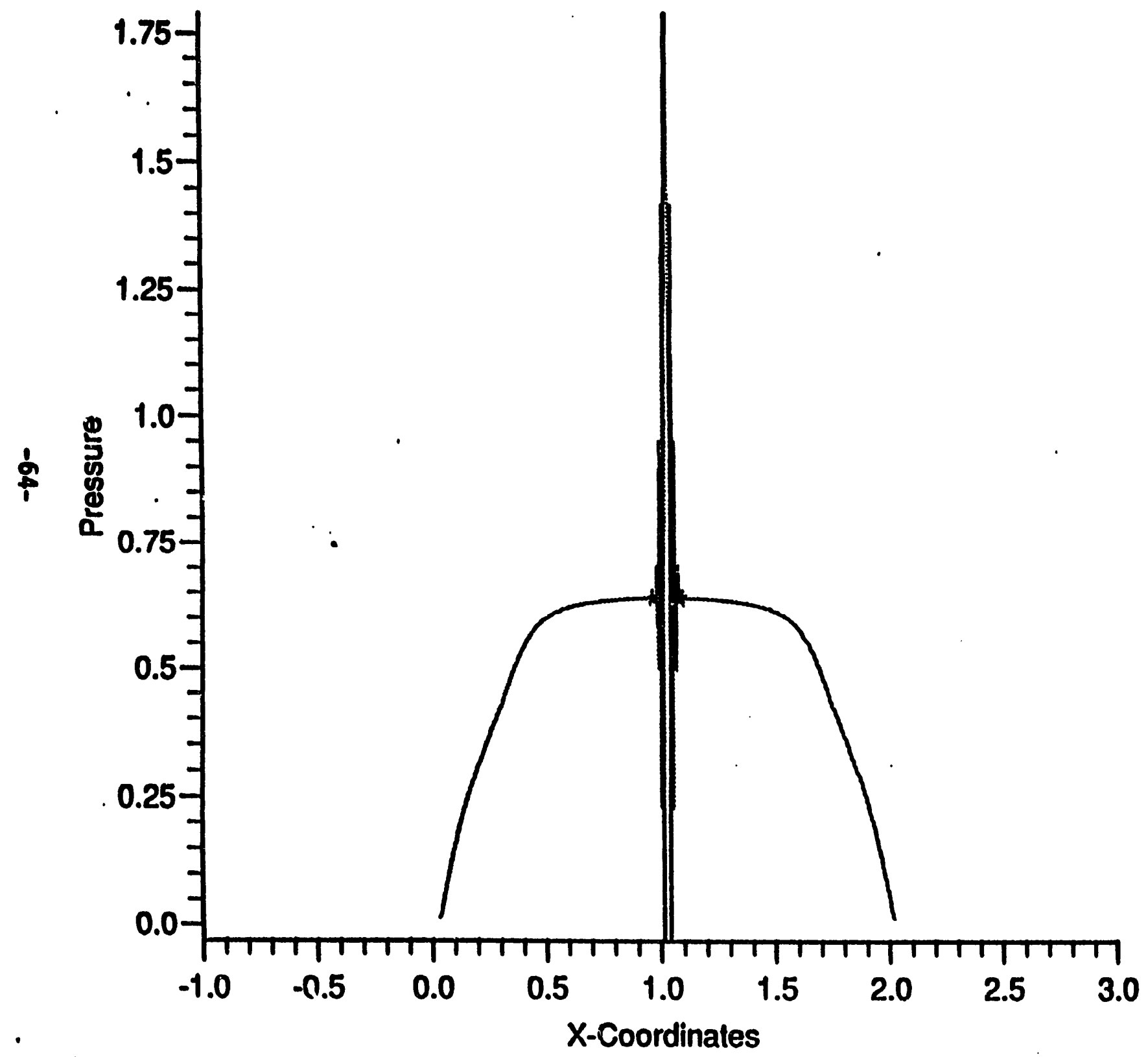

Max Pressure $=\mathbf{0} .017880$

Min Pressure $=\mathbf{- 0 . 0 0 0 3 2 2}$

Alpha $=2.5000$

Beta $=2.5000$

Eps $=0.1000$

G1 $=0.5000$

G2 $=1.0000$

$\mathrm{CFL}=0.3000$

Calculation

29 December 1992 


$$
\begin{aligned}
& 0,0 \\
& 0.0,0.0,3 \\
& 5000,1 \\
& 2.5,2.5,0.1 \\
& 0.50,1.00 \\
& 50 \\
& 0.3,3.0 \\
& -1.0,3.0 \\
& 0 \\
& \text { retrt.000000 } \\
& 2 \\
& \text { aluminum } \\
& 0.0,1.0 \\
& 2.71,1.0 z-08,0.01 \\
& 0.01,1.5 \\
& \text { aluminum } \\
& 1.0,2.0 \\
& 2.71,1.0 \text { - } 08,0.0 \\
& 0.01,1.5 \\
& 2 \\
& 1.0 \\
& 2.0 \\
& \text { rstrt }
\end{aligned}
$$

run G5

[TrOD ( $0=0$ - iginal, 1=Yode1, 2=Yode2), IVARH ( $0=$ Fixed, 1=Var)]

[H (ignored), NPH (ignored), IEOS (1=PerGas, 2=Grun, 3=Mie-Grun)]

[Number of Iterations, Density ( $0=$ sum m, 2 -Cont)]

[Alpha, Beta, Epsilon, (Artificial viscosity)]

[G1, G2 (Wall Heating)]

[Interval]

[CFL No.., Stop Time]

[Xmin, Xmas (Problem Domain)]

[Restart $(0=10,1=$ Yes $)$ ]

[Number of Iiries]

[Material]

[Start and End]

[Density, Internal Energy, and Velocity]

[B,NPH]

[Material]

[Start and End]

[Density, Internal Energy, and Velocity]

[H, KPH]

[Number of Time Dump Intervals]

$\operatorname{run} 65$

Original method

contact impact

Al on Al at $0.1 k \mathrm{kr} / \mathrm{sec}$

1 sec after impact

$-65-$ 

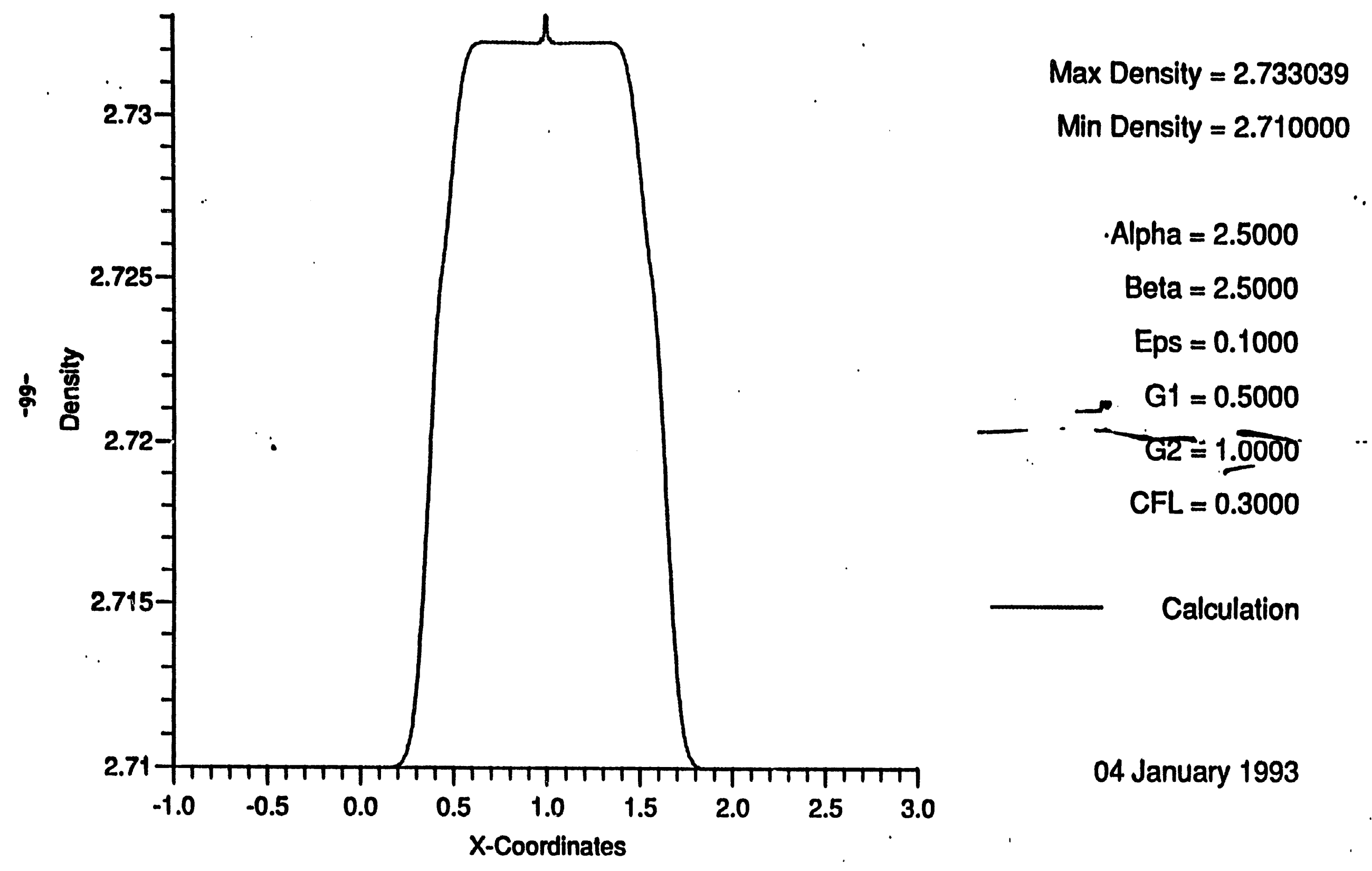
$\cdot 10^{\circ}$

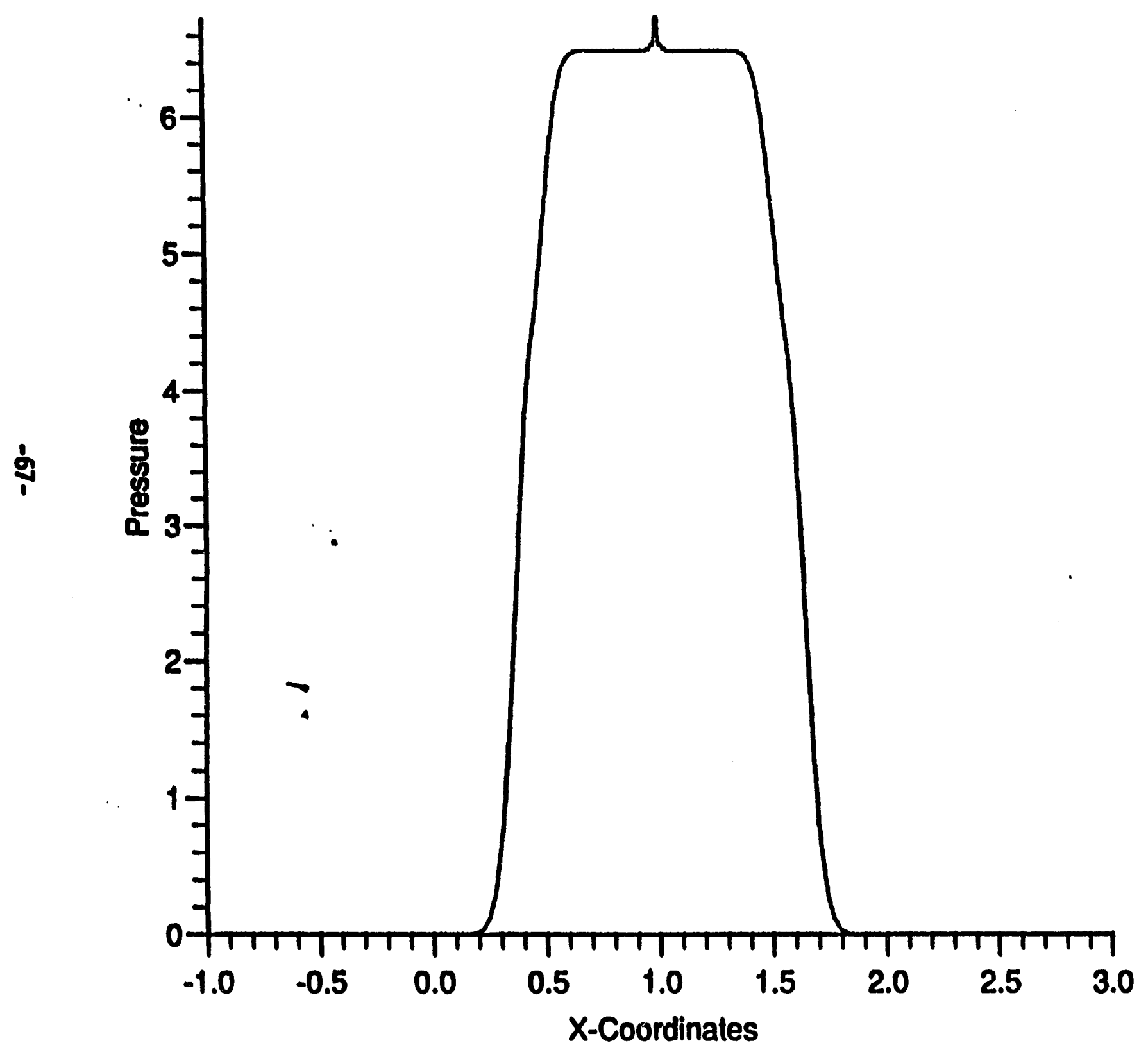

Max Pressure $=0.006720$

Min Pressure $=0.000000$

Alpha $=2.5000$

Beta $=2.5000$

$E p s=0.1000$

G1 $=0.5000$

$G 2=1.0000$

$C F L=0.3000$

Calculation

04 January 1993 
$r u n k / r 1$

$$
\begin{aligned}
& 0,0 \\
& 1.0,0.667 \\
& 0.0,0.0,3 \\
& 5000,1 \\
& 2.5,2.5,0.1 \\
& 0.50,1.00 \\
& 50 \\
& 0.3,3.0 \\
& -1.0,3.0 \\
& 0 \\
& \text { rstrt.000000 } \\
& 2 \\
& \text { aluminum } \\
& 0.0,1.0 \\
& 2.71,1.08-08,0.01 \\
& 0.01,1.5 \\
& \text { aluminum } \\
& 1.02,2.02 \\
& 2.71,1.08-08,0.0 \\
& 0.01,1.5 \\
& 1 \\
& 2.0 \\
& \text { rstrt }
\end{aligned}
$$

[IMOD (0=0rigina], 1=Yode1, 2=Mode2), IVARH ( $0=$ Fixed, 1=Var)]

[BMULT (1. 0-usual), DXOBnX (2. 0-usual)]

[H (ignored), NPH (ignored), IEOS (1-PerGas, 2=Grun, 3=Mie-Grun)]

[Number of Iterations, Density ( $0=$ Sum m, 1=Cont)]

[Alpha, Beta, Epsilon, (Artificial viscosity)]

[G1, G2 (Wall Heating)]

[Interval]

[CFL No.. Stop Time]

[Xmin, Xmas (Problem Domain)]

[Restart $(0=\mathrm{NO}, 1=\mathrm{Yes})$ ]

[Number of Lines]

[Material]

[Start and End]

[Density. Internal Energy, and Velocity]

[H,NPH]

[Material]

[Start and End]

[Density, Internal Energy, and Velocity]

[B,NPH]

[Number of Time Dump Intervals]

$$
\operatorname{rank} / r_{1}
$$

cutoff method

Separated impact

Al on $\mathrm{Al}$ at $0.1 \mathrm{~km} / \mathrm{sec}$

1 user after impact

$-68-$ 
Density vs. $X[N P=300$, Time $=3.0000$, liter $=539]$

rank $/ \mathrm{rl}$

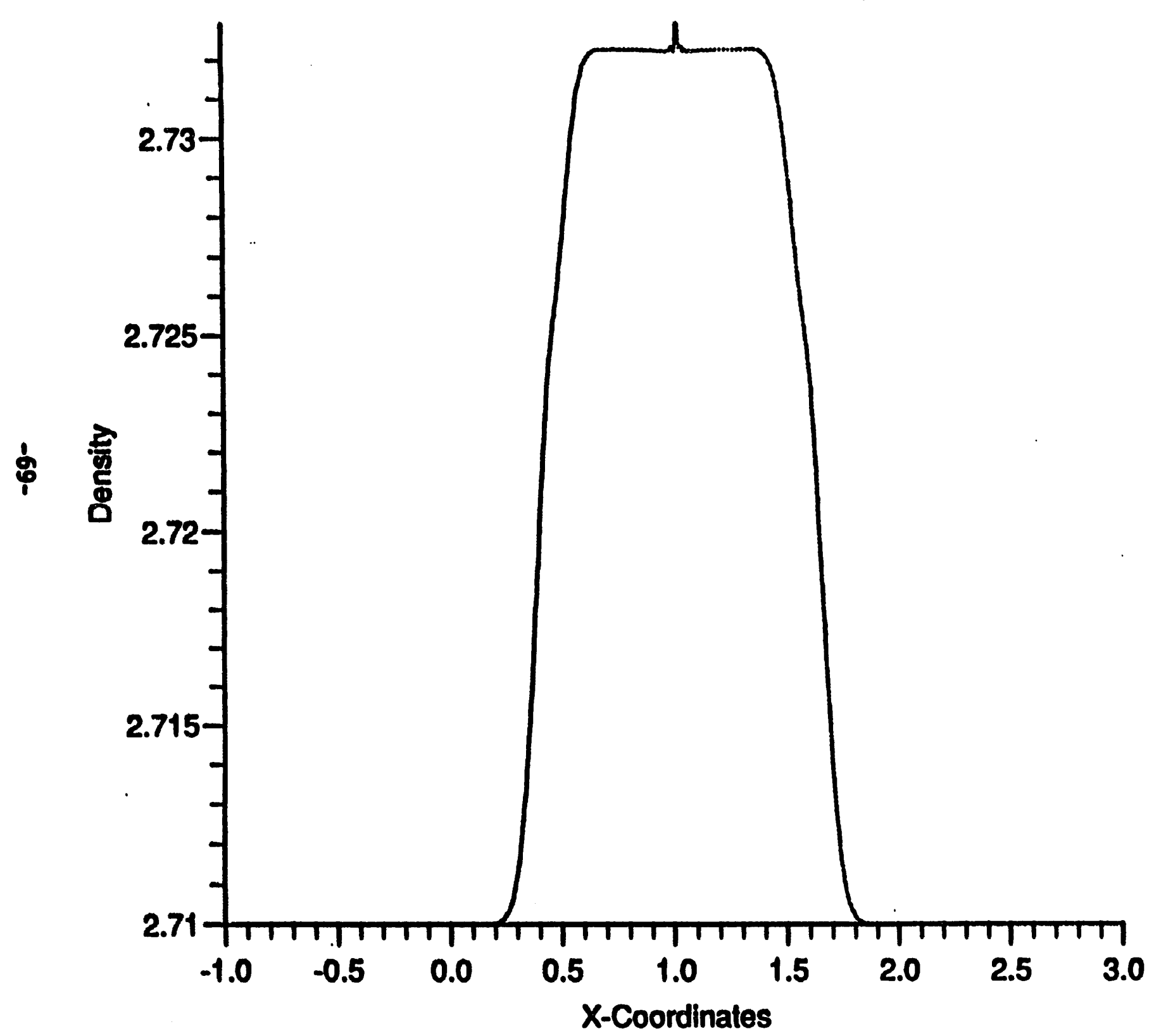

Max Density $=2.732930$

Min Density $=2.710000$

Alpha $=2.5000$

Beta $=2.5000$

Fps $=0.1000$

GI $=0.5000$

$G 2=1.0000$

$\mathrm{CFL}=0.3000$

Calculation

12 July 1993 
$.10^{\circ}$

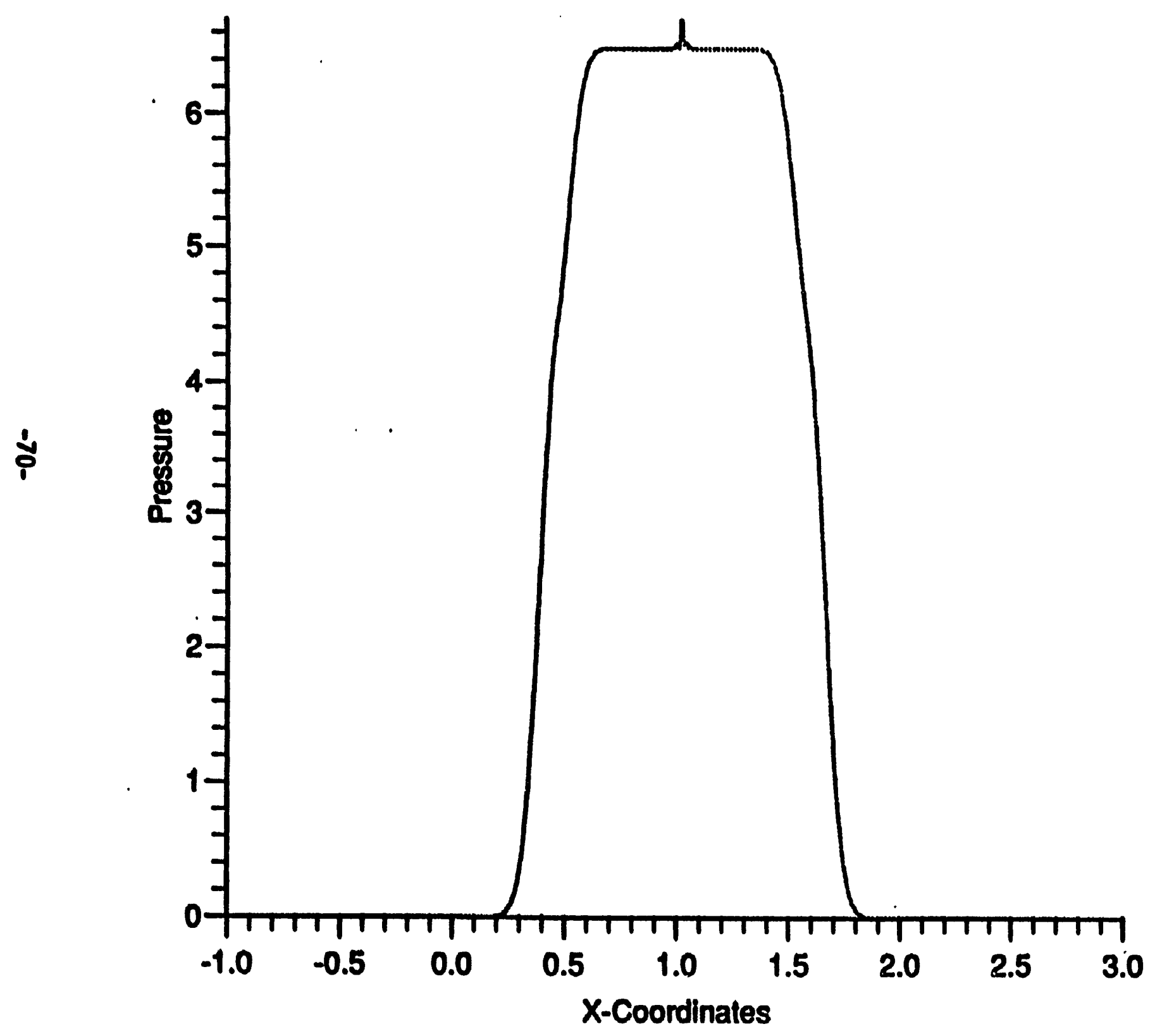

$$
\begin{aligned}
\text { Max Pressure } & =0.006695 \\
\text { Min Pressure } & =0.000000
\end{aligned}
$$

Alpha $=2.5000$

Beta $=2.5000$

Eps $=0.1000$

G1 $=0.5000$

G2 $=1.0000$

$C F L=0.3000$

Calculation

12 July 1993 


\section{DENSITY DEVIATION CANCELLATION}

Continuity Equation:

$$
d \rho_{i} / d t=-\rho_{i}(\nabla \cdot v)_{i}
$$

with

$$
(\nabla \cdot v)_{i}=-\rho_{i}^{-1} \Sigma_{j} m_{j}\left(\mathbf{v}_{i}-\mathbf{v}_{j}\right) \cdot \nabla_{i} w_{i j}
$$

gives

$$
d \rho_{i} / d t=\sum_{j} m_{j}\left(\mathbf{v}_{i}-\mathbf{v}_{j}\right) \cdot \nabla_{i} w_{i j}
$$

New velocity divergence:

$(\nabla \cdot v)_{i}=-\left(\sum_{j} m_{j} w_{i j}\right)^{-1} \sum_{j} m_{j}\left(\mathbf{v}_{i}-\mathbf{v}_{j}\right) \cdot \nabla_{i} w_{i j}$

Deviation factors due to an interface with a vacuum cancel out. 
New Continuity Equation:

$$
d \rho_{i} / d t=\rho_{i}\left(\sum_{j} m_{j} w_{i j}\right)^{-1} \sum_{j} m_{j}\left(\mathbf{v}_{i}-\mathbf{v}_{j}\right) \cdot \nabla_{i} w_{i j}
$$




\section{EFFECTIVE RELATIVE VELOCITY SCHEME}

Usual Continuity Equation:

$$
d \rho_{i} / d t=\sum_{j} m_{j}\left(\mathbf{v}_{i}-\mathbf{v}_{j}\right) \cdot \nabla_{i} w_{i j}
$$

Replace $m_{j}$ with $m_{i}$.

$\underset{\text { (for particle }}{\text { Use }}$ eff. $_{\text {fel. }} 2 m_{j}\left(m_{i}+m_{j}\right)^{-1}\left(\mathbf{v}_{i}-\mathbf{v}_{j}\right)$

Let

$$
M_{i j}=2 m_{i} m_{j}\left(m_{i}+m_{j}\right)^{-1}
$$

New Continuity Equation:

$$
d \rho_{i} / d t=\sum_{j} M_{i j}\left(\mathbf{v}_{i}-\mathbf{v}_{j}\right) \cdot \nabla_{i} w_{i j}
$$

New density by summation:

$$
R_{i}=\sum_{j} M_{i j} w_{i j}
$$


Comparison with usual density by sum:

$$
\sum_{j} M_{i j} w_{i j} \quad \sum_{j} m_{j} w_{i j}
$$

If $m_{j}=m_{i}, \quad \frac{M_{i j}}{m_{j}}=\frac{m_{j}}{m_{j} \text { (just right) }}$

If $m_{j} \gg m_{i}, 2 m_{i} \leq m_{j}$ (too large)

If $m_{j}<<m_{i}, \quad 2 m_{j} \geq m_{j}$ (too small)

Continuity Equation with density deviation cancellation:

$$
d \rho_{i} / d t=\rho_{i}\left(\sum_{j} M_{i j} w_{i j}\right)^{-1} \sum_{j} M_{i j}\left(\mathbf{v}_{i}-\mathbf{v}_{j}\right) \cdot \nabla_{i} w_{i j}
$$




\section{The resulting SPH equations.}

Let

$$
N_{i j}=2 m_{i}\left(m_{i}+m_{j}\right)^{-1}
$$

Density by summation:

$$
R_{i}=\sum_{j} N_{i j} m_{j} w_{i j}
$$

Velocity Divergence:

$$
(\nabla \cdot \mathrm{v})_{i}=-R_{i}^{-1} \sum_{j} N_{i j} m_{j}\left(\mathbf{v}_{i}-\mathbf{v}_{j}\right) \cdot \nabla_{i} w_{i j}
$$

Velocity Gradient:

$$
\mathbf{L}_{i} \equiv(\nabla \mathbf{v})_{i}^{T}=-R_{i}^{-1} \sum_{j} N_{i j} m_{j}\left(\mathbf{v}_{i}-\mathbf{v}_{j}\right) \otimes \nabla_{i} w_{i j}
$$


Continuity Equations:

$$
\begin{aligned}
d \rho_{i} / d t & =-\rho_{i}(\nabla \cdot \mathbf{v})_{i} \\
& =\rho_{i} R_{i}^{-1} \sum_{j} N_{i j} m_{j}\left(\mathbf{v}_{i}-\mathbf{v}_{j}\right) \cdot \nabla_{i} w_{i j}
\end{aligned}
$$

Energy Equations:

$$
\begin{aligned}
d e_{i} / d t & =\rho_{i}^{-1} \mathbf{T}_{i}: \mathbf{L}_{i} \\
& =-\left(\rho_{i} R_{i}\right)^{-1} \sum_{j} N_{i j} m_{j}\left(\mathbf{v}_{i}-\mathbf{v}_{j}\right) \cdot \mathbf{T}_{i} \cdot \nabla_{i} w_{i j}
\end{aligned}
$$

Momentum Equations:

$$
\begin{aligned}
d \mathrm{v}_{i} / d t=\sum_{j} m_{j}[ & N_{i j}\left(\rho_{i} R_{i}\right)^{-1} \mathbf{T}_{i} \cdot \nabla_{i} w_{i j} \\
& \left.+N_{j i}\left(\rho_{j} R_{j}\right)^{-1} \mathbf{T}_{j} \cdot \nabla_{i} w_{j i}\right]
\end{aligned}
$$




\section{Comments:}

The above equations are for the "Effective Relative Velocity" scheme combined with the "Density Deviation Cancellation" idea.

To get the equations without the "Density Deviation Cancellation" idea, replace all $R_{i}$ and $R_{j}$ (on the right side of the equations) with $\rho_{i}$ and $\rho_{j}$ respectively.

To get the equations without the "Effective Relative Velocity" scheme, replace $N_{i j}$ and $N_{j i}$ with 1 everywhere. 


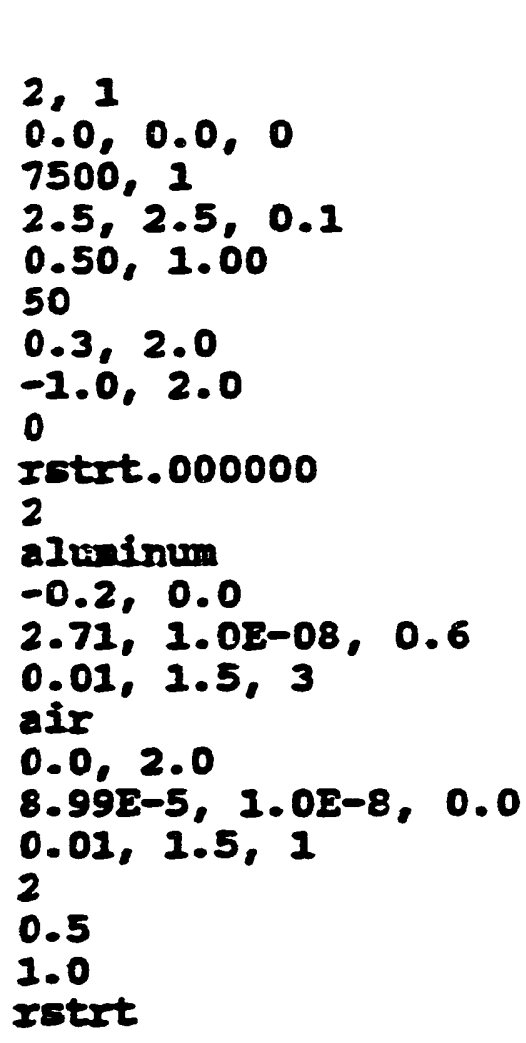

run le

[MOD ( $0=$ Orig, 1 I-IOUB, 2=EffVeI), IVARH (0=Fixed, 1=Var)]

[H (ign), NPH (jg nr), IEOS ( Ign r) (1=PerGas, 2=Grun, 3=Mie-Grun)]

[Number of Iterations, Density $(0=$ Sump, $1=$ Cont)]

[Alpha, Beta, Epsilon, (Artificial viscosity)]

[G1, G2 (Wall Heating)]

[Interval]

[CFI No., stop Time]

[Yin, Xmas (Problem Domain)]

[Restart $(0=\mathrm{NO}, 1=\mathrm{Yes})]$

[Number of Lines]

[Material]

[Start and End]

[Density, Internal Energy, and Velocity]

[H, NPH, IEOS (1=PerGas, 2=GrUn, 3=Mie-Grun)]

[Material]

[Start and End]

[Density, Internal Energy, and Velocity]

[H, NPH, IEOS (1=PerGas, 2=Grun, 3=Mie-Grun)]

[Number of Time Dump Intervals]

run L6 PRELIMINARY RESULTS

Eff, Rel, Vel. method with Dens. Der. Canc.

Piston Problem

Al on air at $6 \mathrm{~km} / \mathrm{sec}$

0.2 used from start

(Density by continuity equations)

$-78-$ 
Density vs. $X[N P=330, T i m e=0.2067$, lter=1000] run $: .6$

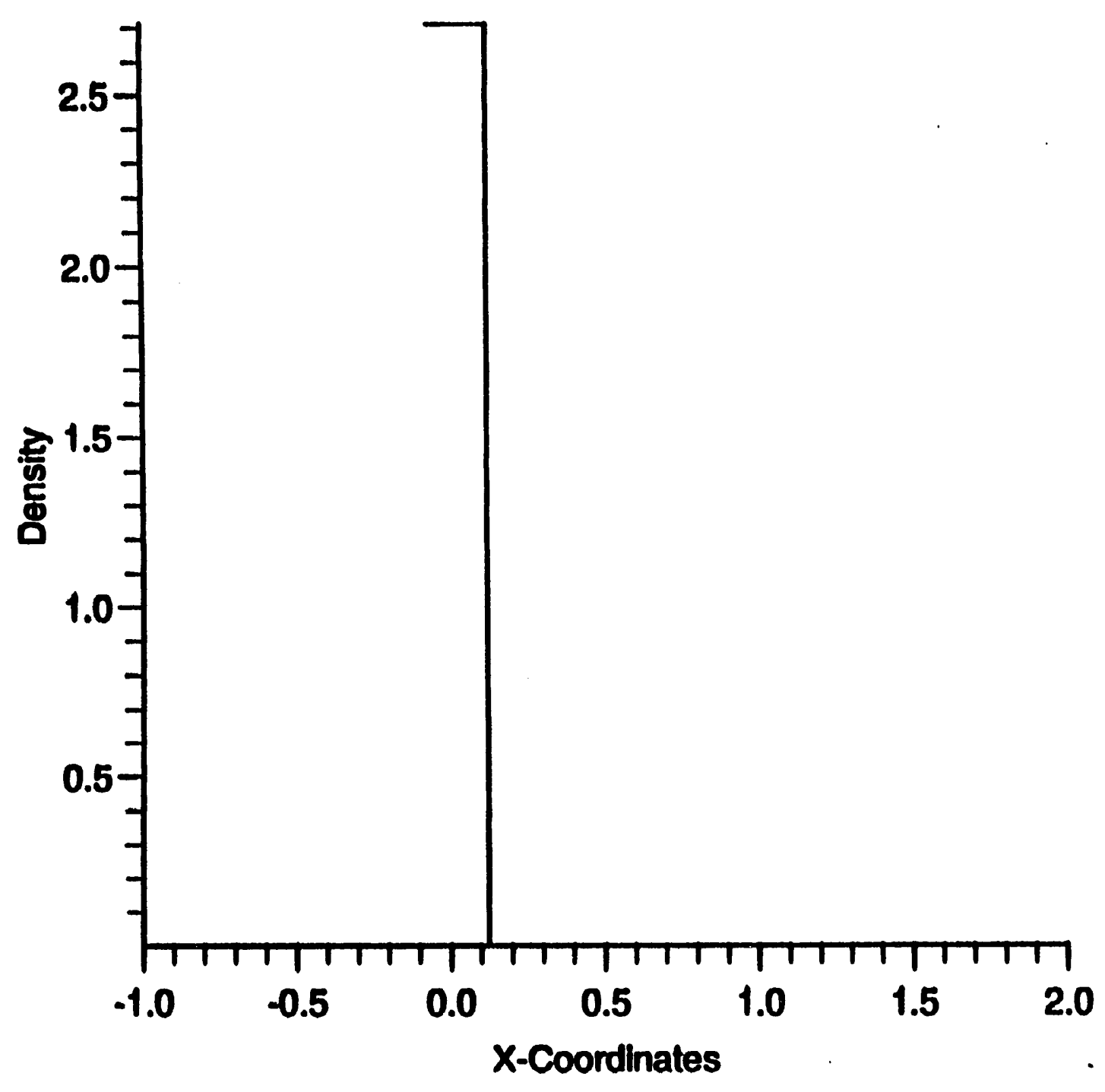

Max Density $=2.710074$

Min Density $=0.000090$

Alpha $=\mathbf{2 . 5 0 0 0}$

Beta $=2.5000$

Eps $=0.1000$

G1 $=0.5000$

$G 2=1.0000$

$\mathrm{CFL}=0.3000$

Calculation

30 August 1993 
Pressure vs. $X[N P=330$, Time $=0.2067$, lier $=1000] \operatorname{run} L 6$

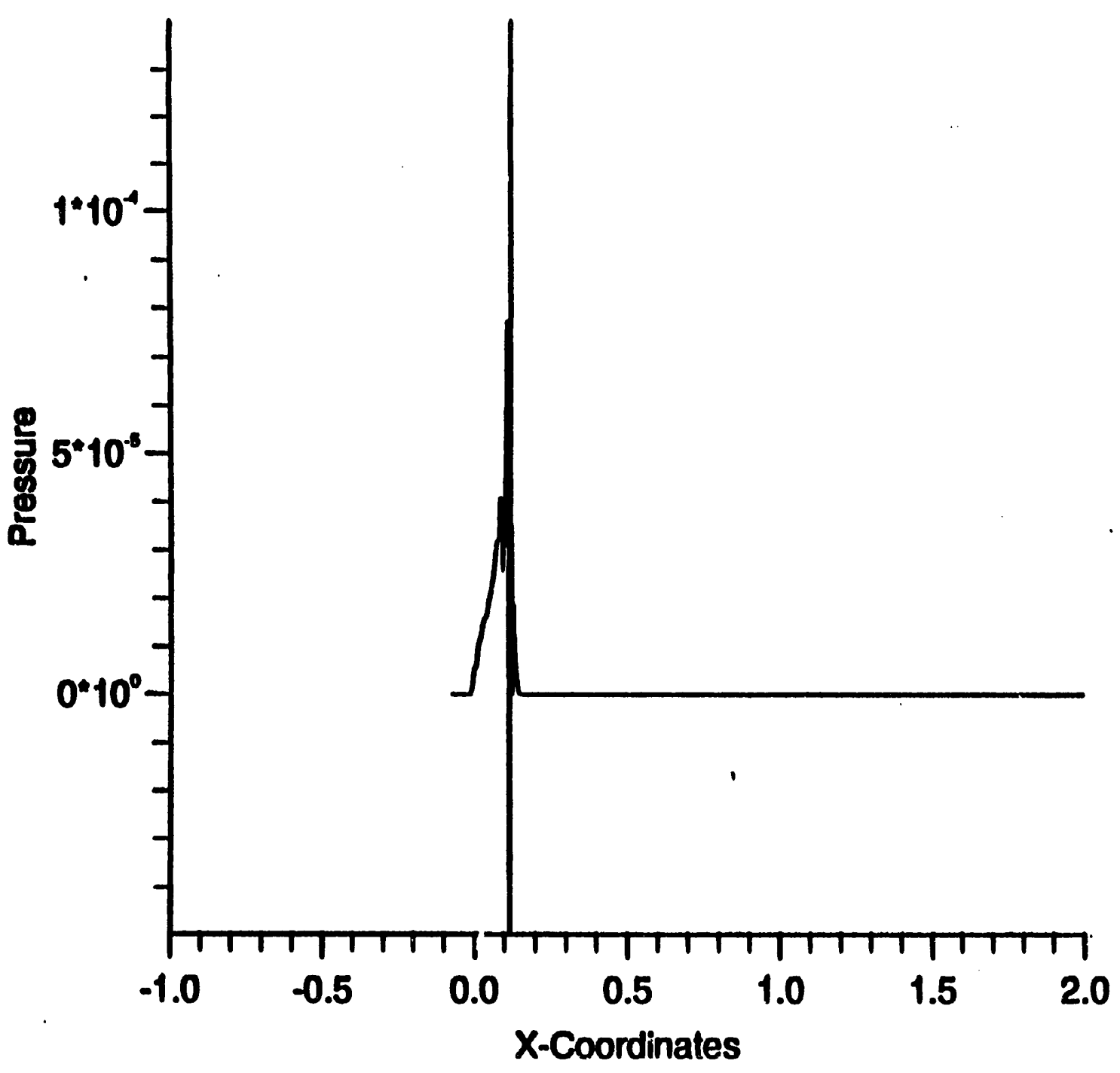

$$
\begin{aligned}
& \text { Max Pressure }=0.000140 \\
& \text { Min Pressure }=-0.000050
\end{aligned}
$$

Alpha $=2.5000$

Beta $=2.5000$

Eps $=0.10 n 0$

G1 $=0.5000$

$G 2=1.0000$

$\mathrm{CFL}=0.3000$.

Calculation

30 August 1993 
$\operatorname{run} L 8$

$$
\begin{aligned}
& 2,1 \\
& 0.0,0.0 ; 0 \\
& 10000,1 \\
& 2.5,2.5,0.1 \\
& 0.00,0.00 \\
& 10,0.2 \\
& 0.3,1,1.1 \\
& -0.1,0 \\
& 0 \\
& \text { retrt. } 000000 \\
& 2 \\
& \text { air } \\
& 0.0,0.5 \\
& 1.00,2.5,0.0 \\
& 0.0025,1.5,1 \\
& \text { air } \\
& 0.5,1.0 \\
& 0.125,2.0,0.0 \\
& 0.0025,1.5,1 \\
& 1 \\
& 0.1 \\
& \text { restart }
\end{aligned}
$$

[IXOD (0=orig, 1=LOUB, 2=EffVel), IVARH (0=Fixed, 1=Var)]

[H (ign), NPH ( Ign), IroN (lg nr) ( I=PerGas, 2=Grun, 3=Hie-Grun)]

[Number of Iterations, Density (0=Summ, 1=Cont)]

[Alpha, Beta, Epsilon, (Artificial Viscosity)]

[G1, G2 (HaIl Heating)]

[Interval]

[CFI No.. Stop Time]

[Yin, max (Problem Domain)]

[Restart (ONo, 1=Yes)]

[Number of Ines]

[Material]

[Start and ind]

[Density. Internal Energy, and Velocity]

[H, NPH, IEOS (1=PerGas, 2=Grun, 3=Hie-Grun)]

[Material]

[start and End]

[Density. Internal Energy, and Velocity]

[H, NPH, IEOS (1=PerGas, 2=Grun, 3=Hie-Grun)]

[Number of Time Dump Intervals]

run L8 PRELIMINARY RESULTS

Eff. Rel. Vel. method with Dens. Der. Canc.

Shock tube problem

Air and air, density ratio 8 , initially

0.2 muse from start

at rest

(Density by continuity equation)

$-81-$ 


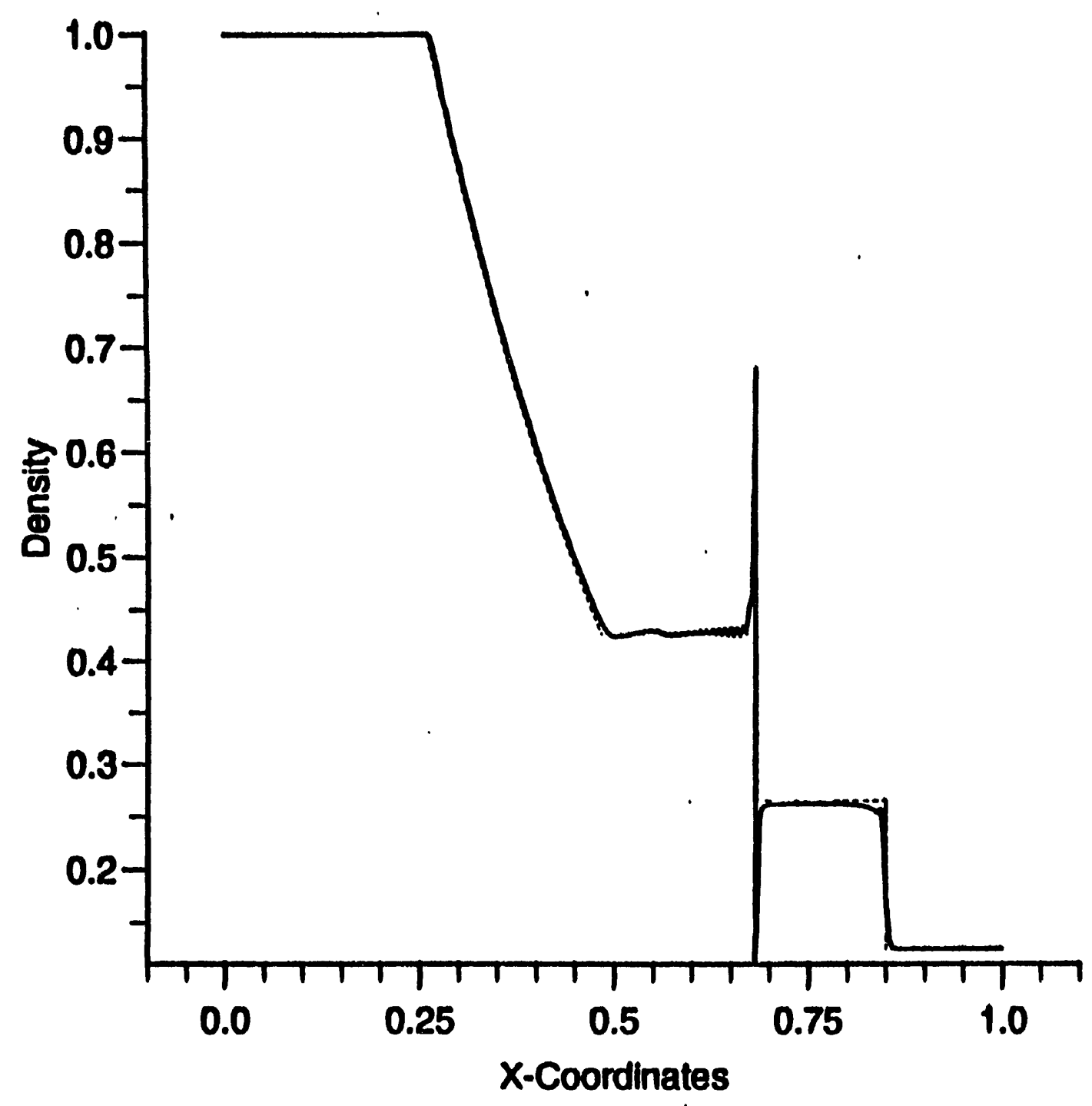

Max Density $=1.000974$

Min Density $=0.110774$

Alpha $=2.5000$ Beta $=2.5000$ Eps $=0.1000$ $\mathbf{G 1}=0.0000$ $\mathrm{G} 2=0.0000$ $C F L=0.3000$

Calculation Analytic

20 September 1993 
Pressure vs. $X[N P=600$, Tim $: e=0.2000$, Iter $=904] \quad$ runL 8

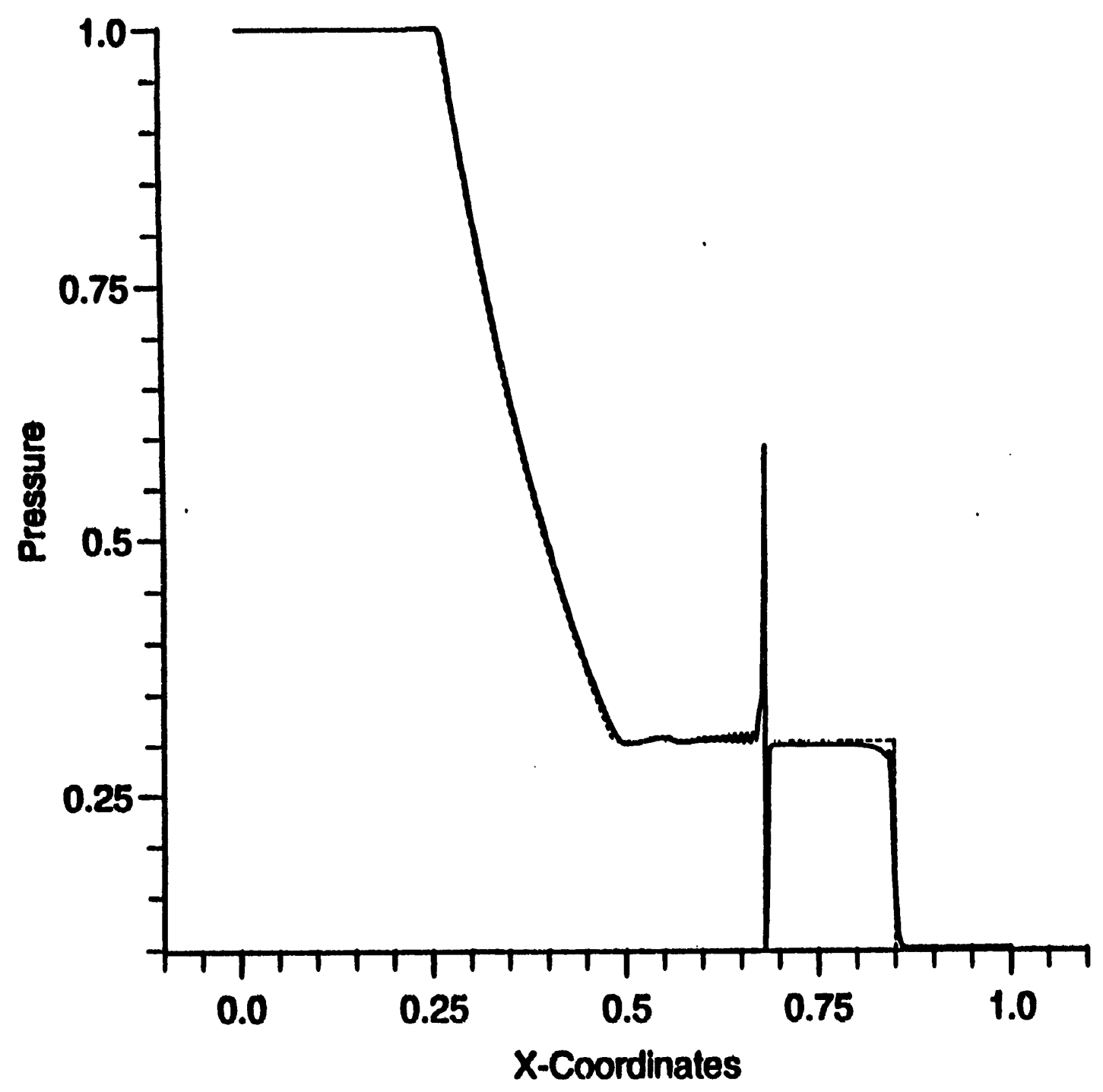

Max Pressure $=1.001363$

Min Pressure $=0.097294$

Alpha $=2.5000$

Beta $=2.5000$

Eps $=0.1000$

G1 $=0.0000$

$G 2=0.0000$

$\mathrm{CFL}=0.3000$

Calculation

Analytic

20 September 1993 


\section{VELOCITY DIVERGENCE BASED ON ESTIMATES USED IN ARTIFICIAL VISCOSITY THEORY}

Estimate of contrib. to vel. div. at particle $i$ due to particle $j$ :

$$
\left|\mathbf{r}_{i}-\mathbf{r}_{j}\right|^{-2}\left(\mathbf{r}_{i}-\mathbf{r}_{j}\right) \cdot\left(\mathbf{v}_{i}-\mathbf{v}_{j}\right)
$$

An averaging procedure weighted by $m_{j} w_{i j}$ gives:

$$
\begin{aligned}
(\nabla \cdot v)_{i}=-\left(\sum_{j} m_{j} w_{i j}\right)^{-1} \sum_{j}\left\{m_{j}\left(\mathbf{v}_{i}-\mathbf{v}_{j}\right)\right. \\
\left.\cdot\left[-\left|\mathbf{r}_{i}-\mathbf{r}_{j}\right|^{-2}\left(\mathbf{r}_{i}-\mathbf{r}_{j}\right) w_{i j}\right]\right\}
\end{aligned}
$$

The result: In the SPH governing equations, $\nabla_{i} w_{i j}$ is replaced by

$$
-\left|\mathbf{r}_{i}-\mathbf{r}_{j}\right|^{-2}\left(\mathbf{r}_{i}-\mathbf{r}_{j}\right) w_{i j}
$$




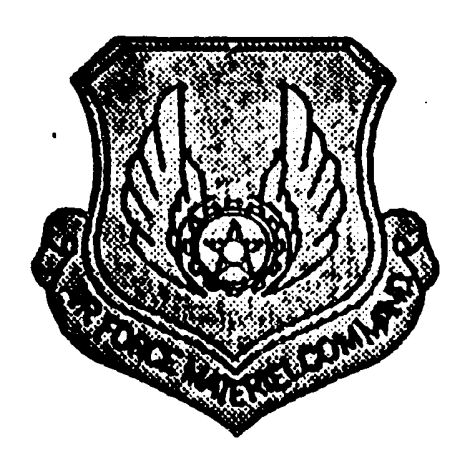

\title{
Boundaries and Interfaces in SPH
}

$\dot{\phi}$

\author{
Louis Baker \\ Dagonet Software
}




\section{Problem: \\ Robust SPH for \\ large density differences various EOS}

Riemann Shock Tube Aluminum Impact Test Cases 


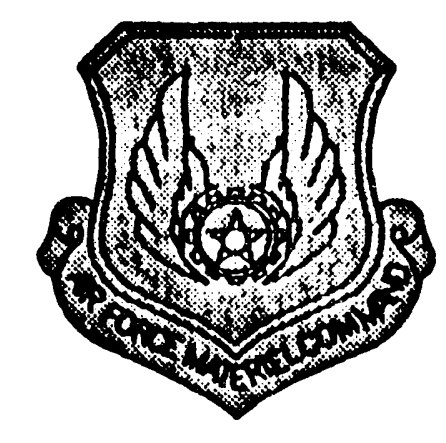

\section{SPH Interpolation}

Gibbs Phenomena at Discontinuities Kernel: Cardinal B-Splines

Ok for uniform spacing, no boundaries

Choice: either

1) ensure smoothness

2) modify interpolation for jumps 


\section{Cubic Cardinal B-Splines}

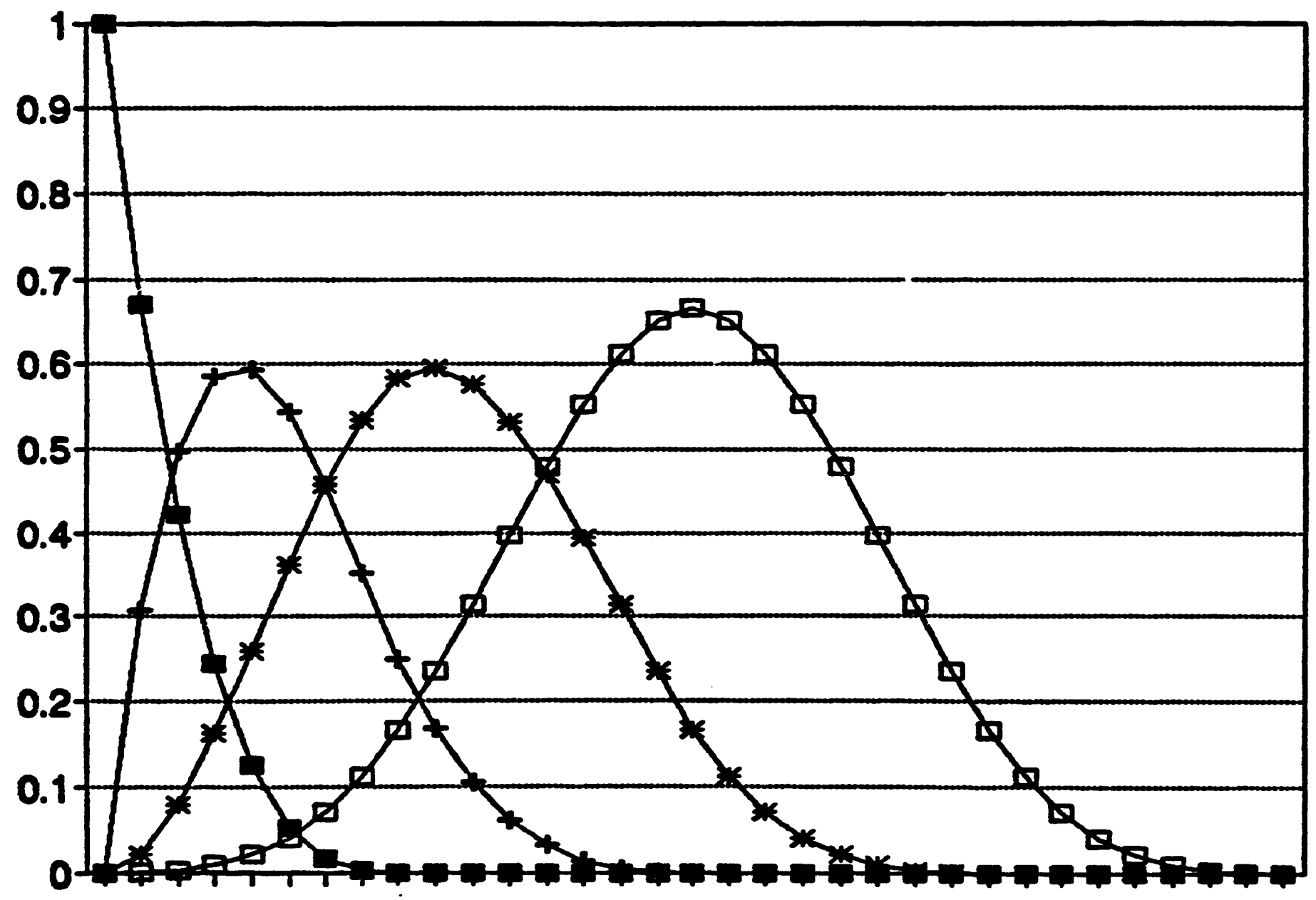




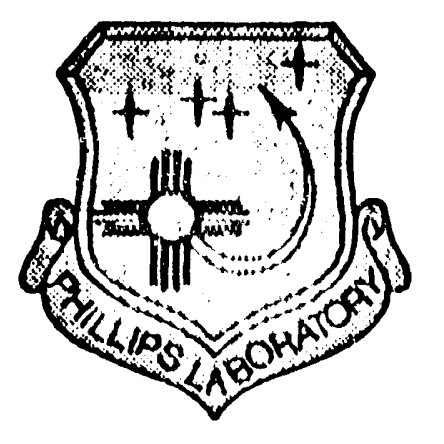

\section{DONT'S}

\$

Mass Match Mass Adjust Ratio Zone 

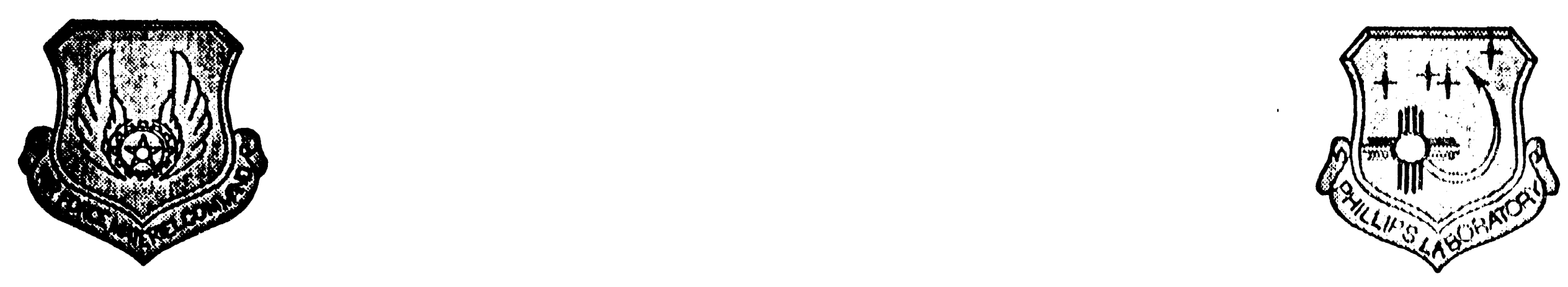

\section{DO's}

;

\section{Use Summation, Not Continuity}

Need to re-normalize density for EOS

Volume Match 
Density v8. X [NP=800, Time=0.1000, lter=1706]

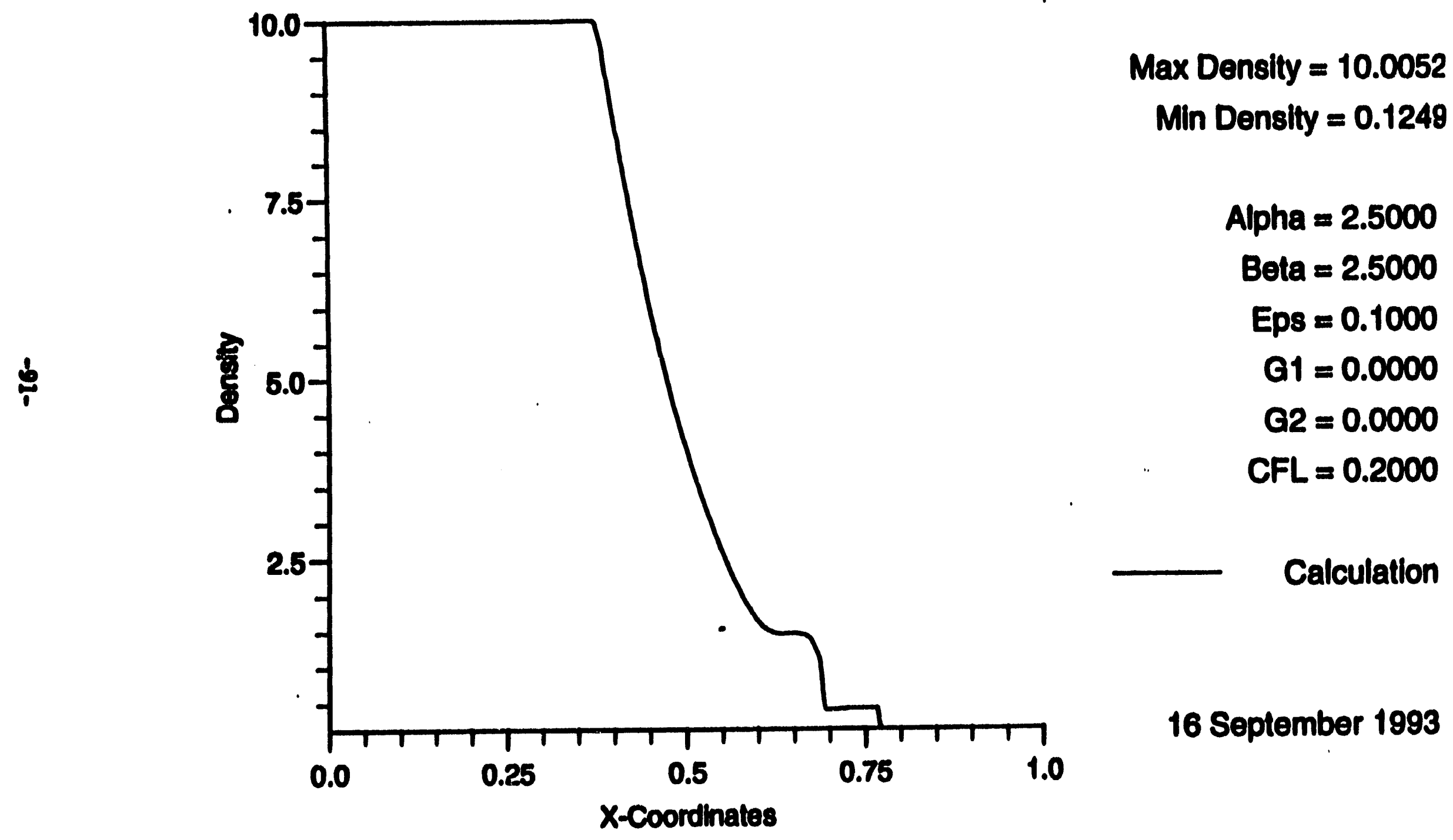

80il Shock Tube 

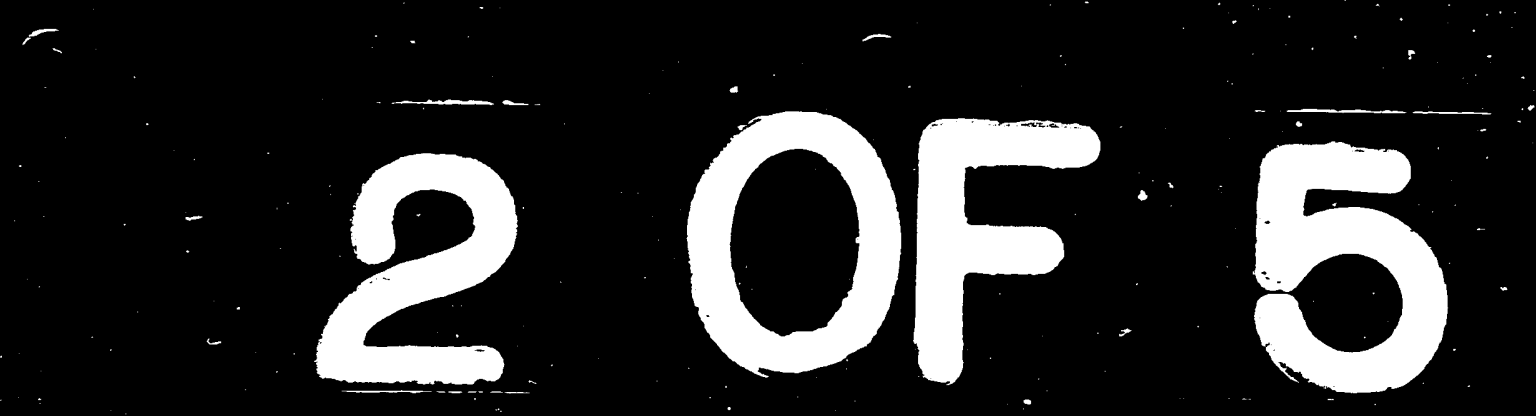

1. 


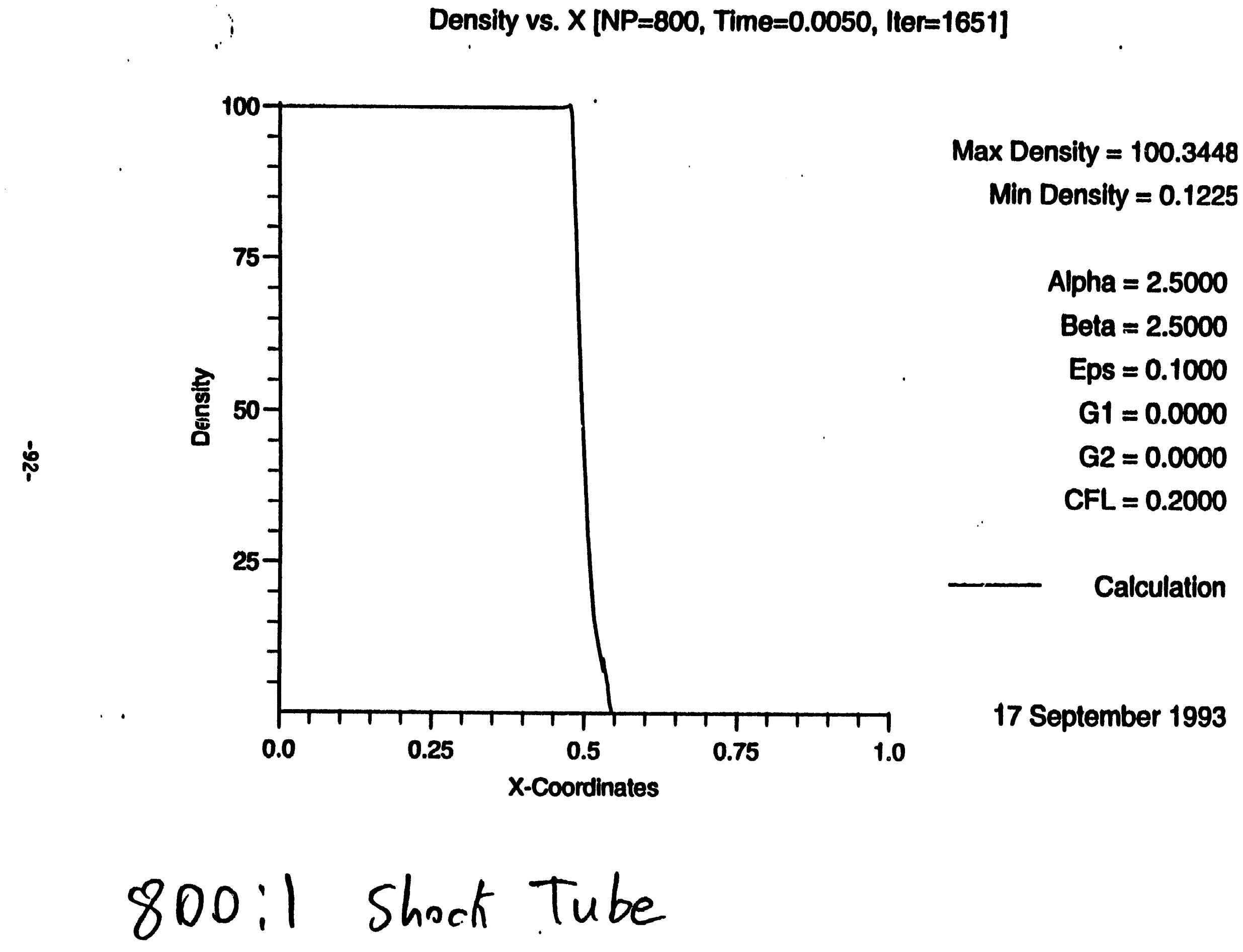


Density vs. $X[N P=450$, Time $=1.0000$, Iter $=409]$

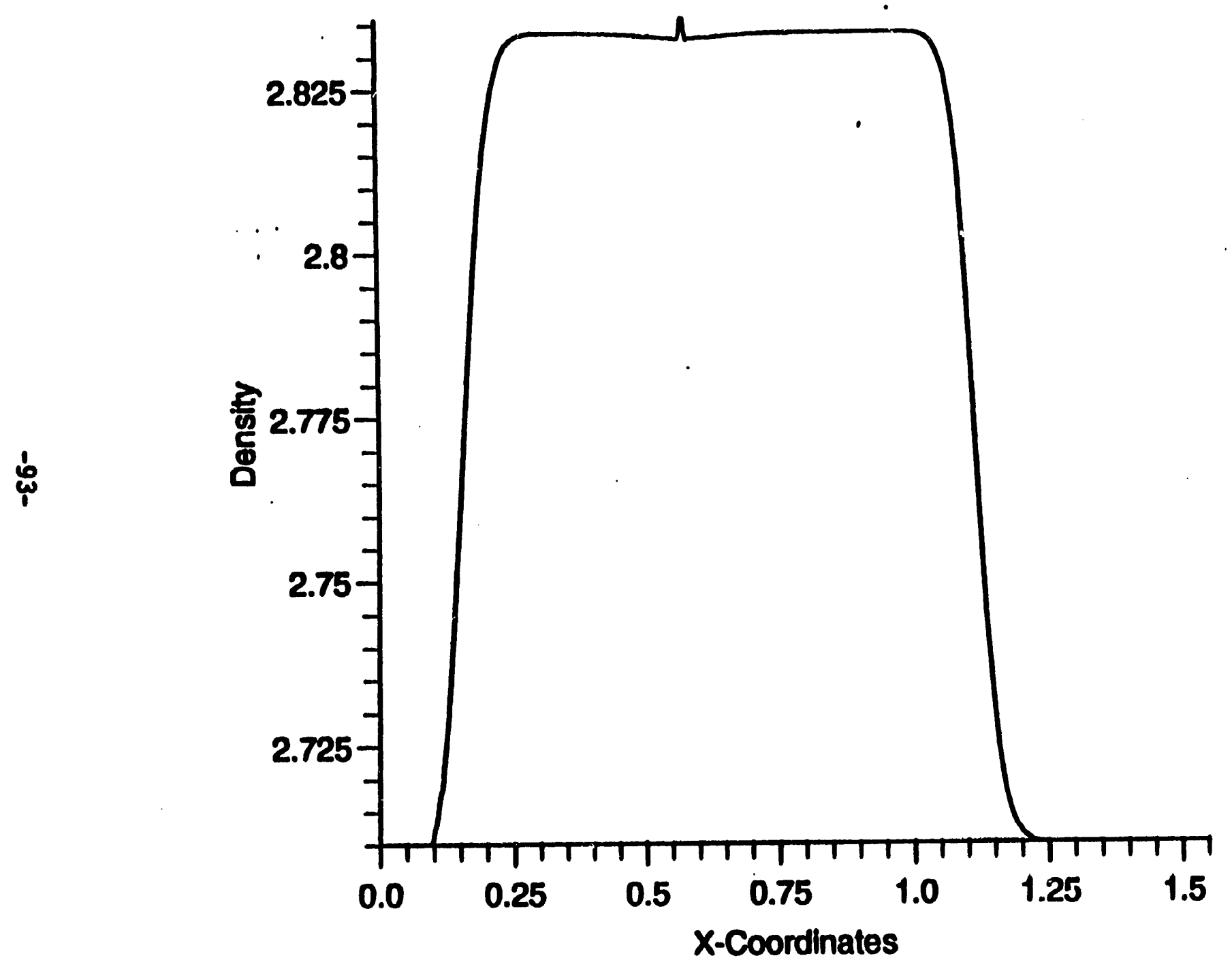

Max Density $=2.8356$

Min Density $=2.7100$

Alphe $=2.5000$

Beta $=2.5000$

Eps $=0.1000$

G1 $=0.5000$

$\mathrm{G} 2=1.0000$

$\mathrm{CFL}=0.3000$

Calculation

13 September 1993

\section{Al Impact-Continuity}


Velocity vs. $X[N P=450$, Tim $\theta=1.0000$, Iter $=409]$

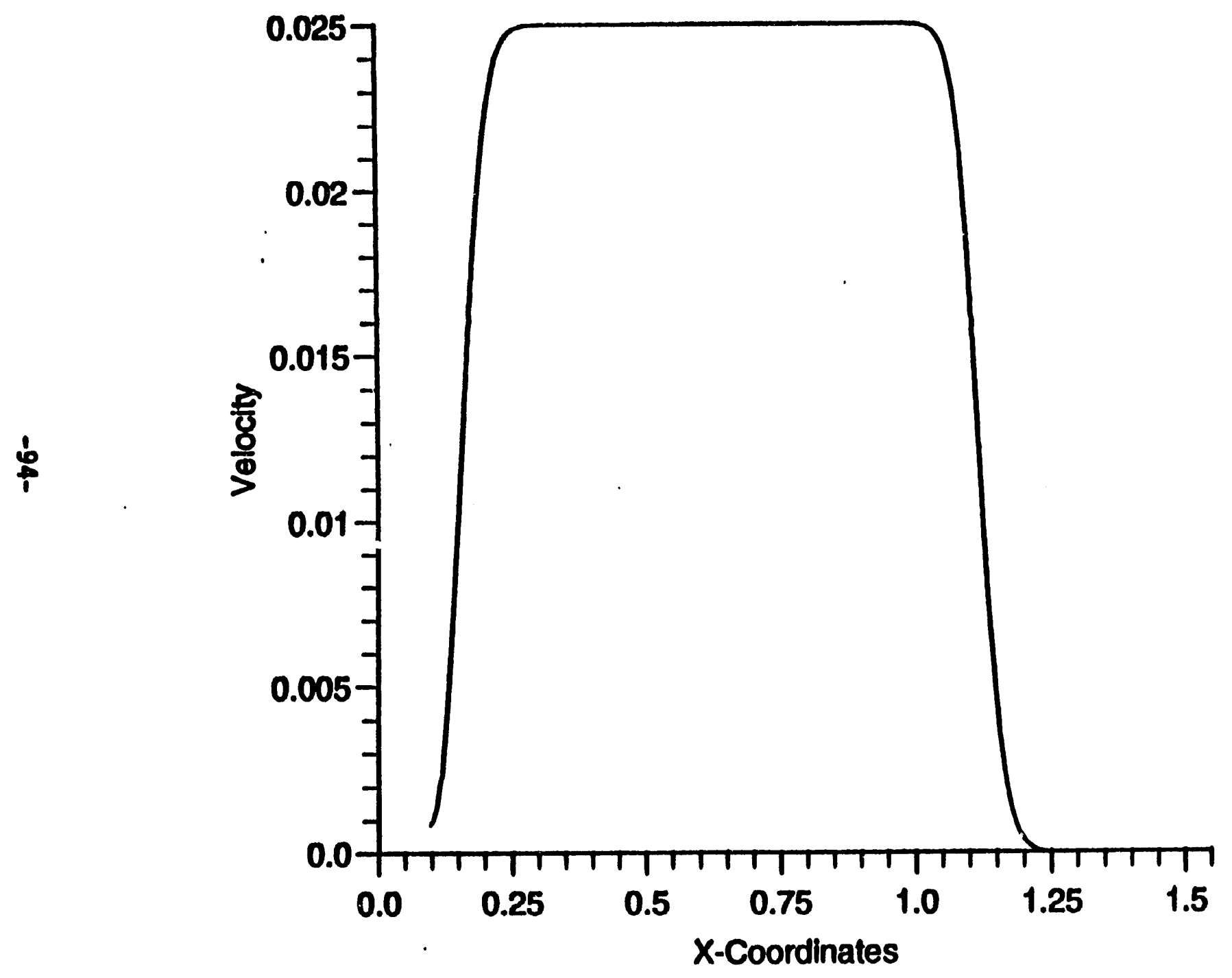

$$
\begin{aligned}
& \text { Max Velocity }=0.0250 \\
& \text { Min Velocity }=0.0000 \\
& \text { Alpha }=2.5000 \\
& \text { Beta }=2.5000 \\
& \text { Eps }=0.1000 \\
& G 1=0.5000 \\
& G 2=1.0000 \\
& \text { CFL }=0.3000 \\
& \text { Calculation }
\end{aligned}
$$

13 September 1993 
Pressure vs. $X[N P=450$, Time $=1.0000$, Her $=409]$

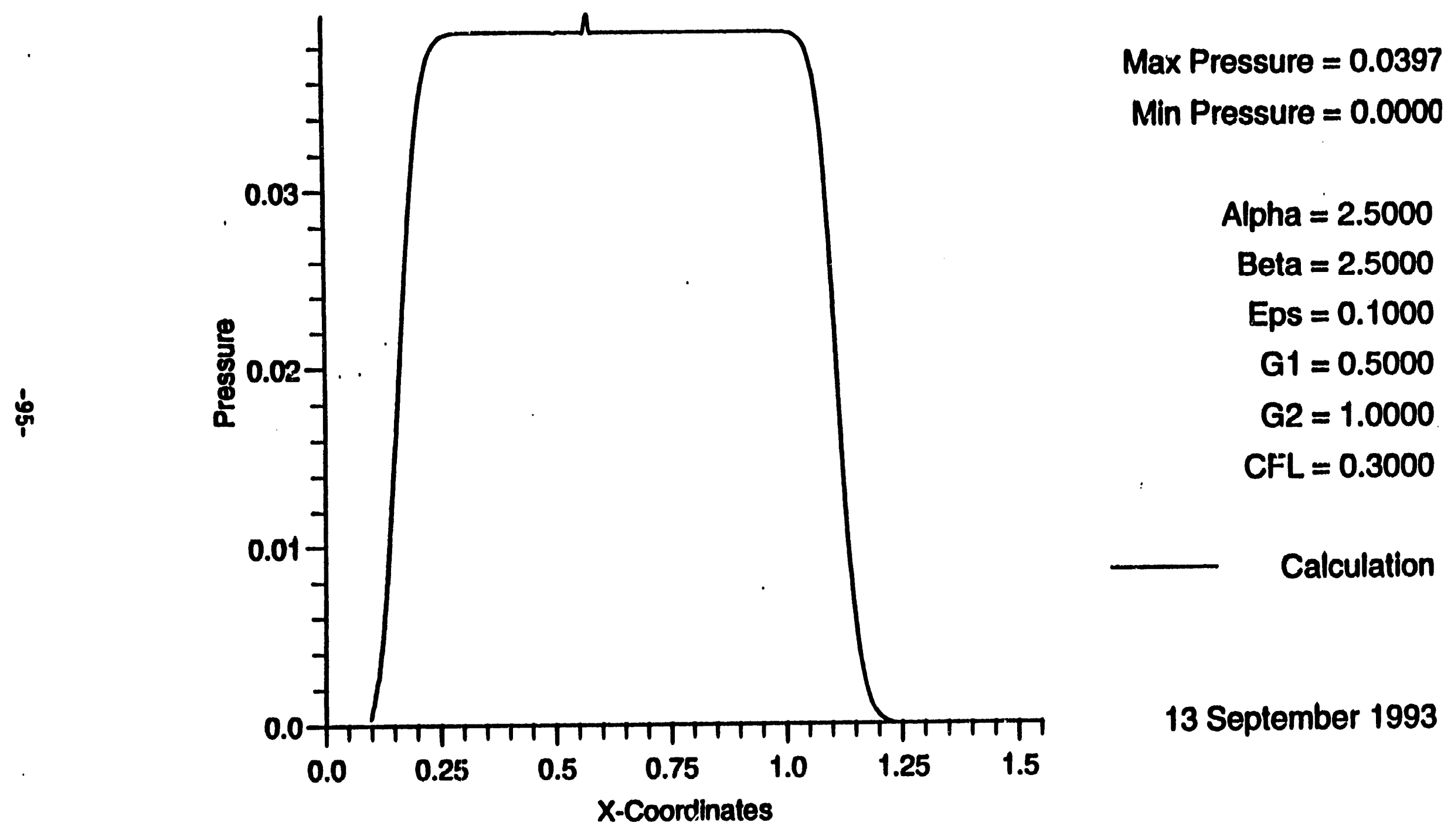


Density vs. $X[N P=450$, Time $=1.0000$, Iter $=505]$

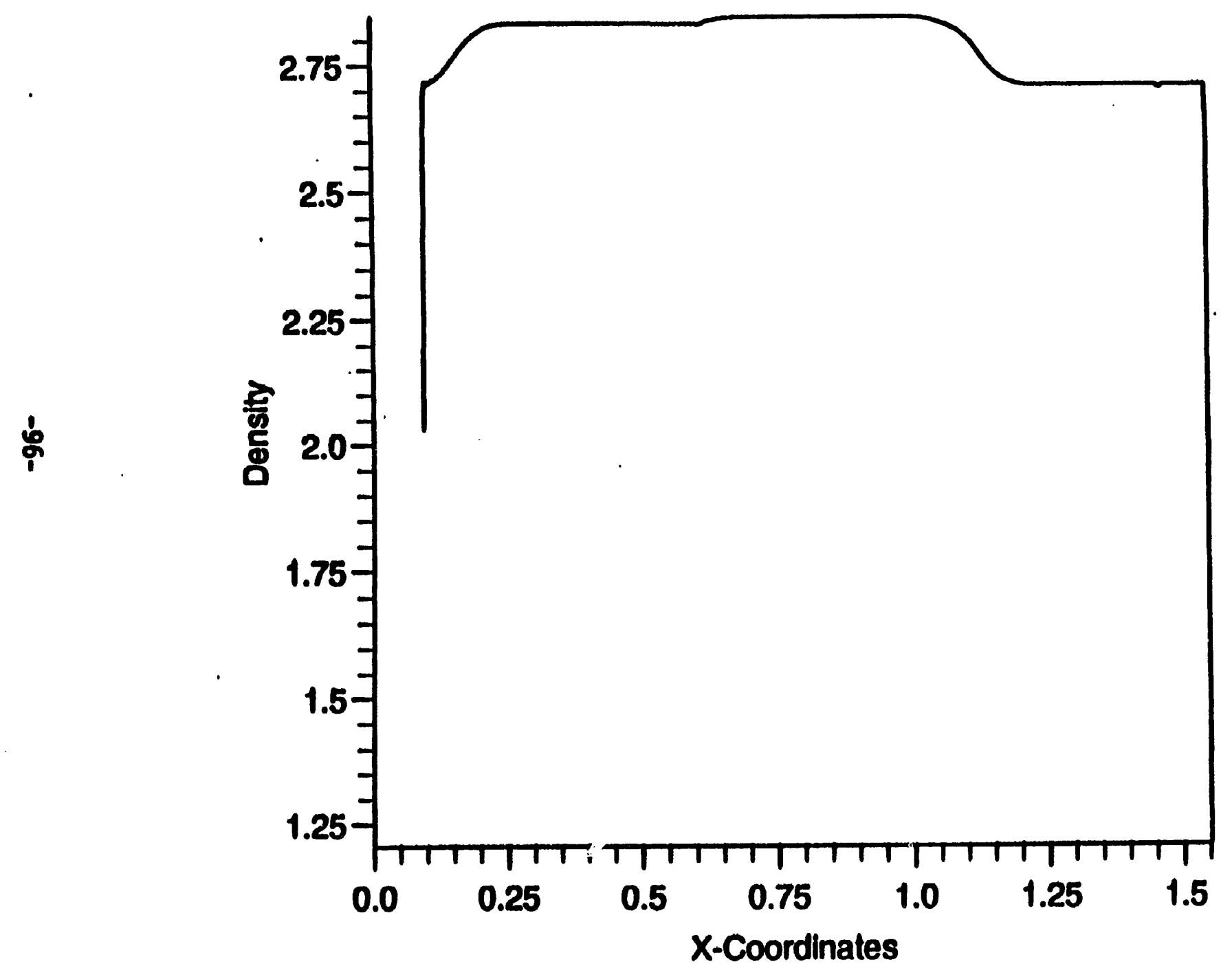

$$
\begin{aligned}
\text { Max Density } & =2.8454 \\
\text { Min Density } & =1.2057
\end{aligned}
$$$$
\text { Alpha }=2.5000
$$$$
\text { Beta }=2.5000
$$$$
\text { Eps }=0.1000
$$$$
\mathrm{G1}=0.5000
$$$$
\mathrm{G} 2=1.0000
$$$$
C F L=0.3000
$$

Calculation

16 September 1993

Al Impact - Naive Summation 
Velocity vs. $X[N P=450$, Time $=1.0000$, Iter $=505]$

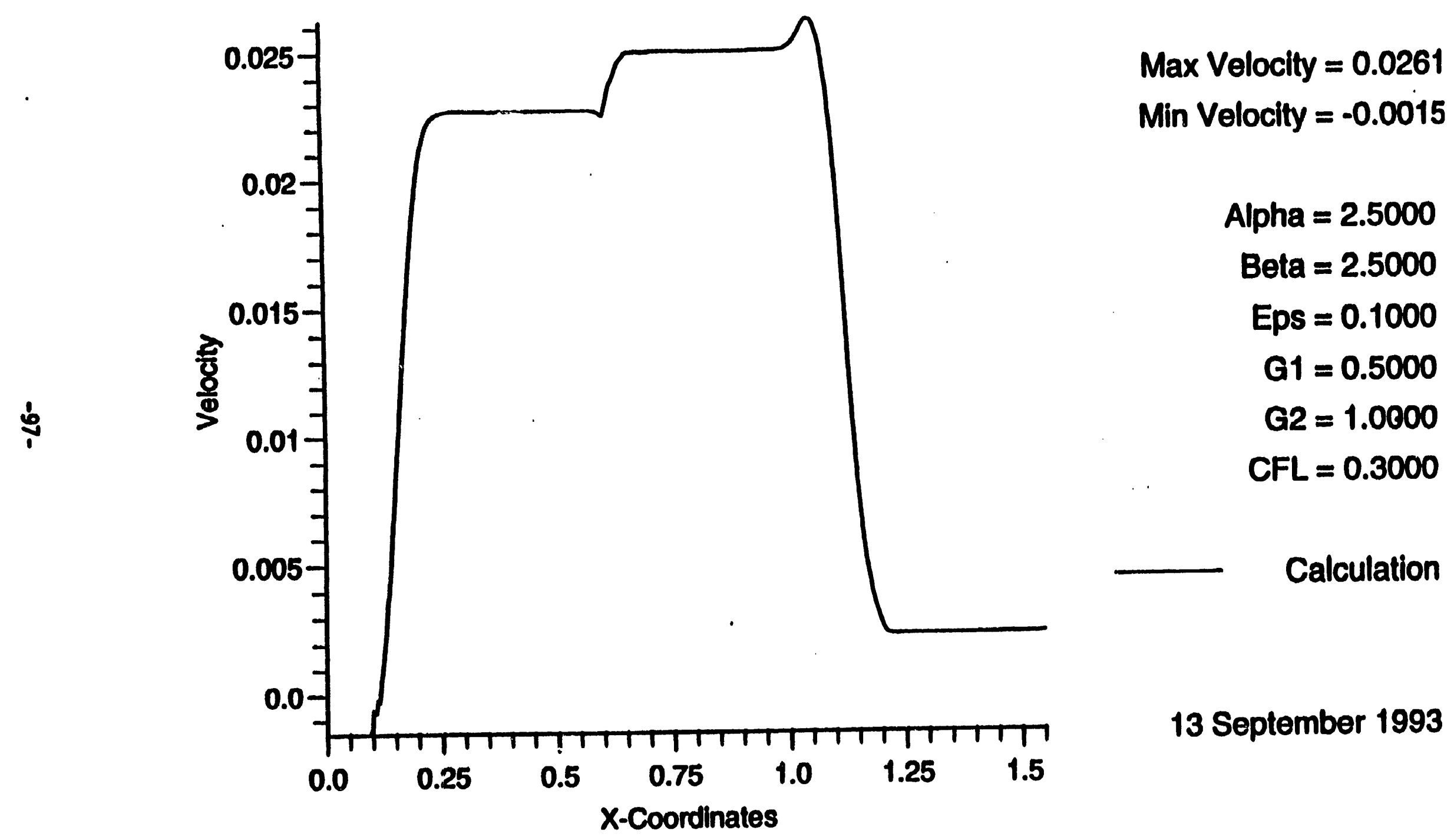


Pressure vs. $X[N P=450$, Time $=1.0000$, lter $=505]$
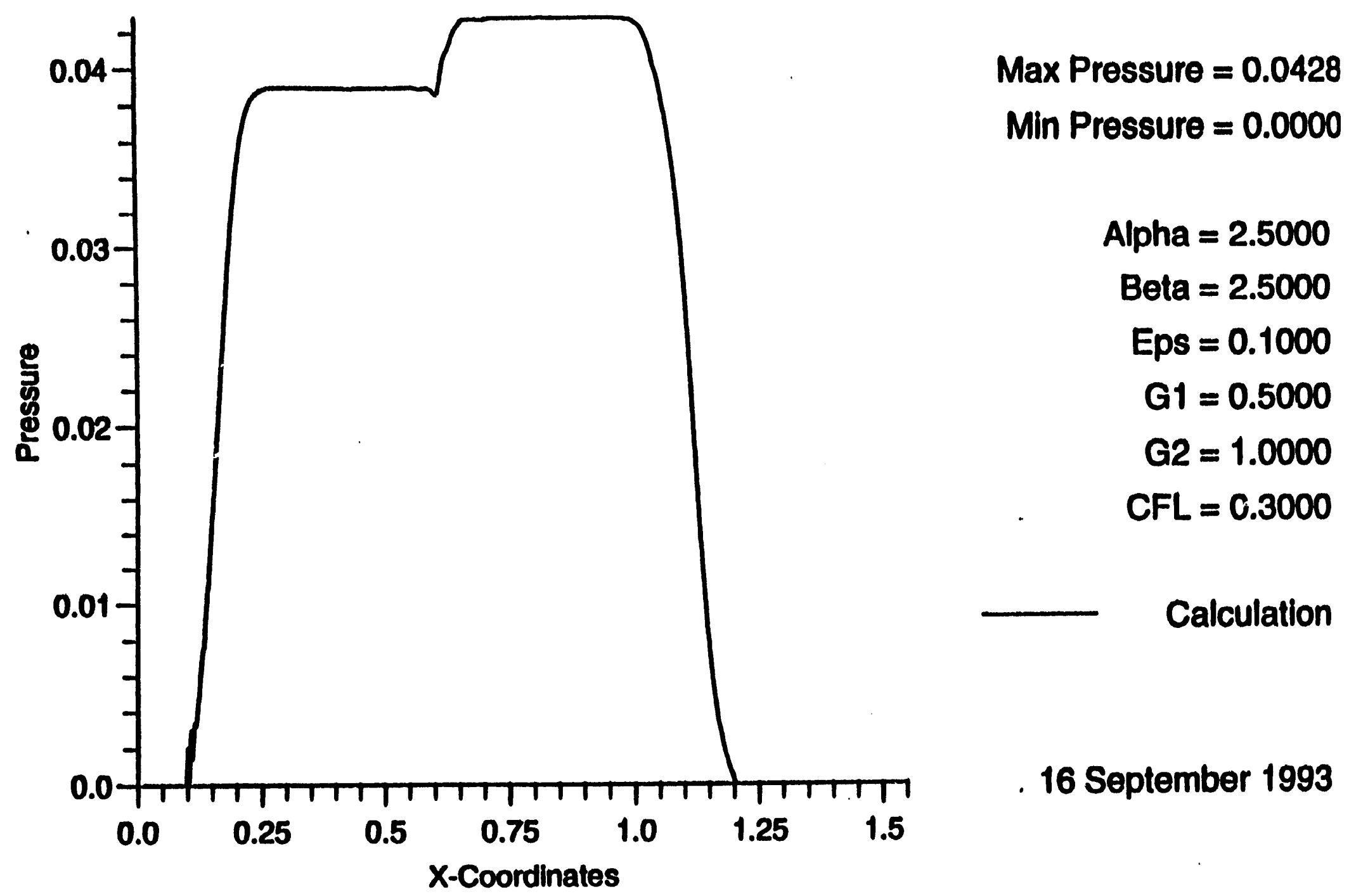
Density vs. $X[N P=450$, Time $=1.0000$, Iter=413]

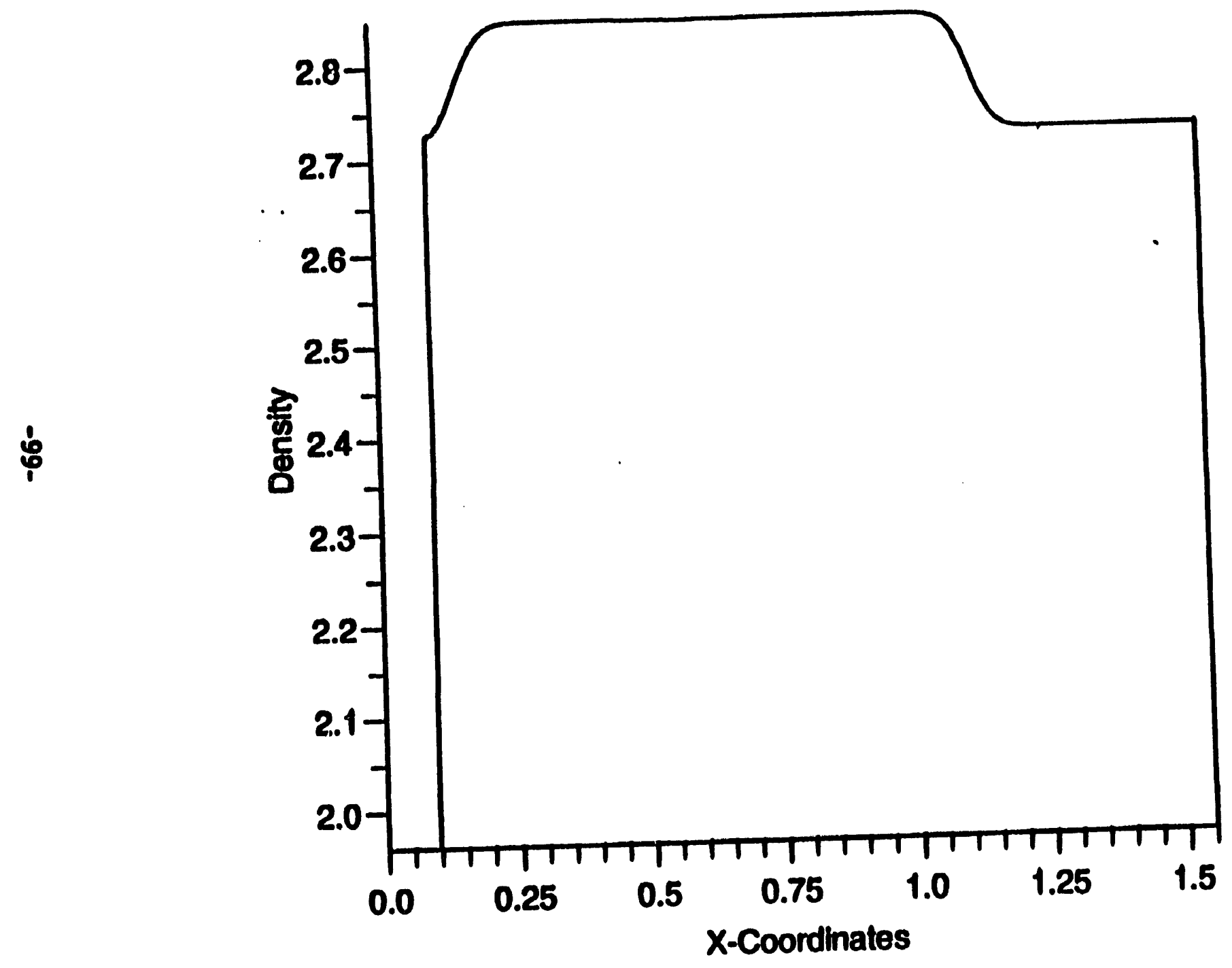

$$
\begin{aligned}
& \text { Max Density }=2.8463 \\
& \text { Min Density }=1.9618 \\
& \text { Alpha }=2.5000 \\
& \text { Beta }=2.5000 \\
& \text { Eps }=0.1000 \\
& \text { G1 }=0.5000 \\
& \text { G2 }=1.0000 \\
& \text { CFL }=0.3000 \\
& \text { Calculation }
\end{aligned}
$$

Al Impact-Summation w. Renorm. 
Velocity vs. $X$ [NP=450, Time=1.0000, Iter=413]

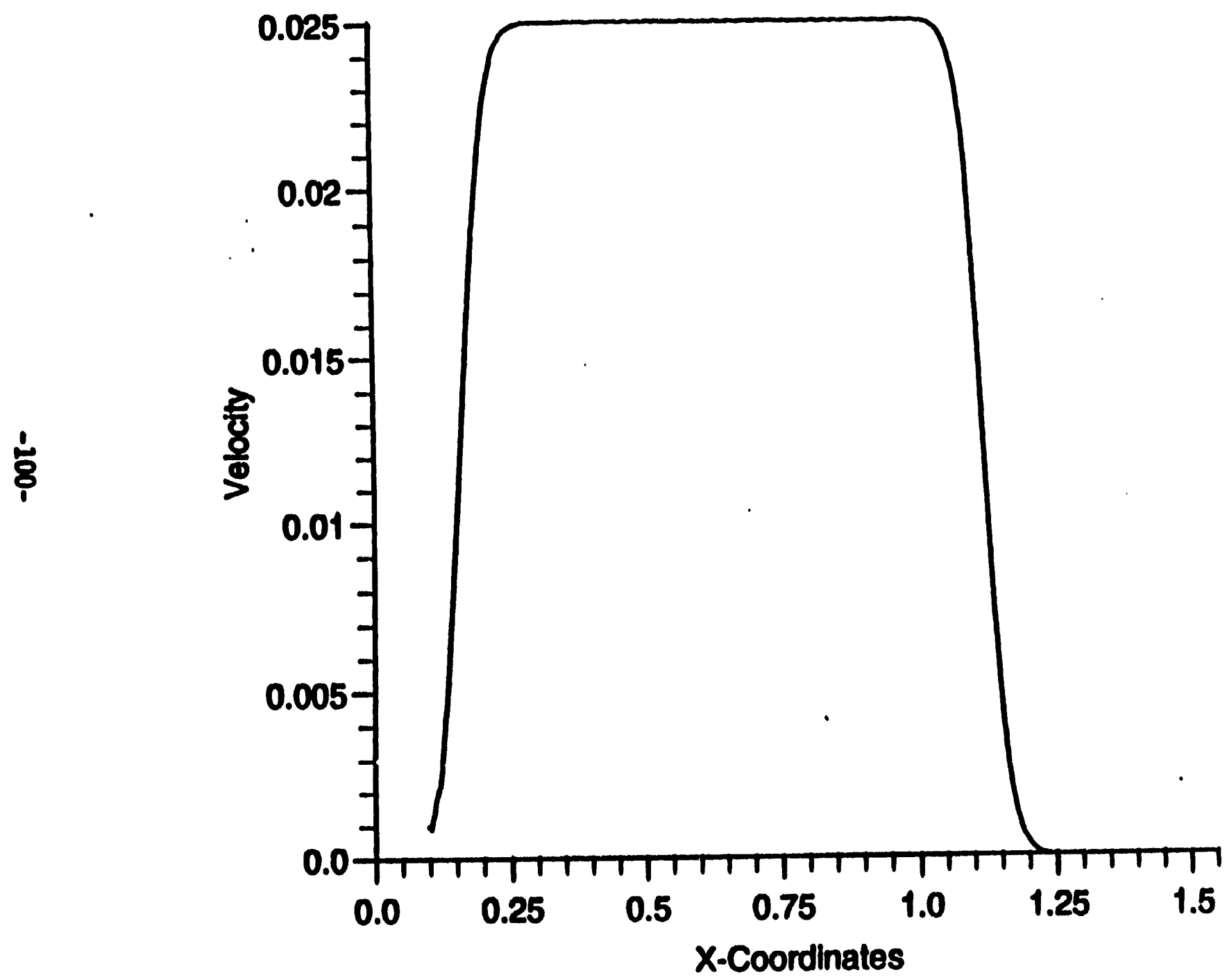

Max Velocity $=0.0250$

Min Velocity $=0.0000$

Alpha $=2.5000$

Beta $=2.5000$

Eps $=0.1000$

G1 $=0.5000$

$G 2=1.0000$

$C F L=0.3000$

Calculation

13 Septembor 1993 
Pressure vs. $X[N P=450$, Tim $\theta=1.0000$, Iter $=413]$

5

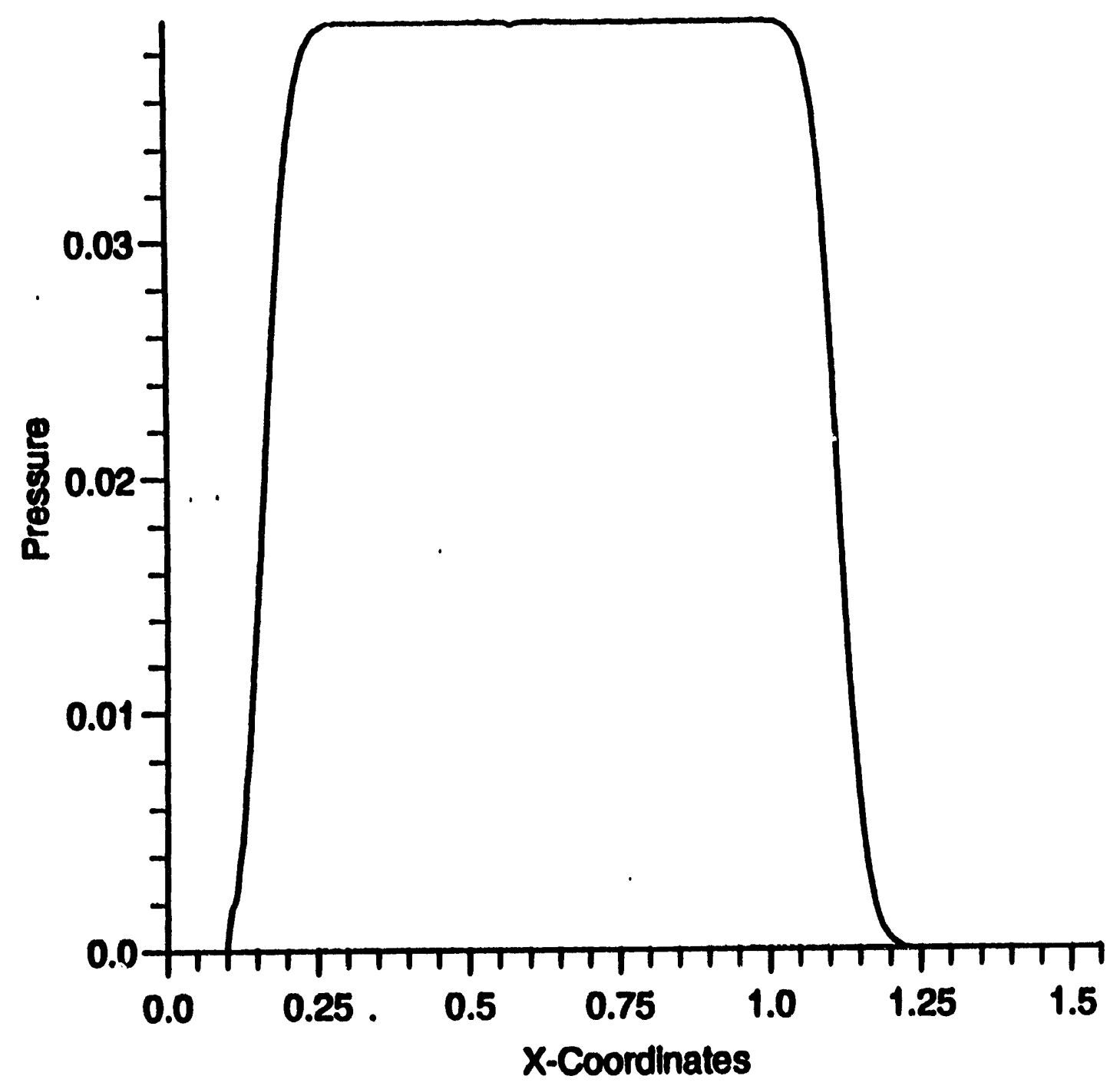

Max Pressure $=0.0392$

Min Pressure $=0.0000$

Alpha $=2.5000$

Beta $=2.5000$

Eps $=0.1000$

$\mathrm{G1}=0.5000$

$\mathrm{G} 2=1.0000$

$\mathrm{CFL}=0.3000$

Calculation

16 September 1993 
Density vs. $X[N P=285$, Time $=1.0000$, Iter $=2810]$

ํㅗำ

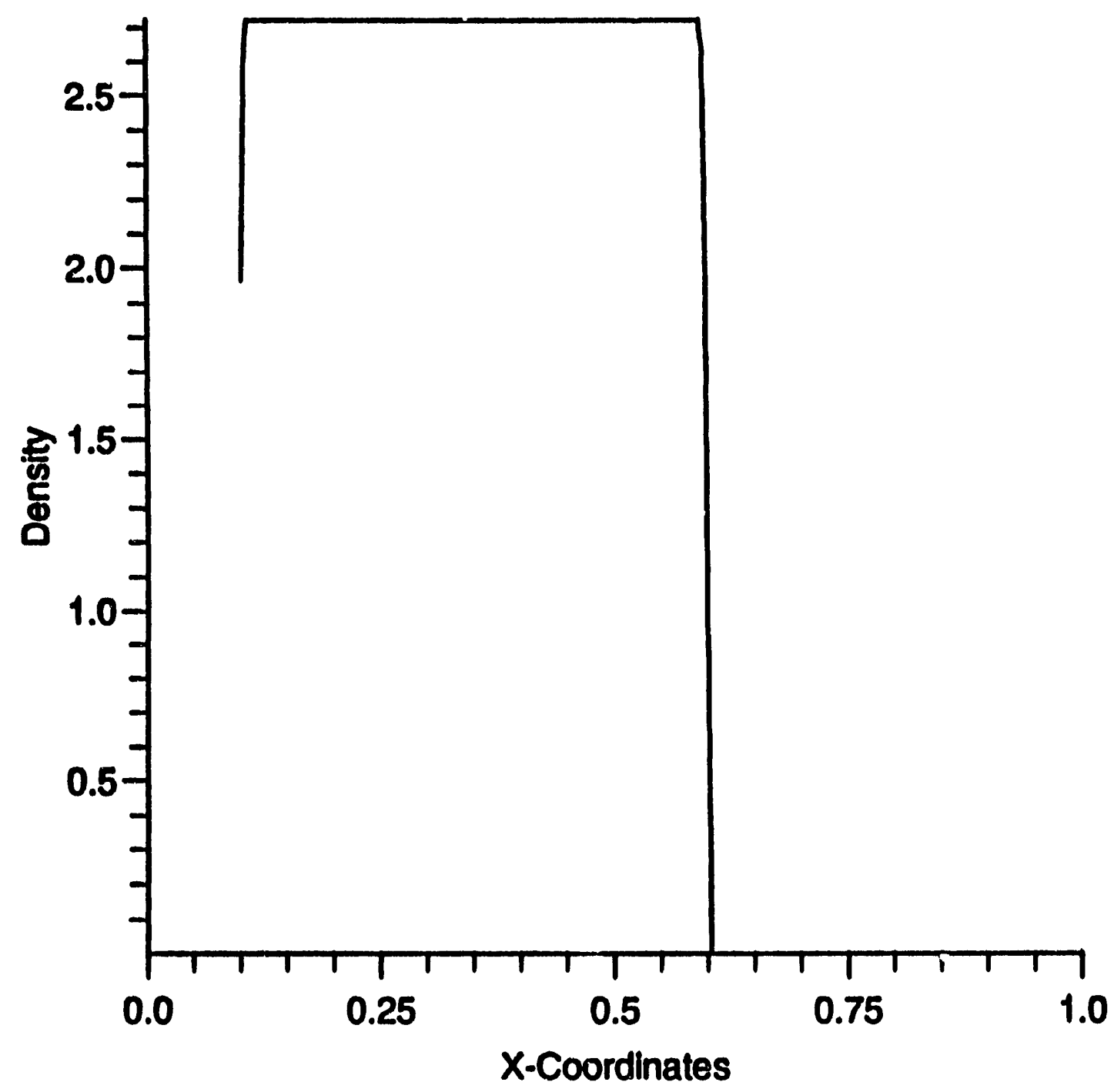

$$
\begin{aligned}
\text { Max Density } & =2.7213 \\
\text { Min Density } & =0.0001
\end{aligned}
$$$$
\text { Alpha }=2.5000
$$$$
\text { Beta }=2.5000
$$$$
\text { Eps }=0.1000
$$$$
\mathrm{G} 1=0.5000
$$$$
\mathrm{G} 2=1.0000
$$$$
\mathrm{CFL}=0.1000
$$

Calculation

20 September 1993

Al piston pushing $A_{i r} \frac{P_{A 2}}{r_{\text {ar }}} \cong 22,000$ 
Velocity vs. $X[N P=285$, Time $=1.0000$, Iter $=2810]$

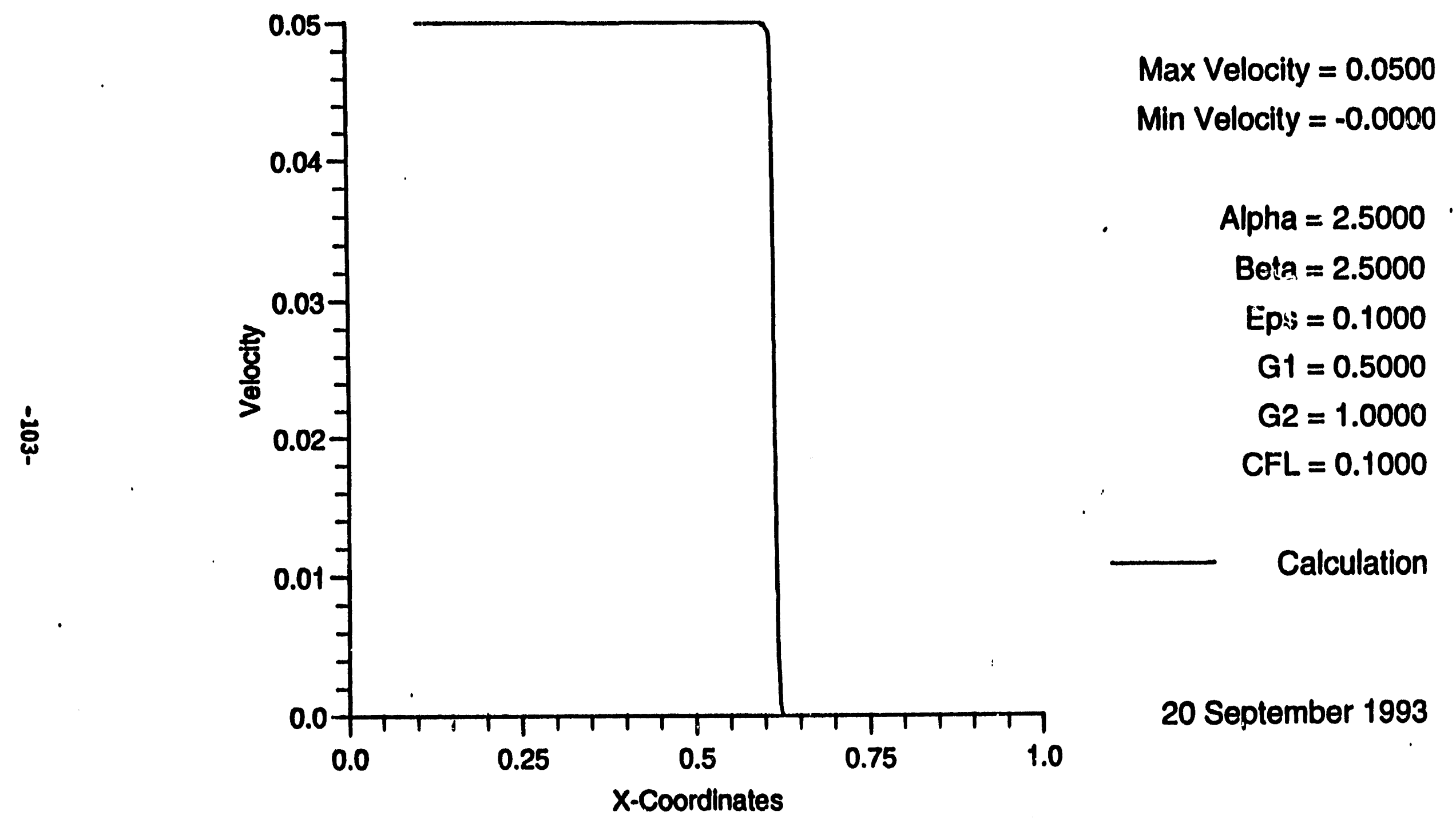


Internal Energy vs. $X[N P=285$, Time $=1.0000$, Iter $=2810]$

$10^{*}$

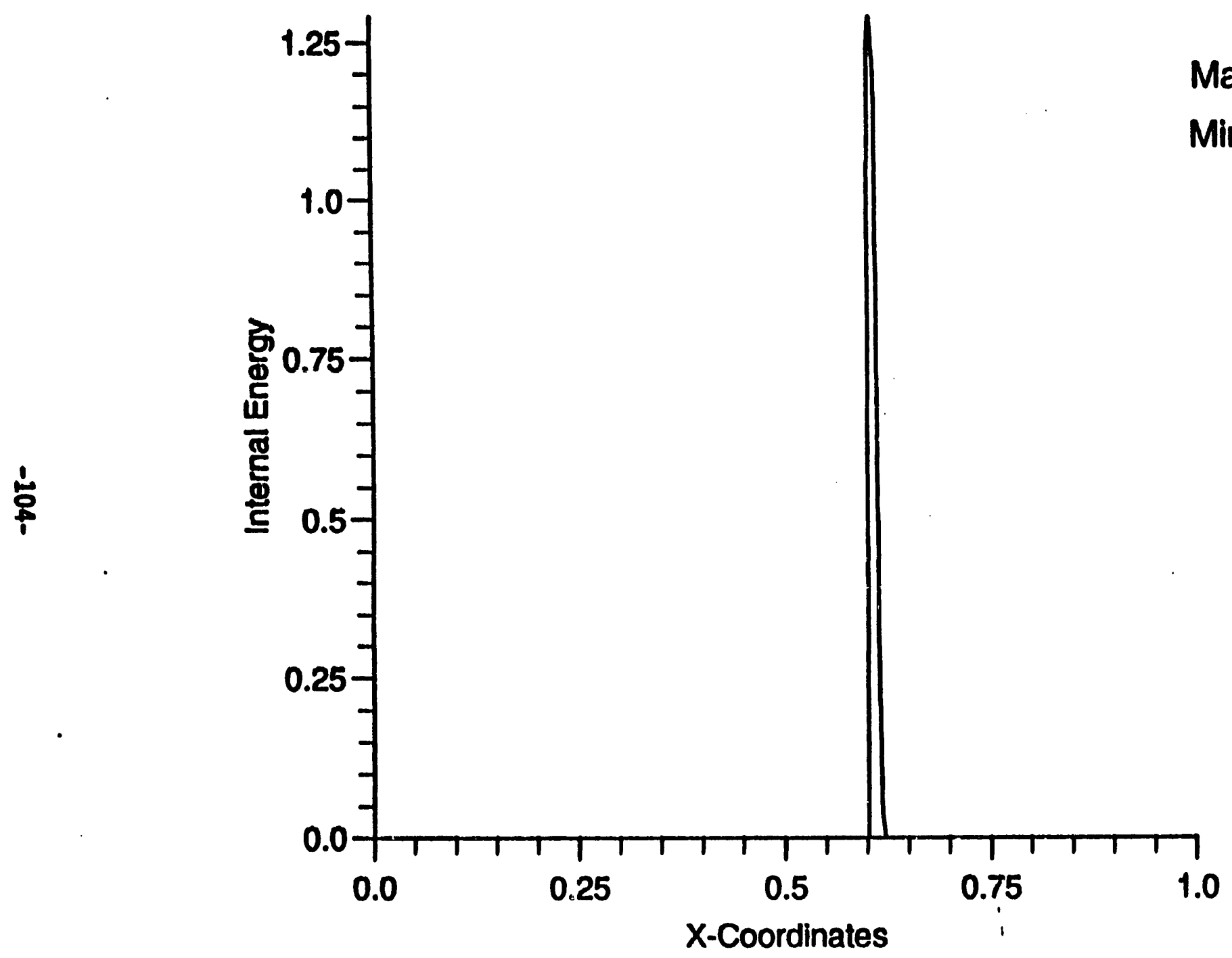

Max Internal Energy $=0.0012$

Min Internal Energy $=-0.0000$

Alpha $=2.5000$

Beta $=2.5000$

Eps $=0.1000$

$\mathrm{G1}=0.5000$

$\mathrm{G} 2=1.0000$

$\mathrm{CFL}=0.1000$

Calculation

20 September 1993 


\title{
Methodology and Tests of Adaptive SPH
}

\author{
Mike Owen * Jens Villumsen ${ }^{\dagger}$ Paul Shapiro \\ Hugo Martell §
}

September 17, 1993

\begin{abstract}
- Deple of Aresceschy, Ohio State Univerity
IDept. of Artreaceny, Ohio State Univarity

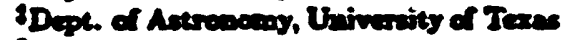

SDept. of Antrosemy, Univerity of Teras
\end{abstract}




\section{Caveat}

The Ohio group (Mike Owen and Jens Villumsen) and the Texas group (Paul Shapiro and Hugo Martell) have collaborated on the basic ideas and goals for the development of Asph, but the two groups have independently derived their own Asph theories and algorithms as well as developeci entirely independent codes based on those ideas. The bodies of work of both of these groups are based on the same basic principles and should give similar results. All the work presented today is the result of our development in Ohio and any questions on this material should be directed to either Mike Owen or Jens Villumsen; questions pertaining to the Texas development should be referred to Paul Shapiro or Hugo Martell. 


\section{Definitions}

Standard Smoothed Particle Hydrodynamics (Sph)

- Utilizes a single isotropic smoothing scale per particle.

- Interpolation kernel $W(\vec{r}, h)$ may be expressed as

$$
W\left(\vec{r}, h_{s}\right)=W\left(\frac{|\vec{r}|}{h_{s}}\right)
$$

- Smoothing scale allowed to vary spatially from particle to particle as well as temporally.

- Artificial viscosity is implemented for any convergent flow within the gas.

\section{Adaptive Smoothed Particle Hydrodynamics (Asph)}

- Utilizes an anisotropic smoothing scale per particle, as embodied by the $\mathbf{H}$ tensor.

- The smoothing tensor is allowed to vary both spatially and temporally, as in Standard Sph.

- The interpolation kernel $W(\vec{r}, \mathbf{H})$ may be expressed as

$$
W(\vec{r}, \mathbf{H})=W(\mathbf{H} \vec{r})
$$

- There are two current variations of Asph, unsuppressed and suppressed: 
- Unsuppressed Asph utilizes the artificial viscosity for any convergent flow, as in Standard Sph.

- Suppressed Asph employs a more restrictive algorithm for implementing the artificial viscosity. 
3 Why use Asph?

- Through the use of aspherical kernels, phenomena that are nonisotropic can be better represented (better resolution for a given number of Sph nodes).

- Standard Sph: $h_{s} \propto \rho^{-\frac{1}{2}}$ in $2 \mathrm{~d} ; h_{s} \propto \rho^{-\frac{1}{3}}$ in 3d.

- Asph: $\mathbf{H}$ is allowed to adapt in arbitrary direction to nonisotropic density evolution, thereby improving resolution; so long as the radius of the anisotropy (shock) is greater than its typical thickness, Asph will have the advantage.

- We are primarily interested in simulating the evolution of structure in the early universe, where in general gravitational instability drives highly nonisotropic evolution. Asph has been tuned to solve the Zeldovich pancake problem as well as possible, as we consider this our canonical problem.

- Asph is in a sense more Lagrangian than Standard Sph, as we are able to more accurately track and maintain a consistent set of neighbors for a given Asph node. The goal of the Asph algorithm is to maintain an equal number of neighboring nodes in all directions from the node of interest. 
- Spurious heating resulting from overuse of the artificial viscosity can artificially interfere with the collapse of the Sph gas and influence the development of the fine structure we wish to examine (galaxies, clusters, etc). 


\section{Disadvantages to Asph}

- Asph is more computationally expensive than Standard Sph for a given number of nodes; this is hopefully balanced by the improved resolution.

- Asph is not guaranteed angular momentum conservation since forces are not in general radial. We believe in fact angular momentum conservation will be met so long as the shape of the smoothing kernel faithfully follows the shape of the underlying fluid element, but this remains to be demonstrated; regardless angular momentum conservation is not rigorously guaranteed as in Standard Sph.

- Our current artificial viscosity suppression algorithm mildly violates energy conservation, but this is a small effect. 
5 The $\mathrm{H}$ tensor transformation

- The $\mathbf{H}$ transformation maps from real position space to normalized position space:

- Standard Sph: $\vec{h}=\vec{r} / h_{s}$

- Asph: $\vec{h}=\mathbf{H} \vec{r}$

- Shape of anisotropic smoothing volume as defined by $\mathbf{H}$ is restricted to be elliptical in $2 \mathrm{~d}$ or ellipsoidal in $3 \mathrm{~d}$ :

$-2 \mathrm{~d}$ : $\mathbf{H}$ is a symmetric $2 \times 2$ matrix

$-3 \mathrm{~d}$ : $\mathbf{H}$ is a symmetric $3 \times 3$ matrix

6 Evolving the $\mathrm{H}$ transformation

- The smoothing volume for an individual Asph node is treated analogously to a fluid element, and is therefore evolved in accordance with the changes in the local velocity field as embodied by the rate-of-strain tensor $\sigma=\partial \vec{v} / \partial \vec{r}$

- $\mathbf{H}$ is evolved through a first order treatment of the local velocity field.

$$
\Delta \vec{v}=\sigma \Delta \vec{r}
$$

Restriction to first order transformations on $\mathbf{H}$ guarantees $\mathbf{H}$ will remain symmetric and therefore elliptical (ellipsoidal). 


\section{Artificial Viscosity Suppression}

We are attempting to develop a more restrictive criterion for the use of the artificial viscosity than the traditional use for any convergent flow. Our reasoning behind this effort is:

- The traditional criterion for the artificial viscosity switch on is overkill in the sense the the artificial viscosity will be active for gas which is not undergoing shocking.

- Overuse of the artificial viscosity can spuriously heat the system, which is potentially a serious problem for collapse simulations.

Some characteristics of our new (and experimental) criterion for the artificial viscosity:

- It distinguishes between the application of the artificial viscosity in the momentum and energy equations.

- It is parameterized in terms of the resolution over the scale of interest and the local velocities-basically a closing time criterion on a pair-by-pair basis. 
- The new criterion does not explicitly rely on characteristics of Asph and could in principle be applied in Sph as well. However, it is uncertain whether the lower effective resolution of Sph would negate the usefulness of the criterion; Asph's improved resolution should give it better stopping power (larger $|\partial \vec{v} / \partial \vec{r}|)$.

- Our current parameterization of the criterion has been purely empirically cailbrated against the $2 \mathrm{~d}$ Zeldovich pancake problem.

- Much more work is needed here to extend the usefulness (and testing) of the criterion. 
8 Technical aspects of the code

- Our current (A)Sph code at Ohio State is maintained under a make system and is configurable through preprocessor flags at compile time for a variety of situations. These include:

$-1,2$, or $3 d$

- Standard Sph or Asph

- Cosmological or Standard space dynamical equations

- Choice of Cosmological models

- With or Without gravity

- Suppressed or Unsuppressed Artificial Viscosity

- A choice of symmetrization schemes in the dynamical equations

- Individual smoothing scales $\left(\boldsymbol{h}_{s}\right)$ or $\mathbf{H}$ transformations are maintained for each Sph/Asph node.

- The code features an asynchronous integrator based on a second order Runge-Kutta scheme; this allows us to maintain individual times and timesteps for each Sph node. The asynchronous algorithm has been designed with the following characteristics:

- implemented with assumption that on "local" scales (scales the size of a local smoothing kernel) times and timesteps will be similar. 
- Basic algorithm:

* Every particle is assigned a current time $\left(t_{i}\right)$ and a target time $\left(t_{i}^{\text {targ }}=t_{i}+\Delta t_{i}\right)$.

* The integrator looks globally for the smallest target time.

* All particles with this smallest target time are selected and integrated (in local groups as batches, if possible). * The newly integrated particles are assigned their new current and target times, and the cycle repeats.

- When compiled for cosmology the code integrates all quantities in terms of a povser of the expansion factor rather than time $\left(p=a^{\alpha}\right)$.

- The code has been optimized for use on the Cray YMP at the Ohio SuperComputer Center-typically hits calculation speeds on the order of 100 Mflops or over (problem dependent).

- Current status in development of code:

- Standard Sph fully implemented and tested in 1, 2, and $3 d$.

- Asph fully implemented in 2d; partially in 3d. 


\section{Tests of Asph and the code}

We have run a variety of test problems for the sake of testing both our code and the technique of Asph. In standard space for both Standard Sph and Asph we have run:

- the Riemann shocktube problem in 1,2, and 3d

- the Sedov blastwave solution for 1d and 2d

In cosmological scenerios we have run (both Sph and Asph):

- the Zeldovich pancake solution for $1 \mathrm{~d}$ and $2 \mathrm{~d}$

- the growth of a void in $1 \mathrm{~d}$ and $2 \mathrm{~d}$ (essentially a gravitationally driven blastwave)

- a 2d Hot Dark Matter model

We have focussed on the Zeldovich pancake as our canonical problem, as we expect this to generically represent the sort of situation we are interested in. 
10 2d Shocktube Simulation

- High Density region initial conditions:

$$
-\rho_{i}=1, P_{i}=1, v_{i}=0 \forall x<0
$$

- Low Density region initial conditions:

$$
-\rho_{i}=0.25, P_{i}=0.1795, v_{i}=0 \quad \forall x>0
$$

- $t_{i}=0, t_{f}=0.15$

- Periodic sysiem $(x, y) \in([-1,1],[-1,1])$

- Particles seeded on initial grid

- $\gamma=c_{P} / c_{V}=1.4$

\section{Numerical Simulation Parameters:}

- $N_{\text {beryon }}=25000$

- High density region: $N_{x}=100, N_{y}=200$

- Low density region: $N_{x}=50, N_{y}=100$

- $d t \in\left[5 \times 10^{-6}, 0.005\right]$

- $h_{s} \in[0.005,0.05]$ 
2d Shocktube @ t=0.15: Asph
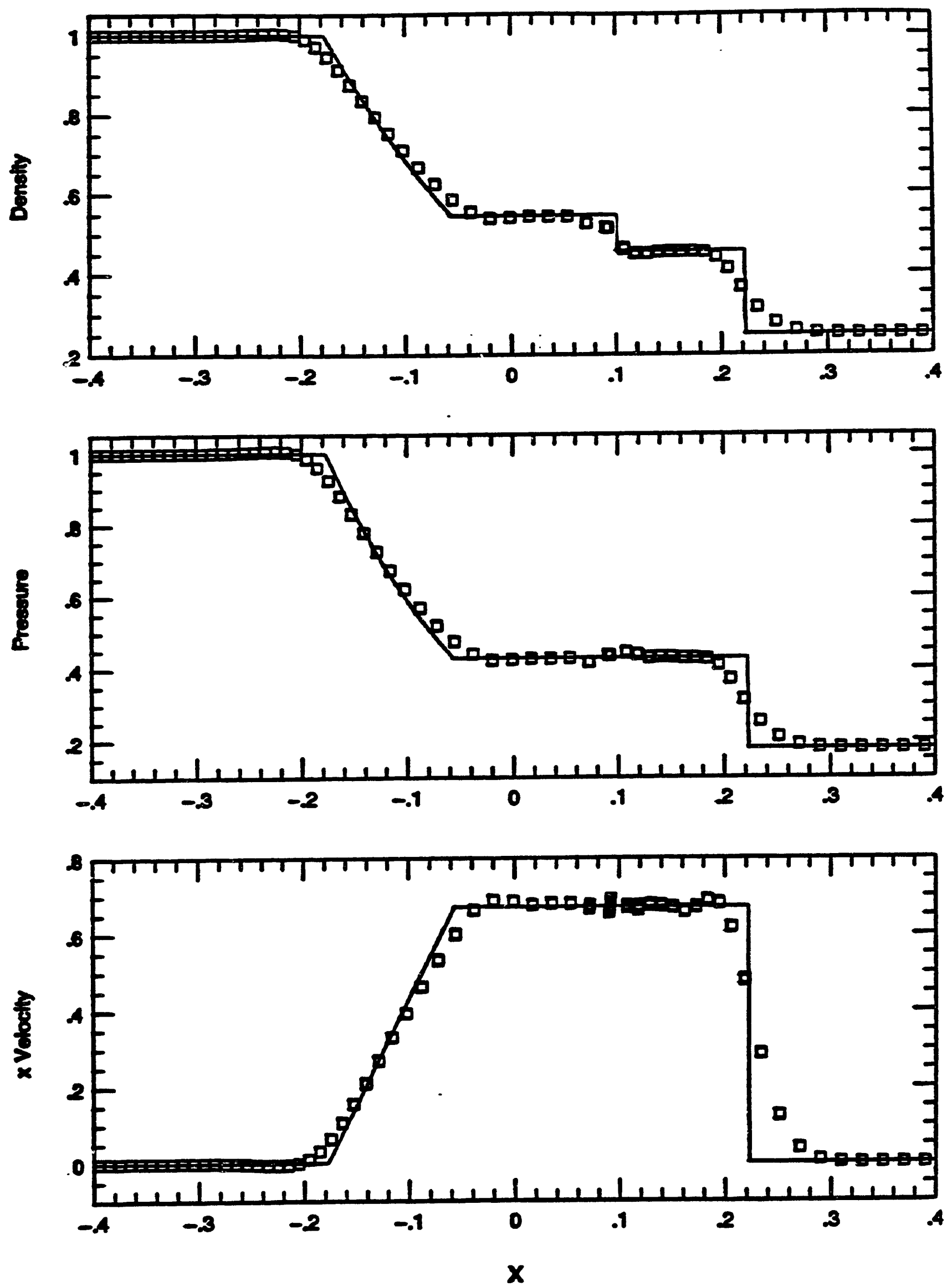
11 Single Wave Planar 2d Zeldovich Pancake Simulations

Background Cosmological and Physical Parameters:

- Einstein-DeSitter cosmology $(\Omega=1, \Lambda=0)$

- $\Omega_{\text {beryon }}=\Omega_{d m}=0.5$

- $H_{0}=50 \mathrm{~km} / \mathrm{sec} / \mathrm{Mpc}$

- $a_{\text {initial }}=1, a_{\text {erunch }}=4, a_{\text {final }}=5, a_{0}=1000$

- $z_{\text {initial }}=999, z_{\text {erunch }}=249, z_{\text {final }}=199$

- $l_{b o x}=10 \mathrm{Mpc} @ a=1 \Rightarrow l_{b o s}=50 \mathrm{Mpc} @ a=5$

- $k_{x}=0, k_{y}=1$

- $T_{i}=3000 \mathrm{~K} \Rightarrow \epsilon_{i}=1.49 \times 10^{-13}$

- $\gamma=c_{P} / c_{V}=5 / 3$

- Pure Hydrogen gas $(\mu=1)$

- No Radiative Cooling implemented

Numerical Simulation Parameters:

- $N_{\text {baryon }}=N_{d m}=4096$

- $\alpha=1,\left(p=a^{\alpha}\right)$

- $d p \in\left[10^{-4}, 0.05\right]$

- $h_{s} \in\left[10^{-5}, 0.1\right]$

- For Asph smoothing kernel axis ratios limited to $h_{2} / h_{1}>0.01$ 


\section{2d Single Wavelength Zeldovich pancake: Baryon positions @ $a=5.0\left(a_{\text {orumet }}=4.0\right)$}

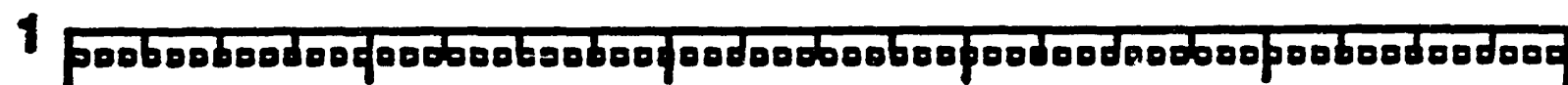

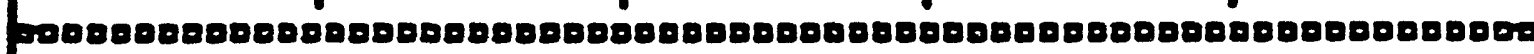

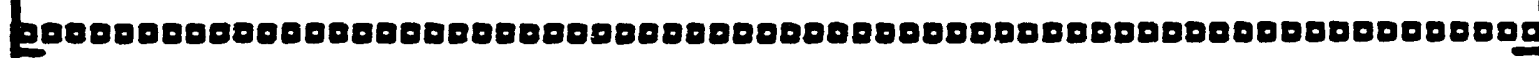

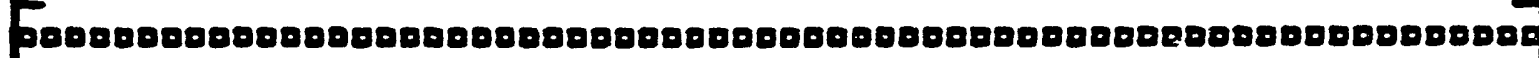

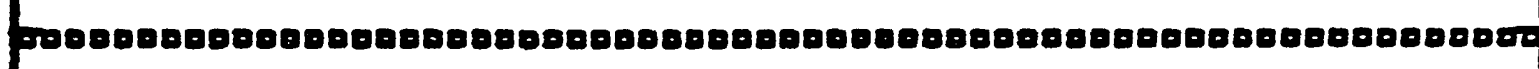

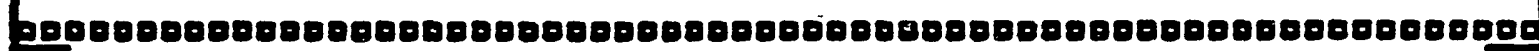

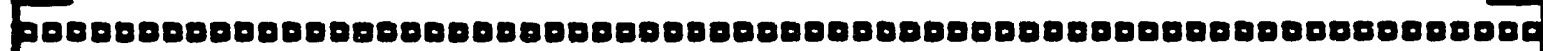

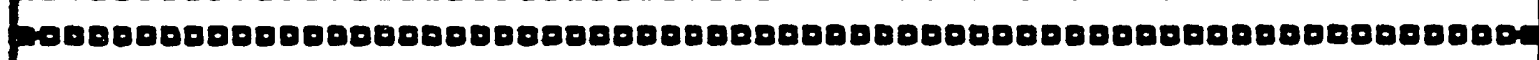

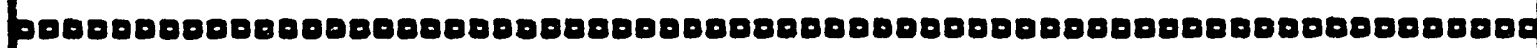

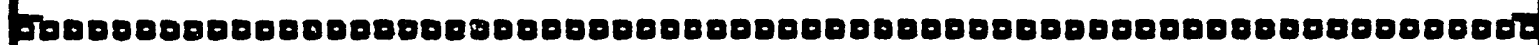

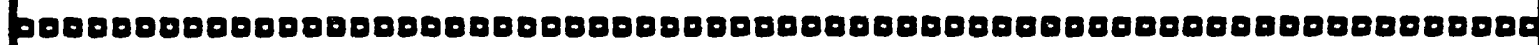

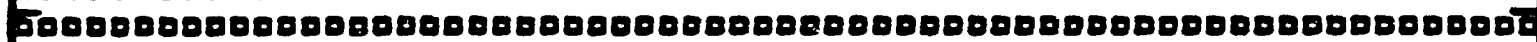
200000000000000800000000000000000000000080000800000000000000800.

.6 F00000000000000000000000000000000000000000000000000000000000000 5000000000000000000000000000000000000000000000000000000000000000

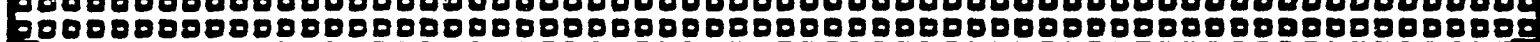

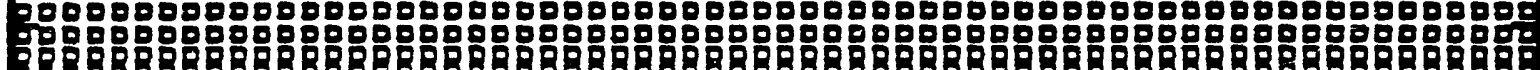

E.

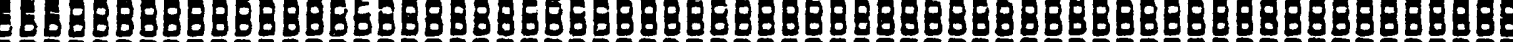
○

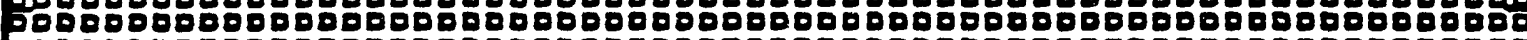
3000080000000000000000000000000000000000000000000800000000080000

. h מ

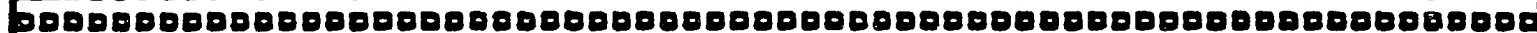

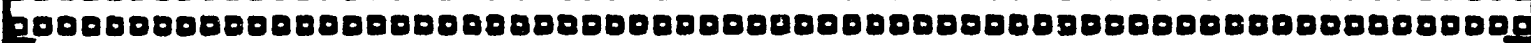
Sอ

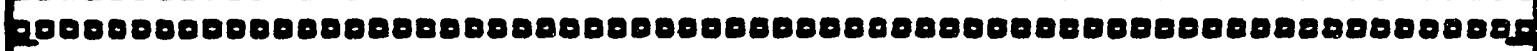

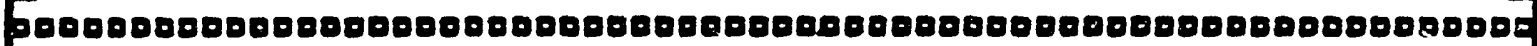

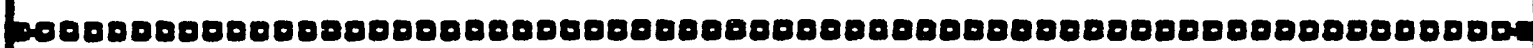

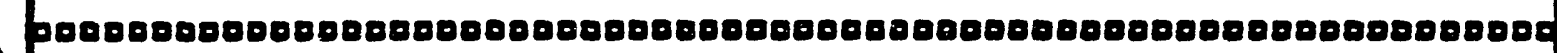

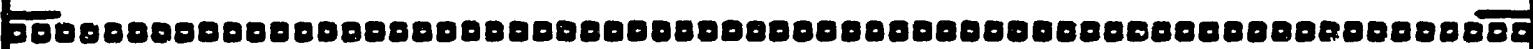

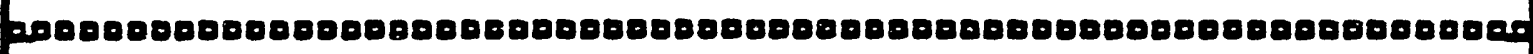
6008000008000000800800000000808000800800000800008008000000000000

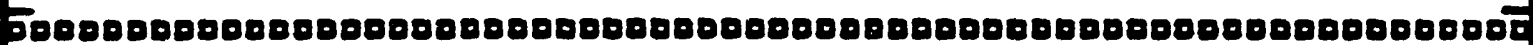
2008000000008000000080000800000000000000000800000008000000080800 0

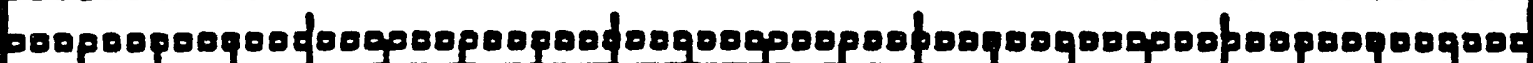




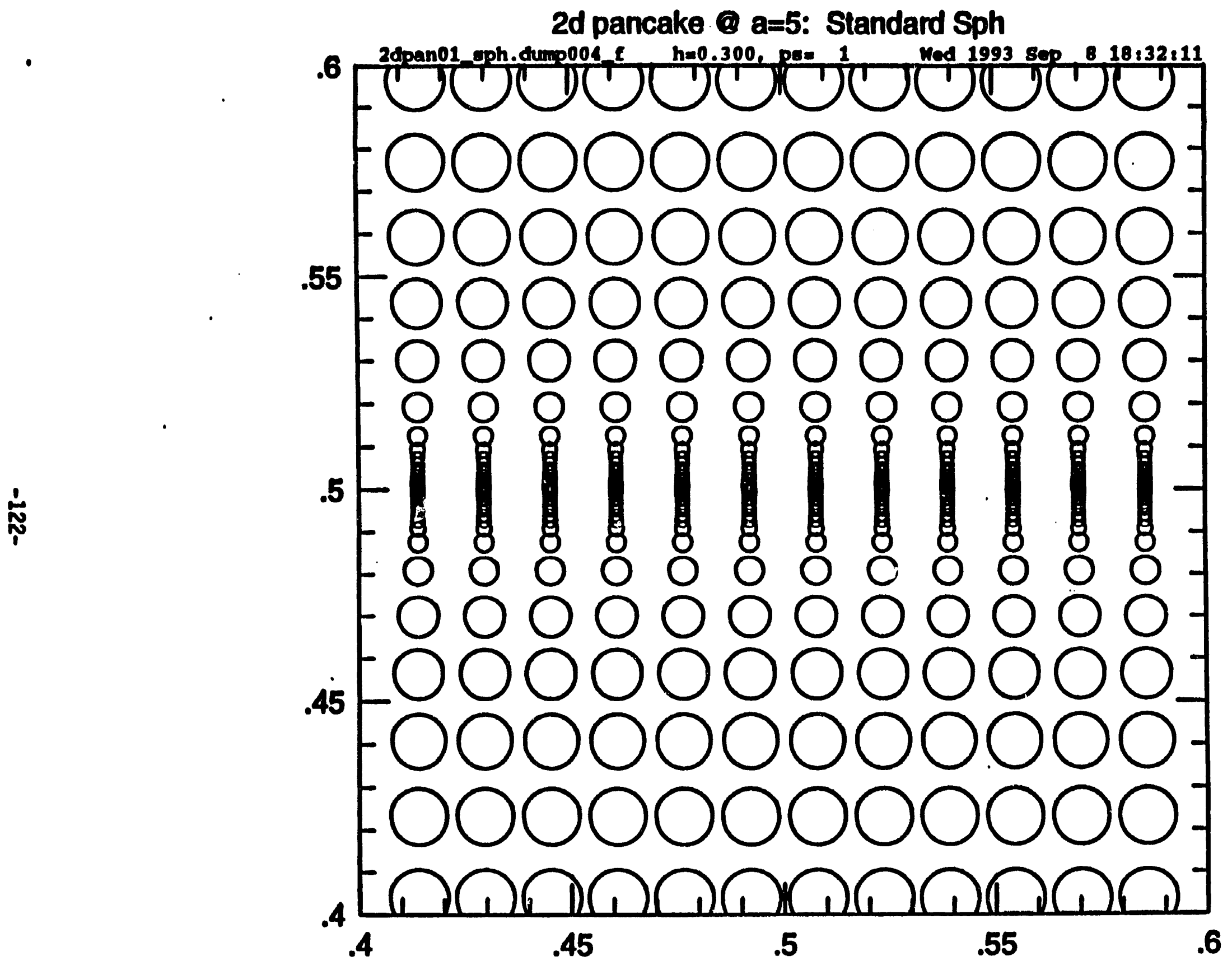




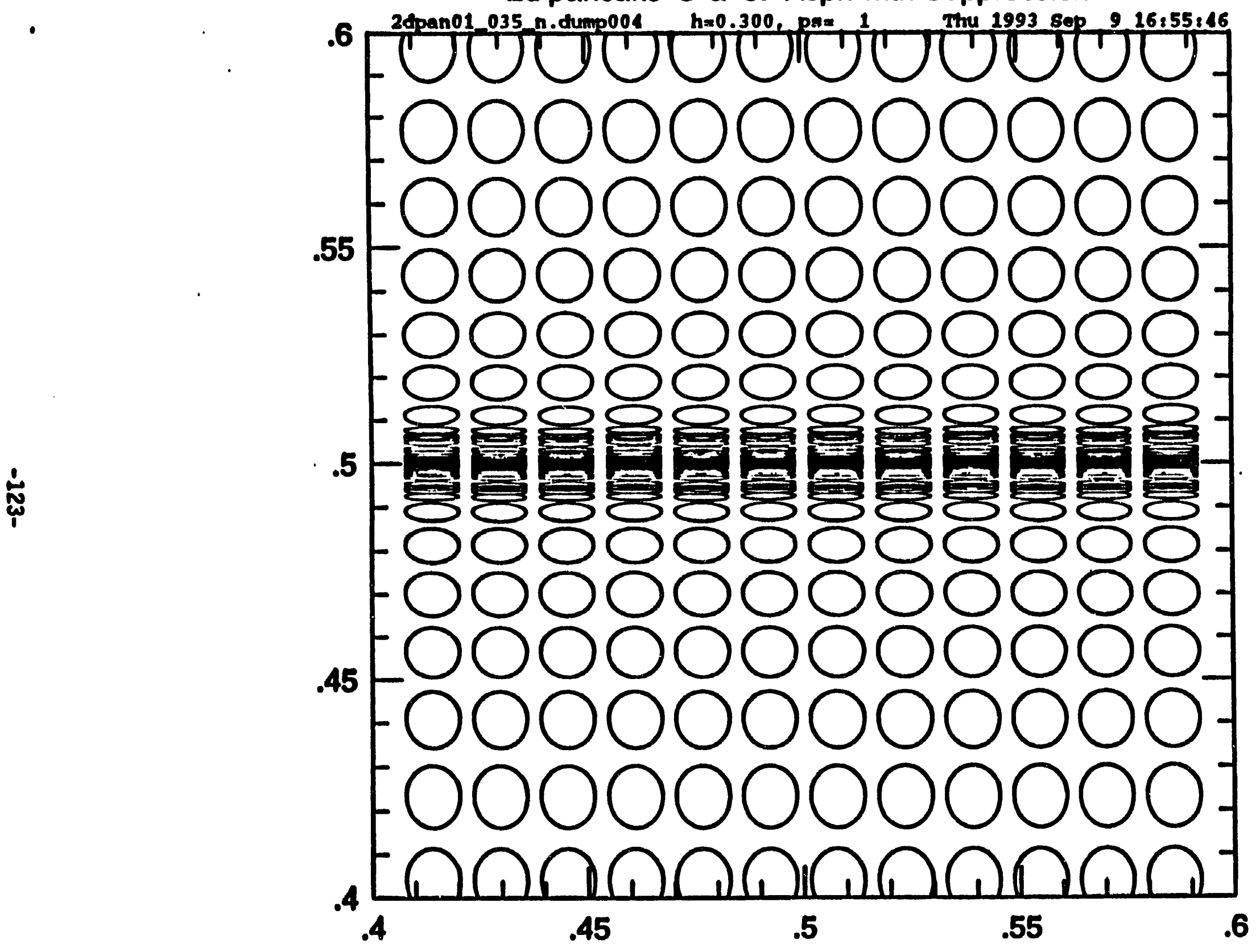


2d pancake $Q a=5\left(a_{\text {cunch }}=4\right):$ Standard Sph
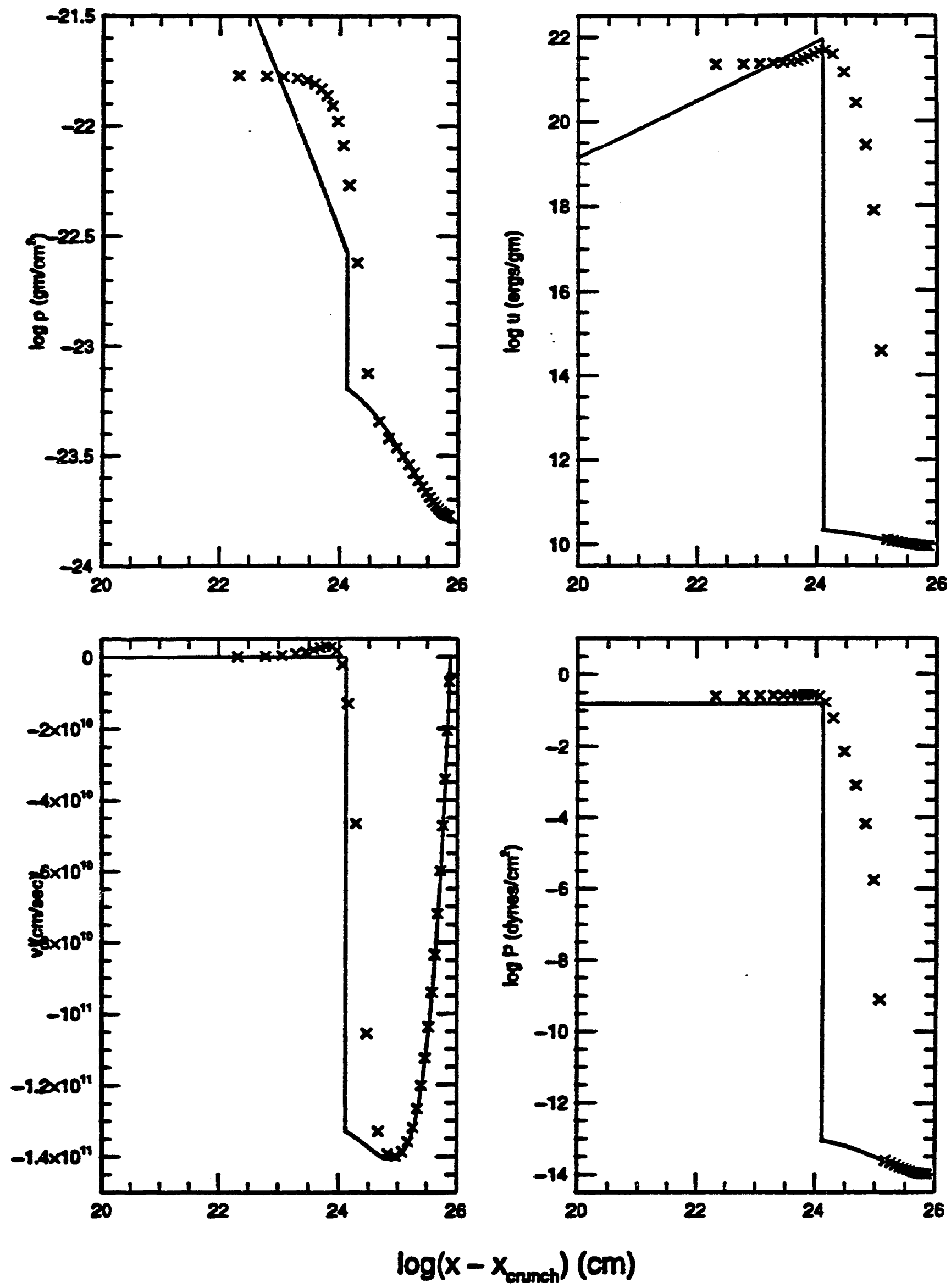
2d pancake @ $a=5\left(a_{\text {crumch }}=4\right):$ Unsuppressed Asph
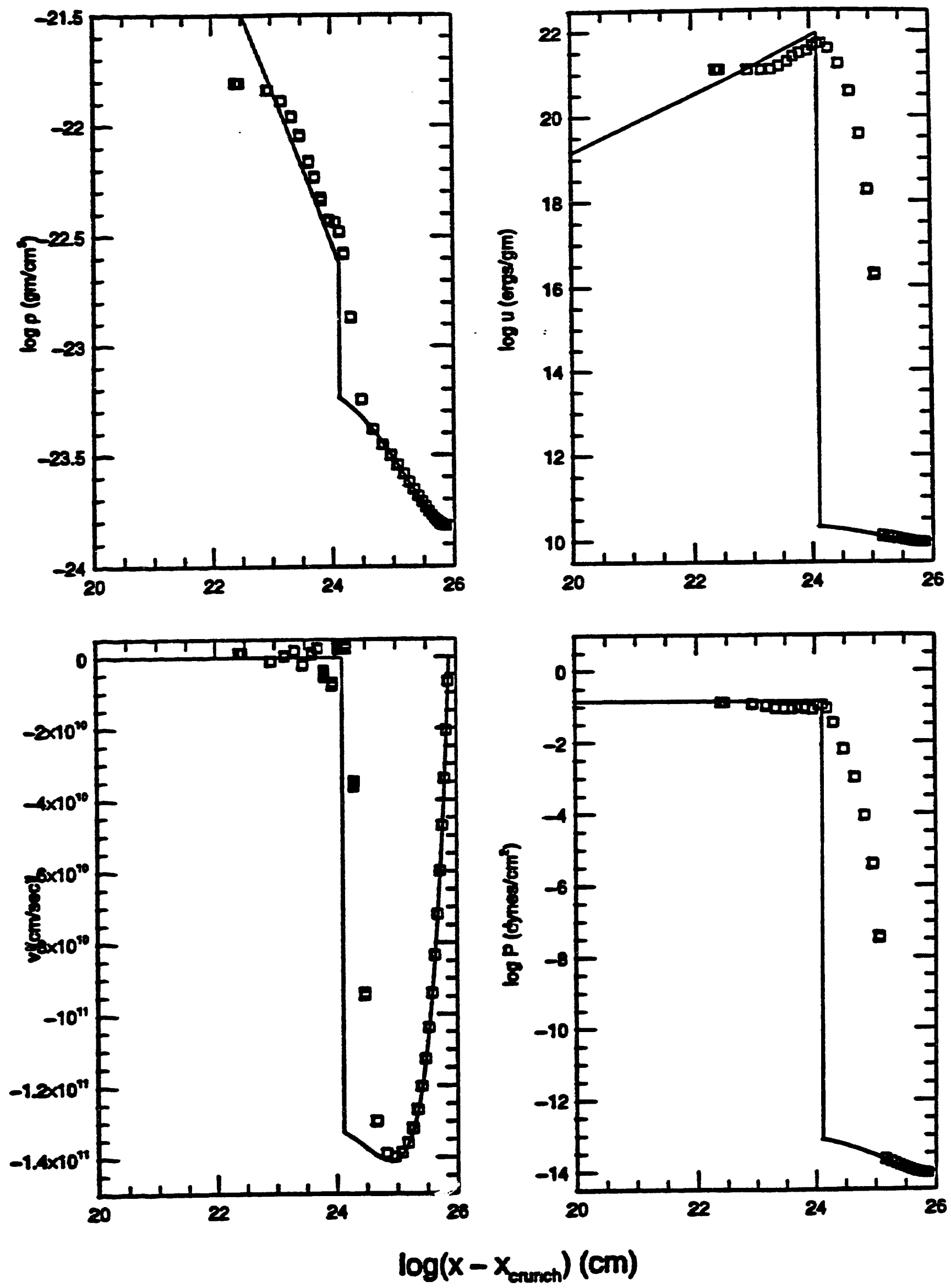


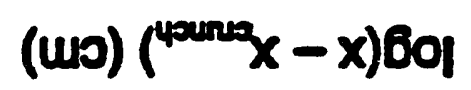
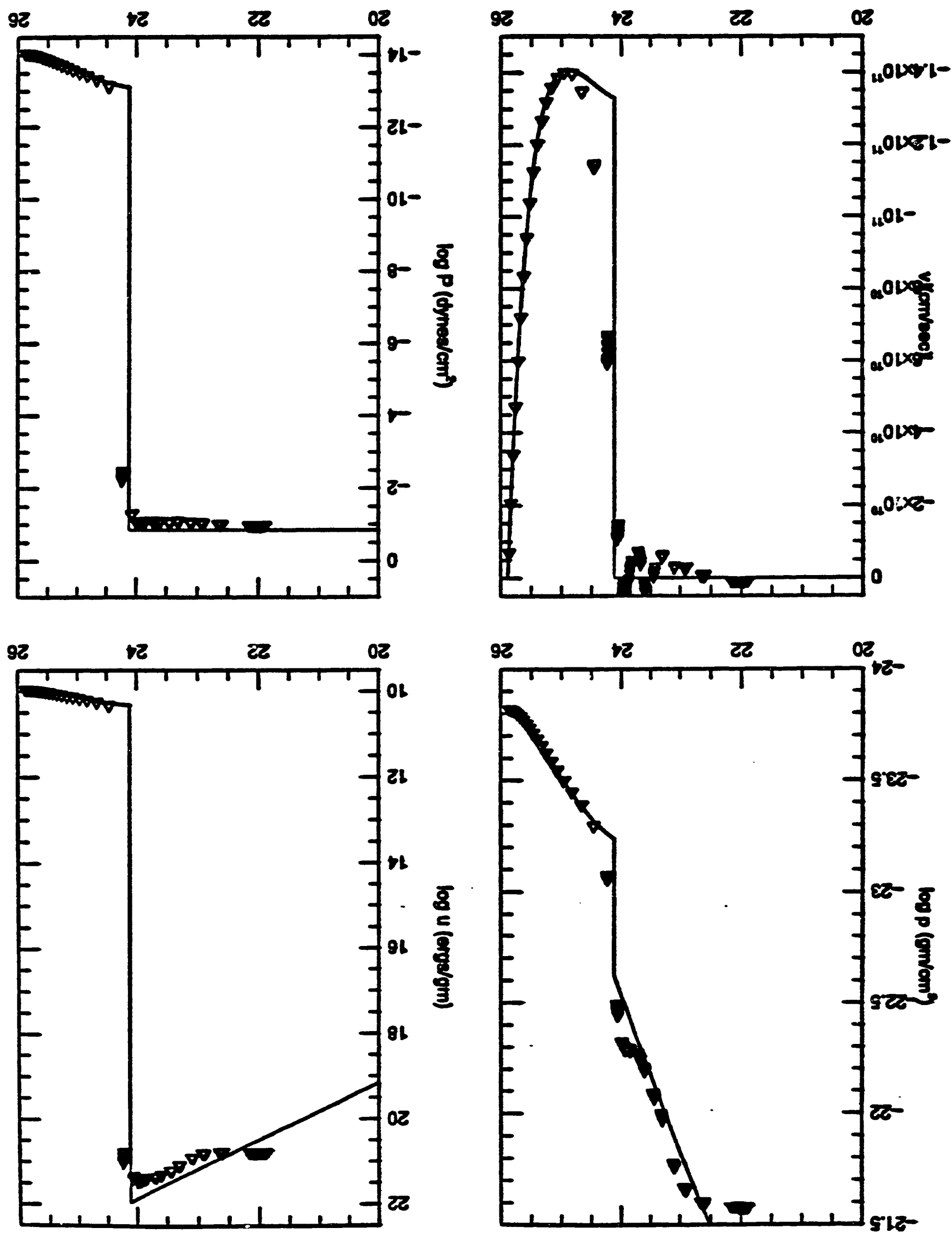

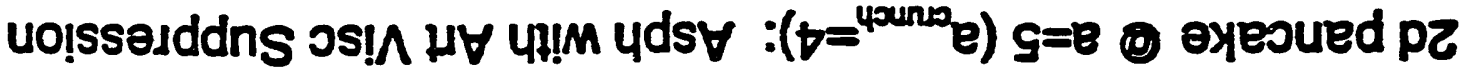


12 Tilted-Shifted Planar 2d Zeldovich Pancake Simulations Background Cosmological and Physical Parameters:

- Einstein-DeSitter cosmology $(\Omega=1, \Lambda=0)$

- $\Omega_{\text {beryon }}=0.1 \Omega_{d m}=0.9$

- $H_{0}=50 \mathrm{~km} / \mathrm{sec} / \mathrm{Mpc}$

- $a_{\text {initial }}=1, a_{\text {crunch }}=4, a_{\text {final }}=5, a_{0}=1000$

- $z_{\text {initial }}=999, z_{\text {erunch }}=249, z_{\text {final }}=199$

- $l_{b a x}=10 \mathrm{Mpc} @ a=1 \Rightarrow l_{b o x}=50 \mathrm{Mpc} @ a=5$

- $k_{x}=2, k_{y}=1$

- $T_{i}=3000 K \Rightarrow \epsilon_{i}=1.49 \times 10^{-13}$

- $\gamma=c_{P} / c_{V}=5 / 3$

- Pure Hydrogen gas $(\mu=1)$

- No Radiative Cooling implemented

Numerical Simulation Parameters:

- $N_{\text {baryon }}=N_{d m}=4096$

- $\alpha=1,\left(p=a^{\alpha}\right)$

- $d p \in\left[10^{-4}, 0.05\right]$

- $h_{z} \in\left[10^{-5}, 0.1\right]$

- For Asph smoothing kernel axis ratios limited to $h_{2} / h_{1}>0.01$ 
2d tilted-shifted pancake: Baryon particle positions $@ a=5\left(a_{\text {crunch }}=4\right)$

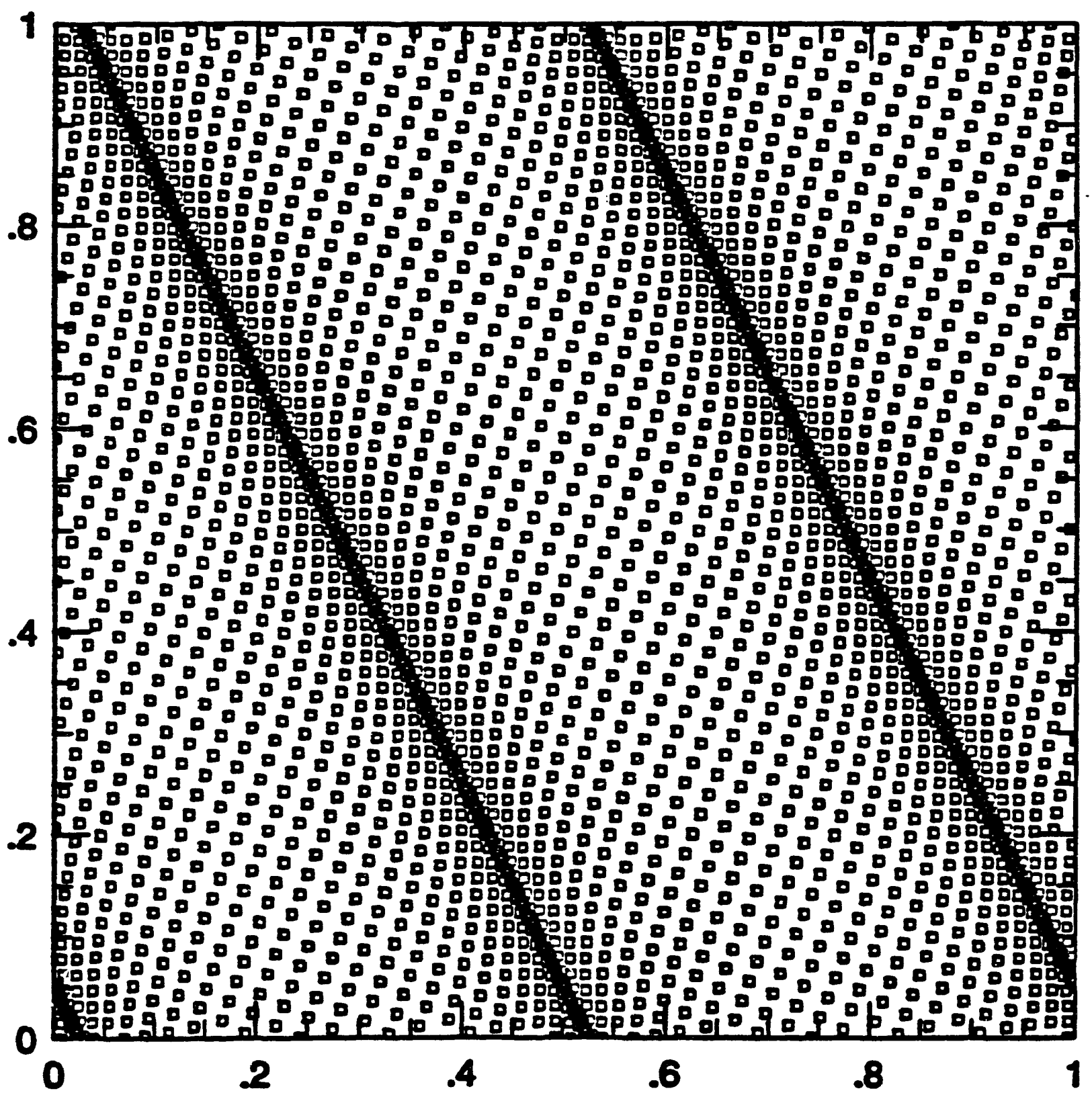


2d tilted-shifted pancake a=5: Standard Sph

$\stackrel{1}{8}$

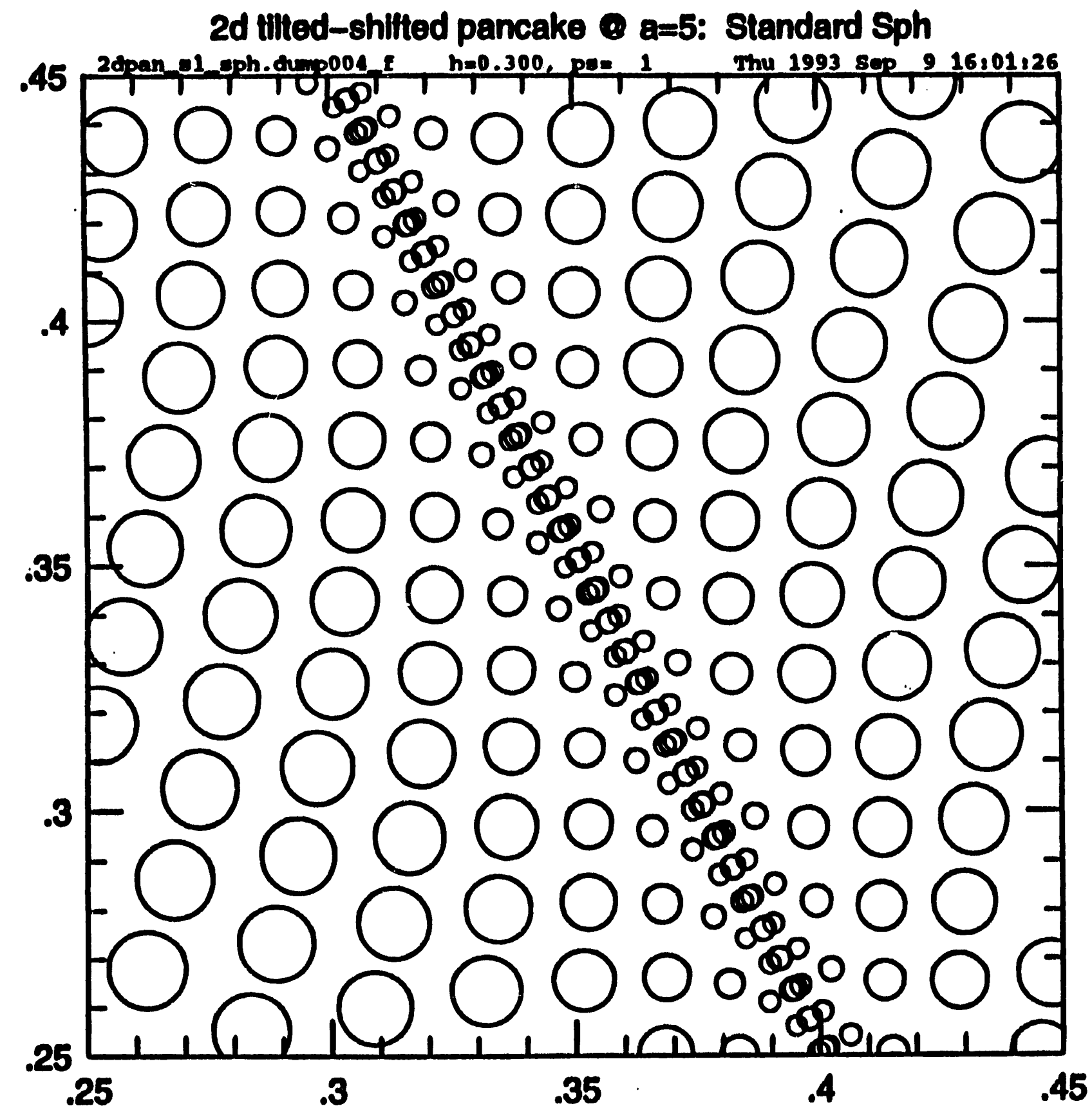




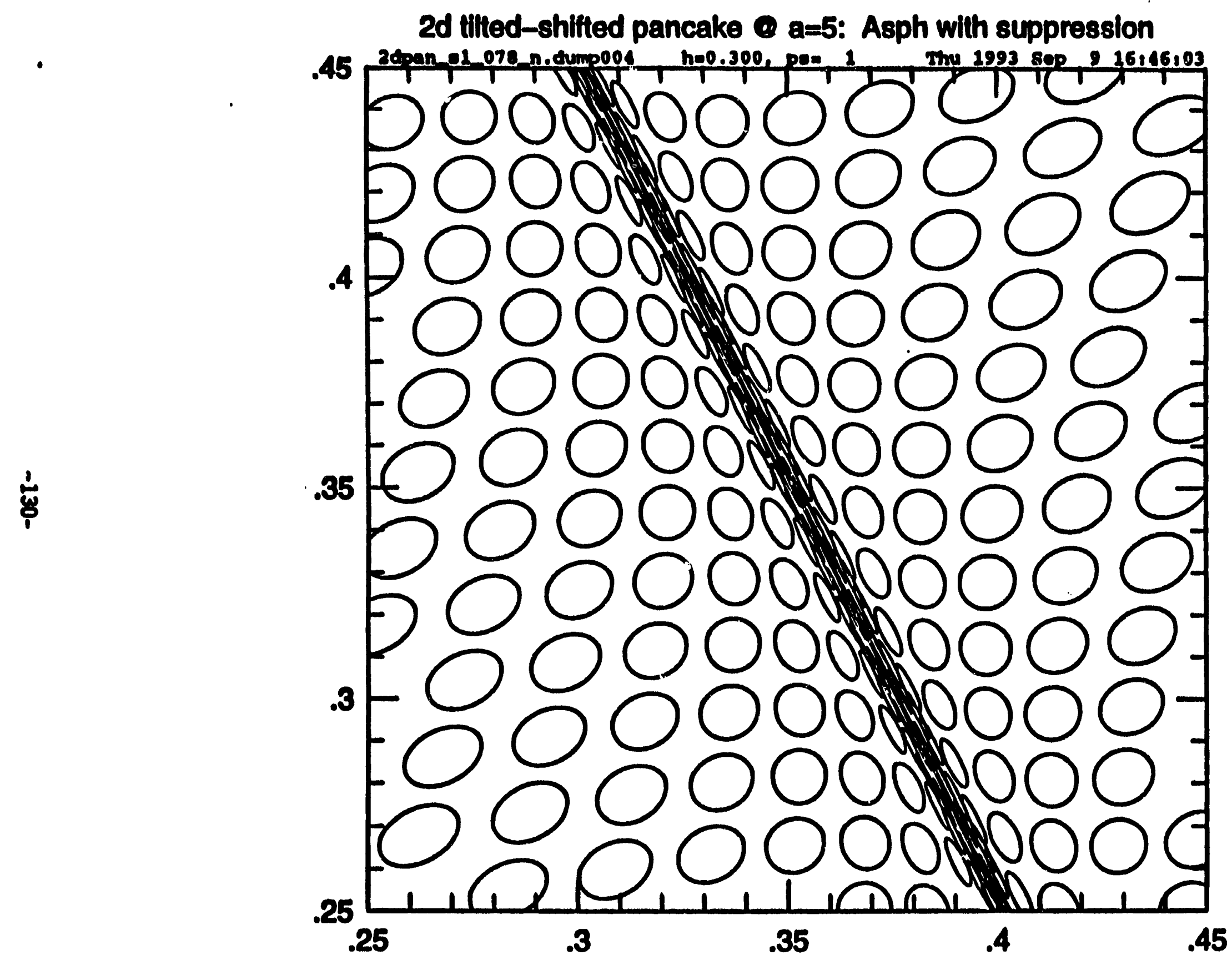


2d tilted-shifted pancake $a=5\left(a_{\text {erunch }}=4\right)$ : Standard Sph
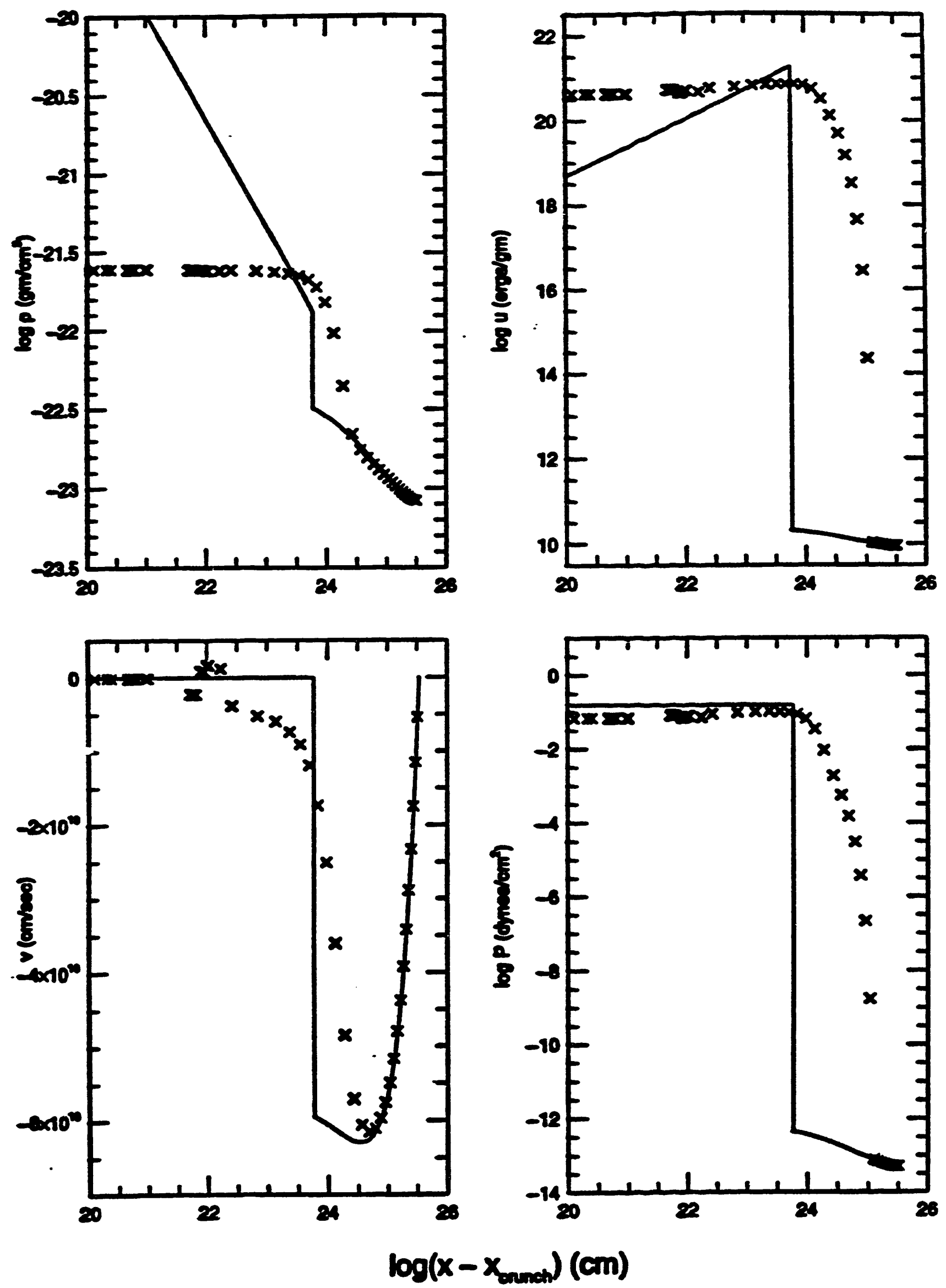

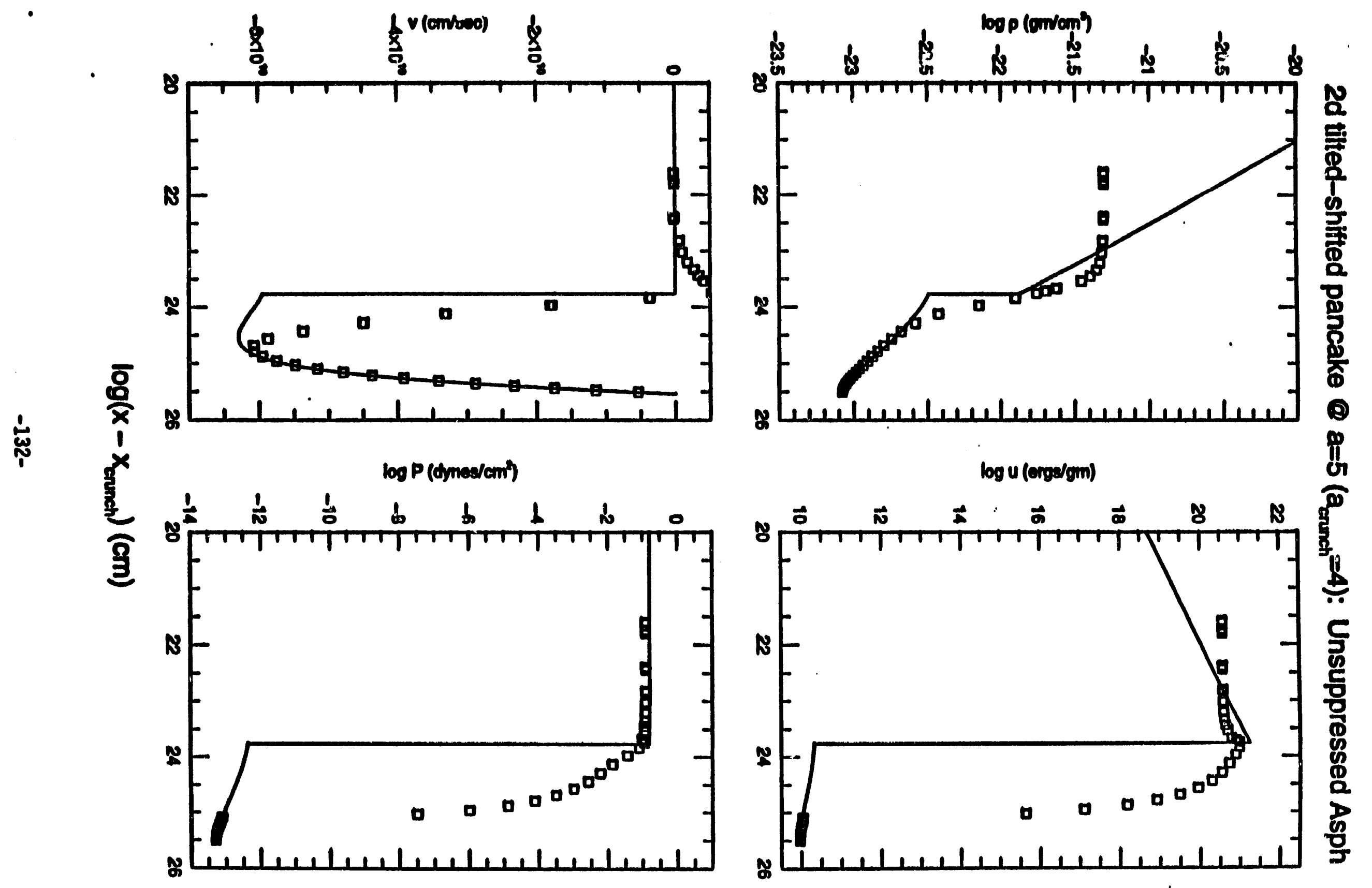
$2 d$ tilted-shifted pancake $a=5\left(a_{\text {erunch }}=4\right)$ : Asph with Art Visc Suppression
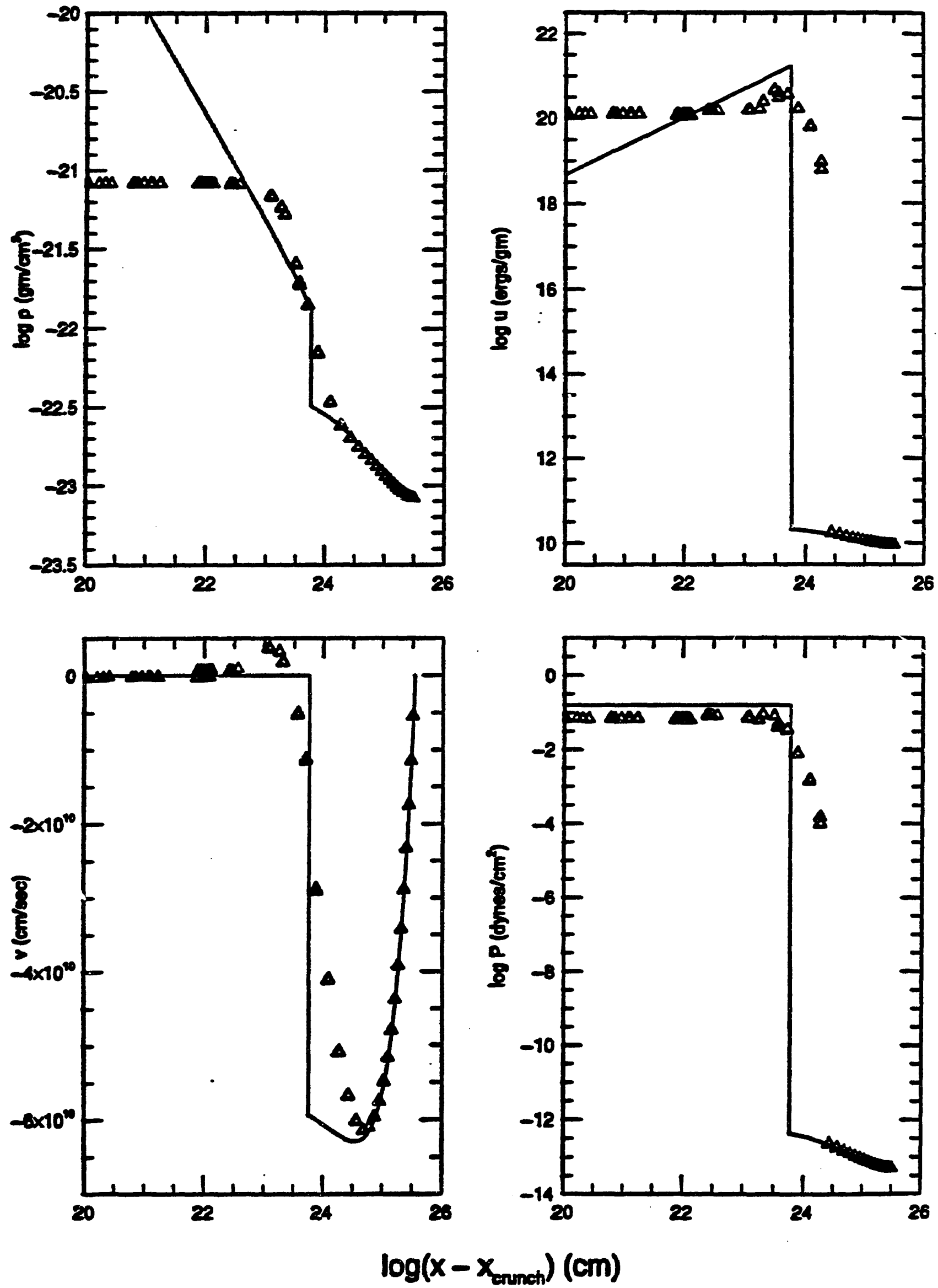
13 2d Hot Dark Matter Simulations

Background Cosmological and Physical Parameters:

- Einstein-DeSitter cosmology $(\Omega=1, \Lambda=0)$

- $\Omega_{\text {baryon }}=0.1 \Omega_{d m}=0.9$

- $H_{0}=50 \mathrm{~km} / \mathrm{sec} / \mathrm{Mpc}$

- $a_{\text {initial }}=1, a_{\text {erunch }}=101.7, a_{\text {final }}=31 a_{0}=31$

- $z_{\text {initial }}=30, z_{\text {crunch }}=-0.7, z_{\text {final }}=0$

- $l_{b o x}=6.45 \mathrm{Mpc} @ a=1 \Rightarrow l_{b o x}=200 \mathrm{Mpc} @ a=31$

- Krove $\in[1,64]$

- 4 fundamental waves across periodic box

- HDM density power spectrum slope $=-4$ with extra factor of $k$ to mimic 3d power spectrum

- Particles displaced from regular grid with waves of random amplitude and phase.

- $T_{i}=2.88 K \Rightarrow \epsilon_{i}=1.156 \times 10^{-11}$

- $\gamma=c_{P} / c_{V}=5 / 3$

- Pure Hydrogen gas $(\mu=1)$

- No Radiative Cooling implemented

Numerical Simulation Parameters:

- $N_{\text {baryon }}=N_{\text {dm }}=16384$

- $\alpha=1,\left(p=a^{\alpha}\right)$

- $d p \in\left[10^{-4}, 0.05\right], h_{s} \in\left[10^{-5}, 0.1\right], h_{2} / h_{1}>0.01$ 


\section{Baryon positions for 2d Suppressed Asph HDM model $a=31.0(z=0.0)$}

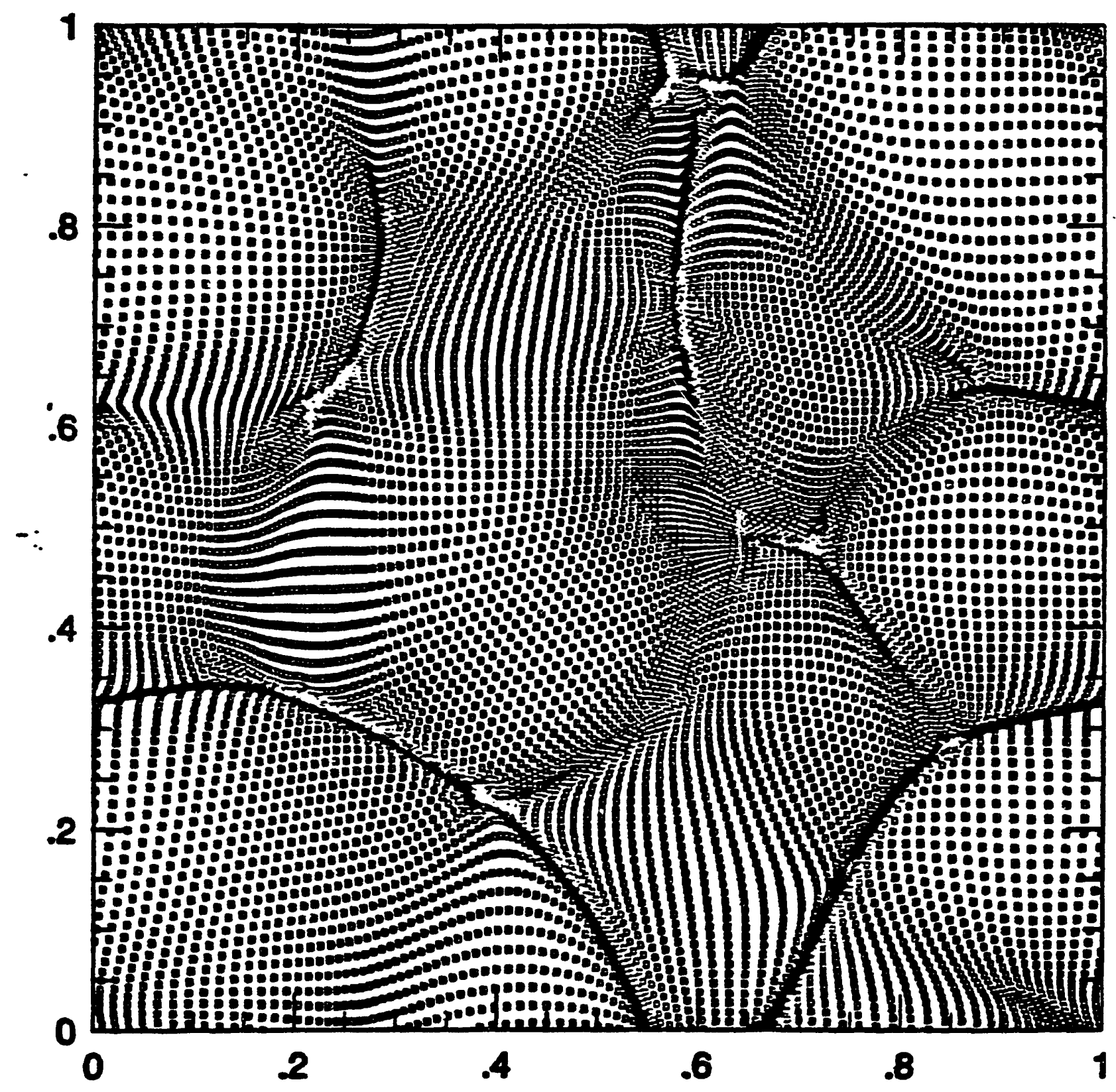




\section{Dark Matter positions for 2d Suppressed Asph HDM model @ $a=31.0(z=0.0)$}

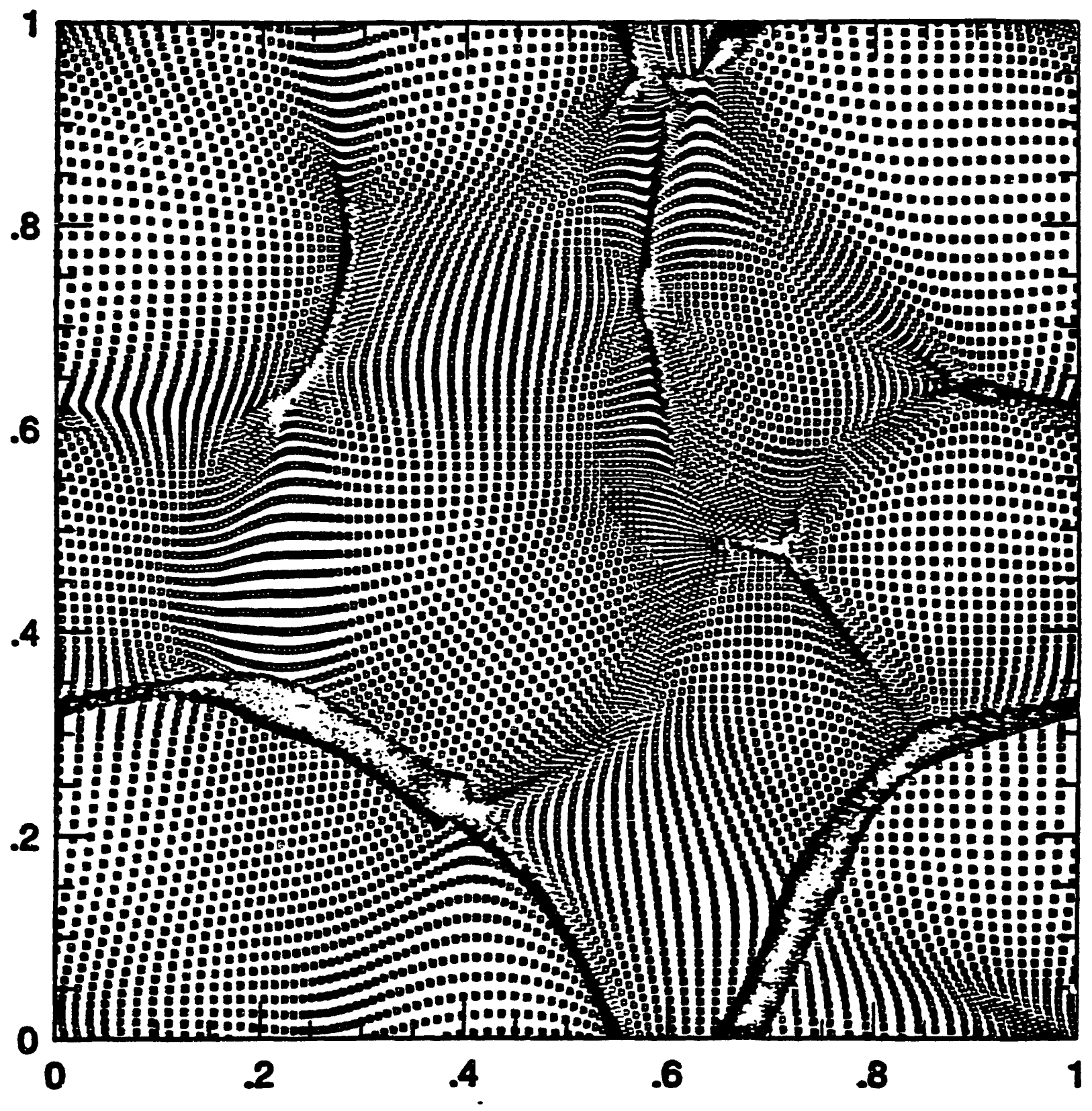




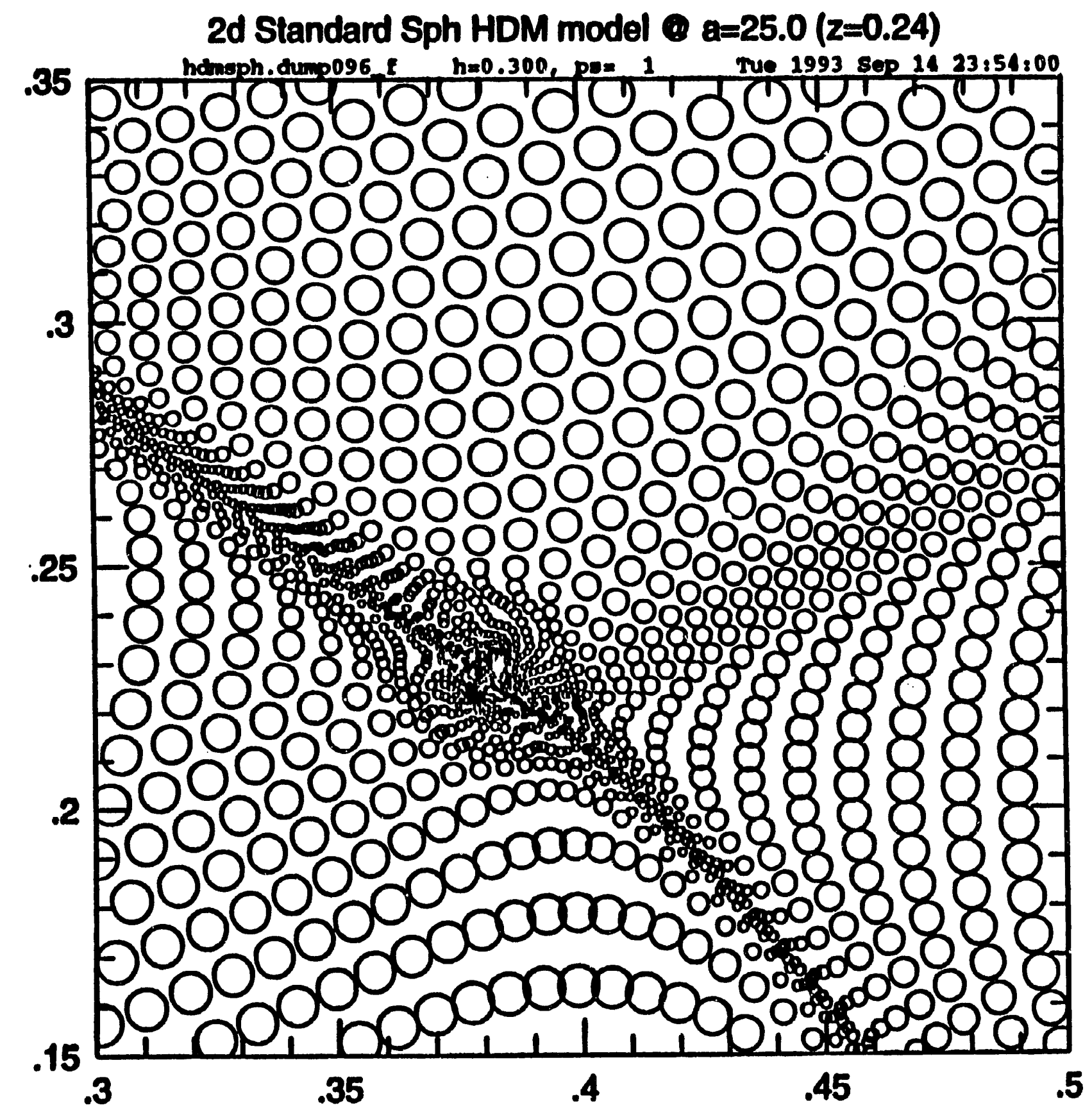


2d Unsuppressed Asph HDM model a $a=25.0$ ( $z=0.24)$

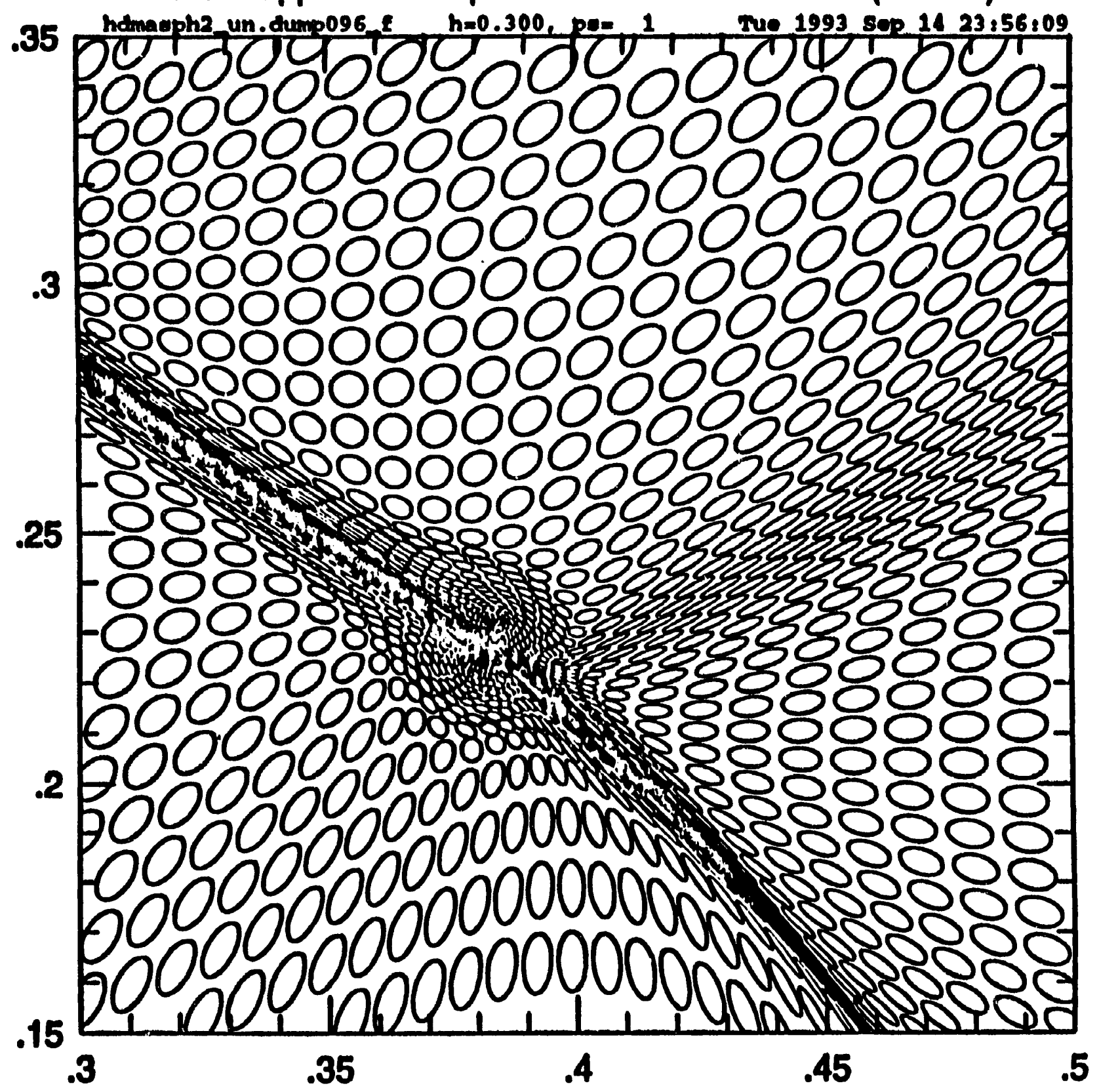




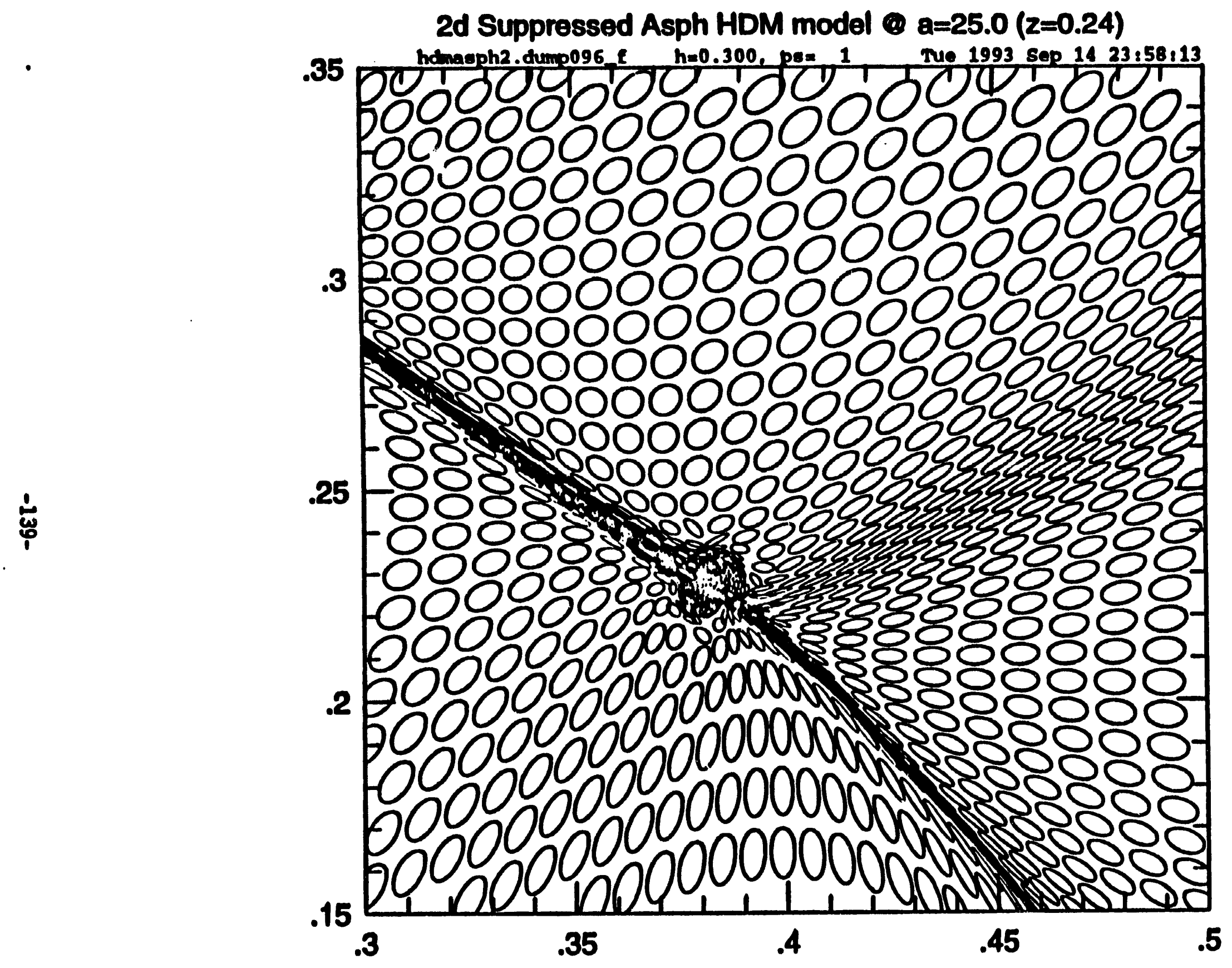




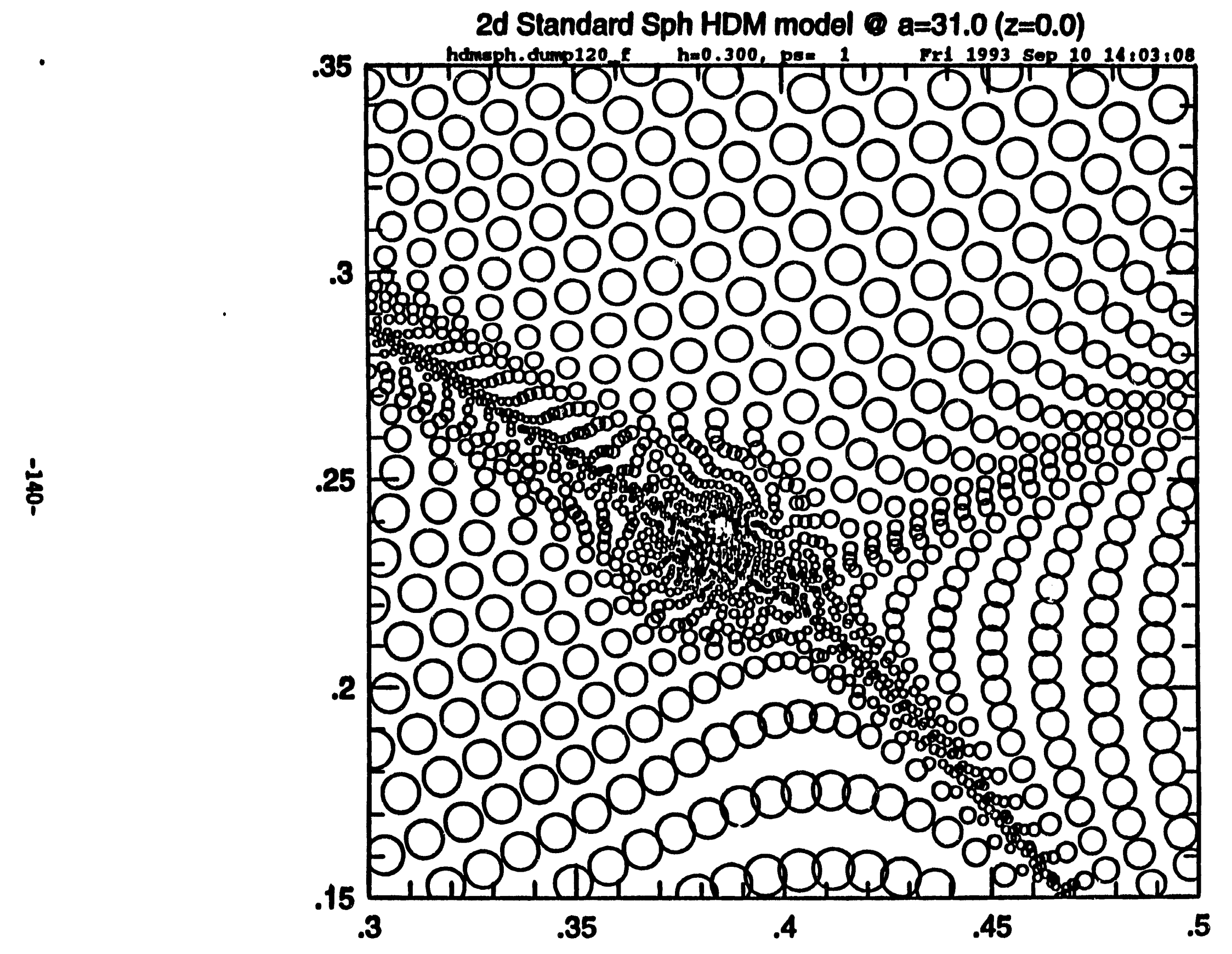


2d Unsuppressed Asph HDM model (a $a=31.0$ ( $z=0.0)$

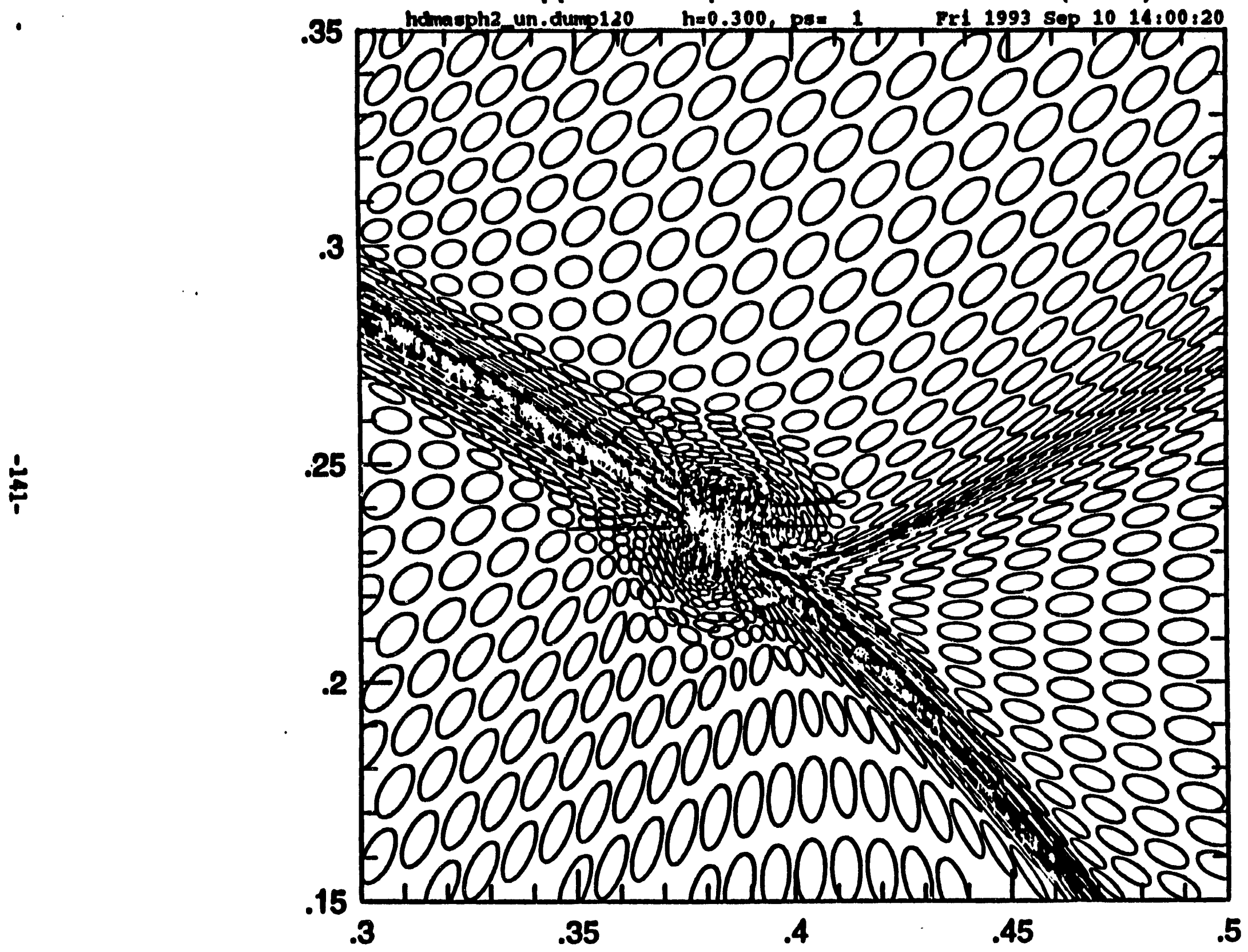




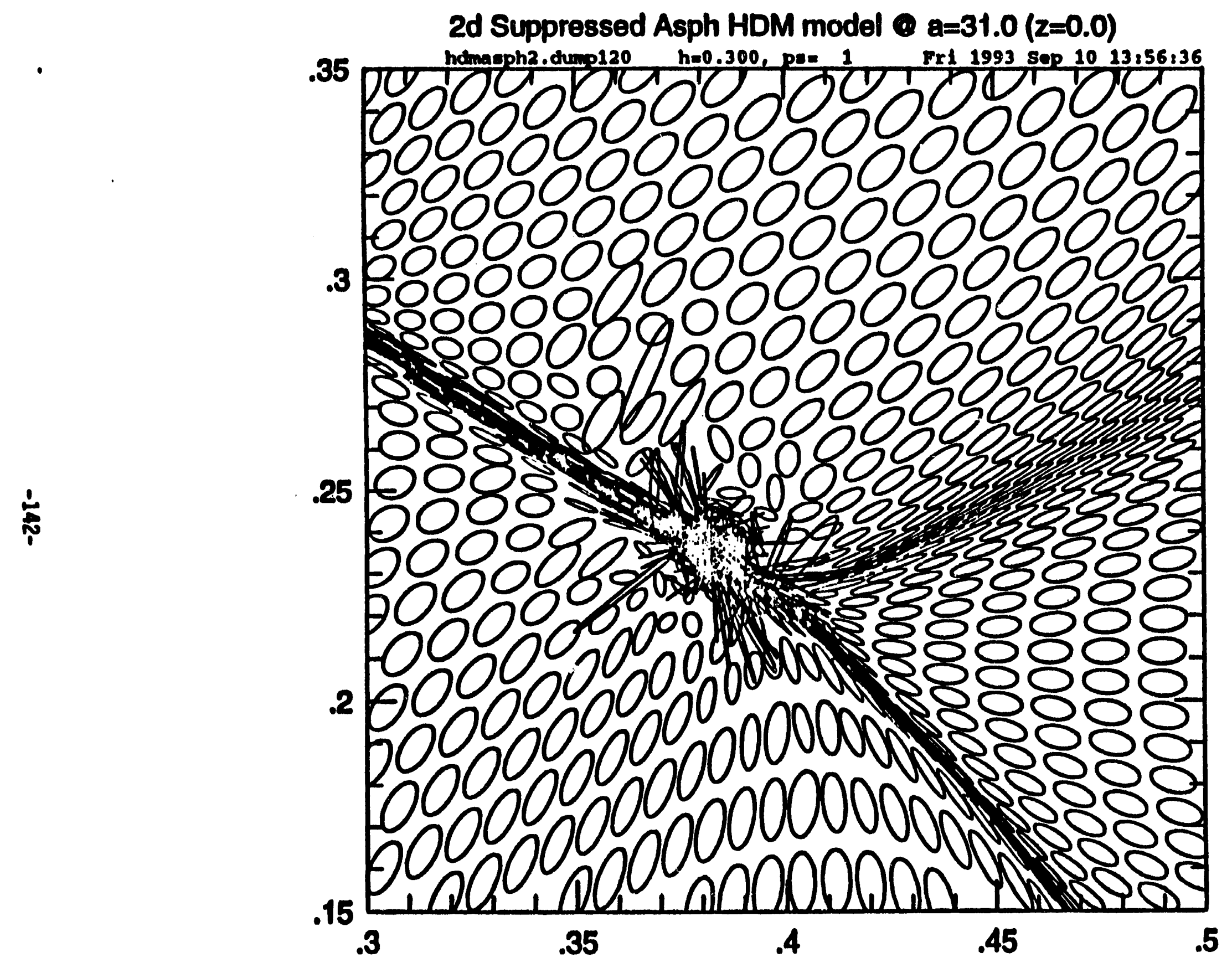


2d HDM Temperature isocontours for Standard Sph @ a=25

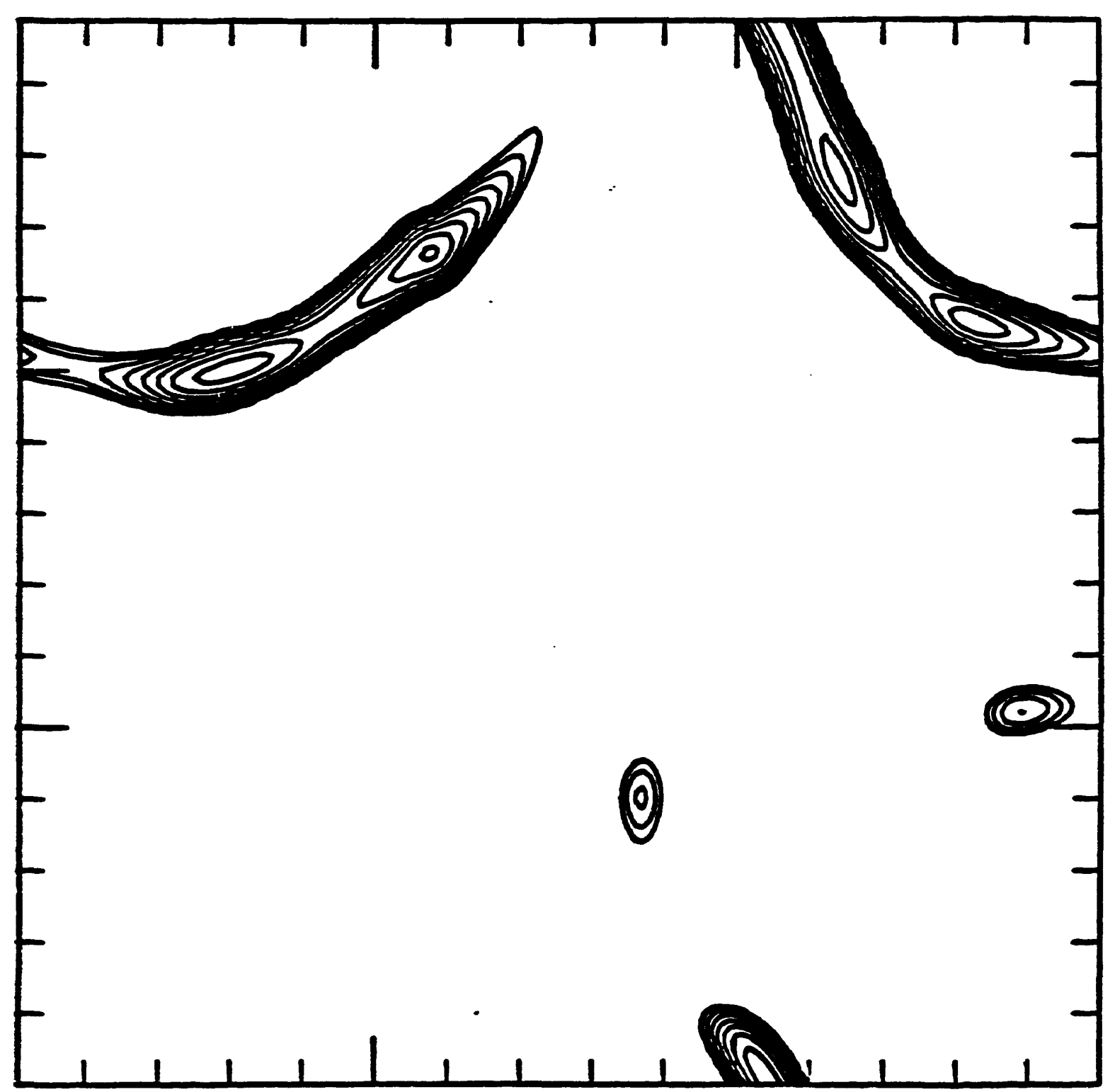

$$
\begin{aligned}
& \text { Low contour }=10^{5} \mathrm{~K} \\
& \text { Contour Ratio }=2
\end{aligned}
$$


2d HDM Temperature isocontours for Unsuppressed Asp @ a=25

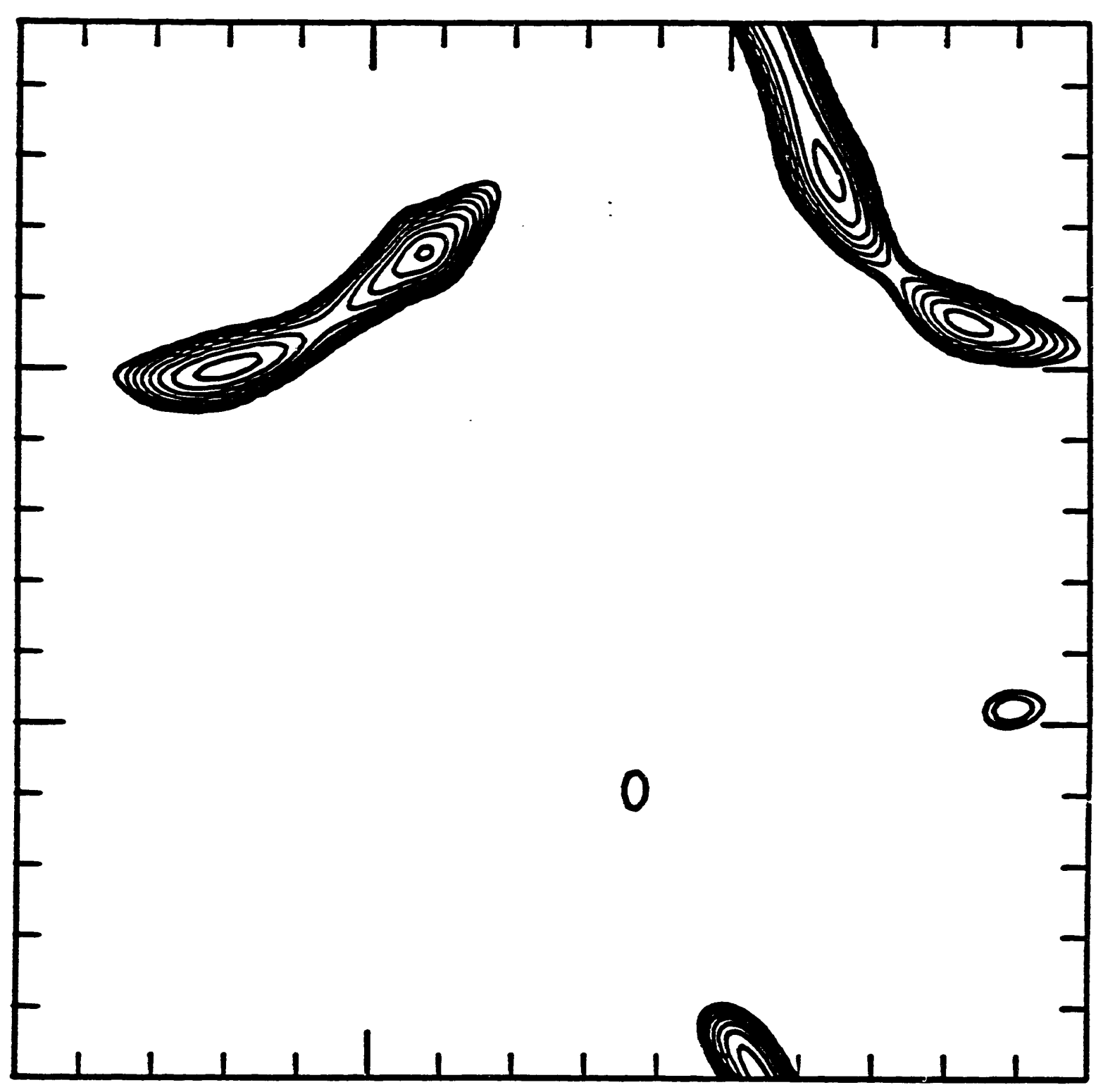

Low contour $=10^{5} \mathrm{~K}$

Contour Ratio $=2$

$-144-$ 
2d HDM Temperature isocontours for Suppressed Asph @ a=25

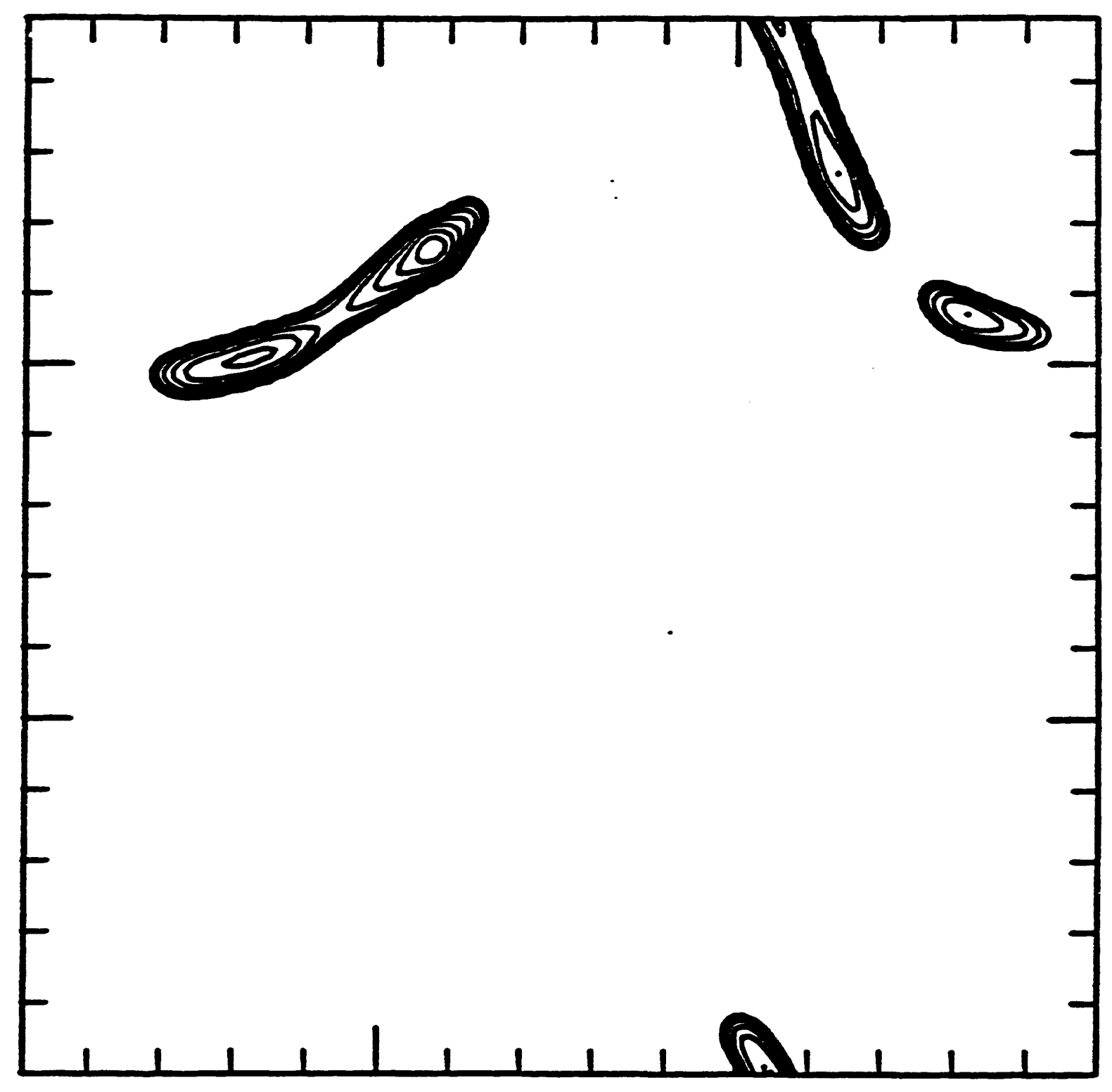

Low Contour $=10^{5} \mathrm{~K}$

Contorr Ratio : 2

$-145-$ 


\section{Conclusions}

- Asph shows promise in general for allowing improved resolution within a simulation for a given number of Asph nodes vs. Standard Sph, particularly in intrinsically anisotropic situations.

- The development of an improved criterion for the use of the artificial viscosity in order to reduce spurious heating in combination with the improved resolution of Asph will hopefully allow us to investigate a wider range of scales in cosmological structure formation scenarios.

Future work and considerations:

- The general principles of Asph need to be tried out in a wider range of test problems, in order to explore the benefits and limits of the current technique.

- The question of angular momentum conservation under Asph needs further investigation, since it would seem angular momentum conservation under Asph is dependent upon the $\mathbf{H}$ tensor properly following the fluid element approximation.

- The artificial viscosity suppression algorithm needs much .Jre refinement in order to extend its applicability and reliability; most notably it should be converted to an entirely local criterion. 


\title{
Energy Conservation in \\ Viscous Flows
}

\author{
Mike Fisher
}

\author{
Battelle
}




\section{Continulity Equation:}

$$
\frac{d p_{i}}{d t}=\sum_{j} m_{j}\left(v_{i}^{\alpha}-v_{j}^{\alpha}\right) \frac{\partial W_{i j}}{\partial x_{i}^{\alpha}}-\rho_{B}\left(v_{i}^{\alpha}-v_{B}^{\alpha}\right) n_{B}^{\alpha} W_{i B} A_{B}
$$

a dut

(4)

官

Momentum Equation:

$\frac{d v_{i}^{\alpha}}{d t}=\sum_{j} m_{j}\left(\frac{\Pi_{j}^{\alpha \beta}}{\rho_{j}^{2}}+\frac{\Pi_{i}^{\alpha \beta}}{\rho_{i}^{2}}\right) \frac{\partial W_{i j}}{\partial x_{i}^{\beta}}$

$-\rho_{B}\left(\frac{\Pi_{B}^{\alpha \beta}}{\rho_{B}^{2}}+\frac{\Pi_{i}^{\alpha \beta}}{\rho_{i}^{2}}\right) n_{B}^{\beta} W_{i B} A_{B}$ 


\section{Example Problem:}

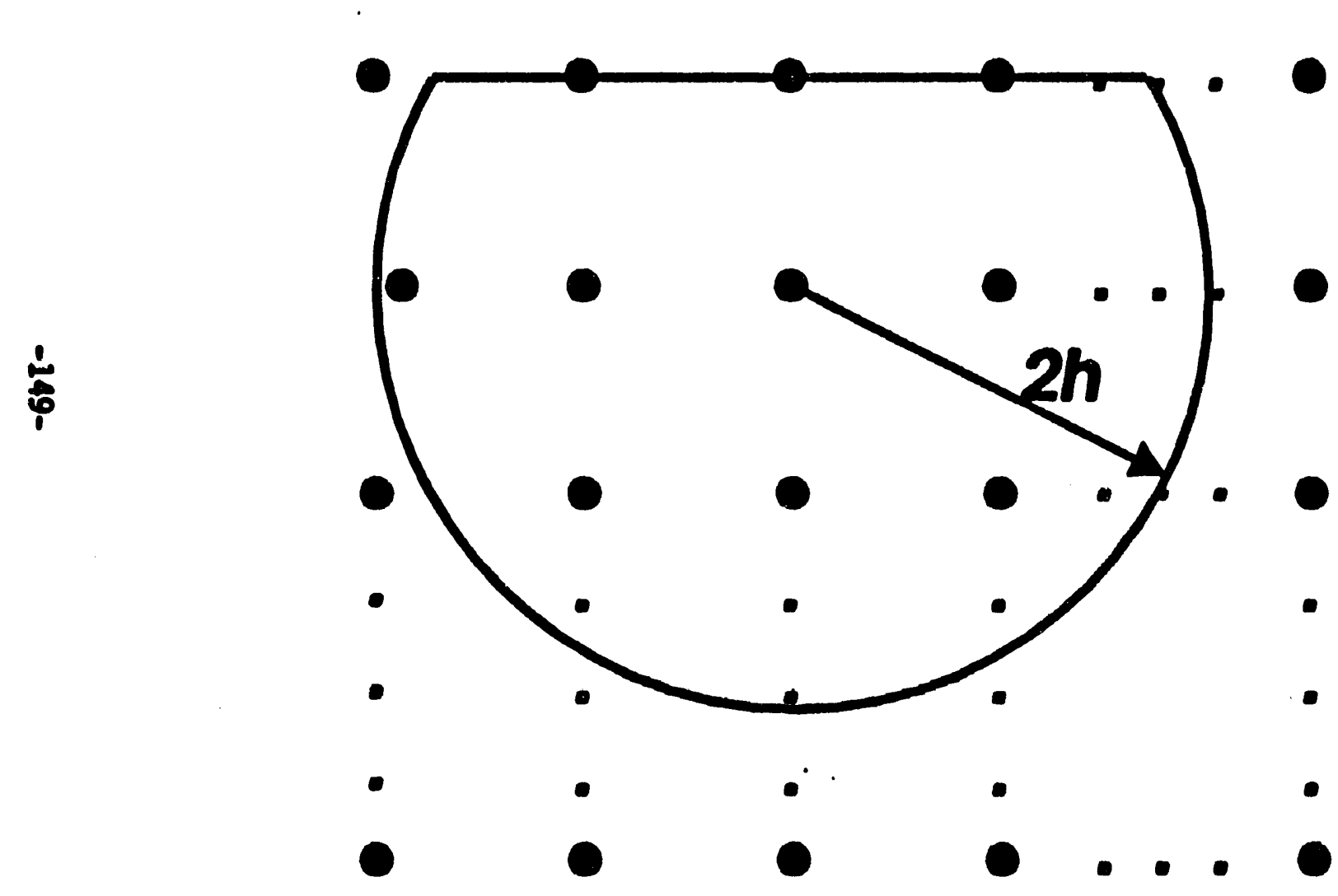




\section{Preliminary Results}

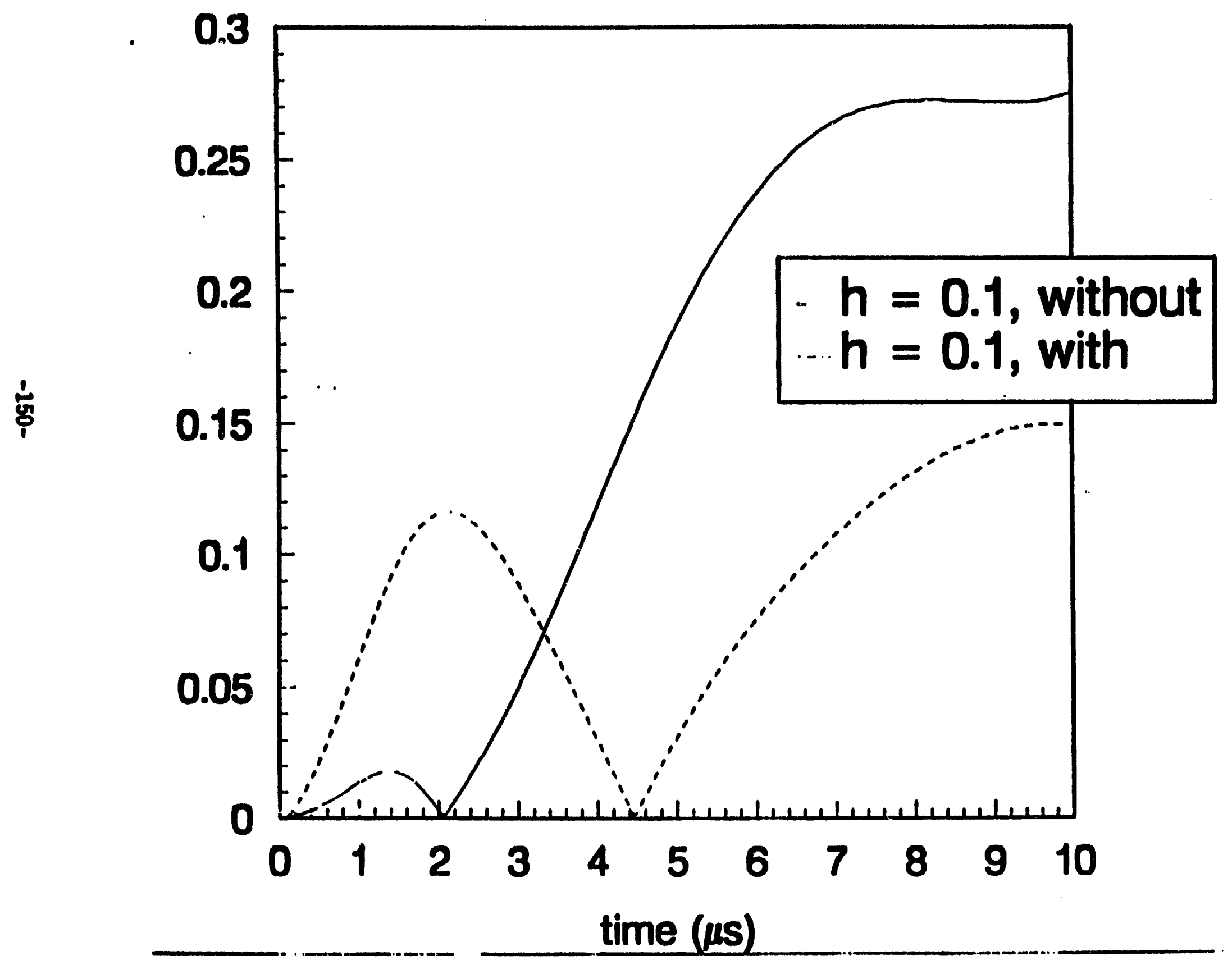




\section{Preliminary Results}

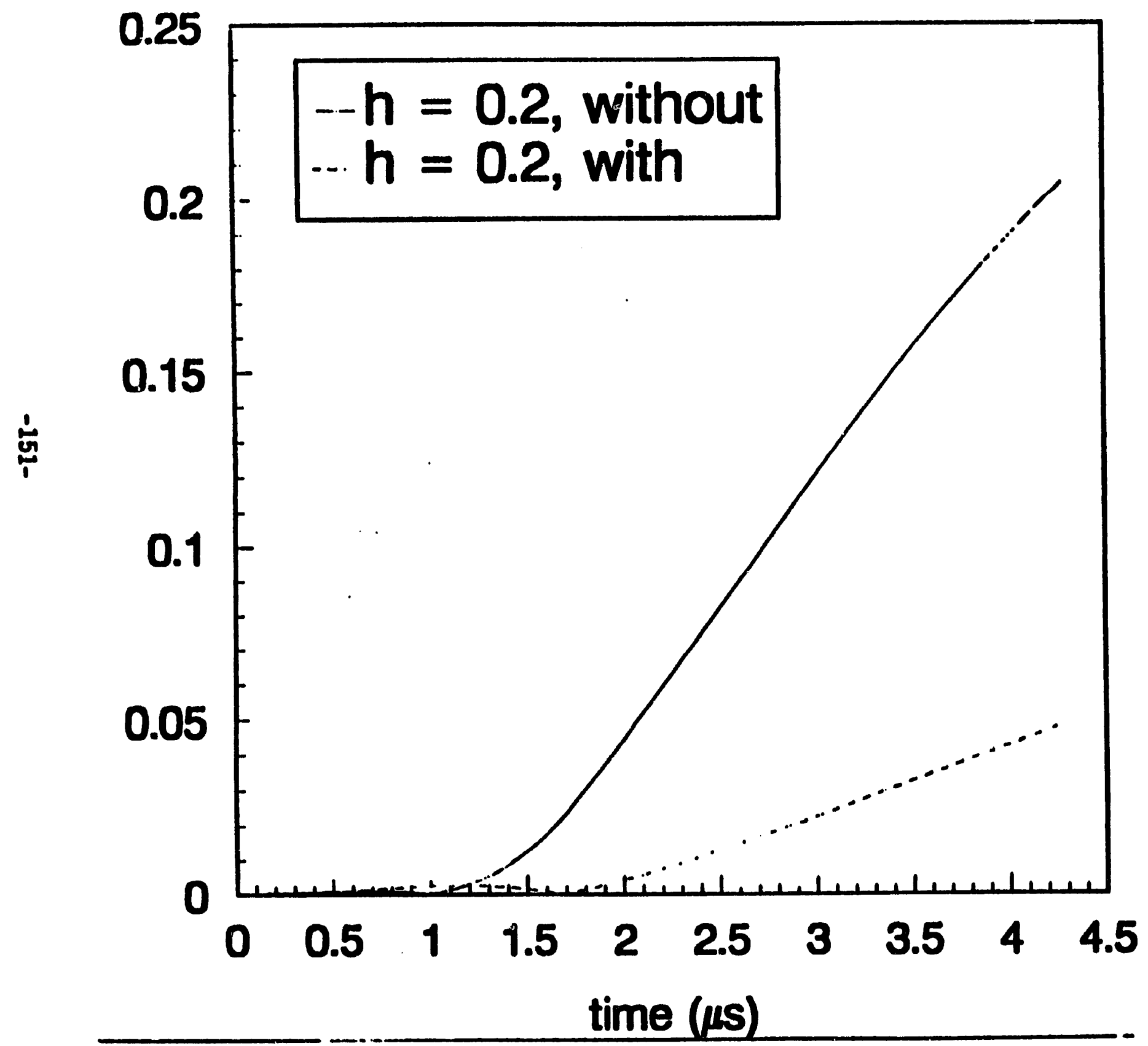




\title{
Linked Penetration Computations
}

\author{
Gordon R. Johnson \\ Alliant Techsystems \\ Hopkins, Minnesota USA
}

SPH Workshop

Los Alamos

September 23, 1993 


\section{Outline}

- Motivation and discussion

- Background

- Lagrangian code structure for SPH nodes and standard elements

- Examples and discussion

- SPH only

- SPH nodes attached to standard elements

- SPH nodes sliding on standard elements

- SPH nodes automatically generated from standard elements

- Summary and conclusions 


\section{MOTIVATION AND DISCUSSION}

- Desirable to have a Lagrangian code which could accurately handle severe distortions

- SPH can handle severe distortions in a Lagrangian framework - But what about accuracy and computing time?

- It may be a good compromise between Standard Lagrangian codes and Eulerian codes

- Linking SPH to a Standard Lagrangian code could have great potential

- Capability

- Accuracy

-Efficiency (Computing time) 


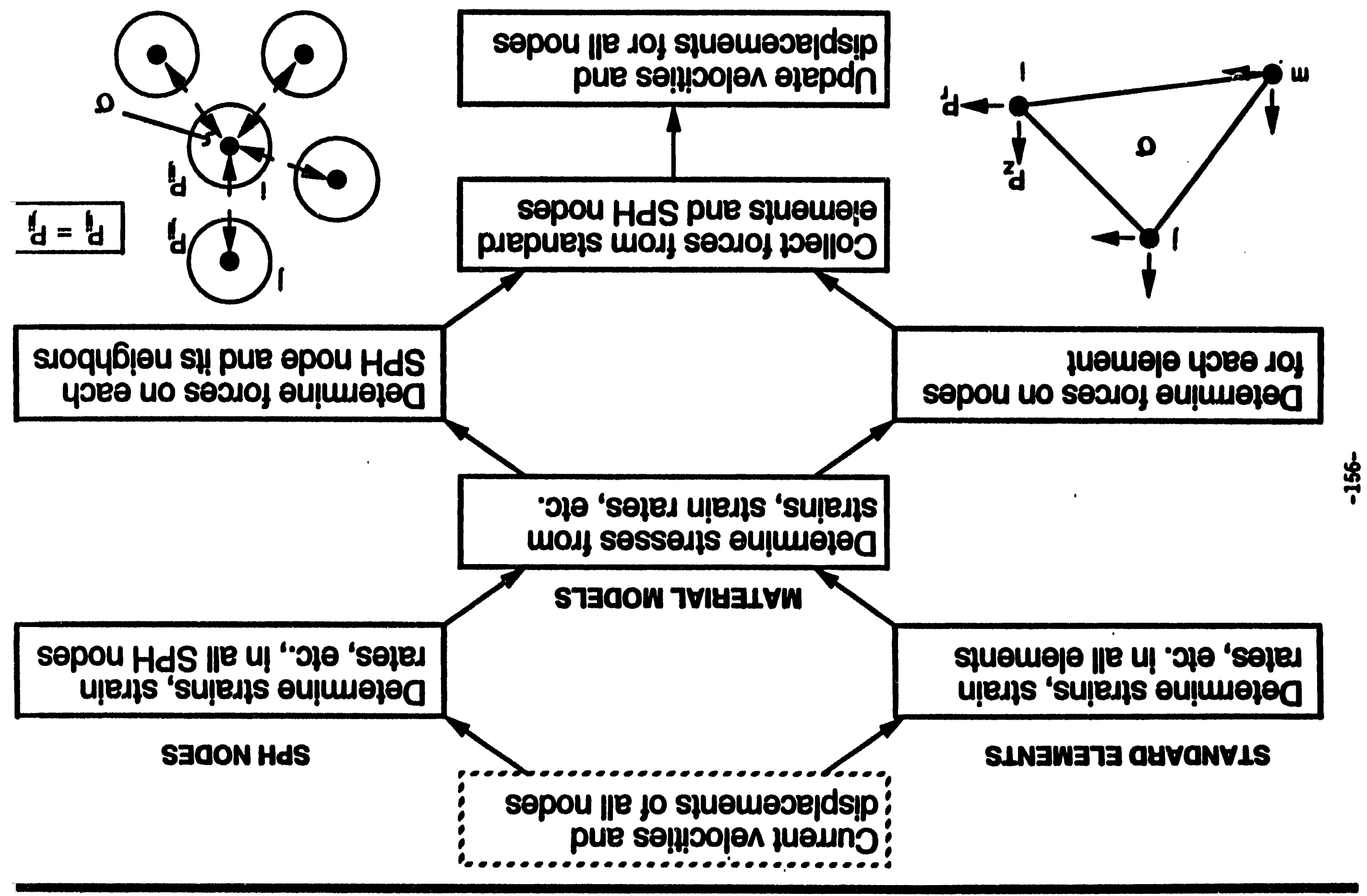

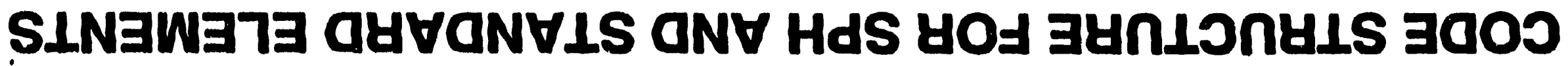




\section{SPH and Standard Element Results}
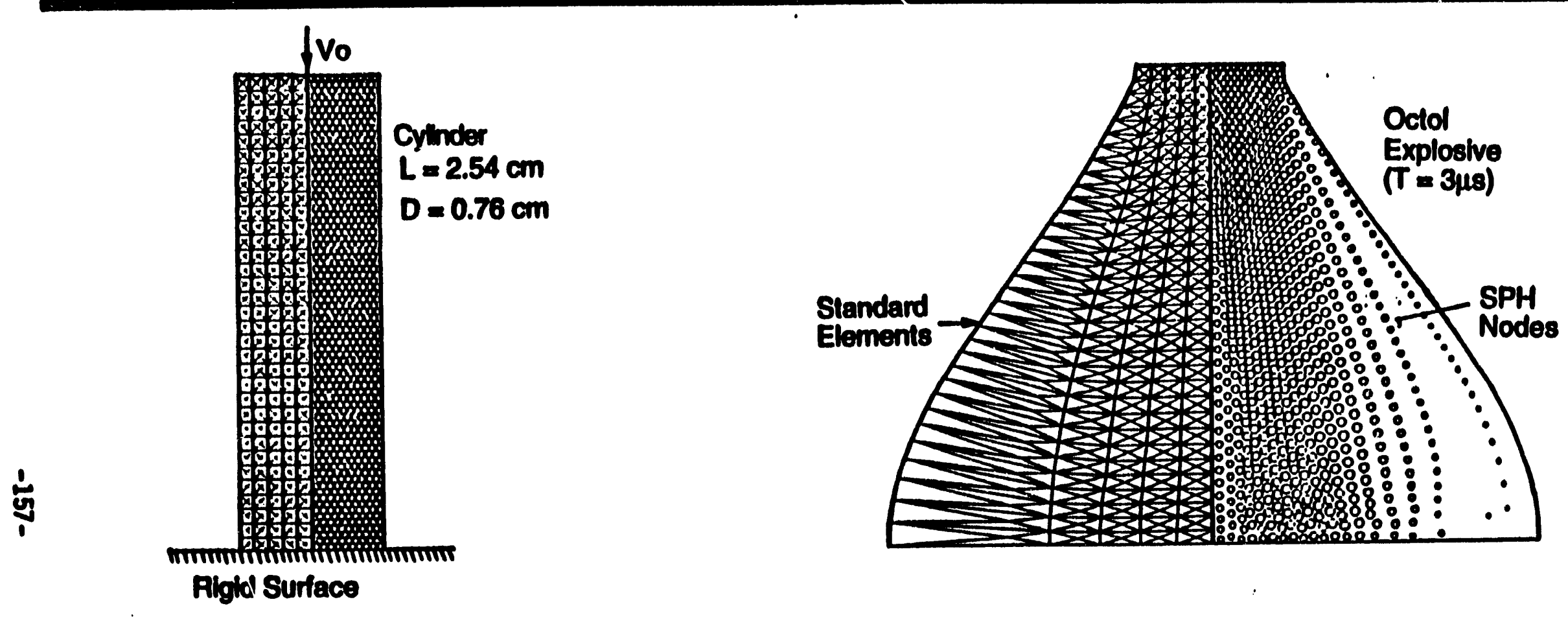

Algtal Surface
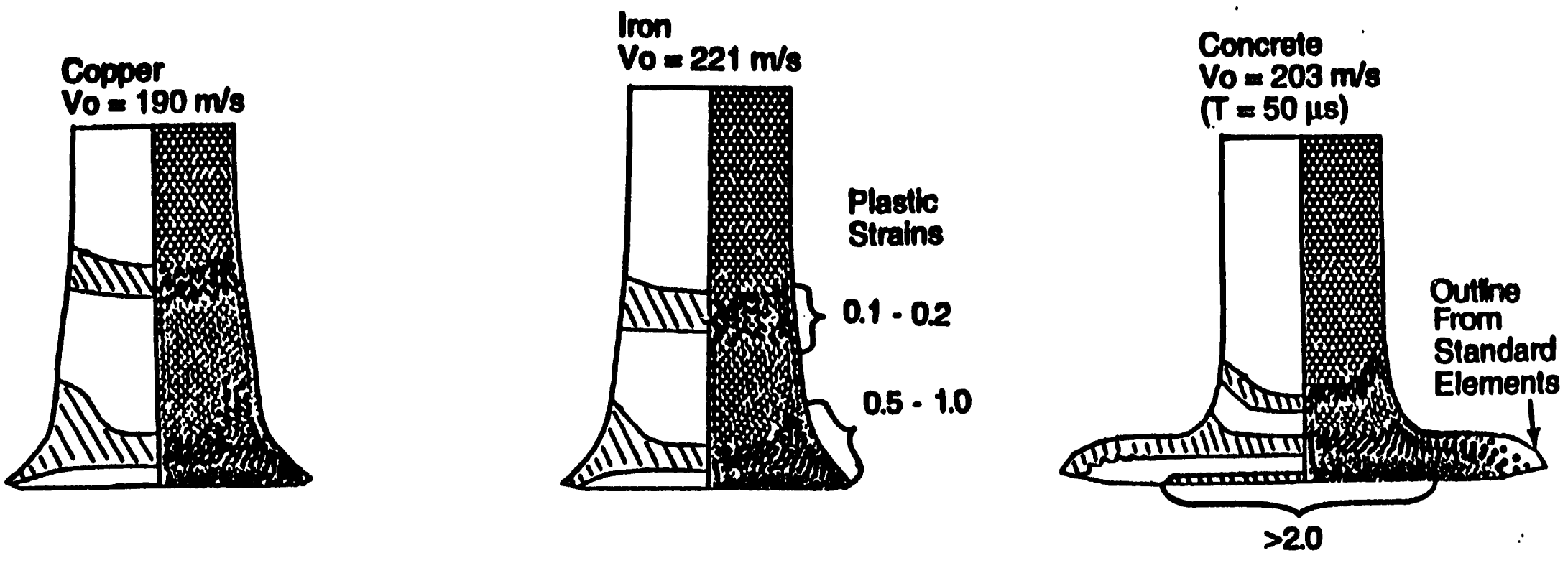


\section{SPH PERFORATION COMPUTATIONS}

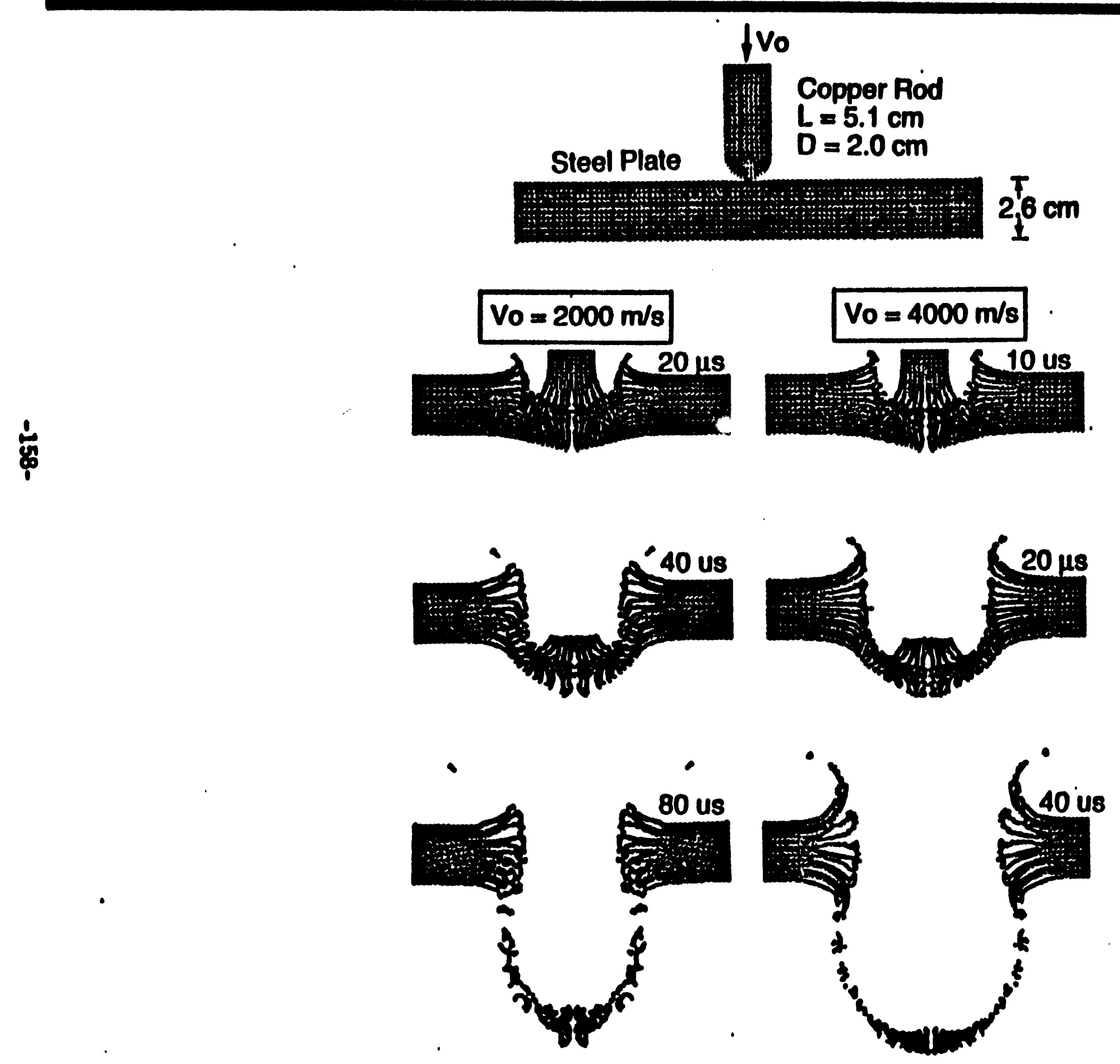




\section{SPH PENETRATION COMPUTATION}

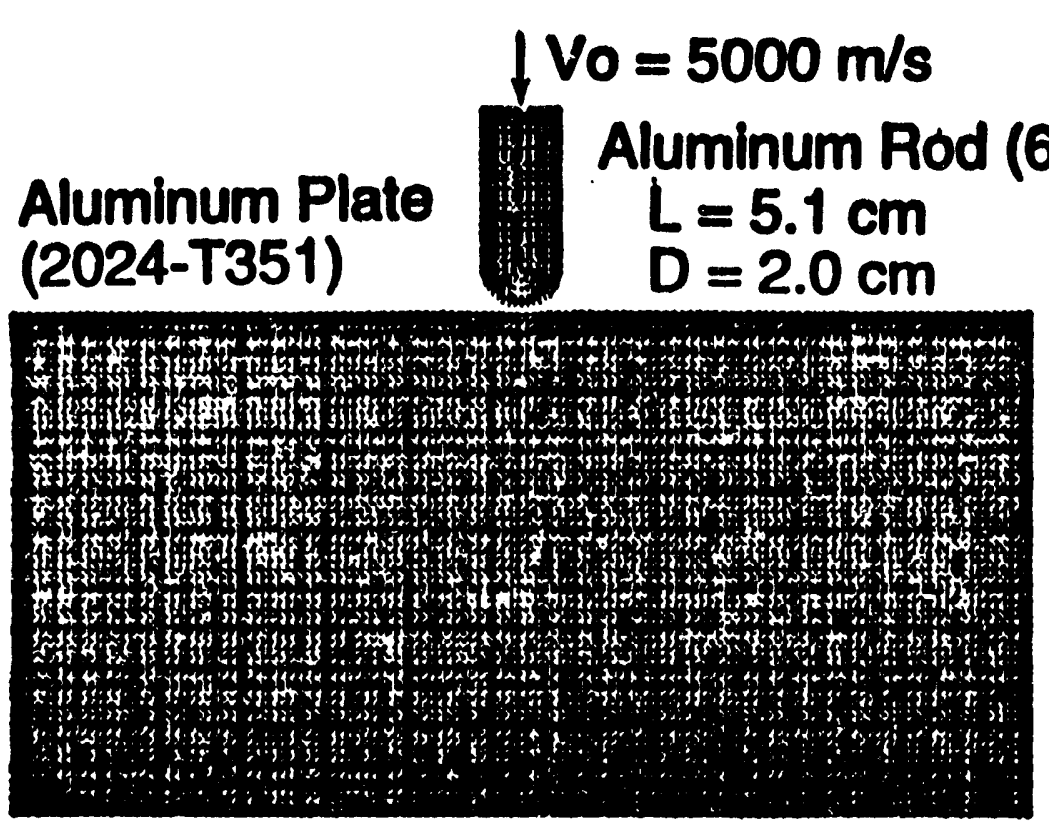

Plate Thickness $=12.7 \mathrm{~cm}$
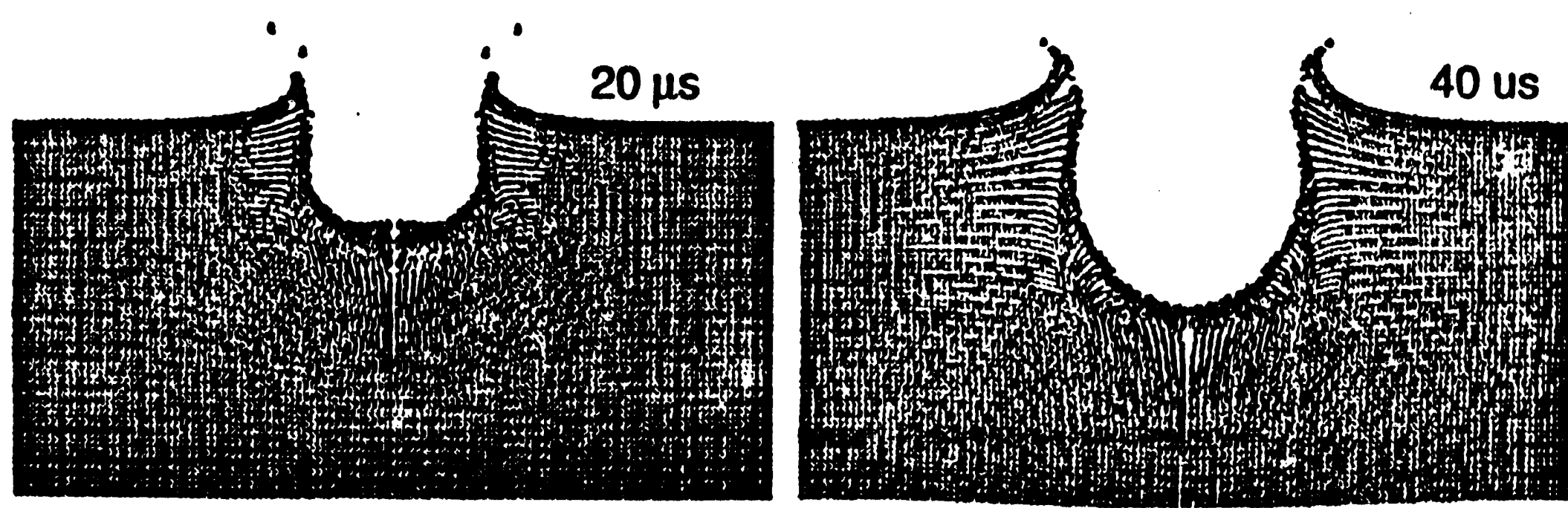


\section{SPH SPALL COMPUTATION}

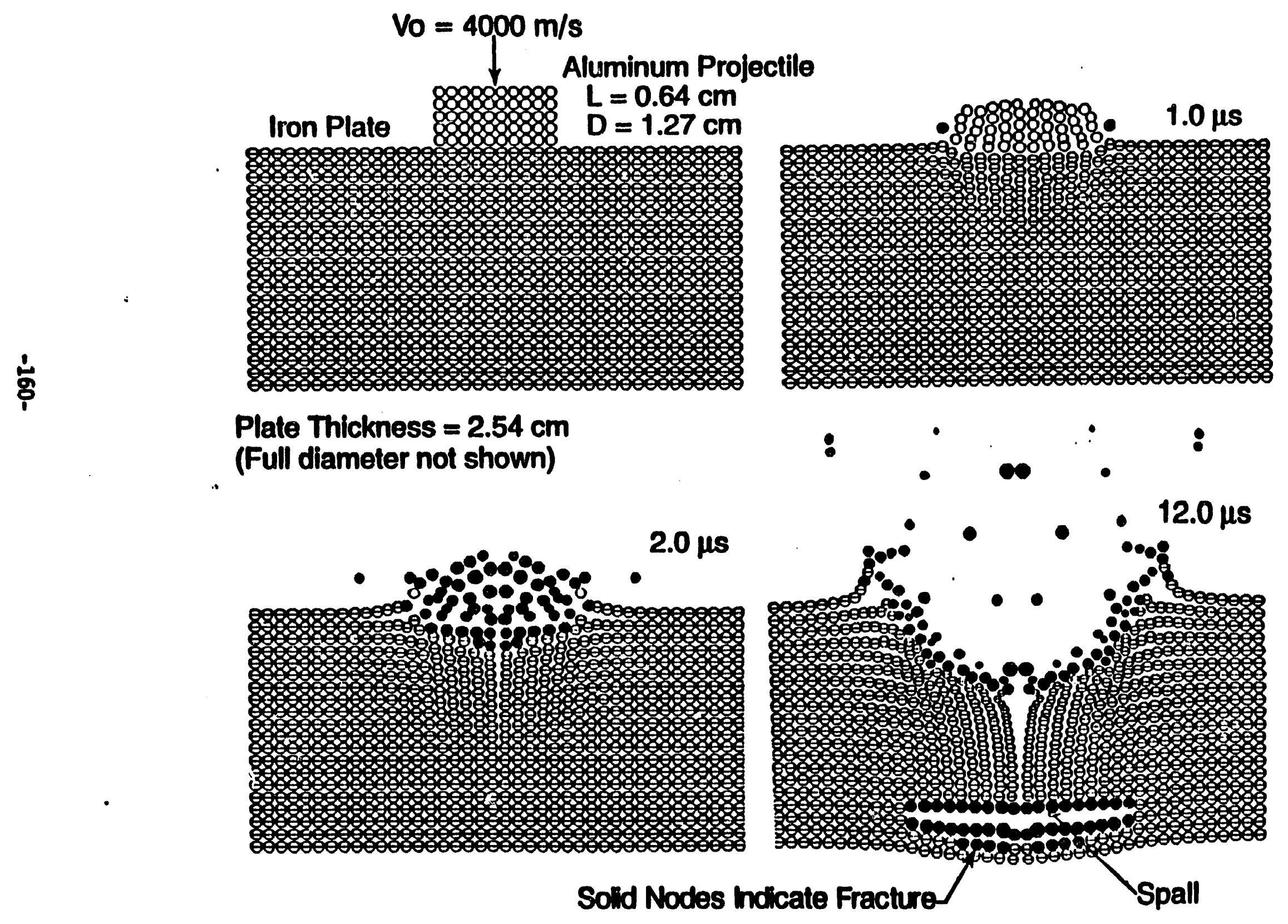




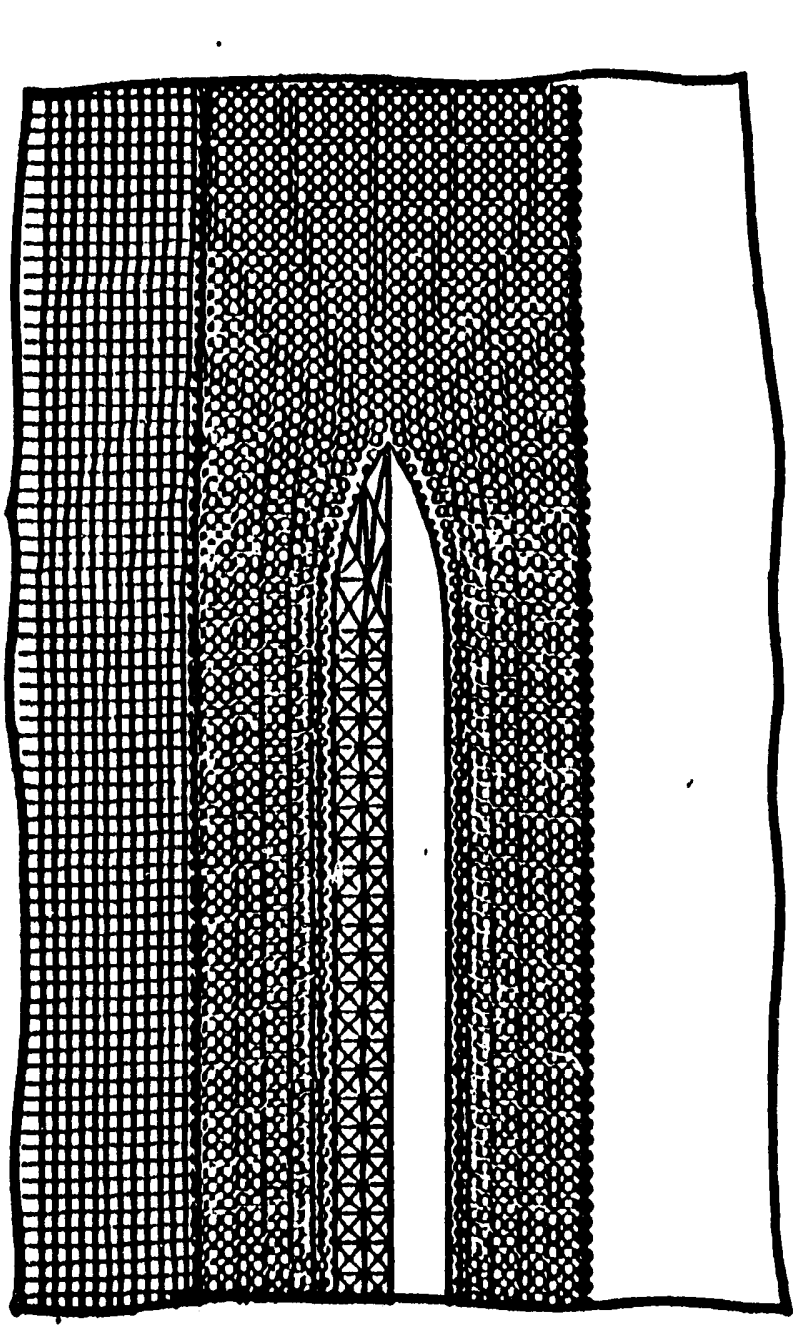

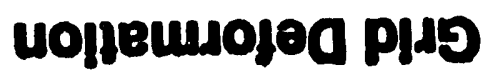

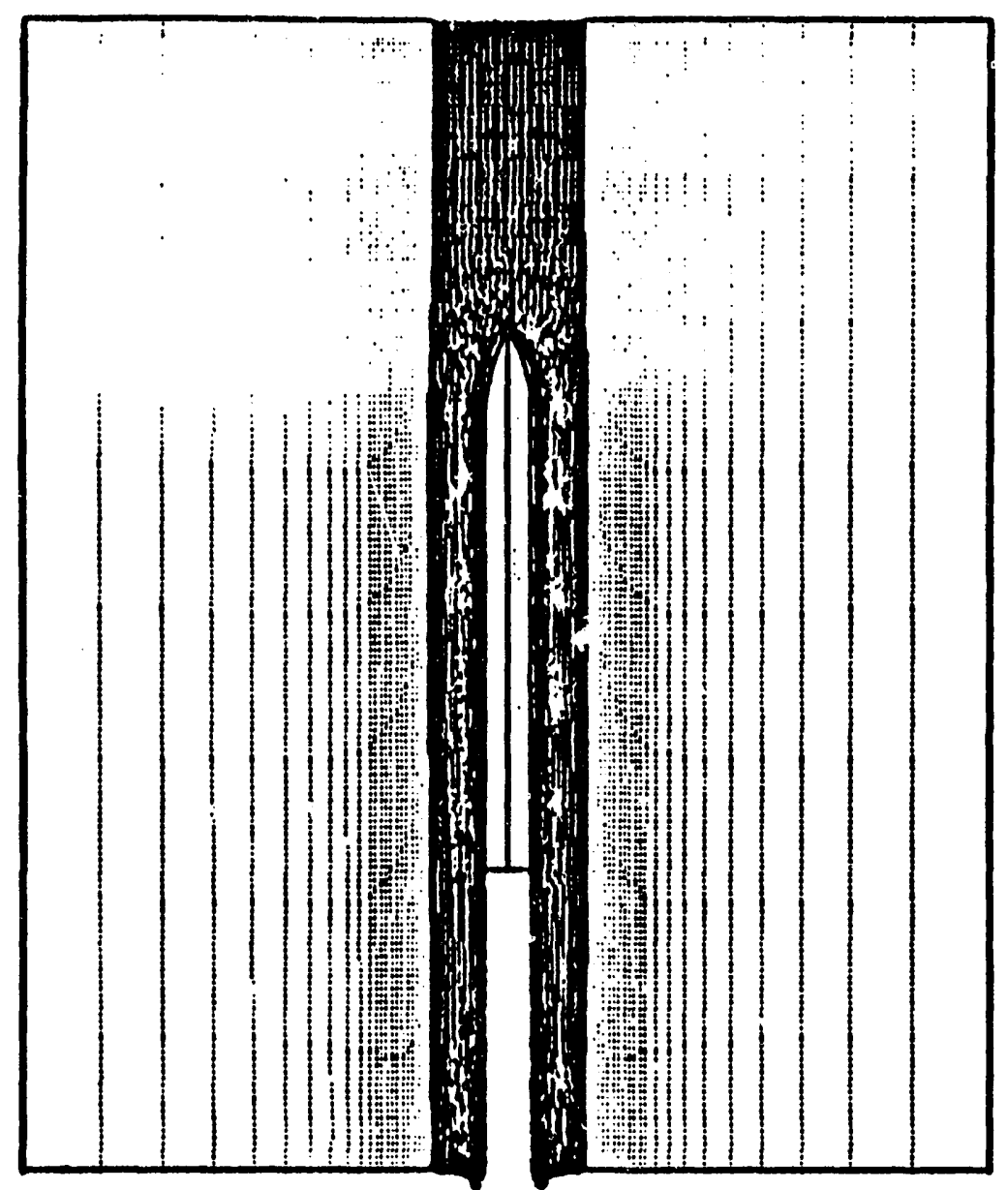

s/ul $826={ }^{\circ} \wedge$ dof uopgenoued fBul]

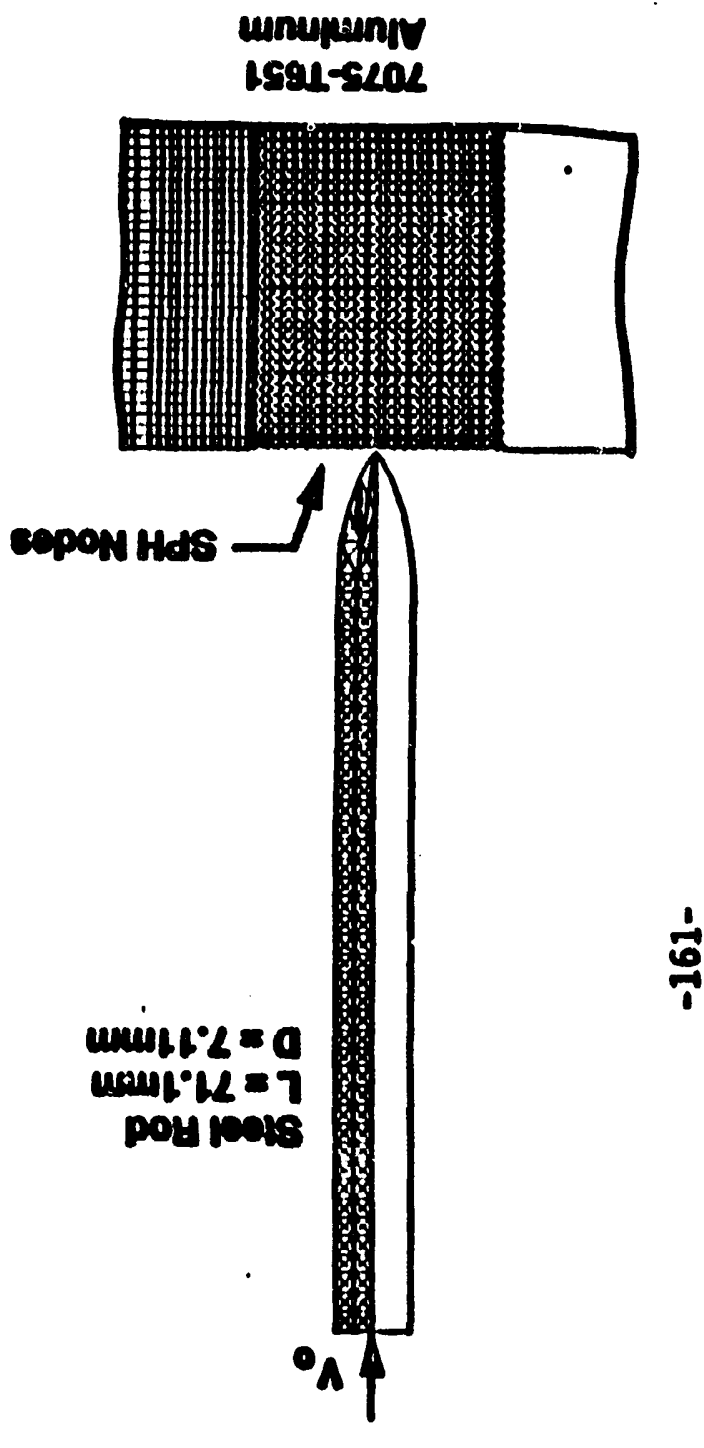

Anoworo fв|yu|

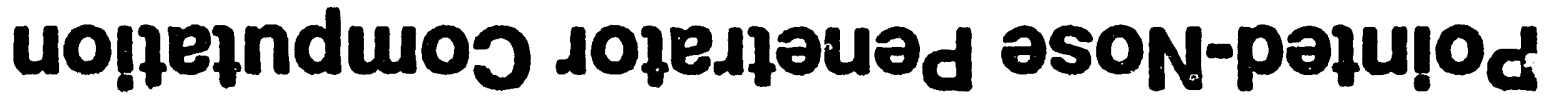




\section{Linked SPH Results Compared to Test Data}

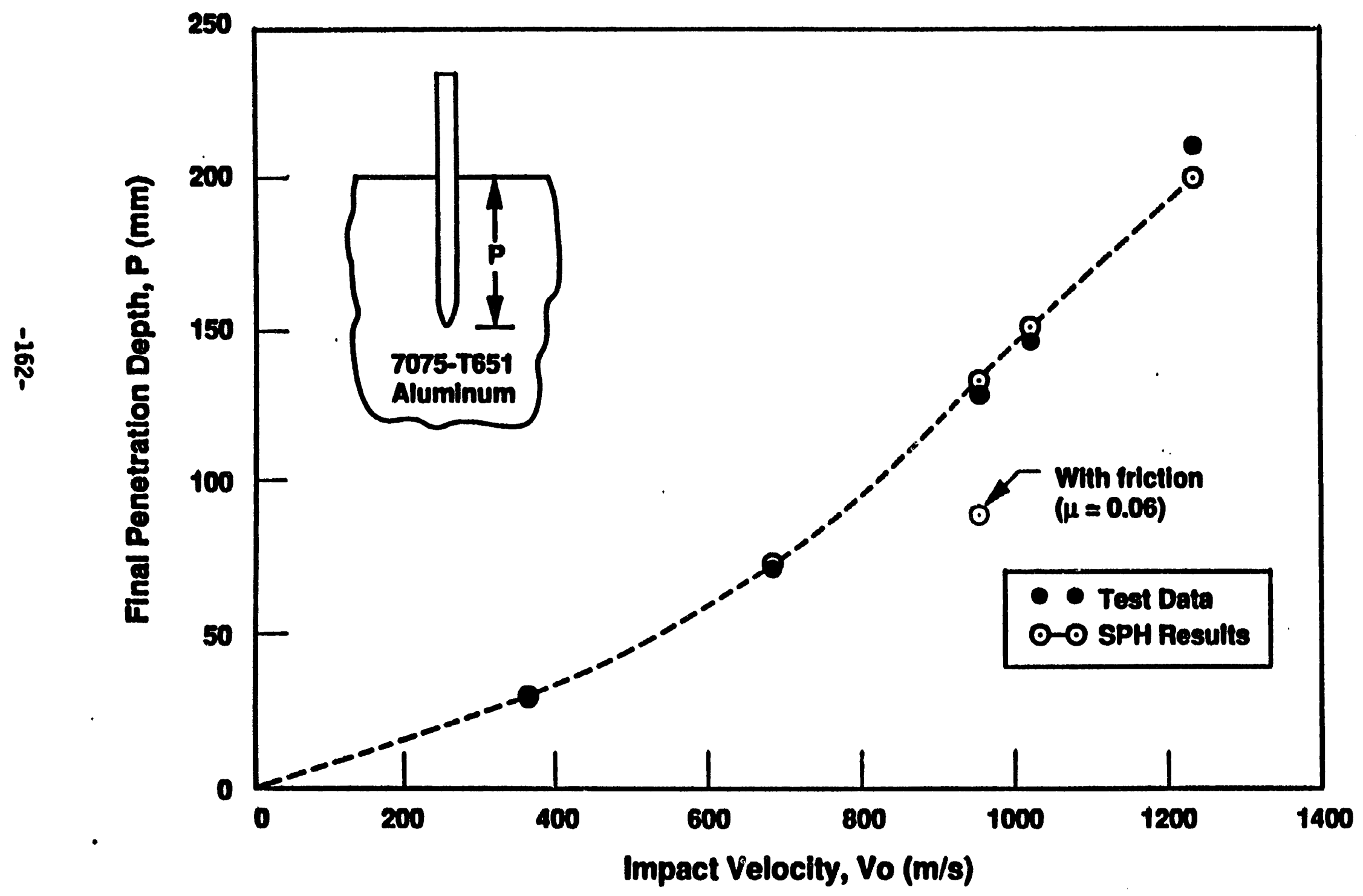

GR110 


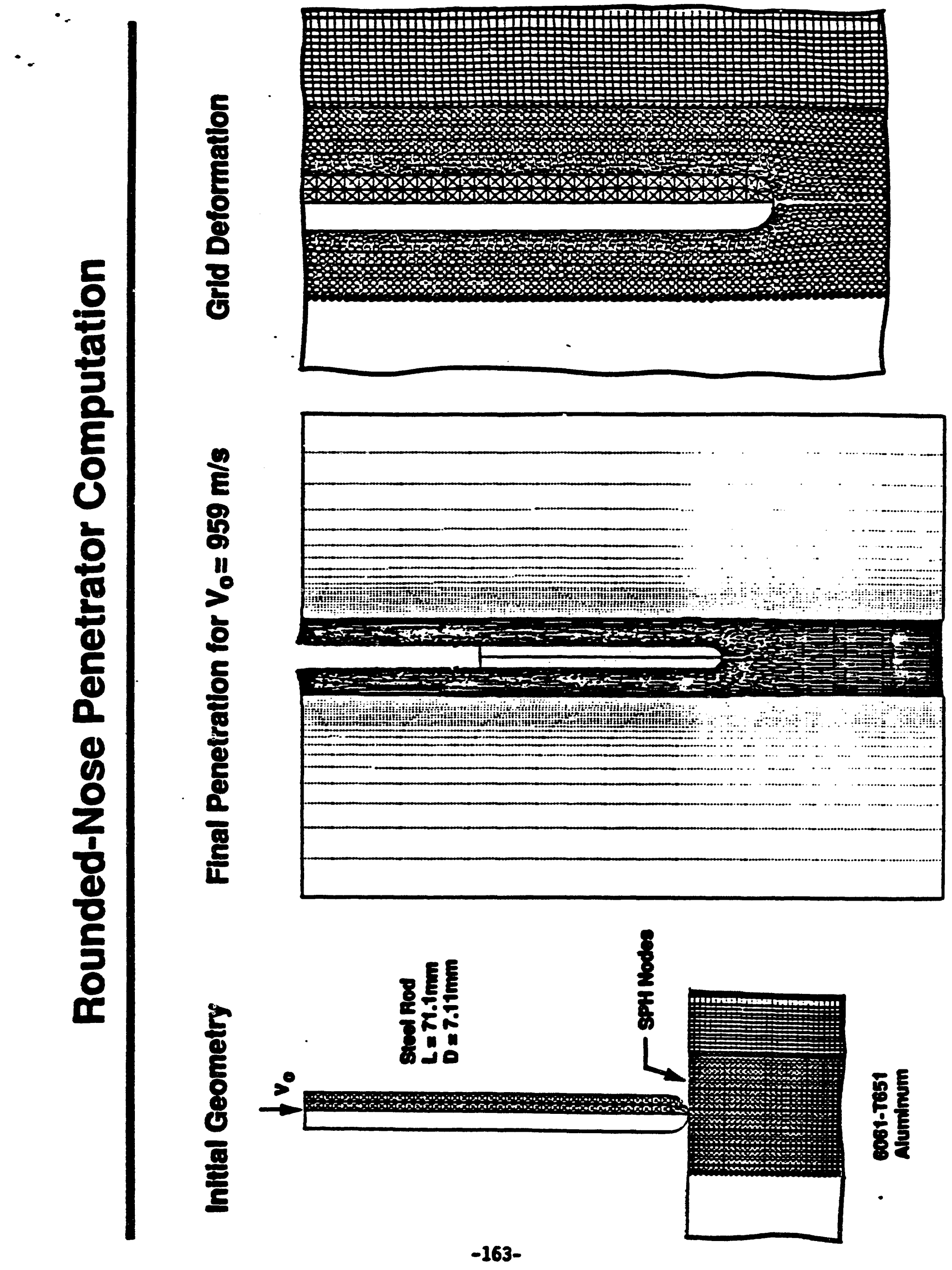




\section{Linked SPH Results Compared to Test Data}

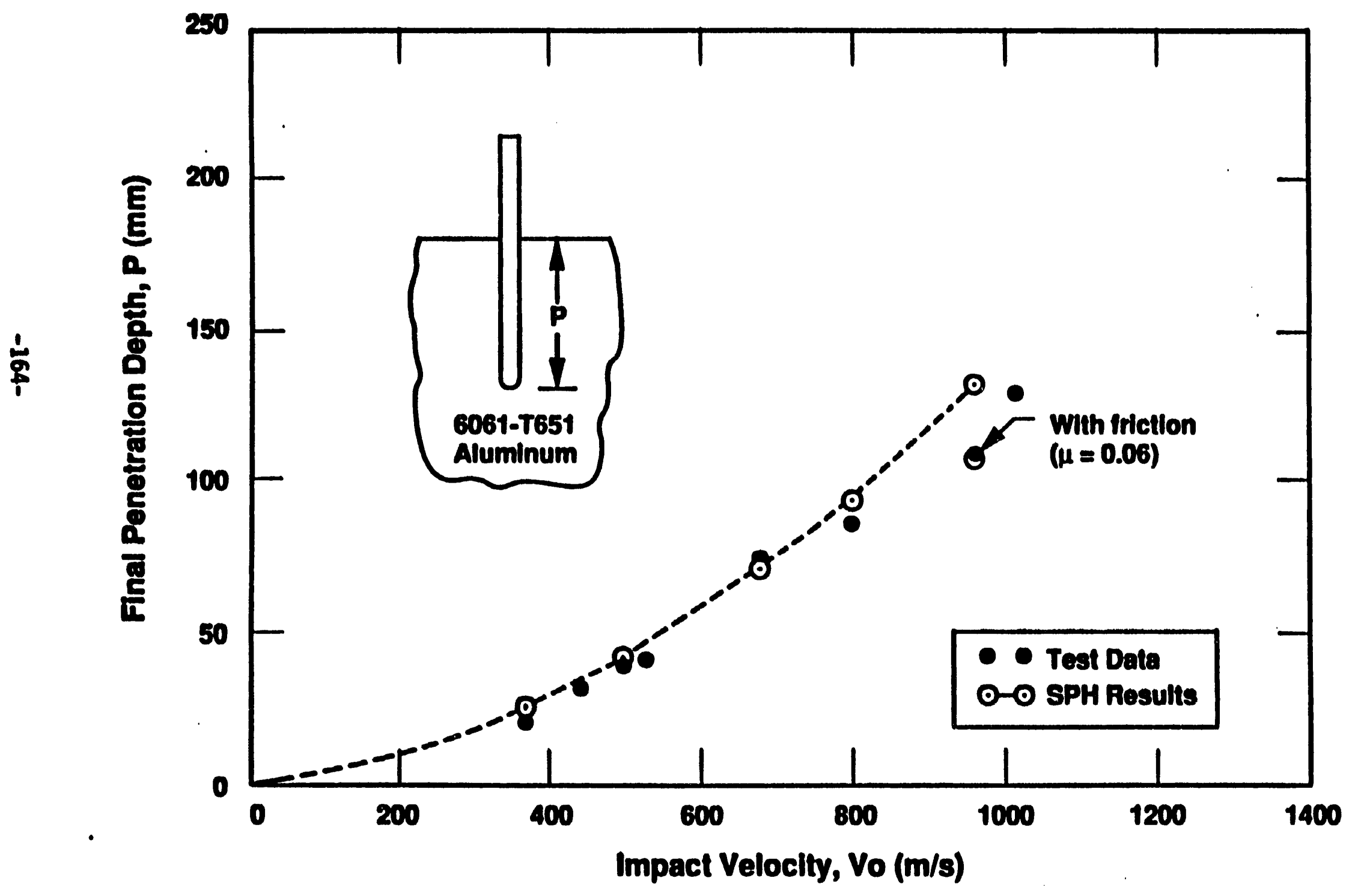

GRS2 


\section{SPH Attachment to Standard Grid}

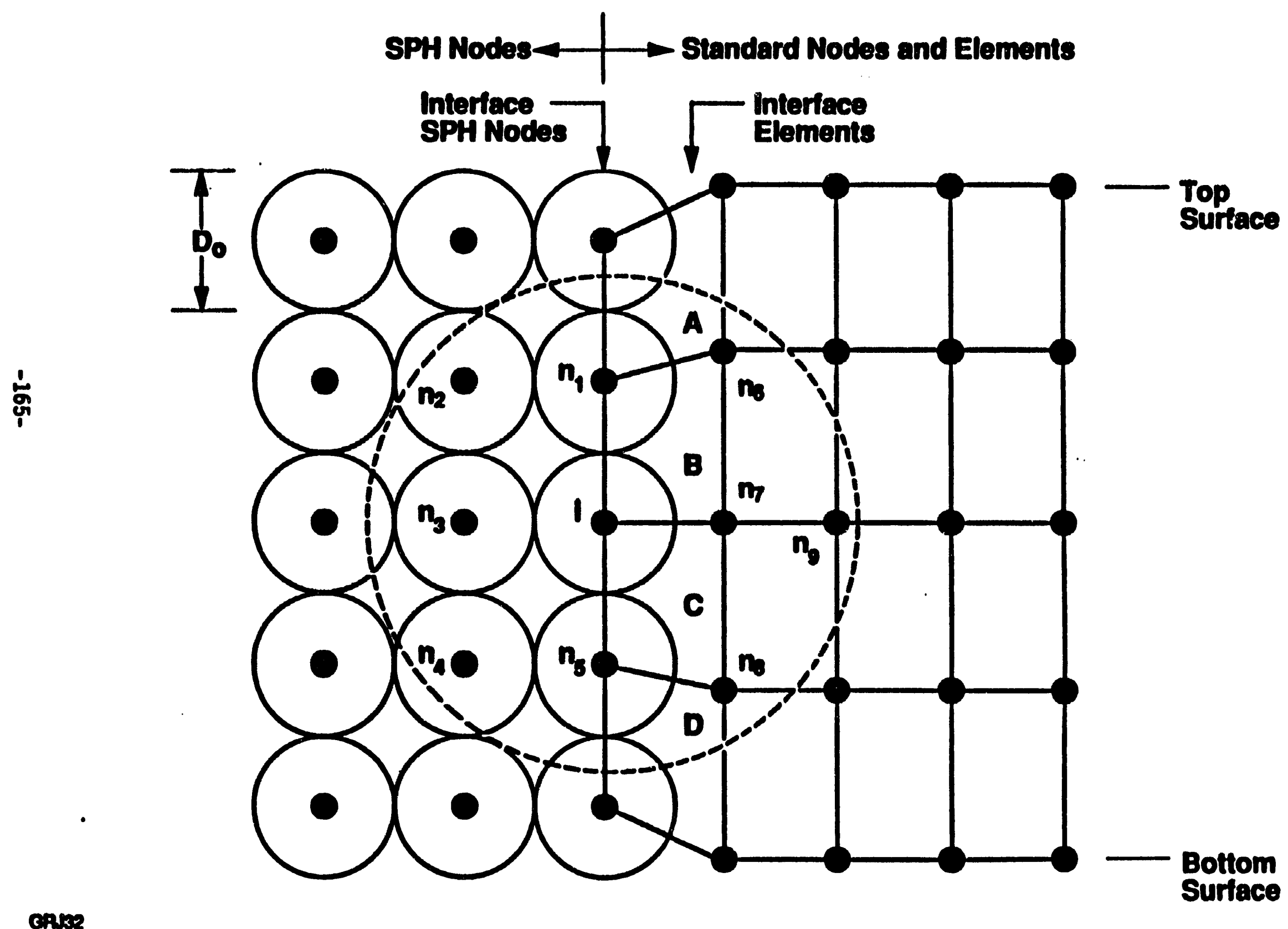




\section{SPH Sliding on Standard Grid}

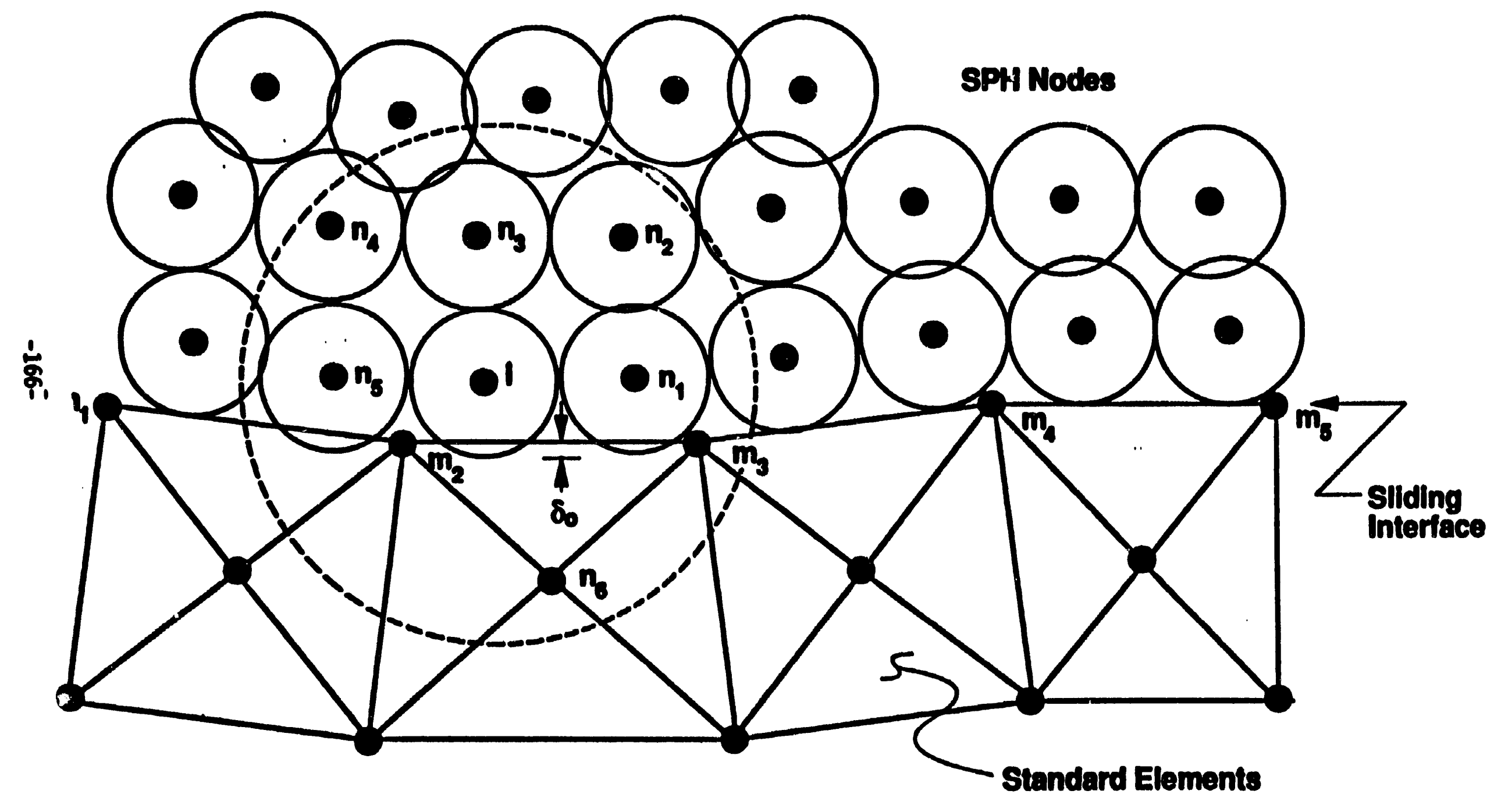

- SPH nodes are slave nodes

- Master surface defined by standard nodes $m_{1} \ldots m_{8}$ 


\section{Eroding Penetrator Computation}
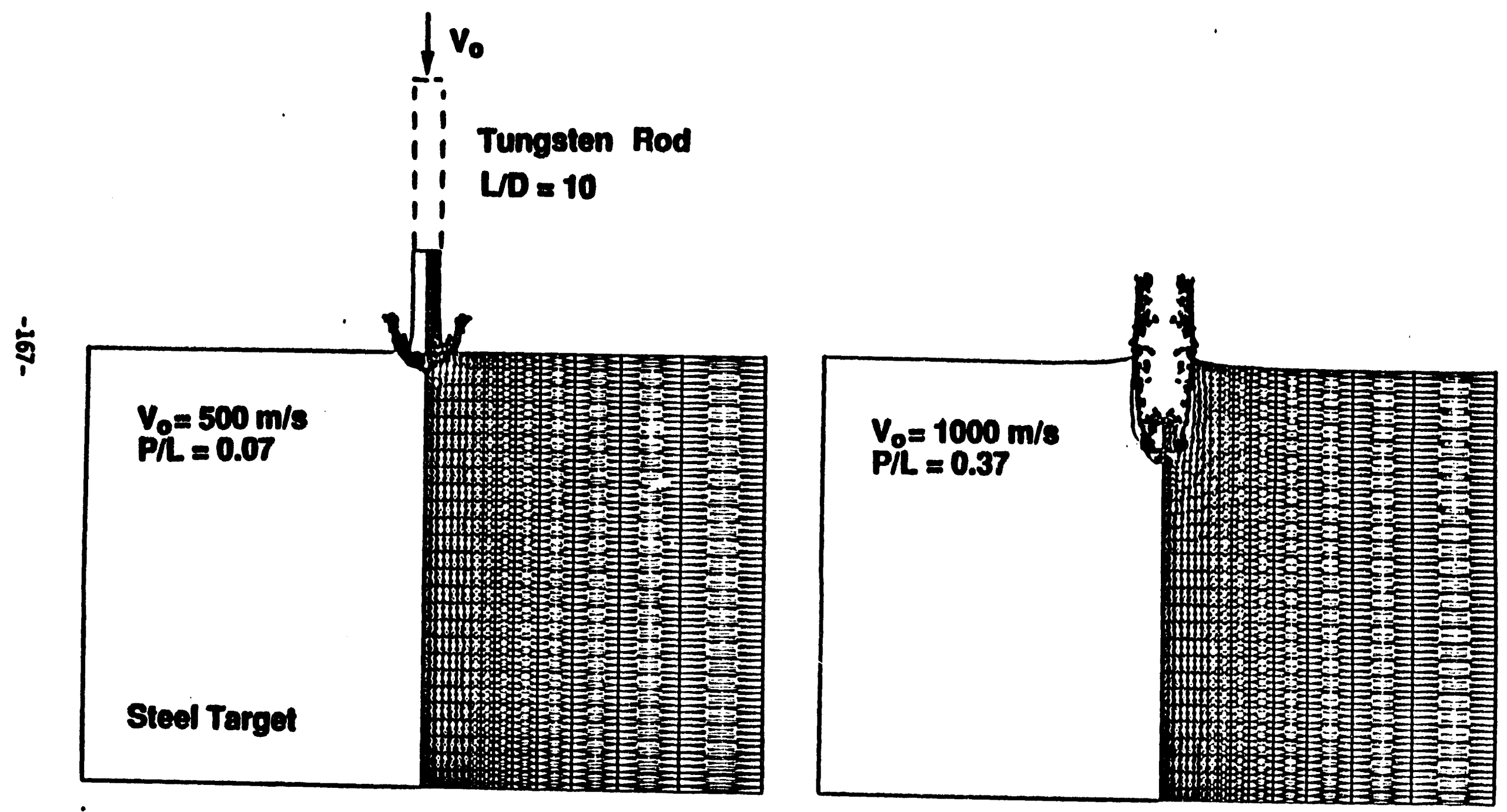


\section{Eroding Penetrator Computation}
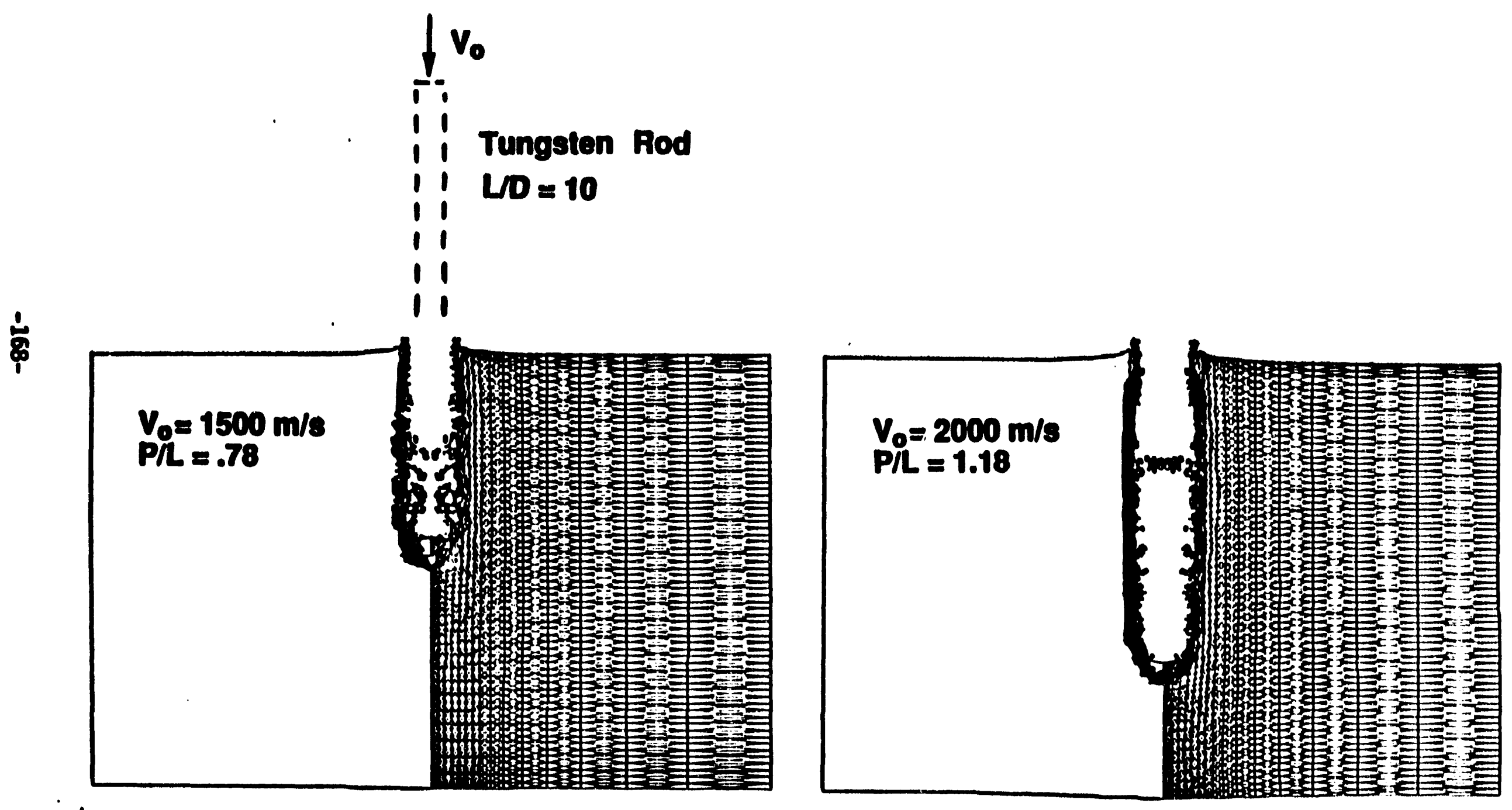

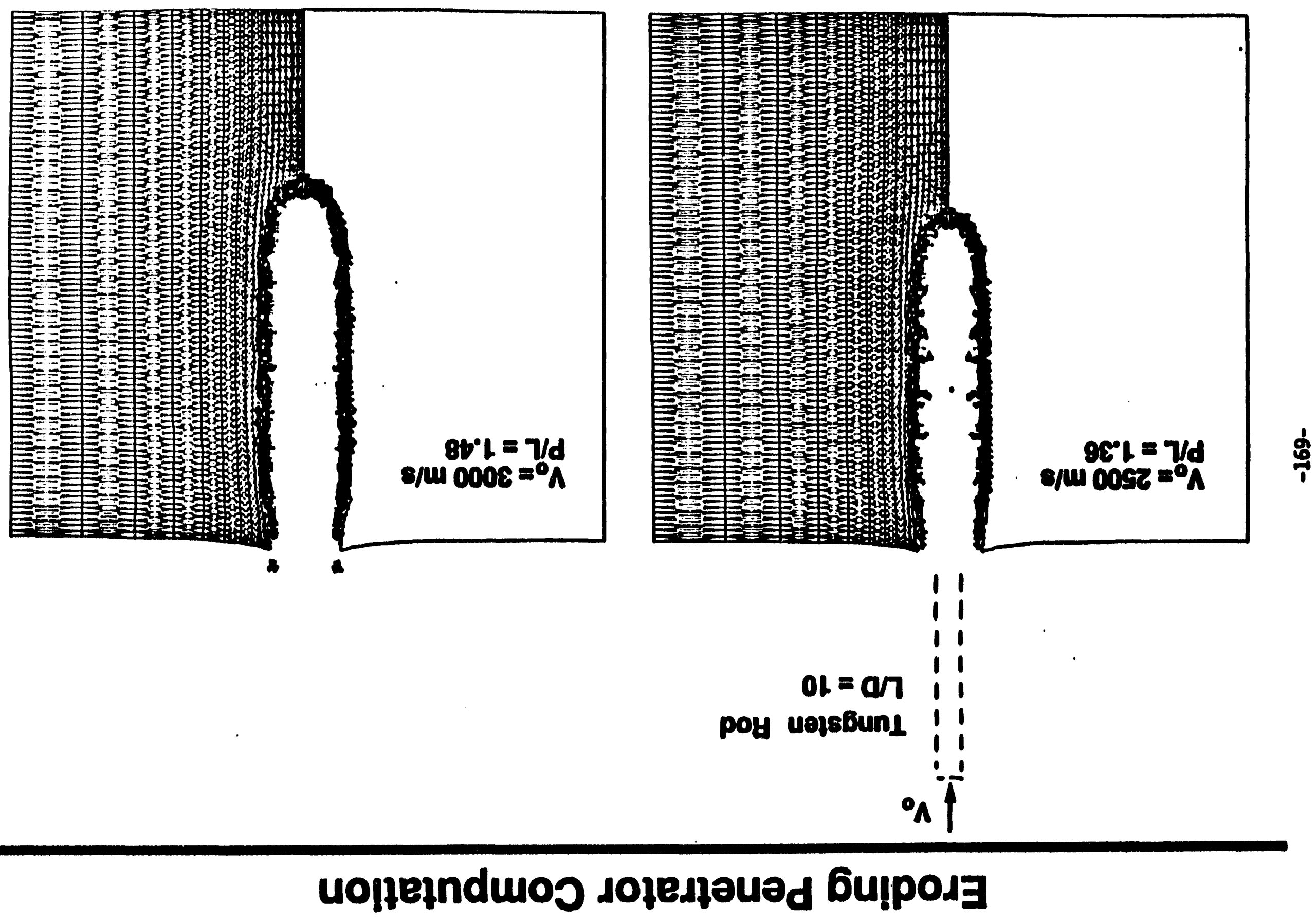

uopfełndwos sopedpoued bu!podg 


\section{Linked SPH Results Compared to Test Data}

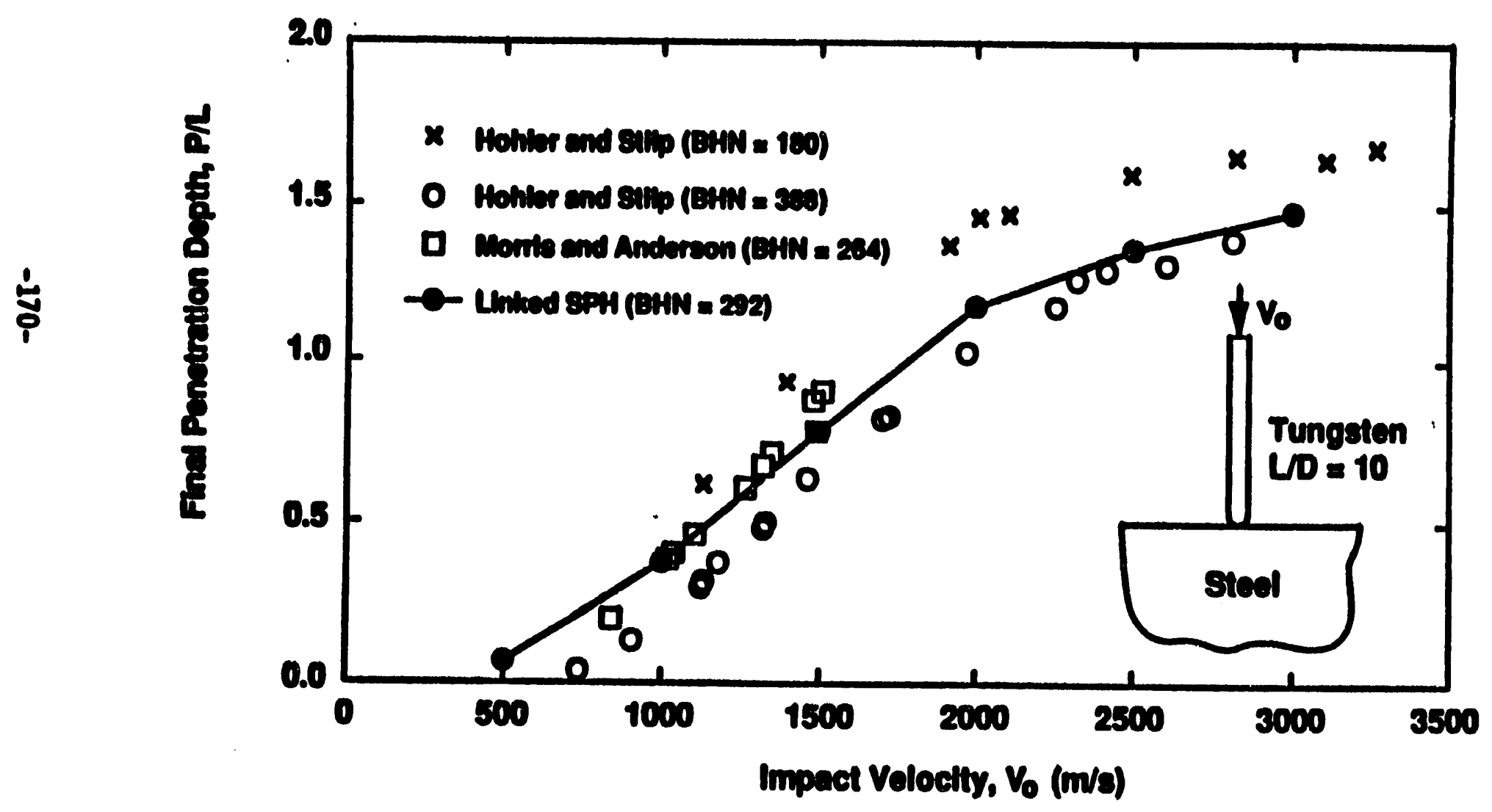

GANOO 


\section{AUTOMATIC SPH NODE GENERATION}

\section{STANDARD EROSION ALGORITHM}

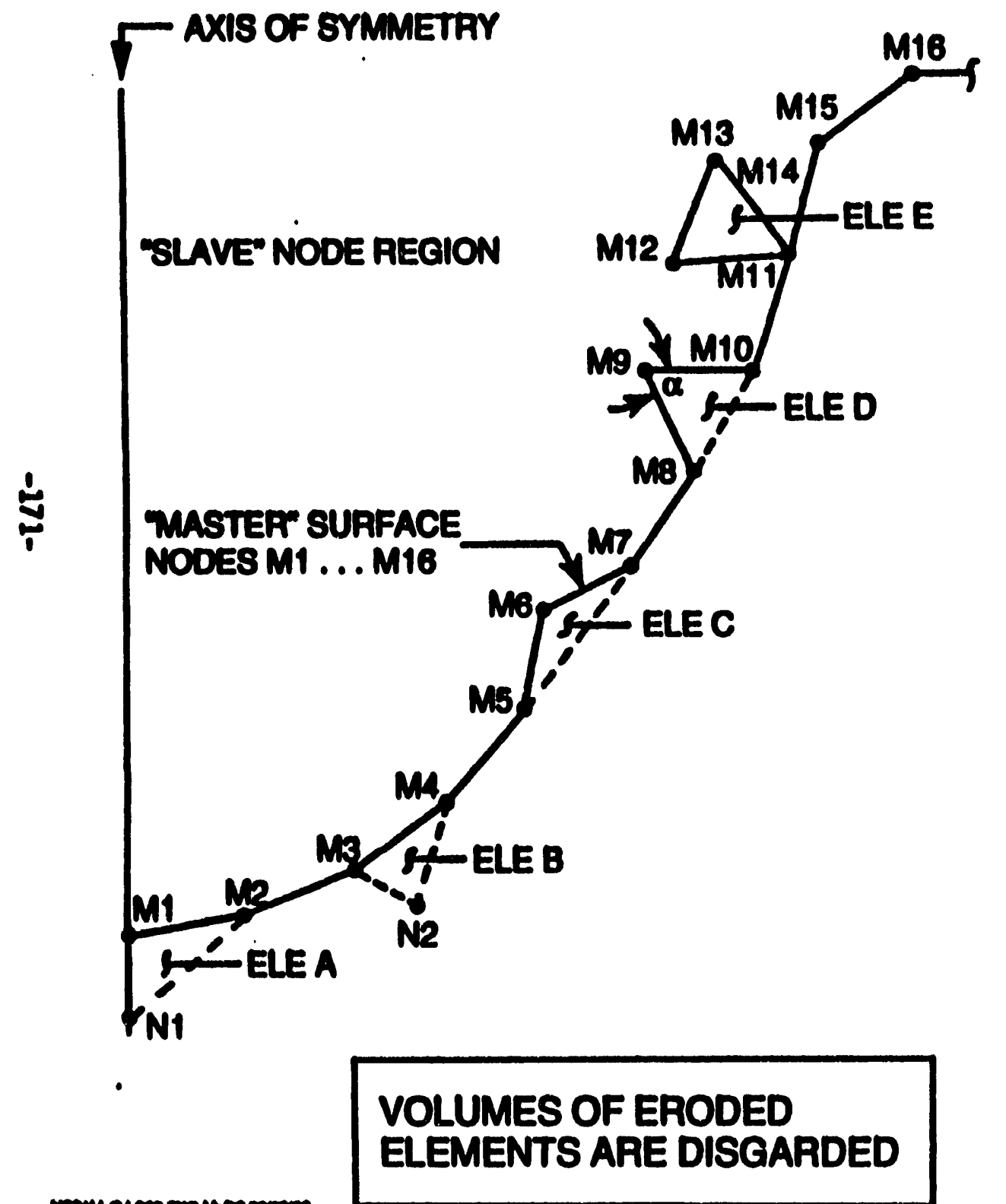

\section{SPH NODE GENERATION}

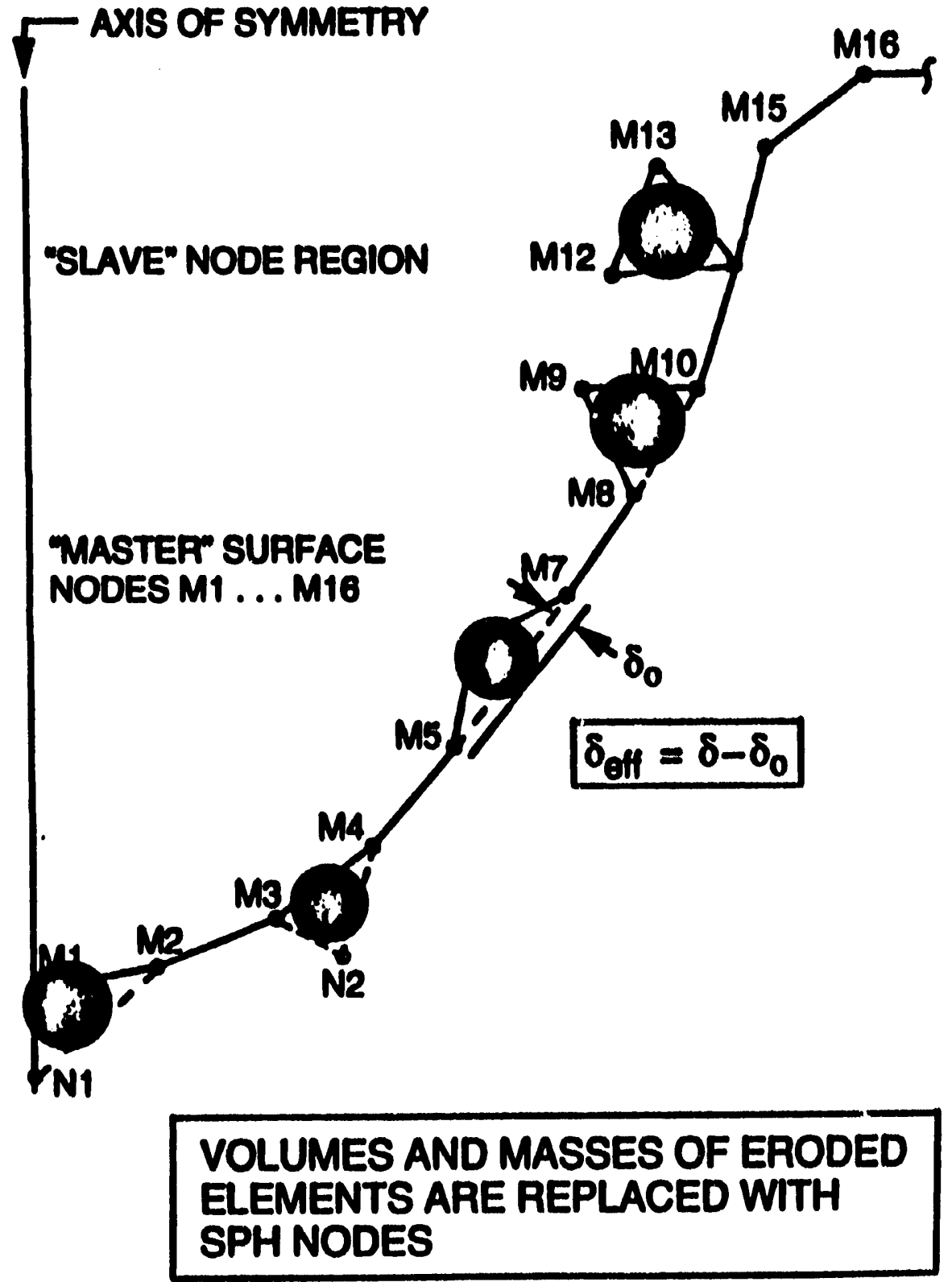




\section{Summary and Conclusions}

- Linked SPH computations show good agreement with test data for three sets of problems

- Linked SPH techniques show great potential for some classes of problems

- More evaluation and development is required for linked SPH

- Capability

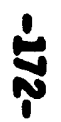

- Accuracy (material interfaces)

- Efficlency (computing time) 


\title{
Topics in SPH
}

\author{
Chuck Wingate
}

\section{Los Alamos National Laboratory}




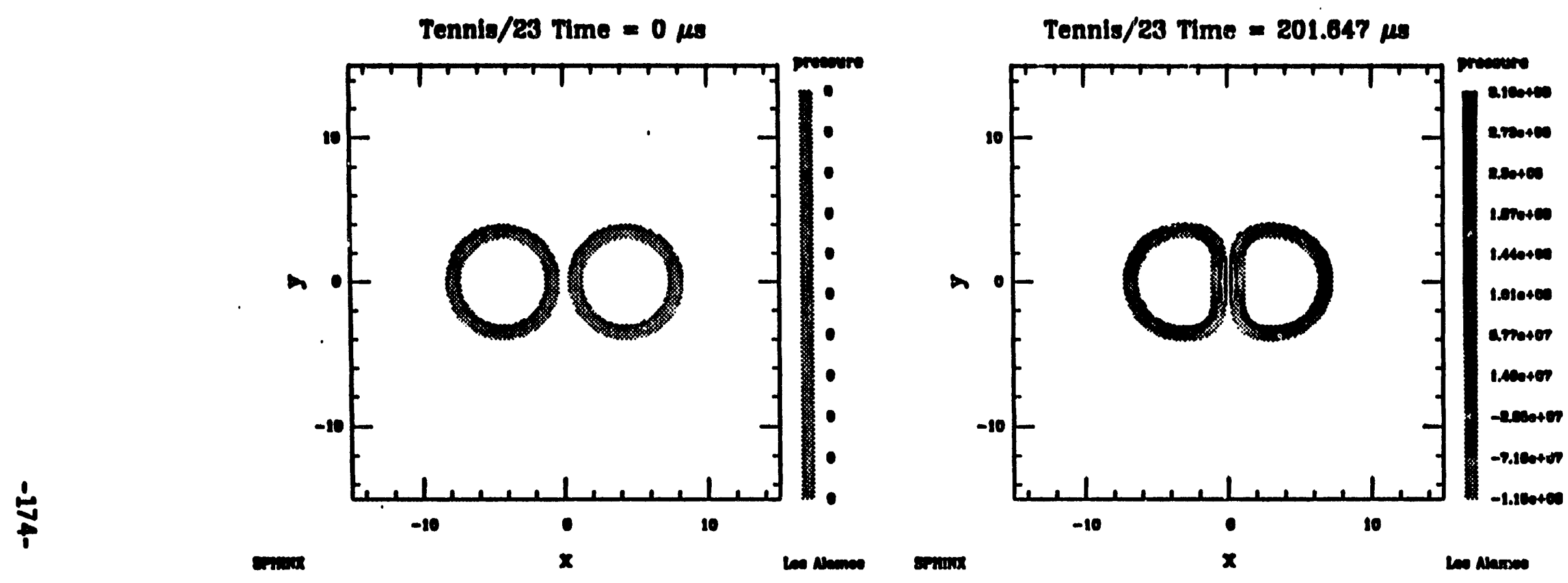

Tennis/23 Time $=400.564 \mu \mathrm{s}$

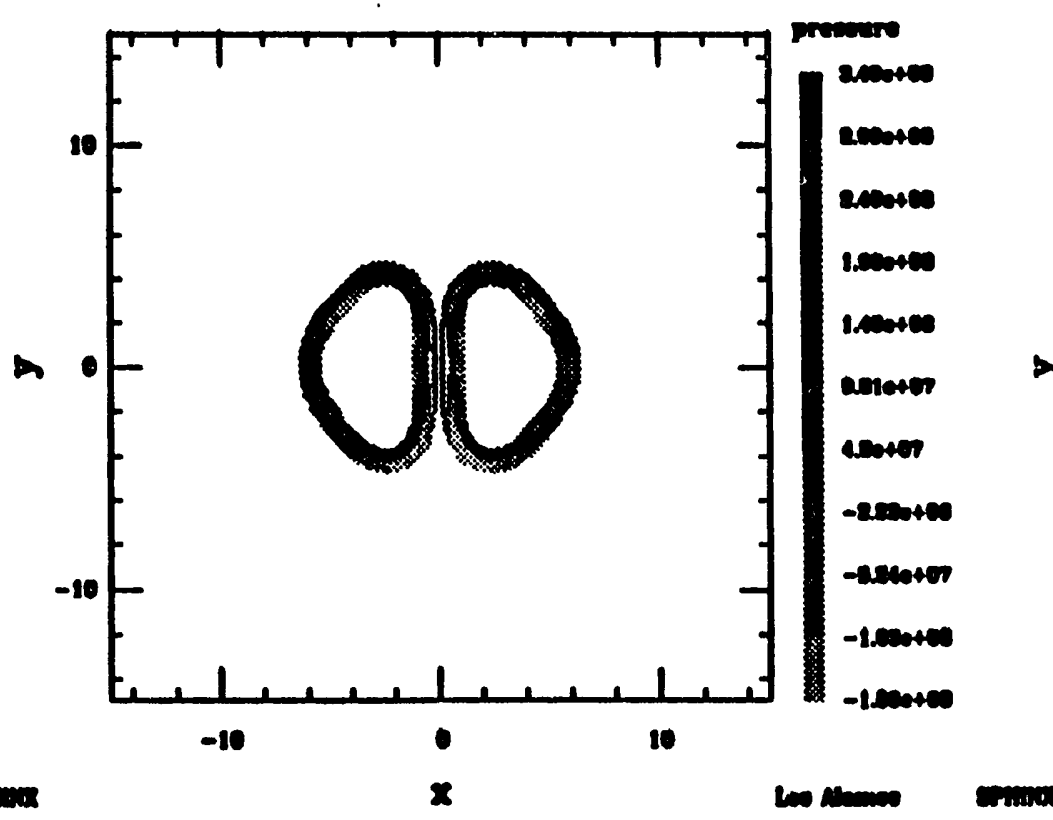

Tennis/23 Time $=603.947 \mu$

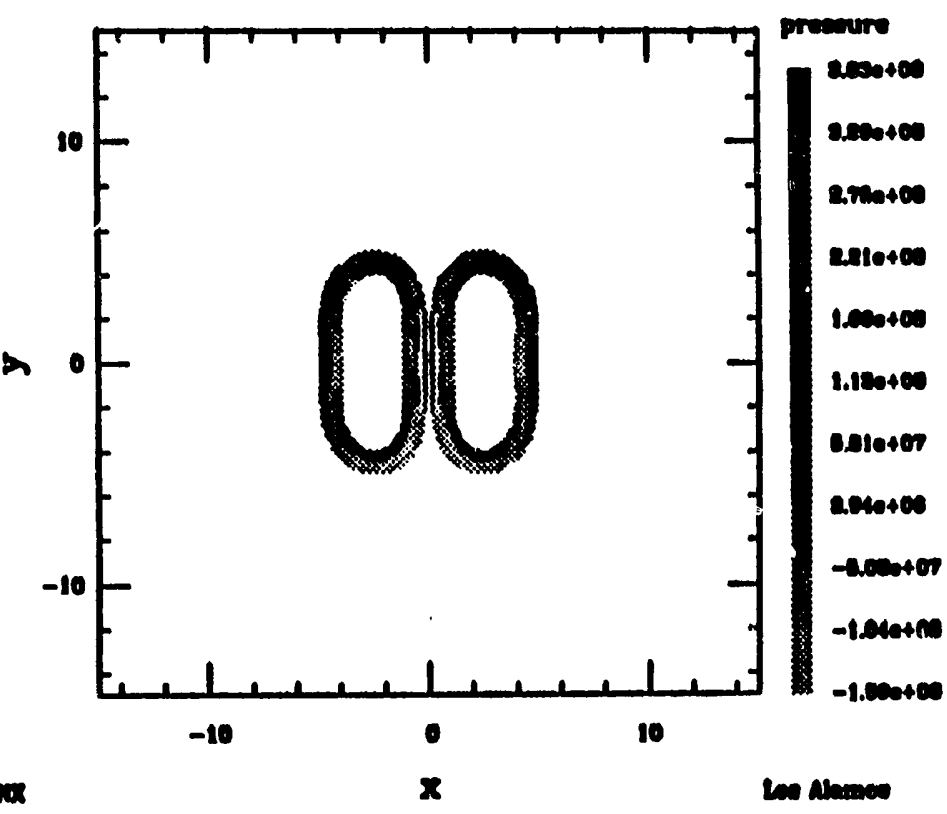


Tennis/23 Time $=802.315 \mu \mathrm{s}$

है

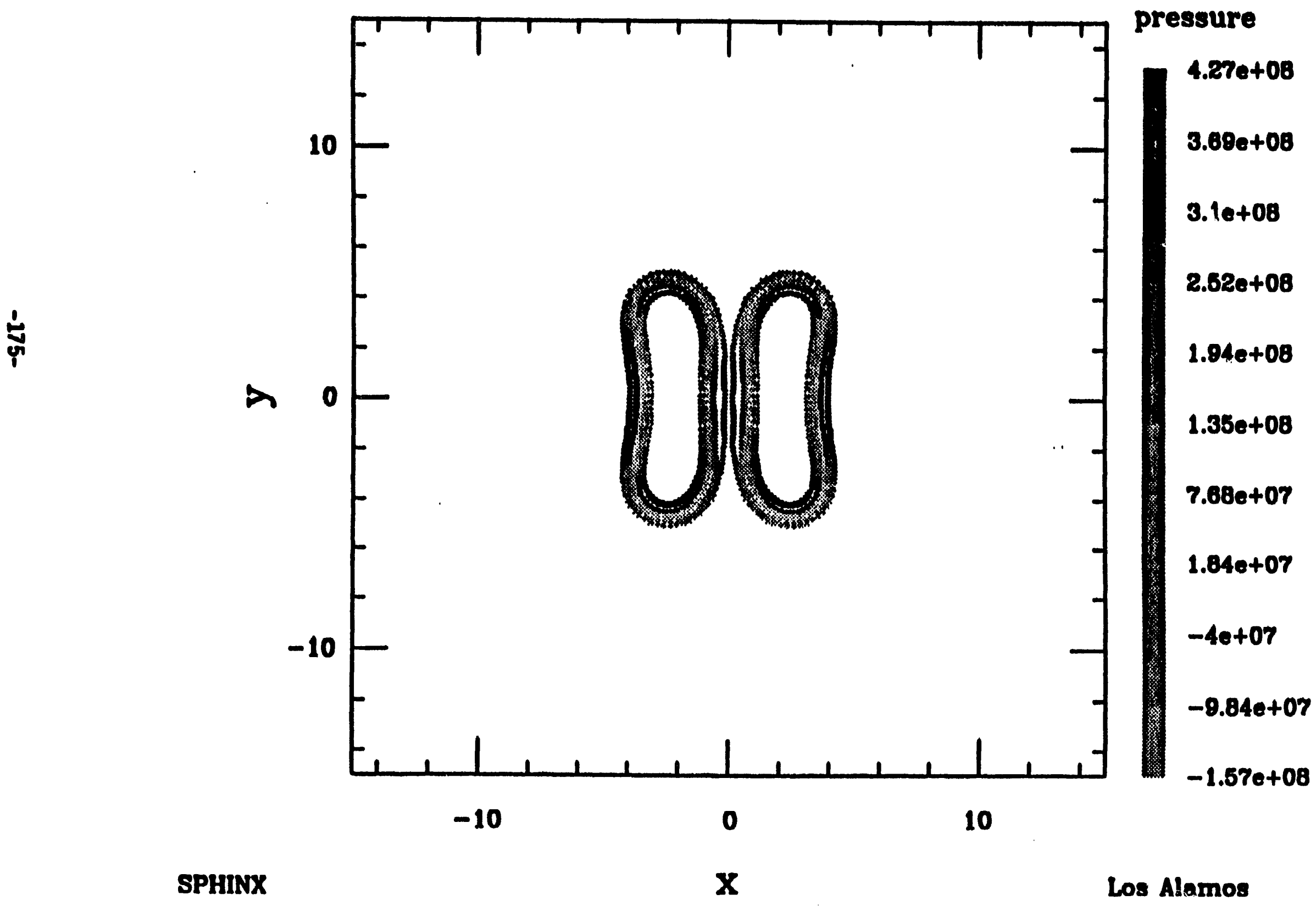



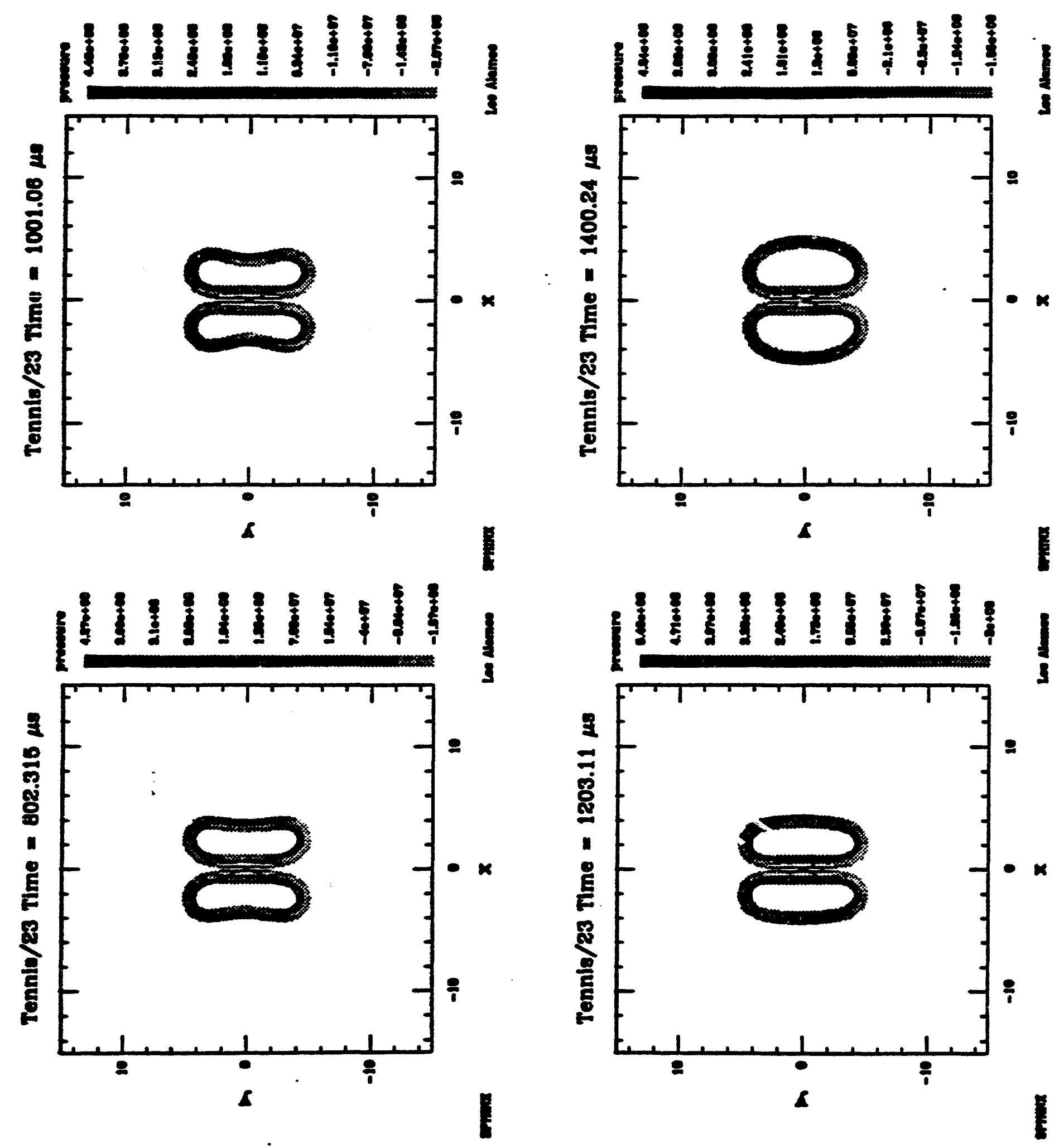

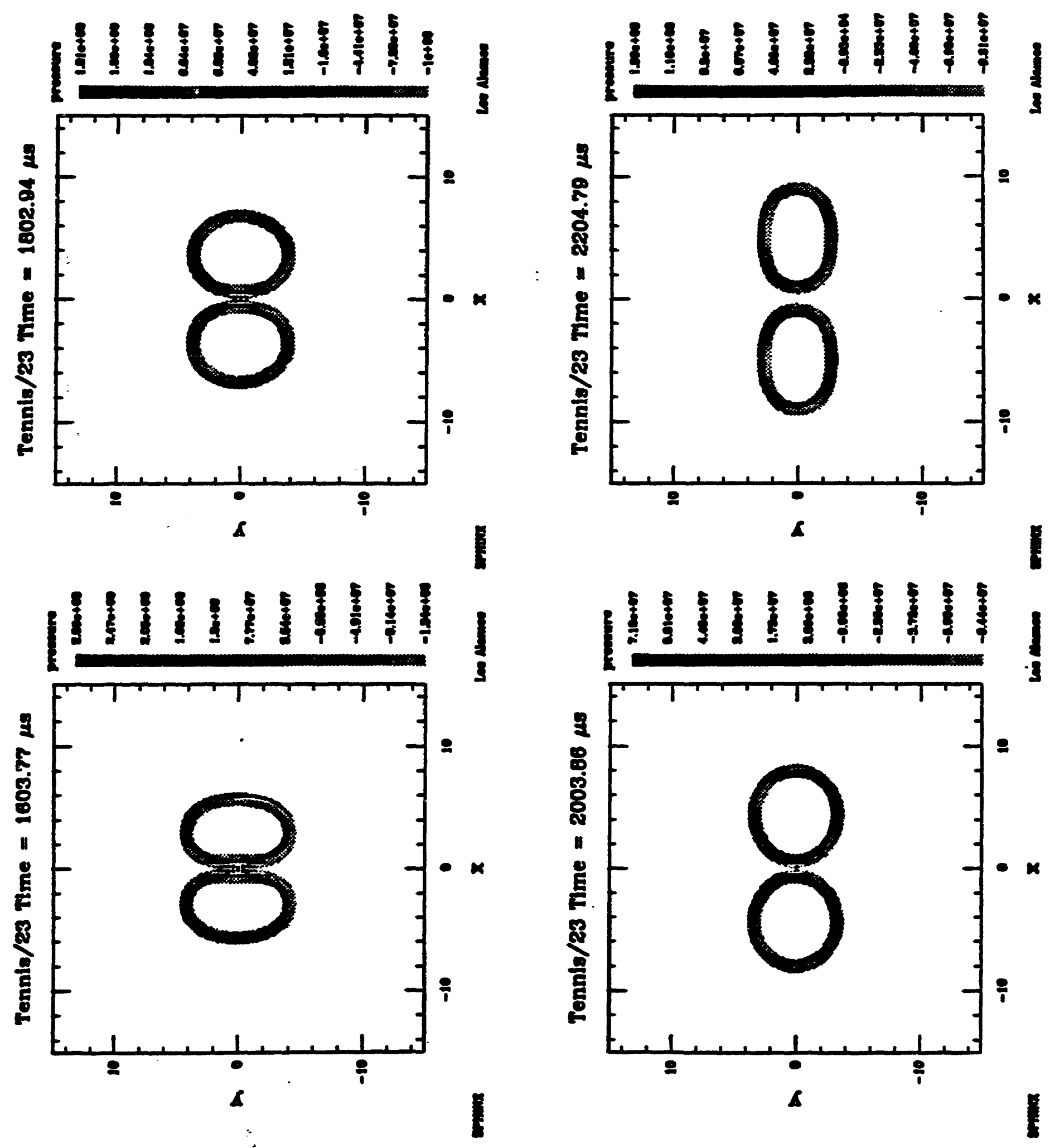

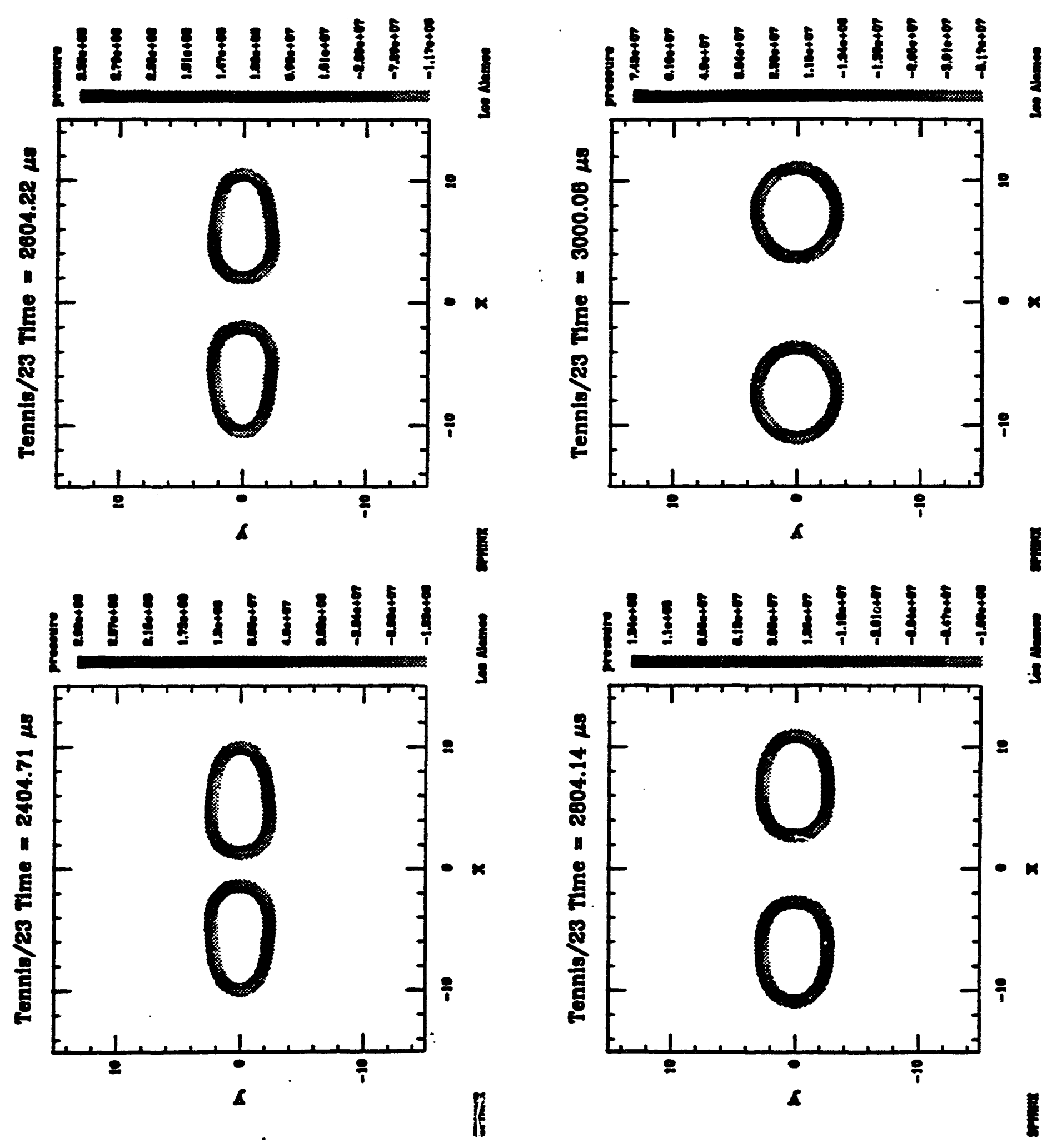

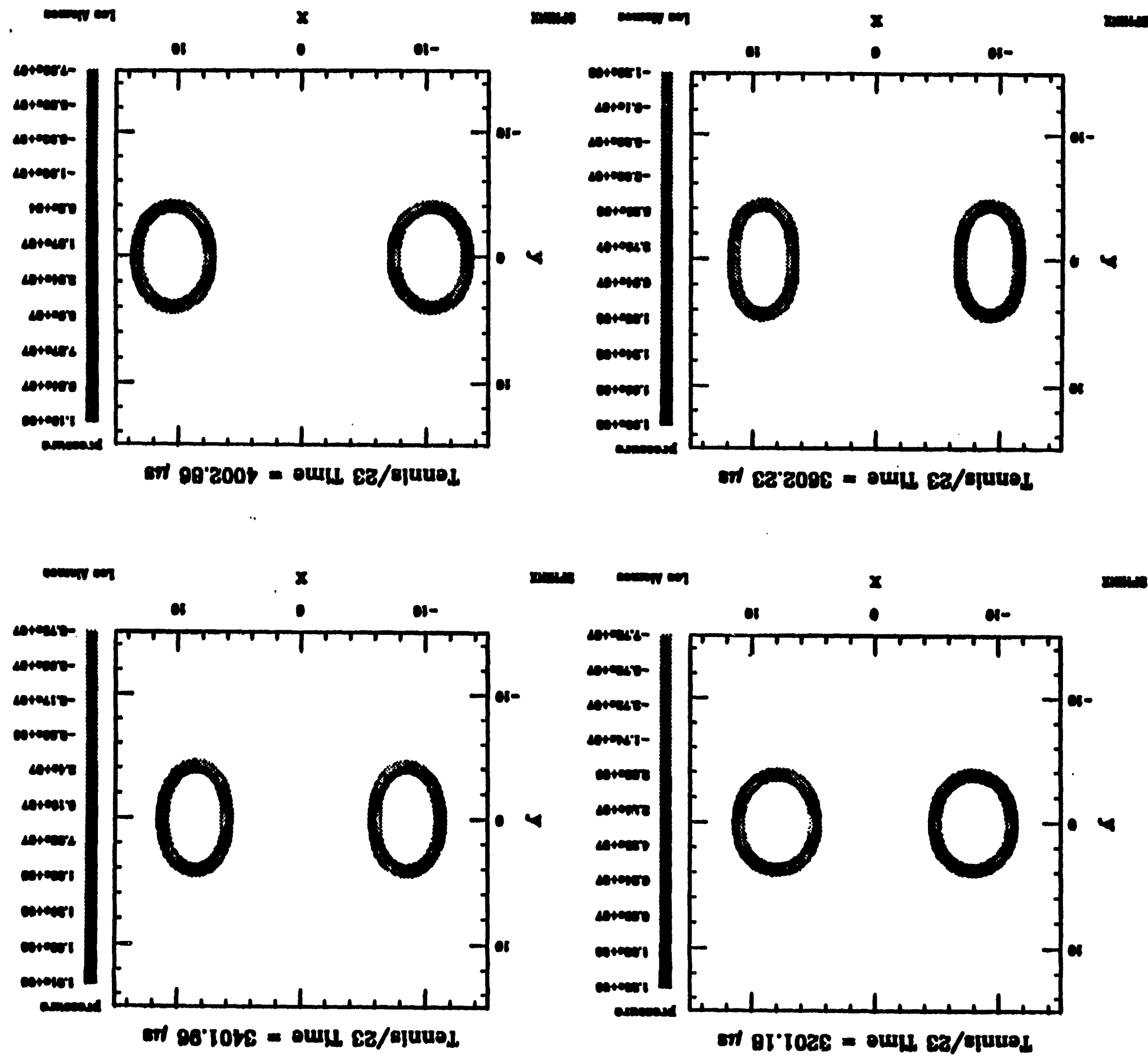
Tennis $/ 16$ Time $=2000.03 \mu \mathrm{s}$

8

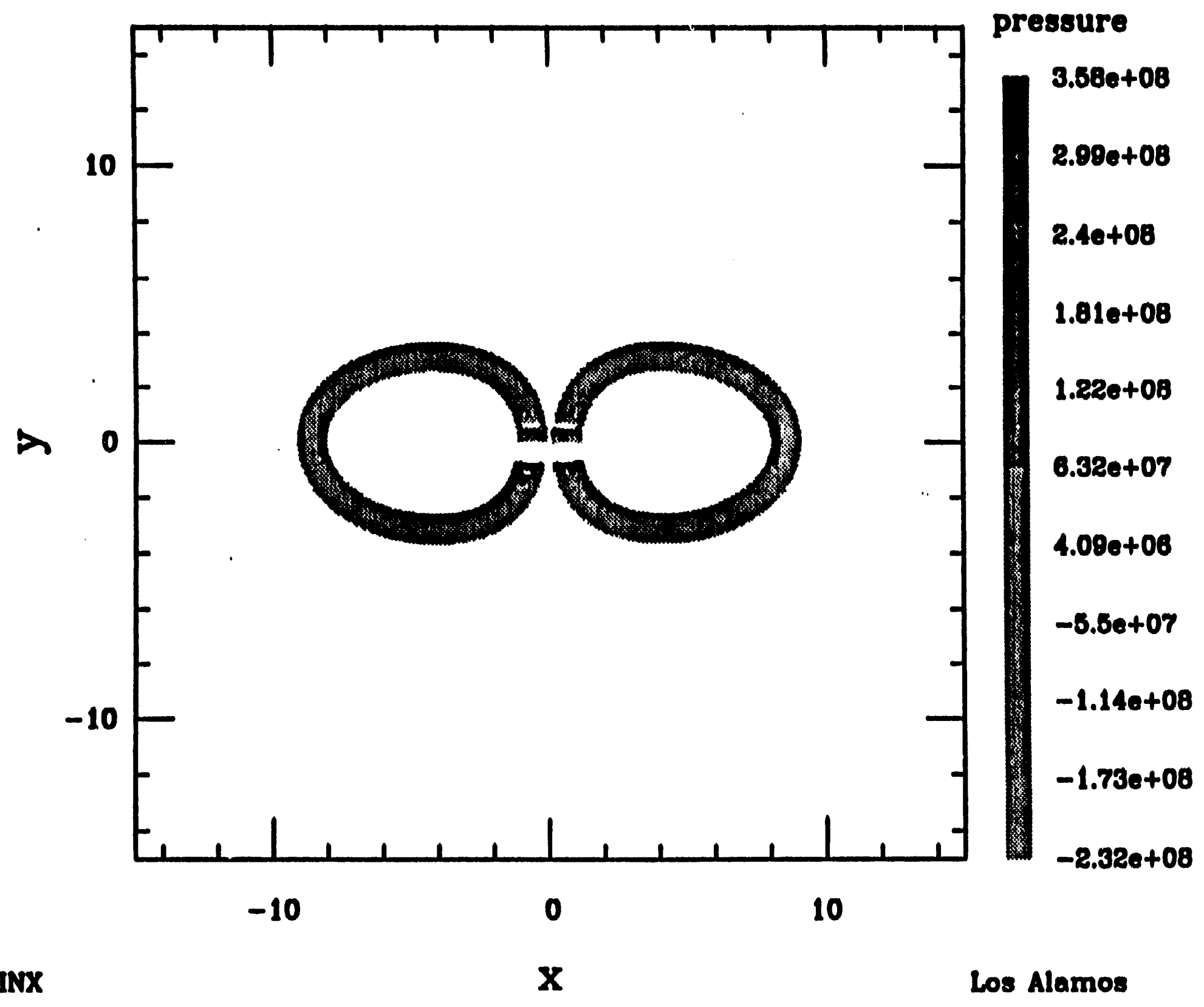




\section{Tennis/22 Time $=2001.14 \mu \mathrm{s}$}

$\frac{1}{1}$

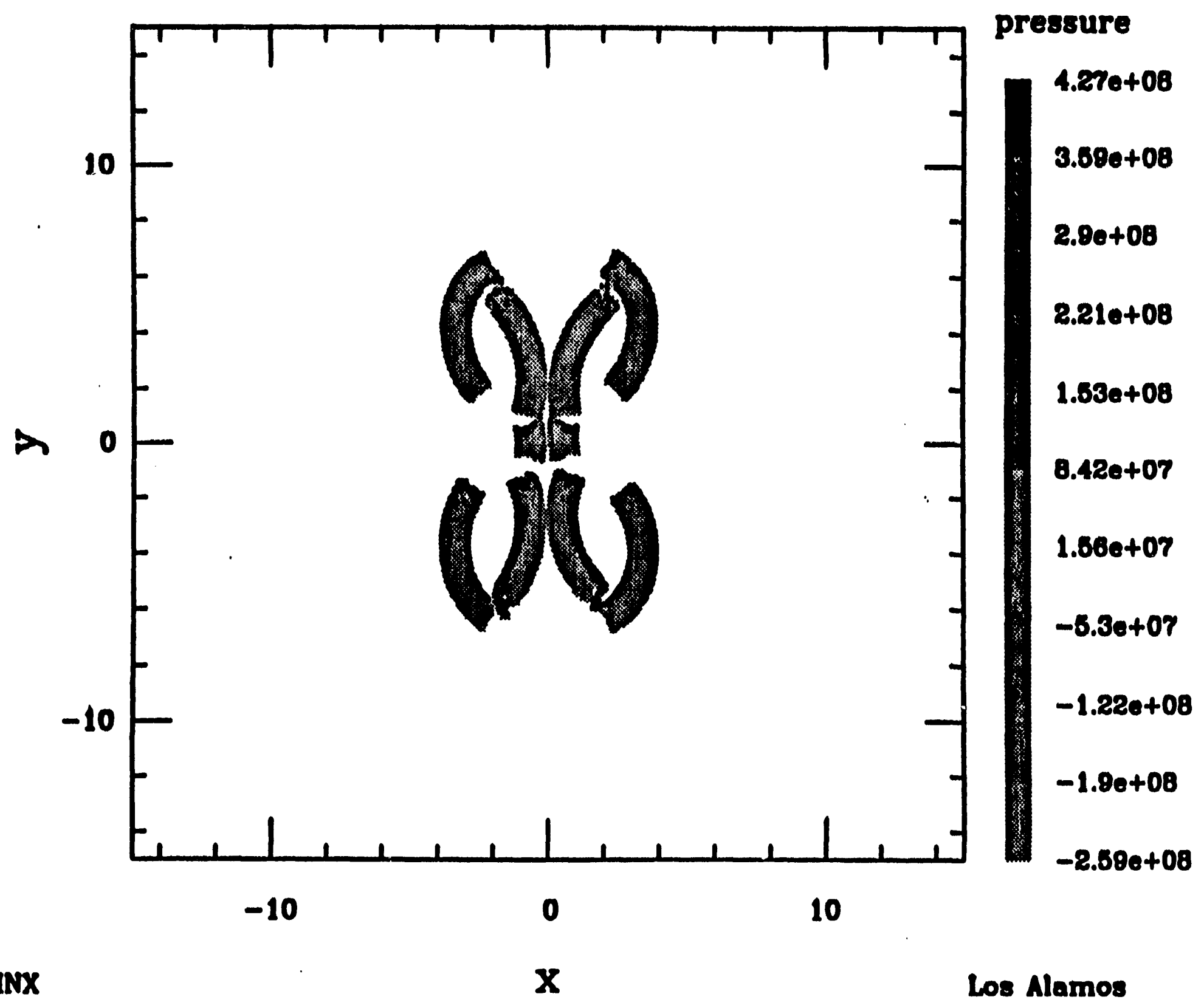




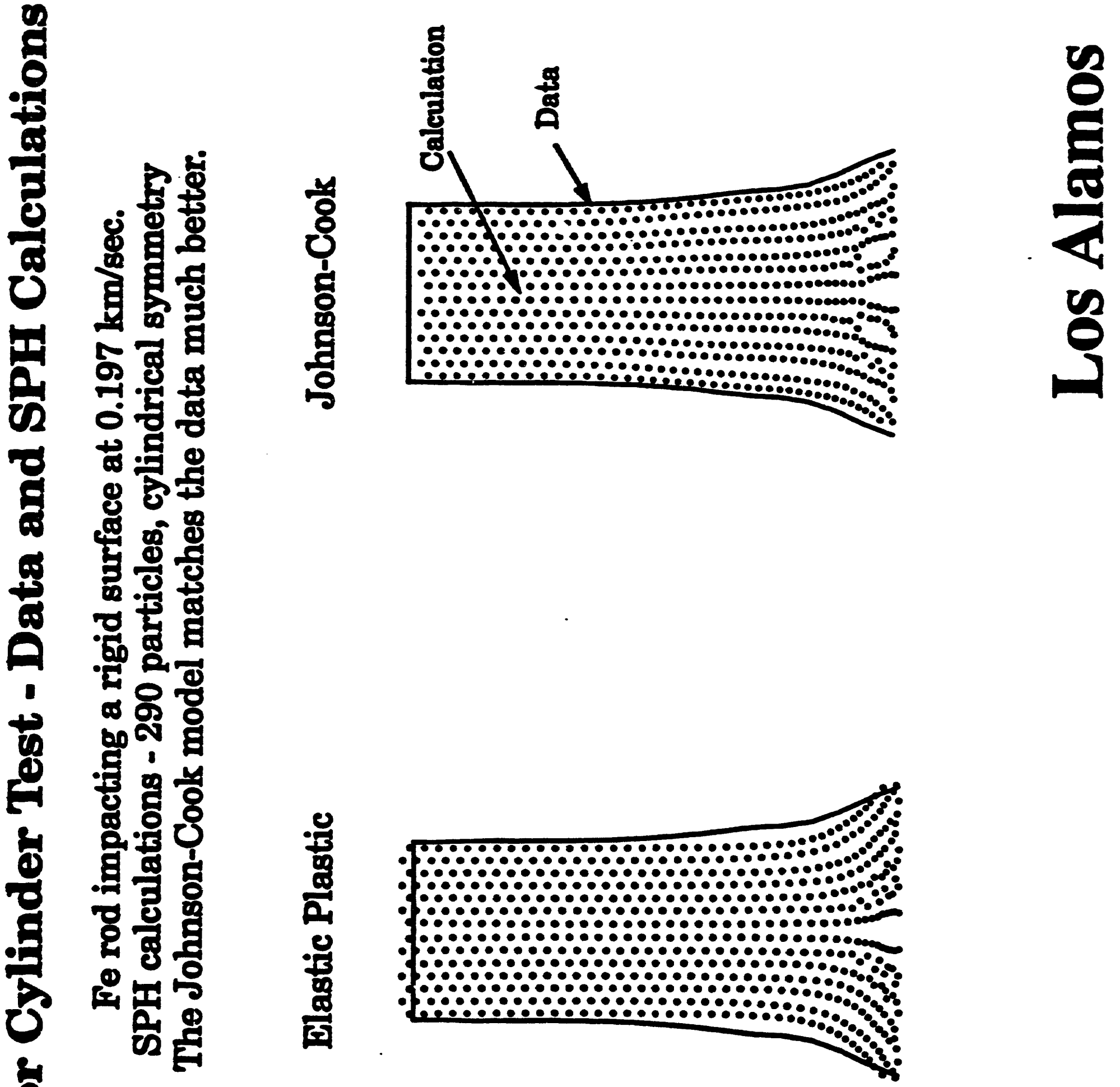




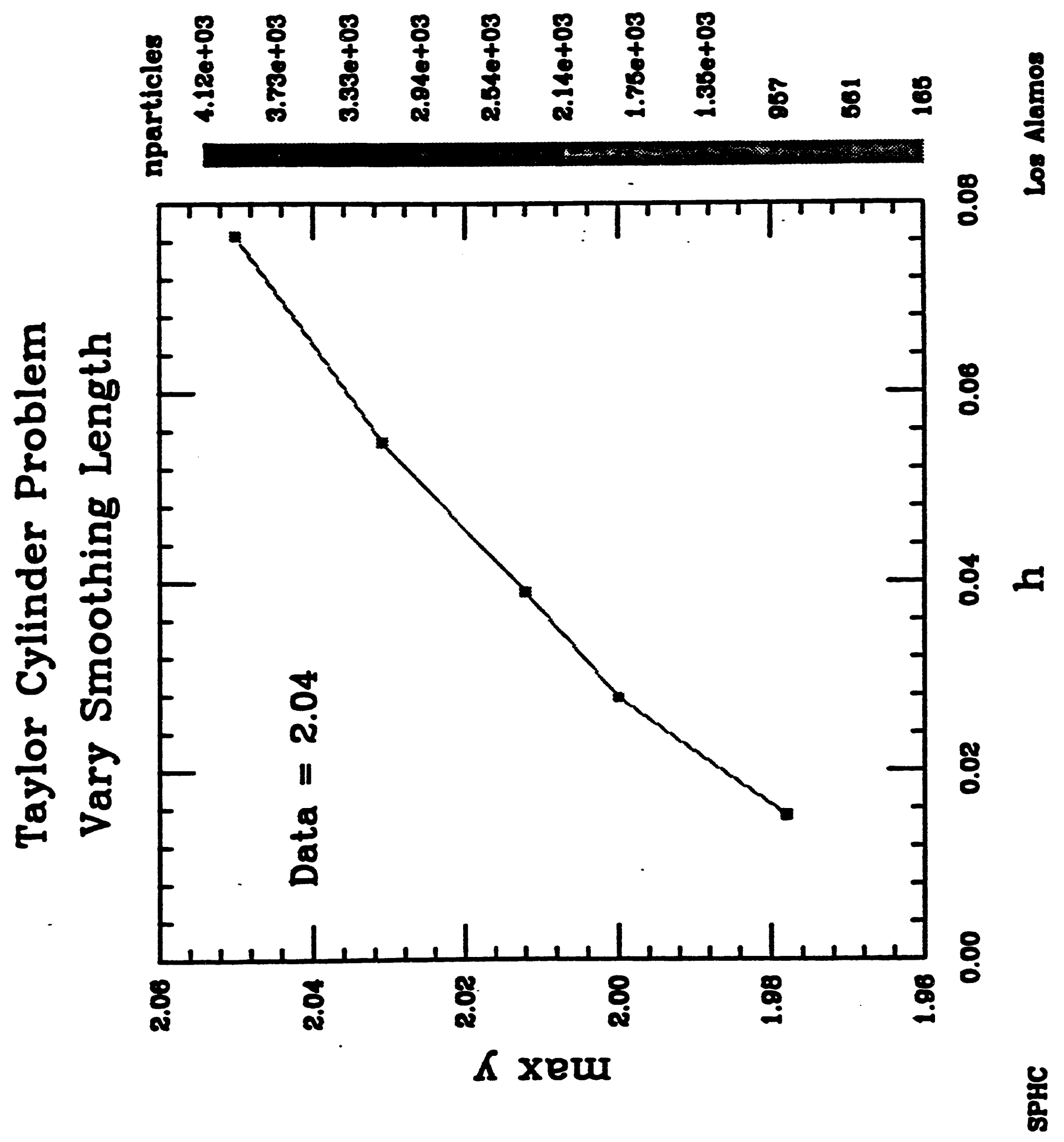


Shock Tube (Density Jump $=4$ ) - SPH Results $90,180,360$ particles and analytic

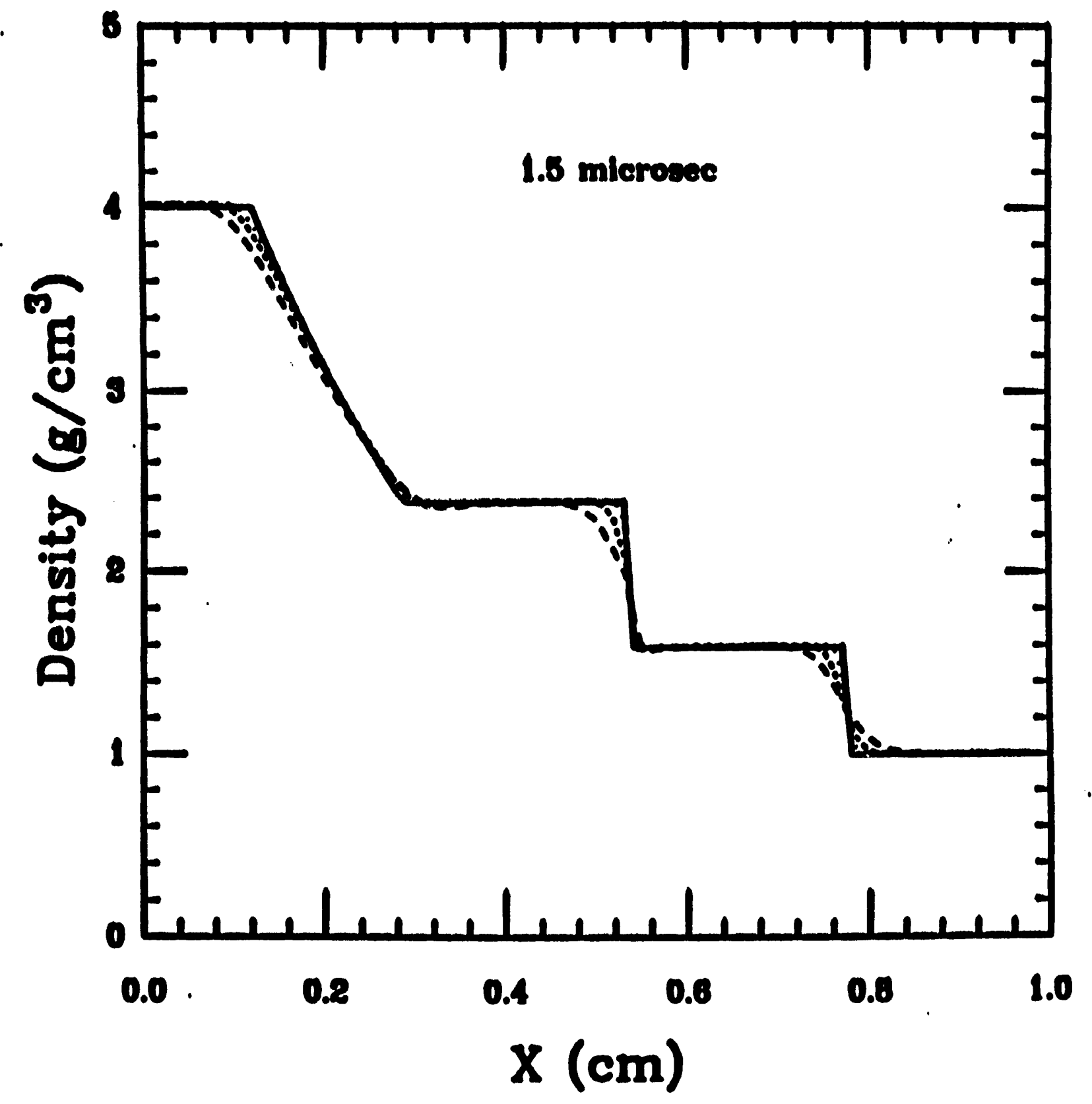


Strong Shock Tube (TP37C) - SPH Results 360 particles

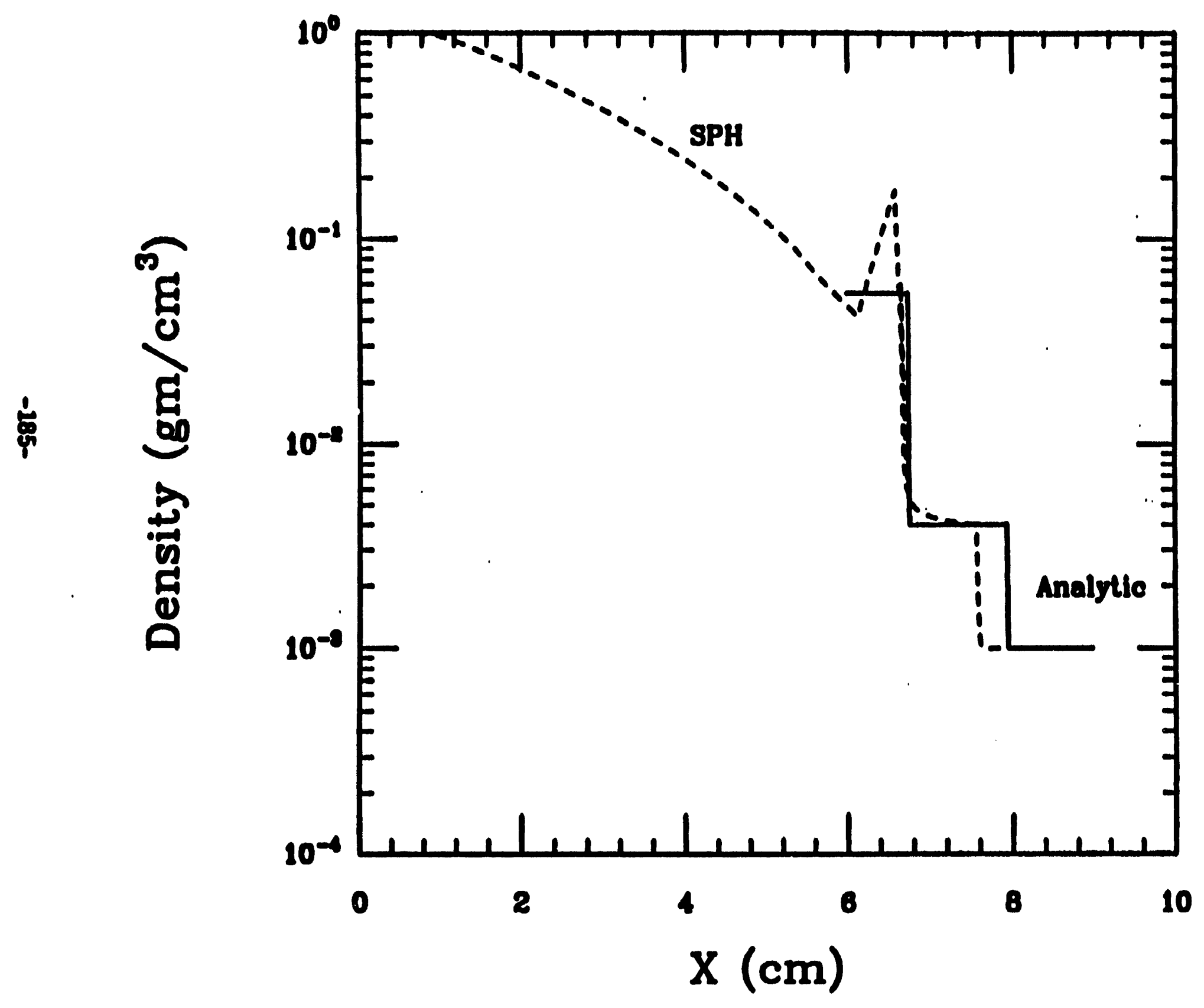



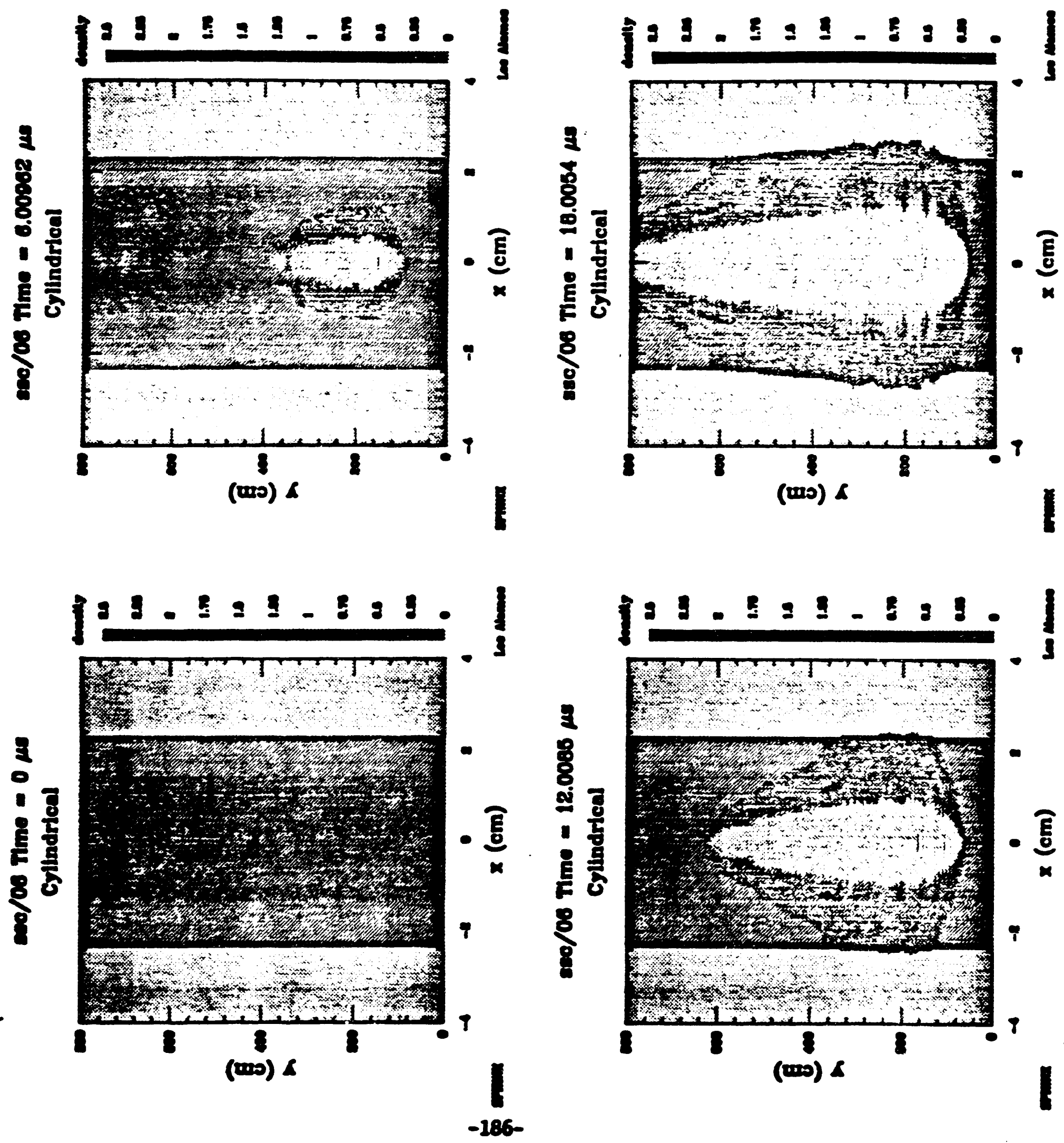

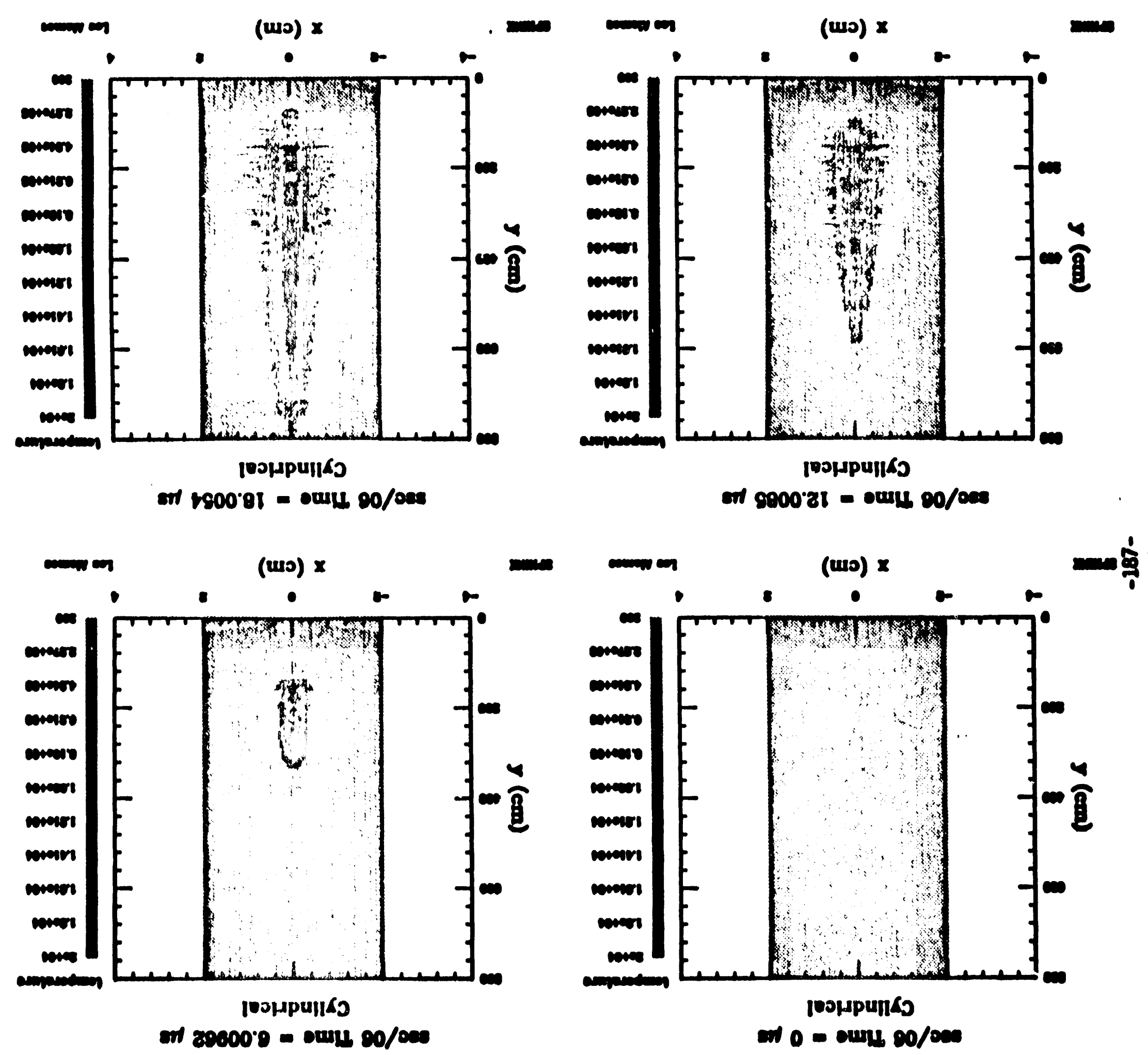


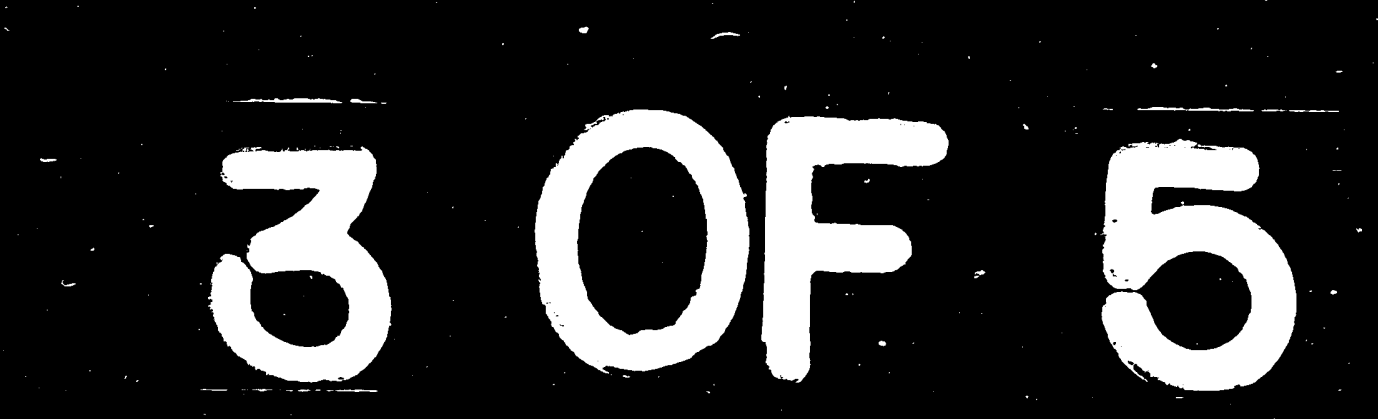



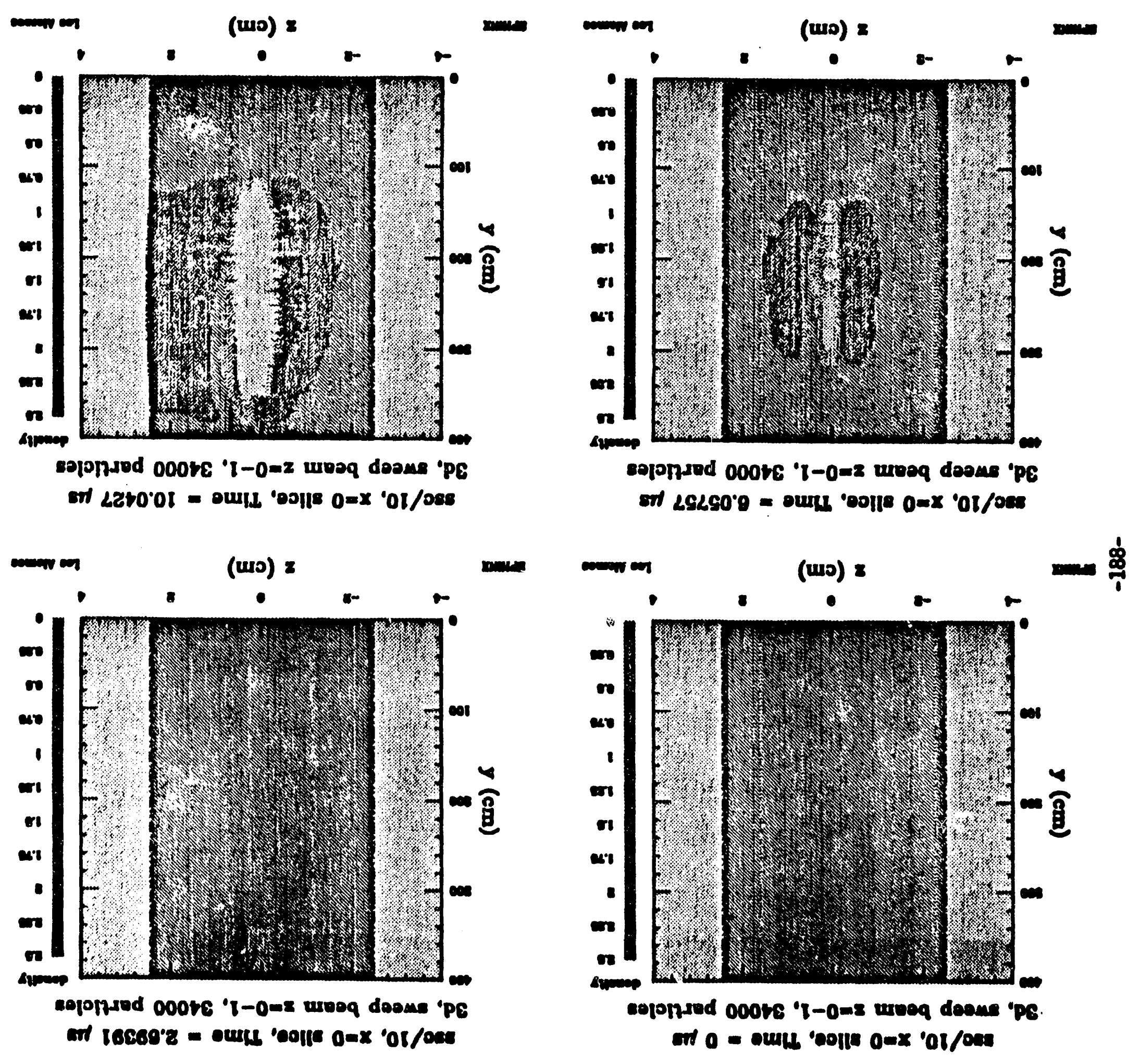


\section{STABILITY AND CONSISTENCY OF THE SPH METHOD}

J. W. Swegle* and D. L. Hicks ${ }^{\&}$

"Sandia National Laboratories

\&Michigan Technological University

Presented at the

Workshop on Advances in Smoothed Particle Hydrodynamics LANL, September 21-22, 1993 


\section{Outline}

1. Short review of stability criterion

2. Stability, the kernel function, and area vectors

3. Consistency of SPH

4. Adjustable factors in SPH numerics 


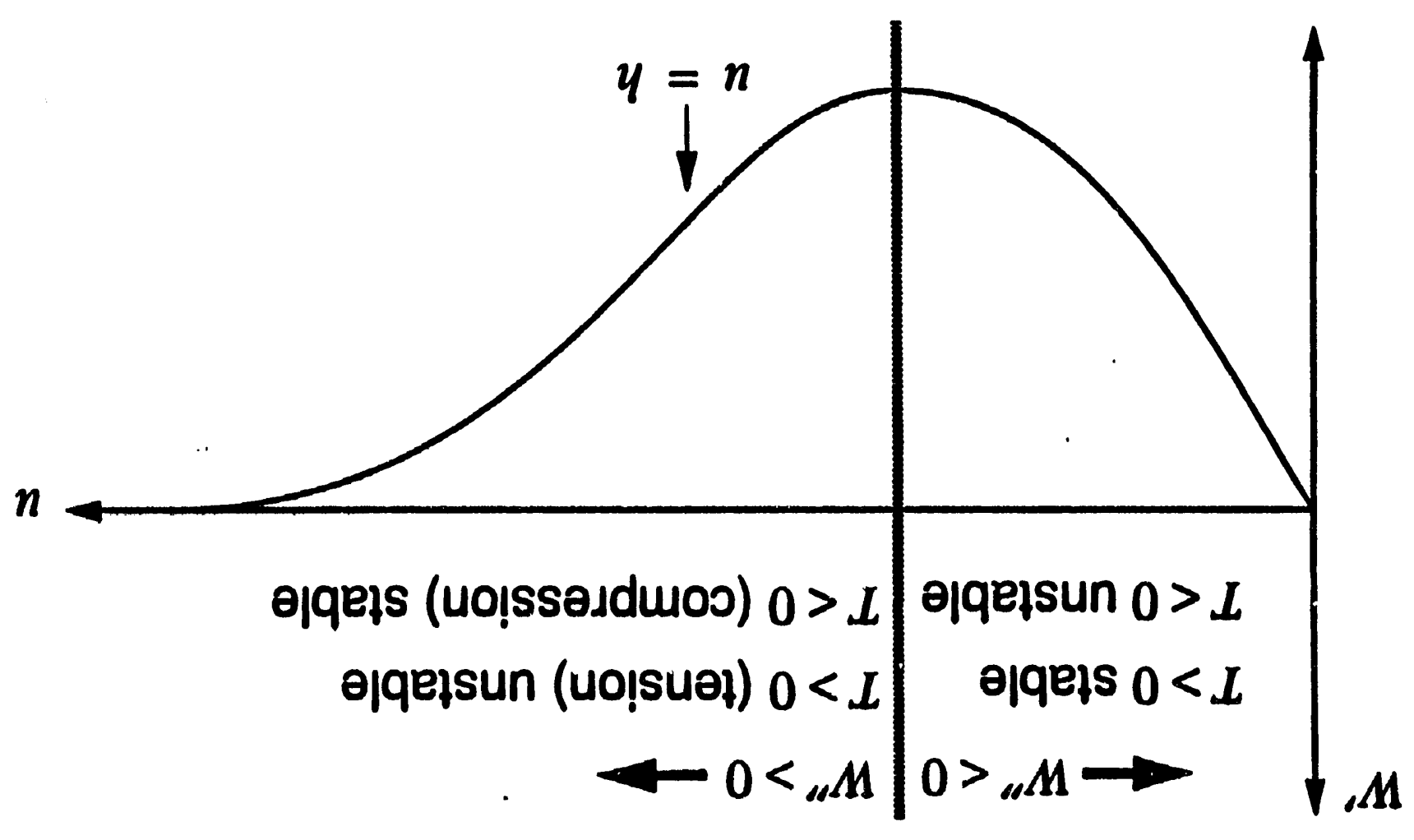

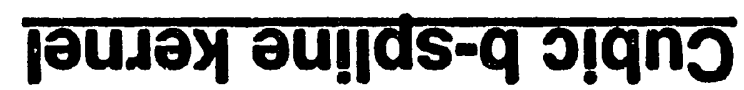

$$
\begin{aligned}
& \text { ㅋq리 } \Leftarrow \quad 0>I_{\text {, } M} \\
& \text { ө|qeisun } \Leftarrow \quad 0<L_{\text {, } M}
\end{aligned}
$$

\section{sou!boy K!!!!qeis}




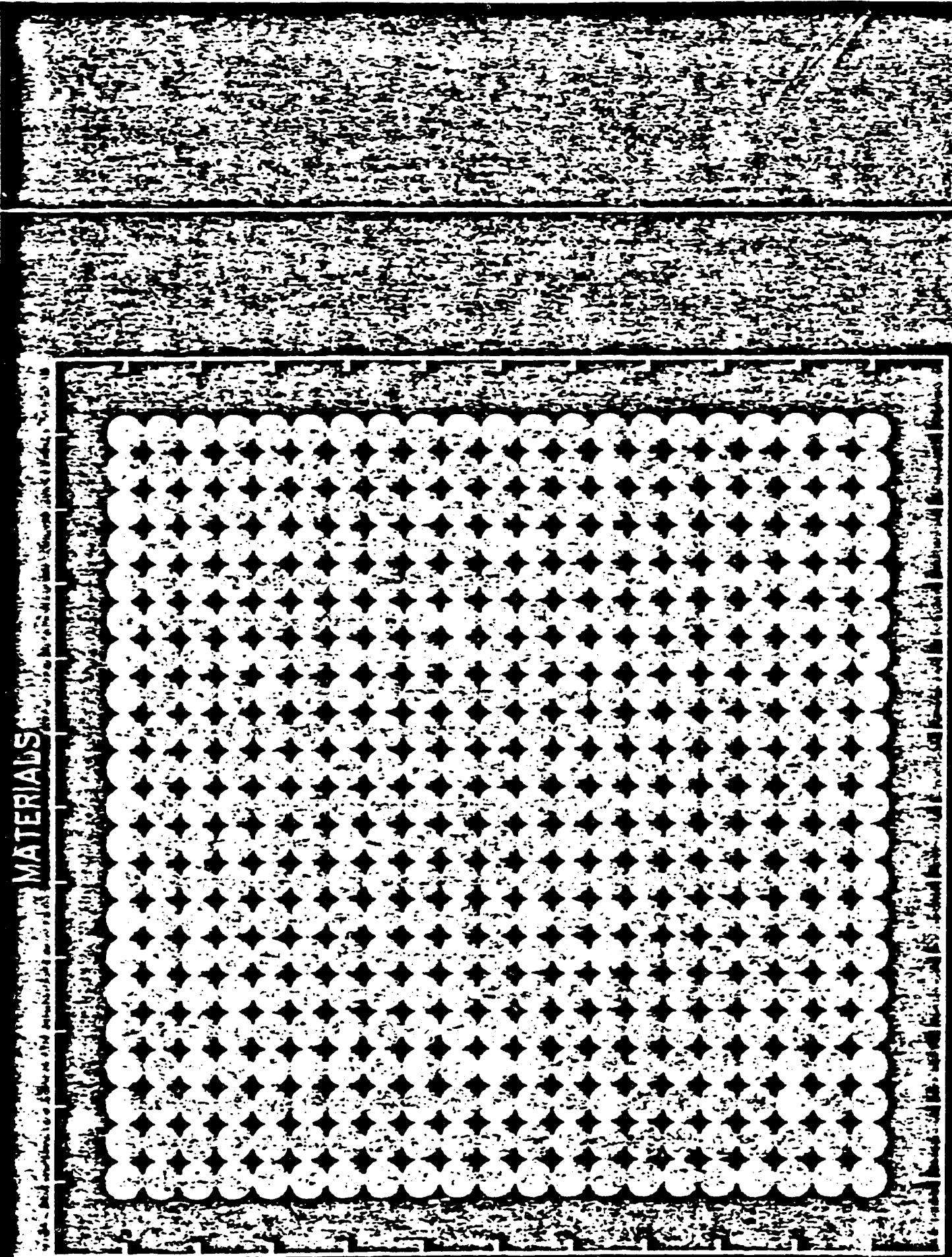

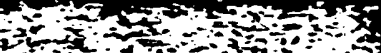

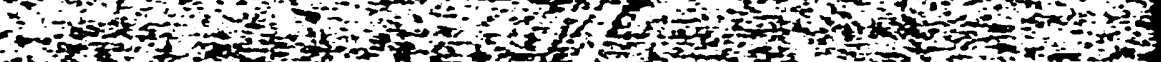

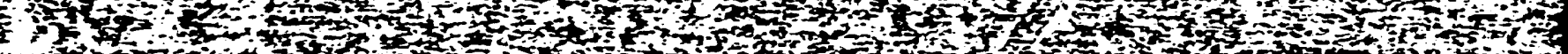
-

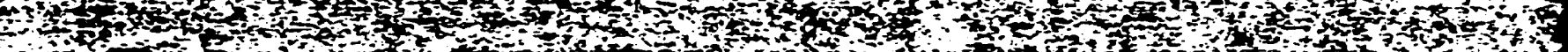
-

S. L

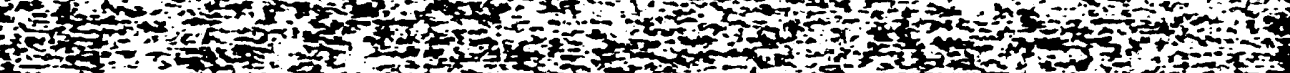

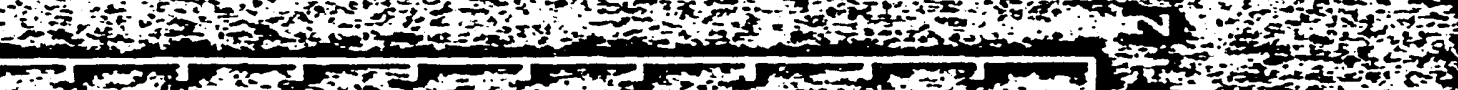
a $y$ J

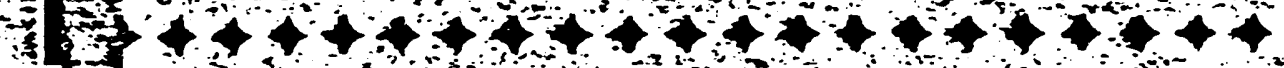

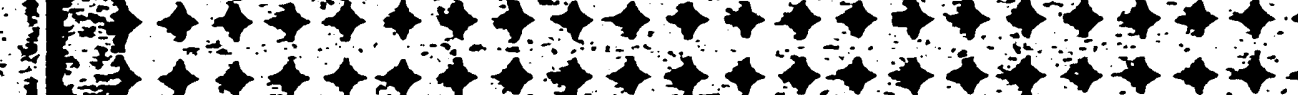
1) $+t+t+t+t+t+t+t+t+t+t+t$

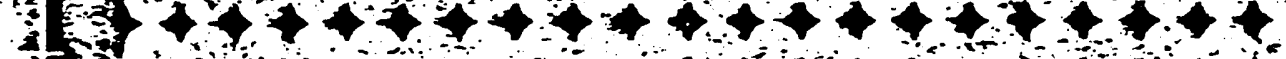

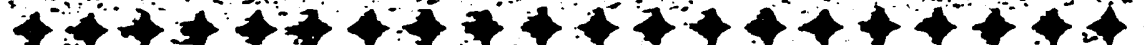
W a

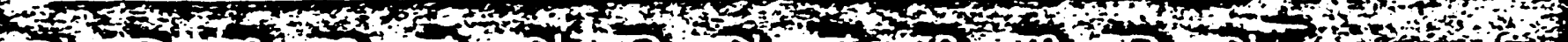

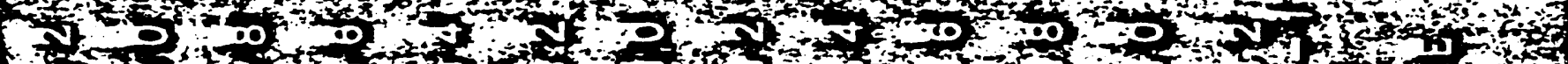

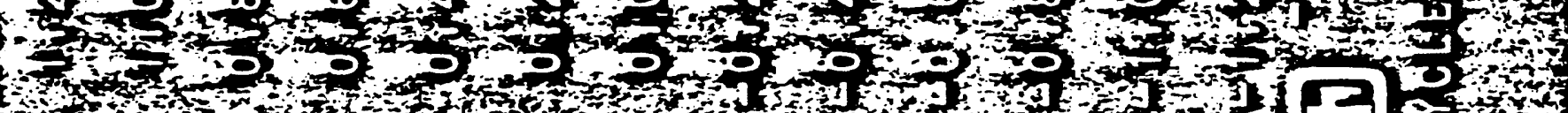
作 107.

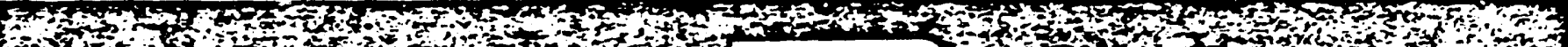

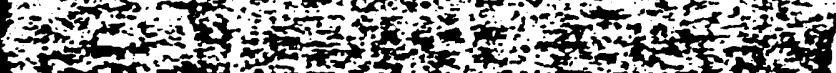
15. 1. 1.

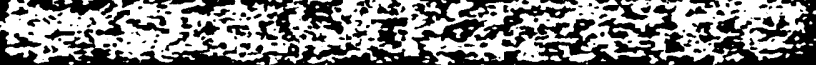

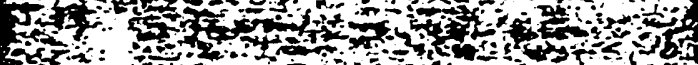

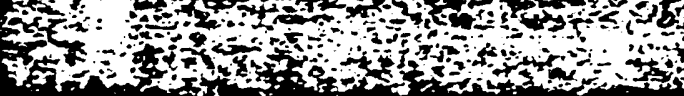




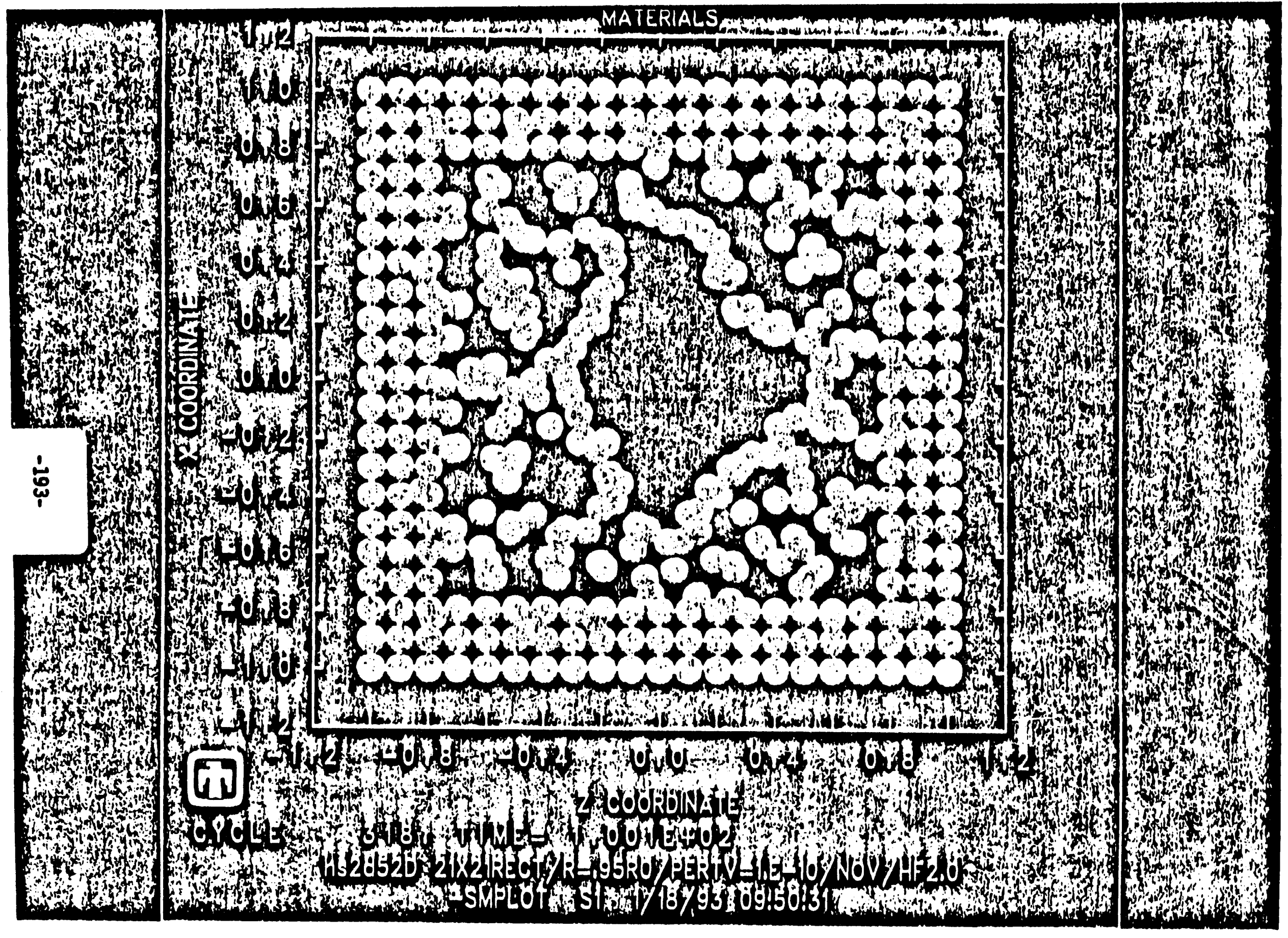




\section{Physical Basis of Instability - Effective Stress}

Finite difference eqt of motion

$\ddot{x}=\frac{\partial \sigma}{\rho_{0} \partial X} \approx \frac{\Delta \sigma}{\rho_{0} \Delta X}=\frac{\Delta \sigma}{m}$

\section{SPH eqt of motion}

$\ddot{x} \sim \frac{\Delta\left(-\sigma W^{\prime}\right)}{m}$

$\Rightarrow$ effective stress is $-\sigma(\varepsilon) W^{\prime}(\varepsilon)$

Kernel can cause instabilities

- frequency dependence not taken into account

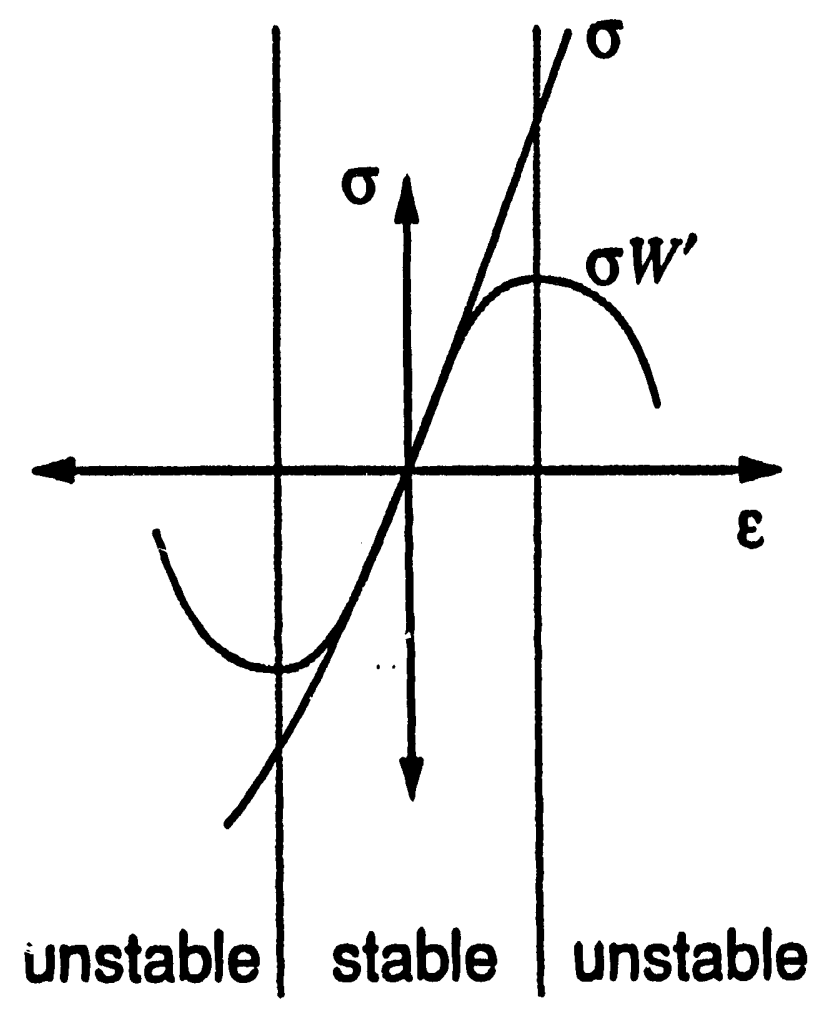




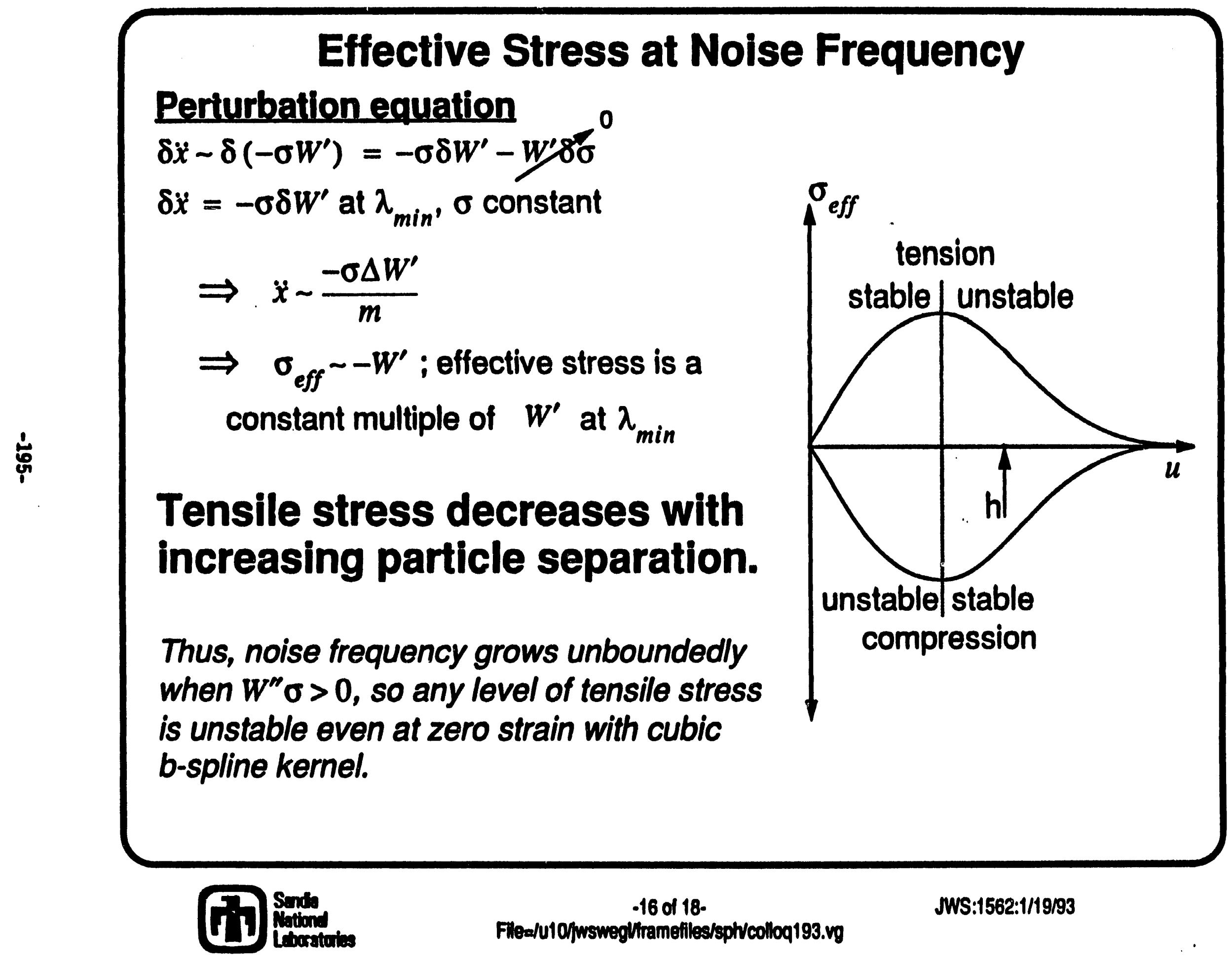




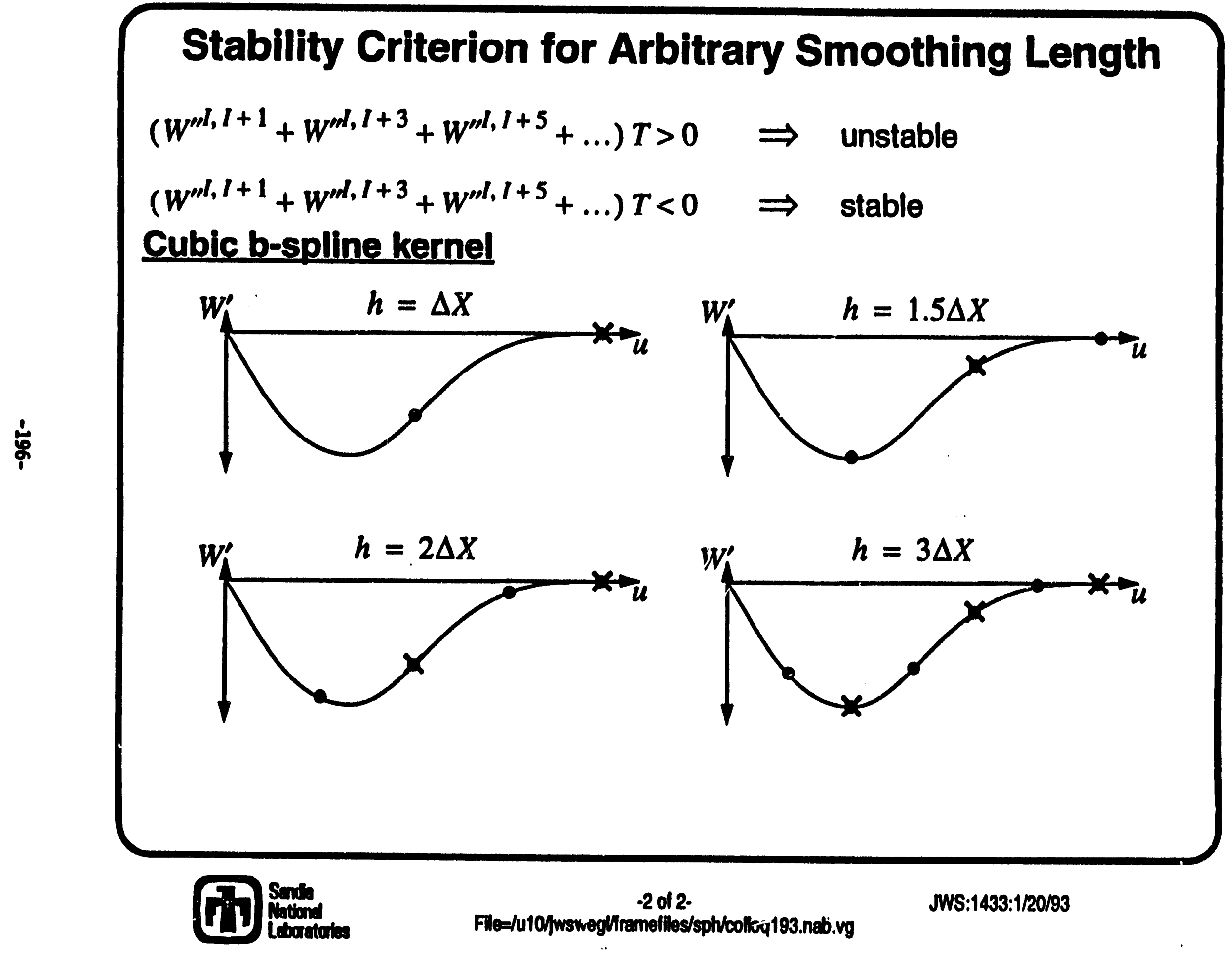




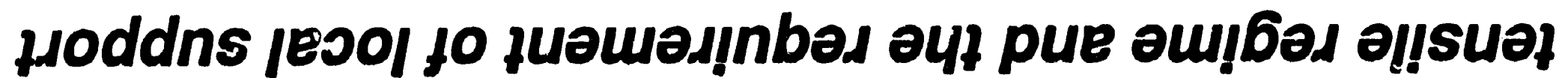

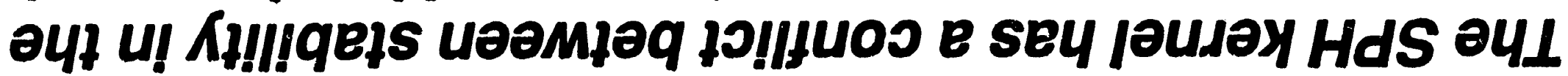

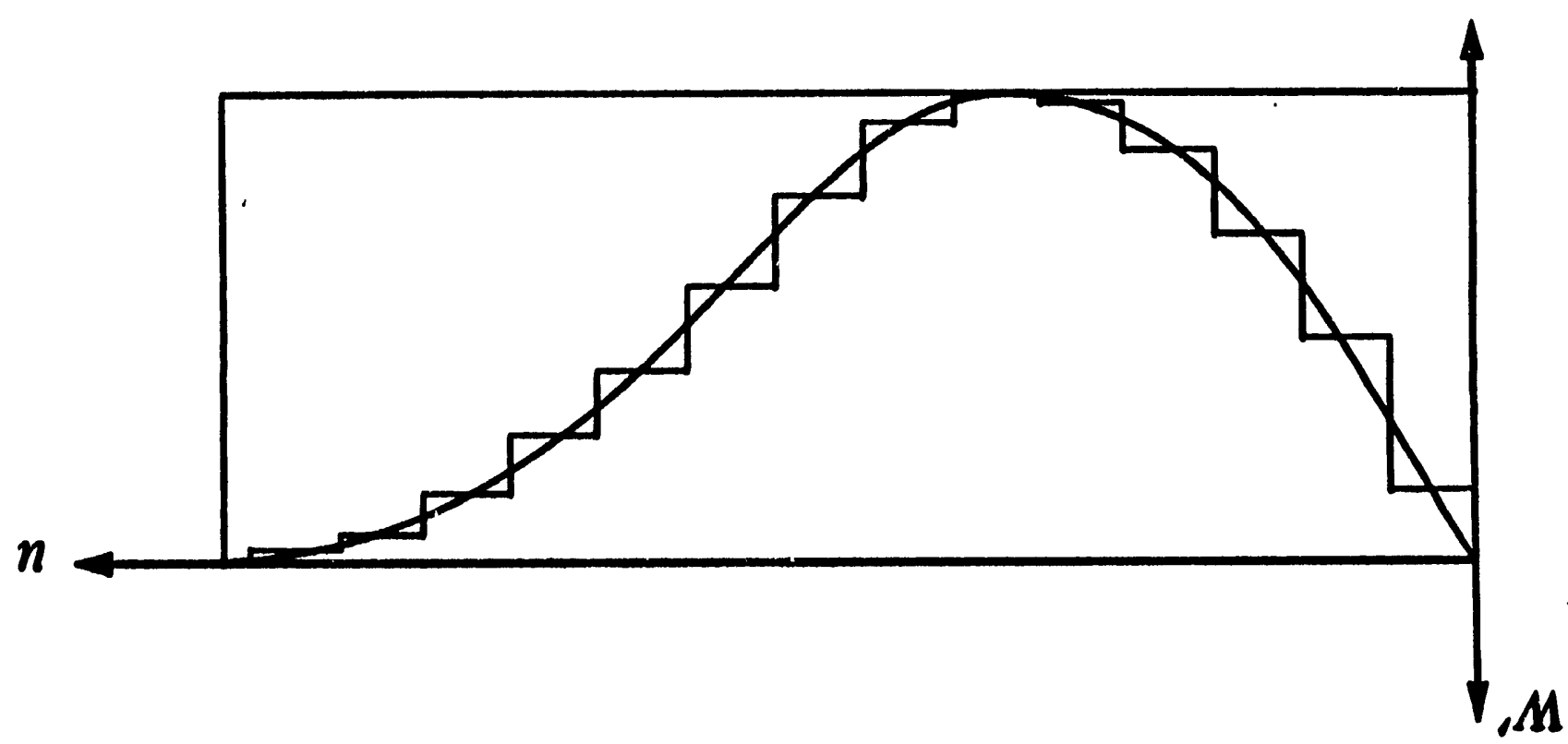

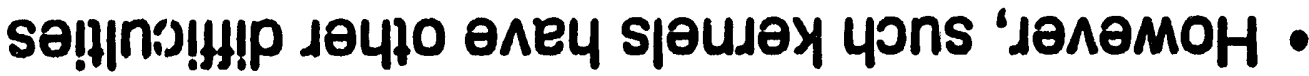

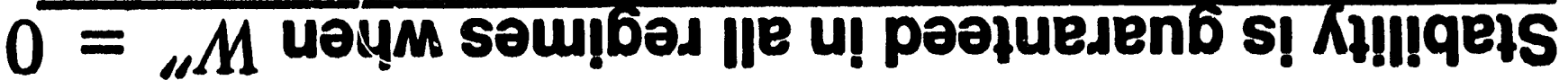

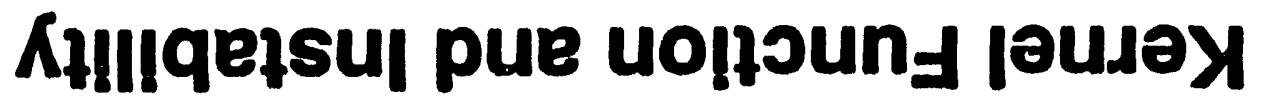




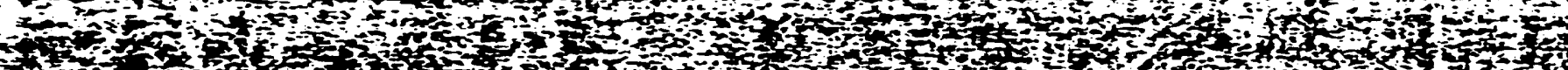

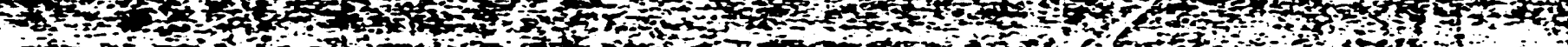

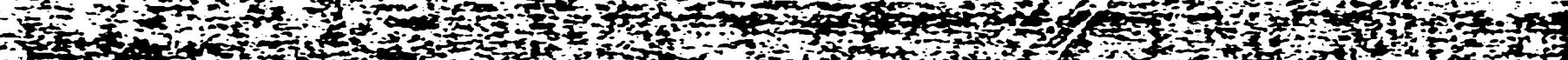

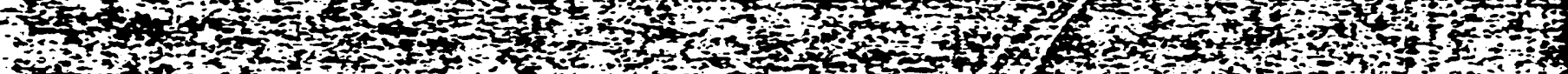
(1)

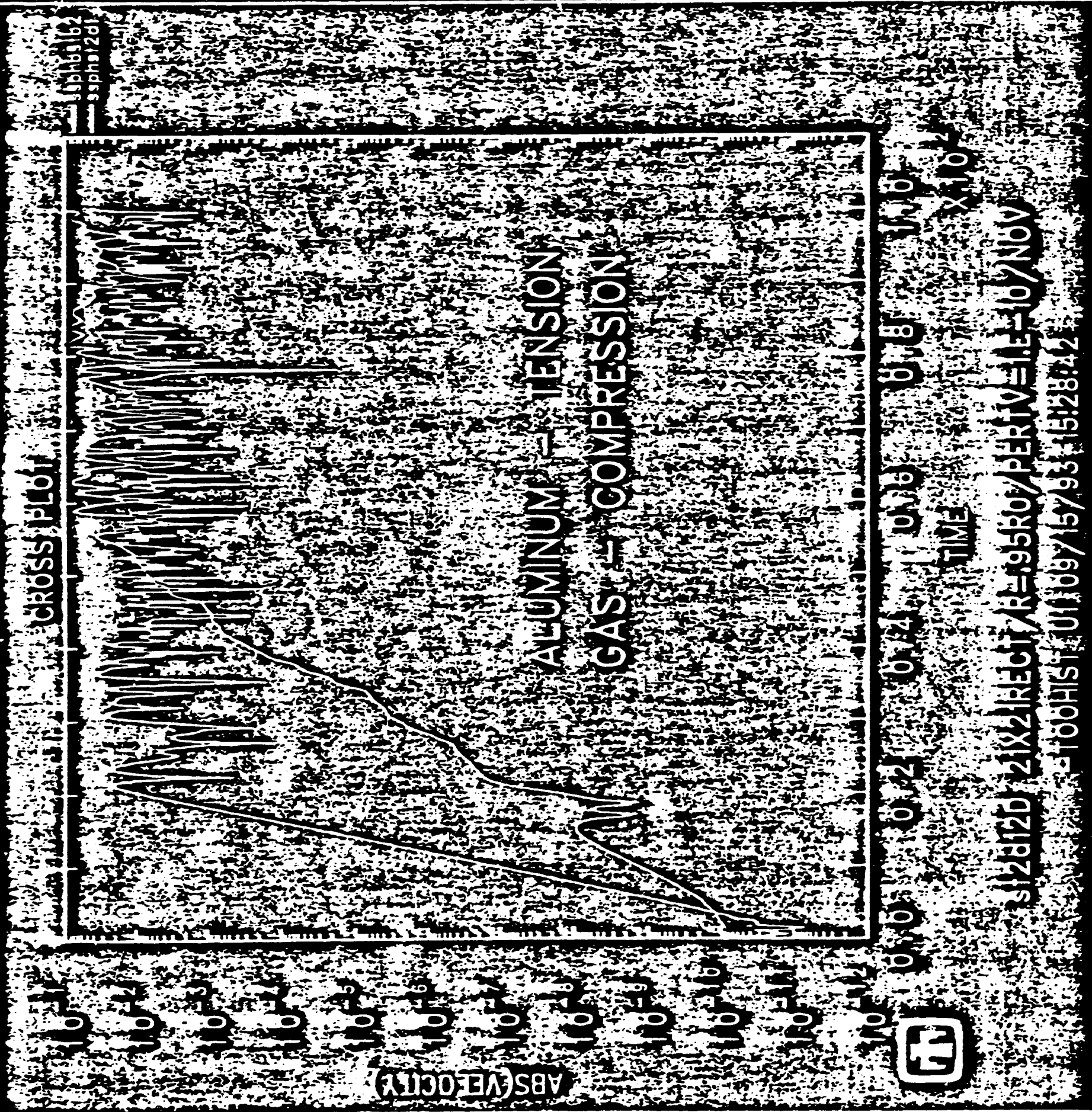

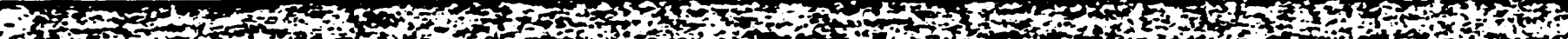

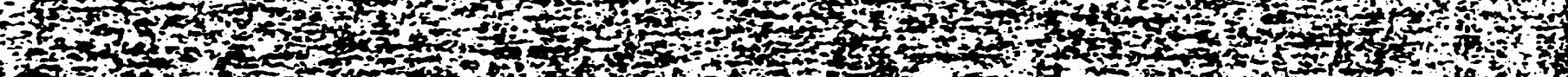

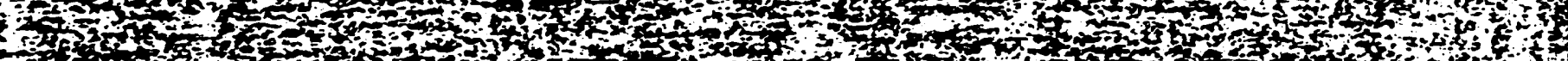

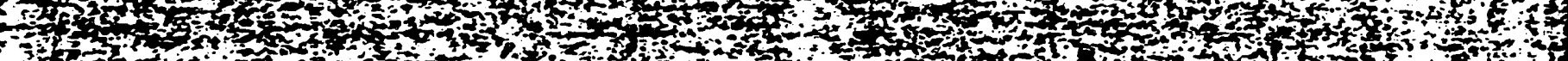

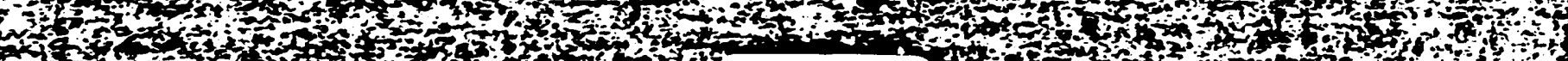

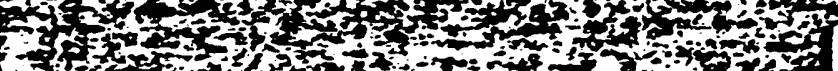

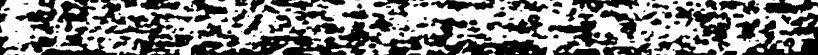




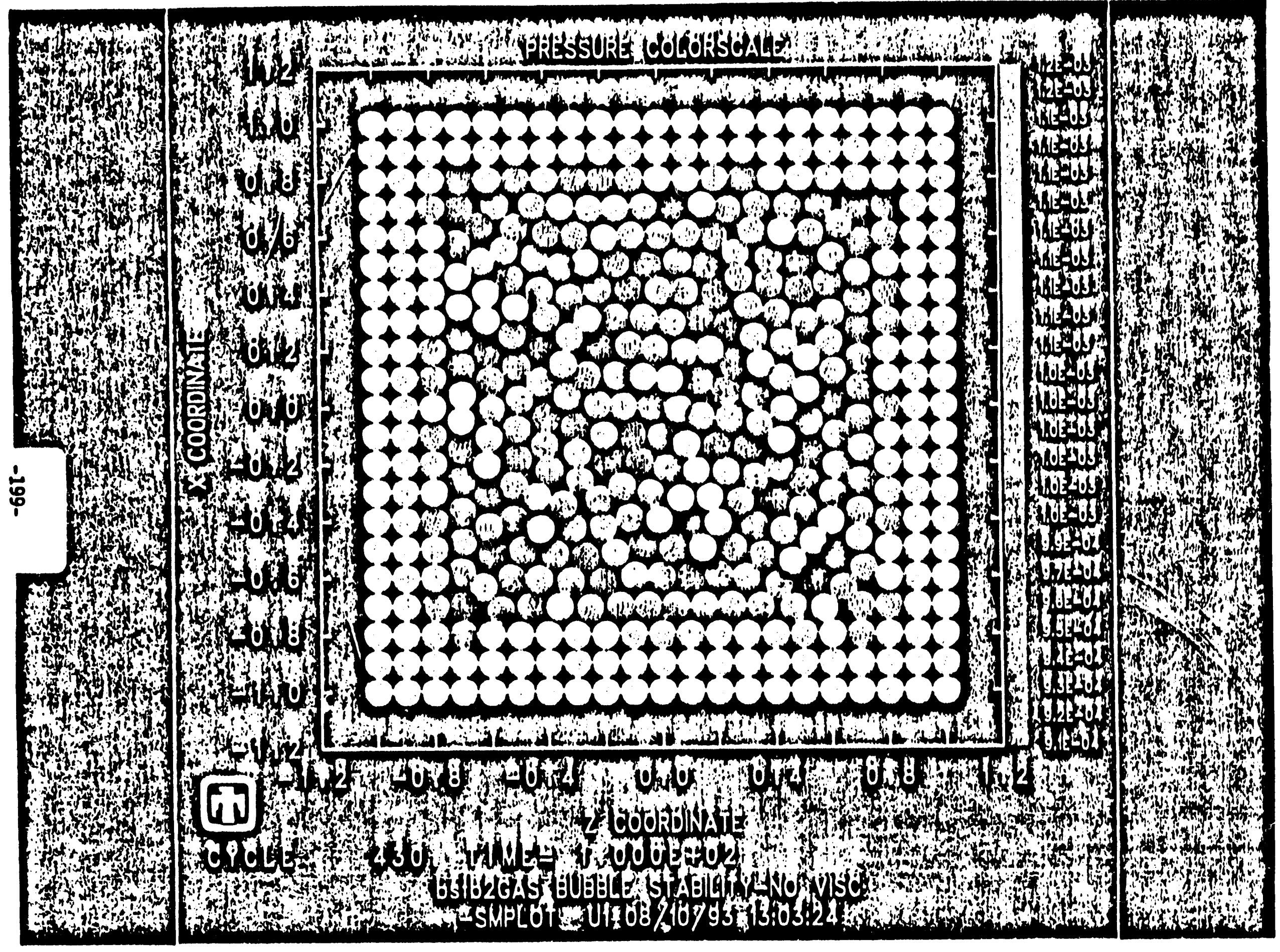




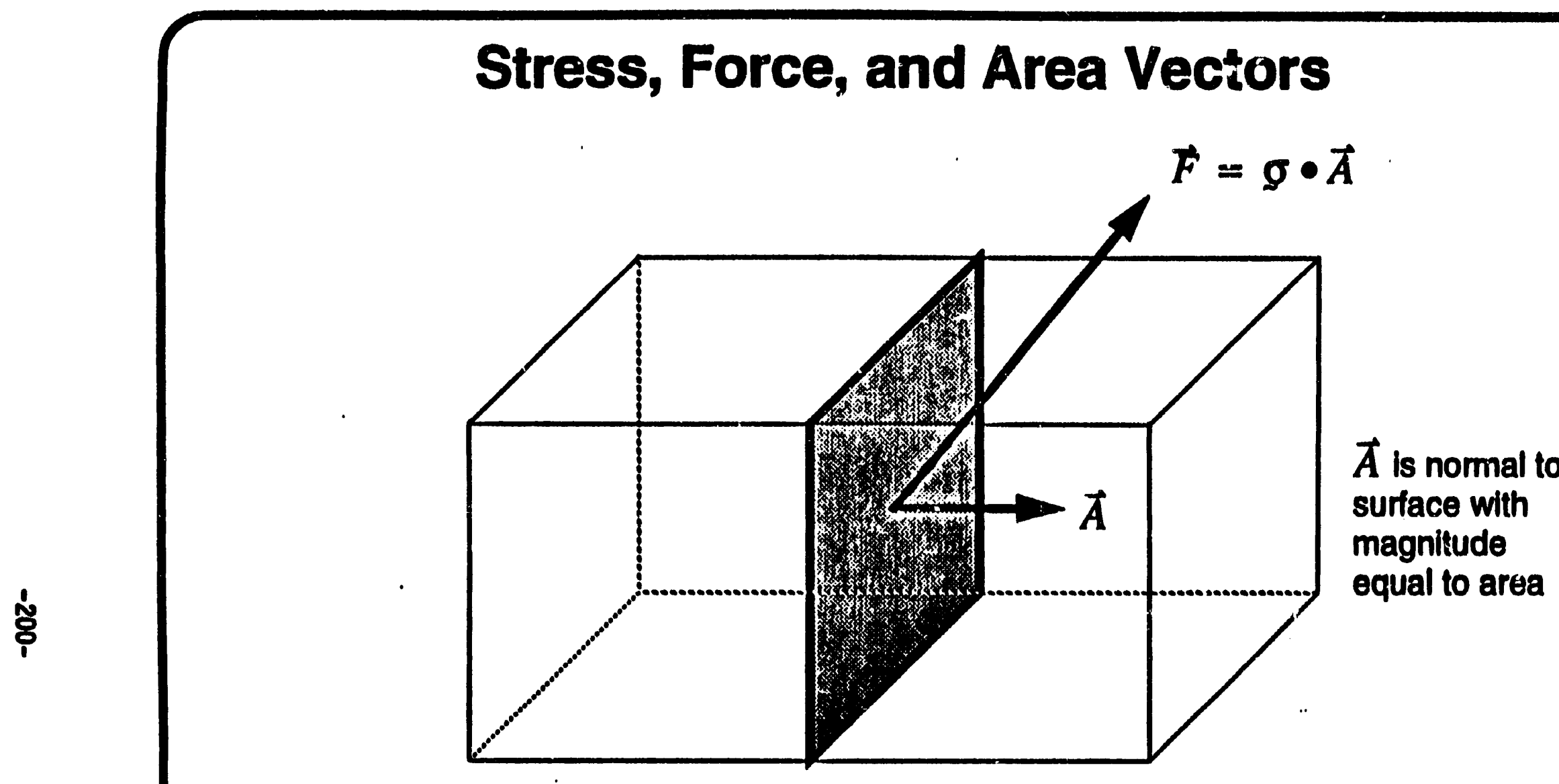

Total force: sum over all areas

$$
\vec{F}_{t}=\sum_{i=1}^{n} g_{i} \cdot \vec{A}_{i}
$$




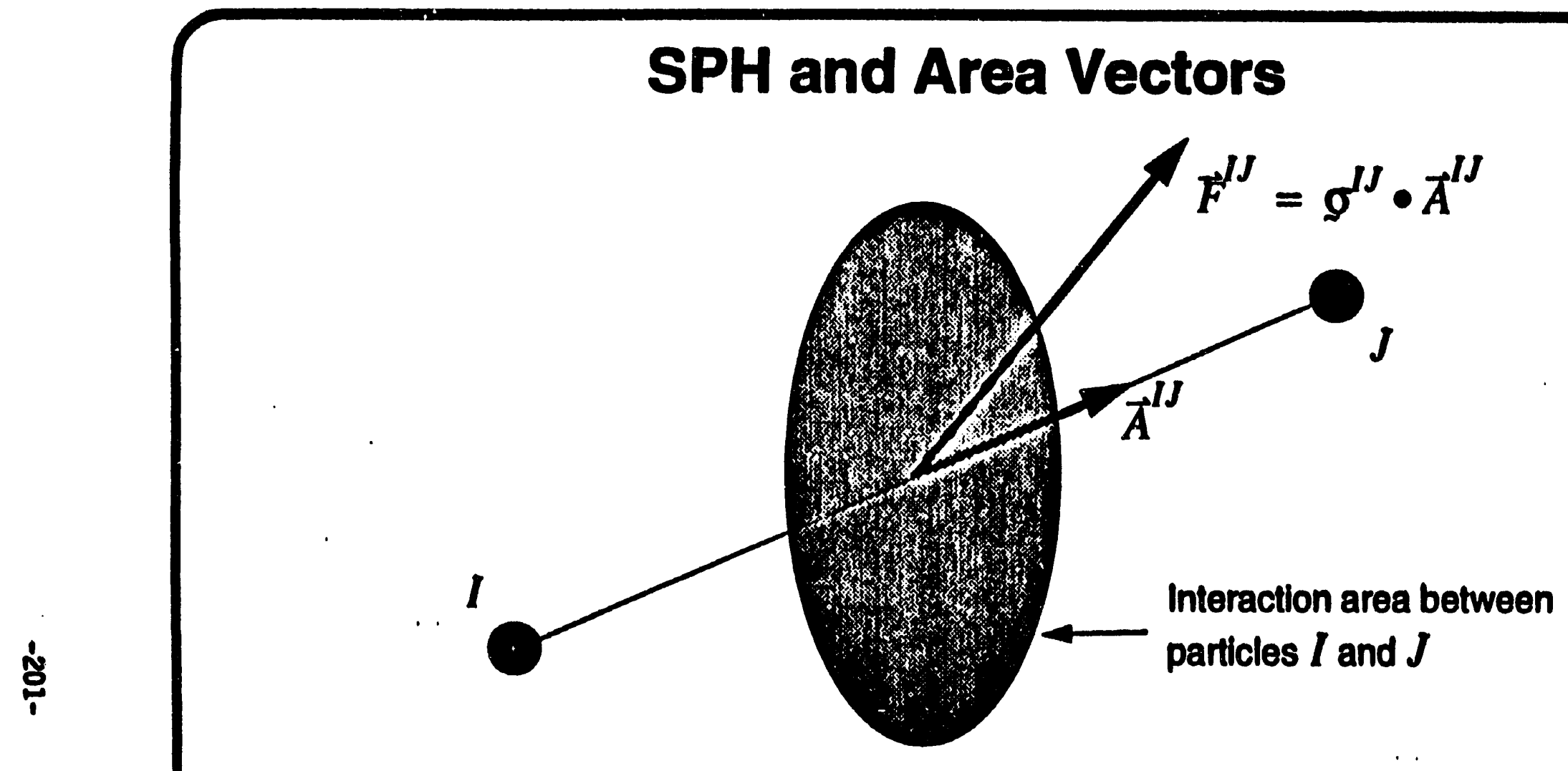

Kernel function defines area vectors

$$
\begin{aligned}
\vec{F}^{\prime}= & m^{I} \vec{a}^{I}=-\sum_{J=1}^{N} \frac{m^{I} m^{J}}{\rho^{I} \rho^{J}} g^{I J} \cdot \nabla_{J} W=\sum_{J=1}^{N} g^{I J} \cdot \vec{A}^{I J} \\
& \Rightarrow \vec{A}^{I J}=-\frac{m^{I} m^{J}}{\rho^{I} \rho^{J}} \nabla_{J} W=-\operatorname{Vol}^{I} \operatorname{Vol}^{J} \nabla_{J} W
\end{aligned}
$$




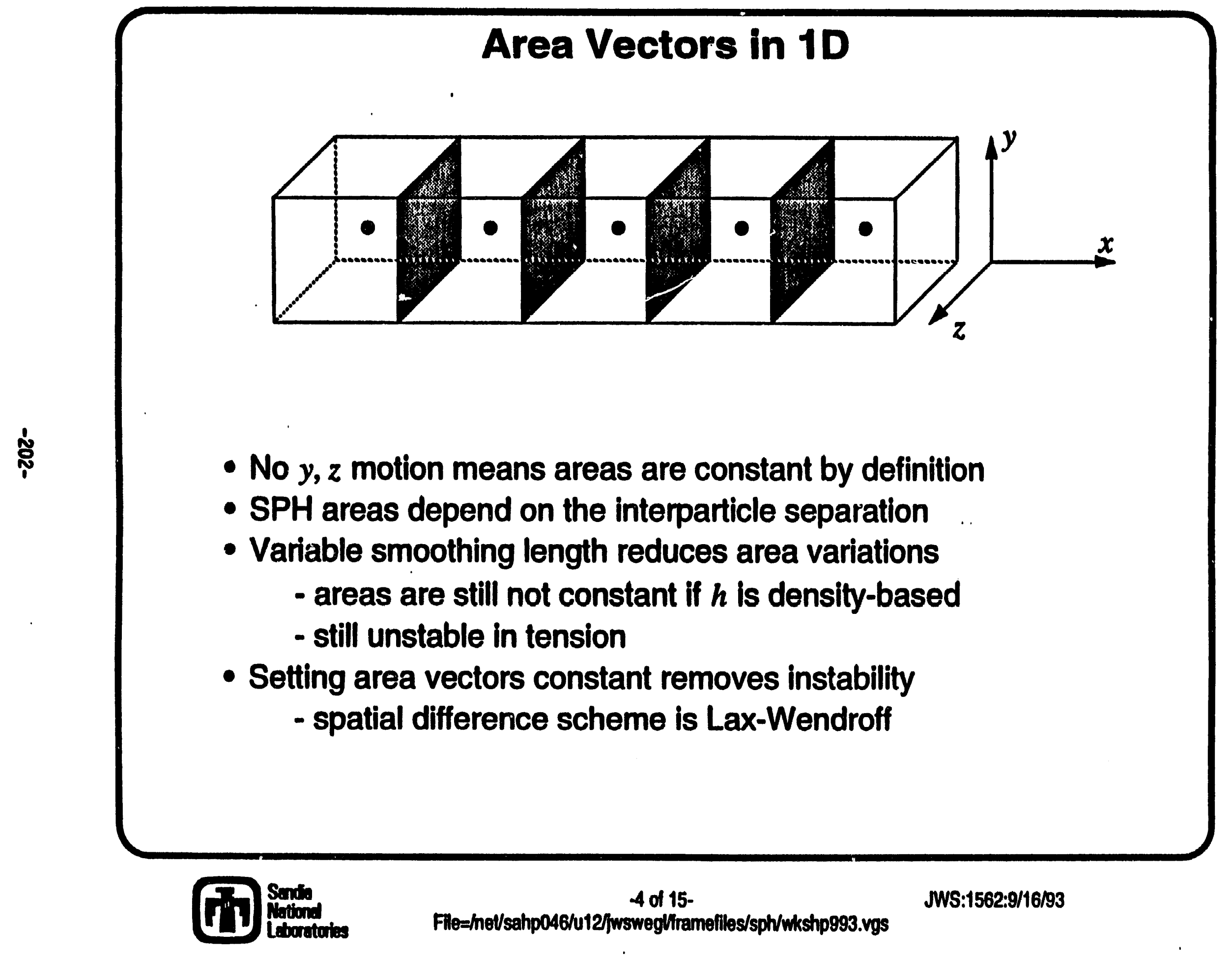




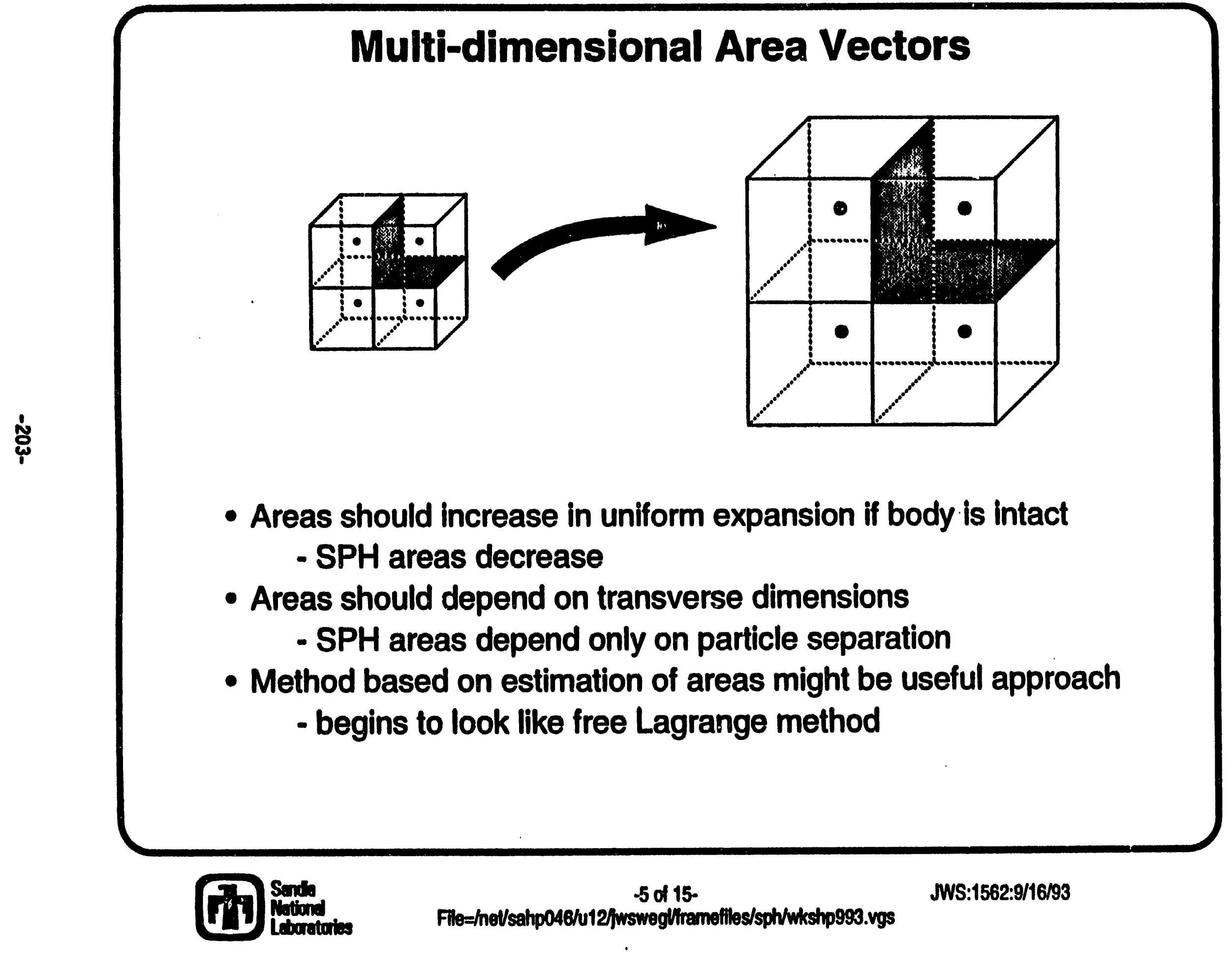




\section{Consistency}

Definition of consistency: the discretized forms reduce to the differential forms

Continuum equation of motion

$$
\rho \dot{\vec{x}}^{J}=\nabla \bullet \sigma
$$

Symmetrized SPH equation of motion

$$
\ddot{\vec{x}}^{J}=-\sum_{J=1}^{N} m^{J}\left[\left(\frac{g}{\rho^{2}}\right)^{J}+\left(\frac{g}{\rho^{2}}\right)^{I}\right] \bullet \nabla_{\jmath} W
$$

The above SPH form of the equation of motion is not consistent with the continuum equation. 


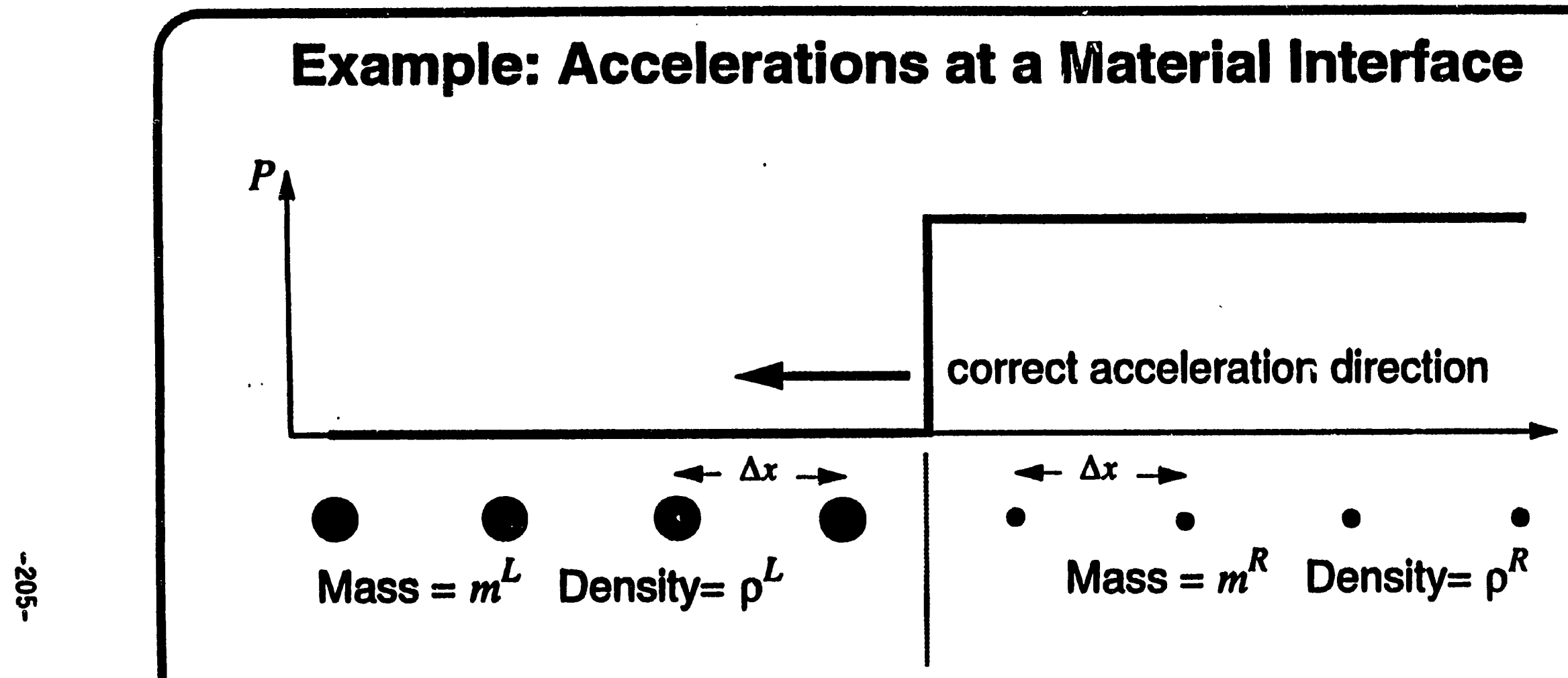

- Standard SPH accelerations do not correspond to $-\Delta P /(\rho \Delta x)$

- Low mass particle is accelerated in wrong direction if $m^{L}>2 m^{R}$

- Accelerations are obtained even if $P$ is constant across interface 


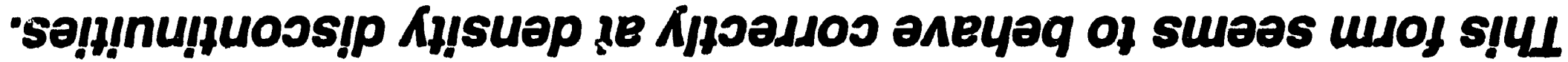

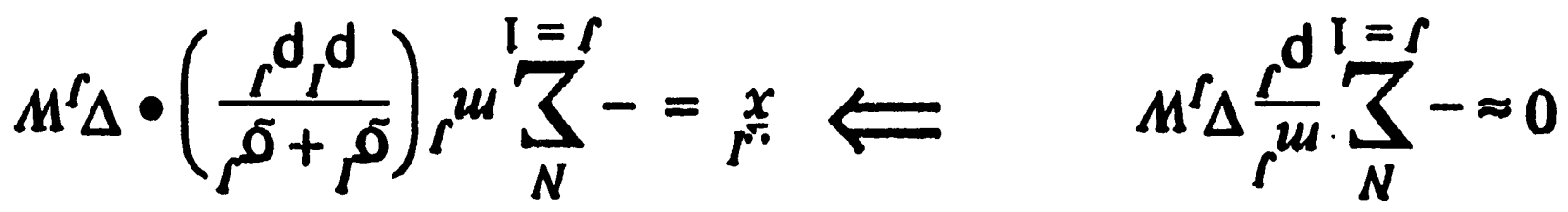

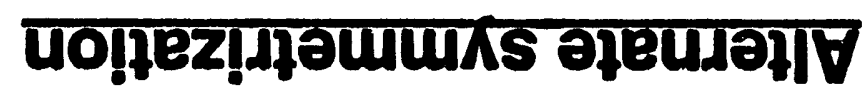

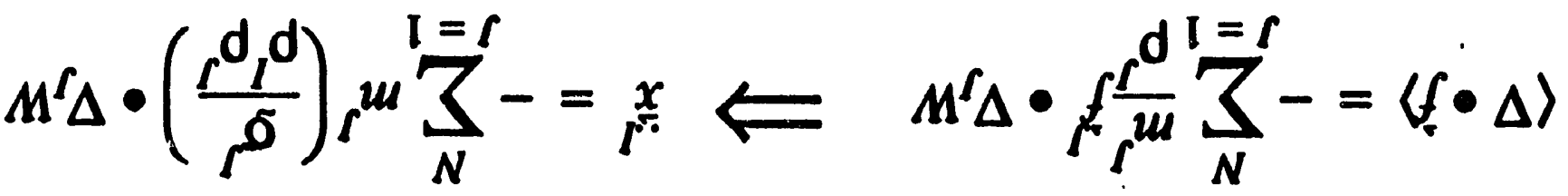

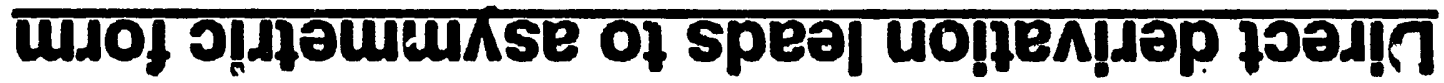

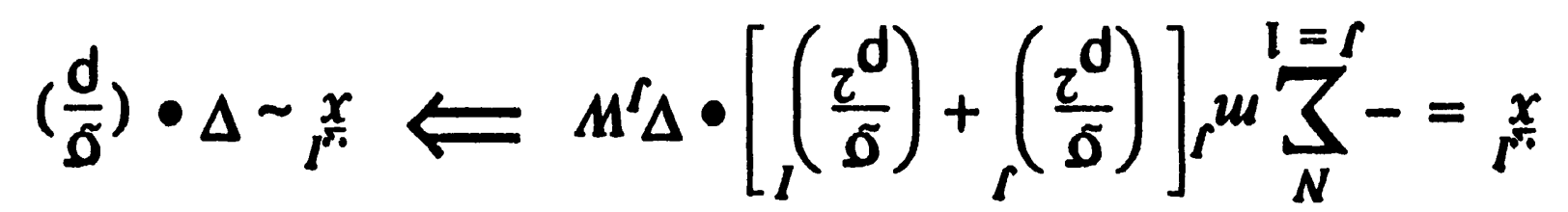

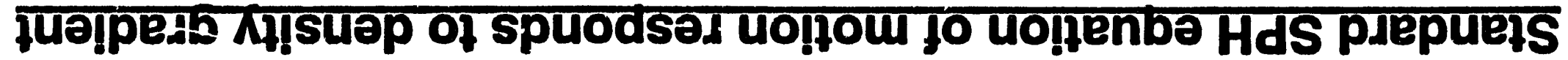

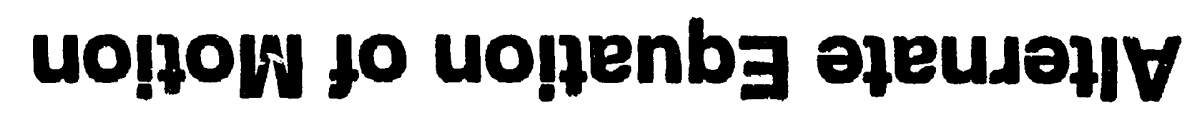




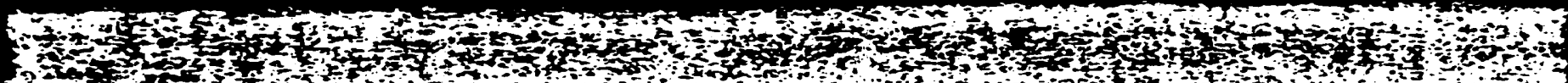

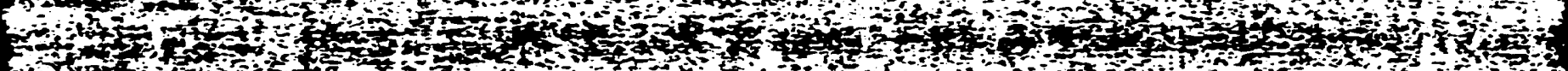

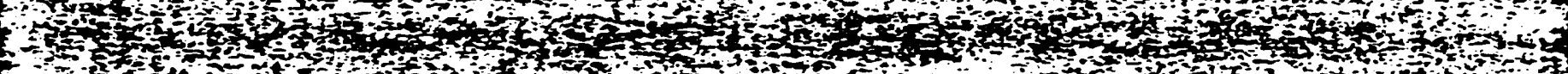
of

H. (1)

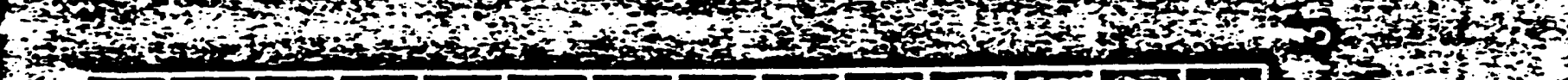

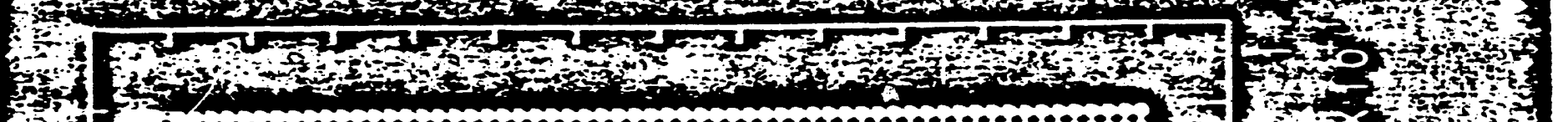

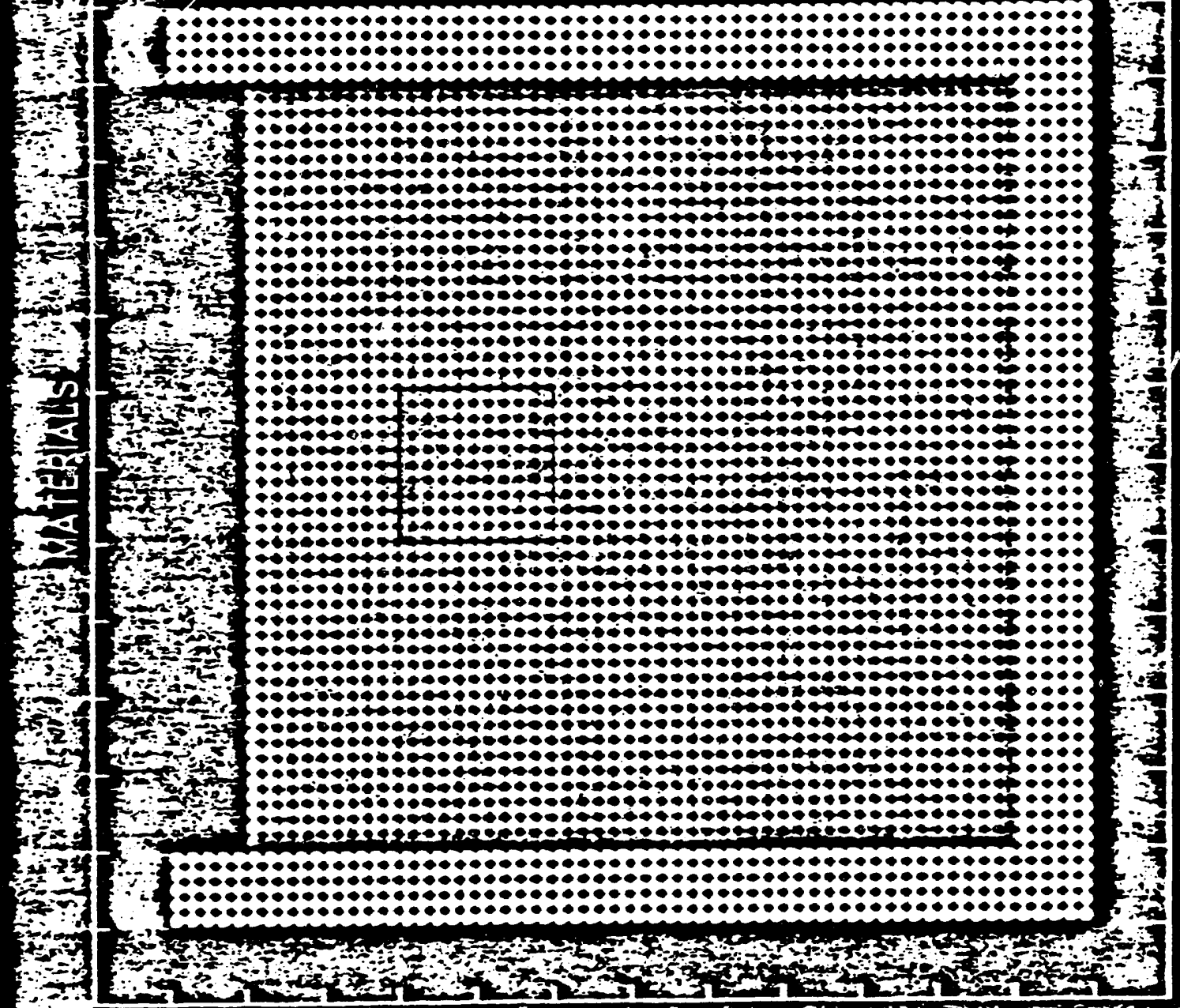

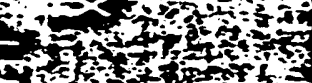

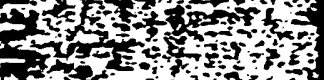

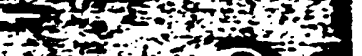

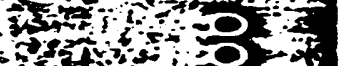
$00+\frac{2}{20}$

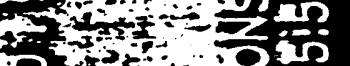

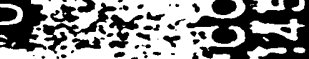
$+0=0 \frac{10}{10}$ $N^{2}=5 \frac{x}{20}$

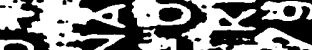
ato

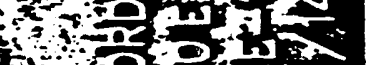
in 00 \%

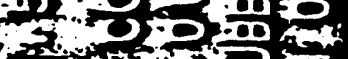
$0=0$

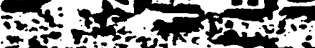
f ond $0 \times-5 \times \frac{12}{2}$ $\frac{40+20}{4020}$

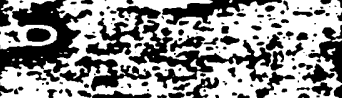
$40 x+4$

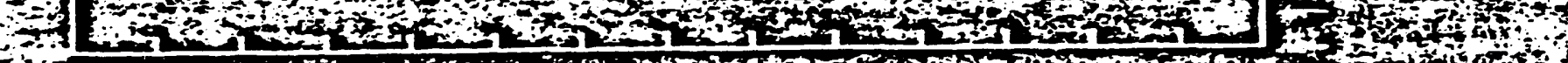

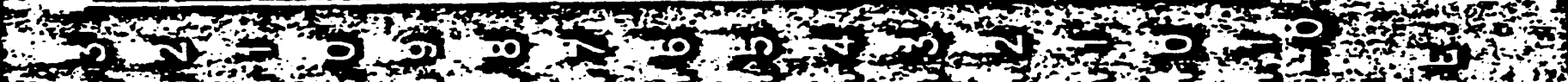
40 on

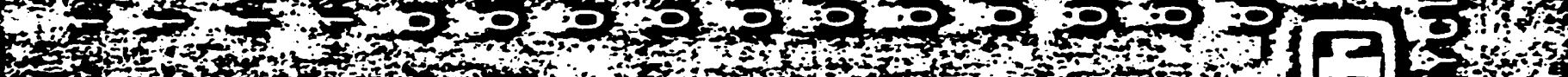

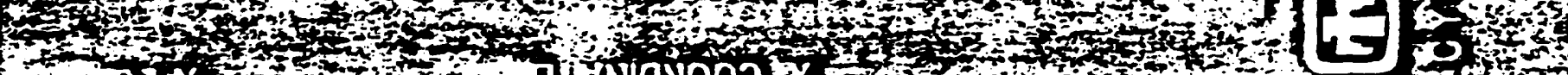

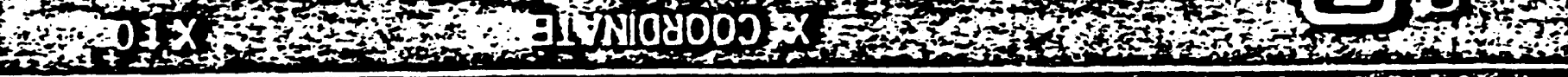

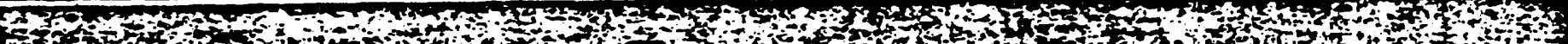

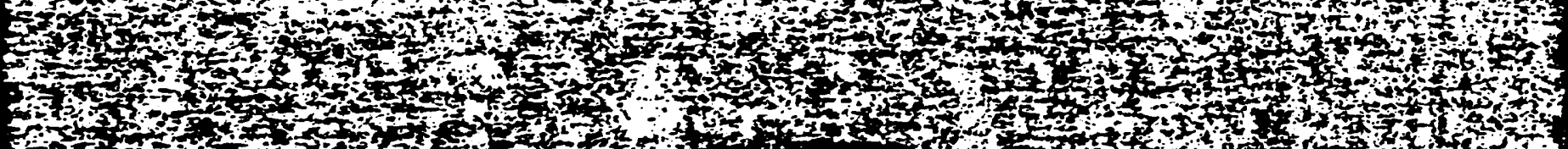

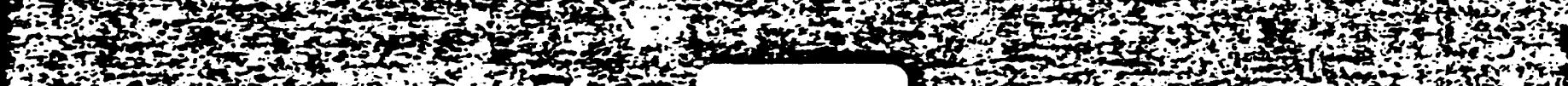
a 


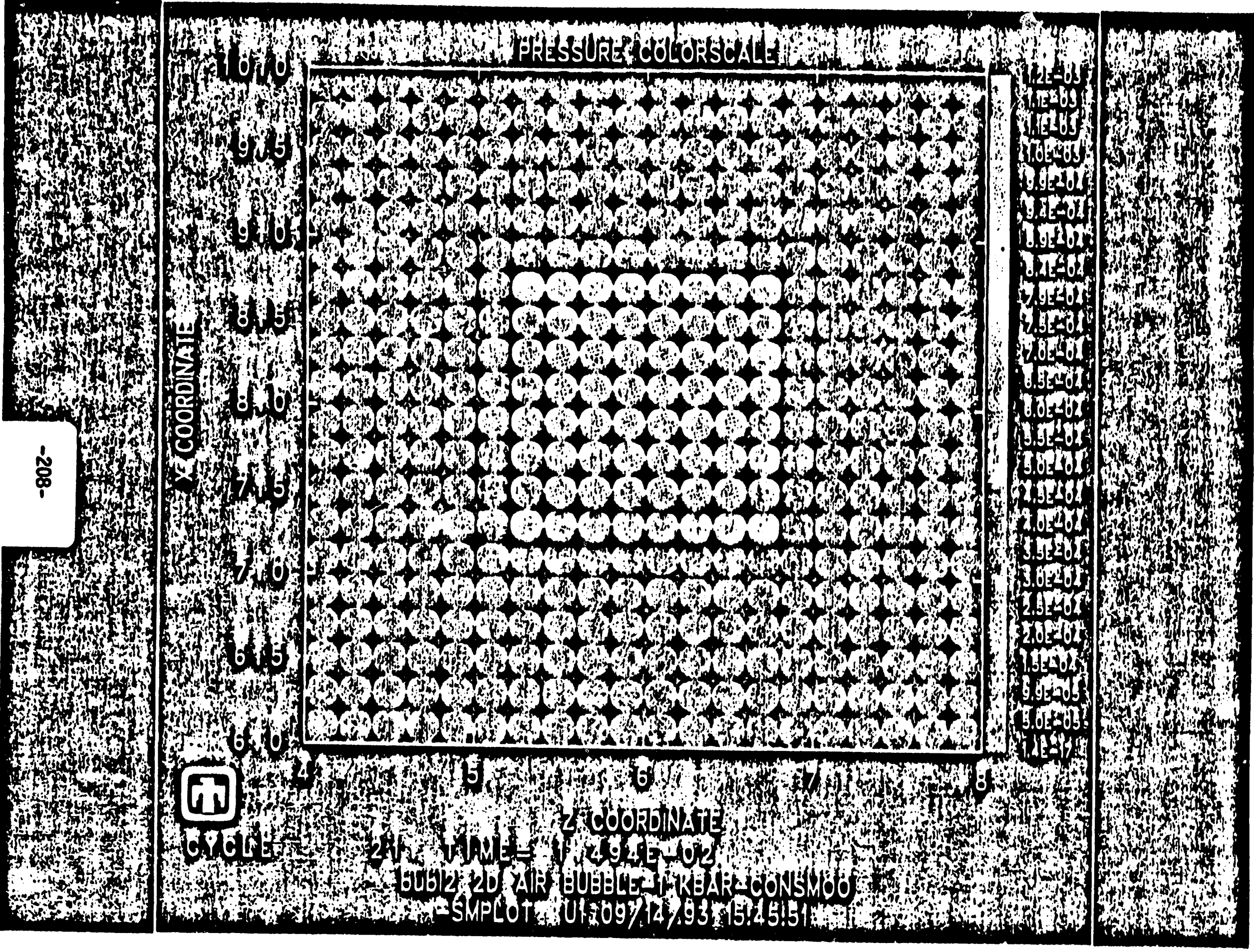




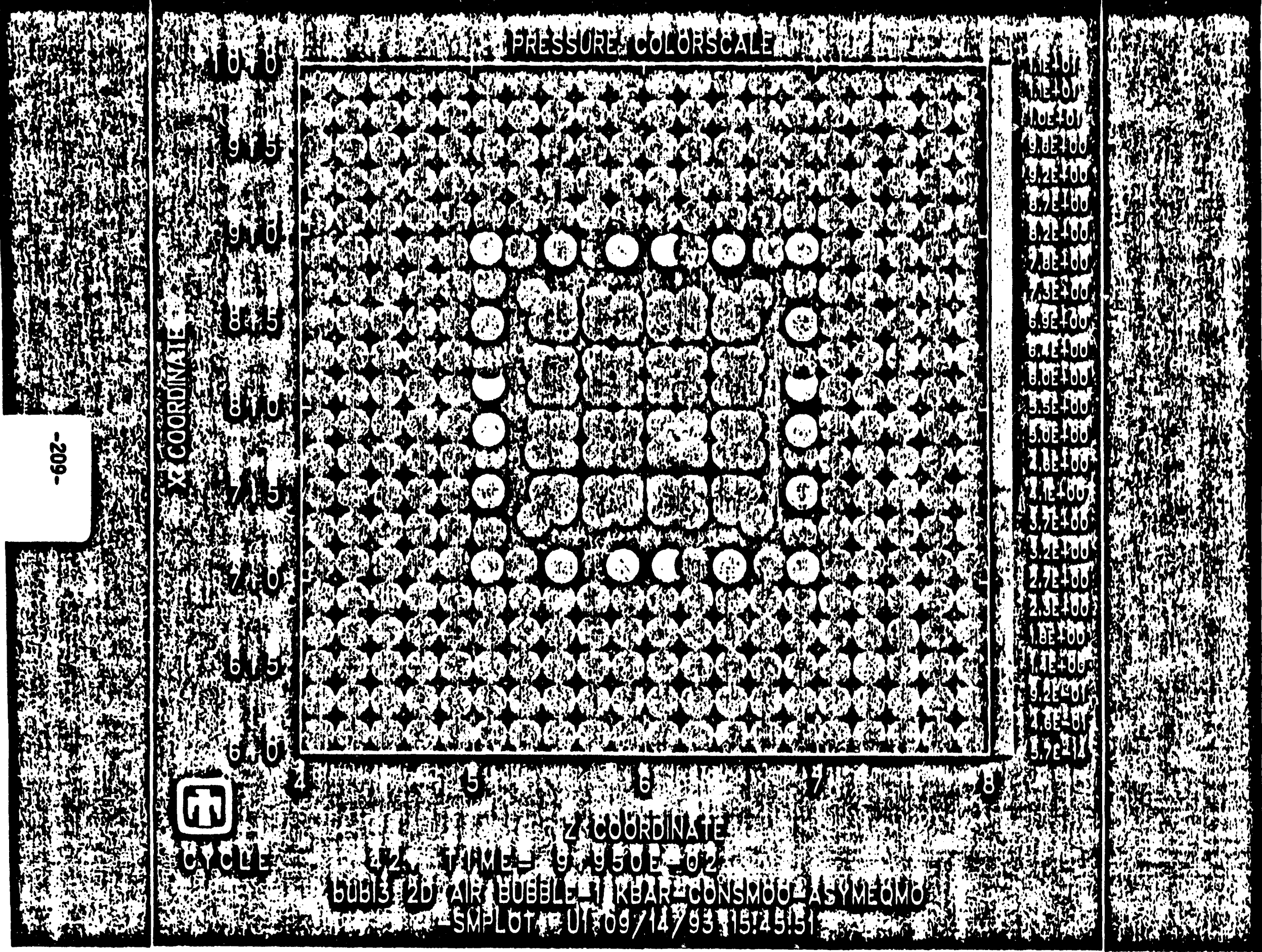




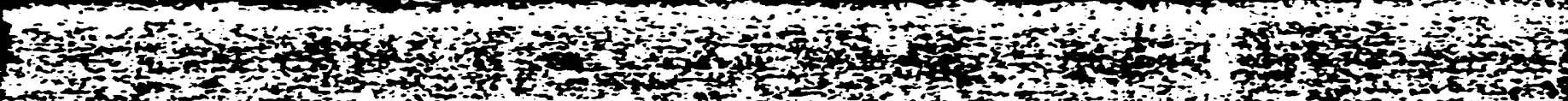

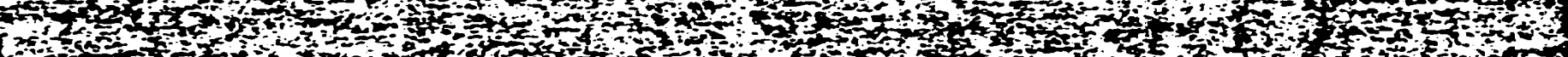
(3) Fol

Fe 3 (n)

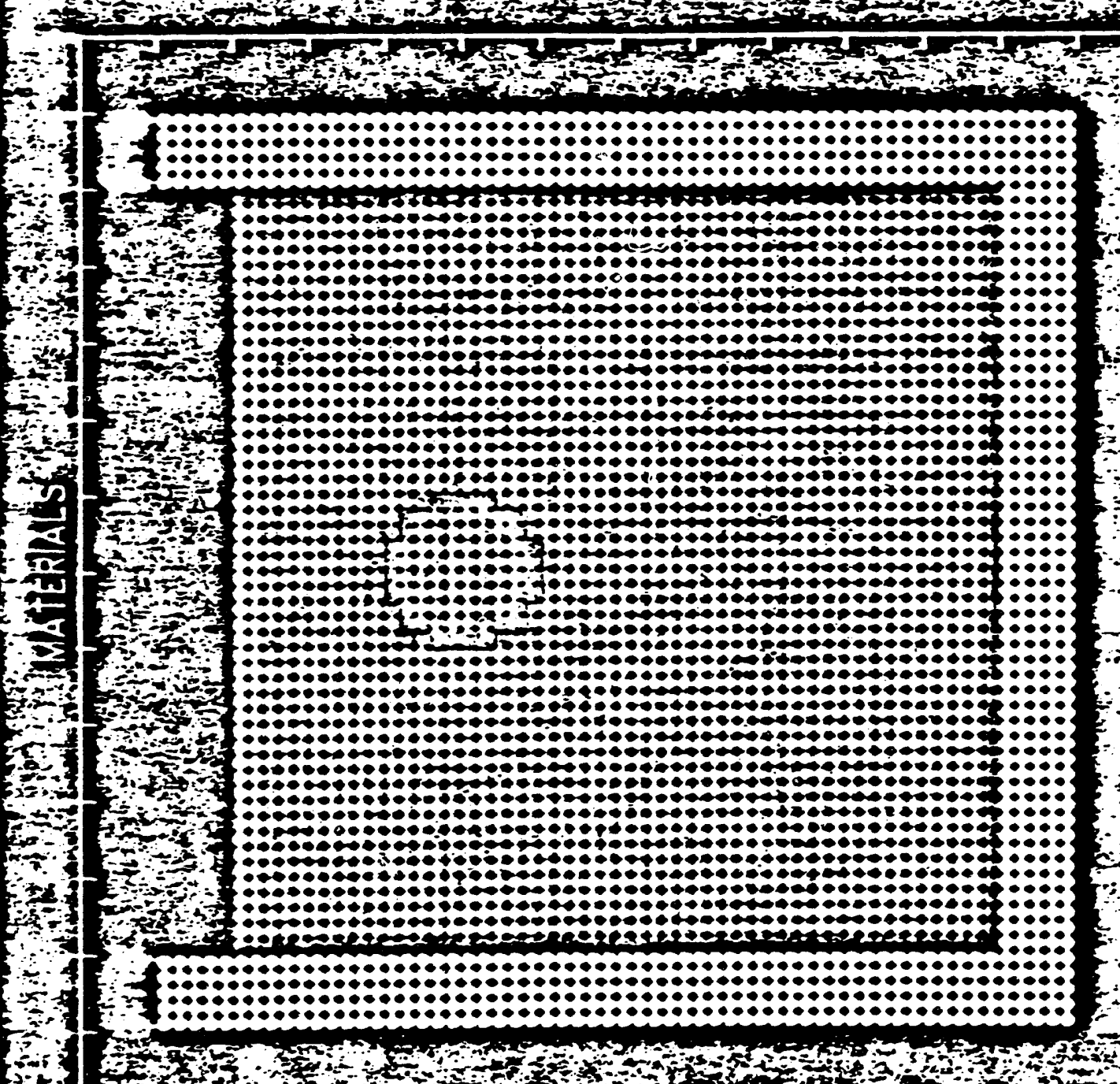

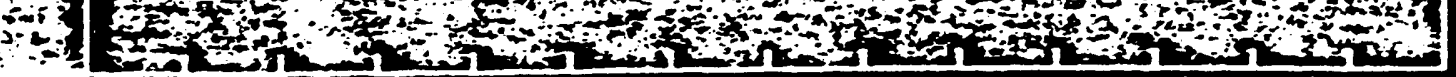

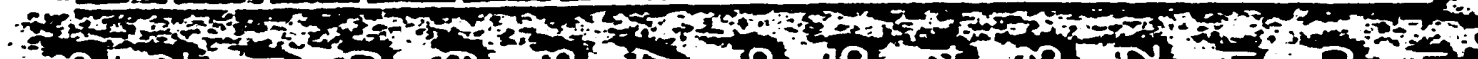

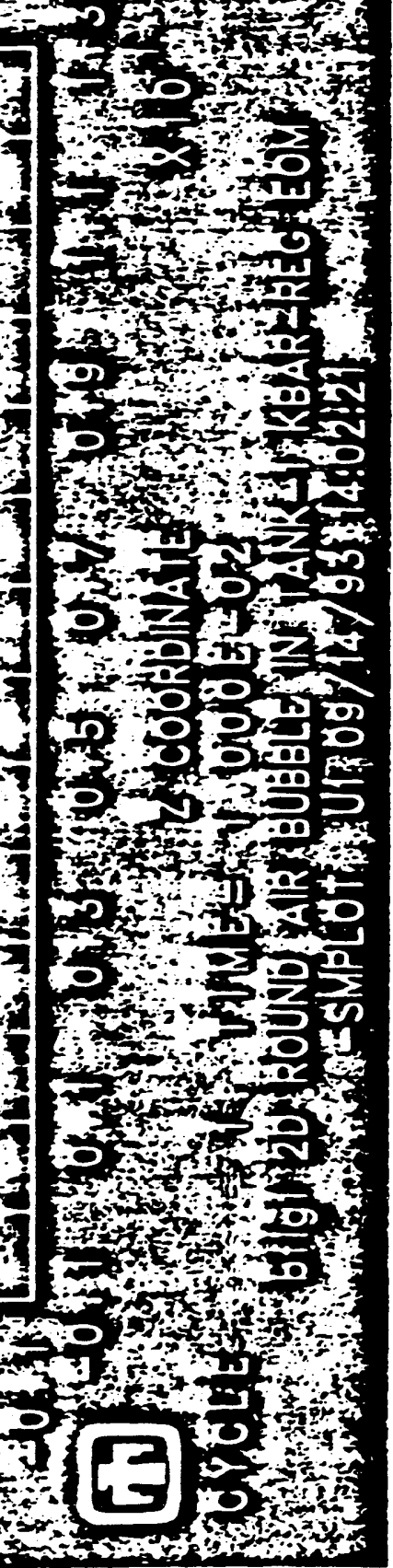

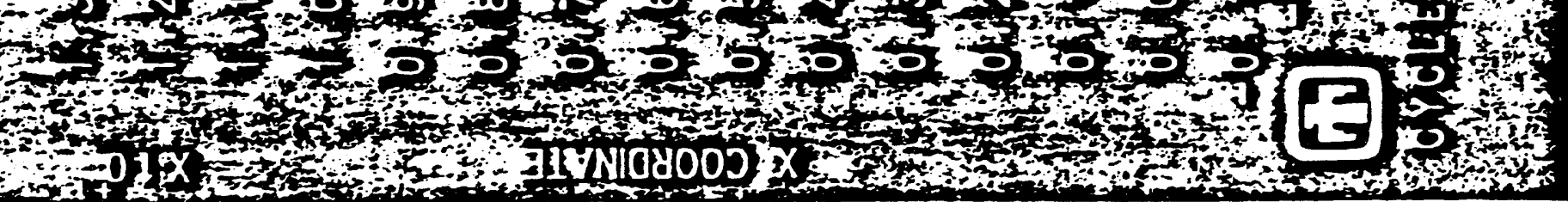

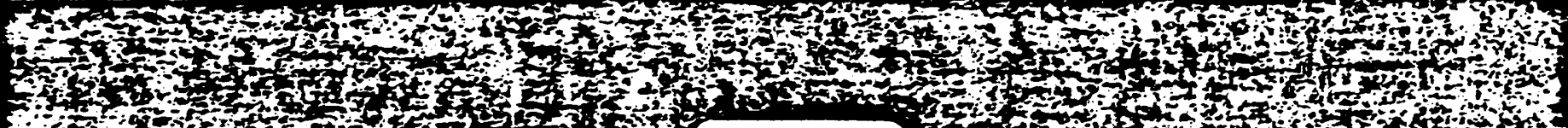

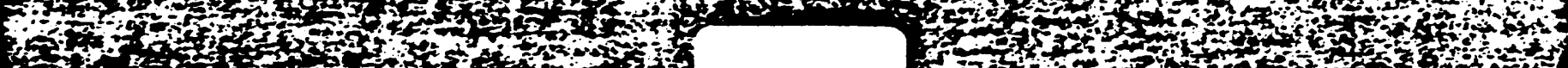

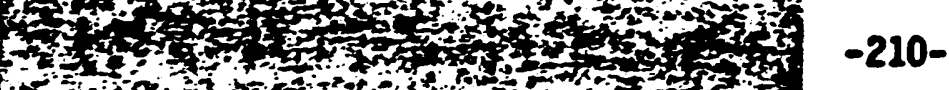

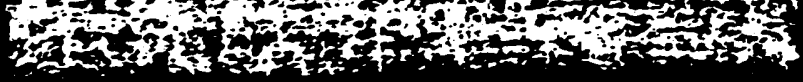
(n) 
Txis

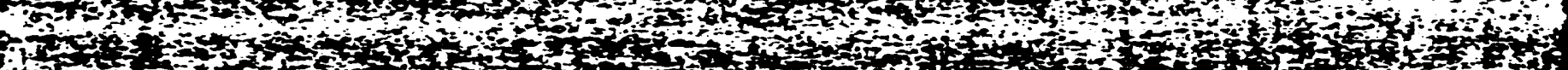

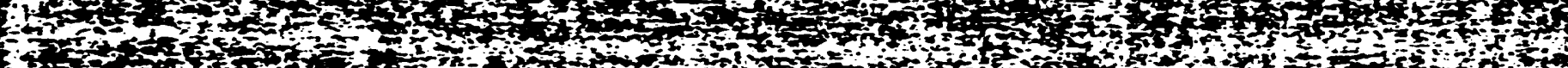

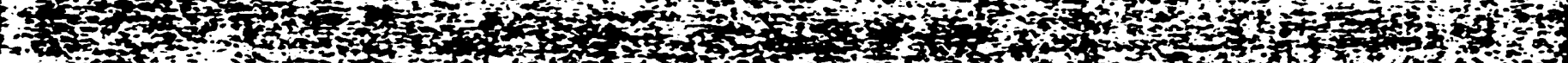
exisy

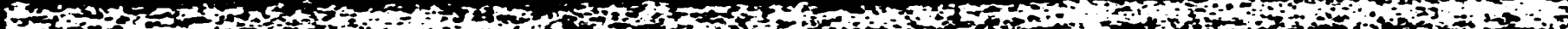
Sy 20,
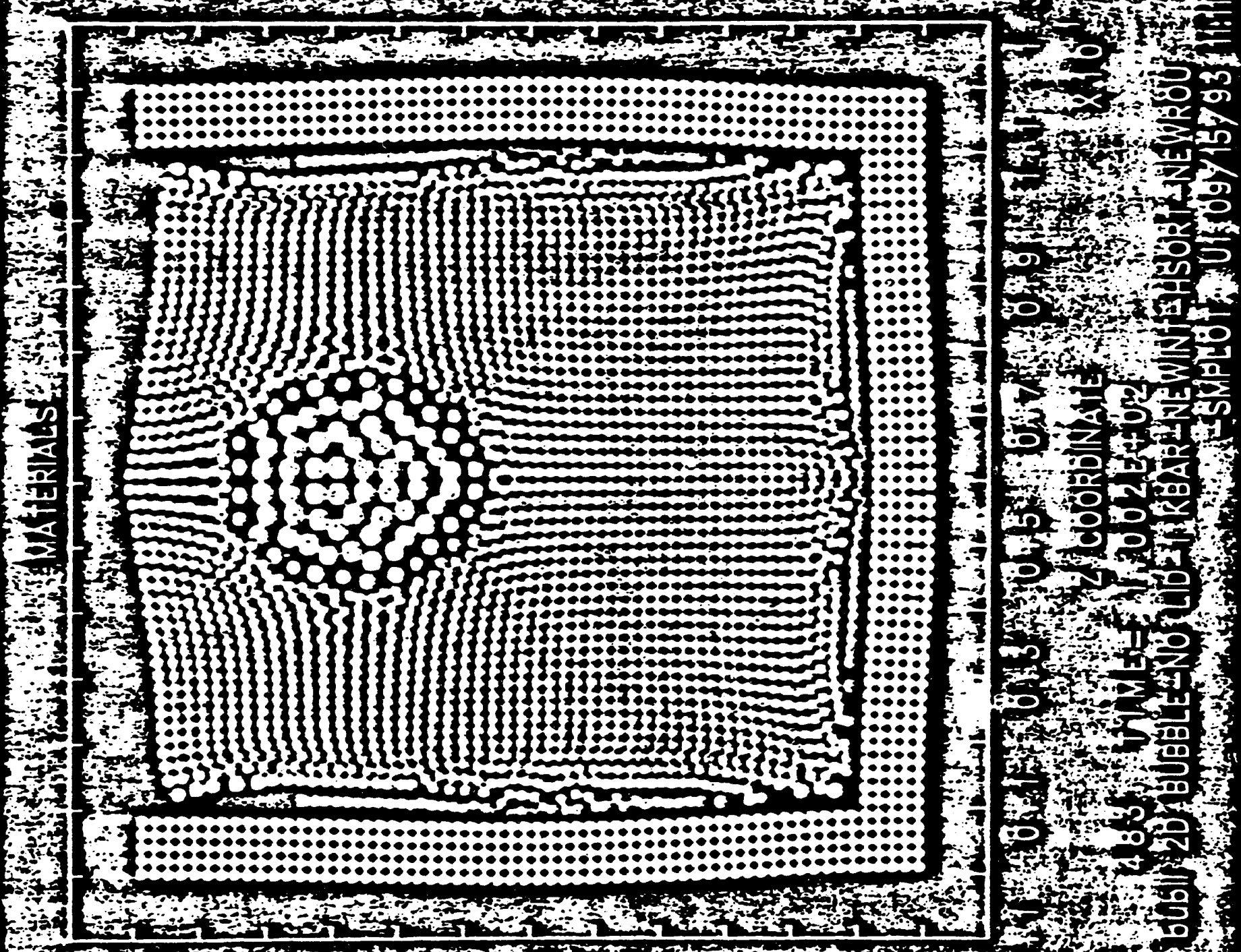

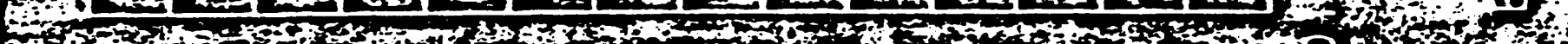
W

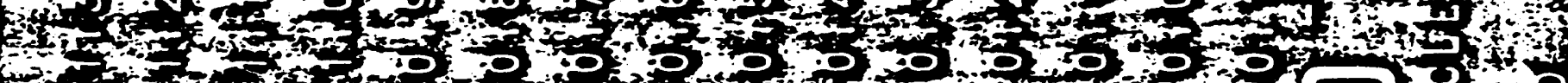

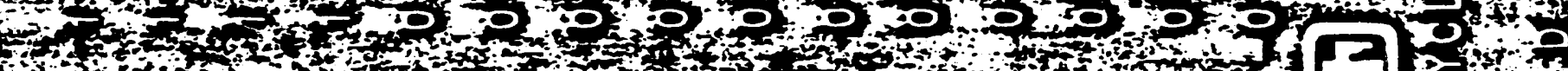
5 -

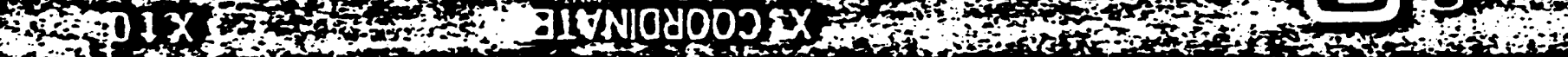

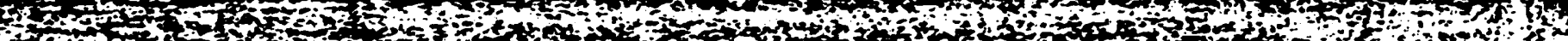

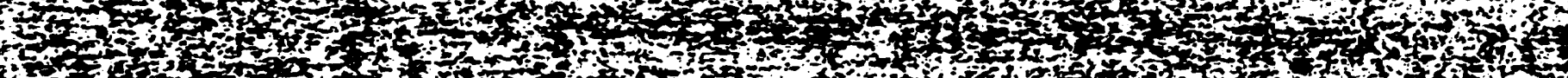

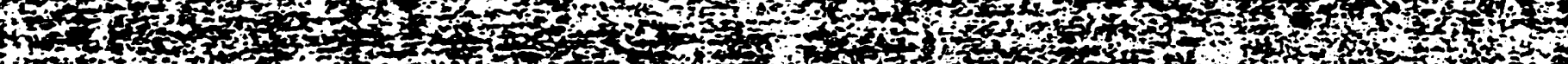

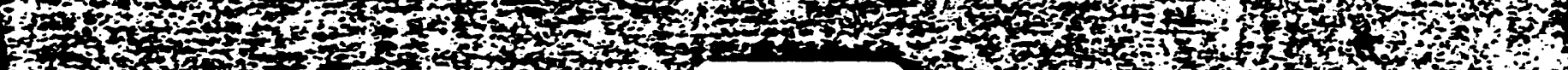

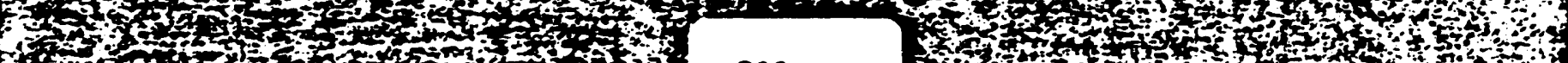
$12+211-$ 2013 


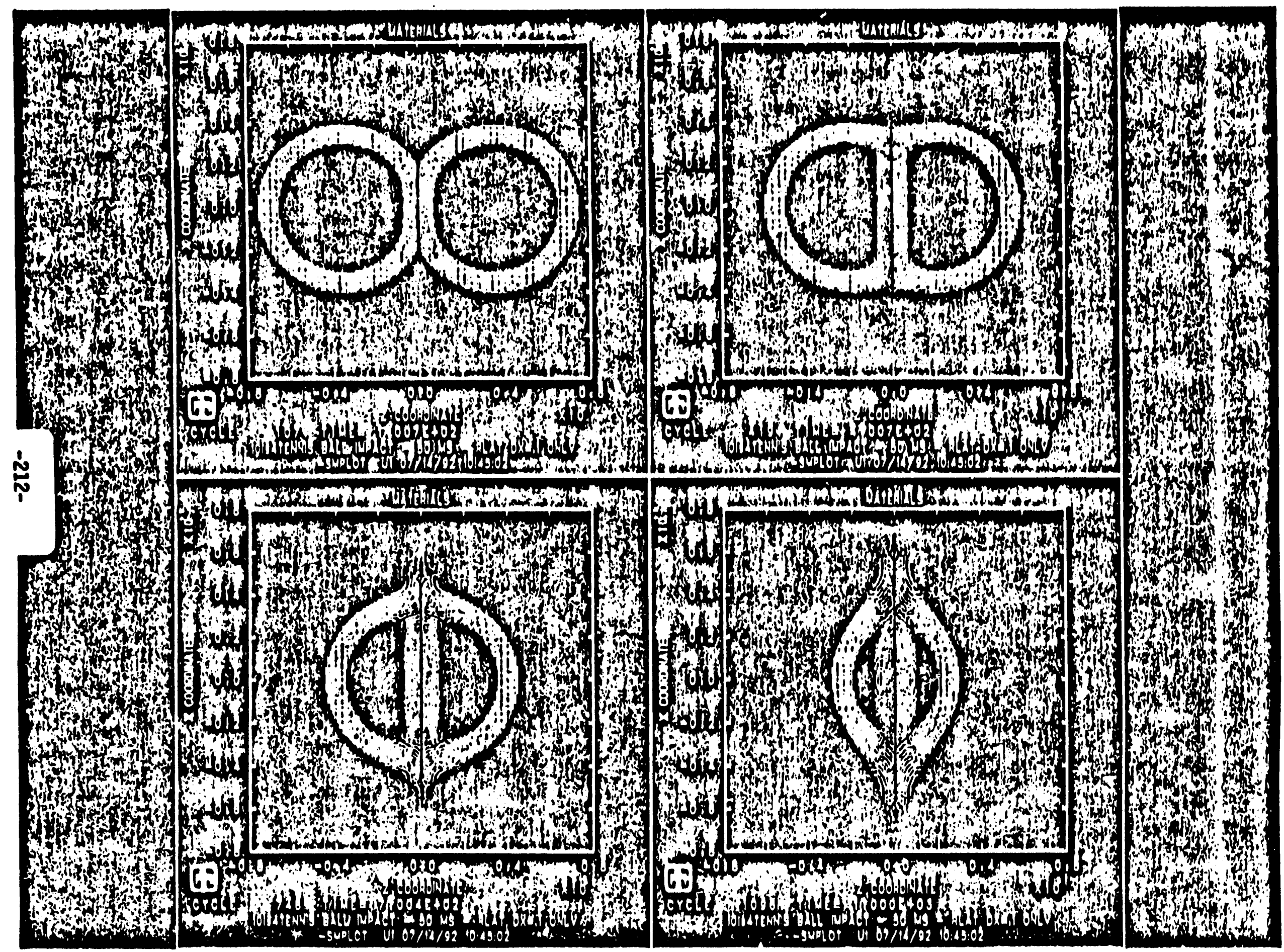




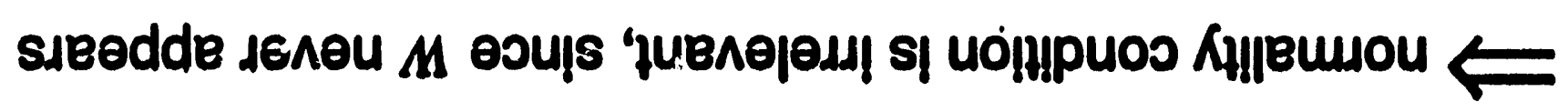

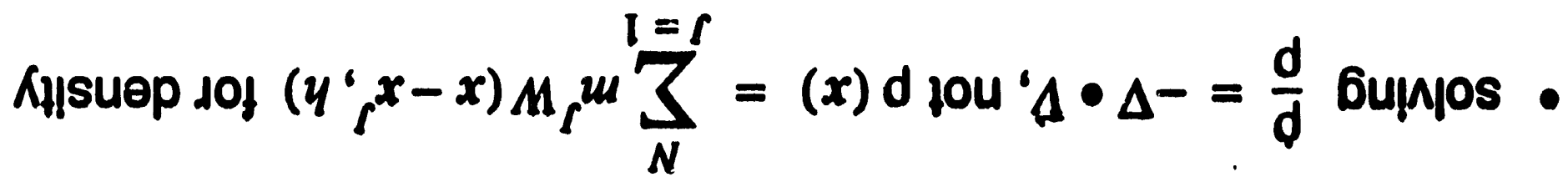

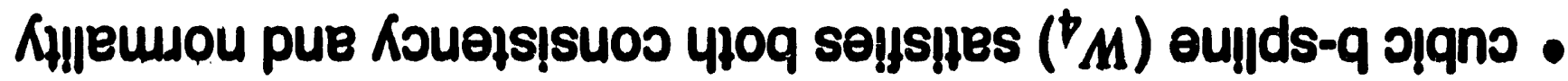

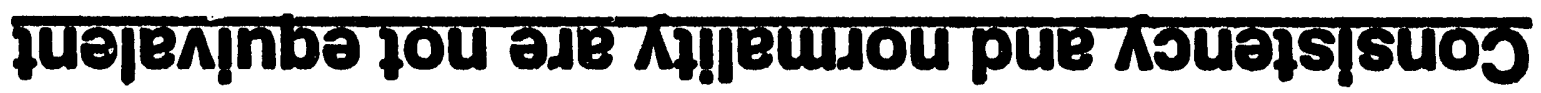

$$
\frac{\tau^{4 Z}}{I}-=(y=x), M
$$

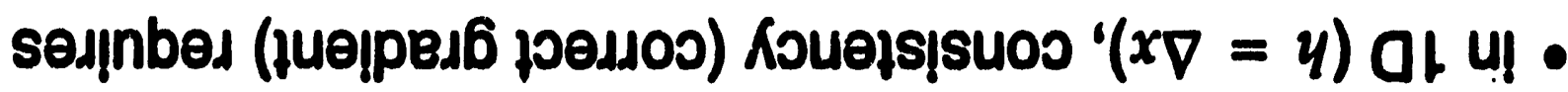

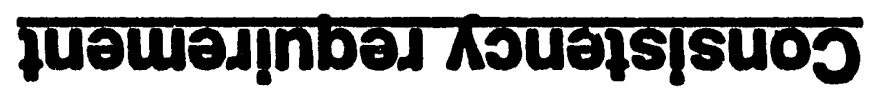

$$
I=\operatorname{xp}\left(y^{\prime} x\right) M \int
$$

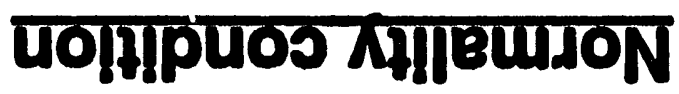

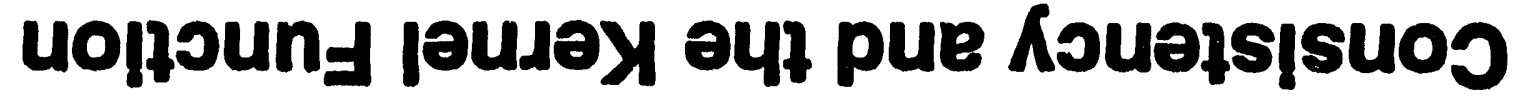




\section{Adjustable Factors in SPH Numerics}

- Resolution

- Viscosity coefficients

- Stability criterion (stable time step factor)

- Smoothing length/particle spacing ratio (3)

- Constant smoothing length vs. variable smoothing length (2)

- Kernel sum density vs. continuity equation (2)

- Von Nuemann Richtmyer viscosity vs. SPH (Monaghan) viscosity (2)

- Initial particle packing (rectangular, hexagonal, radial) (2)

- Ordered particle distribution vs. perturbed particle distribution (2)

- Equation of motion symmetrization $\left(\left(\frac{\sigma}{\rho^{2}}\right)^{I}+\left(\frac{\sigma}{\rho^{2}}\right)^{J}, \frac{\sigma^{\prime}+\sigma^{J}}{\rho^{I} \rho^{J}}\right.$, etc. ) (2)

- Velocity gradient symmetrization $\left(\frac{m^{J}}{\rho^{I}}\left(V_{i}^{l}-V_{i}^{J}\right) \frac{\partial W}{\partial x_{j}^{J}}, \frac{m^{J}}{\rho^{J}}\left(V_{i}^{l}-V_{i}^{J}\right) \frac{\partial W}{\partial x_{j}^{\prime}}\right)$ (2)

- Kernel function (splines, exponential, gaussian, super-gaussian) (3)

Number of combinations $=3 \times 2 \times 2 \times 2 \times 2 \times 2 \times 2 \times 2 \times 3=1152$ 


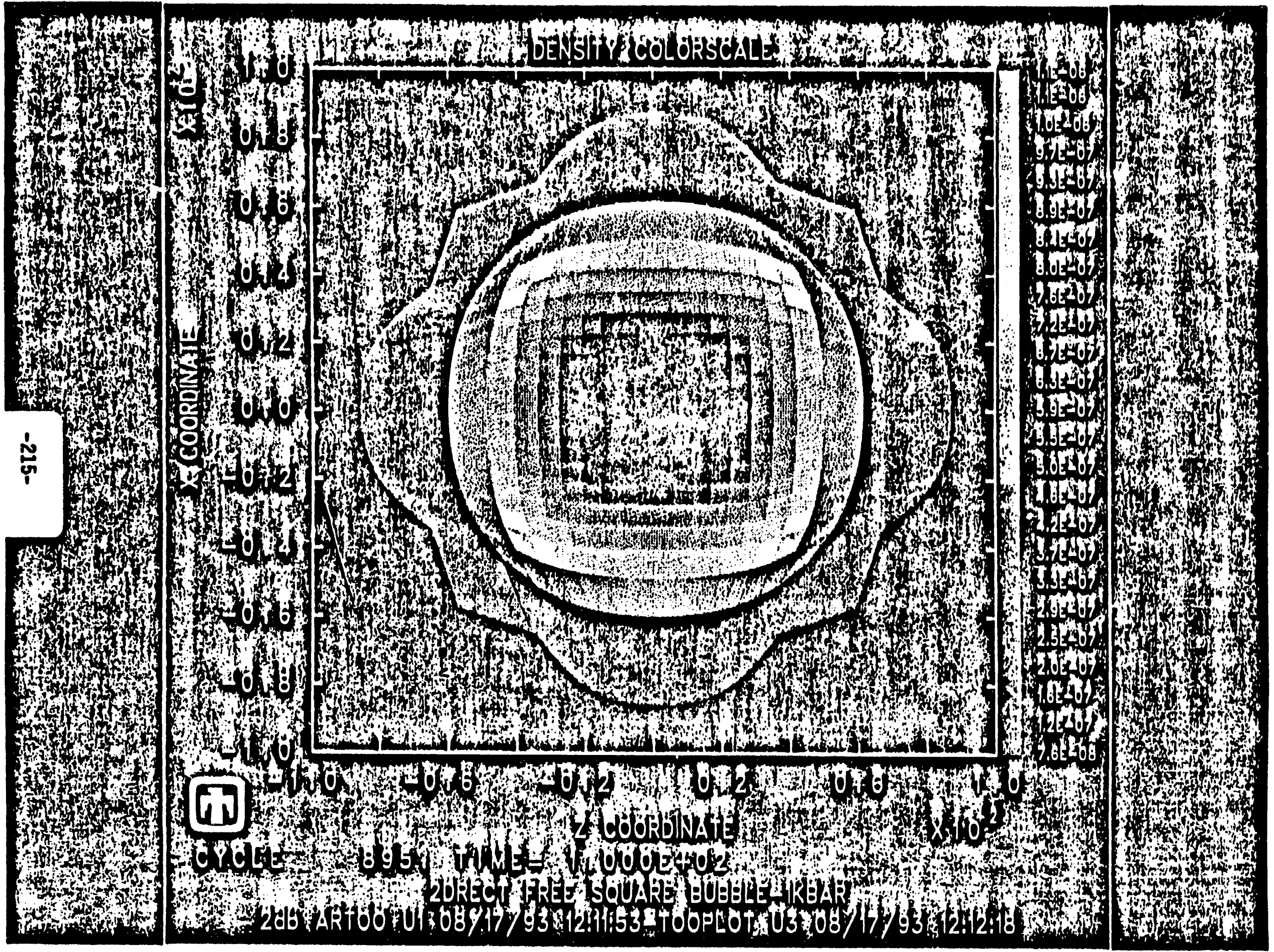




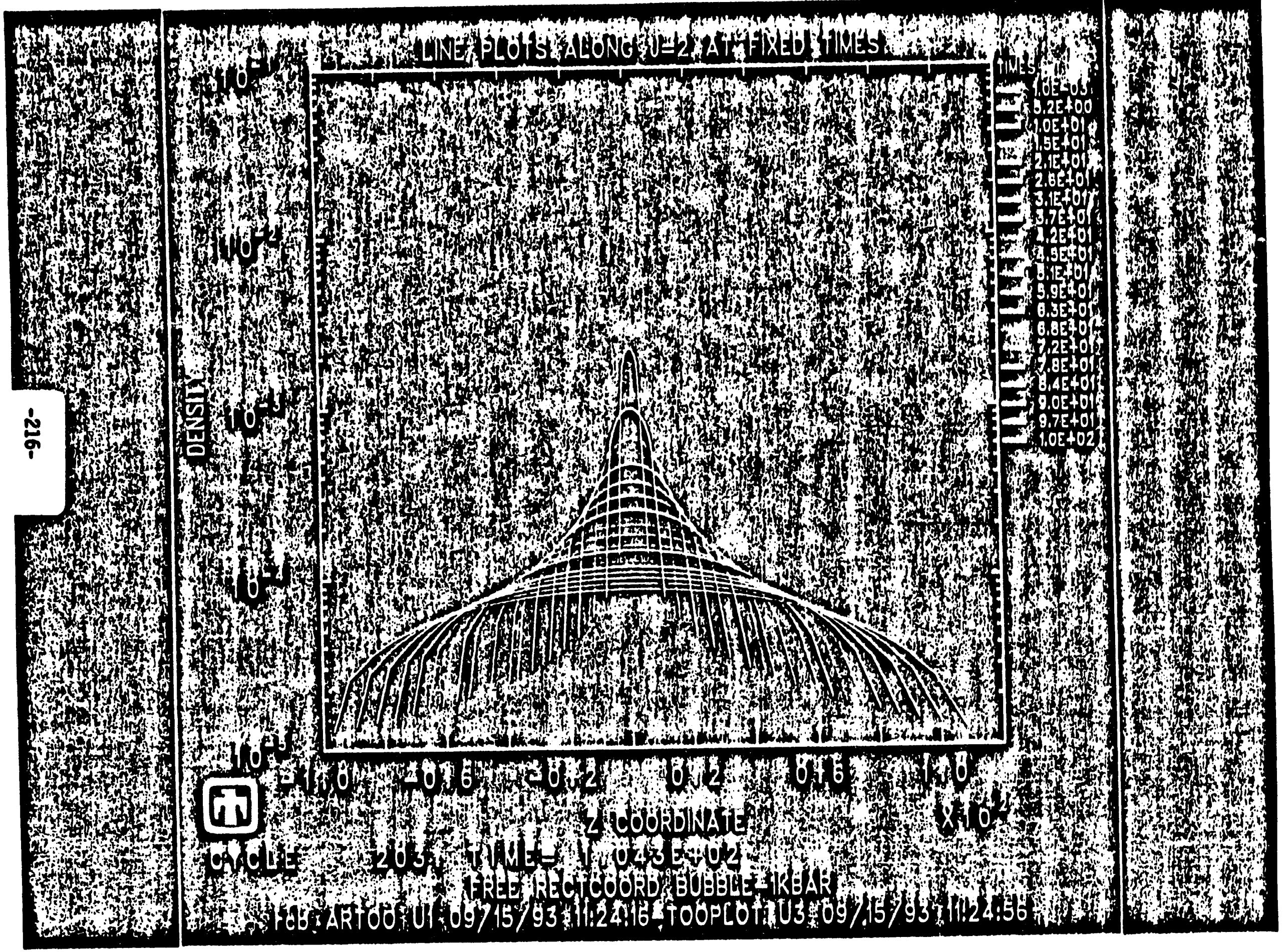




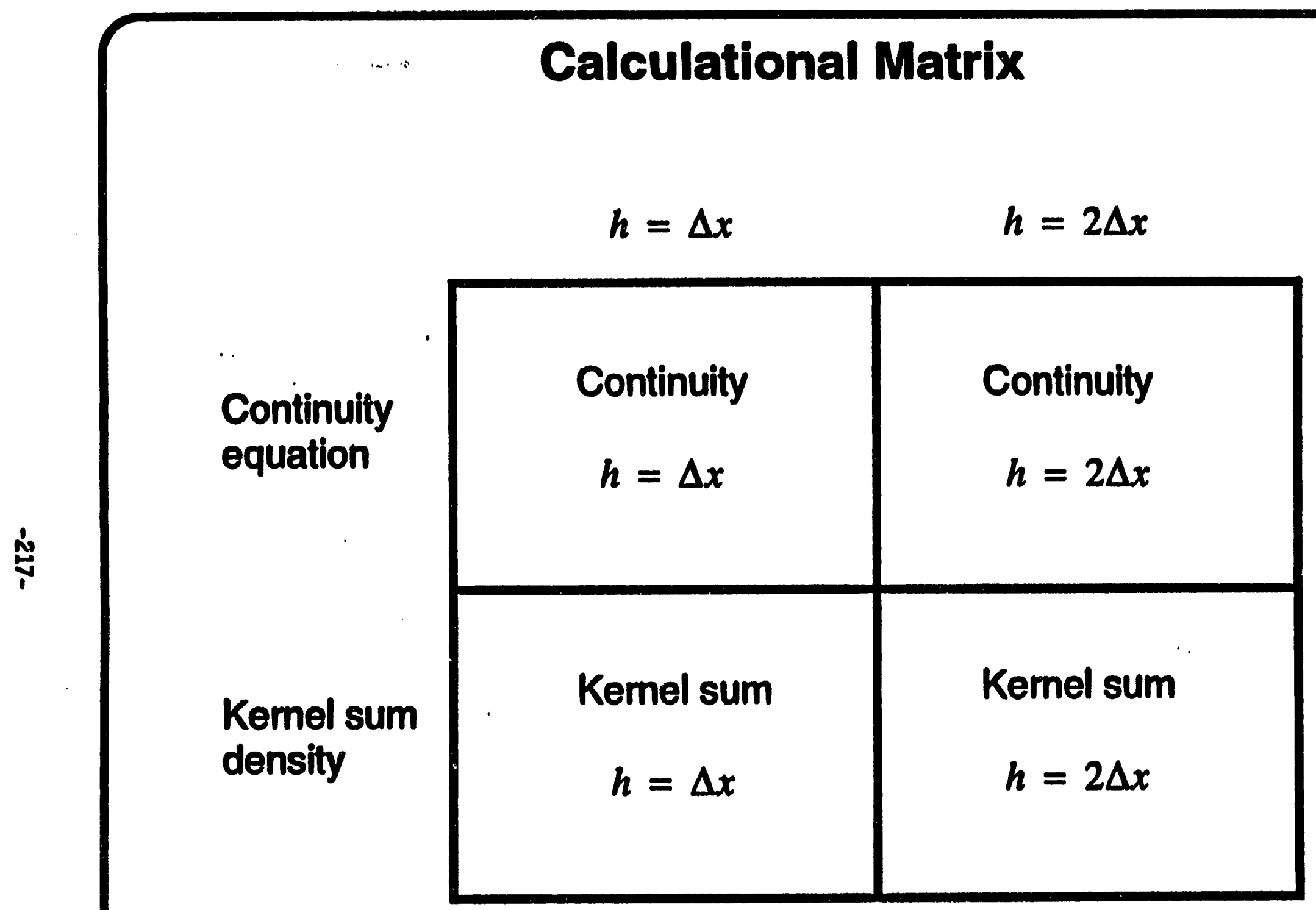




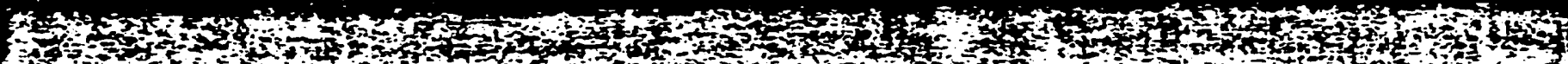

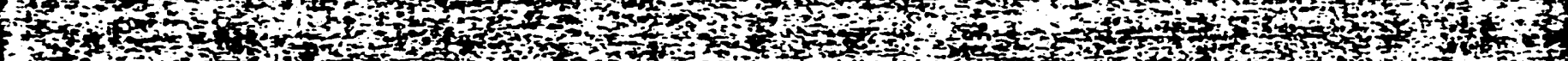

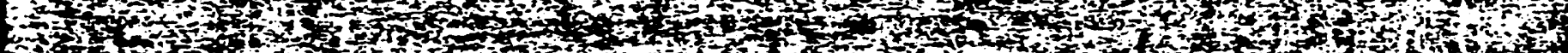
7 .

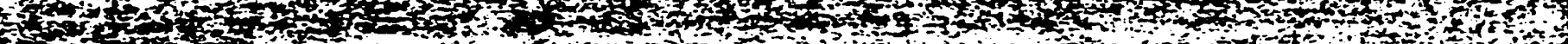

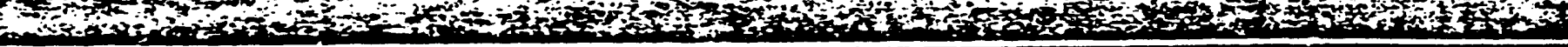
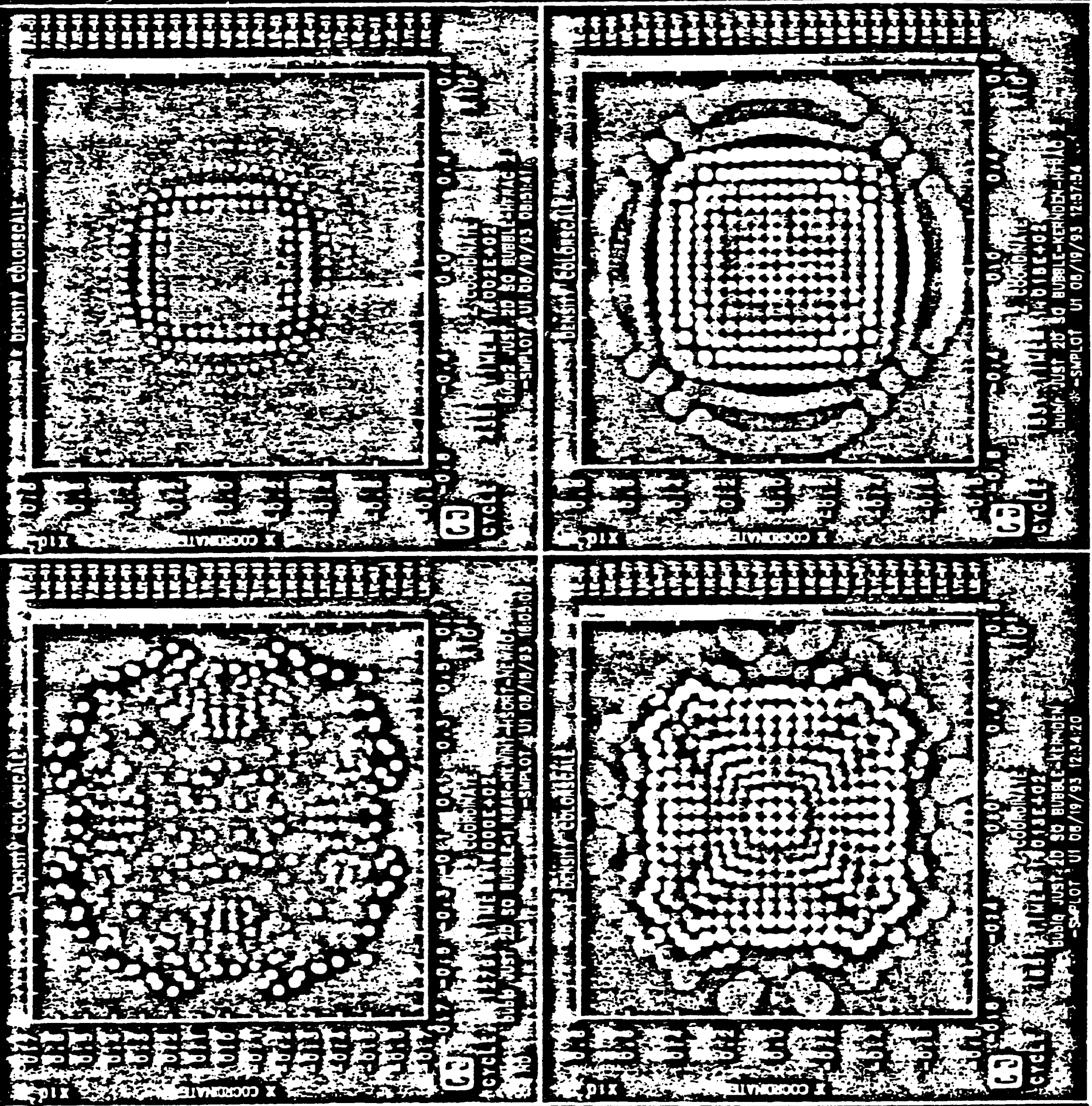

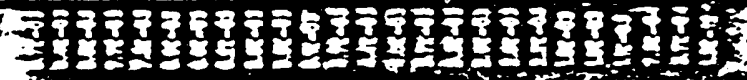

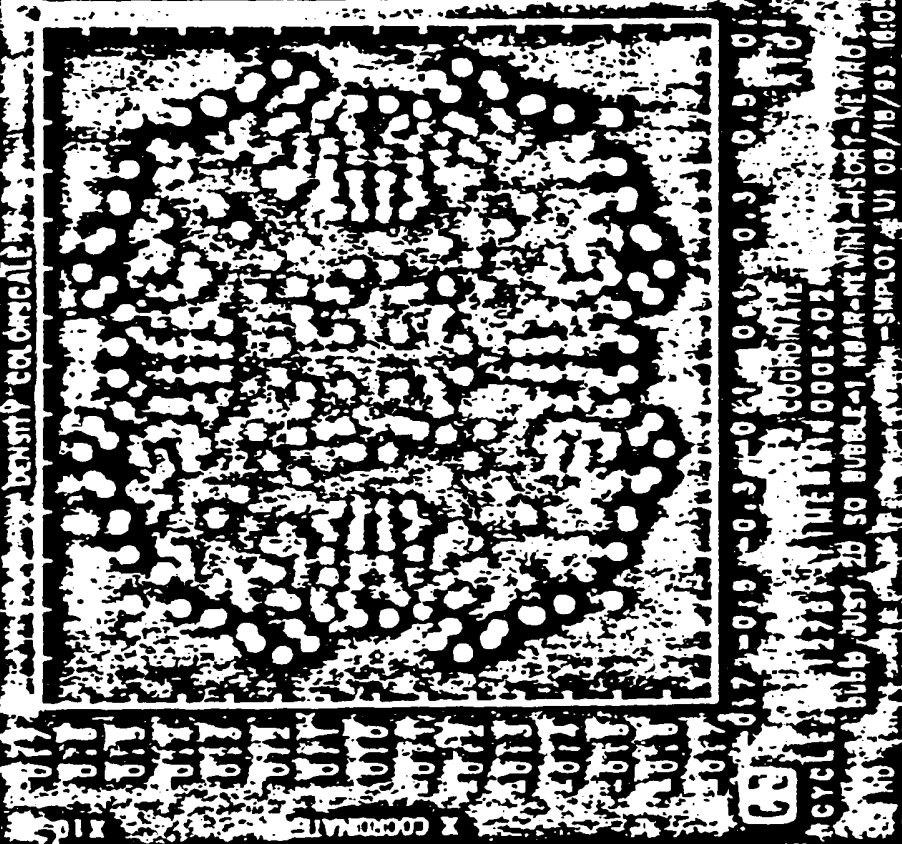

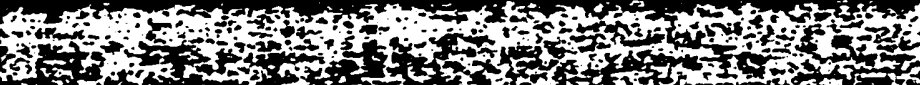

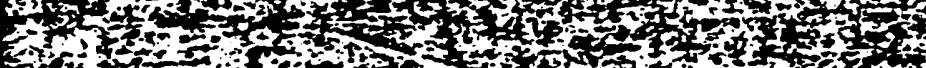

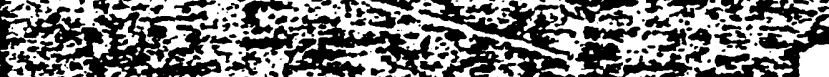

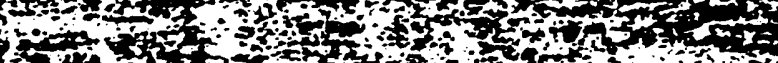

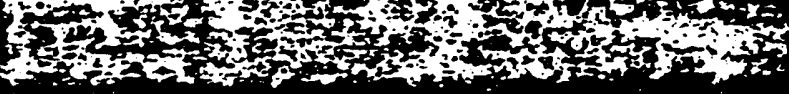

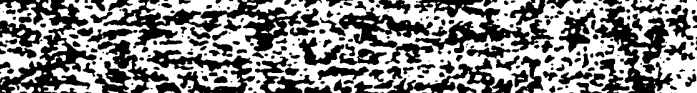
intis 


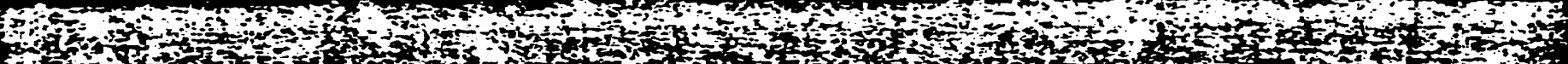
130 (3)

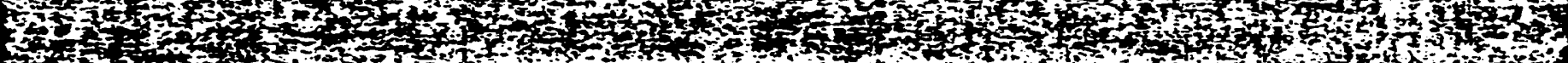
(3)

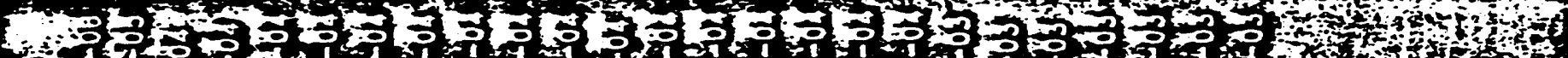

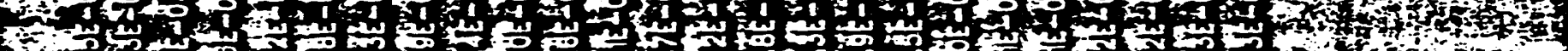

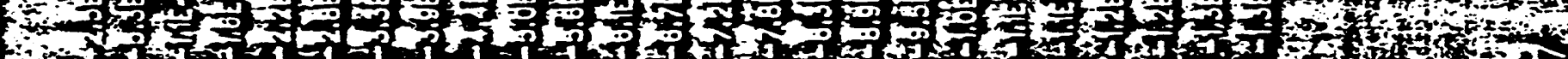
(1)

(f)

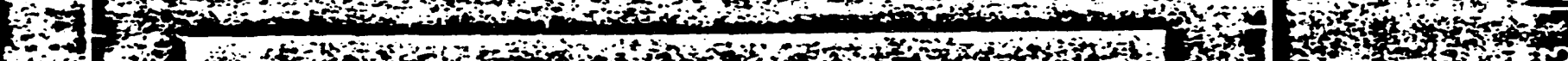

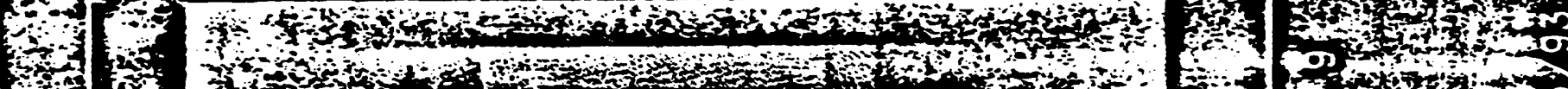

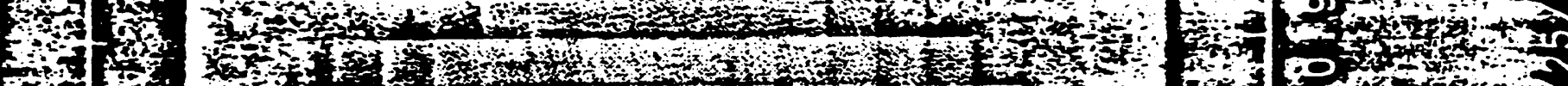
W

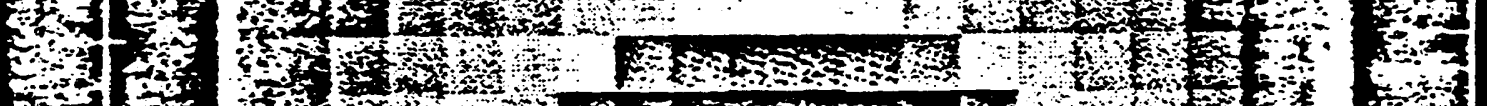

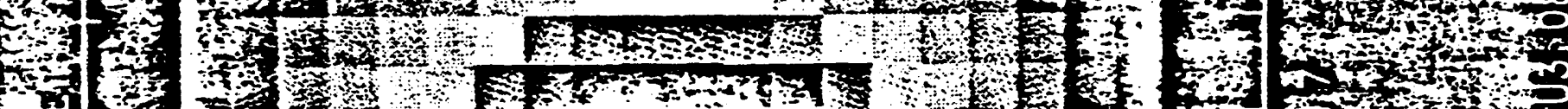
$=\frac{1}{4}$ th

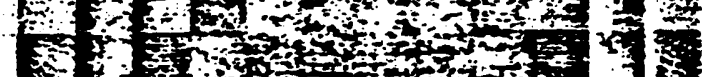

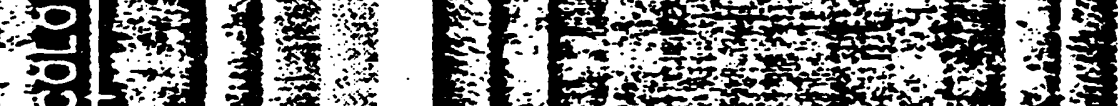
iw

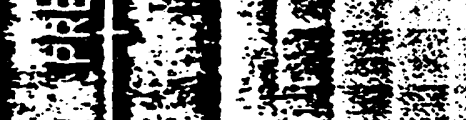
$2-1$ $=0-4$ $=$ ( 40

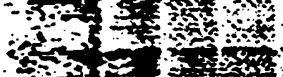
군 $1+3=0$ $2=150$

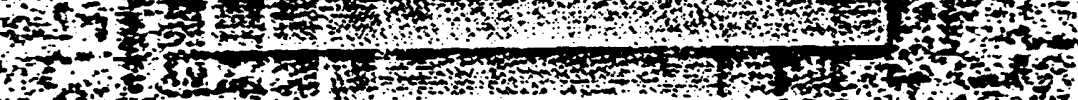

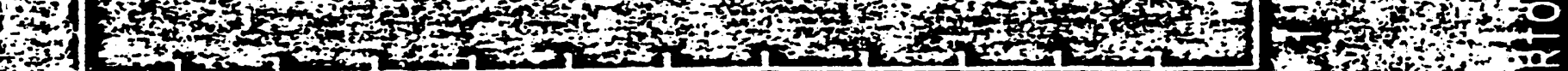
W2t

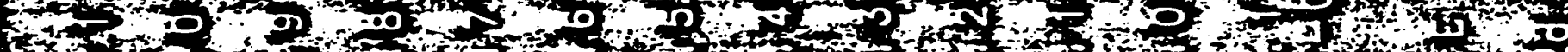

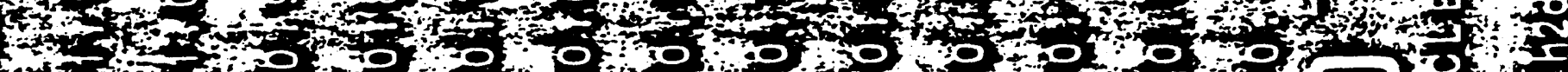

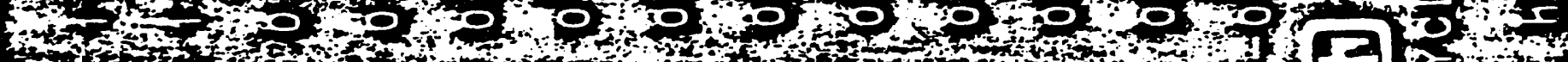

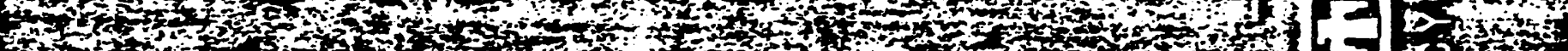

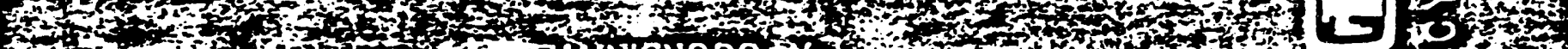
7.

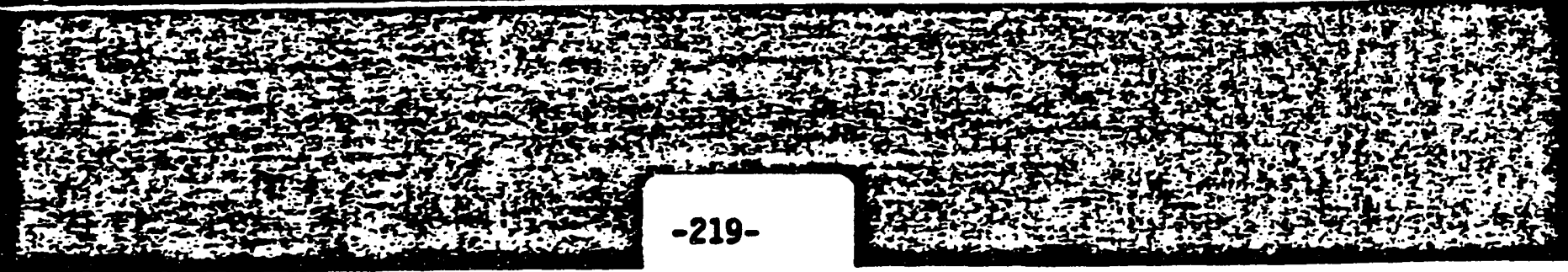


(n)

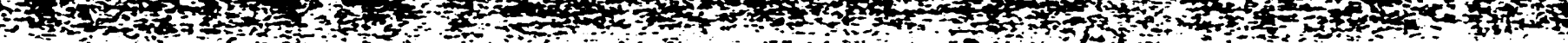
10

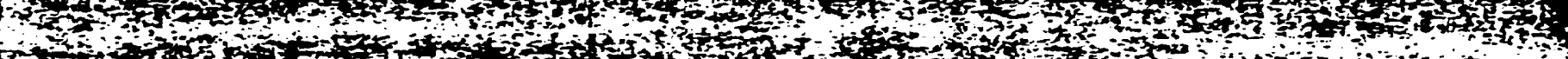
23

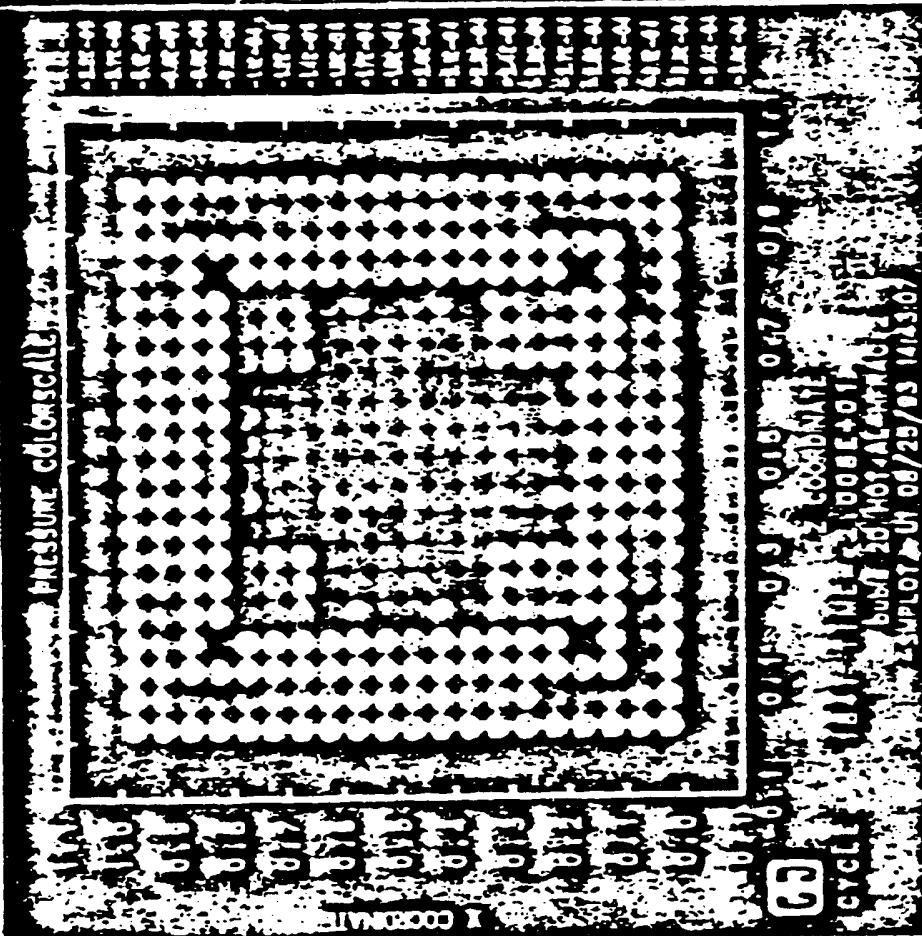

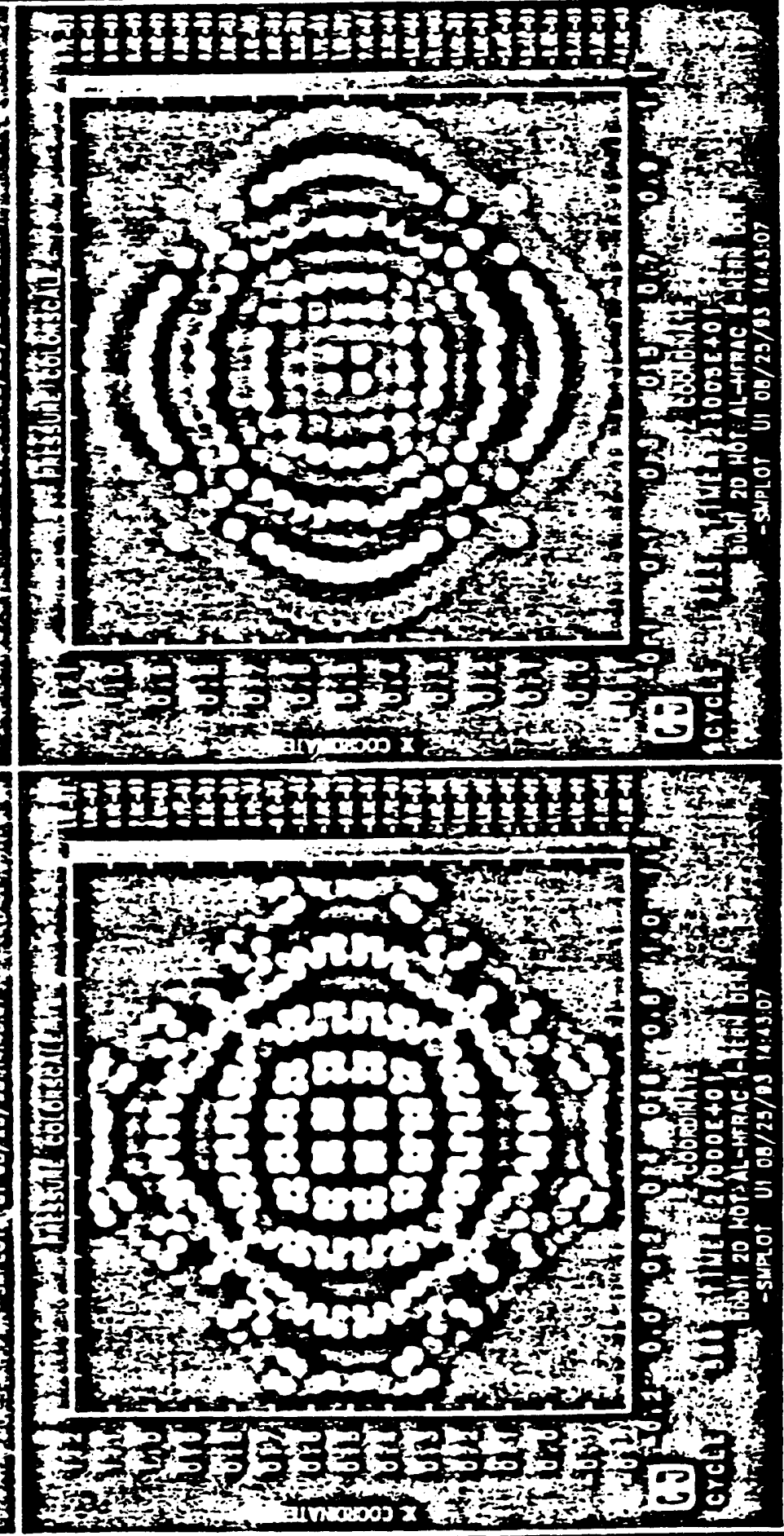

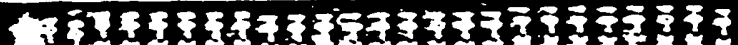

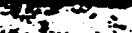

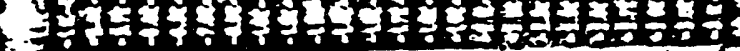

-

(

$3+4+4+1,13$

$=4+4-4+4+4<-4$

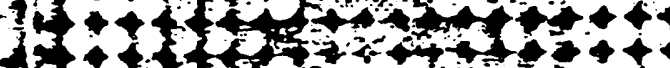

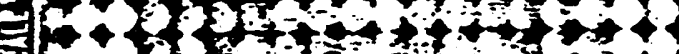

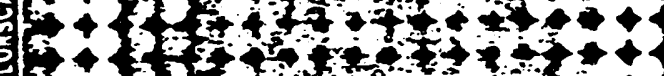

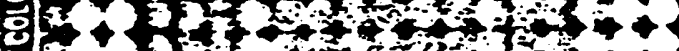

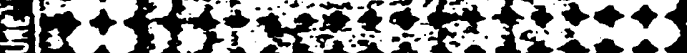

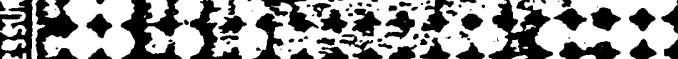

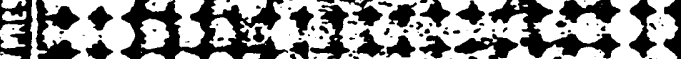

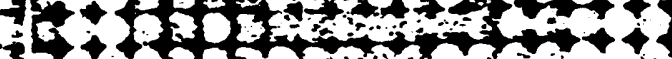

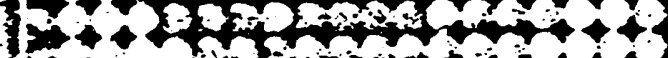

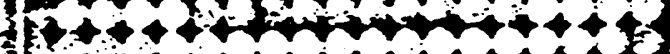

$3+4+4+44+4-4+4.4$

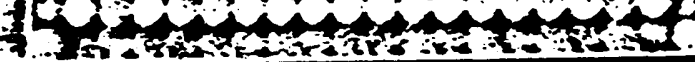
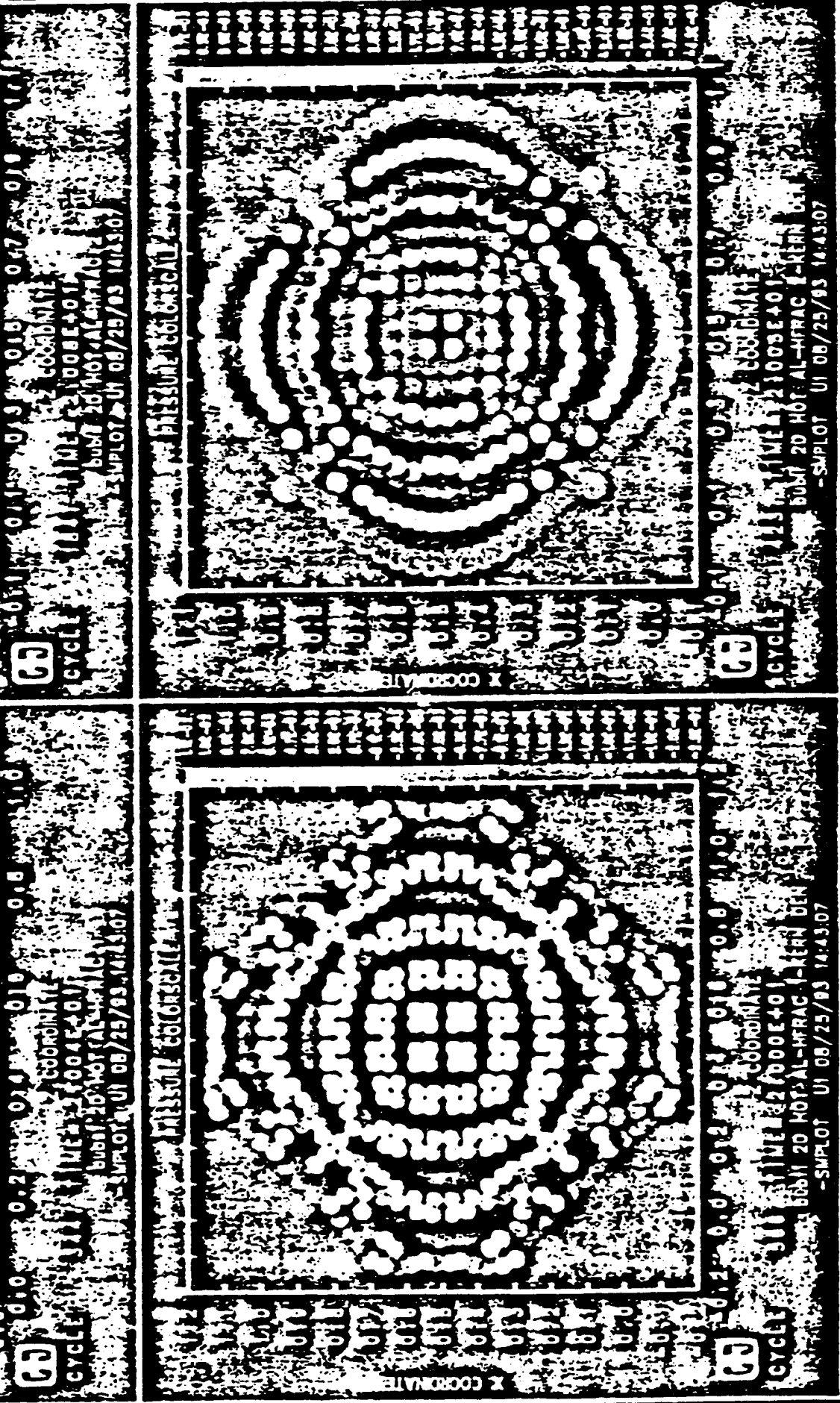

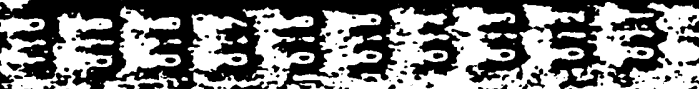

H

to

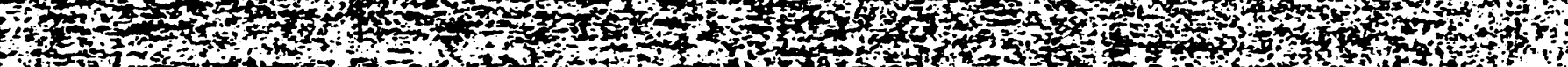
(1) -

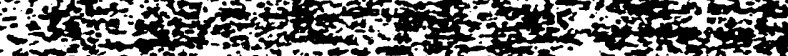
30 


\section{Conclusions}

A numerical method must be:

- Stable

- Consistent

- Conservative

Satisfying one or two of these conditions is relatively easy. The challenge is to make SPH meet all three simultaneously. 
\& 


\title{
SPH: Instabilities, Wall Heating, and Conservative Smoothing
}

\author{
by \\ D.L. Hicks, J.W. Swegle, S.W. Attaway \\ (September 22, 1993)
}




\section{Outline}

\section{Instabilities in SPH.}

II. Conservative Smoothing can stabilize SPH.

But is there anything left except FLATLAND?

\section{Test Problems}




\section{Instabilities in SPH}

Sufficient condition for instabilities:

- Nearest neighbor case:

$$
\sigma W^{\prime \prime}<0
$$

- Many neighbors case:

$$
\sigma \sum_{\text {odd } \Omega} W_{l}^{\prime \prime}<0
$$

where $W_{\ell}^{\prime \prime}=\left(W_{j,+\ell}^{\prime \prime}+W_{j,-\ell}^{\prime \prime}\right) / 2$.

This analysis is for a uniform stress:

$$
\sigma=p+q
$$

Note: instabilities are possible for both cases

$$
\left\{\begin{array}{l}
\sigma>0, \text { positive } \\
\sigma<0, \text { negative }
\end{array} .\right.
$$




\section{Conservative smoothing ${ }^{*}$ can stabilize SPH.}

But is there anything left except

$$
\text { FLATLAND? }
$$

- Conservative smoothing on the s.mom. of the artifical viscosity type:

$$
\begin{aligned}
\text { smom }_{j}= & \operatorname{smom}_{j} \\
& +\operatorname{csm}_{j+\frac{1}{2}} *\left(\operatorname{smom}_{j+1}-\text { smom }_{j}\right) \\
& -c s m_{j-\frac{1}{2}} *\left(\text { smom }_{j}-\text { smom }_{j-1}\right)
\end{aligned}
$$

- Conservative smoothing on the s.i.erg. of the artifical heat conduction type:

$$
\begin{aligned}
\operatorname{sierg}_{j}= & \text { sierg }_{j} \\
& + \text { csie }_{j+\frac{1}{2}} *\left(\text { sierg }_{j+1}-\operatorname{sierg}_{j}\right) \\
& - \text { csie }_{j-\frac{1}{2}} *\left(\text { sierg }_{j}-\operatorname{sierg}_{j-1}\right)
\end{aligned}
$$

* Introduced in 1969 in AFWC-TR-69-20 by D.L.Hleks. 
- Conservative smoothing on the s.vol. of the artifical strain relaxation type:

$$
\begin{aligned}
\text { svol }_{j}= & \text { svol }_{j}: \\
& +c s v_{j+\frac{1}{2}} *\left(\text { svol }_{j+1}-s v o l_{j}\right) \\
& -c s v_{j-\frac{1}{2}} *\left(s v o l_{j}-s v o l_{j-1}\right)
\end{aligned}
$$

Our analysis shows that we can stabilize SPH with conservative smoothing of the type described above. The result boils down, approximately, to the following timestep restriction for an ideal gas $(\gamma=1.4)$ in compression:

$$
C_{F L} \leq \frac{1}{2}
$$

Full blown conservative smoothing ${ }^{*}\left(c s_{j+\frac{1}{2}}=\right.$ 0.25) was assumed to get this result. We decided to test this theory with some numerical experiments.

* $0 \leq c s \leq .25$ 


\section{Test Problems}

Several years ago. we developed a set of hydrocode test problems [Hicks \& Pelzl (1968): AFWLTR-68-112].

These test problems have seven categories:

SCTP I Single Shock Wave Problems

SCTP II Single Rarefaction Wave Problems

SCTP III Accelerating Wave Problems

SCTP IV Decelerating Wave Problems

SCTP V Riemann's Resolution of the Discontinuity Problems

SCTP VI Shock Collision Problems

SCTP VII Shock Overtake Shock Problems 
Along with these test problems (whose solution's are known exactly) we developed a procedure for quantitatively comparing hydrocodes using the $\ell_{1}, \ell_{2}, \ell_{\infty}$ norms to measure the error in various functions. We compared a von Neumann-Richtmyer code with a Lax-Wendroff code.

Next we show some samples of the tables and graphs from that comparison. 
Table $\pi$

errors on scip-I-s

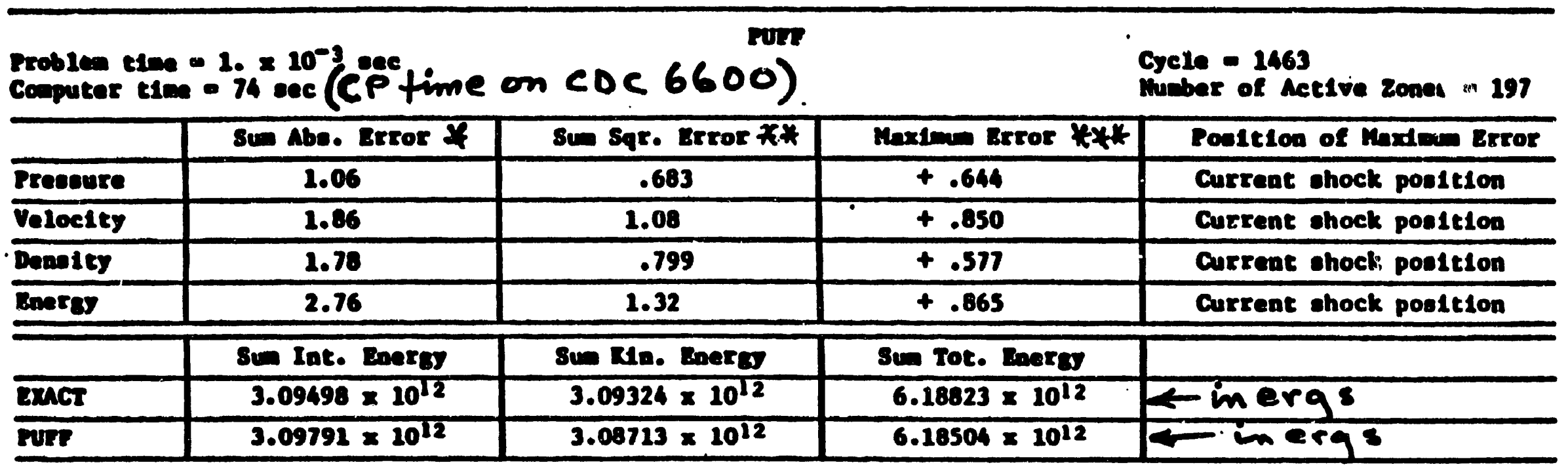

vir-neworof

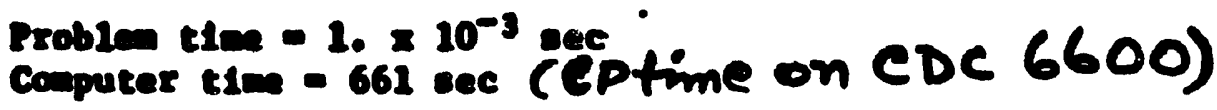

Cycle -1762

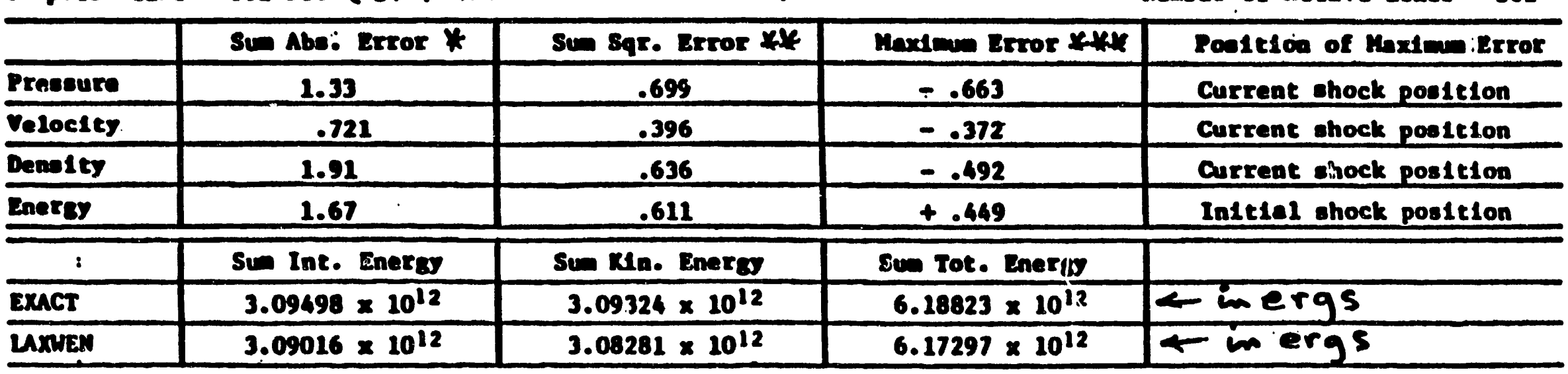

$4 l_{1}$ norm, nondimensionalizel with max. value of exact solution

* $l_{2}$ norms

( ) frit 200 norm,

1

11

11

11

e $1 .<10$ 


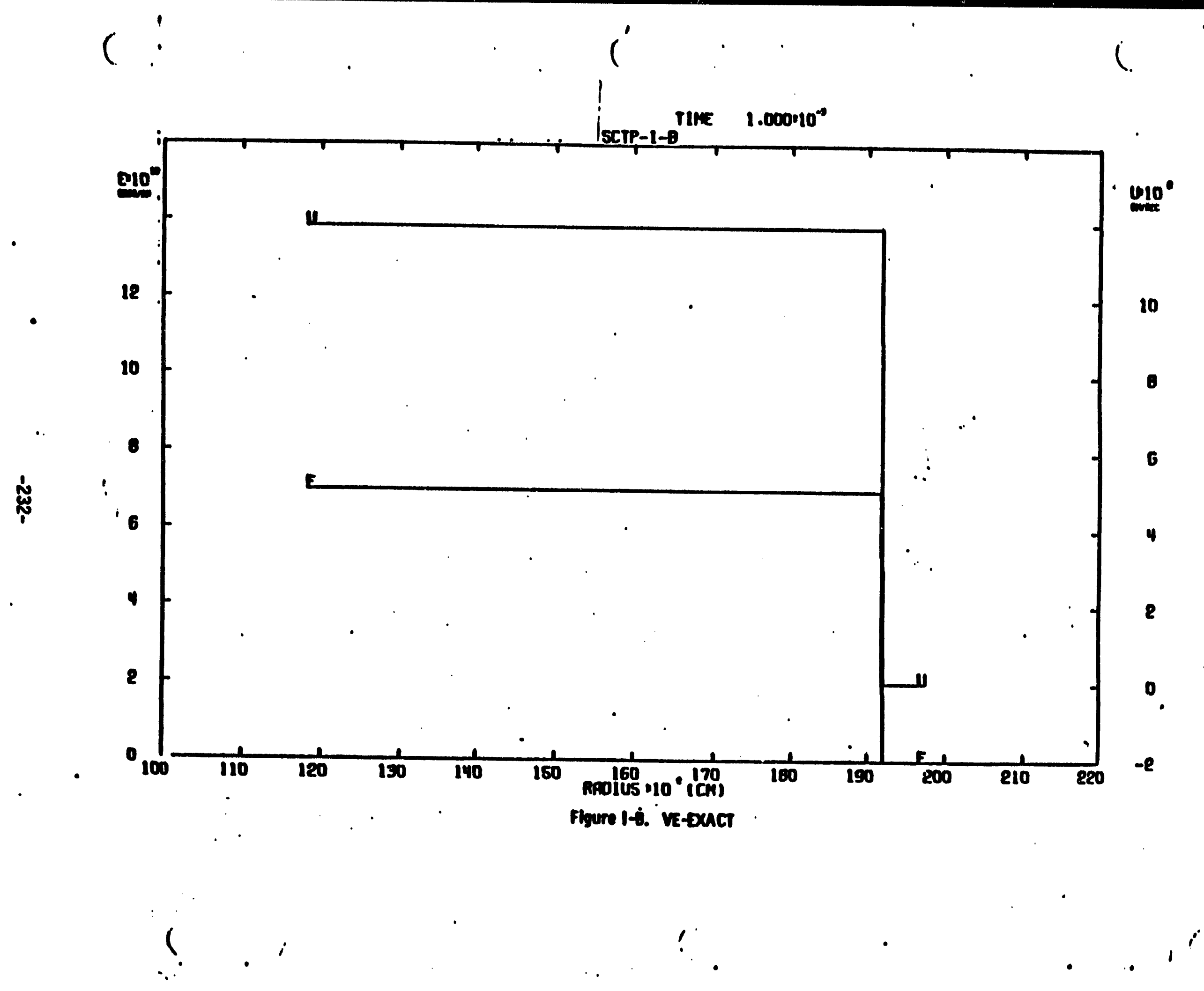




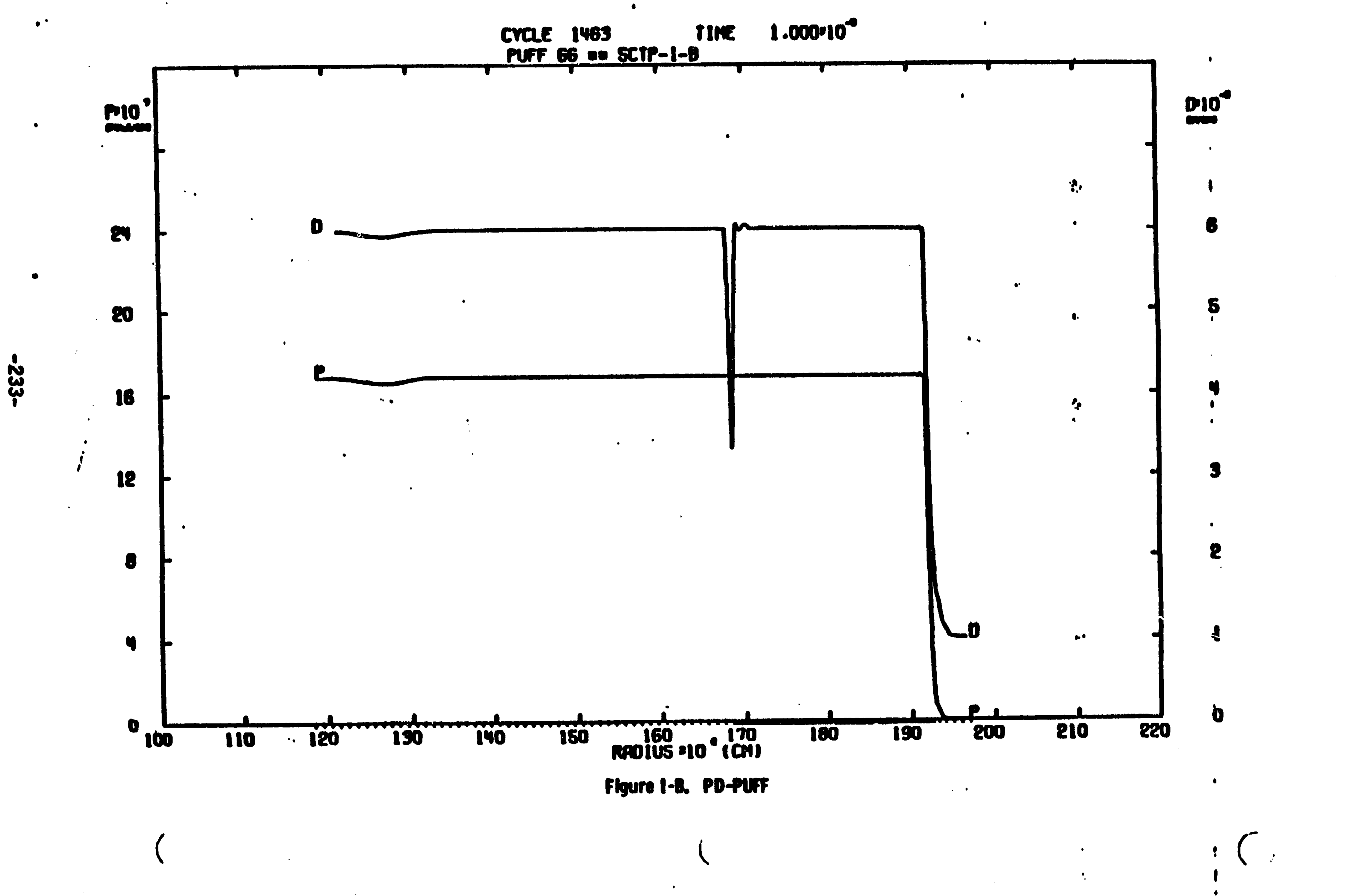




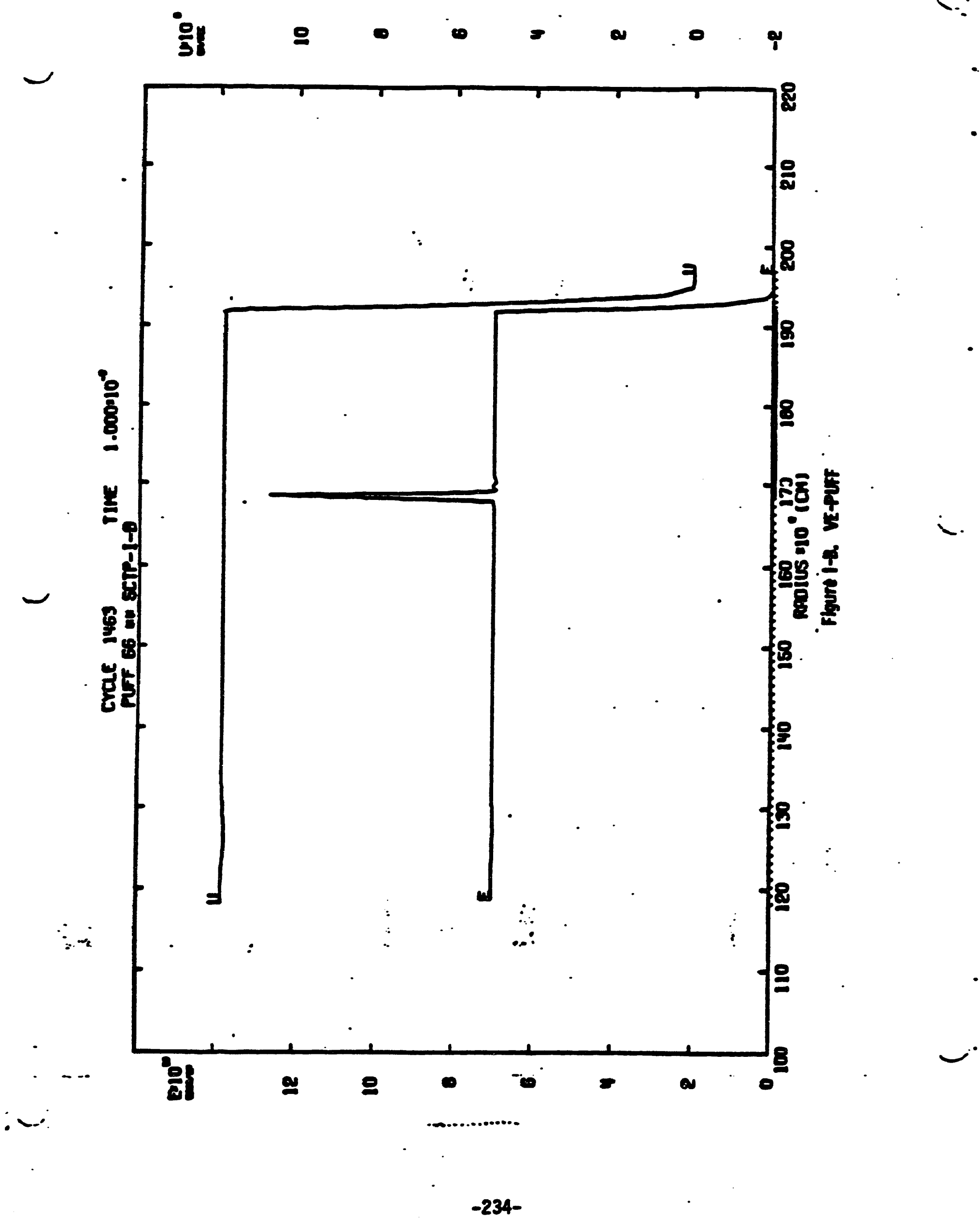



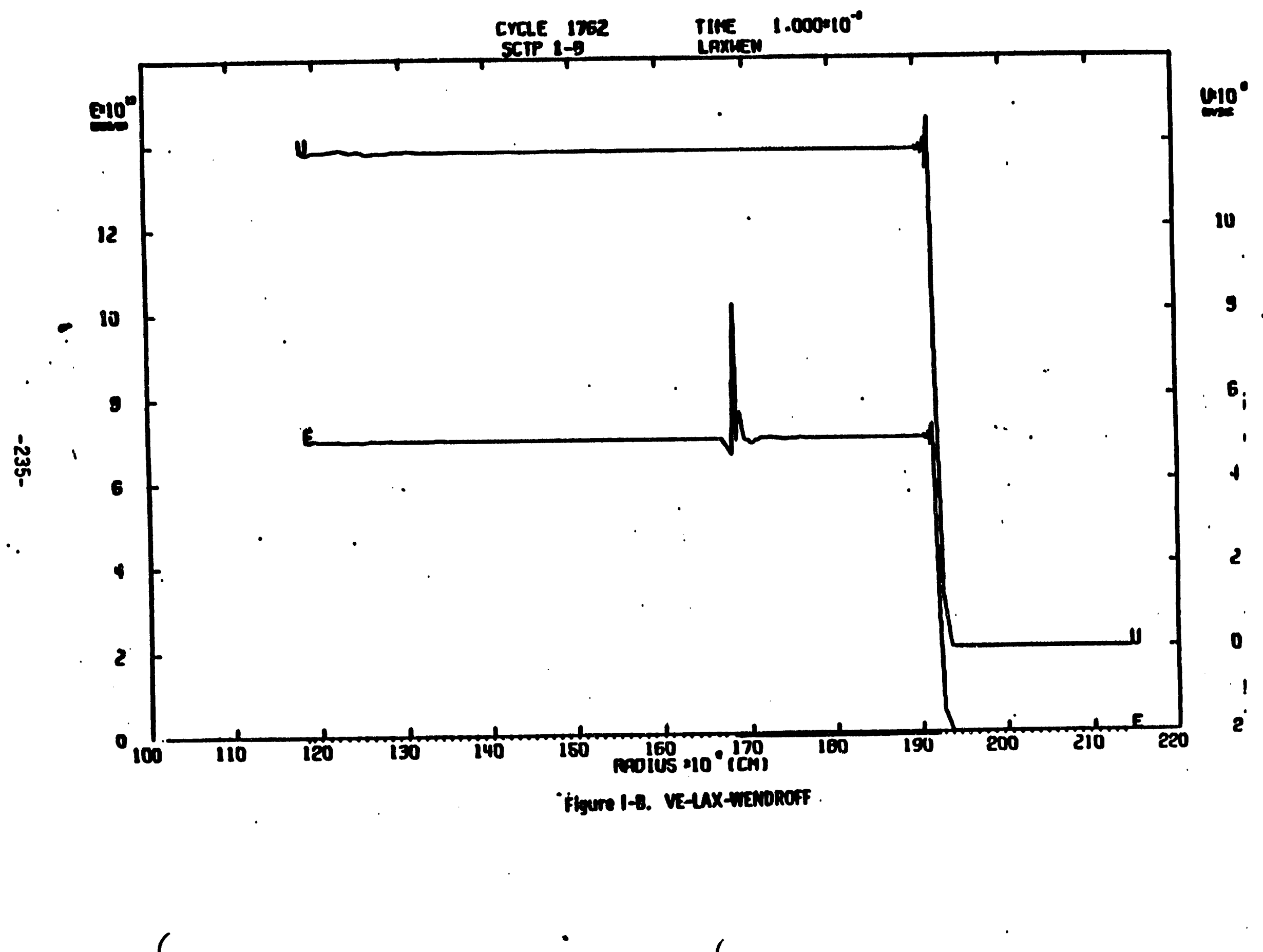


$$
\text { AE }
$$




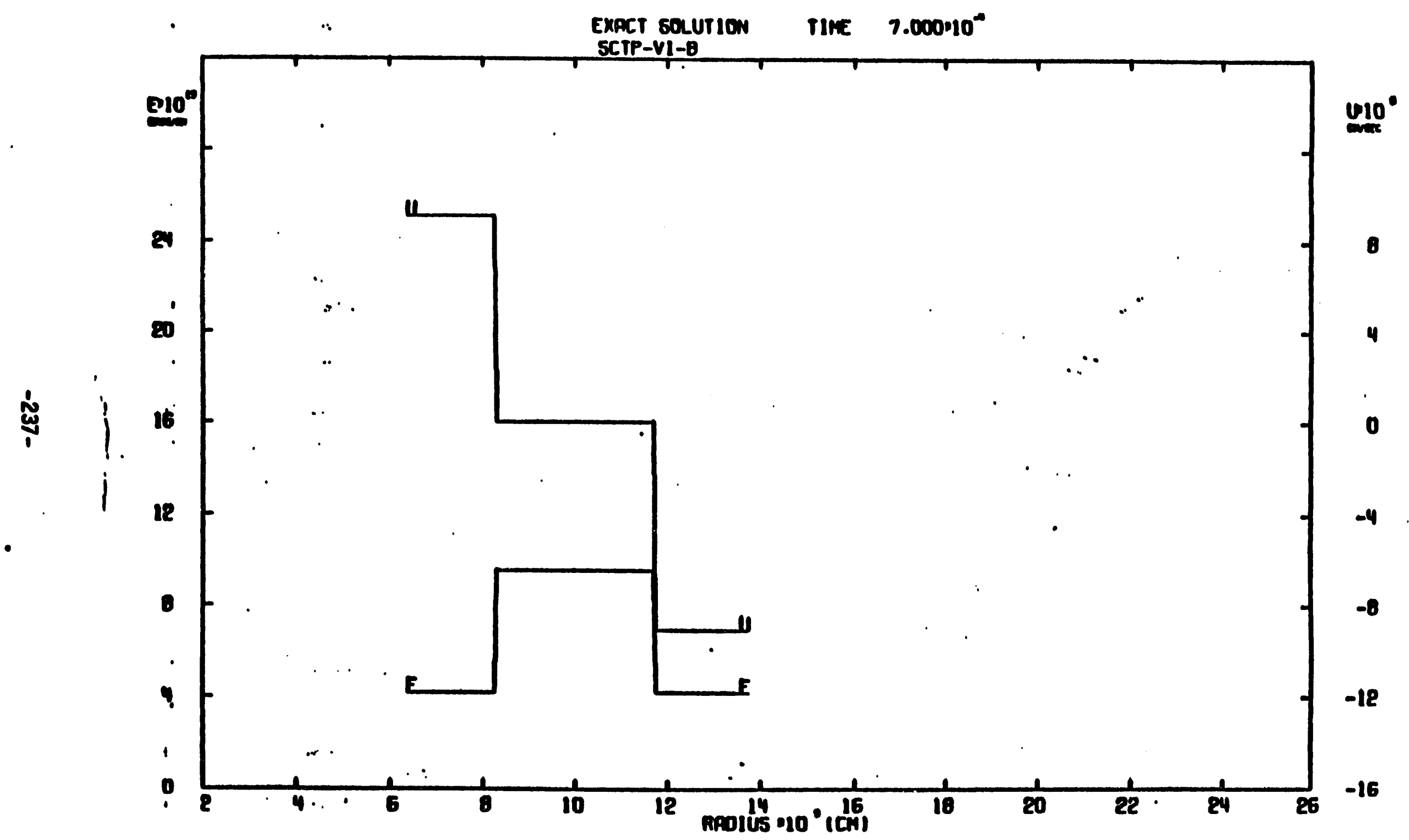

Flgure vita, vetexact 
$\therefore$

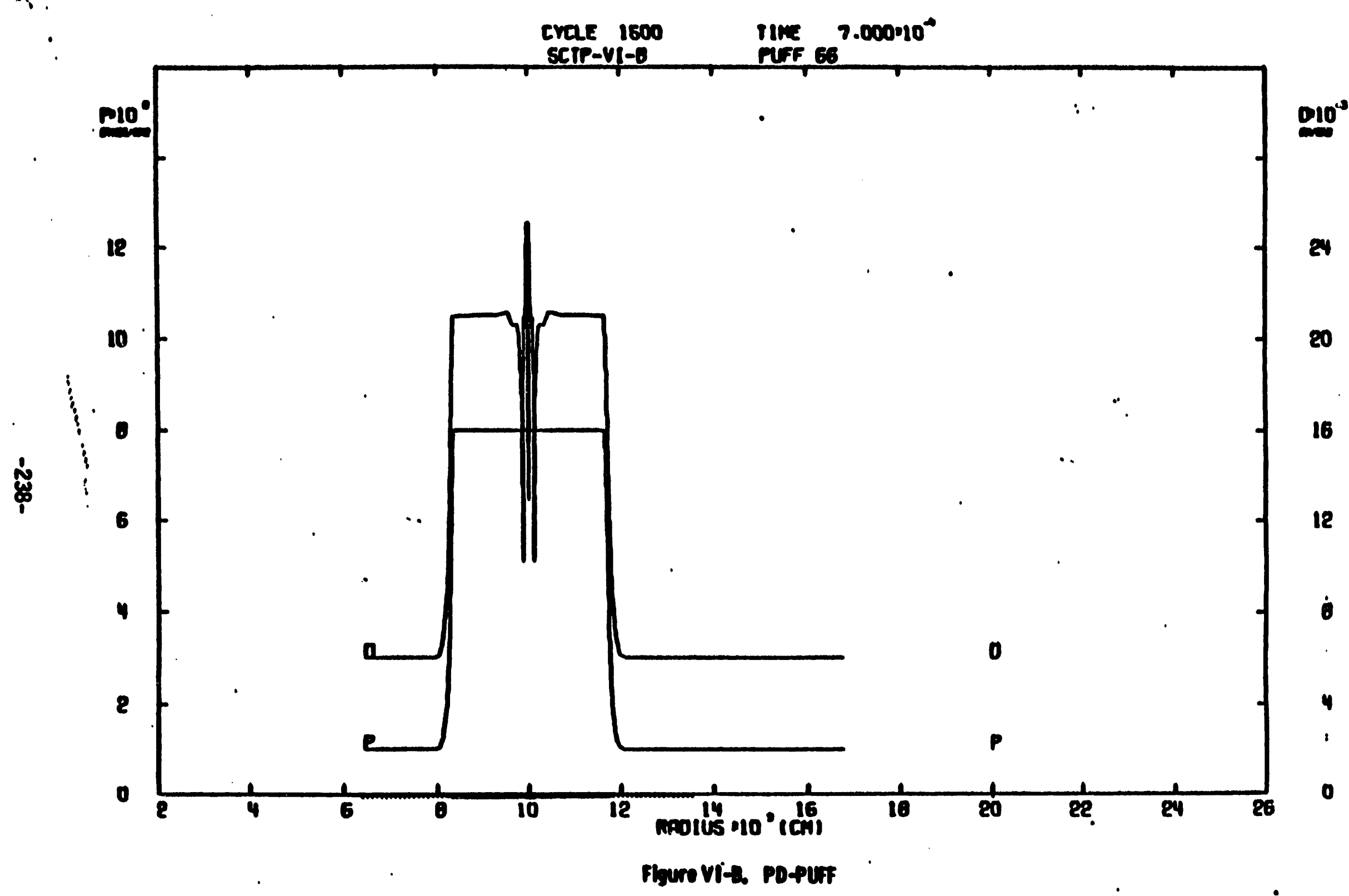

$(1$ 

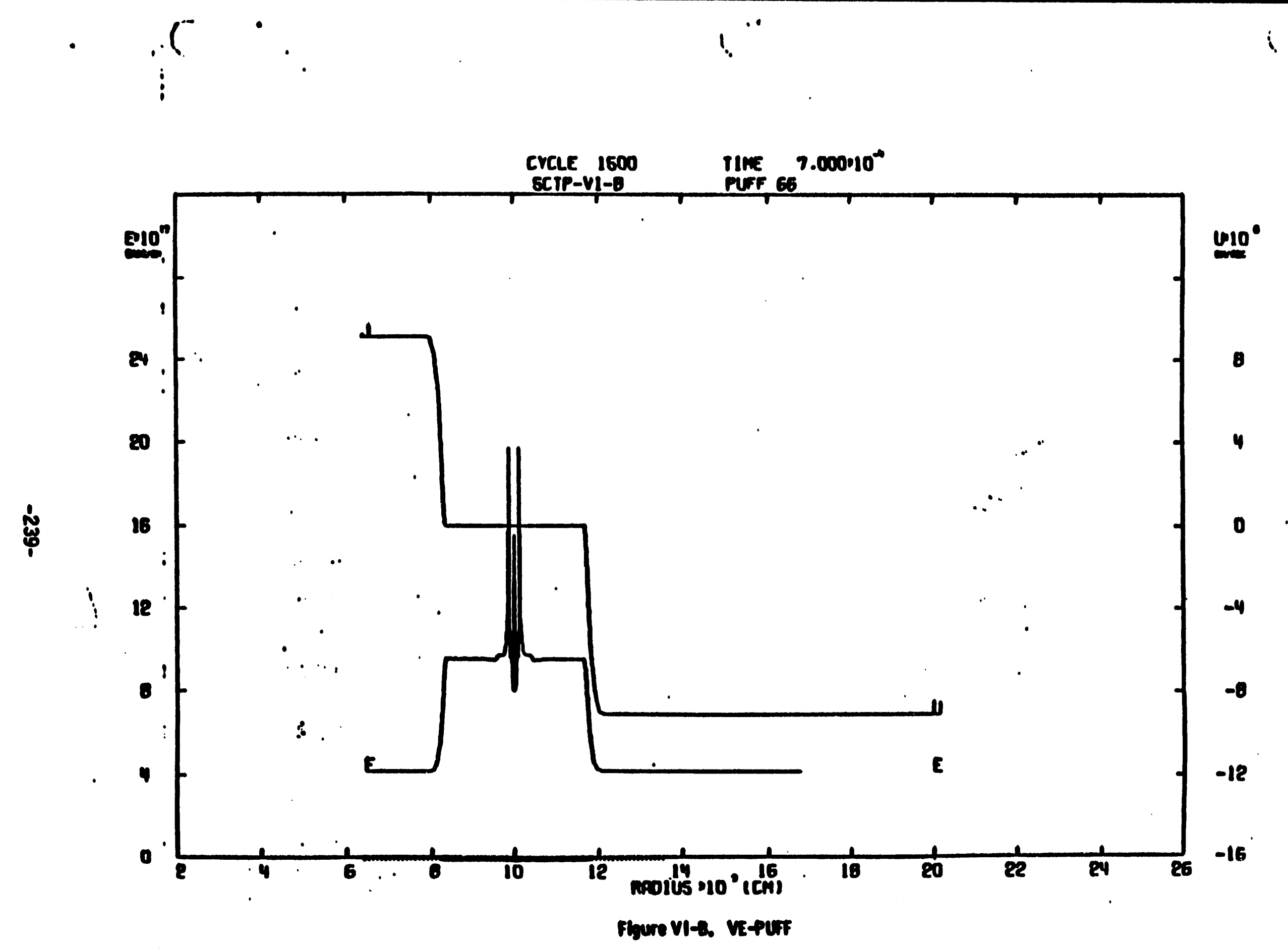

Flyore VI-a, VEturt 


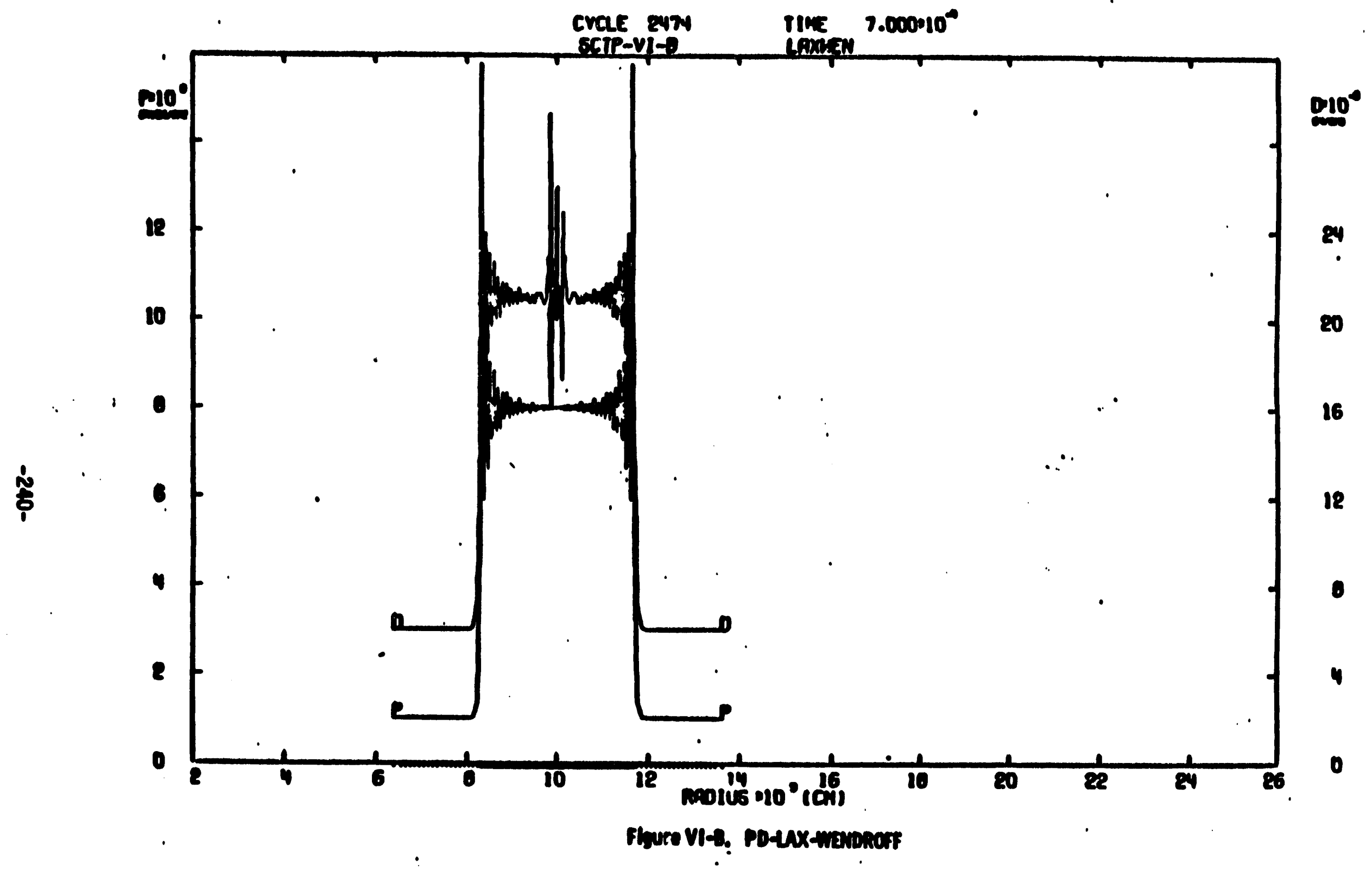

- $C^{\circ}$

$$
(\because .) \quad . . c^{\prime}
$$


) :

).

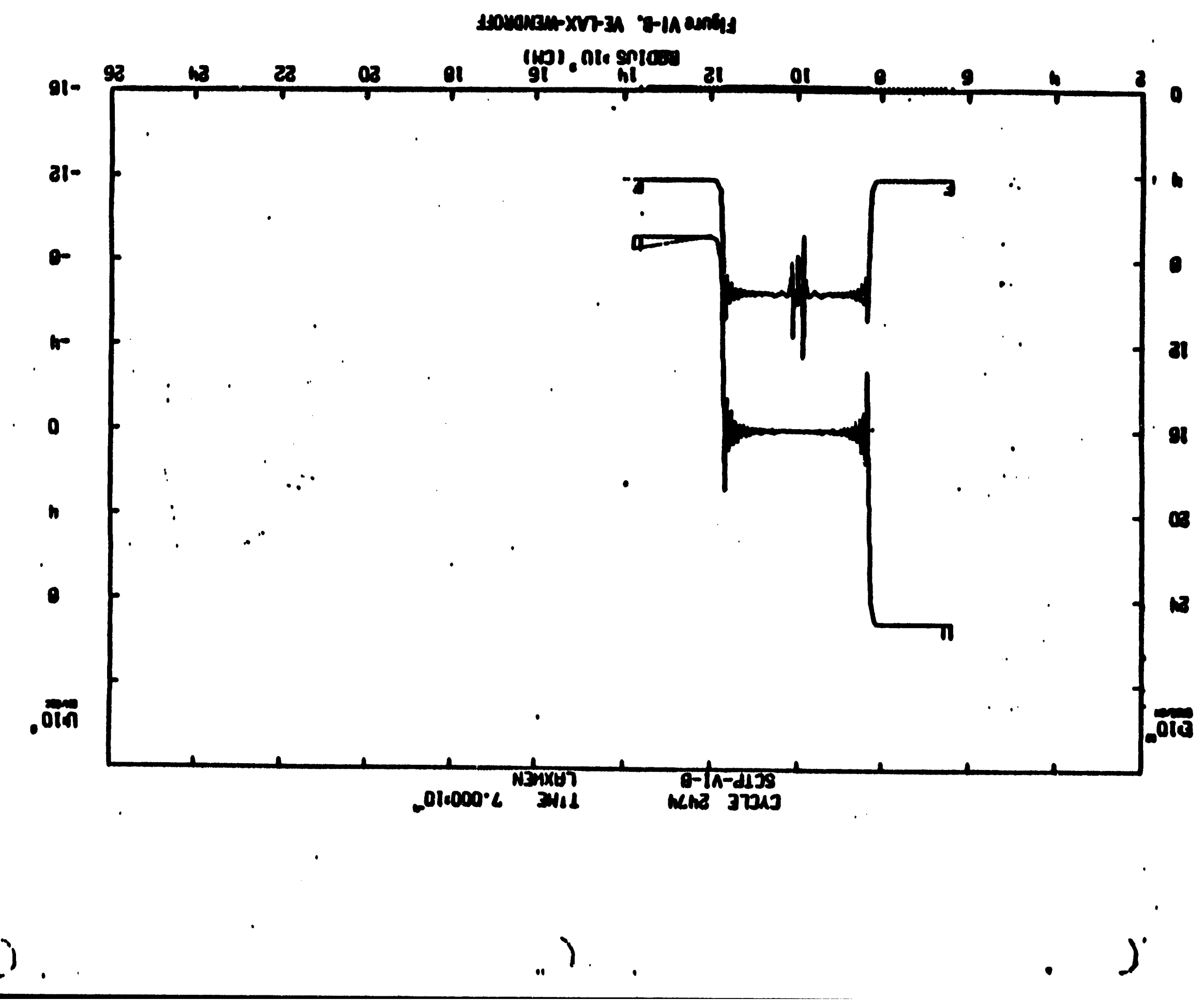


Recently we tried to run an SPH calculation on SCTP VI-B. Our SPH calculation is of the following form: :

$$
\begin{gathered}
\mathbf{U}^{T}=(V, \mathbf{u}, E) \\
\mathbf{F}^{T}=(-\mathbf{u}, \sigma, \mathbf{u} \bullet \sigma) \\
\rho \dot{\mathbf{U}}=-\operatorname{div} \mathbf{F} \\
m_{j} \dot{\mathrm{U}}_{j}=\sum_{i=1}^{N_{p}}\left(\frac{\mathbf{F}\left(x_{i}\right)+\mathbf{F}\left(x_{j}\right)}{2}\right) \bullet \mathbf{A}_{i j} \\
\mathbf{A}_{i j}=2 V o l_{i} V o l_{j} \operatorname{grad}_{x_{i}} W_{i j} \\
W_{i j}=W\left(x_{i}-x_{j}, h\right)
\end{gathered}
$$

The results were as follows. 


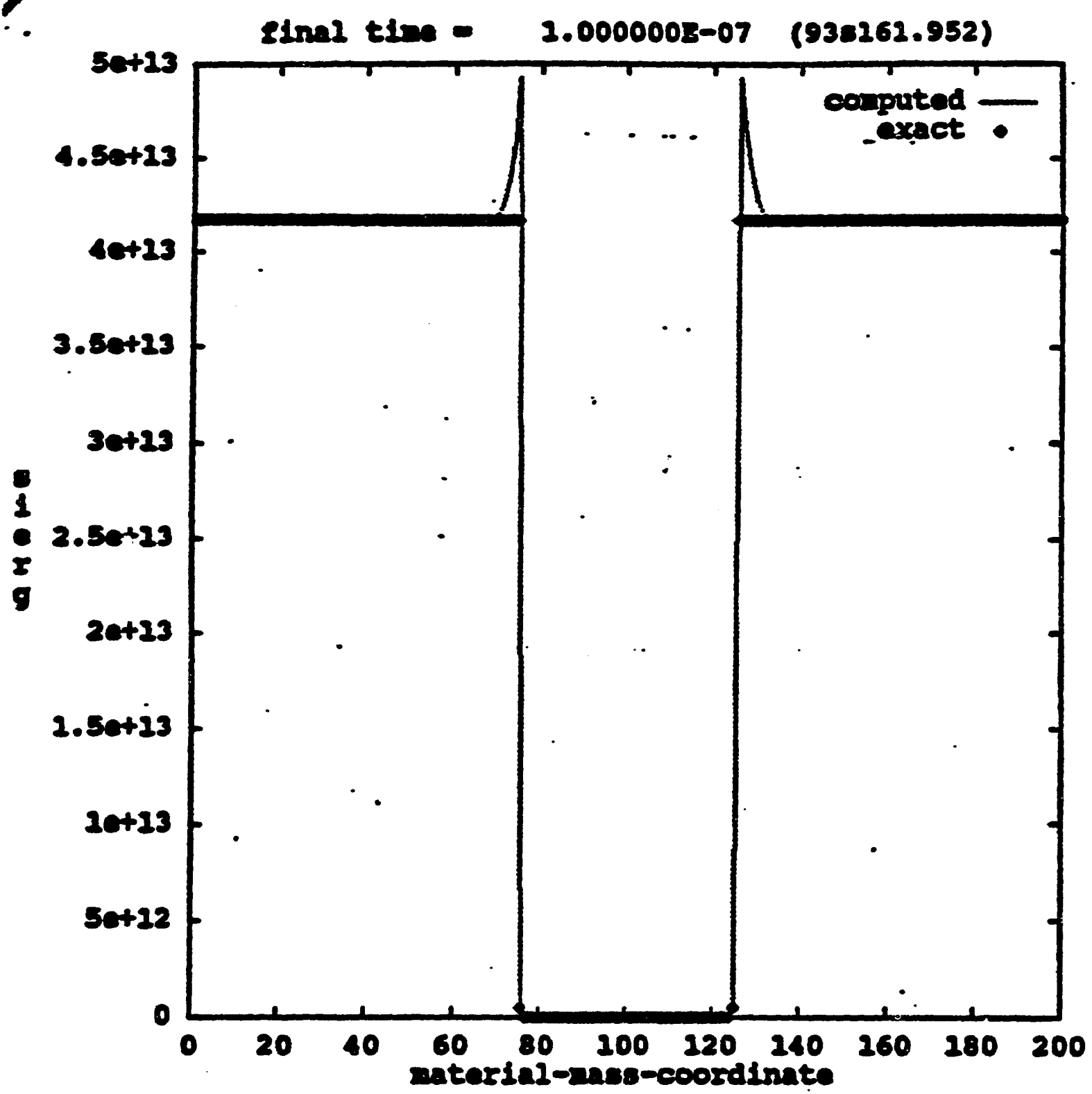

carvis $1=$ carvis $2=3.0$

nzones $=200$

ncycles $=15$

leftshock. started at 75 meters right shode " "125meters

$-243-$ 


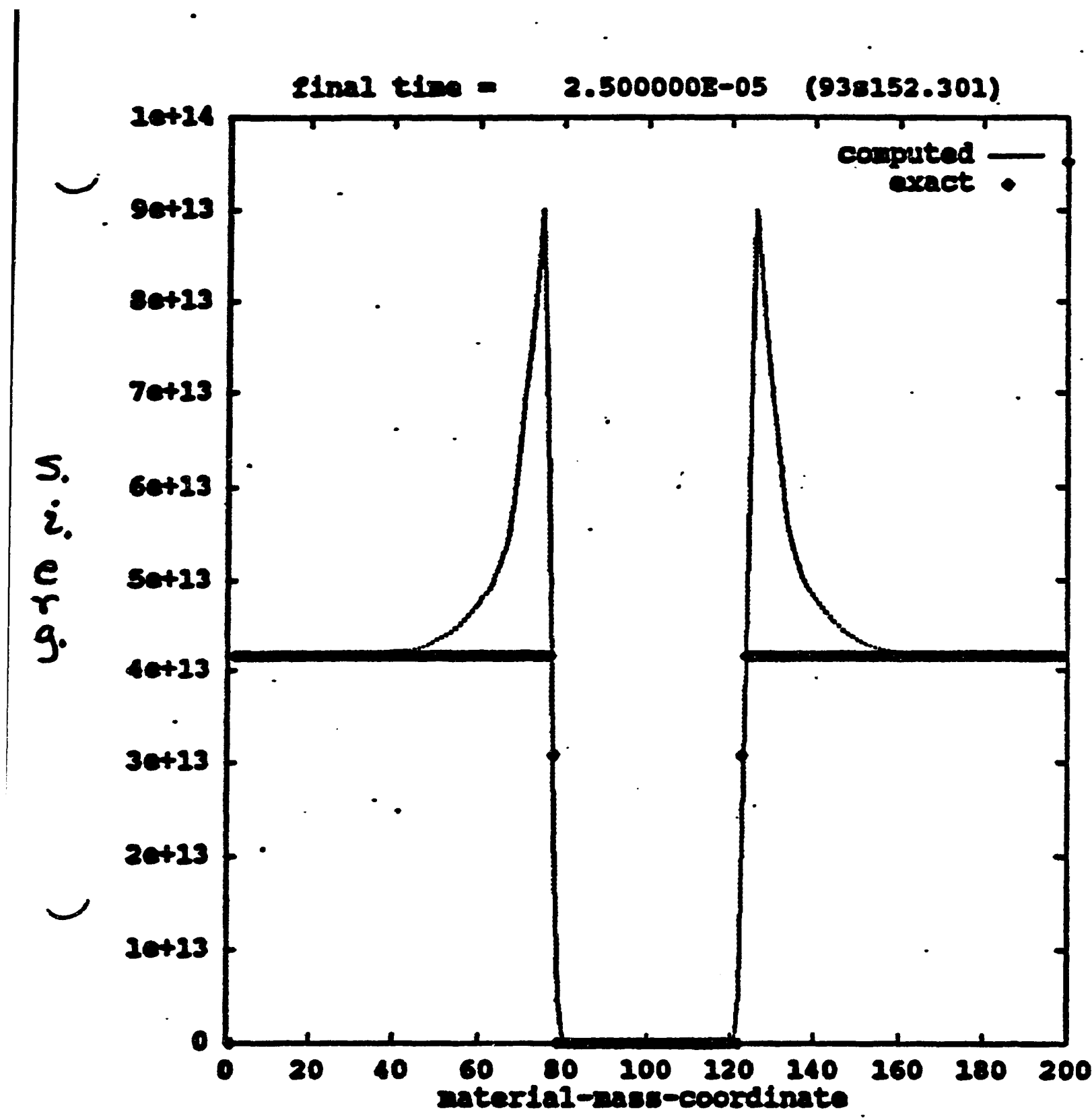

s.i.erg.

ncycles $=382$

$-244-$ 


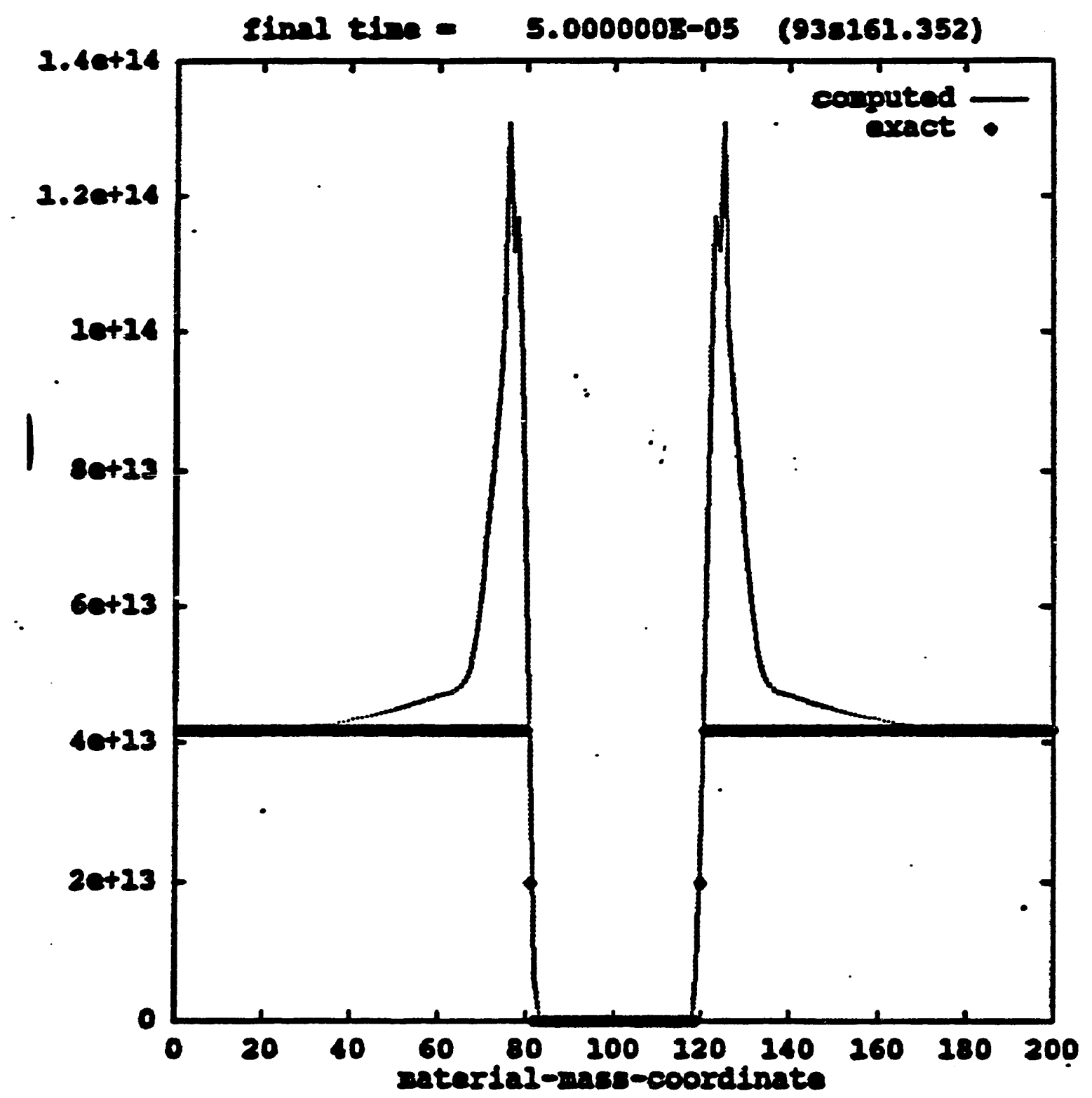

$$
\begin{aligned}
& \text { s.i. erg } \\
& \text { cles }=647
\end{aligned}
$$

ncycles $=647$

$-245-$ 


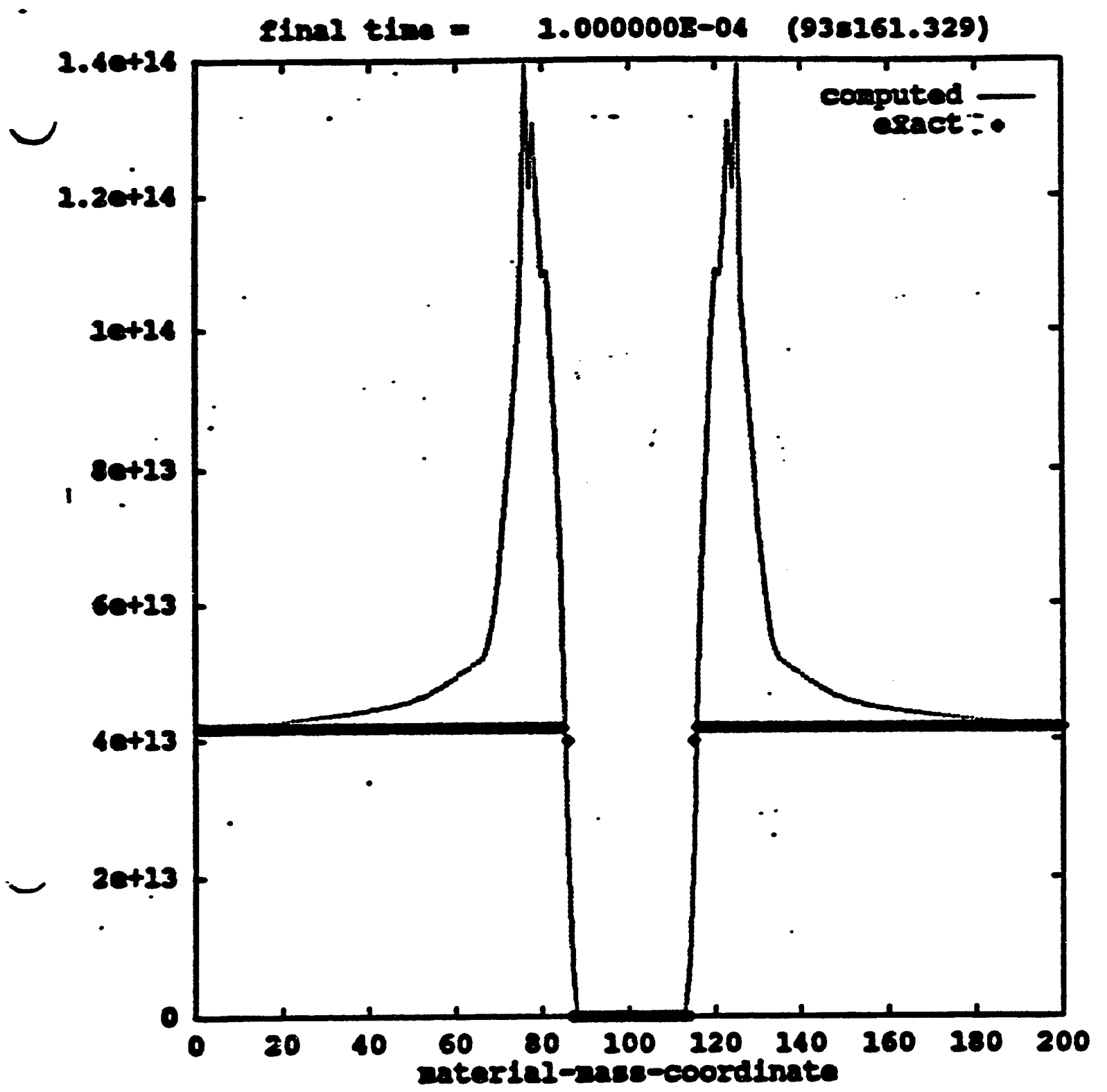

s.i.erg
ncycles $=1518$ 


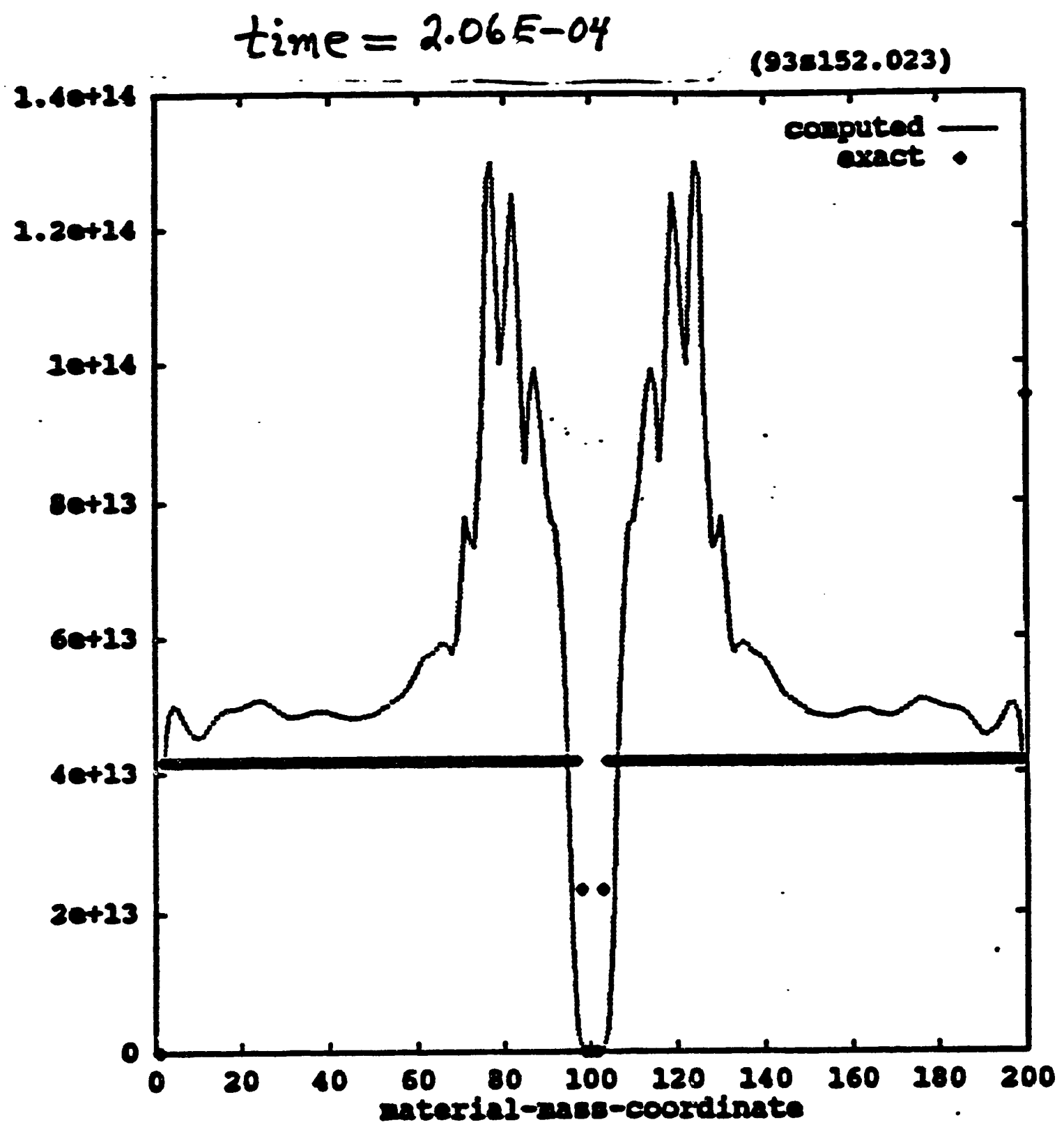

si. erg.

ncycles $=4347$

computation failed: $x(2)>x(3)$

$$
\begin{aligned}
& t_{\text {collision }} \doteq 2.28 e-4 \\
& t_{\text {last }}=7 . e-4
\end{aligned}
$$

Si i.erg. obviously under smoothed :

$-247-$ 


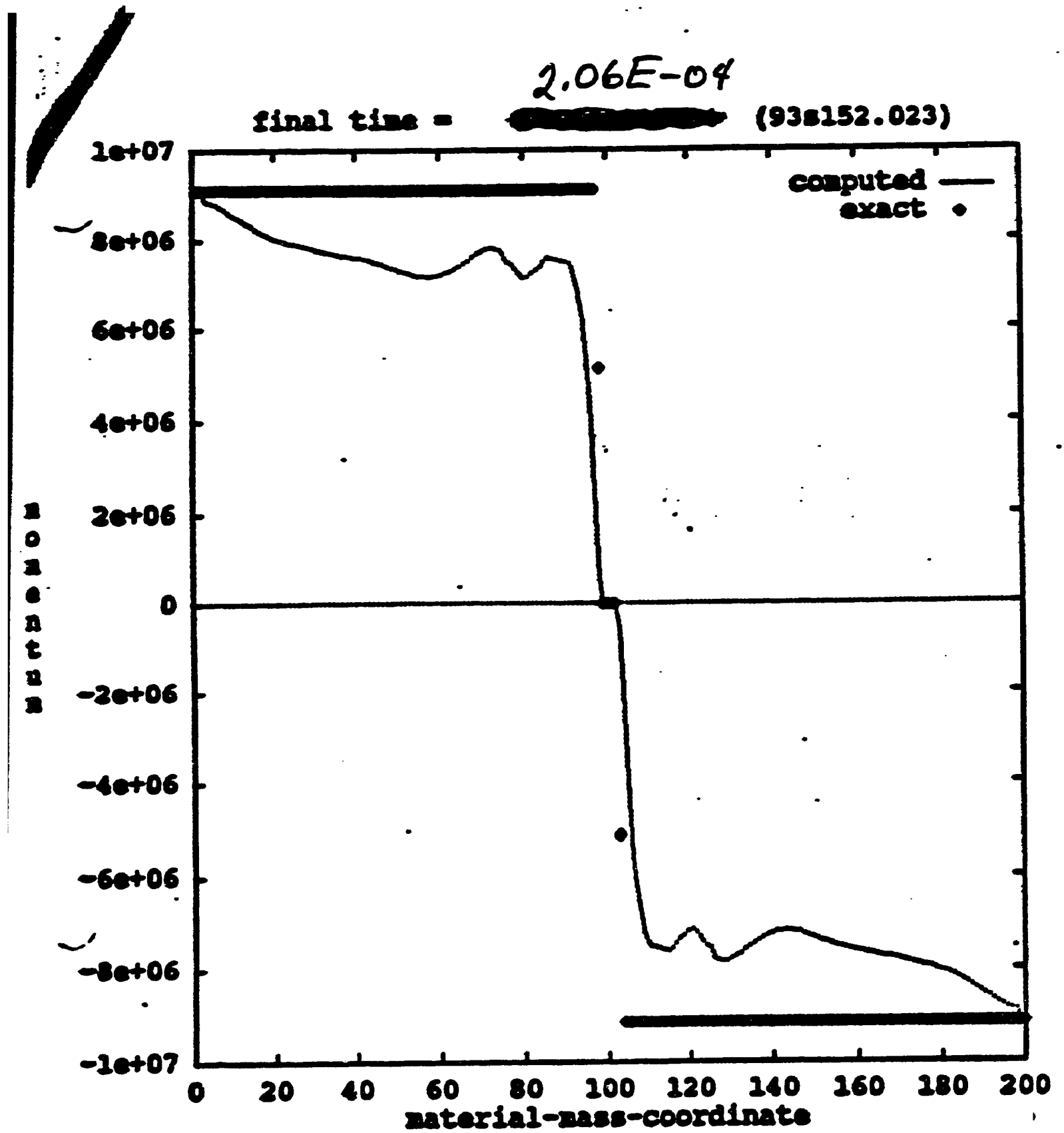

S. mom.

cycles $=4347$.
computation failed: $x(2)>x(3)$.

:

$$
\begin{aligned}
& t_{\text {cod }} \doteq 2,28 e-4 \\
& t_{\text {last }}=7 . e-4
\end{aligned}
$$

- Simoom obviously over smoothed

$-248-$ 
2.06E-04 computation failed
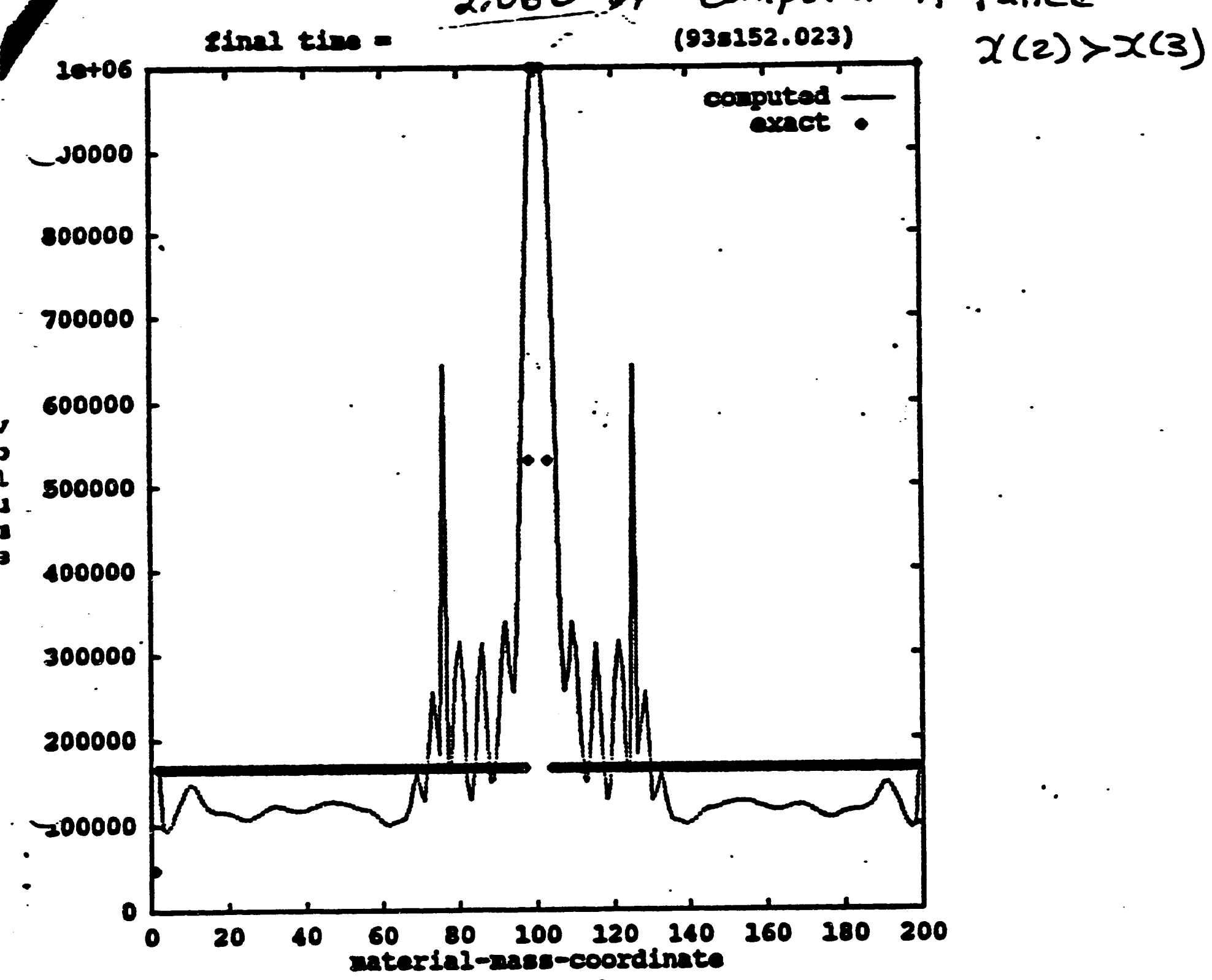

s. vol.

ncycles $=4.347$

computation failed: $x(2)>x(3)$

$$
\begin{aligned}
& t_{\text {col }} \doteq 2.28 e-4 \\
& t_{\text {last }}=7 . e-4
\end{aligned}
$$

s.vol.obiriously under smoothed

$-249-$ 


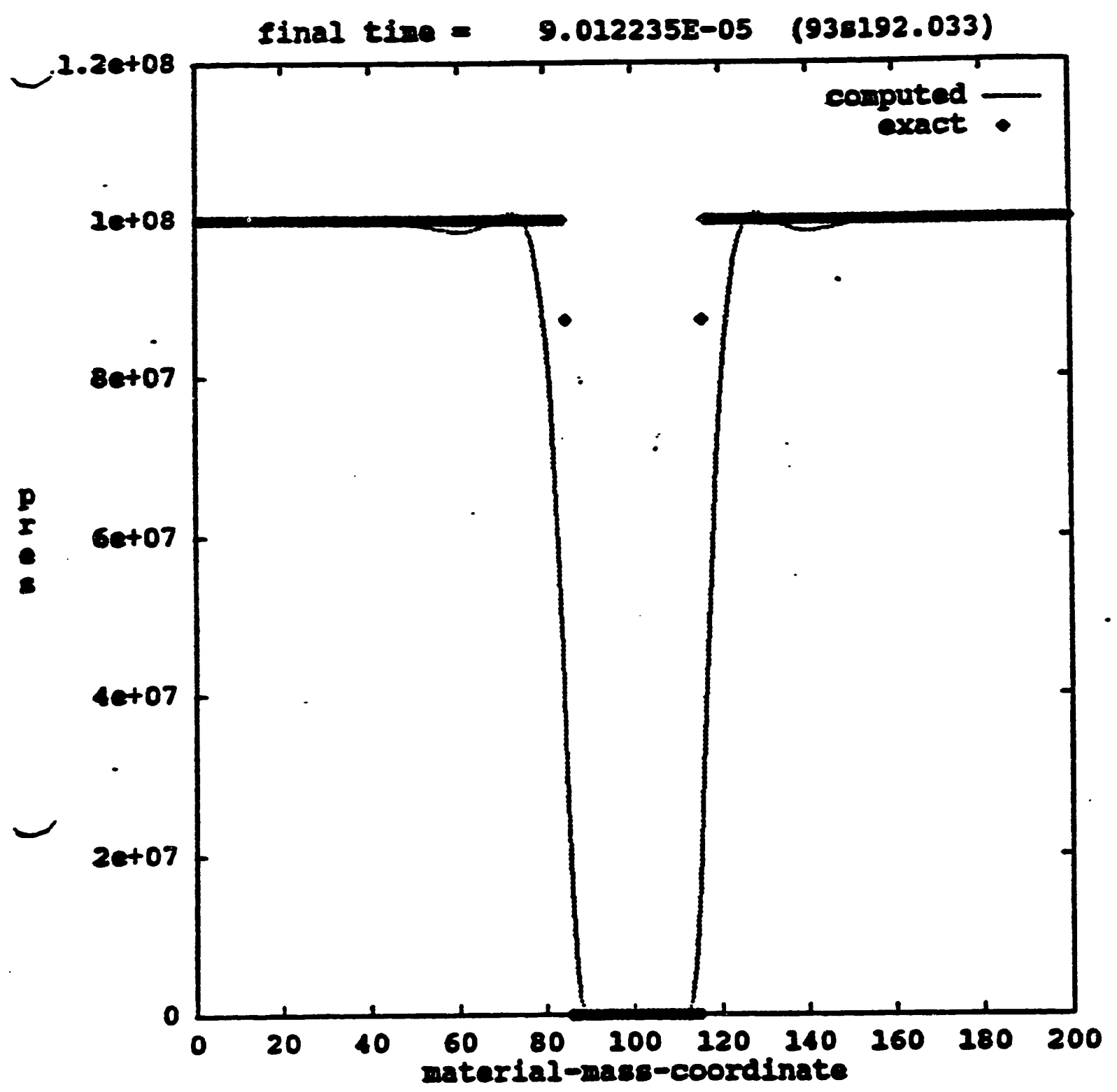

$\underbrace{\text { lozp }}_{\text {shock has travelled (o propagated) } 10 \text { zones }}$

$-250-$ 


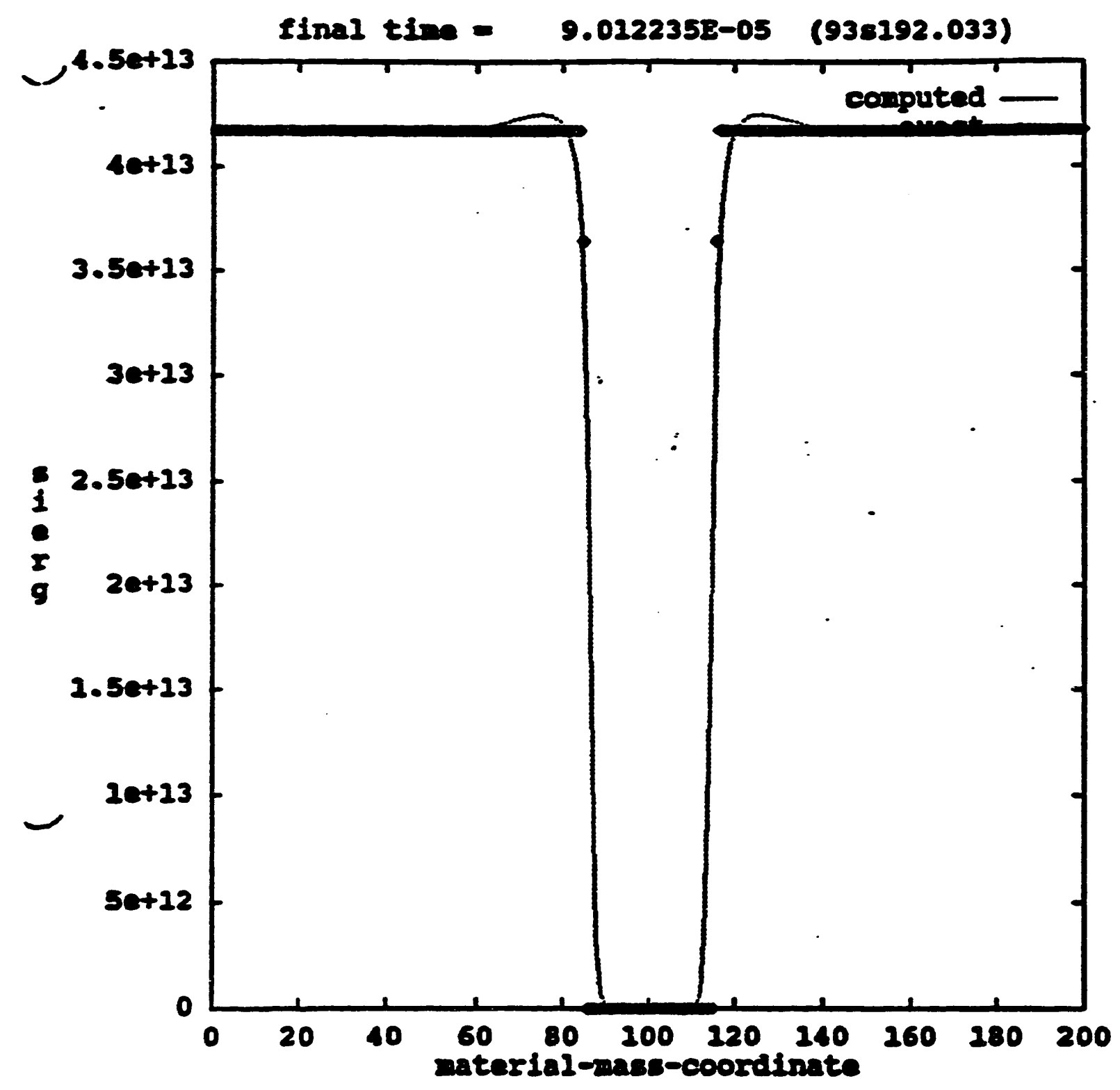




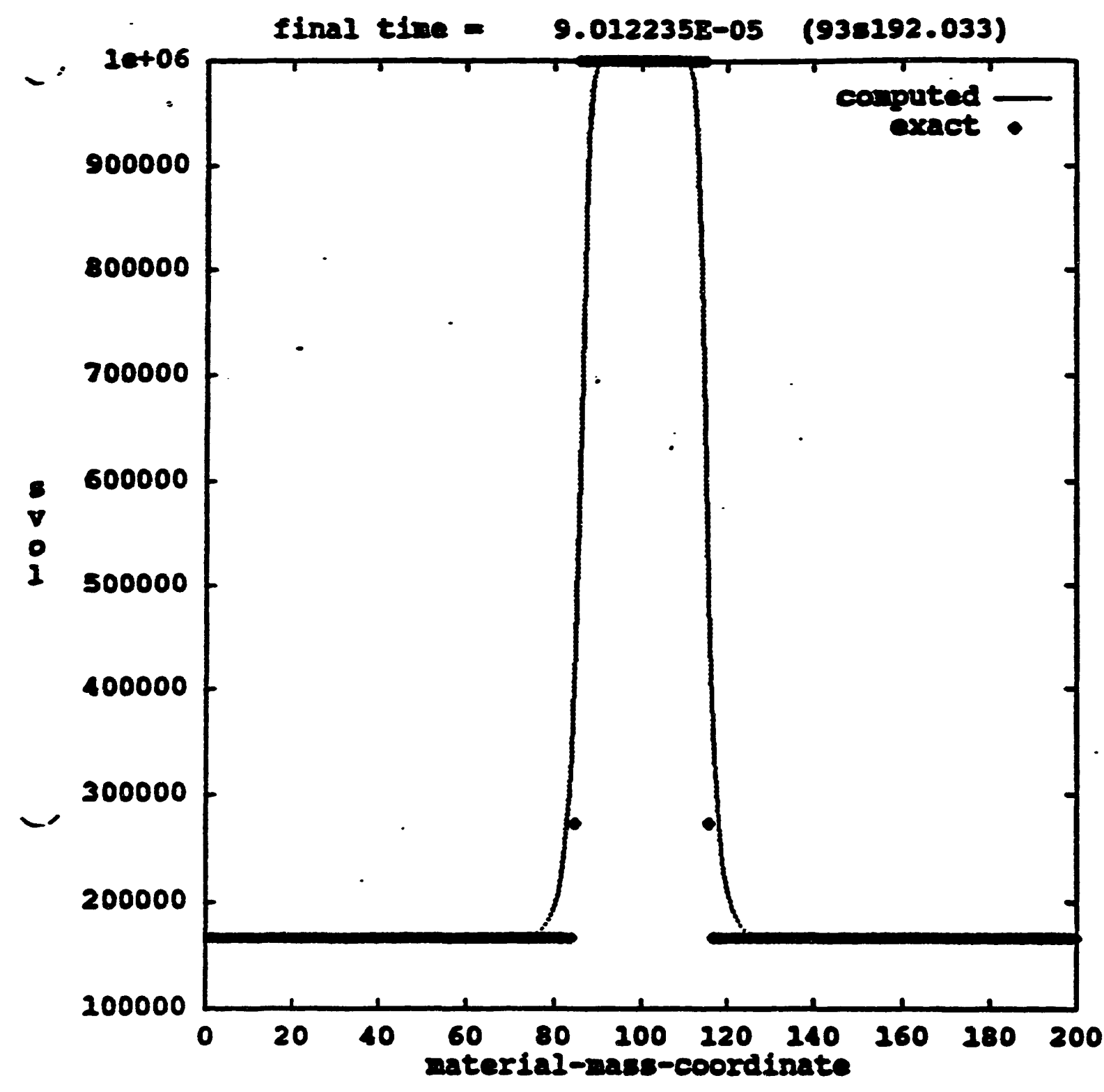




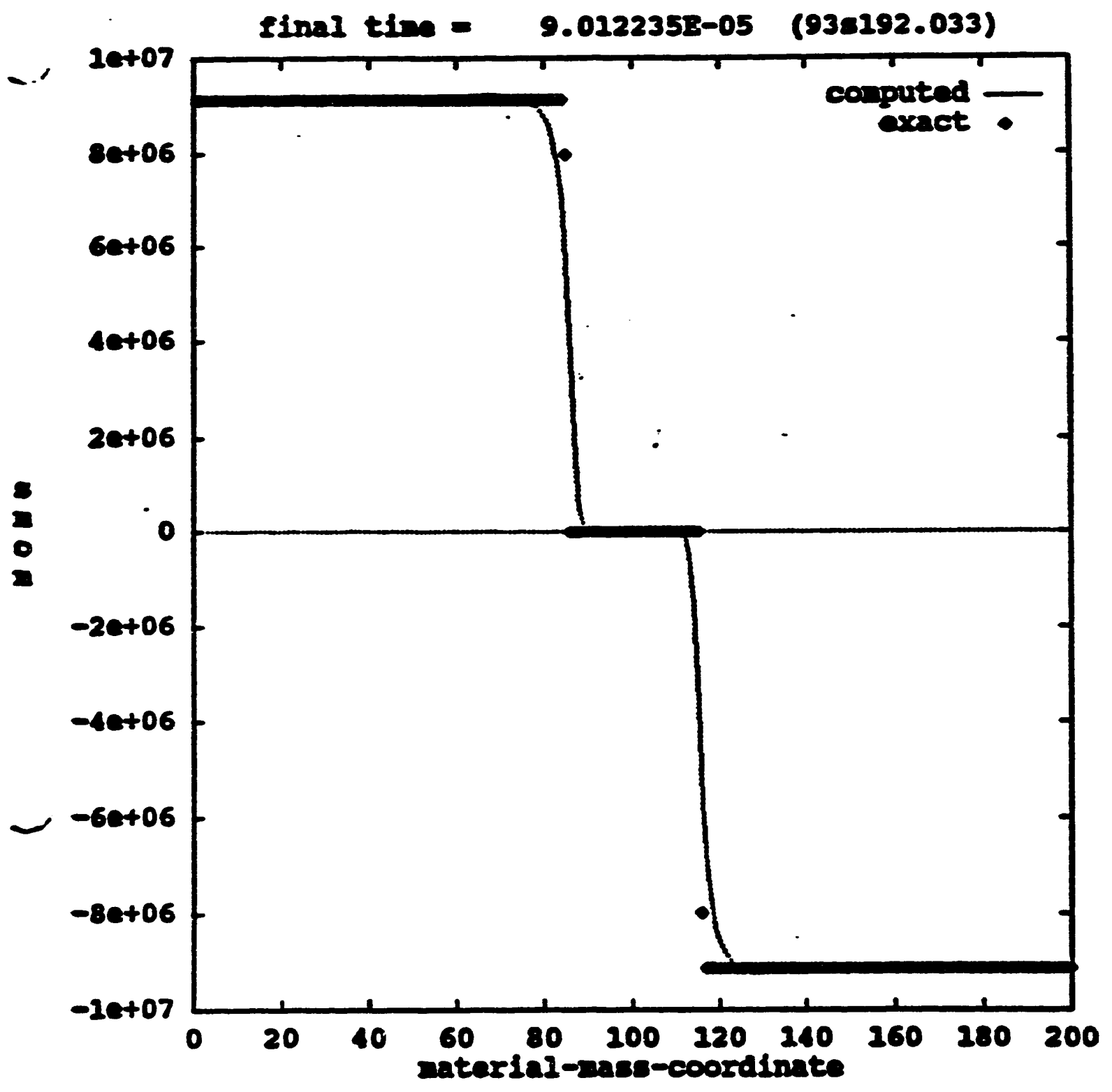




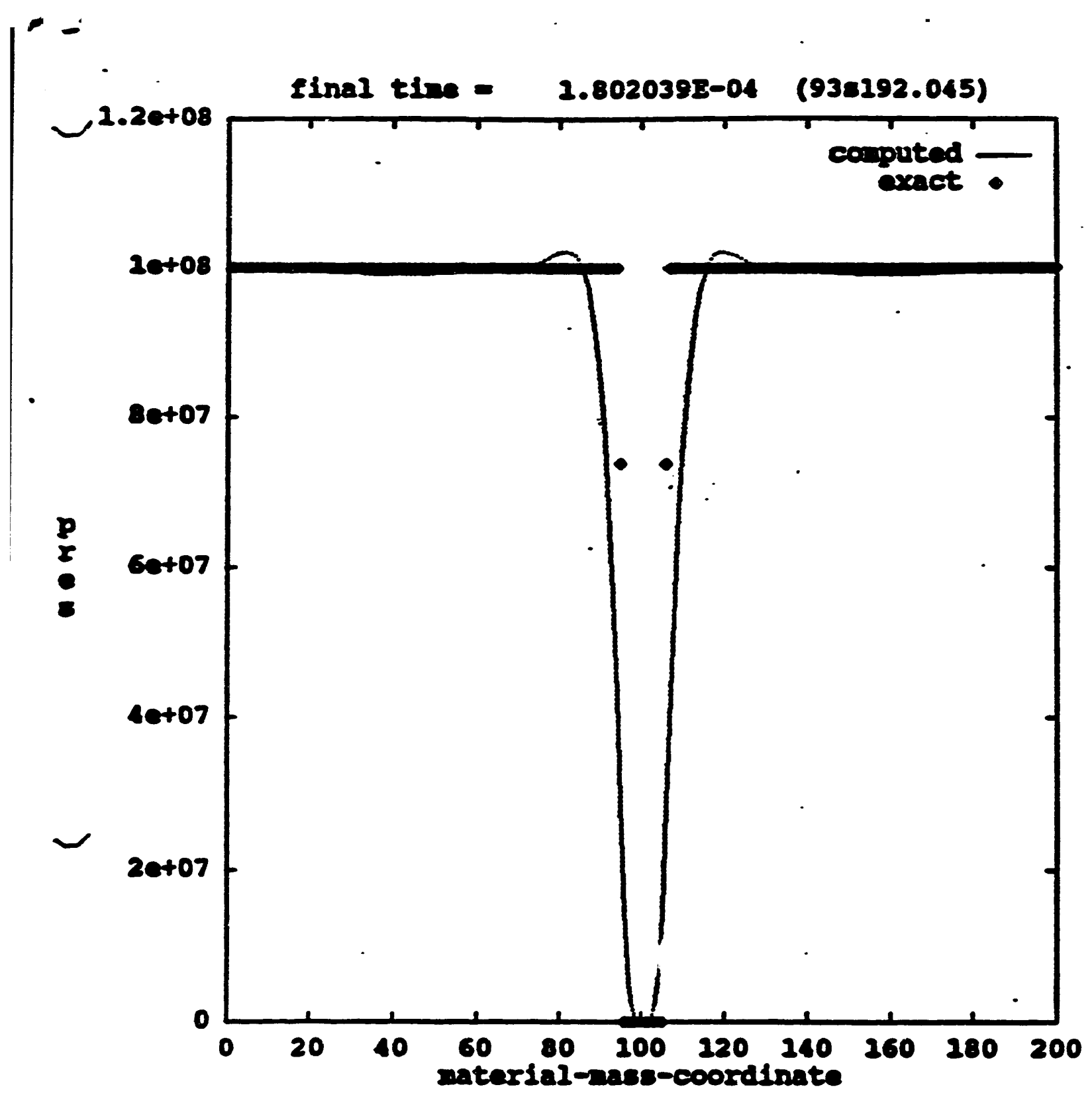

$20 z P$

$-254-$ 


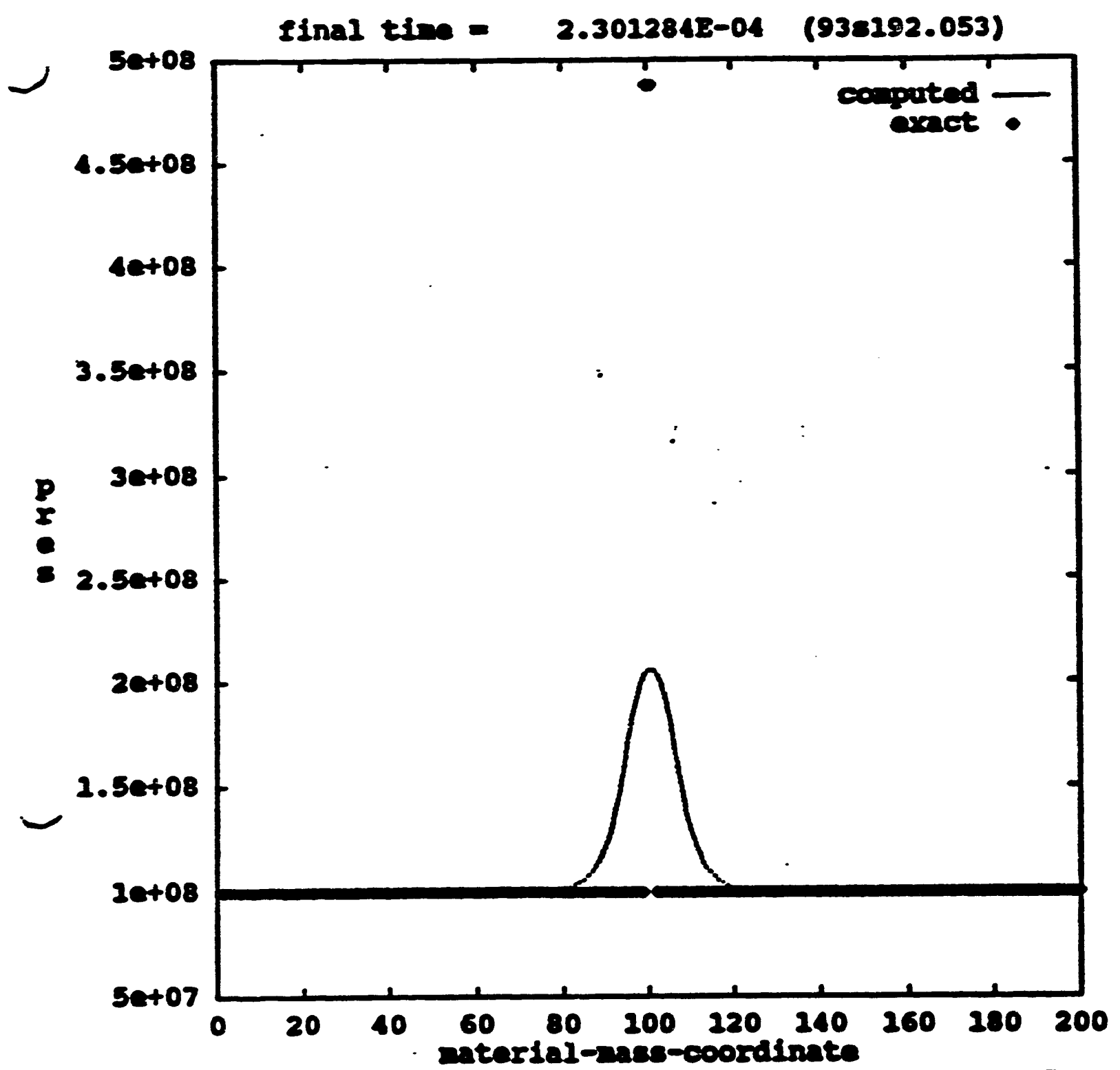

B.I.B.O. E B-spline In, B-spline OzZ

$-255-$ 


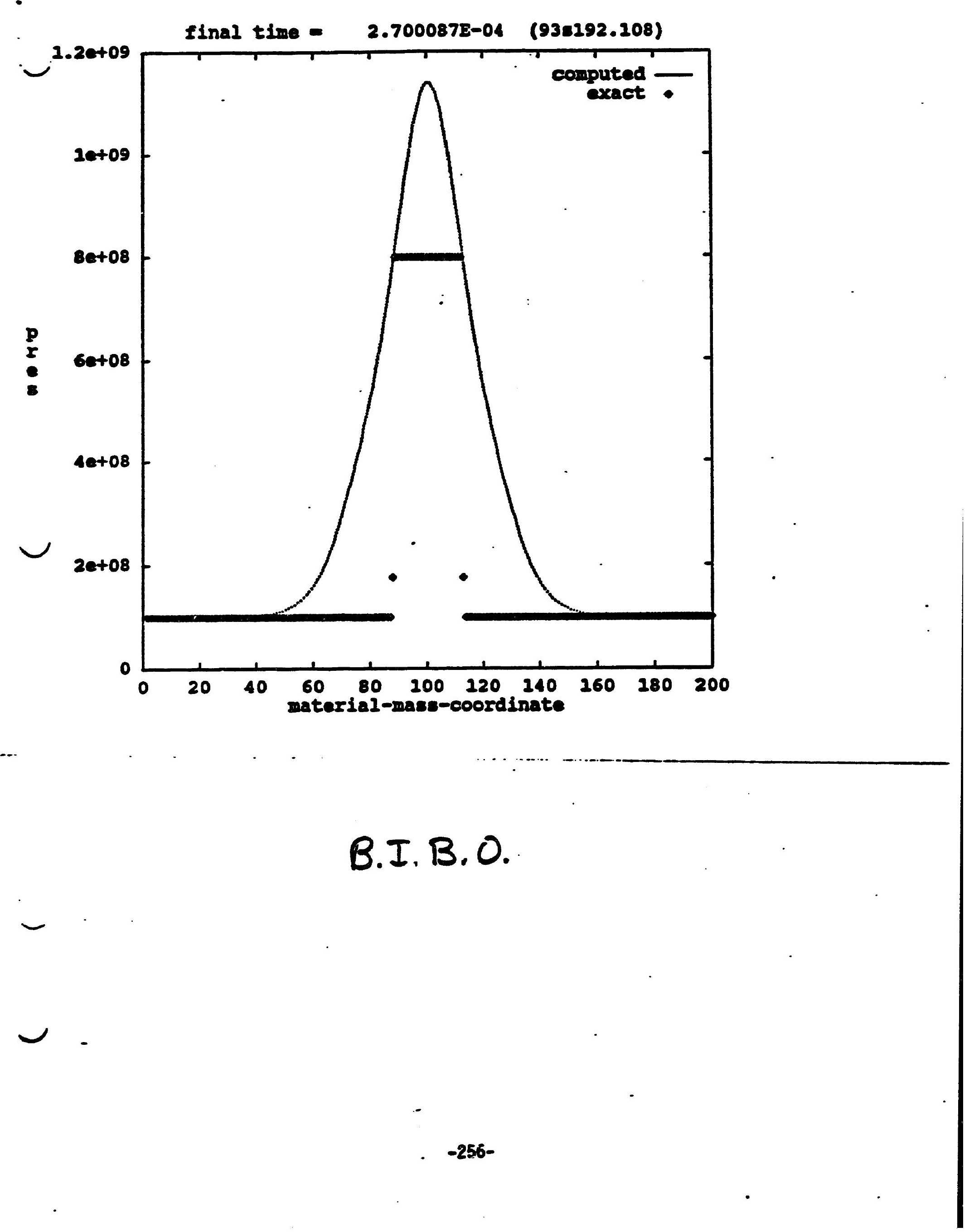


suaspoping (th

\section{$500 /$ (s}

\section{woyaprowajlwI (s}

$5 w 0.400 .4 \%$ i

:5uyfoO

\section{Yoppos'singet $H$ \\ tfon 'zuad $M$}

\section{HAS rOSN3/}




$$
(\varepsilon \delta \sim 4)
$$

iij smoff andayos! sof sag sysom -

$$
(\cdot y+\cdot y) \div=y
$$

uaym onsf wara s! silf : $\overline{y q N}$

- bubbiano joog of

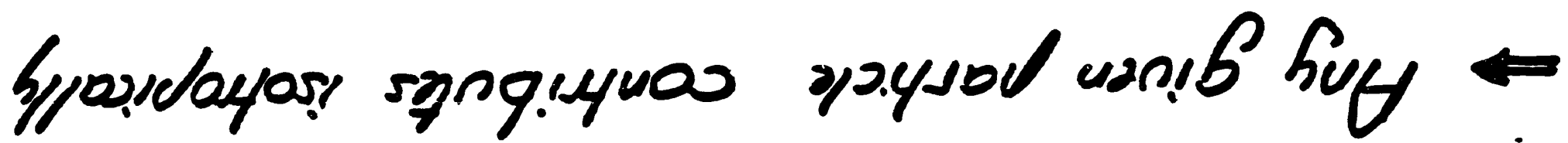

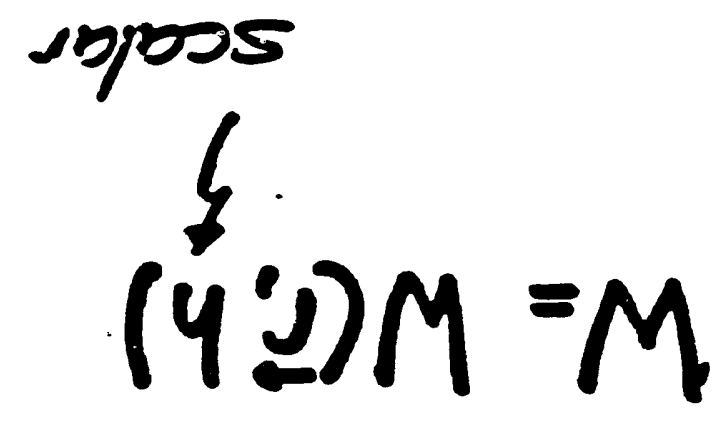

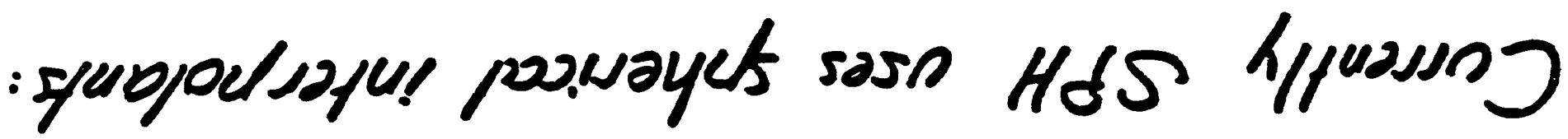

$$
\text { suo.ybn.40 }
$$


However, there is no such thing as an isotropic flow.!'

$\Rightarrow$ Flows have a preferred direction $(x, y, z, r, \ldots)$

$\rightarrow$ increase resolution along that direction.

Good for: - Impacts

- Shock waves

- Objects with aspectrahios $\neq 1$

etc...

Equivalent of a folly adaptive grid! 
Idea:

Replace the scalar h by a tensor $\underline{h}$ and write an eudution equation for each of its components:

$$
\begin{aligned}
& h \rightarrow \underline{h}=\left(\begin{array}{lll}
h_{x x} & h_{x y} & h_{x z} \\
h_{x y} & h_{y y} & h_{y z} \\
h_{x z} & h_{y z} & h_{z z}
\end{array}\right) \\
& \frac{d h}{d t} \rightarrow \frac{d \underline{h}}{d t}
\end{aligned}
$$

Note: Because $h$ has real eigenvalues, the tensor is symmetric!

Spheres $\rightarrow$ Triaxial ellipsoids 


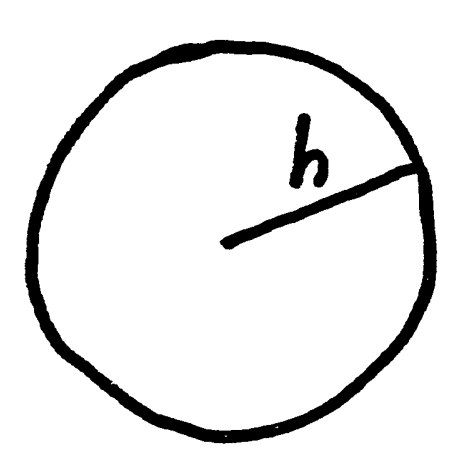

$h$ : radius of sphere

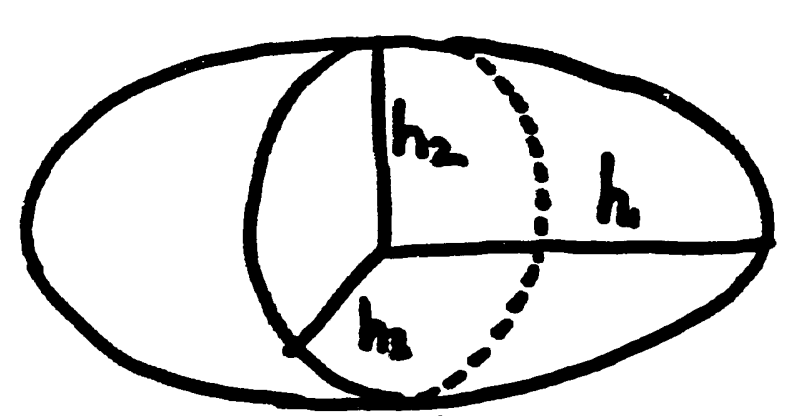

$h_{1}, h_{2}, h_{3}$ princijplater of elpipsort.

$\rightarrow h_{1}, h_{2}, h_{3}$ soak with the flow independently.'

$\rightarrow$ list of neighbors remains nearly constant.

but: added complexity, ie. SPH is no binger as easy as adverts ed!'

$-261-$ 
Implementation

1) Diagonalize $\underline{\underline{h}}$ :

$$
\left(\begin{array}{lll}
h_{z x} & h_{x y} & h_{x i} \\
h_{x y} & h_{y y} & h_{y z} \\
h_{x z} & h_{y z} & h_{z z}
\end{array}\right) \longrightarrow\left(\begin{array}{lll}
h_{1} & & \\
& h_{2} & \\
0 & h_{3}
\end{array}\right)
$$

$h_{3}, h_{2}, h_{3}$ : eigenvalues of $\underline{h}$

The eigenvectors define a transformation from lab. to a frame in which $\underline{h}$ is diagonal.

$$
\begin{aligned}
& \underline{V_{h}}=\left(\begin{array}{lll}
v_{1 x} & v_{1 y} & v_{i z} \\
v_{2 x} & v_{2 y} & v_{2 x} \\
v_{3 x} & v_{i y} & v_{3 z}
\end{array}\right) \\
& \vec{x}^{\prime}=\underline{V_{n}} \cdot \vec{x} \\
& \dot{x}_{\text {kernel frame frame }}
\end{aligned}
$$

$-262-$ 
2) Get kernel values:

$$
W \text { tot }=\frac{1}{2}\left(W_{i}+W_{j}\right)
$$

where $W_{i}$ is the contribution from particle $i$ and $w_{j} \cdot \cdot \cdot \cdot{ }^{\prime}$

- Define $\left(\vec{r}_{j j}\right)_{i}^{\prime}=V_{b}^{i} \vec{r}_{i j}$

herne lis space $1 / a b$. space

$$
\Rightarrow v_{i}=\left|\left(\vec{r}_{i j}\right)_{i}^{\prime}\right|=\left(\frac{x_{i j}^{\prime}}{h_{i}^{i^{2}}}+\frac{y_{i i}^{\prime}}{h_{i}^{i 2}}+\frac{\varepsilon_{i i}^{\prime}}{h_{3}^{\prime 2}}\right)^{\prime / 2}
$$

get $W=W\left(v_{i}, h_{i}^{i}, h_{i}^{i}, h_{2}^{i}\right)$ using B-spline kerne!

Vote: Loci i of iso -Ware really, triaxial ellipsorict.

The procedure s repeated for $W_{j}$.

$-263-$ 
3) Get kernel gradients

$$
\vec{\nabla} W_{\text {tot }}=\frac{1}{2}\left(\vec{\nabla} W_{i}+\vec{\nabla} W_{j}\right)_{\left(\nabla \cdot W_{i j}-\nabla_{i} w_{i j}\right)}
$$

- use same procedure to get $v_{i}$ and $V_{j}$

- use $\vec{\nabla} w=\frac{d w}{d v} \cdot \overrightarrow{\nabla v}$

with $\vec{\nabla} v=\frac{1}{v}\left(\begin{array}{l}x_{i j}^{\prime} / h_{1}^{2} \\ y_{i}^{\prime} / h_{2}^{2} \\ z_{i j}^{\prime} / h_{i}^{2}\end{array}\right) \quad$ (in kernel frame)

Note: unless $h_{1}=h_{2}=h_{3}$, the gradient of $W$ is not aligned with $\vec{r}_{i j}$

- rotate kernel components into lab frame

$$
(\vec{\nabla} W)_{L}=\underline{V}_{i}^{-1}(\vec{\nabla} W)_{i}
$$

The procedure is repeated for $\nabla W_{j}$ 
4) Time evolution of $\underline{h}$

Just as in the spherical case with $h \times N_{n}^{-1 / 3}$ setting $h$ by looking at the neighbor distribution is too rosy. $\Rightarrow$ differential form.

Transformation:

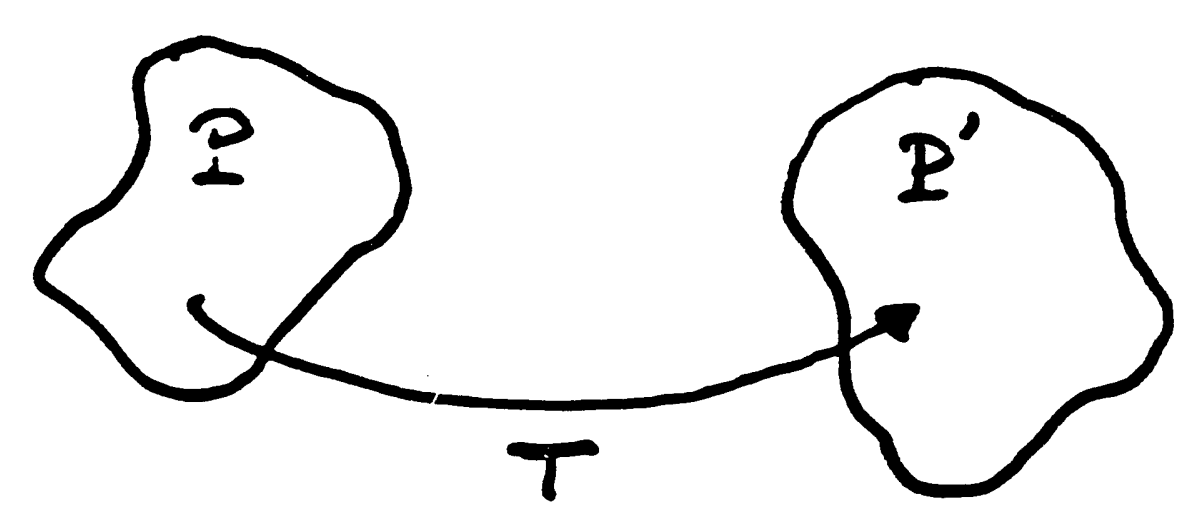

$$
T=D+R
$$

pure deformation $\downarrow_{\text {pure rotation }}$

We assume "small" deformations and rotations (during 1 timestep.)

$-265-$ 
Deformation:

$$
D: \dot{\varepsilon}^{\alpha \beta}=\frac{1}{2}\left(\frac{\partial v^{\alpha}}{\partial x^{\beta}}+\frac{\partial u^{\beta}}{\partial x^{\alpha}}\right)
$$

strain rate tensor

Rotation:

$$
R: \dot{R}^{\alpha \beta}=\frac{1}{2}\left(\frac{\partial v^{\alpha}}{\partial x^{\beta}}-\frac{\partial v^{\beta}}{\partial x^{\alpha}}\right)
$$

rotation rate tensor

Naive idea for deformation:

$$
\dot{h}^{\alpha \beta}=\dot{\varepsilon} \alpha \beta \cdot h^{\alpha \beta} \text { (no sum.') }
$$

Trouble: if $h^{\alpha \beta}=0$ : initially, weill remain 0 at all times.'

$-266-$ 
Solution:

ग) Diagonalize $\dot{\varepsilon}^{\alpha \beta}$

$\dot{\varepsilon}_{1}, \dot{\varepsilon}_{2}, \dot{\varepsilon}_{3}$ : eigenvalues of $\dot{\varepsilon}^{\alpha \beta}$ (real)

$\underline{V}_{\varepsilon}$ : transformation matrix defined by

the eigenvectors associated with

$$
\dot{\varepsilon}_{1}, \dot{\varepsilon}_{2}, \dot{\varepsilon}_{3}
$$

2) Transform $h$ into frame of ref. in which $\dot{\varepsilon}^{\alpha} \beta$ is diagonal

$$
\underline{n}^{\prime}=\underline{V}_{\varepsilon}^{-1} \underline{h} \underline{V_{c}}
$$

3) In this frame, obtain $\underline{h}^{\circ}$

$$
\begin{aligned}
& \dot{h}_{x x}^{\prime}=\dot{\varepsilon}_{1} \cdot h_{x x}^{\prime} \\
& \ddot{h}_{y y}^{\prime}=\dot{\varepsilon}_{2} h_{y y}^{\prime} \\
& h_{z z}^{\prime}=\dot{\varepsilon}_{3} h_{t z}^{\prime}
\end{aligned}
$$

not: inches frame $\underline{h}^{\prime 201}$ 's diagonal! 
4) Rotate $\underline{h}^{\prime}$ into lab. Frame:

$$
\underline{\dot{h}}=\underline{V}_{\varepsilon} \underline{h}^{\prime} V_{\varepsilon}^{-1}
$$

note: $\underline{h}$ is symmetric $=\underline{h}$ will remain symmetric.'

+ Aced rotation terms

$$
\underline{h}^{\alpha \beta}=\ldots .+h^{\alpha \gamma} \cdot R^{\beta r}+h^{\beta r} R^{\alpha \gamma}
$$

"Jauman stress rates equivalent" 
Application: compression of a cylinder

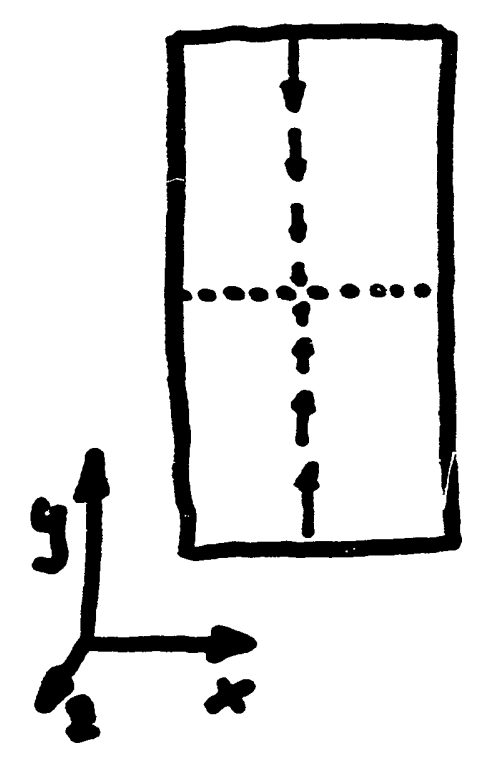

$U_{y} \times y$ : homologow compression.

Analytical sod: constant density

$$
\rho_{t}=\frac{M}{V}=\frac{M}{\pi R^{2}\left(L-v_{j} \cdot t\right)}
$$

Not: At $t=180 \mathrm{~ms}$, we have $\left.\begin{array}{ll}\text { Standard SPH } & h \equiv 5000 \\ \text { Tensor } S P H & h_{y y}=500\end{array}\right\} \Rightarrow 10 \times \begin{aligned} & \text { resolution } \\ & \text { increase ! }\end{aligned}$ but since $h \times N^{-1 / 3}$ standard SPH would need $10^{3}=1000$ times more particle to achieve similar resolution! 


\section{Standard SPM}
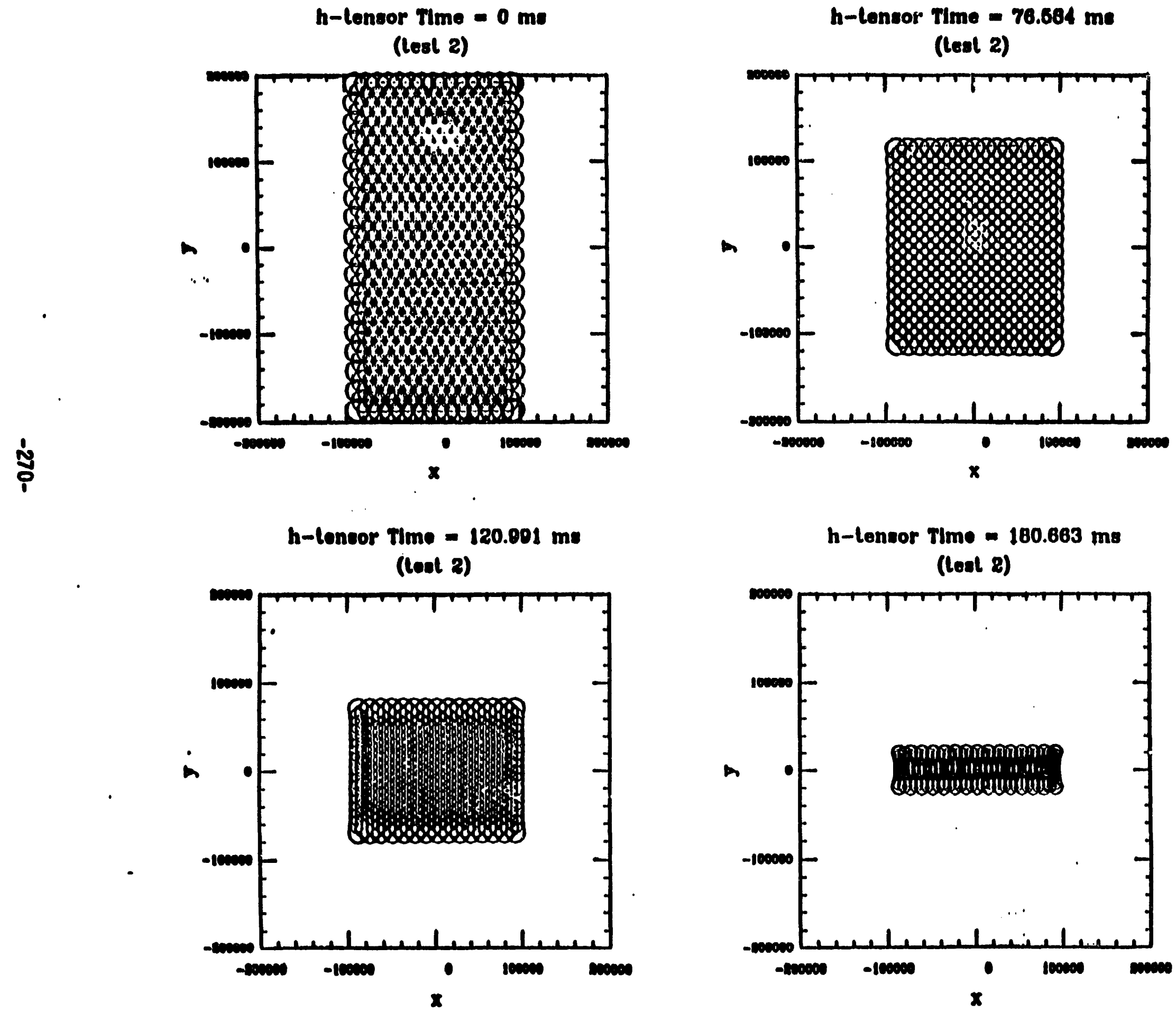


\section{Tensor SPI}
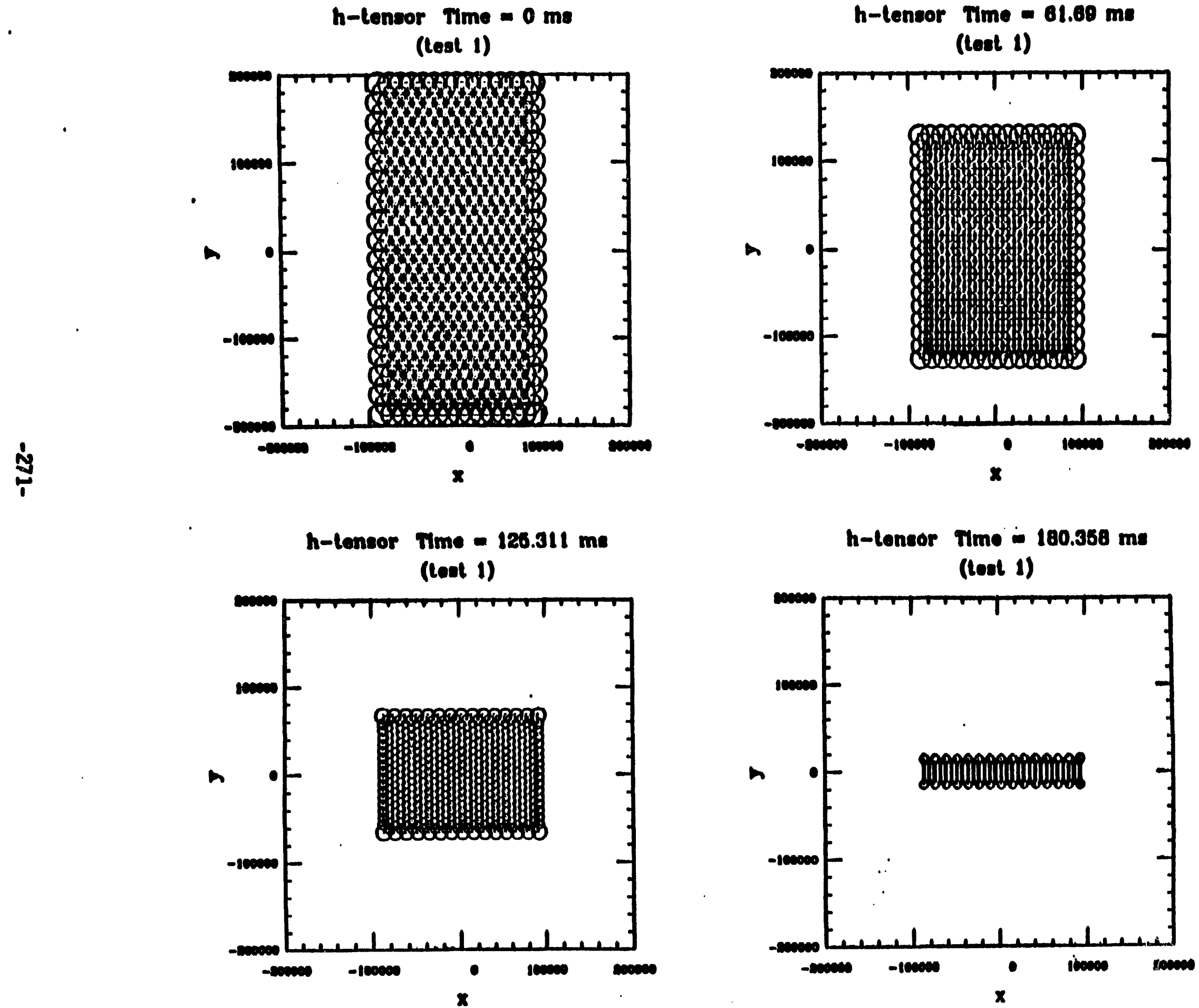


\section{Tensor SPH}
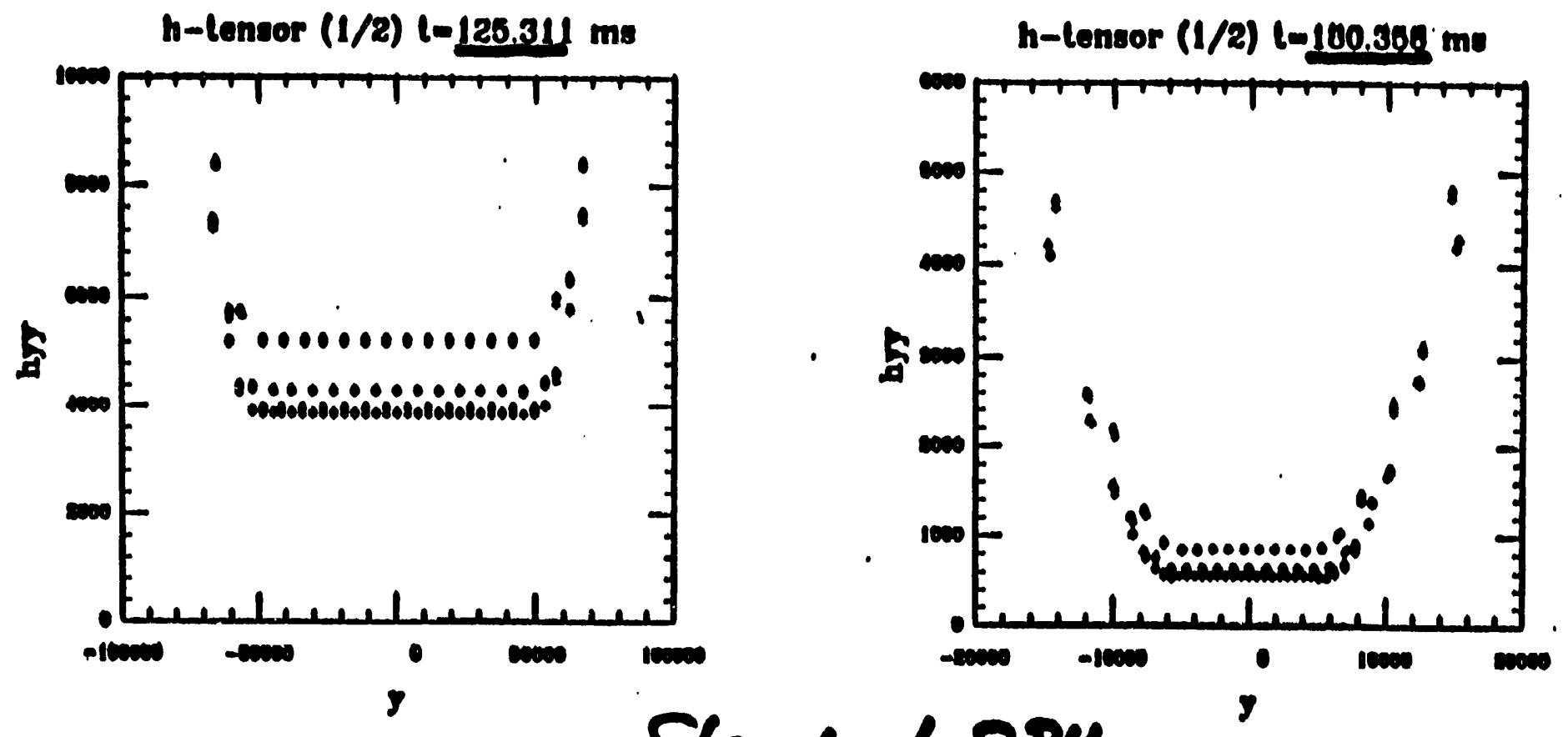

\section{Stanctard SPH}
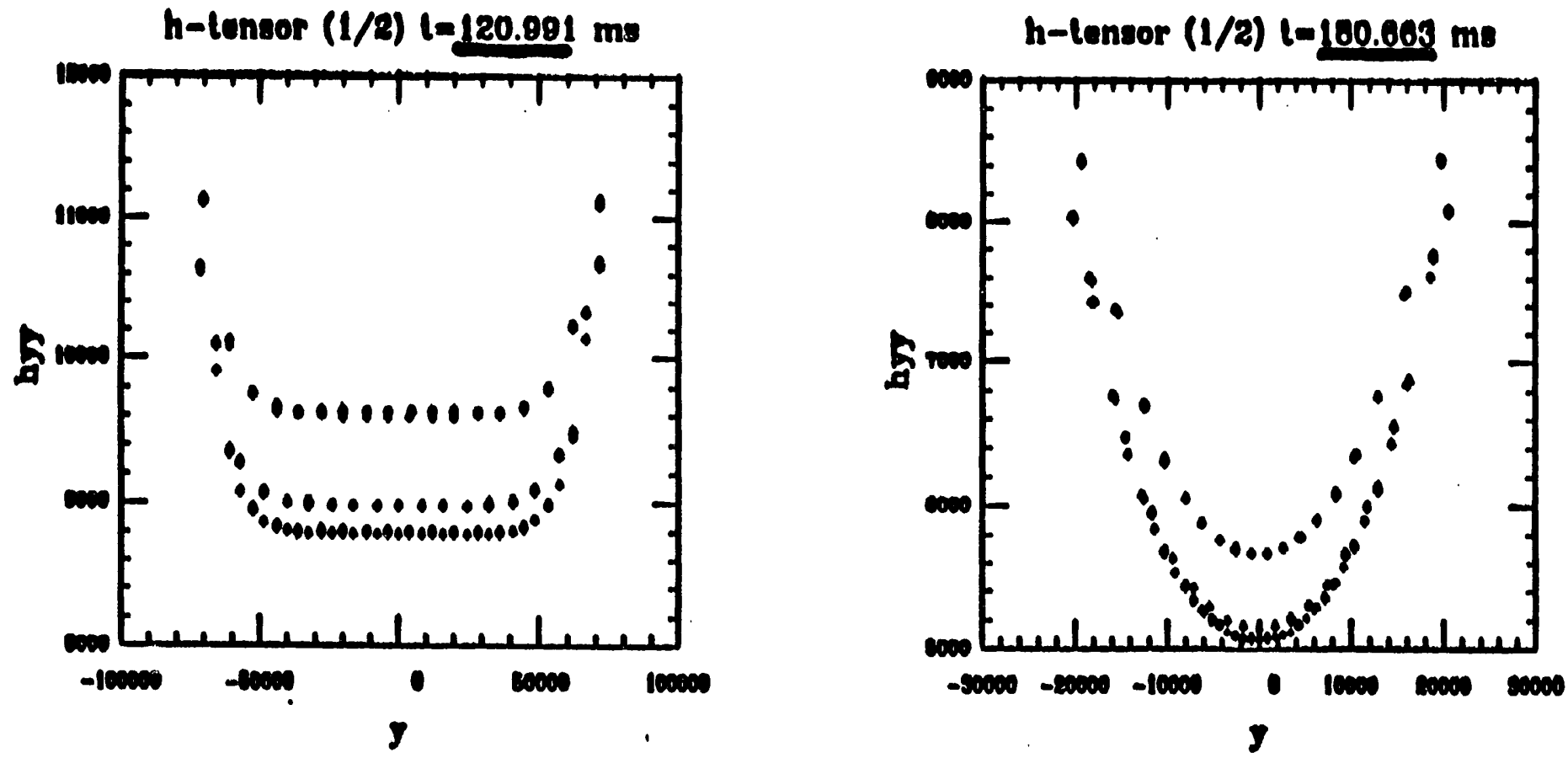


\section{Tenser SPH.}
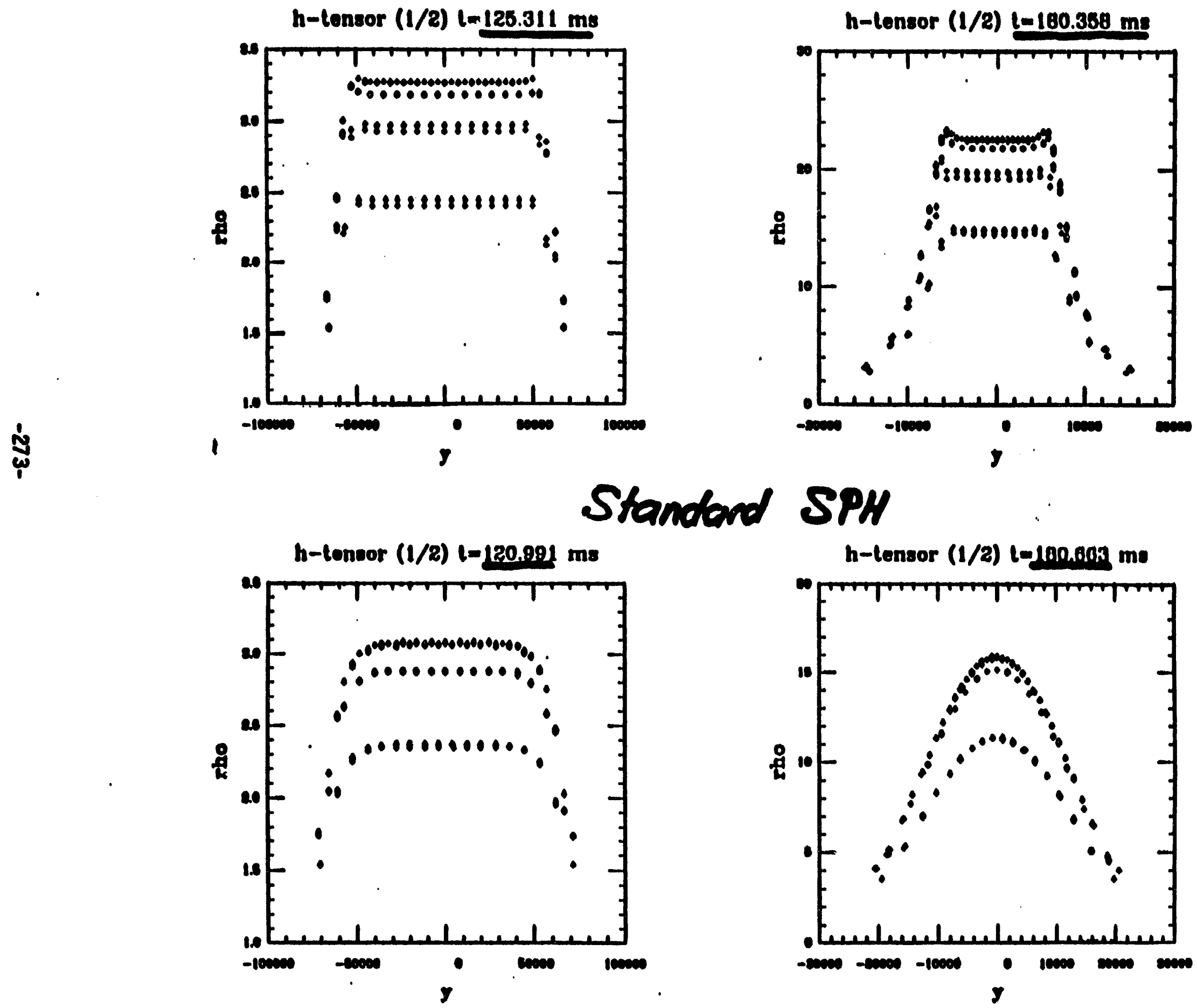

\section{Standard SPH}

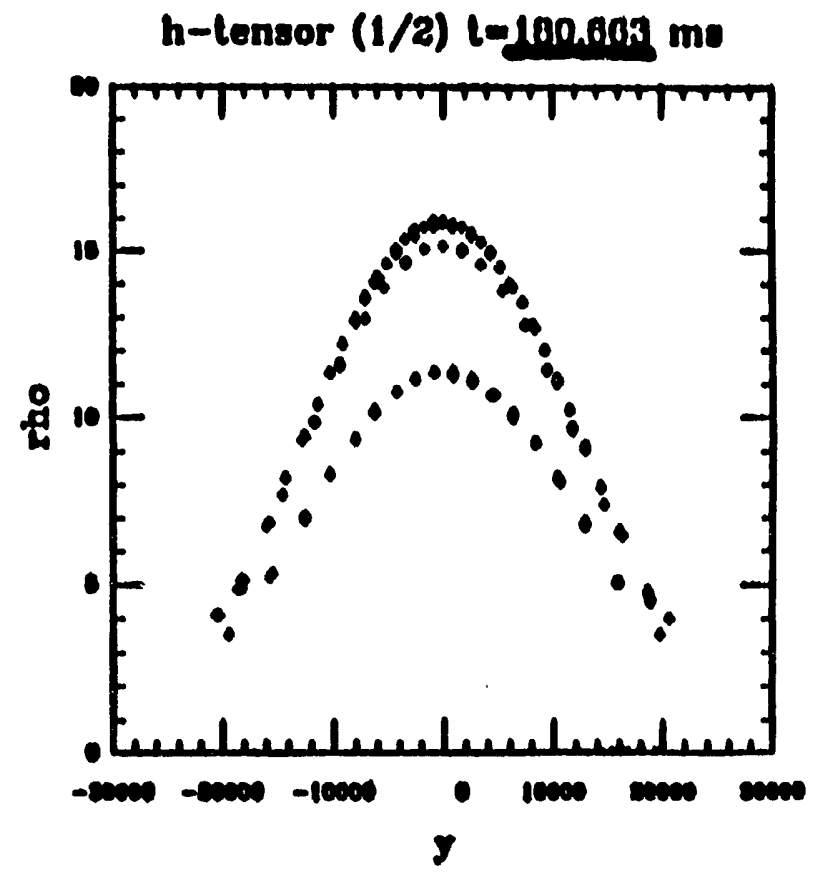


Rotating Disk

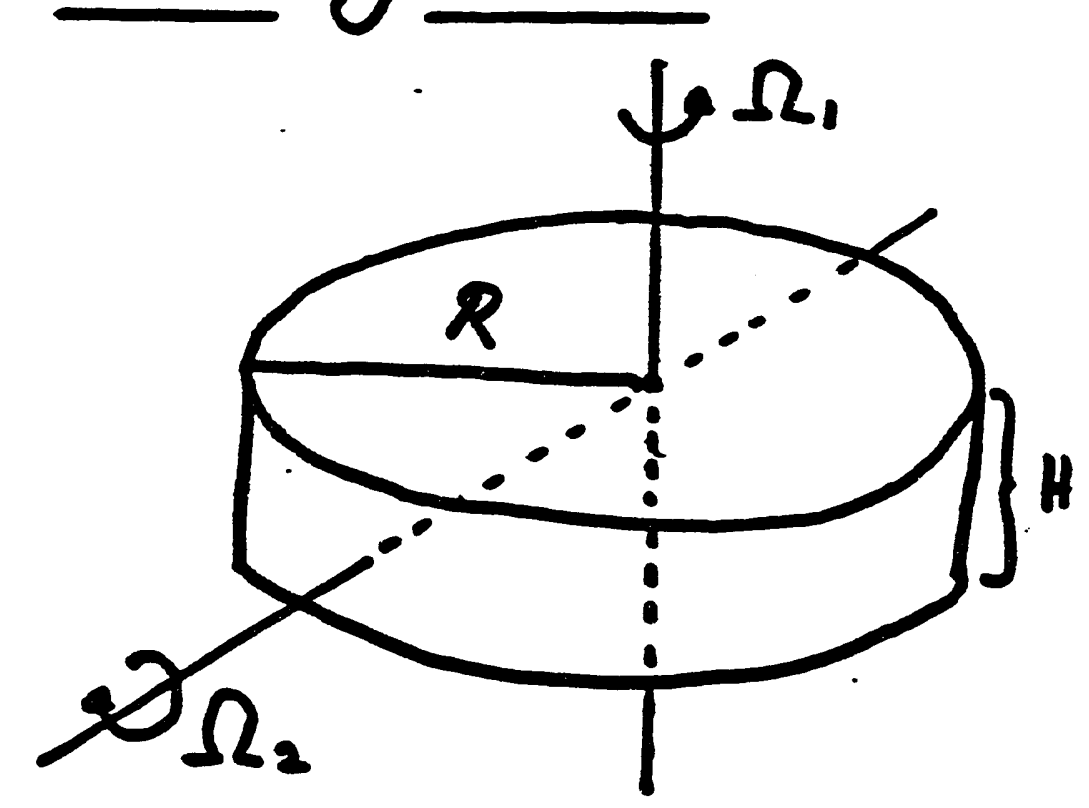

Set : $\frac{H}{R}=0.2$.

$\Omega=2 \pi \cdot 10^{4} \rightarrow P=10^{-4}=$

Tilbtom eos for water.

Result:

1) Rotation around $\Omega_{1}$ conserves momentum and angular momentum exactly

2) Rotation around $\Omega_{2}$ does not conserve angular momentum!

Why? 1) $\vec{\nabla} W$ is not along $\overrightarrow{r_{i}}$

2) Rotation terms for ha are poor!

$-274-$ 

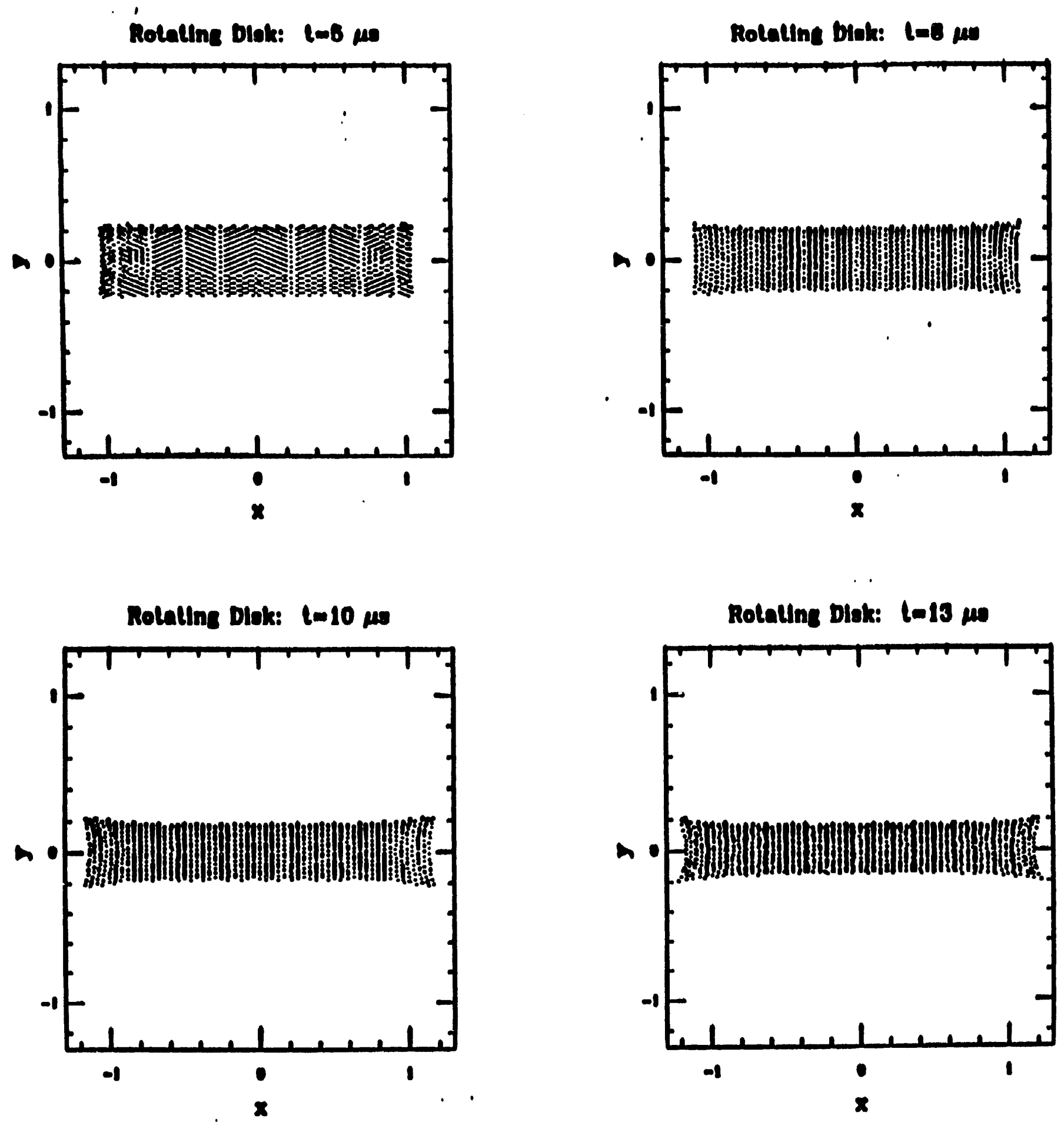


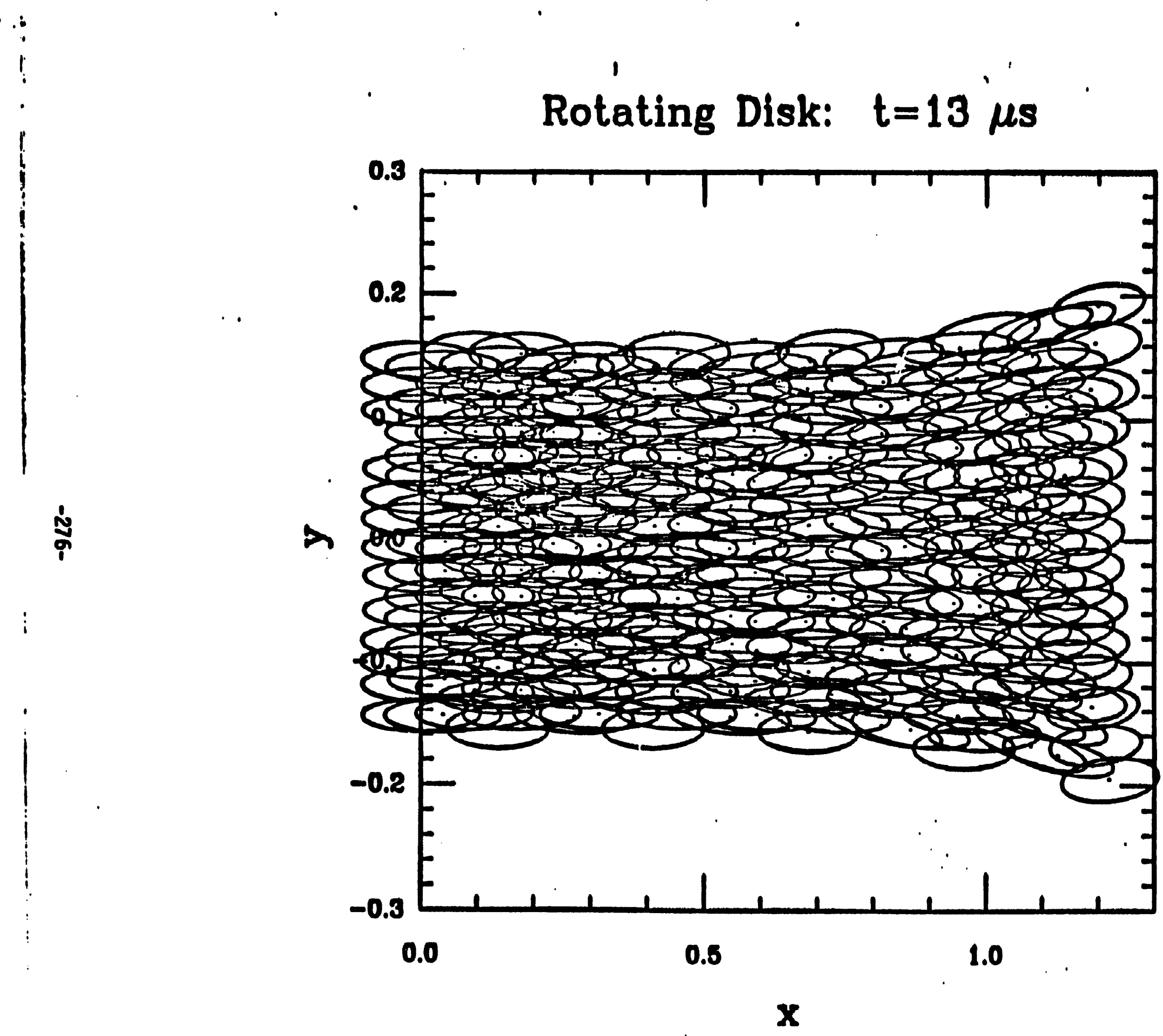



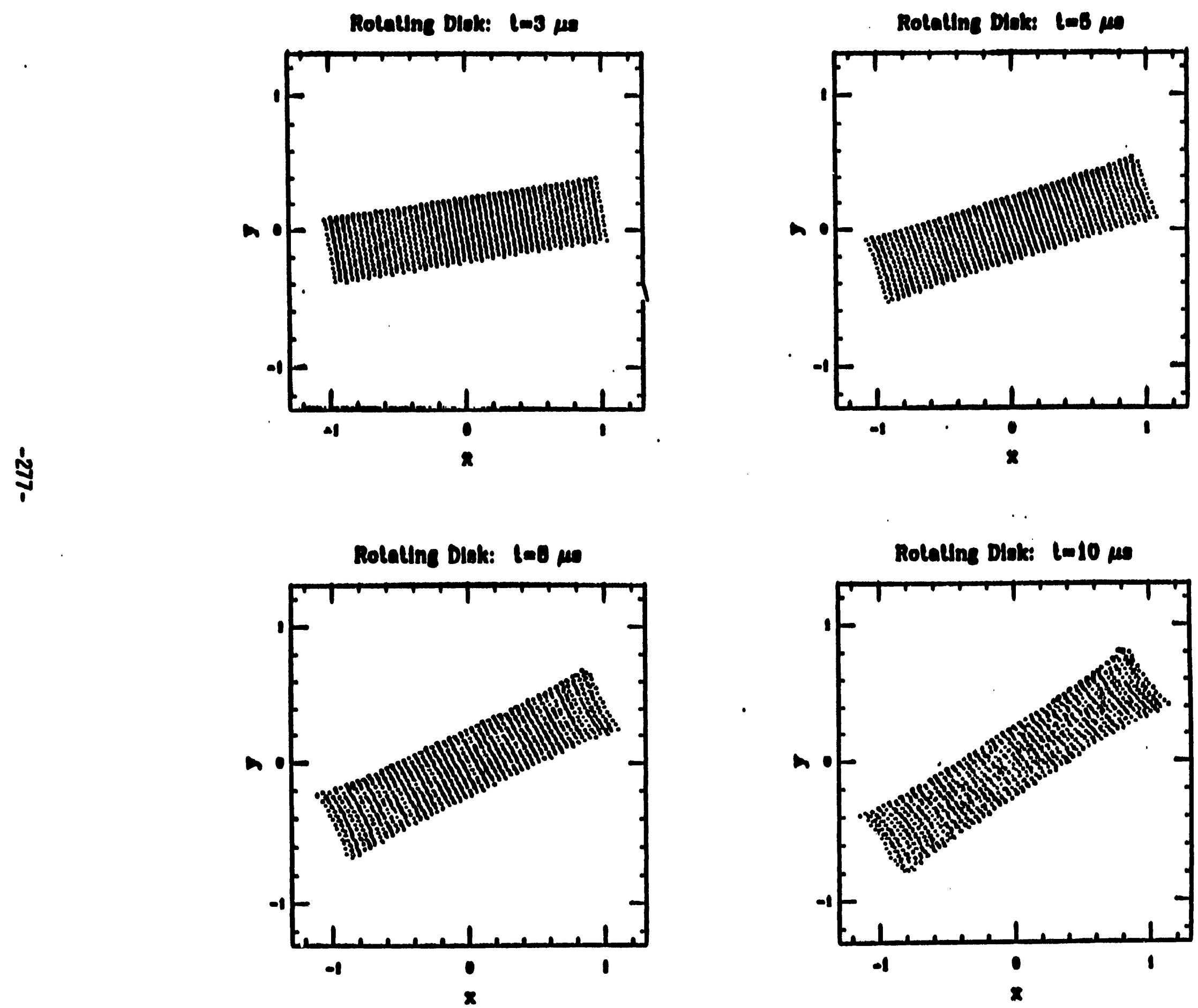


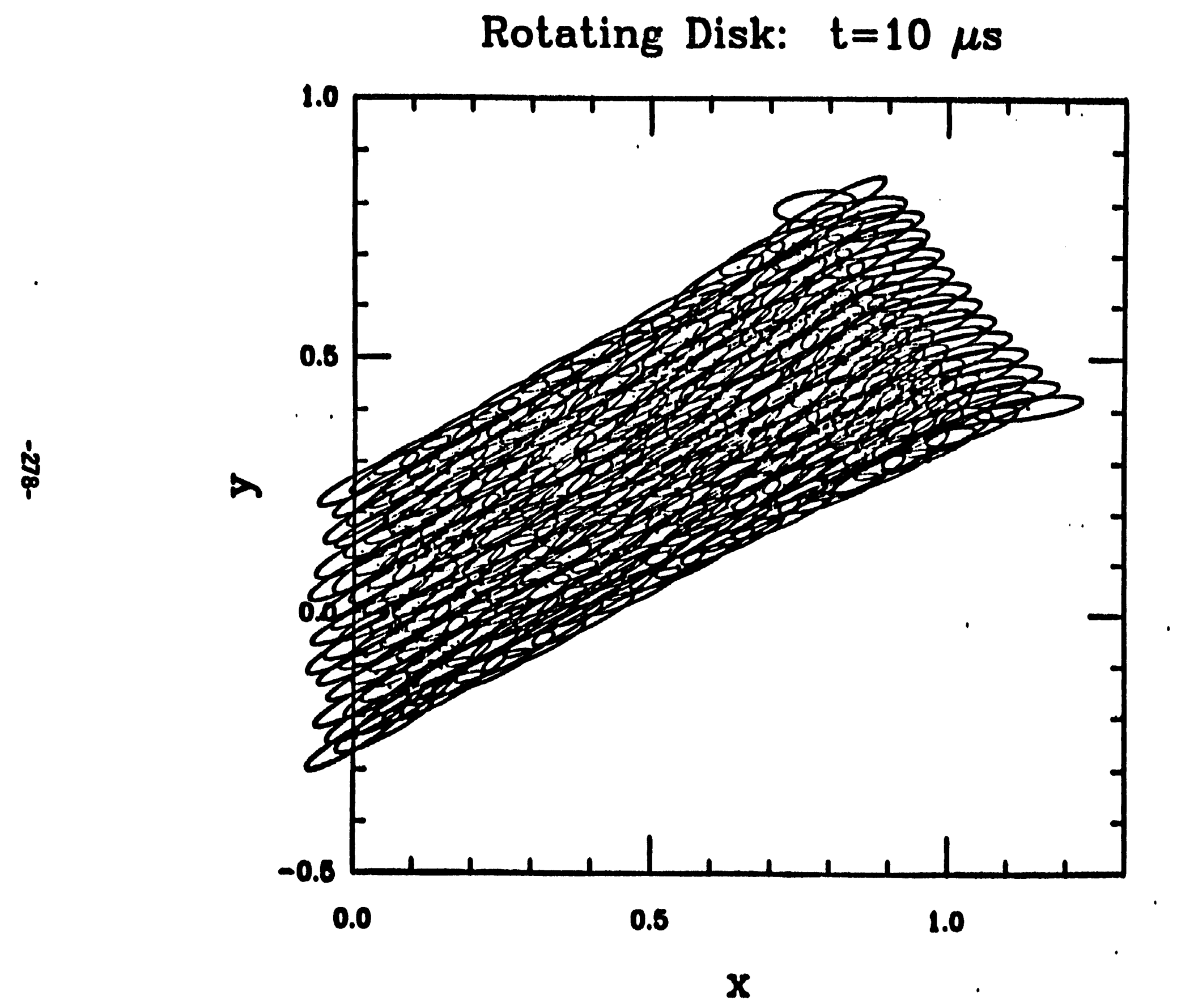




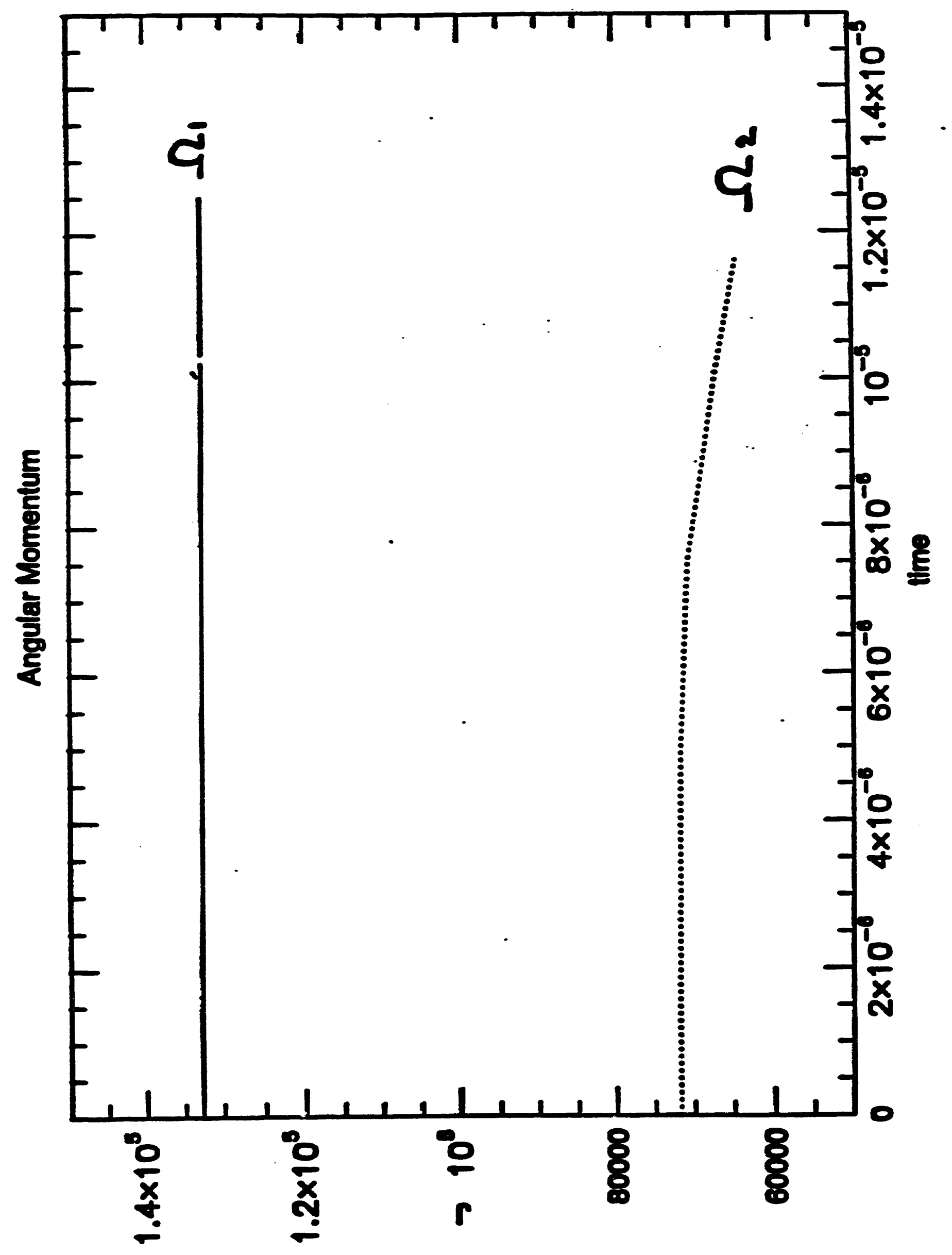


Conservation of Angular Momentum

Total angular momentum:

$$
J=\sum_{a} m_{a} \overrightarrow{r_{a}} \times \overrightarrow{v_{a}}
$$

Conservation implies $\therefore \frac{d J}{d t}=0$

$$
\rightarrow \frac{d}{d t} \sum_{a} m_{a} \overrightarrow{r_{a}} \times \overrightarrow{v_{a}}=\sum_{a} m_{a} \overrightarrow{r_{a}} \times \frac{d \overrightarrow{v_{a}}}{d t}
$$

Assume 2D + gaussian kernel

$y_{a} \cdot \frac{\partial u_{a}^{x}}{\partial t}=-\sum_{b} m_{b} \sigma_{a b} \frac{\left(x_{a}-x_{b}\right)}{h_{x}^{2}} W_{a b}$

$$
x_{a} \frac{\partial v_{a}^{y}}{\partial t}=-\sum_{b} m_{b} \sigma_{a b} \frac{\left(y_{a}-y_{b}\right)}{h_{y}^{2}} W_{a b}
$$

So that conservation becomes

$$
\frac{d y}{d t}=\sum_{a} m_{a}\left(y_{a} \dot{v}_{a}^{+}-x_{a} i_{a}^{y}\right)
$$

$-280-$ 
puling everything together:

$$
\frac{d J}{d t}=-\sum_{a} \sum_{b} m_{a} m b \sigma_{a b} W_{a b}\left[\frac{y_{a}\left(x_{a}-x_{b}\right)}{h_{x}^{a^{2}}}-\frac{x_{a}\left(y_{a}-y_{b}\right)}{h_{z}^{a^{2}}}\right]
$$

rewrite it as

$$
\frac{d J}{d t}=-\sum_{a} m_{a} x_{a} y_{a}\left[\frac{1}{h_{a^{2}}^{a^{2}}}-\frac{1}{h_{y}^{a^{2}}}\right] \cdot \sum_{b} m b T_{a b} W_{a b}\left(\frac{x_{a} y_{b}}{h_{y}^{a^{2}}}-\frac{y_{a} X_{b}}{h_{x}^{b^{2}}}\right)
$$

- clearly if $h_{x=} h_{y} \rightarrow \frac{d y}{d t}=0$

- the $2^{\text {ad }}$ sum can be written os an integral:

$$
\begin{aligned}
& \int \rho_{b} \sigma_{a b} W_{a b}\left(\frac{x_{a} y_{b}}{h_{y}^{a^{2}}}-\frac{y_{a} x_{b}}{h_{x^{2}}^{2}}\right) d r_{b} \\
\rightarrow & \frac{x_{a}}{h_{y}^{2}} \int Q_{a b} \cdot W_{a b} y_{b} d r_{b}-\frac{x_{0}}{h_{b}^{2}}\left(Q_{a b} W_{a b} x_{b} d r_{b}\right. \\
& Q_{a b}=P_{b} \cdot \sigma_{a b}
\end{aligned}
$$

- if $Q a b=$ constant $\rightarrow \frac{d J}{d t}=0$ !

- if Gab $\neq$ constant use Taylor expansion

$$
Q_{a b}=Q_{a b}(a)+\left(x_{b}-x_{a}\right)\left(\frac{\partial Q}{\partial x}\right)_{a}+\left(y_{b}-y_{1}\right)\left(\frac{\partial Q}{\partial b}\right)_{a}+\ldots
$$


All but the second order terms will vanish!'

$\rightarrow$ Error term of order $h^{2}$ !! 
$-888^{-}$

ifmovalmas japas bivo an for of $\leftarrow$.

$$
\begin{aligned}
& \text {; axyoy burbpapon bg wanb so } \\
& \text { wo.y2urep yfim raison pads yougs } \\
& \text {;somoufp Guom } \leftarrow \\
& \text { Pif Siopo si foyf } M \triangleq
\end{aligned}
$$

do frowolwos ayf Givo ase vo.yonsasuos mynawow sopsbus yfirs wapgoul anowas ol

$$
\text { ssom /sp/g }
$$




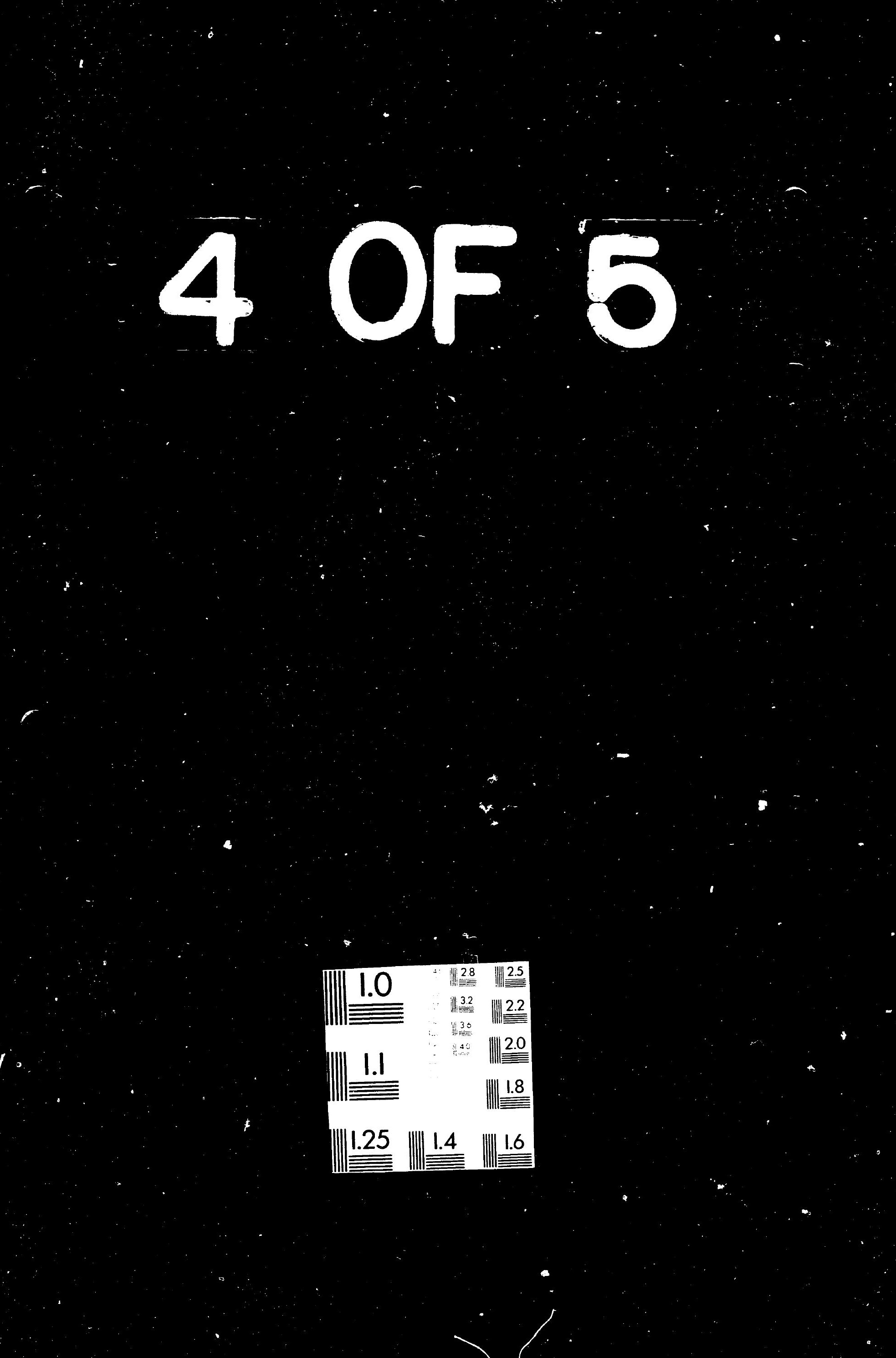



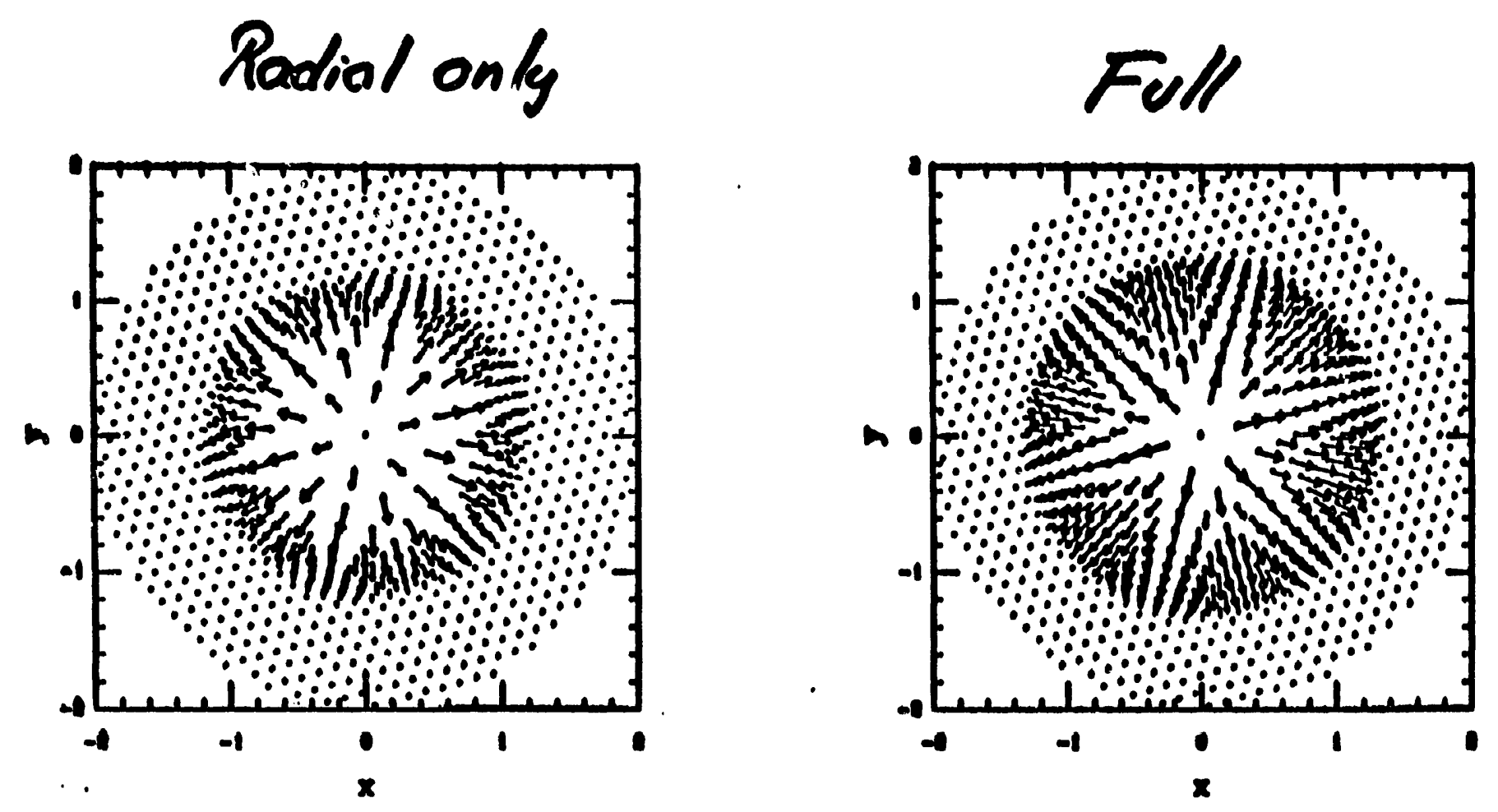

甲

\section{Standard SPH}

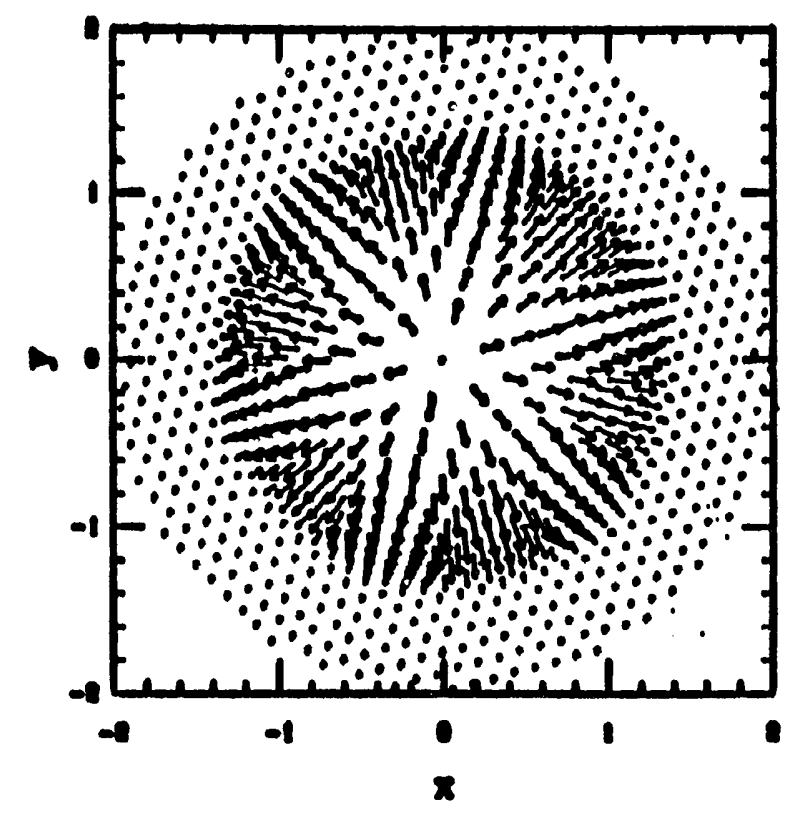



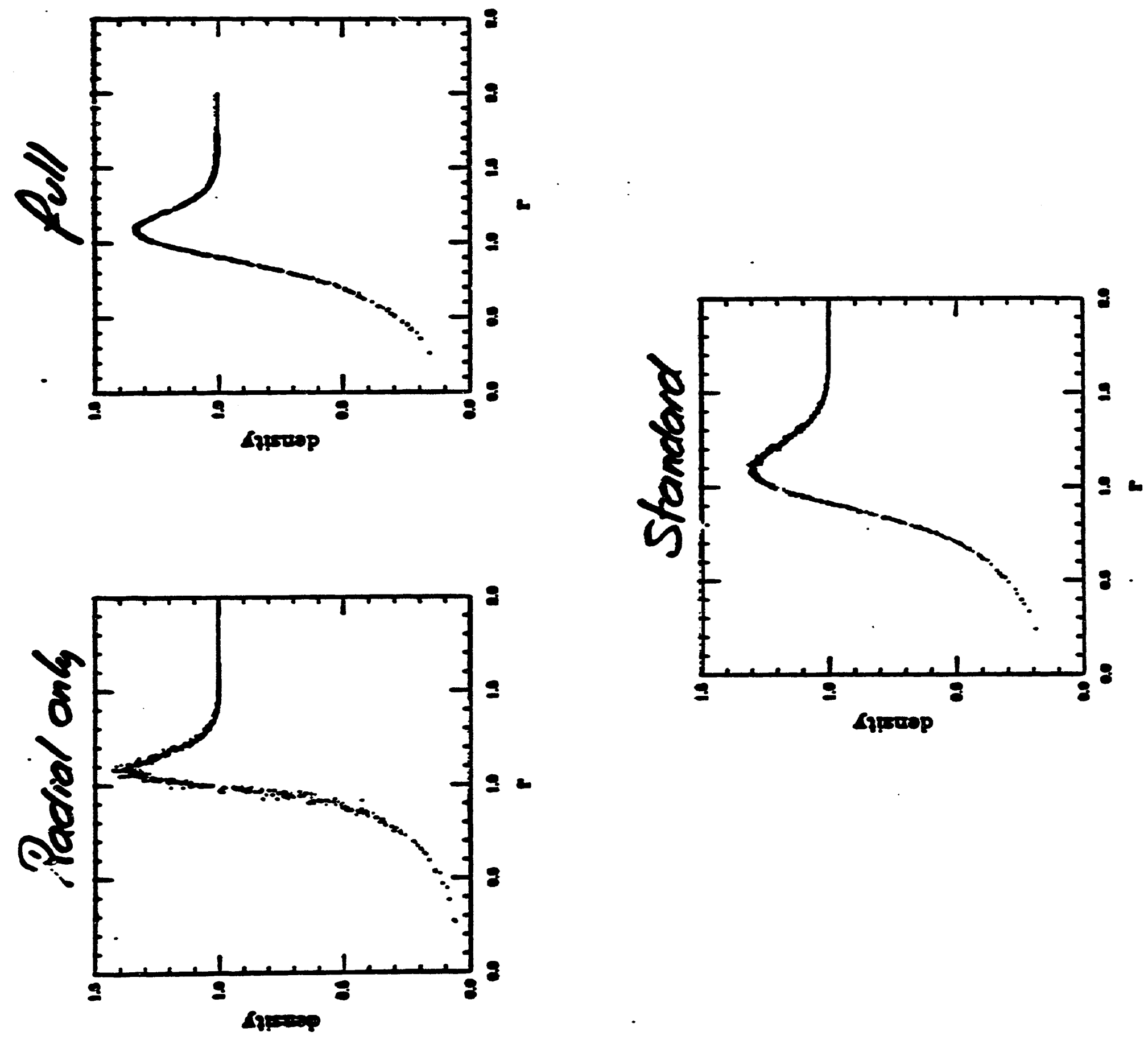

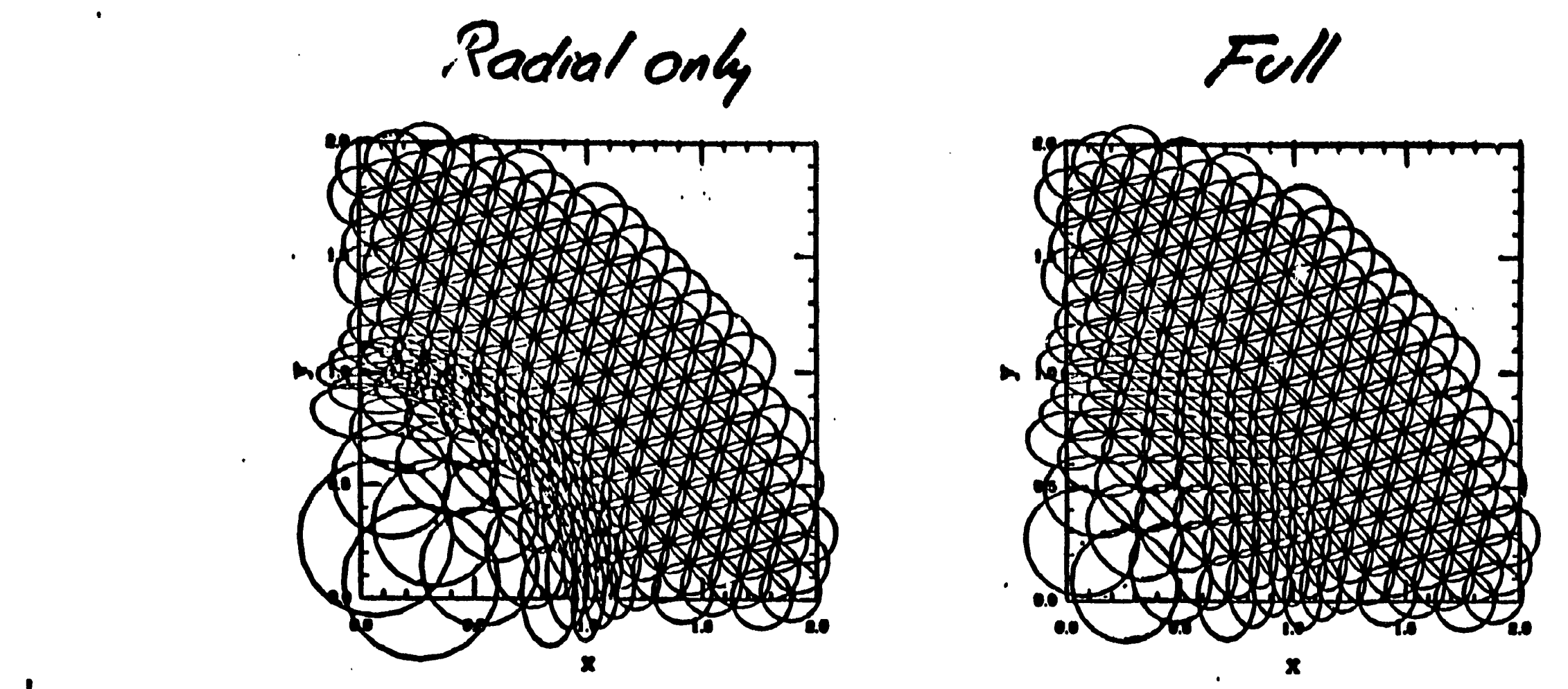

ఖ্ণ

\section{Standard}

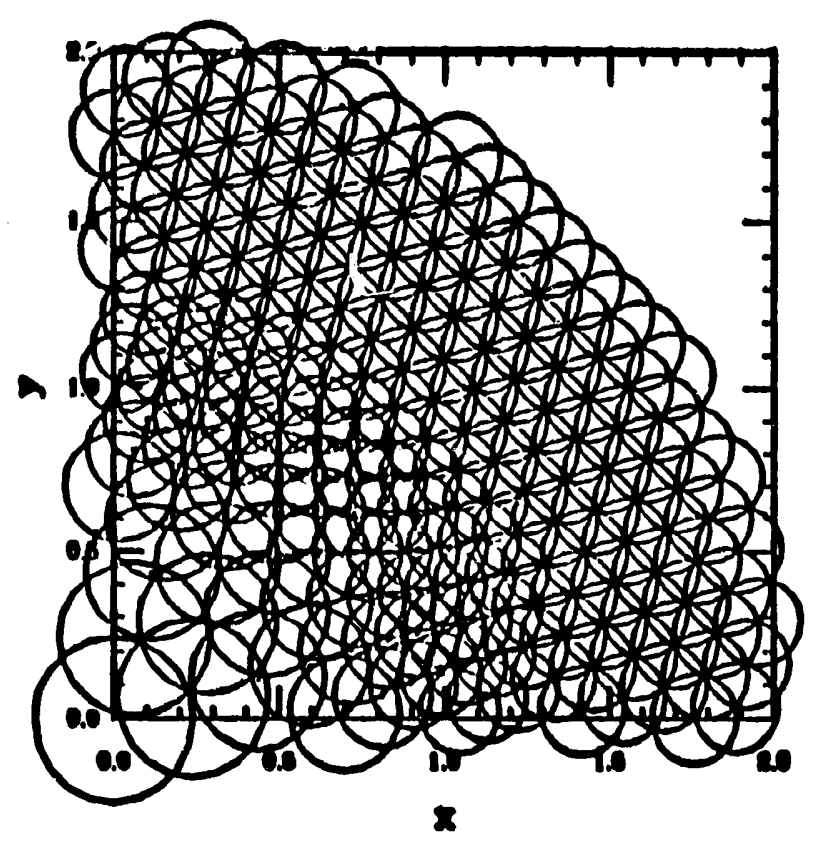


Tidal Disruption of stars

H. Foll bright

Top view:

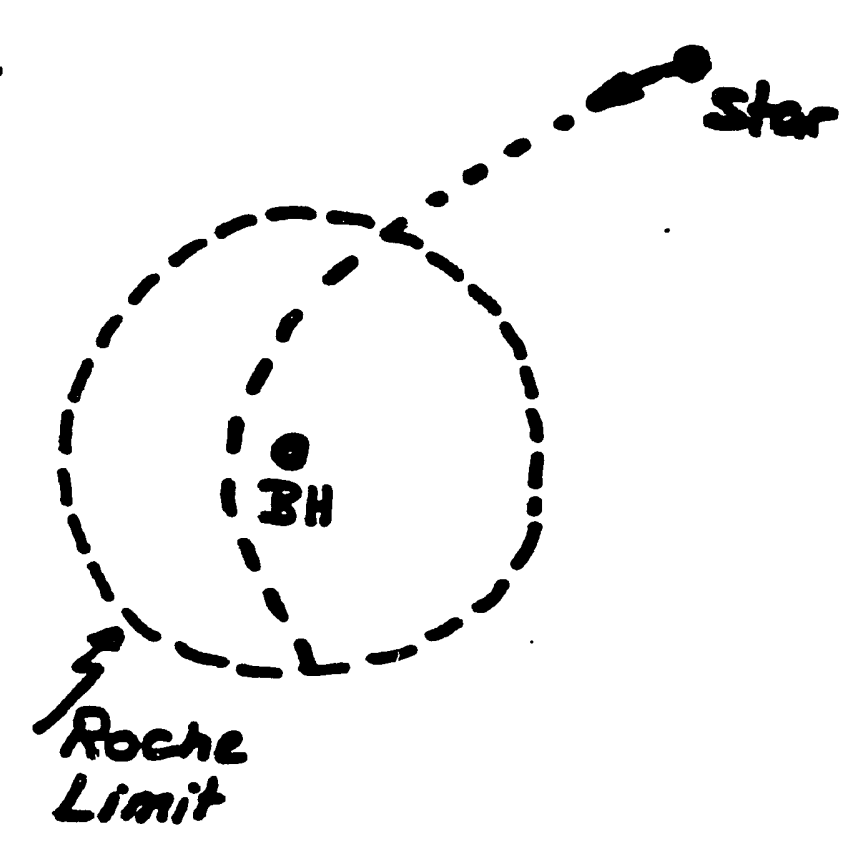

Sick view:

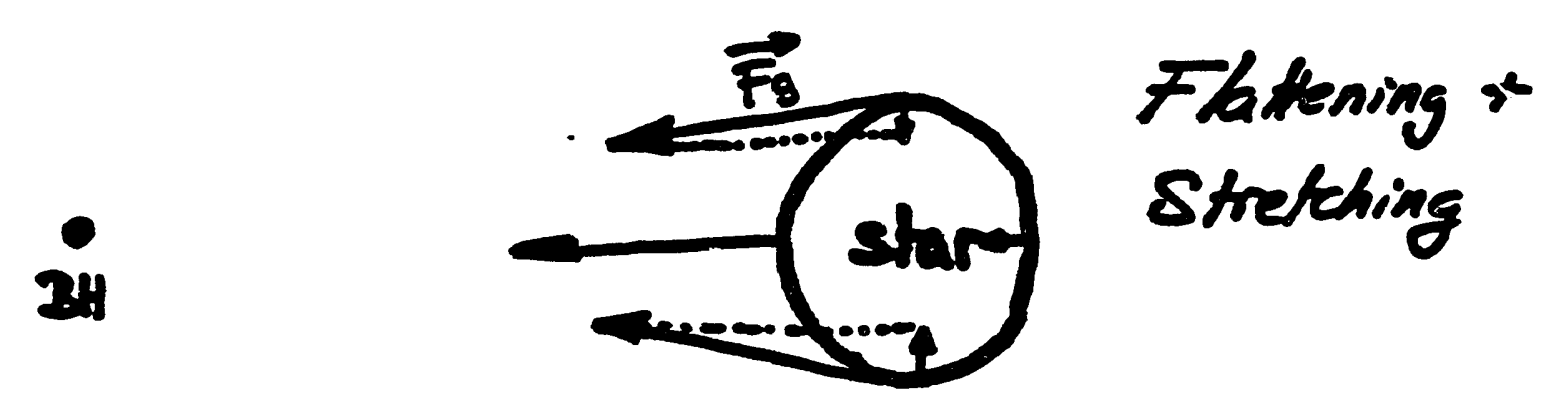

For $\frac{M_{2 N}}{M_{*}} \gg 1$ Very, very strong flattening! 
Question of astrophysical interest:

1) Amount of material captured

2) Ejection velocity and distribution of obis not captured

3) Density spike (red herring!')

Compression faster than stretching

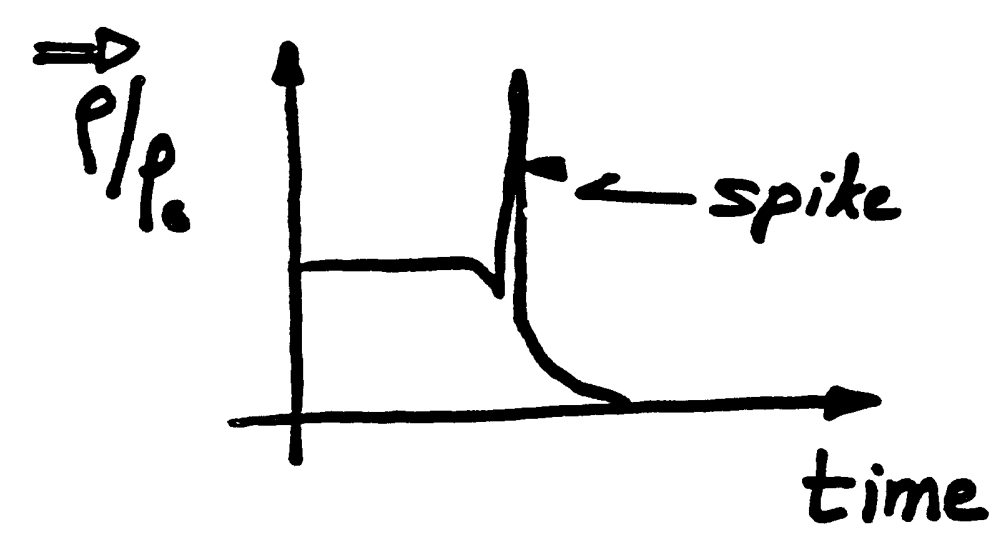

$\rightarrow$ Numerical diffiouthes: ID compression $\frac{z}{R} \ll 1$ in fact $z<h ! !$

$\rightarrow$ ideal case for ellipsoidal kernels -288- (Blchnellt tringotl) 


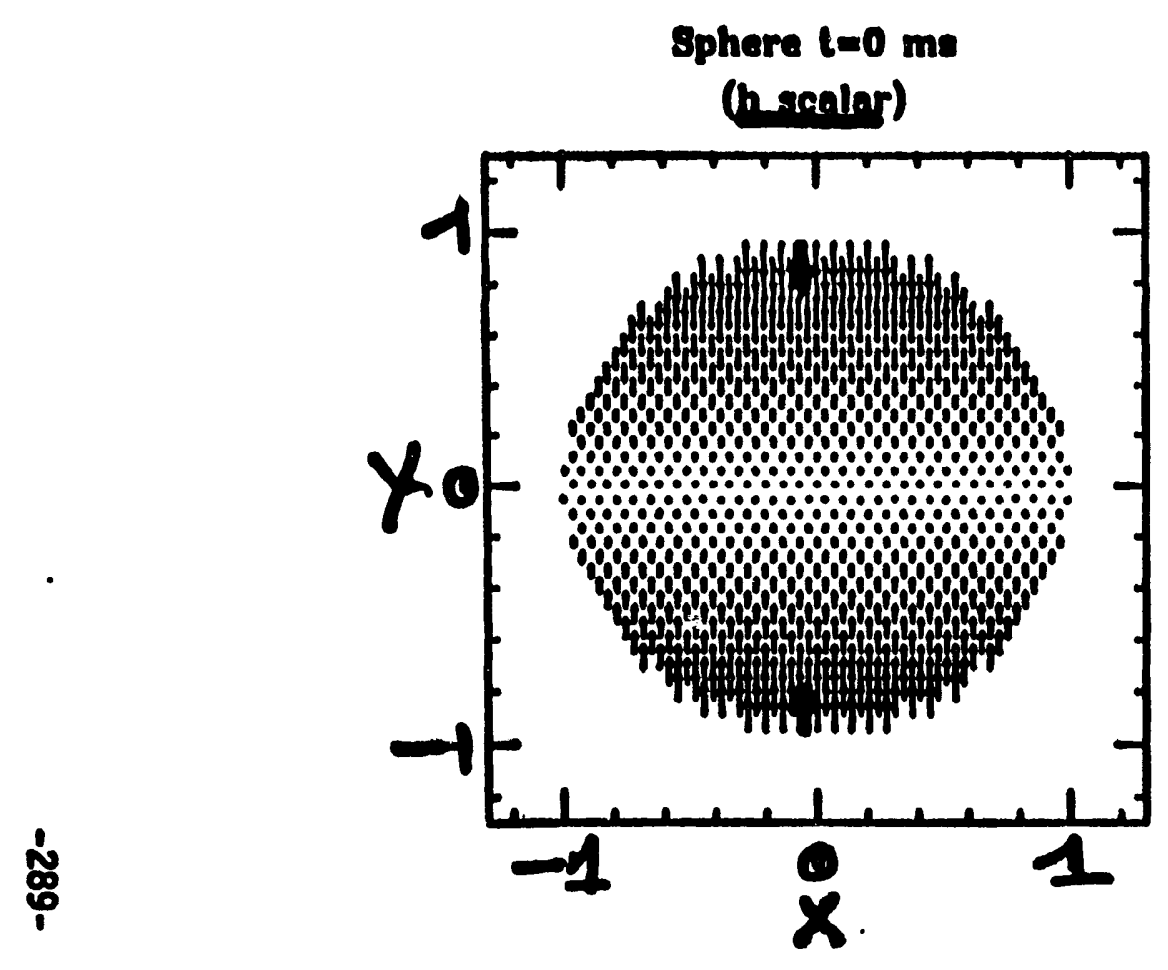

Sphore t-0.237 me

(hecalar)

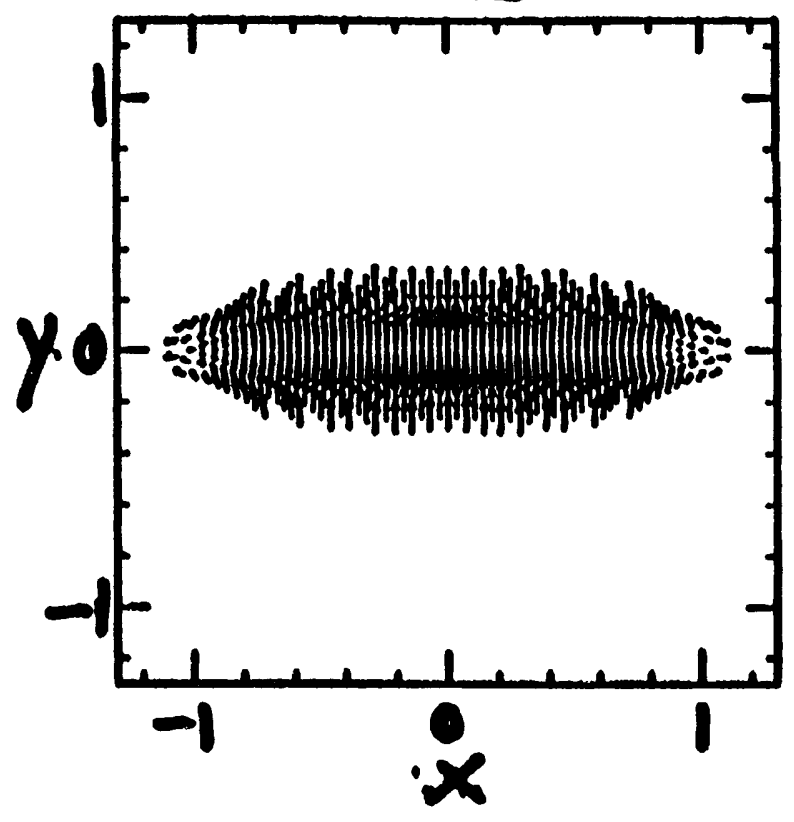

Sphers to6.026 ms

(hresples)

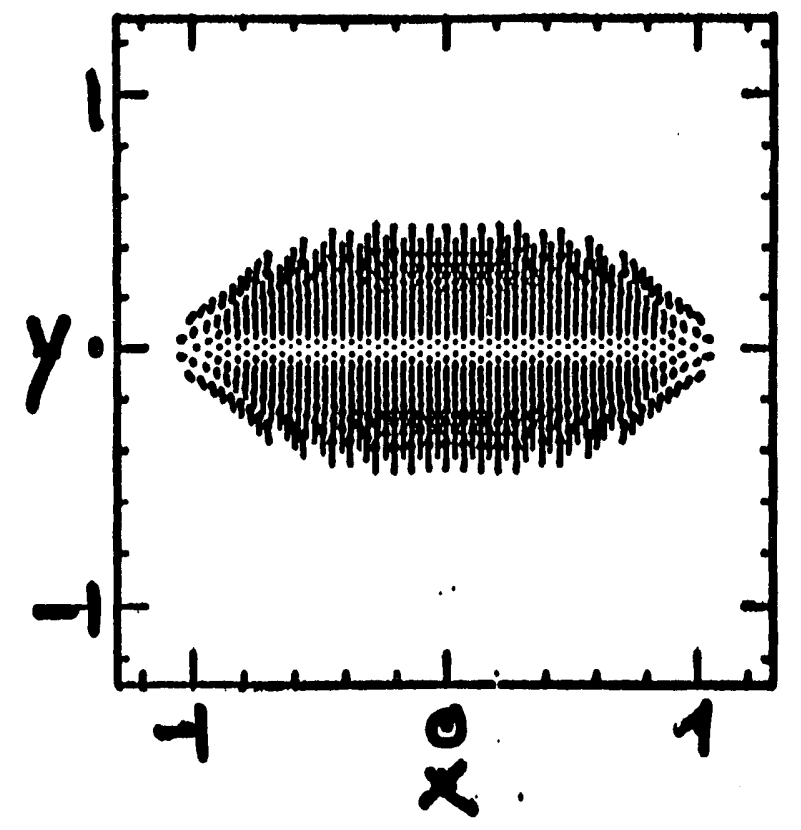

Sphore l-18.014 mo

(hecalar)

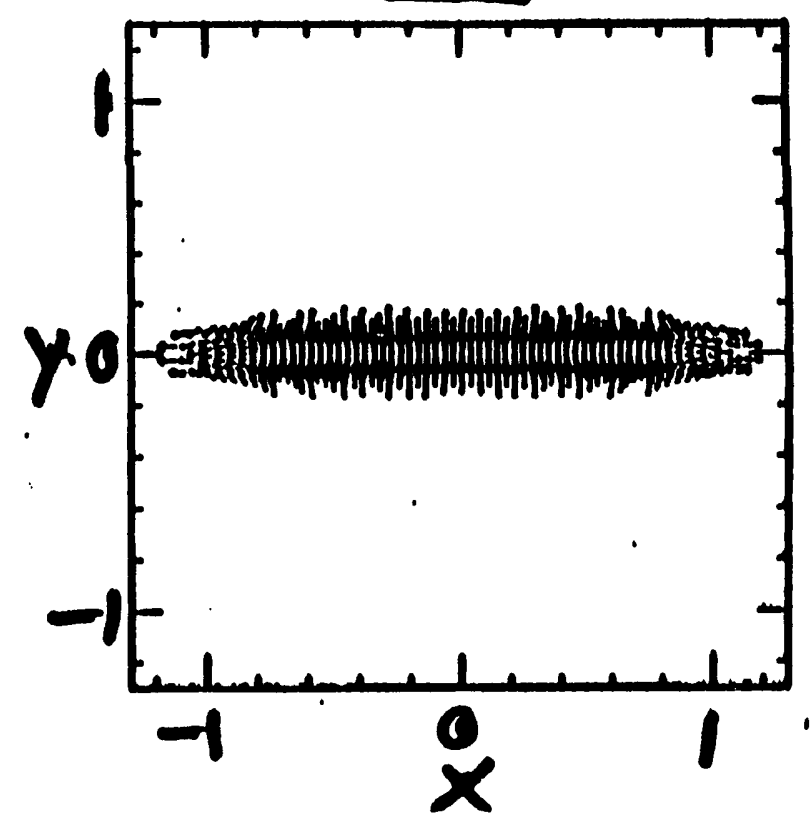



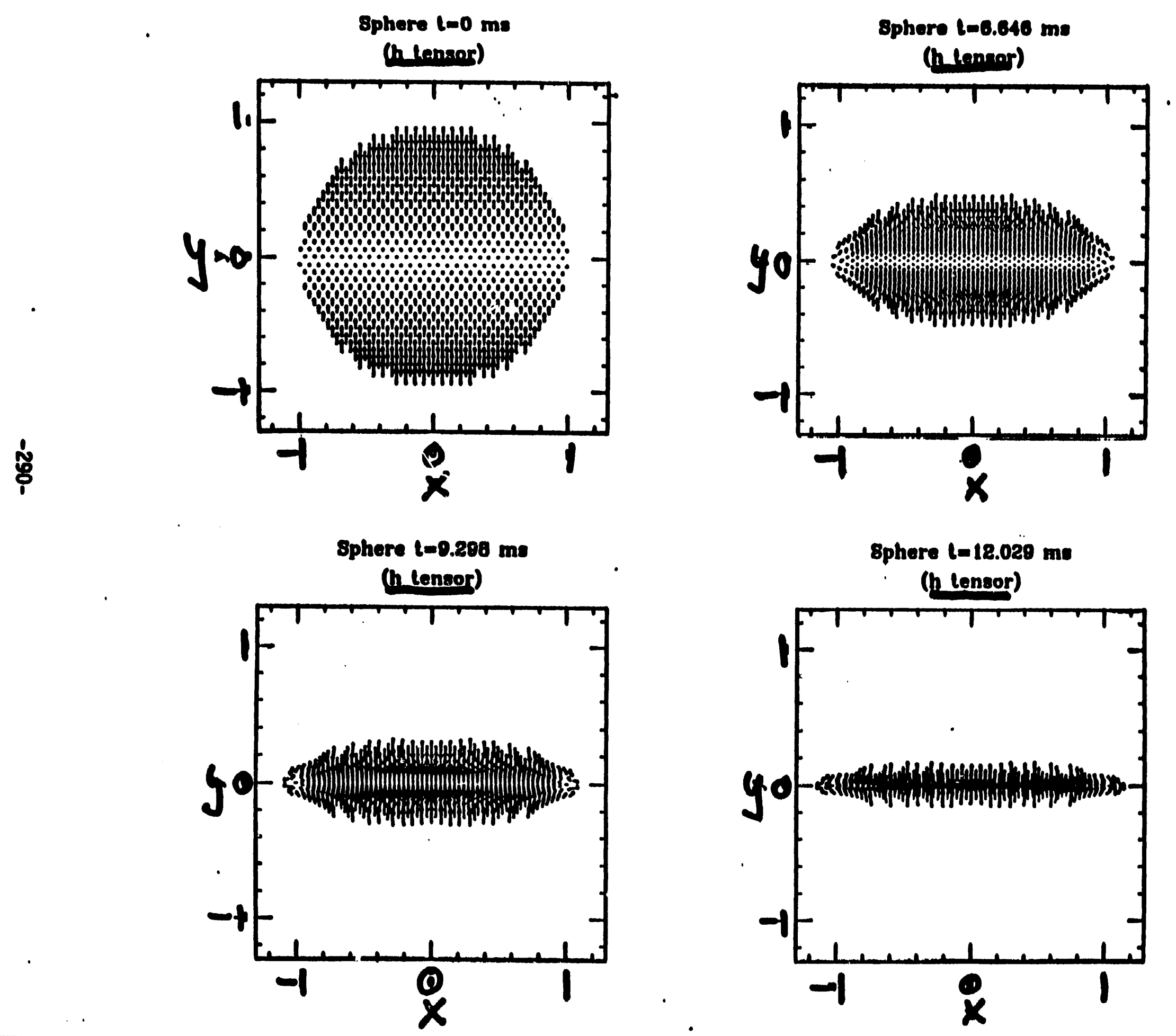


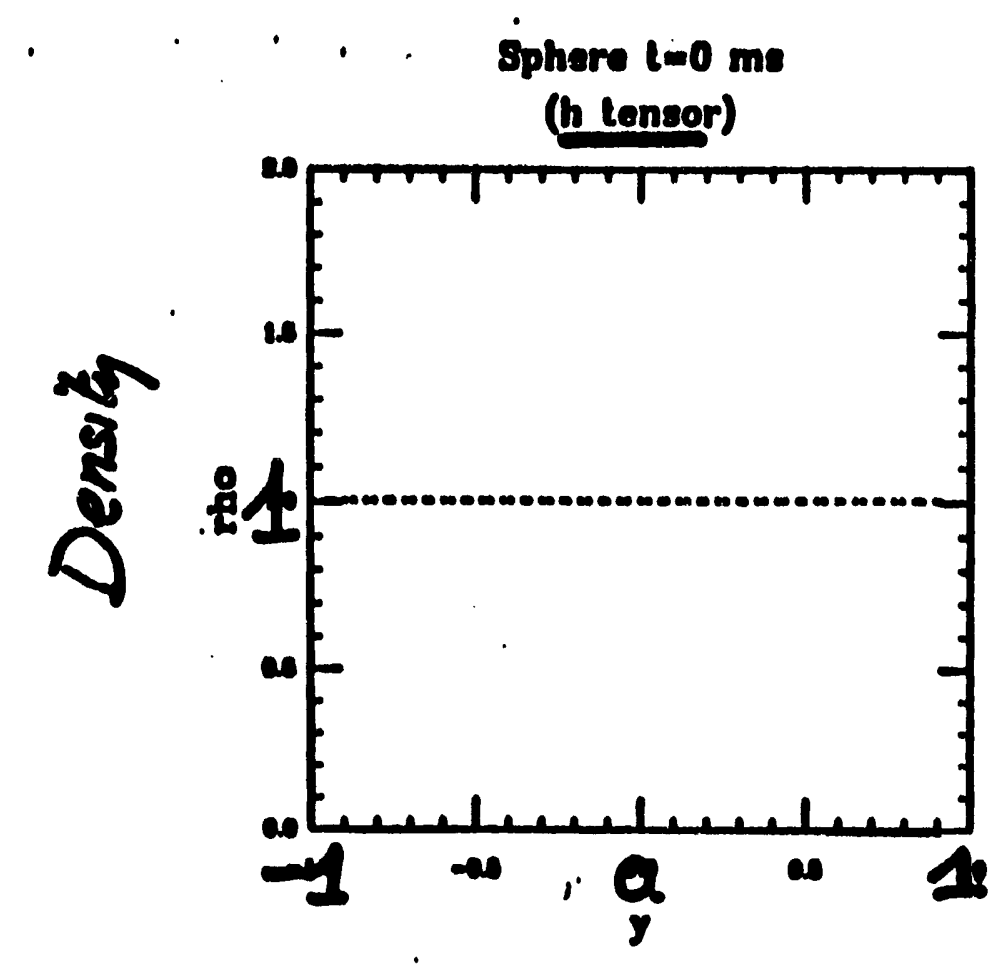

\&

Dphore l-0.200 mo (h lenser)

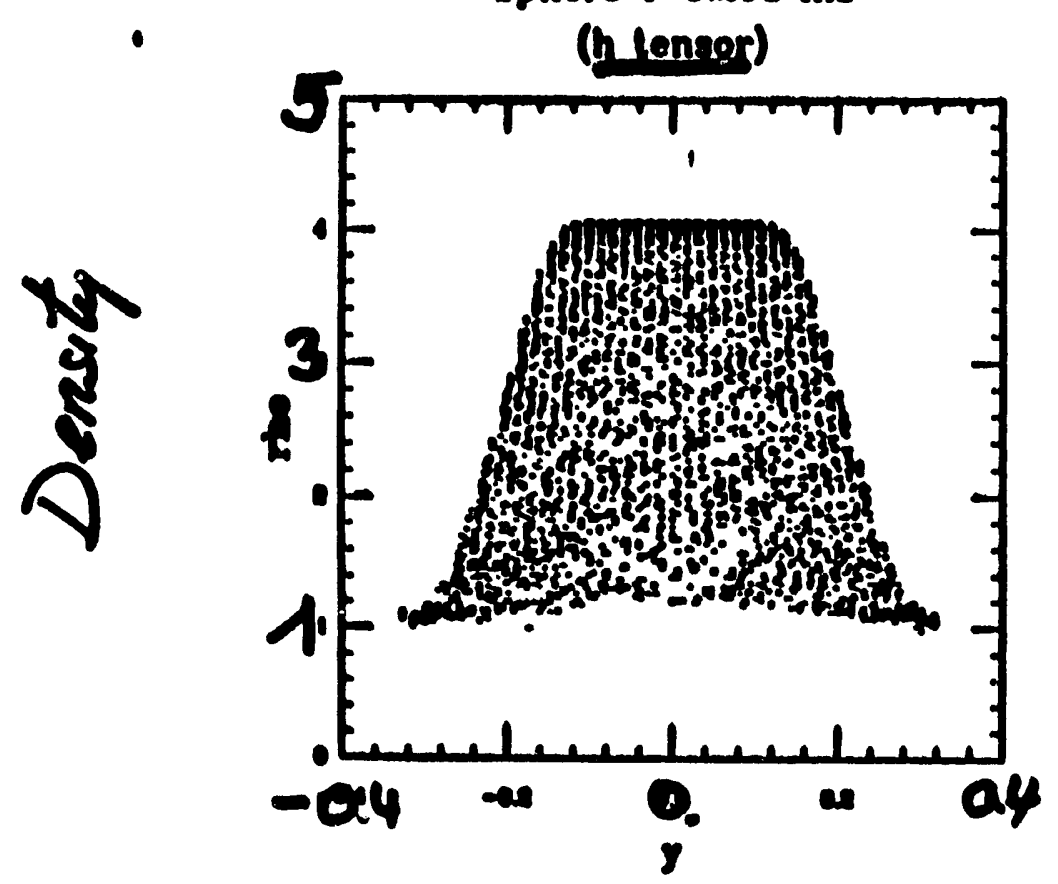

Sphore lo0.048 mo (h lennor)

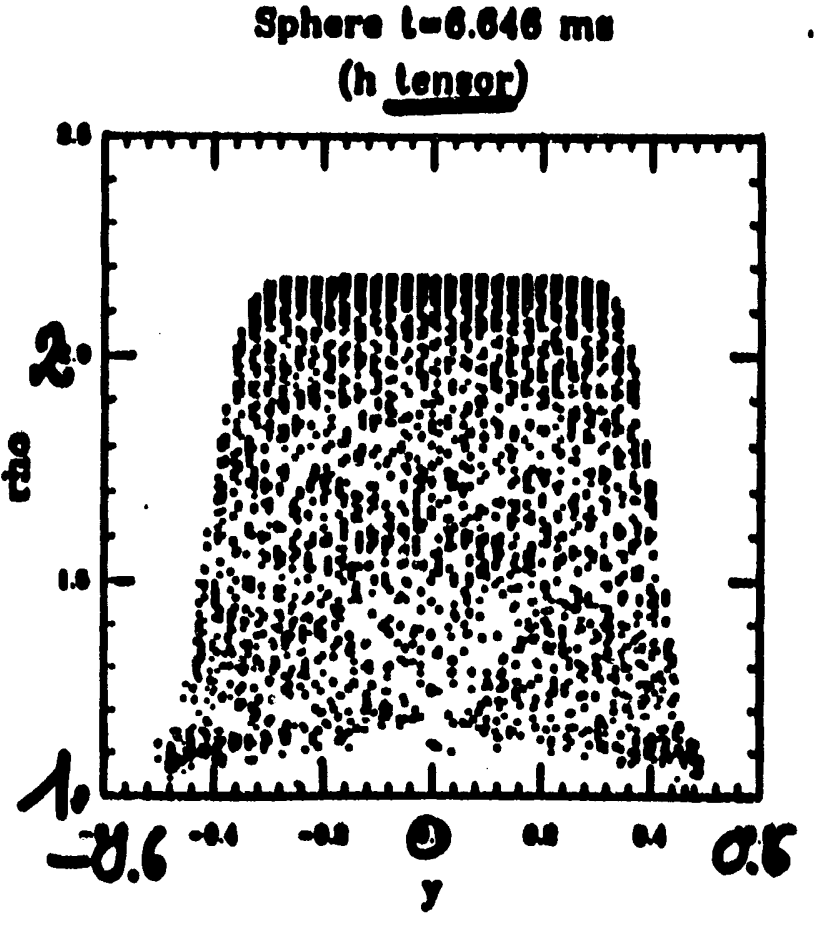

Sphore lel2.020 mo (heennor)

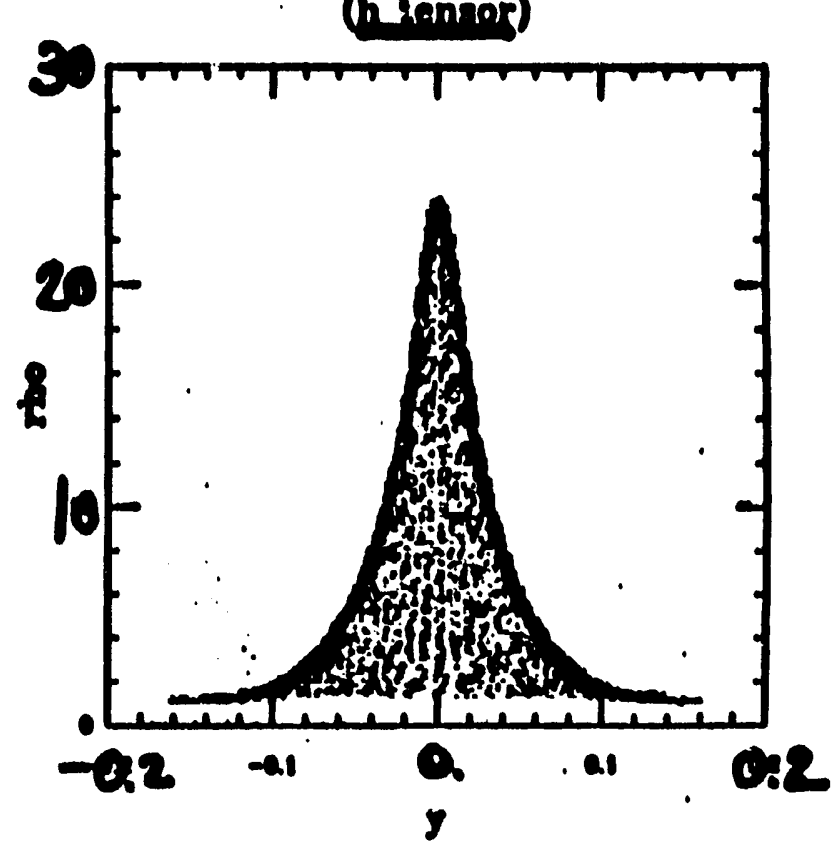



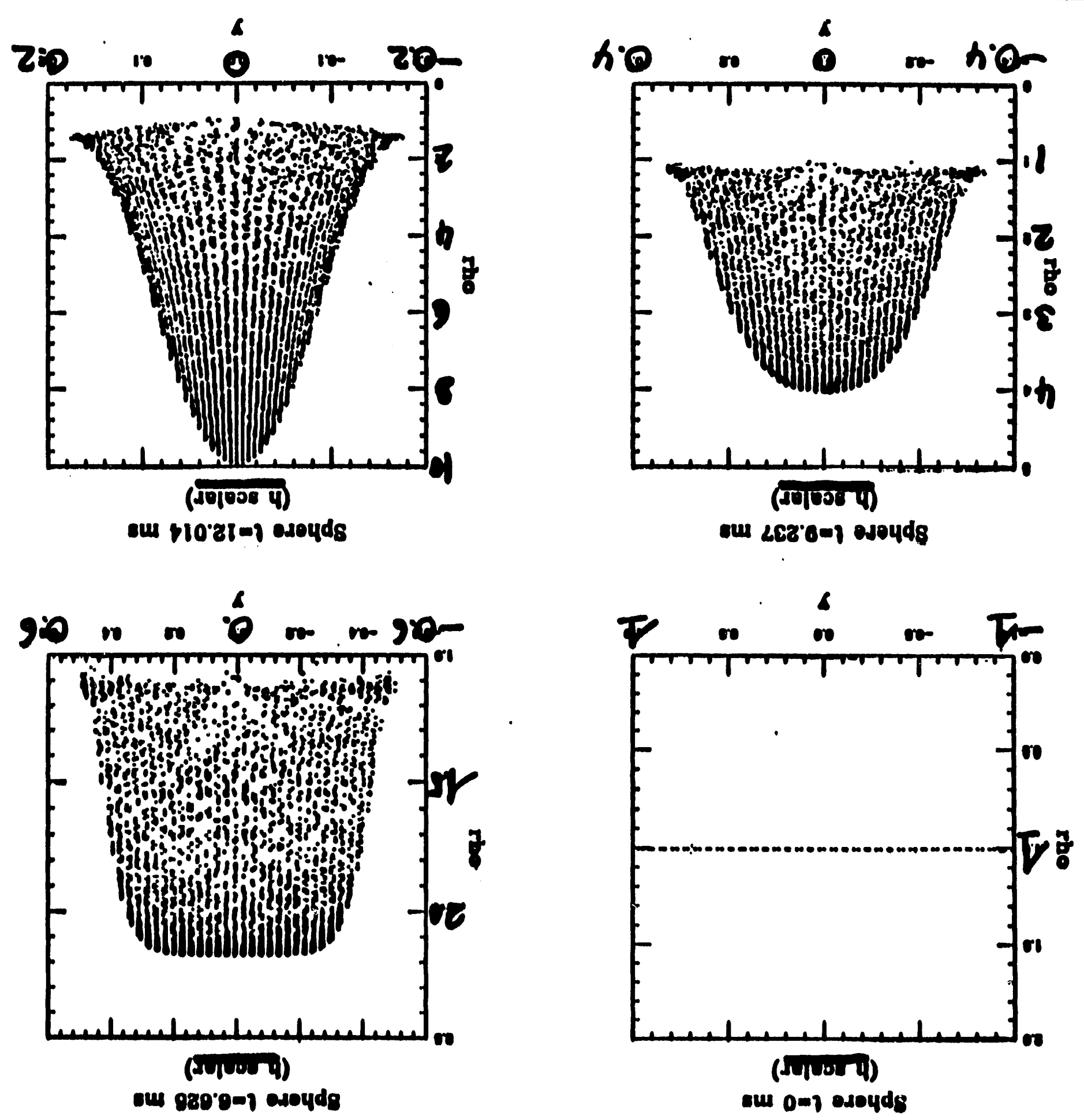


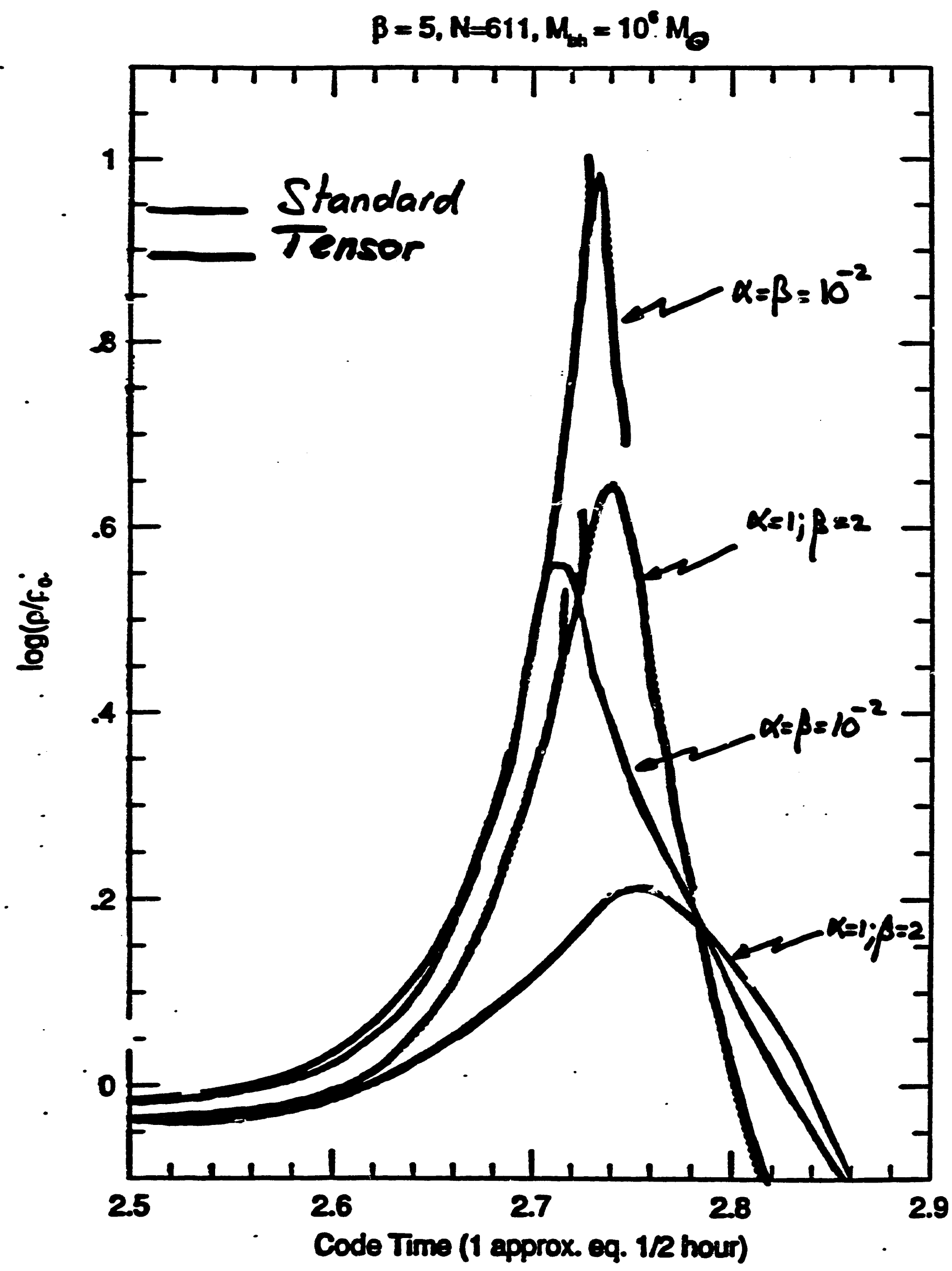

$-293-$ 
Tidal Disruption: critical parameters

1) Resolution

with $N \approx 611 \quad h=0.16$ in standard SPH (at maximum $\rho$ ) $\quad h_{Z z}=0.02$ in tensor SPY I

$\Rightarrow$ resolution increase $\sim 8$

$\Rightarrow$ standard SPH $\sim N_{\sim}$ 31,000 parts.!

2) Artificial Viscosity.

If shocks are present, dissipation will increase entropy $\Rightarrow$ resist further squeezing!

but.... there are no real shocks as the stor gets squeezed homologourly...

$\rightarrow$ results should be indef. of $\alpha, \beta$. but the are not!' 

STANDARD SPH

- sodp/pol

\section{TENSOR SPH}

\section{$\log \left(p / p_{0}\right)$}

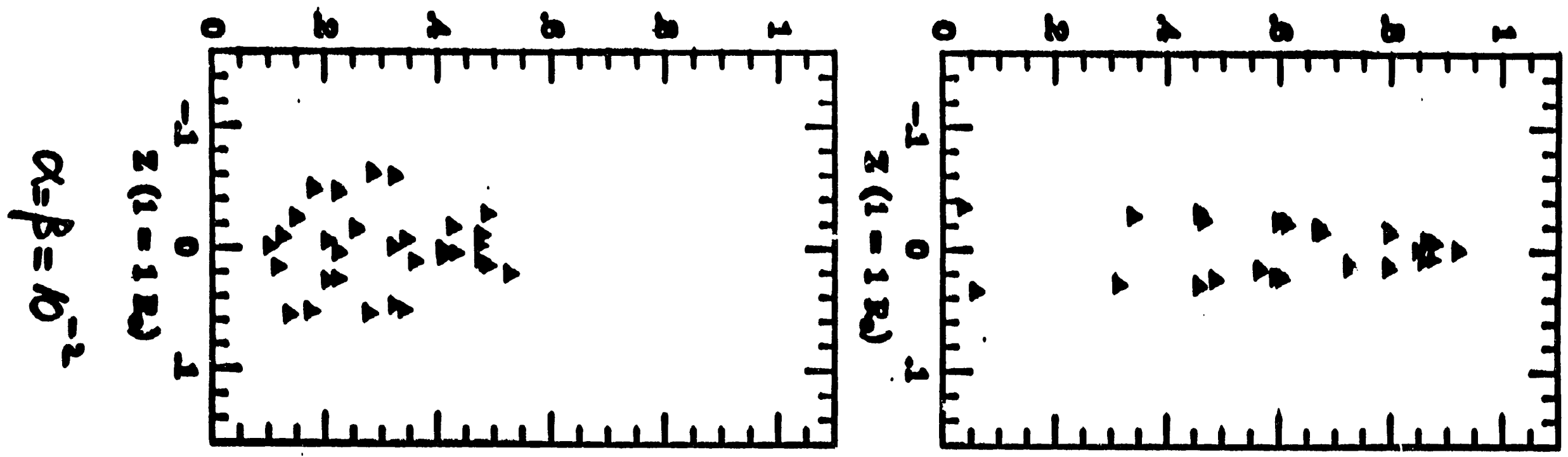

\&ั

$\log (0 / p)$

$100(0 / p)$

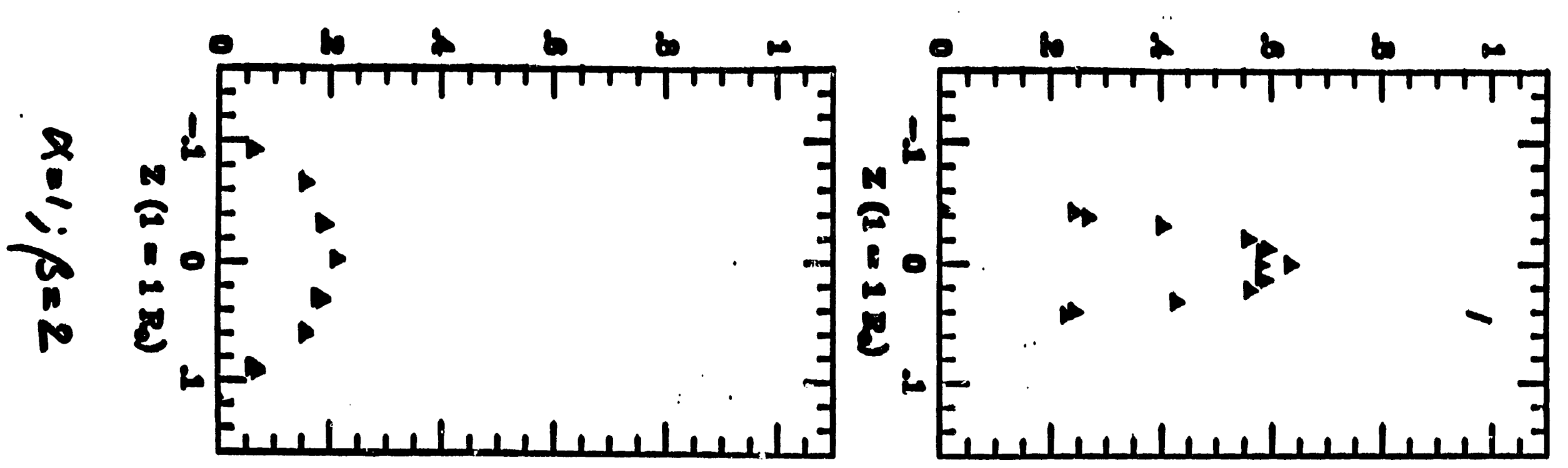




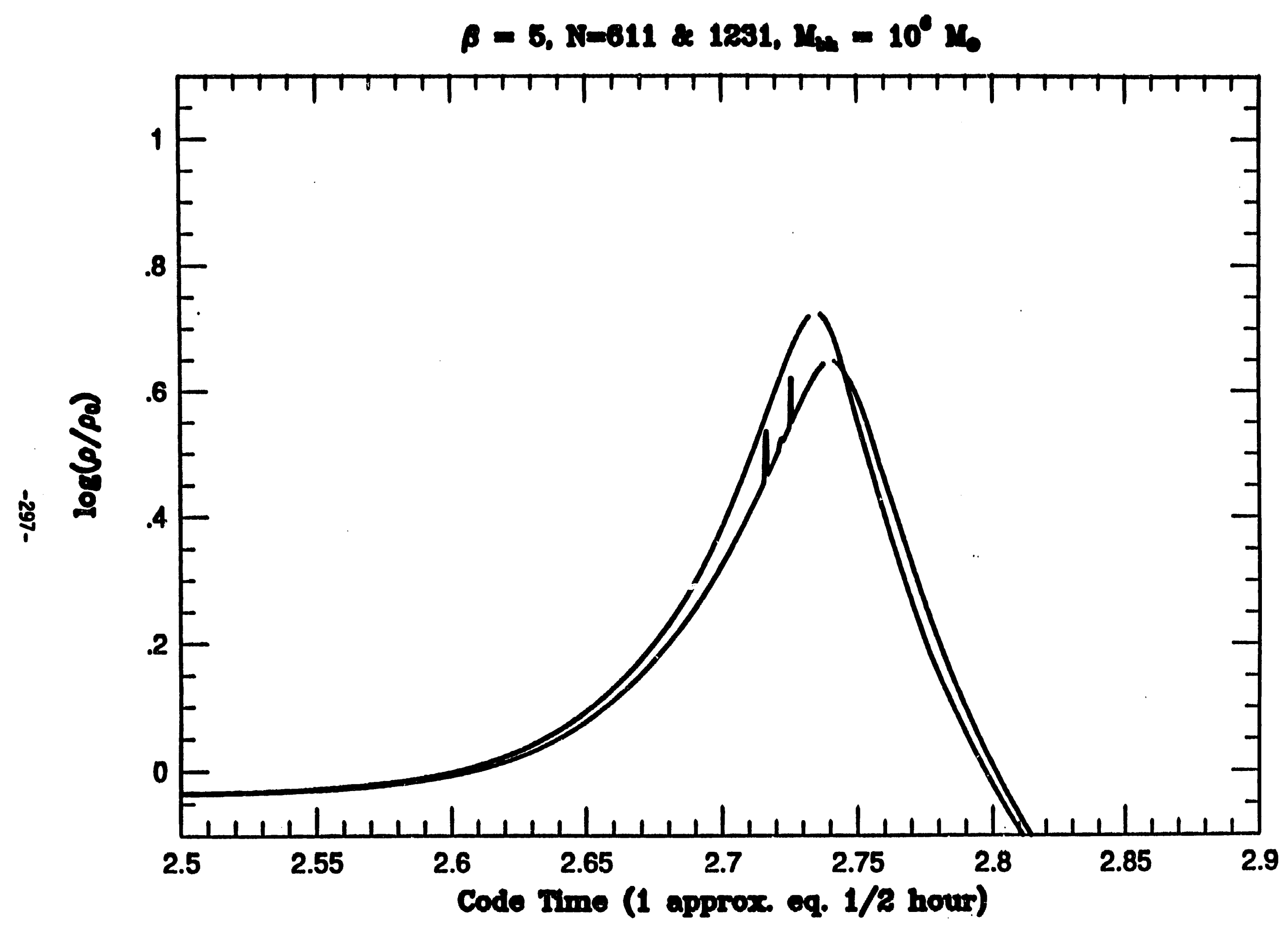


Results:

tighter densities with low values for $\alpha, \beta$.

but... not really hydrodynamics as parties stream through the phone...

Note: Using the "tank stopper" XSPH is not a real solution as this cobs not conserve energy!

$\rightarrow$ Bottom line: Fix artificial viscosity to remove heating in homologous flows!

$-298-$ 


\section{Breaking the 10,000,000 particle limit in SPH}

Mike Warren (LANL T-6, UCSB)

mswleagle.lanl.gov

$\stackrel{1}{8}$

W. H. Zurek, J. G. Hills, W. A. Miller (LANL T-6)

J. K. Salmon, M. B. Davies (Caltech)

P. Laguna (Penn State) 


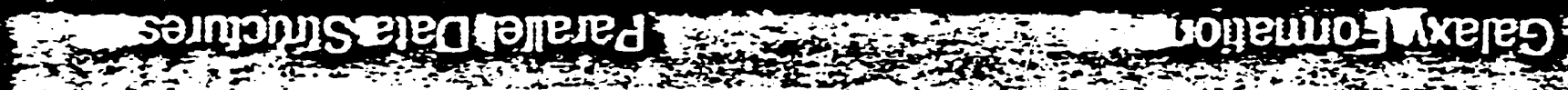

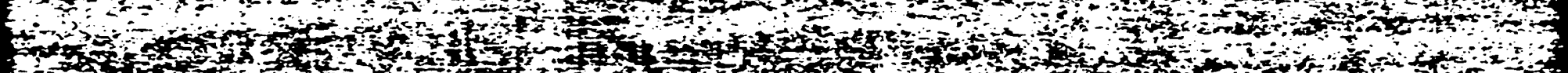

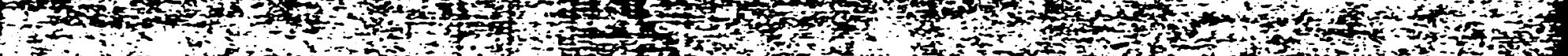

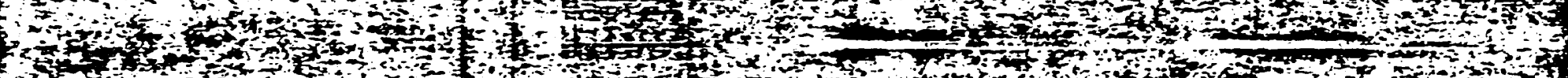

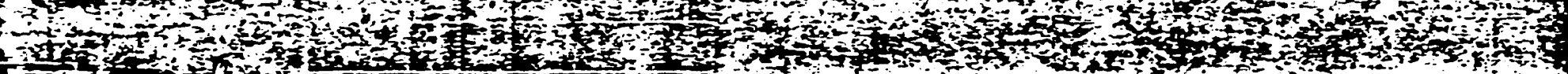

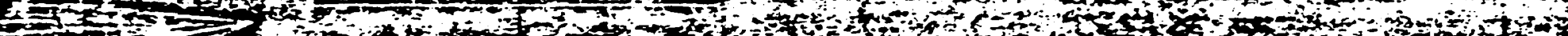

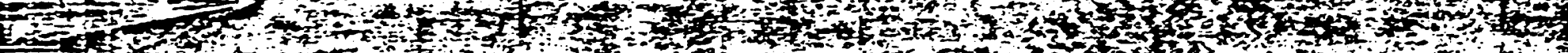

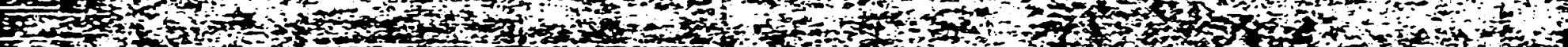

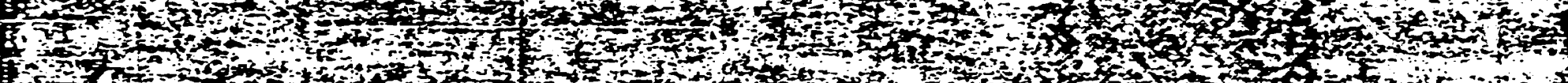

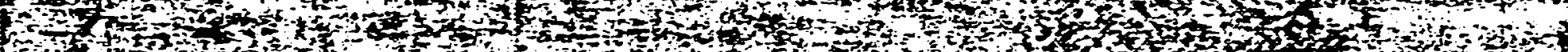

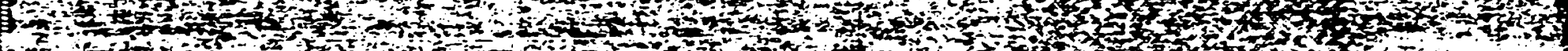

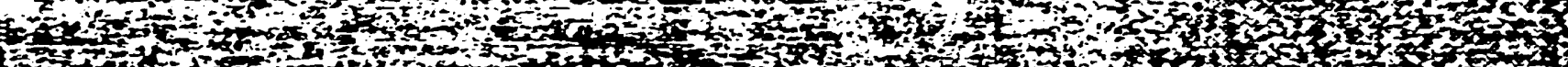

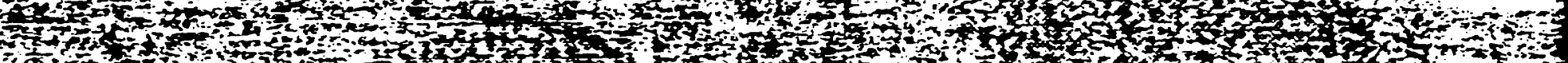

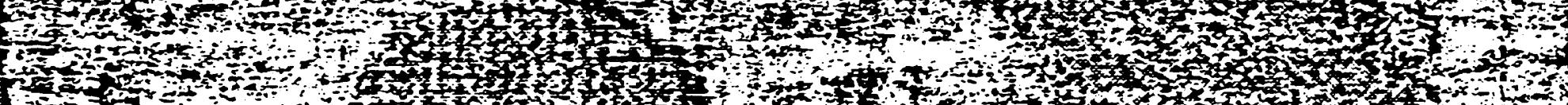

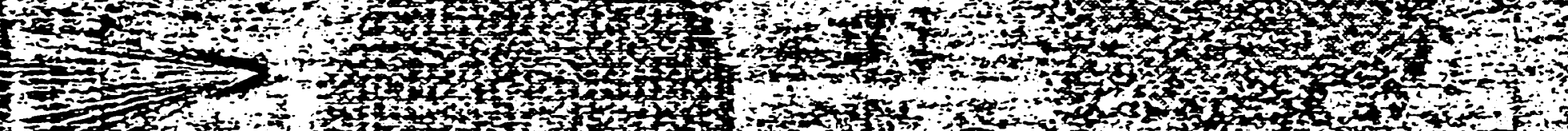

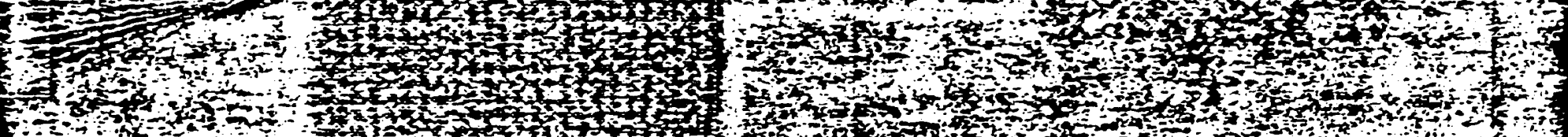

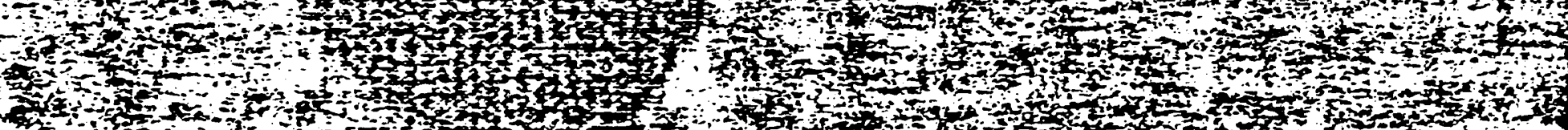

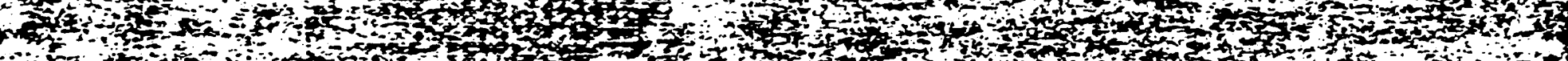

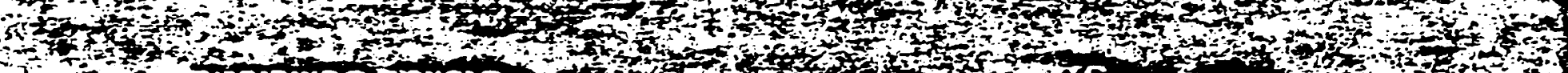

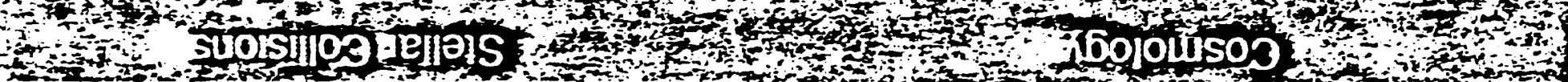

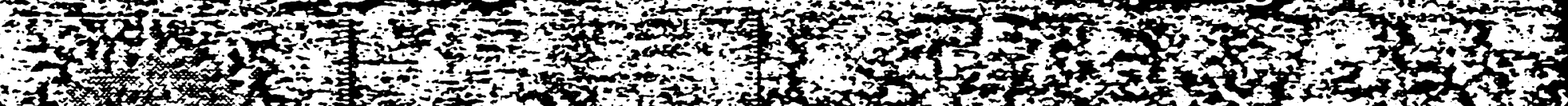

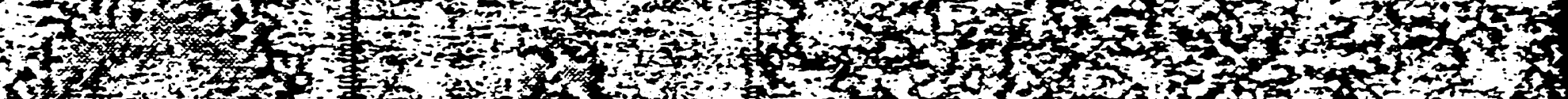

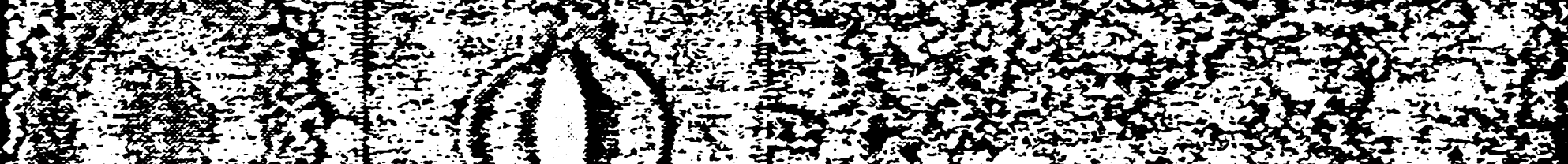

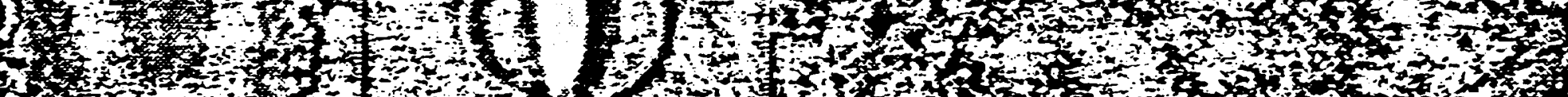

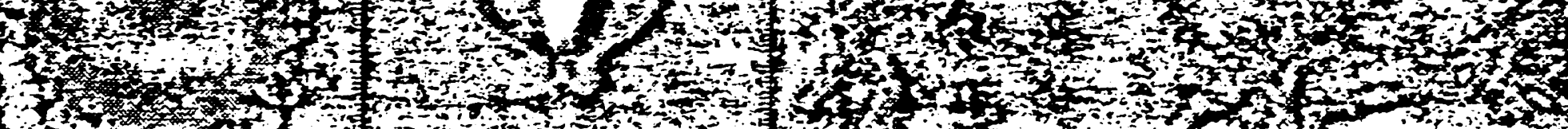

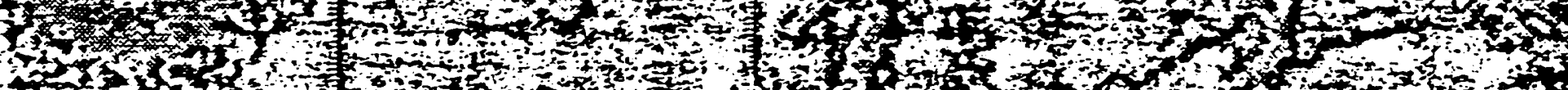

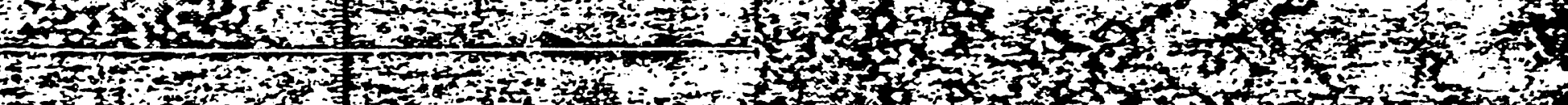

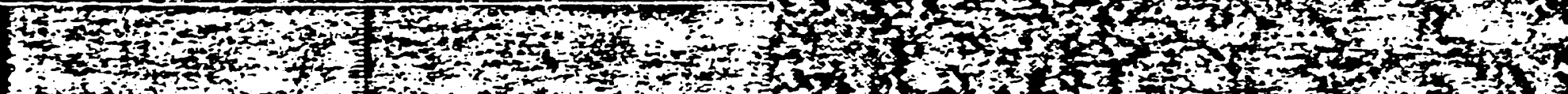

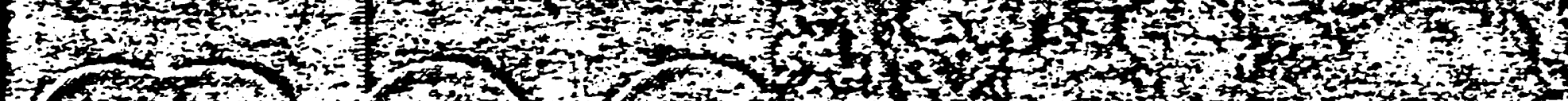
(1) 

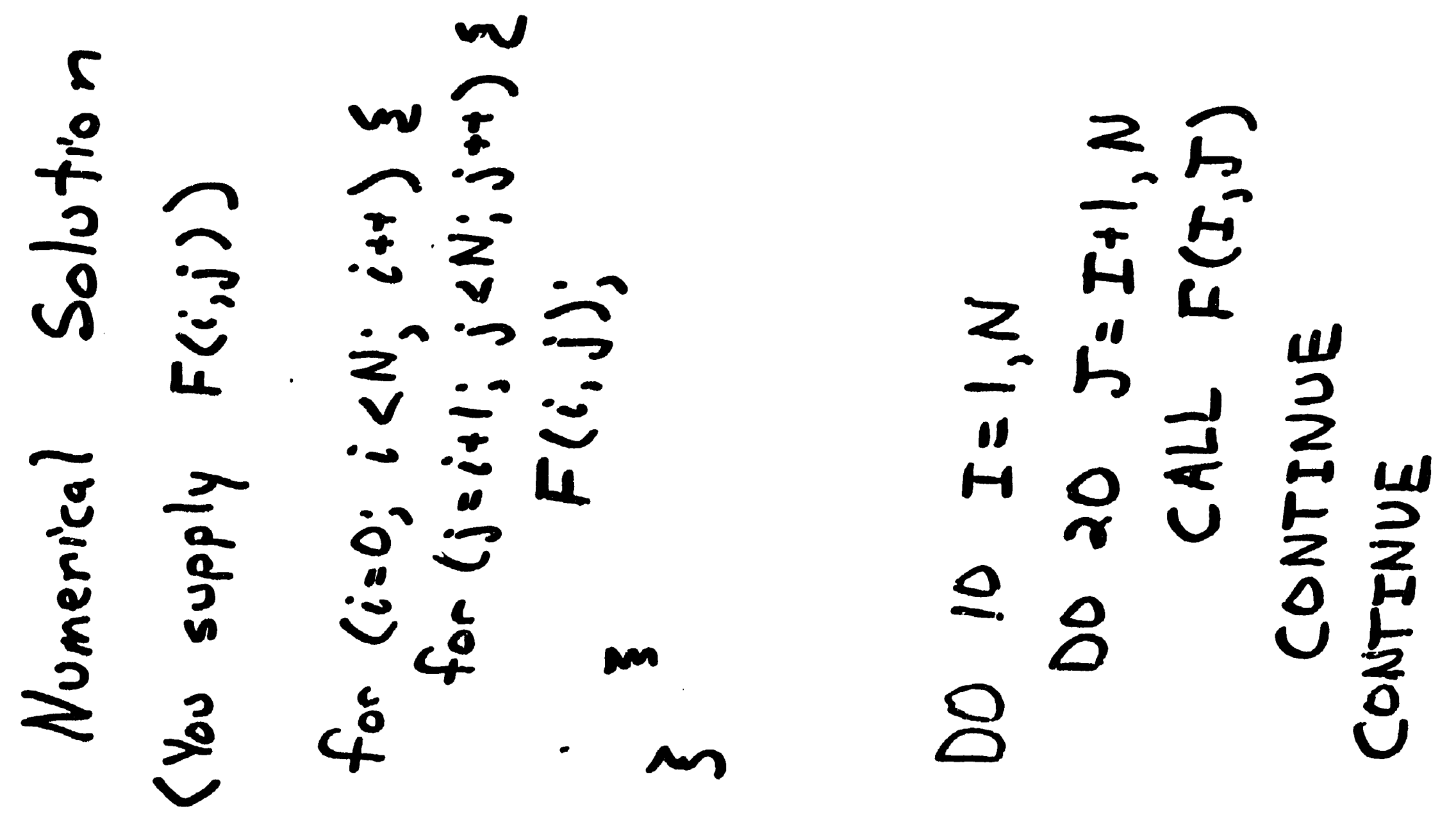

웅 


\section{The N-body Problem}

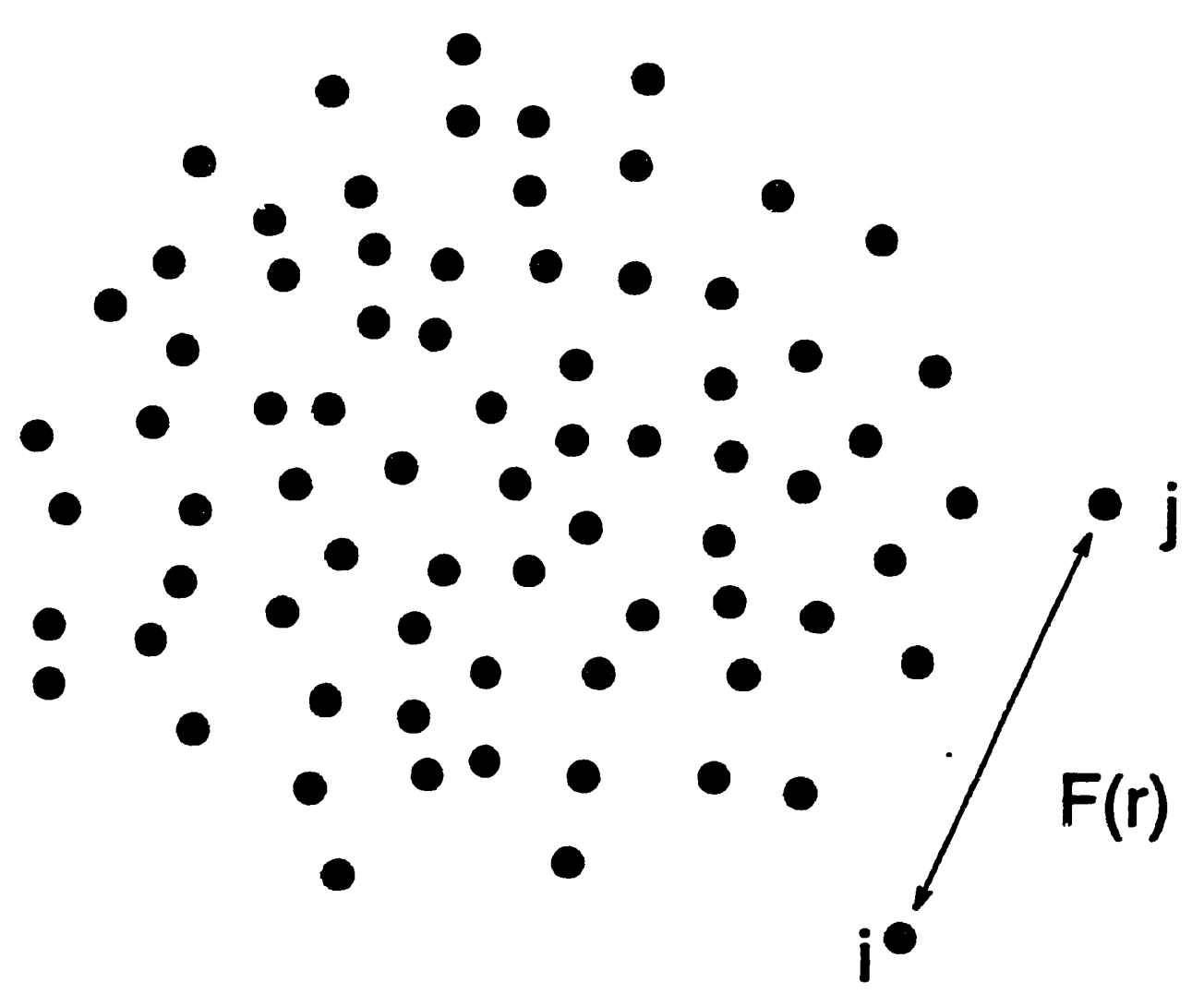




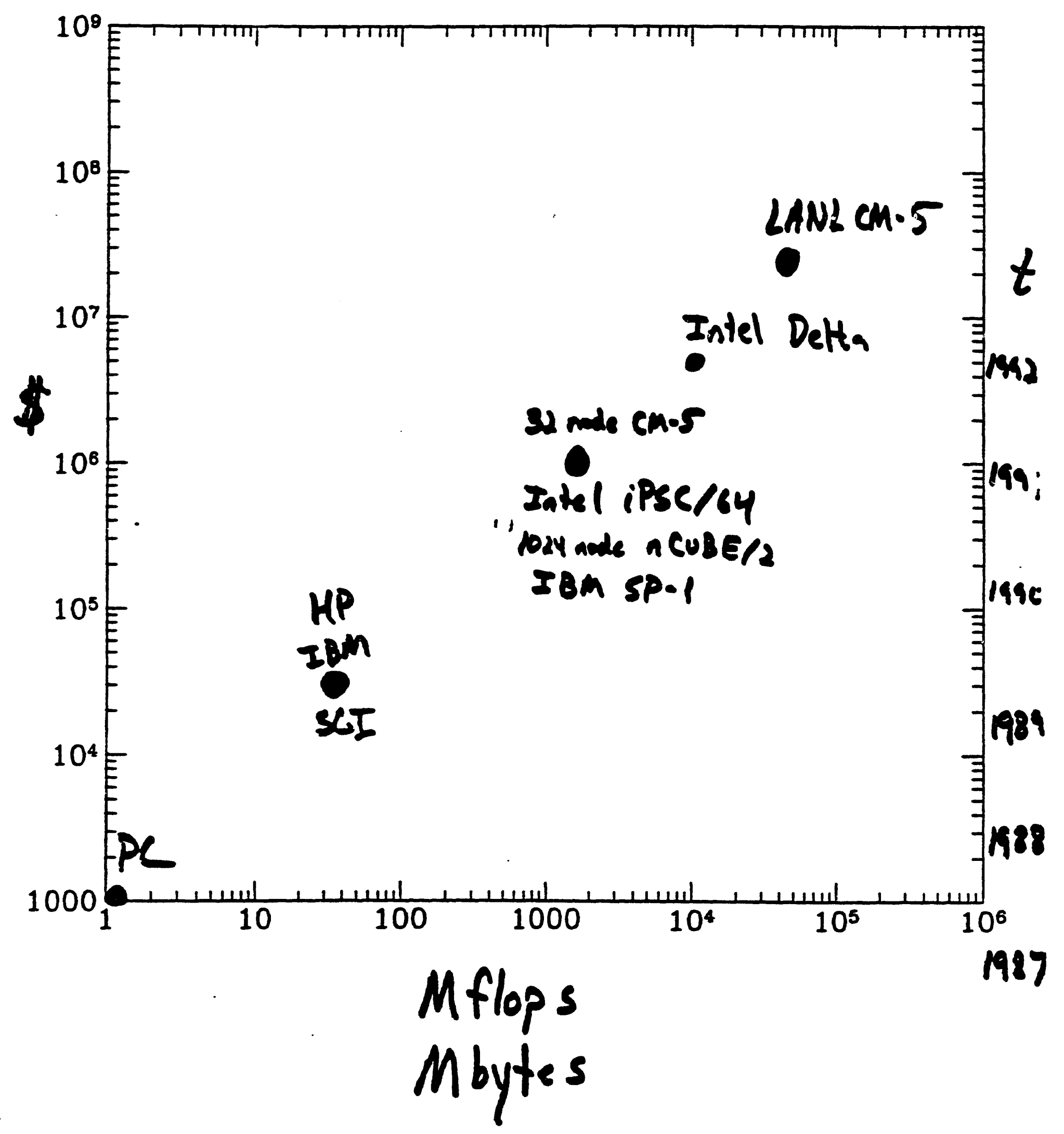




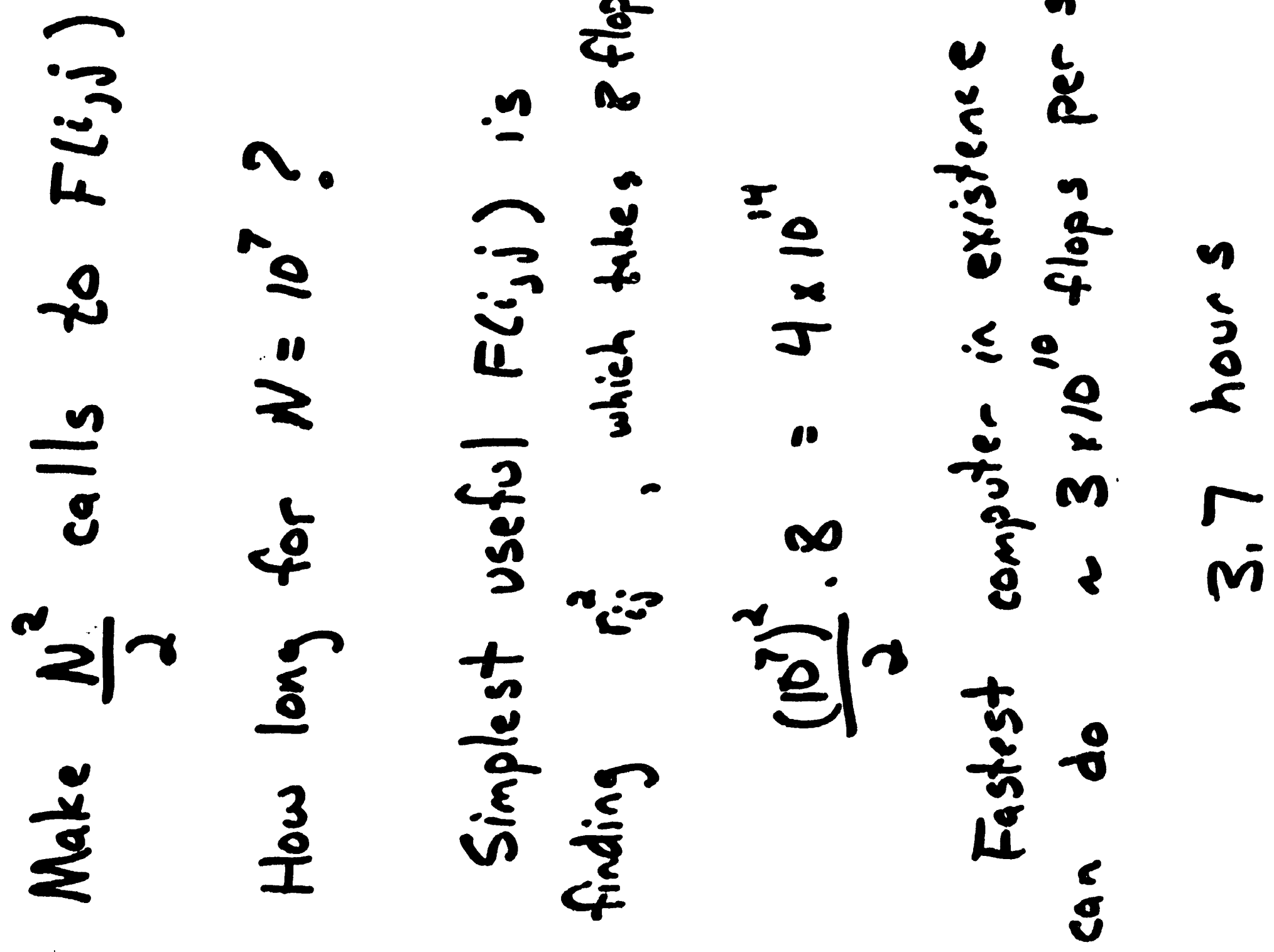




\section{Multipole Approximation}
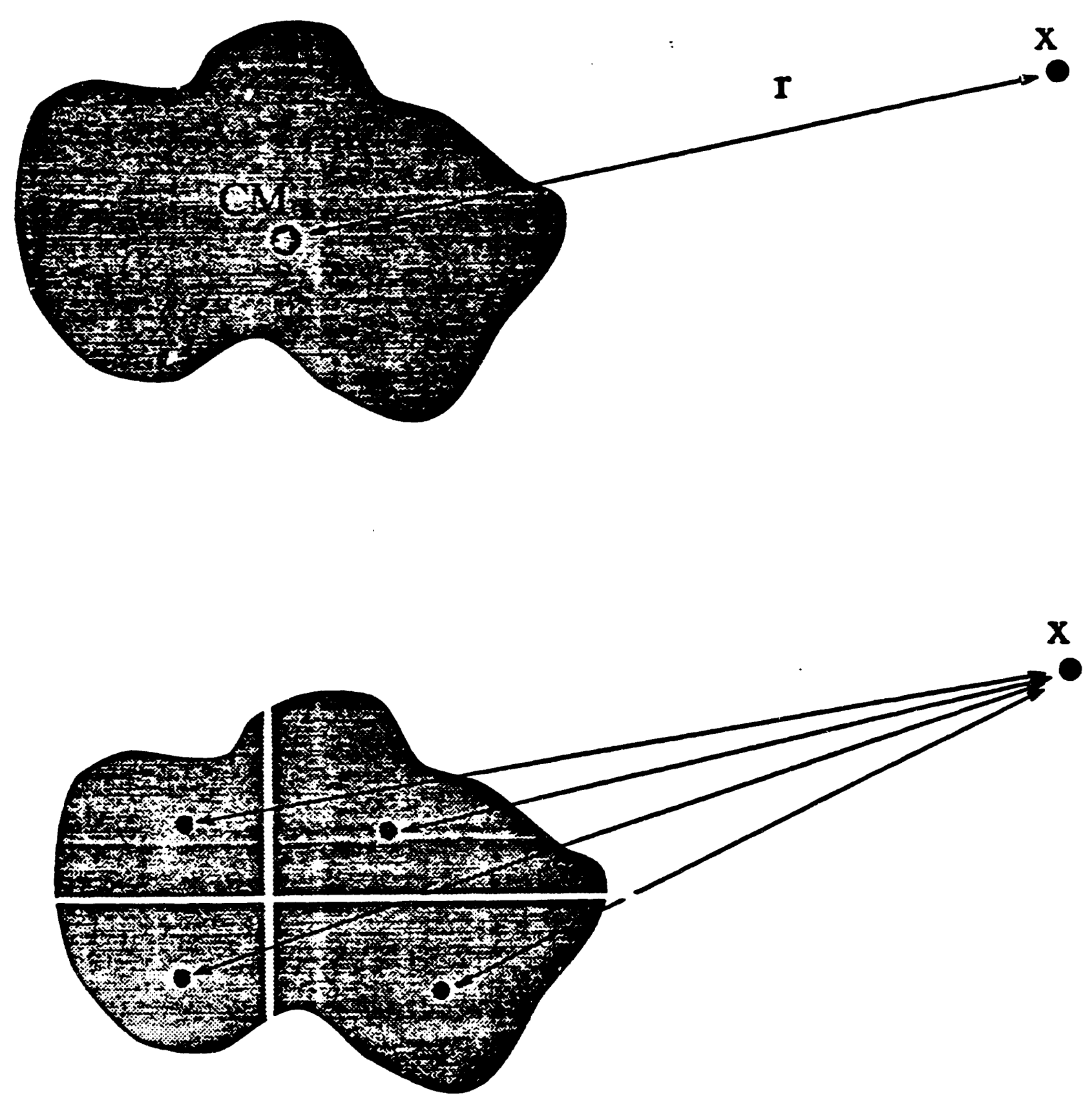


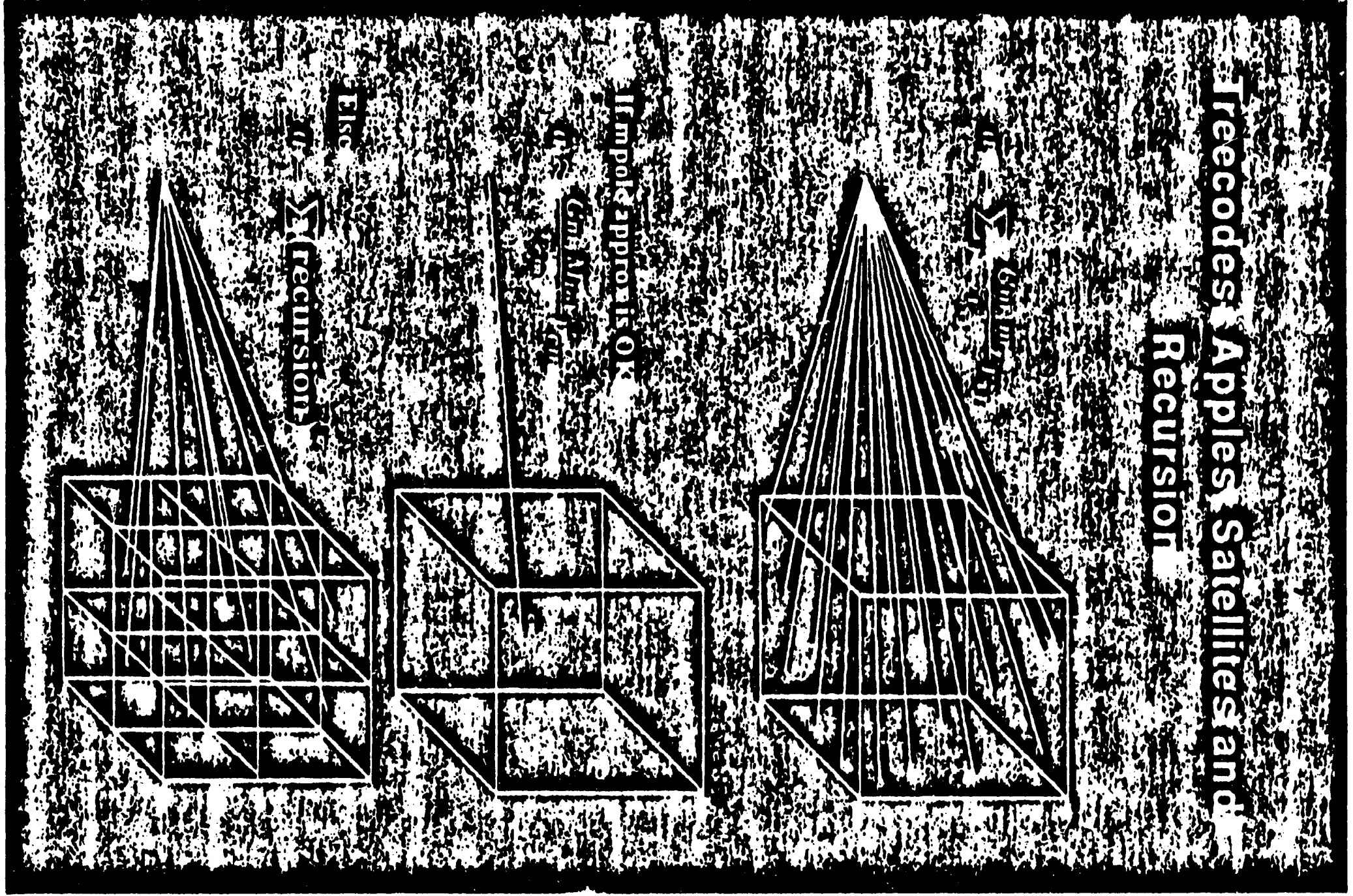




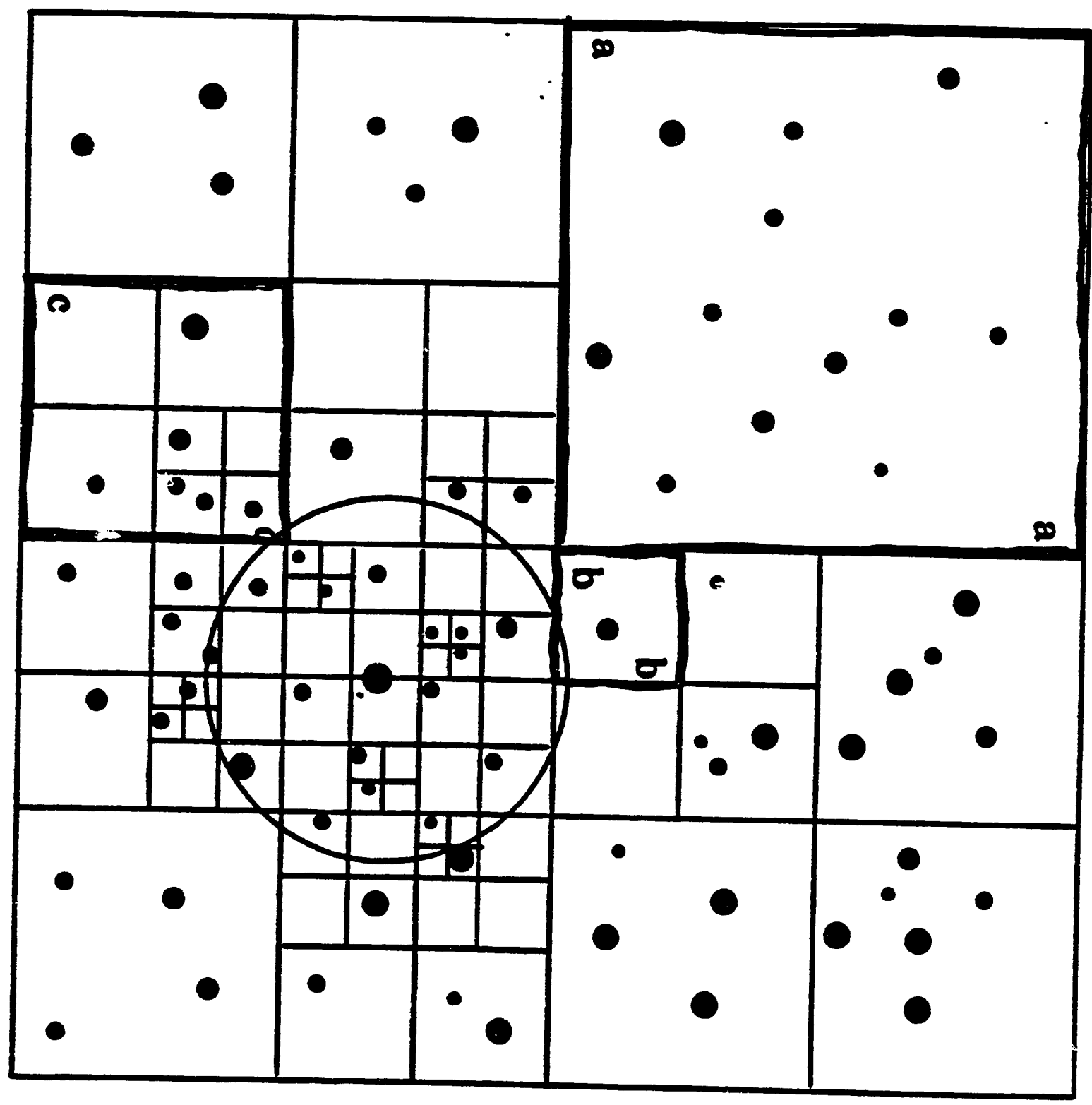




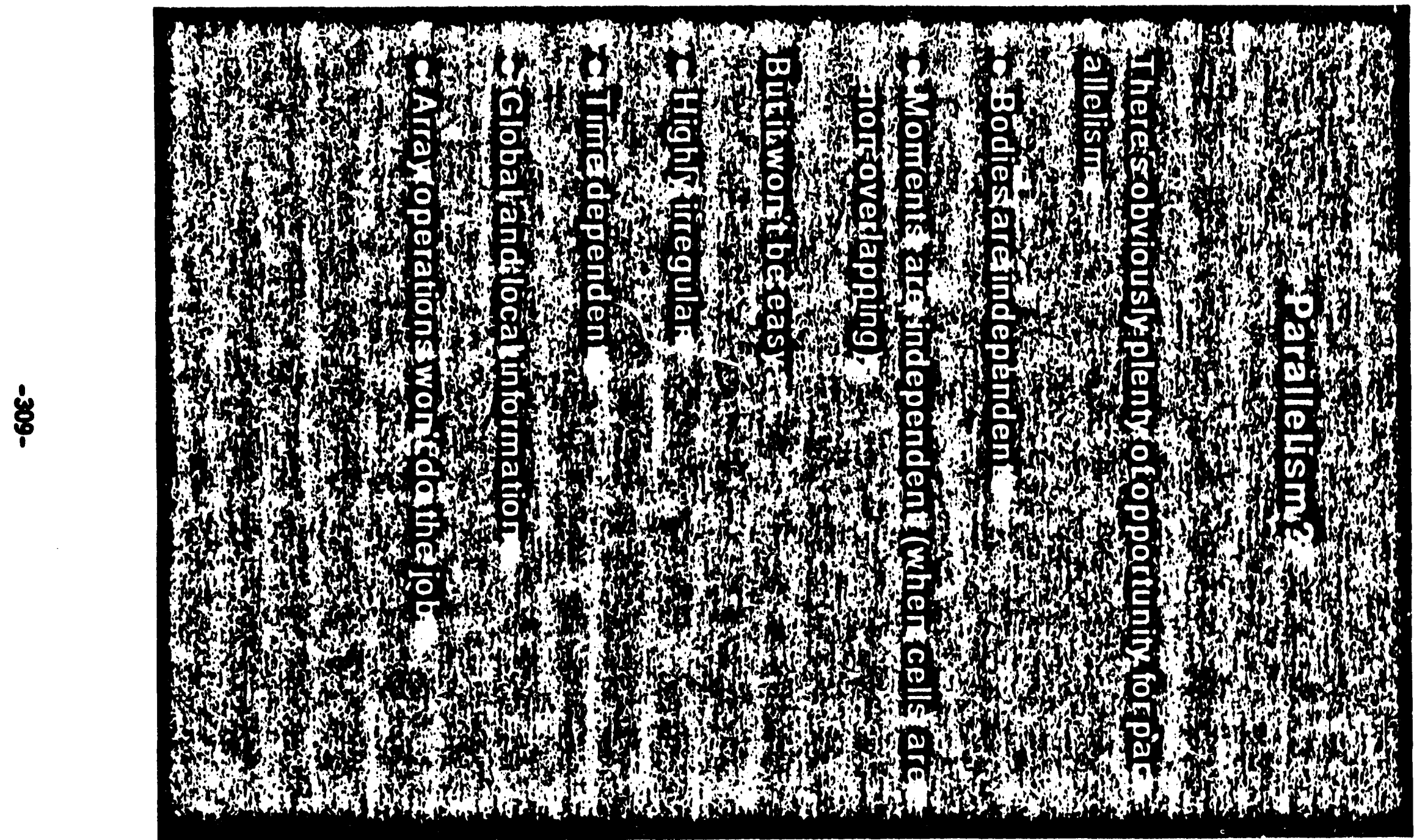




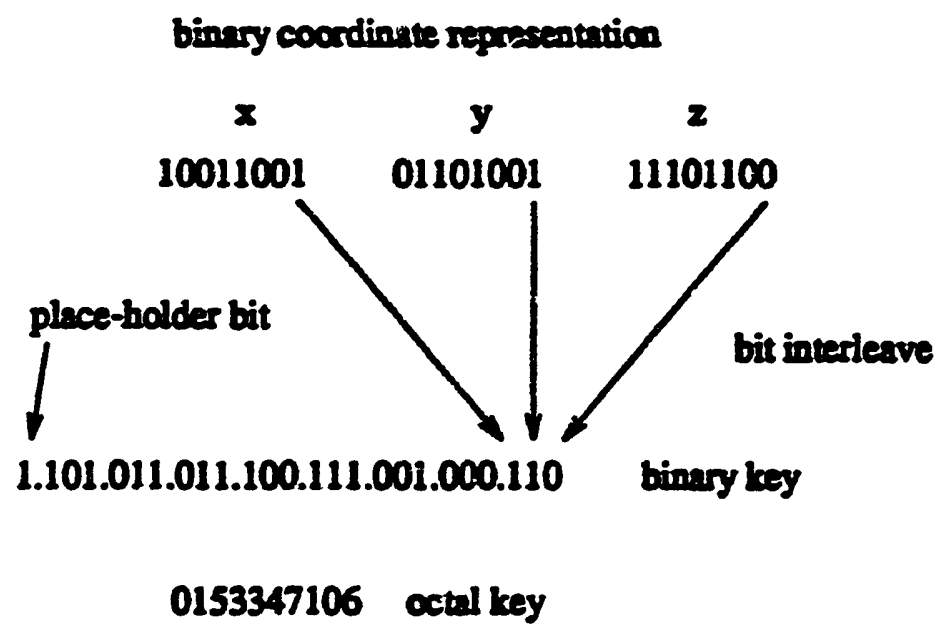



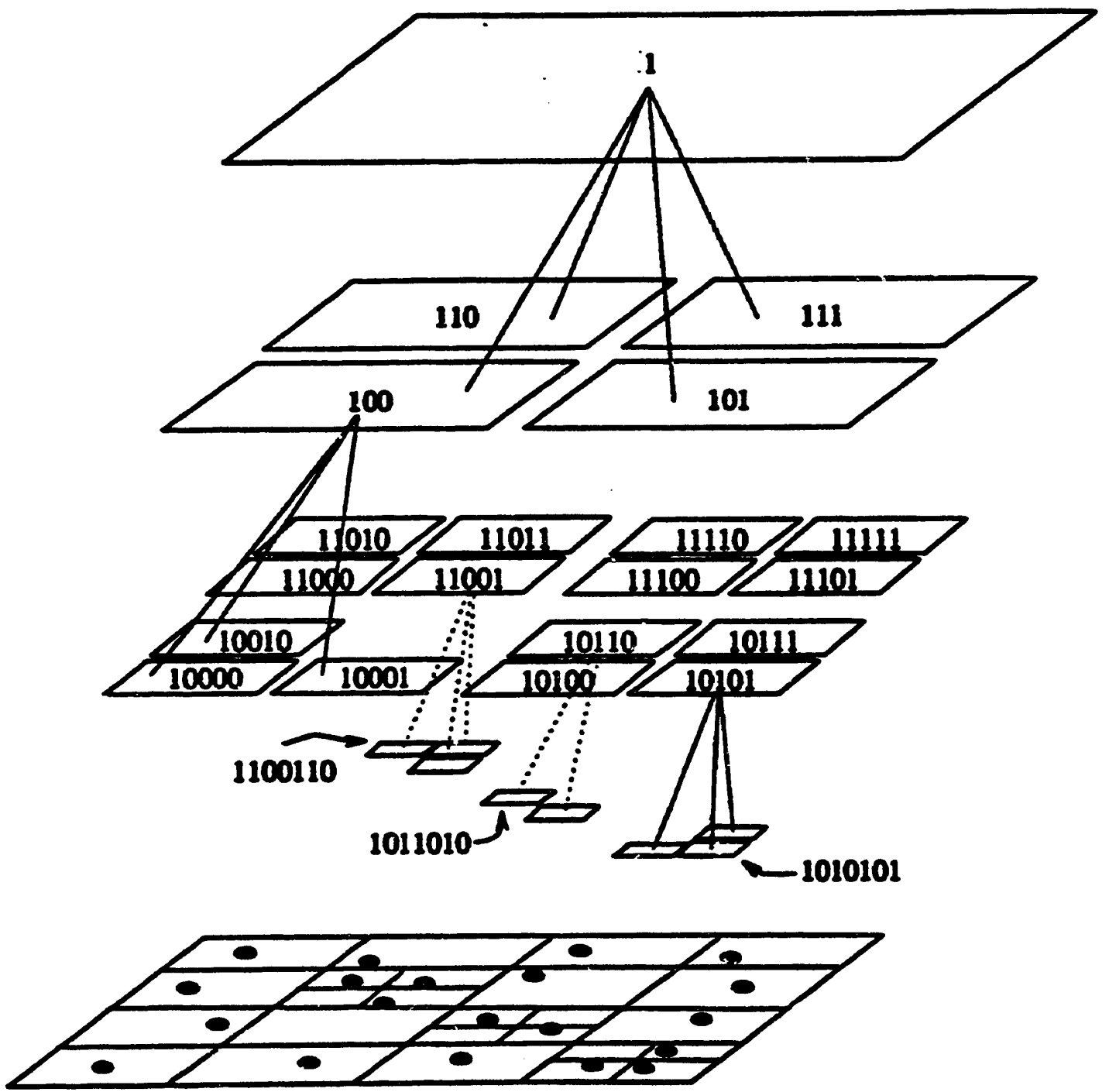


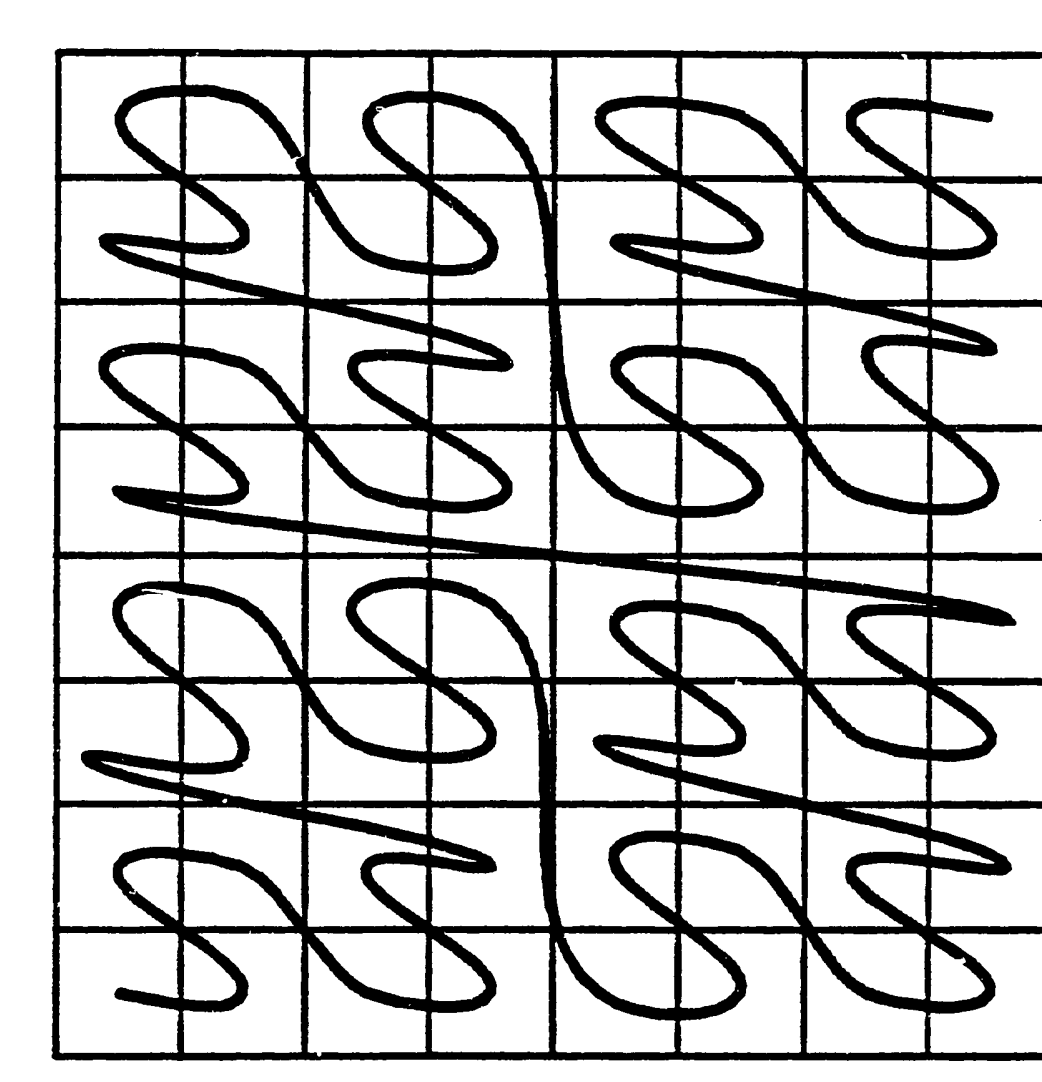




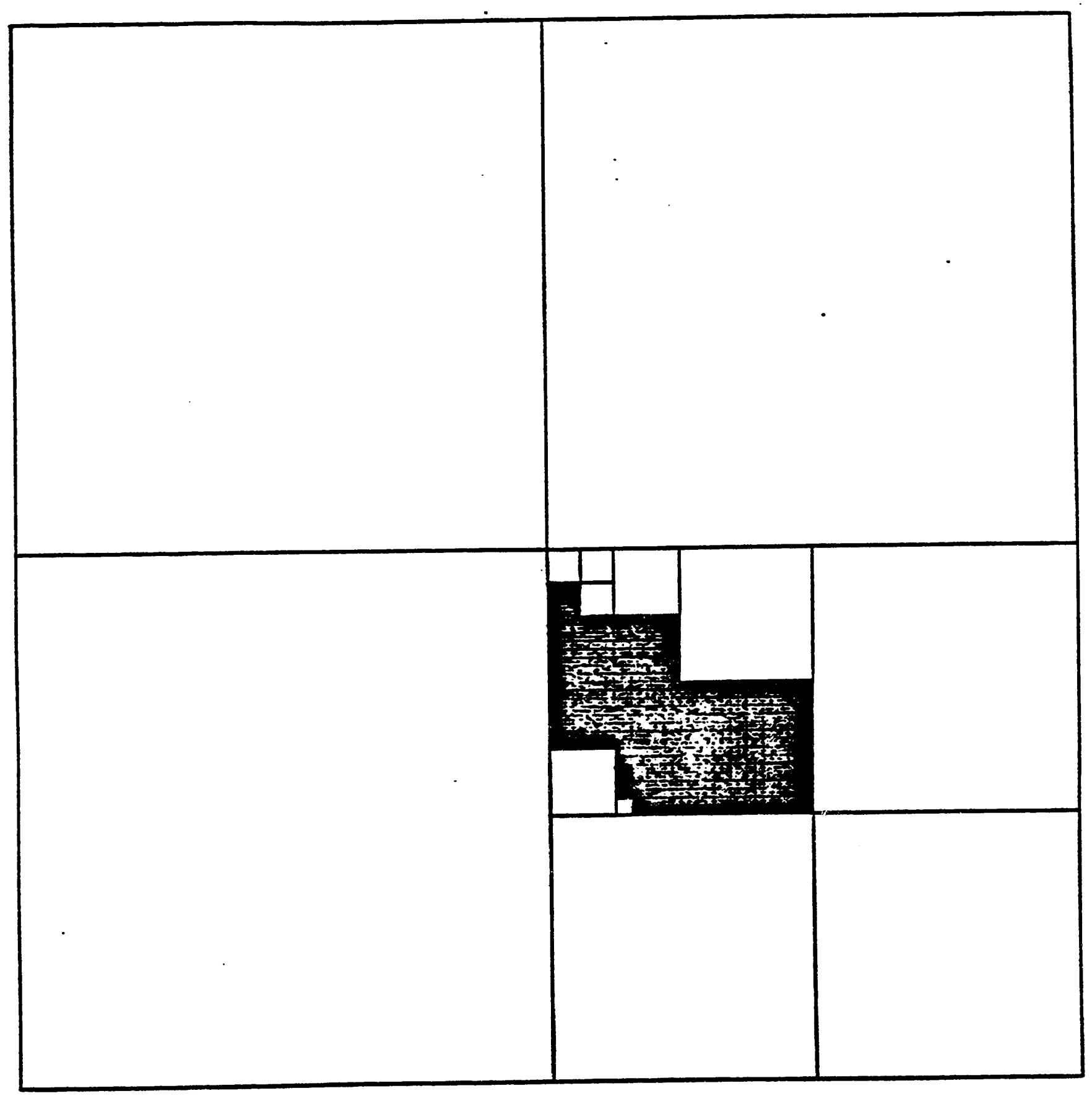

$-313-$ 


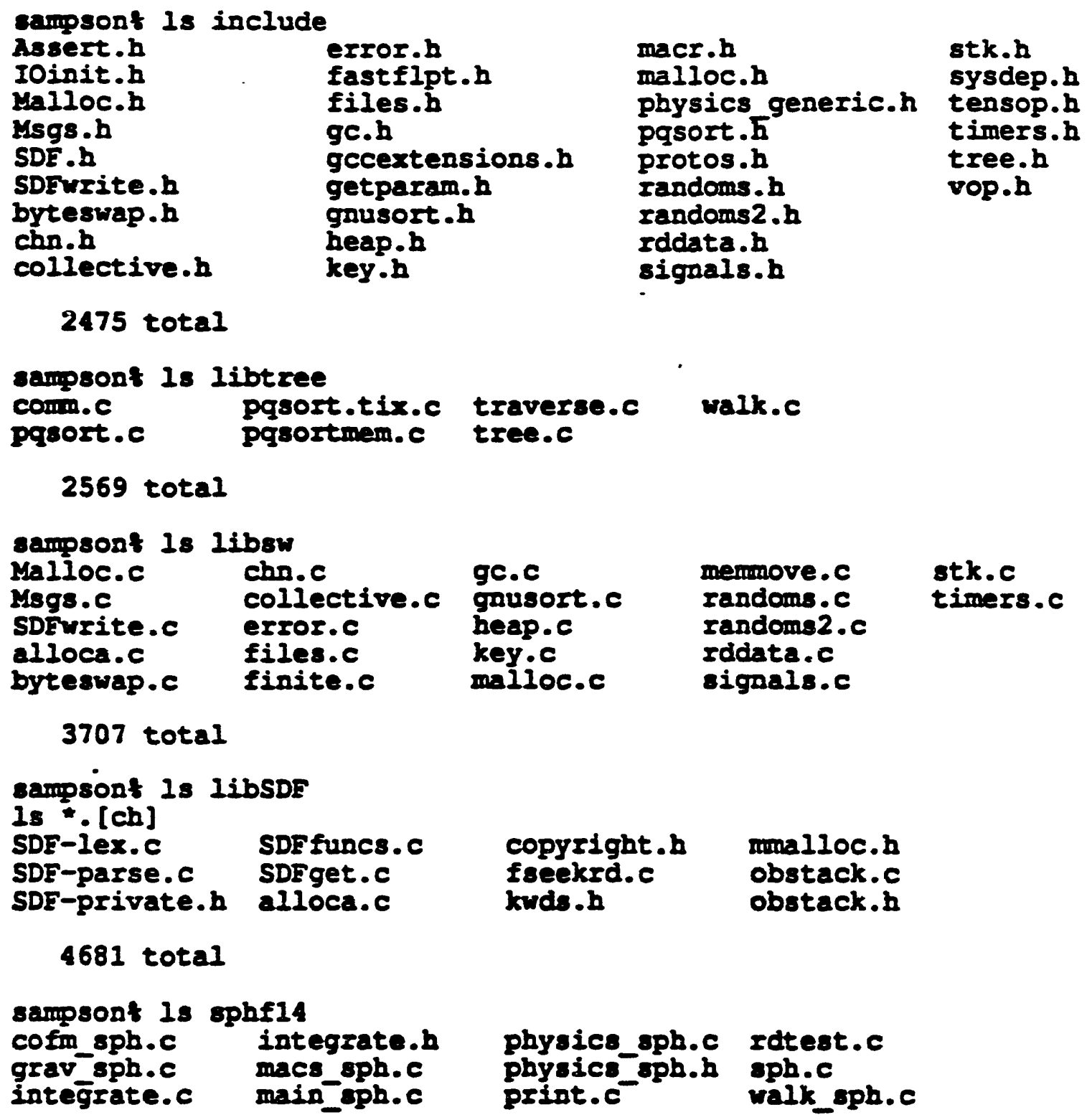

error.b

Eastelpt.h

Eiles.b

gc.h

gccextensions.h

getparam. $\mathbf{b}$

gnusort.h

heap.h

key.h

macr.h

malloc.h

physics generic.h

pqsort. $\bar{h}$

protos.h

randoms . h

randoms $2 . \mathbf{b}$

rddata.h

signals.h

stk.h

sysdep.h

tensop.h

timers.h

tree.h

vop.h

\section{5 total}

gc.c

gnusort.c

heap.c

key.c

malioc.c

3707 total

sampsont 18 libSDE

$18 *$. [ch]

SDE-lex.c

SDF-parse.C

SDEfuncs.C

SDFget.C

SDE-private.h

alloca.c

4681 total

sampsont is sphf14

cofin_sph.c integrate.h

grav_sph.c macs_sph.c

integrate.c

copyright.h freekrd.c kwds. $h$

stk.c

timers.c

\section{5 total}

sampoont 18 gysdep

\section{data.c oyadep_eui.c}

ivfprintf.c sysdep_express.c

singlprinte arv.C

singlPrintéunix.c sysdep_com $5 . \bar{c}$

sysdep_ncube.c

sysdep_nx.c

oysdep_seq.c
oyadep_5r.C timers_clock.c timers cons.c timers-gettimer.c timers_bwelock.c timers mclock. $C$ timers ntime.c timers_readrtc.c

\section{7 total}




\section{SPH Performance}

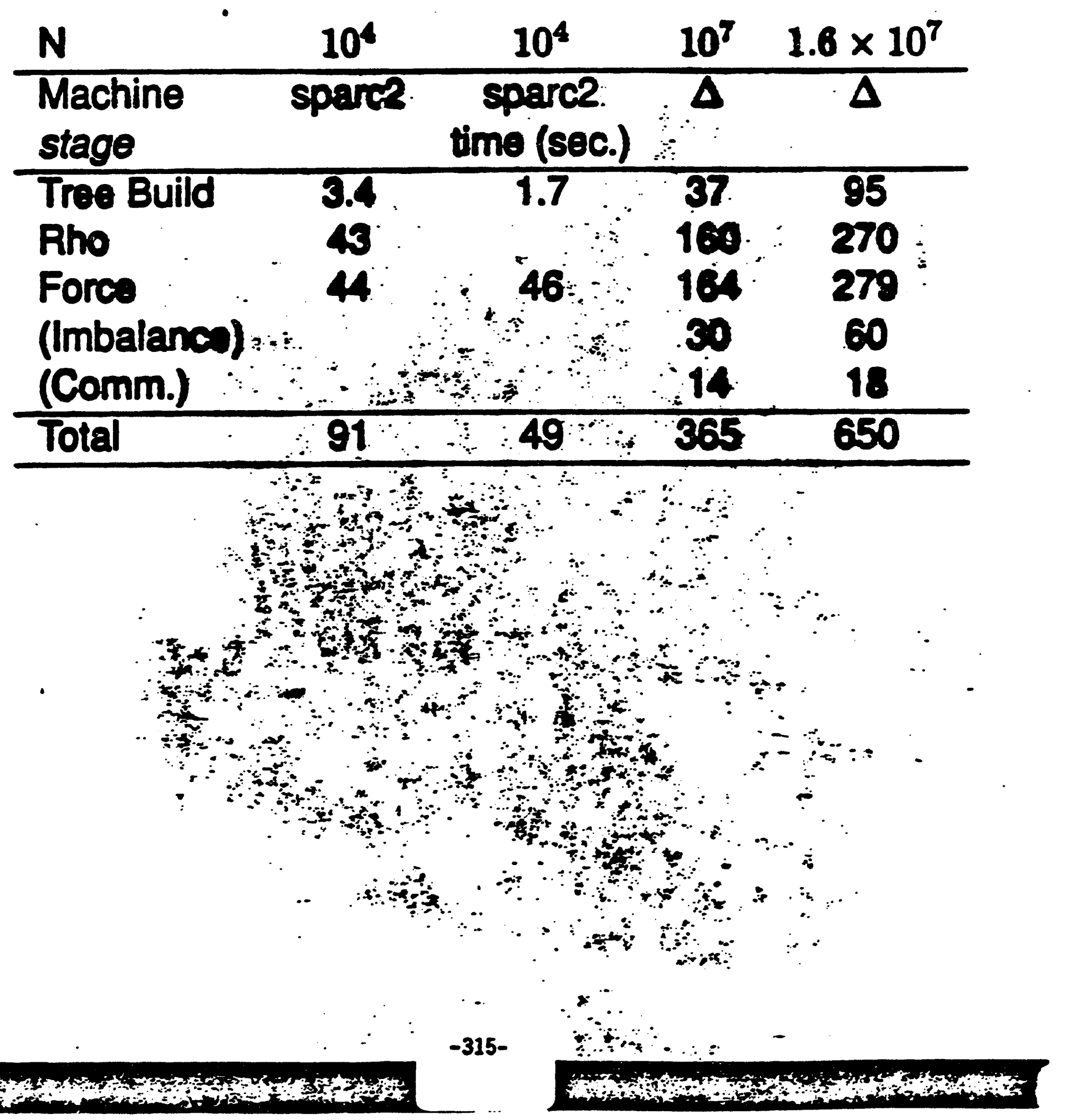




\section{a 120 bytos / particle}

typedef struct \{

float mass;

float pos [NDIM] ;

float vel[NDIM] ;

float $h$;

float rho;

float pr;

float vsound;

float rho est;

Key_t key;

float acc[NDIM] ;

float phi;

float u;

float udot;

float pos last [NDIM] ;

float udot last;

float drho dt;

float hdot;

unsigned int ident;

unsigned int nterms;

unsigned int nbrs;

\} body, *bodyptr; 


\section{Modelling Relativistic Collapse: SPH vs FEM .}

Patrick J. Mann

Department of Applied Mathematics

University of Western Ontario.

Now, here, you see, it takes all the running you can do, to keep in the same place. If you want to get somewhere else, you must run twice as fast as that. "Through the Looking Glass", Lewis Carroll, 1871 


\section{Rationale}

\section{Einstein's Equations}

- Extremely non-linear

- complicated

- few analytic solutions in non-linear regimes

$$
\text { Therefore }
$$

\section{Two Possibilities}

1. Self-Checking codes:

2. Code-Code comparison: $* * * *$ 
Self-Checking Codes

- M. Choptuik: multi-grid methods

- Spherical, Vacuum, Scalar fields.

- Advanced Numerical Methods.

Code-Code Comparison

- Spherical Symmetry: done

- A handful of non-spherical simulations.

- Basic Method finite differences

Alternate Methods

- Spectral Methods J. Marck, S. Bonnazola

- Multi-grid Methods M. Choptuik, A. Lanza

- Finite Element Methods P. Mann

- Particle Methods P. Mann, M. Dubal, W. Miller, A. Kheyfets

Direct Comparison

- FEM vs SPH P. Mann 


\section{Choice of Methods}

- self-gravitating fluid

- Evolution equations + elliptic constraints

- non-spherical

- robust, Easy (at least 2 codes)

Finite Element Method: FEM

- Standard, Well-developed

- Particularly good for non-symmetric problems

- Equations appear only in quadrature routines

Smoothed Particle Hydrodynamics: SPH

- Astrophysical standard

- Easy physical basis

- Wonderful for gravity (clustering) 


\section{Which SPH?}

- Relativity: problem with length. (proper vs coord vs ...)

- Therefore must extend classic SPH

There are two generic approaches

1. Physical particles

- Particles move on fluid characteristics

- Particles move with locally flat coords

- (see Kheyfets, Miller)

2. Numerical Interpolation

- "Particles" parametrize an interpolation

- Interpolation solves set of pde's 


\section{ADM 3+1}

The sph interpolation requires:

- time: initial value

- space: boundary value forget Einstein

- mass: conserved

therefore:

- ADM 3+1 space-time split

- Standard, Universally used 


\section{ADM 3+1 (cont'd)}

J.W.York, "Kinematics and Dynamics of General Relativity" in Sources of Gravitational Radiation, ed. L. Smarr

\section{This Project:}

- Spherical metric

- Schwarzschild Coordinates

- $t=$ time at infinity

- $r=$ areal radius

- $x=r \cos (\theta)$, etc.

- 2 elliptic equations on a time slice

- particles averaged onto 1d, spherical grid

- FEM solver for metric

- metric interpolated onto particles

\section{Metric:}

$d s^{2}=-b^{2}(1-2 m / r) d t^{2}+d r^{2} /(1-2 m / r)+r^{2} d \Omega^{2}$

where $b=b(r, t)$ and $m=m(r, t)$. 


\section{Fluid Equations}

Euler's Equation

$$
\begin{aligned}
& S_{a, t}+\nabla \cdot\left(\vec{v} S_{a}\right)=-b p_{, a}+\frac{1}{2} w D u^{t}[ \\
& 1-\frac{x^{a}}{r}\left(2 b \frac{\partial b}{\partial r}\left(1-\frac{2 m}{r}\right)+\right. \\
& \frac{\partial}{\partial r}\left(1-\frac{2 m}{r}\right) \\
& \left(1-\frac{2 m}{r}\right)^{2} \\
& \left.\quad+2 f \frac{\partial v^{r}}{\partial r} \frac{1}{r}\left(b^{2}\left(1-\frac{2 m}{r}\right)^{2}+\left(v^{r}\right)^{2}\right)\right) \\
& \quad]
\end{aligned}
$$

Internal Energy Equation

$$
E_{, t}+\nabla \cdot(E \vec{v})=-p A \nabla \cdot \vec{v}-p \frac{d A}{d t}
$$

Mass Conservation

$$
D_{, t}+\nabla \cdot(D \vec{v})=0
$$

where

$$
\begin{gathered}
A=u^{t} \sqrt{-g} \quad S_{\alpha}=D w u_{\alpha} \\
\text { 3-velocity }=v^{\alpha}=u^{\alpha} / u^{t} \\
E=A e \quad D=A \rho_{0} \\
\rho=\rho_{0}+e \\
w=(p+\rho) / \rho_{0}
\end{gathered}
$$


S, A, v Relationships

$$
\begin{gathered}
A=\frac{1}{w D} \sqrt{\frac{(w D)^{2}+S^{2}-\frac{2 m}{r} S_{r}^{2}}{1-2 m / r}} \\
v^{a}=\frac{b}{A} \frac{1}{w D}\left(S_{a}-\frac{2 m}{r} S^{r} \frac{x^{a}}{r}\right)
\end{gathered}
$$

- Nasty

- All variables involved (fluid, metric, even pressure)

- Iterate corrector (twice) 


\section{SPH: Density}

$D$ gives a conserved rest mass.

Therefore write

$$
D=\sum_{k} m_{k} W_{k}
$$

where

- $W_{k}=W\left(\left|\vec{r}-\vec{r}_{k}\right|, h_{k}\right)$

- $m_{k}(t)$ : particle rest mass

- $\vec{r}_{k}(t)$ : particle position

- $h_{k}(t)$ : smoothing length 


\section{SPH: Momentum \\ Internal Energy}

$\vec{S}$ is a momentum: $\vec{S}=D \vec{Z}$

Therefore

$$
\vec{S}=\sum_{k} \vec{Z}_{k} m_{k} W_{k}
$$

where $\vec{Z}_{k}(t)$ are interpolation parameters.

Also $E=D \epsilon$ so

$$
E=\sum_{k} \epsilon_{k} m_{k} W_{k}
$$

- mass-weighted interpolations

- Only $\vec{S}$ and $E$ used as physical quantities, not the parameters $\vec{Z}_{k}$ and $\epsilon_{k}$

- no divisions by $D\left(\vec{r}_{i}\right)$ 


\section{Discretization of Fluid Equations Weighted Residual Method}

- Insert approximation in equation

- Multiply by weight $F_{i}(\vec{r}, t)$

- Integrate over slice

$$
\int E_{, t} F_{i} d V=\int(-\nabla \cdot \vec{v} E-\ldots) F_{i} d V
$$

Time Derivative explicit differentiation:

$$
E_{, t}=\sum_{k} \dot{\epsilon}_{k} W_{k}+\epsilon_{k} \frac{d}{d t} W_{k}
$$

Weight Galerkin

- $F_{i}=W\left(\vec{r}-\vec{r}_{i}, h_{i}\right)$

- Lo'cal support: $h_{i}$ 


\section{Integration}

- (Gauss Quadrature: overlapping particles)

- "particle position" quadrature ***

Therefore

$$
\begin{aligned}
\sum_{j}\left(\sum_{k} \dot{\epsilon}_{k} W_{j k}\right) W_{i j}= & -\sum_{j}((\nabla \cdot \vec{v}) E)_{j} W_{i j} \\
& - \text { time derivative terms }
\end{aligned}
$$

- Matrix equation

- Sparse, but varying pattern

- Use condensation ("mass-lumping")

$$
\sum_{j} \sum_{k} \dot{\epsilon}_{k} W_{j k} W_{i j}=\dot{\epsilon}_{i} \sum_{j} \sum_{k} W_{j k} W_{i j}
$$

End up with

$$
\dot{\epsilon}_{i}=\ldots
$$

Similarly for

- $\dot{Z}_{i}(t)$

- $\dot{m}_{i}(t)$ 


\section{Code (Predictor-Corrector)}

1. Define $\dot{\vec{r}}_{i}, \dot{h}_{i}$ and update $h$ and $r$

2. Predict:
(a) Predict $Z_{i}, \epsilon_{i}, m_{i}$
(b) Sum $S_{i}, D_{i}, E_{i}$
(c) Compute $A, \vec{v}_{i}$
(d) Metric solution

3. Correct:
(a) Define $\dot{\vec{r}}_{i}$ and update positions
(b) Compute $\dot{m}_{i}, \dot{Z}_{i}, \dot{\epsilon}_{i}$
(c) Sum $S_{i}, D_{i}, E_{i}$
(d) Compute $A, \vec{v}_{i}$
(e) Metric solution
(f) Re-compute $A, \vec{v}_{i}$

Iterate corrector: " $P(E C)^{2}$ " 
NOTES

- Interpolations used only as interpolations

- Kernel defined in coordinate space

- Choose $\frac{d}{d t} \vec{r}_{i}=\vec{v}_{i}$

- (not necessary)

- $h_{i}$ using standard schemes

- Final $v_{i}$ evaluation is necessary

- Modified weight: include $r^{2} D$, etc. 


\section{C++ vs Fortran}

- Codes in both $\mathrm{C}++$ and Fortran

- FEM C++ is twice as fast as Fortran!!!

- Fortran data structure: $d(n), e(n), \ldots$

- C++ data structure: node[n].d, node[n].e,

- SPH C++ is slightly faster (insignificant)

- Double Sum!

- Same difference in data structure.

- $\mathrm{C}++$ is as advertised

- Carefully thought out objects

- Local objects

- understandable code

- Many more compiler errors, fewer runtime errors

- Reusable (eg. FEM/SPH i/o, Spherical solver) 


\section{Tests}

1. Shock Tube: SPH vs. FEM (done)

2. Spherical Symmetry: SPH vs. FEM (done)

3. 3d Collapse: SPH vs. FEM (in progress)

4. 3d Collision: SPH (in progress) vs. FEM (not yet)

Summary

- Comparable results

- SPH smooths more than FEM

- FEM is more sensitive to instability

- FEM is much faster for equivalent resolution

- (but no binning in SPH yet)

- SPH better during horizon formation

- Robust, Co-moving

- (almost freefall) 


\section{Future}

- Non-spherical Tests in progress

- Gravitational Radiation estimates in progress

- Gravity $\rightarrow$ Perturbed $\rightarrow$ Poisson's Eq'n : Colliding neutron stars

- Gravity $\rightarrow 2 \mathrm{~d}$ Axisymmetric in progress

- Gravity $\rightarrow$ 3d (Huge job here) 


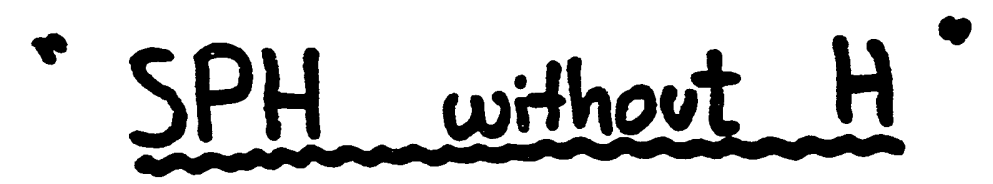

\section{Pablo Laguna Penn State}

I. Introduction (Why SPI?)

II.. SPI $\left\{\begin{array}{l}\text { a.. Tnuncation Ennors . Convengence } \\ \text {. Kennel }\end{array}\right.$ b.. Kennel

III.. Ware Equation $\left\{\begin{array}{l}\text { a.. Eulenian Tests } \\ \text { b.. Lagnangian Tests }\end{array}\right.$

III. Adrection

I.. Conclusions and Futune Wonk 
THEOREM:

Let SPH be smoothed panticle hydnodynamic: with a $C^{(n)}$ kennel $W$, if $H$ is not singulan then SPI (smoothed panticle intenpolation) exists fon $n$-th onden differential eqs.

PROOF:

a.. $H$ non-singulan $\Rightarrow \operatorname{det}(H) \neq 0 \Rightarrow$ $H^{-1}$ exists $\Rightarrow H H^{-1}=I$

b.. $S P H H^{-1}=S P I$

$-3360$ 
SPH :

1.. Computes denivatives without a grid.

2.. Lagnangian natune.

3.. Easy implementation.

$$
\begin{aligned}
& \text { Fully adaptive SPH : }\left\{\begin{array}{c}
\text { Variable } h \\
\text { Non-sphenical W } \\
\Downarrow
\end{array}\right. \\
& \text { (easy implementation?) } \\
& \text { Q.M. }=\left\{\begin{array}{l}
\text { - Panticles } \\
\text { - Waves }
\end{array}\right. \\
& \text { S.P.H. }=\left\{\begin{array}{l}
\text {. Particles (Physies) } \\
\text { - Intenpolation (Numienies) }
\end{array}\right.
\end{aligned}
$$




$$
S P H+\left\{\begin{array}{l}
\text { FD: Hydao + Gaavity } \\
\text { Rasio } \\
\text { FE: Hydno + Gravity } \\
\text { Manum } \\
\text { N-body: HOM + COM Wannen E.ol }
\end{array}\right.
$$

Why not SPH + SPH?

Hydeo + Maguatic Fields Stellingwent? 
Kennel Estimation:

$W$ is spherical

$$
\begin{aligned}
& \langle\phi\rangle=\int \phi^{\prime} W d x^{\prime} \\
& \left\langle\frac{d \phi}{d x}\right\rangle=\int \frac{d \phi^{\prime}}{d x^{\prime}} W d x^{\prime}=\int \phi^{\prime} \frac{d W}{d x} d x^{\prime}=\frac{d}{d x}\langle\phi\rangle \\
& \left\langle\frac{d^{2} \phi}{d x^{2}}\right\rangle=\int \frac{d^{2} \phi^{\prime}}{d x^{\prime}} w d x^{\prime}=\int \phi^{\prime} \frac{d^{2} w}{d x^{2}} d x^{\prime}=\frac{d^{2}}{d x^{2}}\langle\phi\rangle
\end{aligned}
$$

An general $\quad\left\langle\frac{d^{n} \phi}{d x^{n}}\right\rangle=\frac{d^{n}}{d x^{n}}\langle\phi\rangle$

provided $W$ is of class $C^{n}$; that ir, its $n$-th derivatives exist and ane continuous.

Also

$$
\left\langle\frac{d^{n} \phi}{d x^{n}}\right\rangle=\frac{d^{n} \phi}{d x^{n}}+\theta\left(h^{2}\right)
$$

Trivial Jon $W a e^{x}$

-339- 

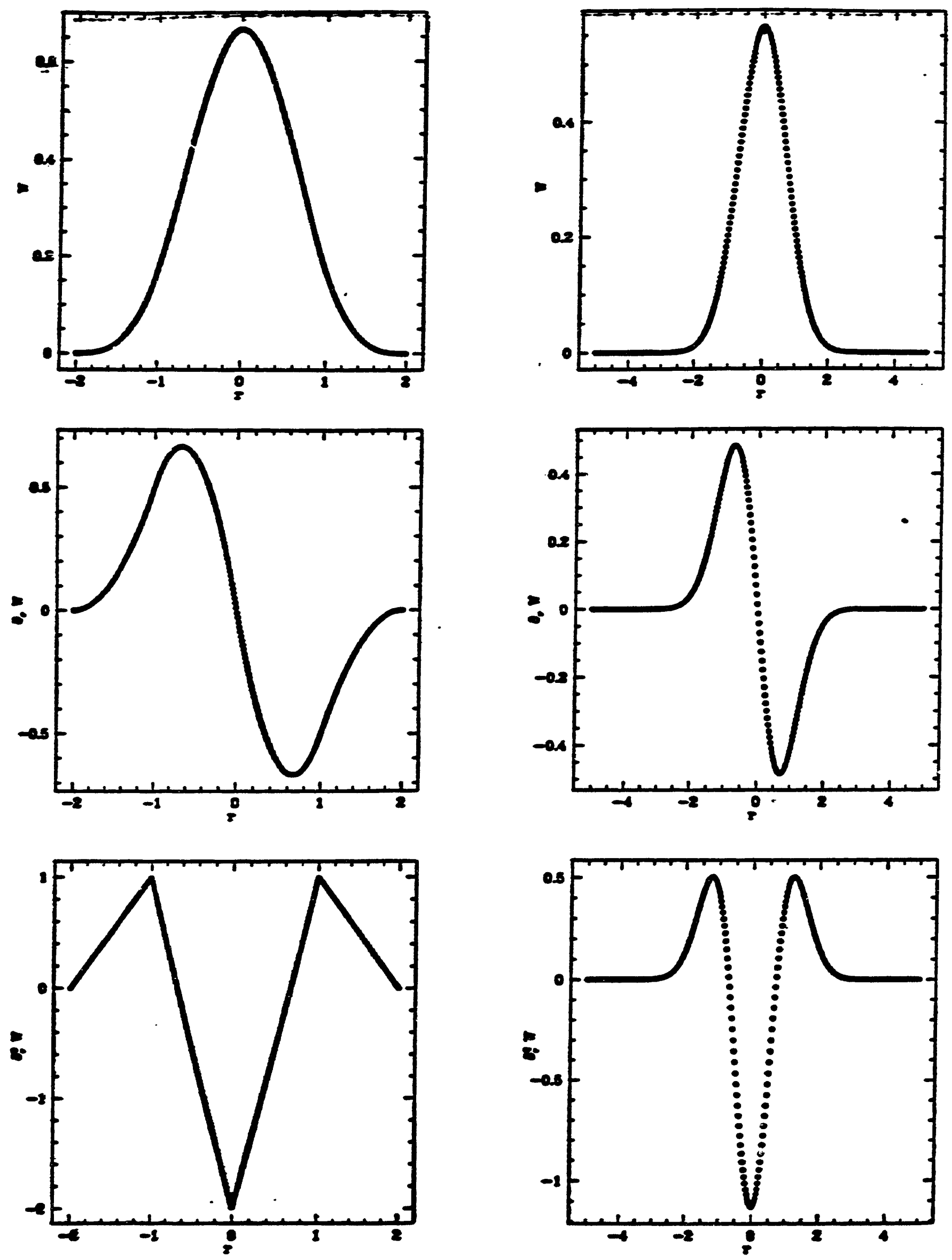

Spline

Gaussian 
ERROPS and CONUERGENCE

$$
\begin{array}{cc}
\mathcal{L} \phi=0 & \mathcal{L}: \text { diffenential operaton } \\
\hat{\mathcal{L}} \hat{\phi}=0 & \hat{\mathcal{L}}:\left[\begin{array}{l}
F E \\
F D \\
\text { SpI }
\end{array}\right] \text { represcutaction of } \mathcal{E} . \\
\text { Tnuncation Ennon } & \hat{\mathcal{J}}=\hat{\mathcal{L}} \phi \\
\text { Solution Ennon } & \hat{e} \equiv \phi-\hat{\phi}
\end{array}
$$

a discretization scheme is OPTIMALLY CONYERGENT 4

$$
\theta(\hat{\varepsilon})=\theta(\hat{\jmath})
$$

Is SPI optimally convengent?

Wave equation YES if the

\# of nieighbons is lange enough. 

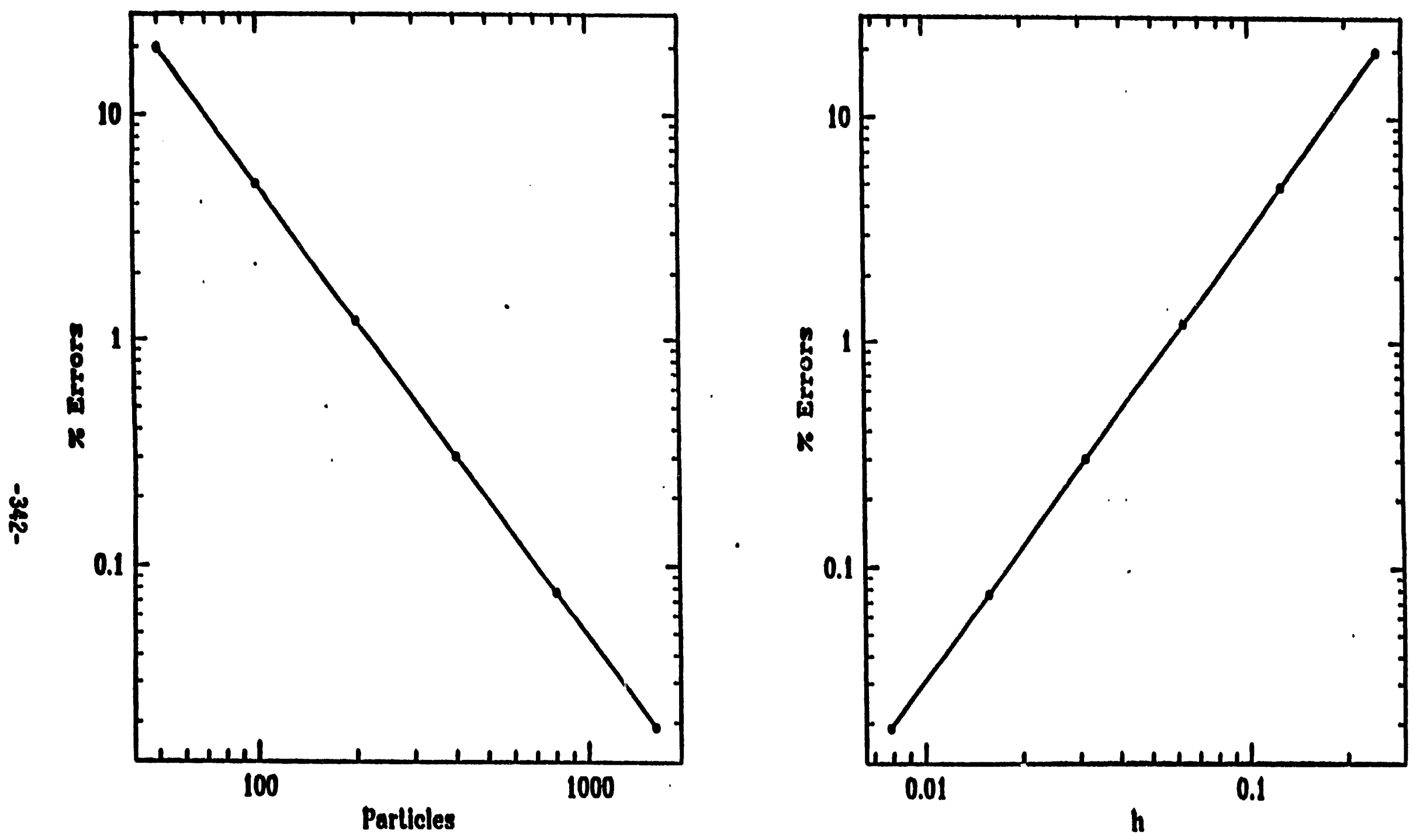

Neighbons $=20 \quad$. 

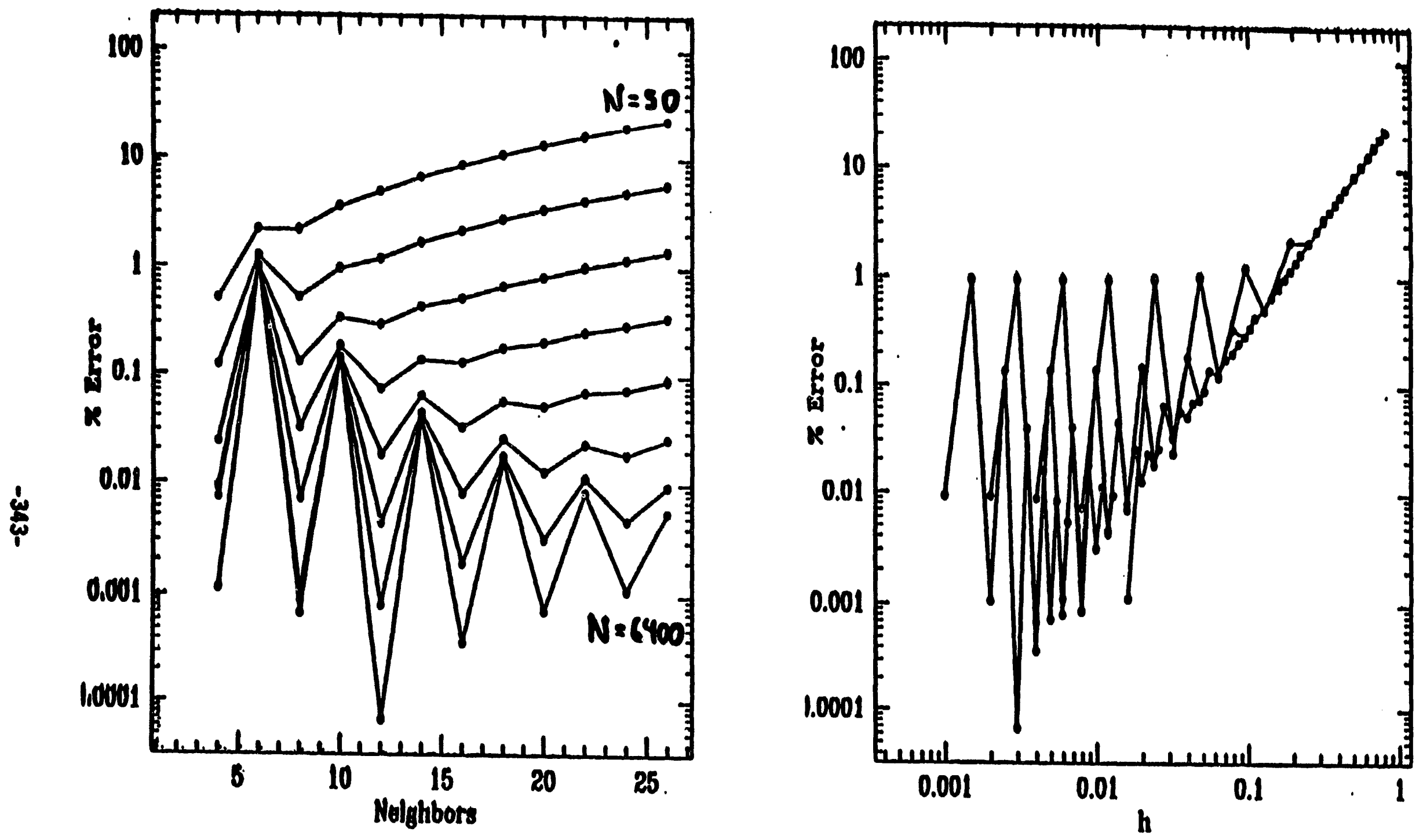

$$
\left\langle\frac{d \phi}{d r}\right\rangle-\frac{d \phi}{d p}=r_{1}
$$

Wespline 

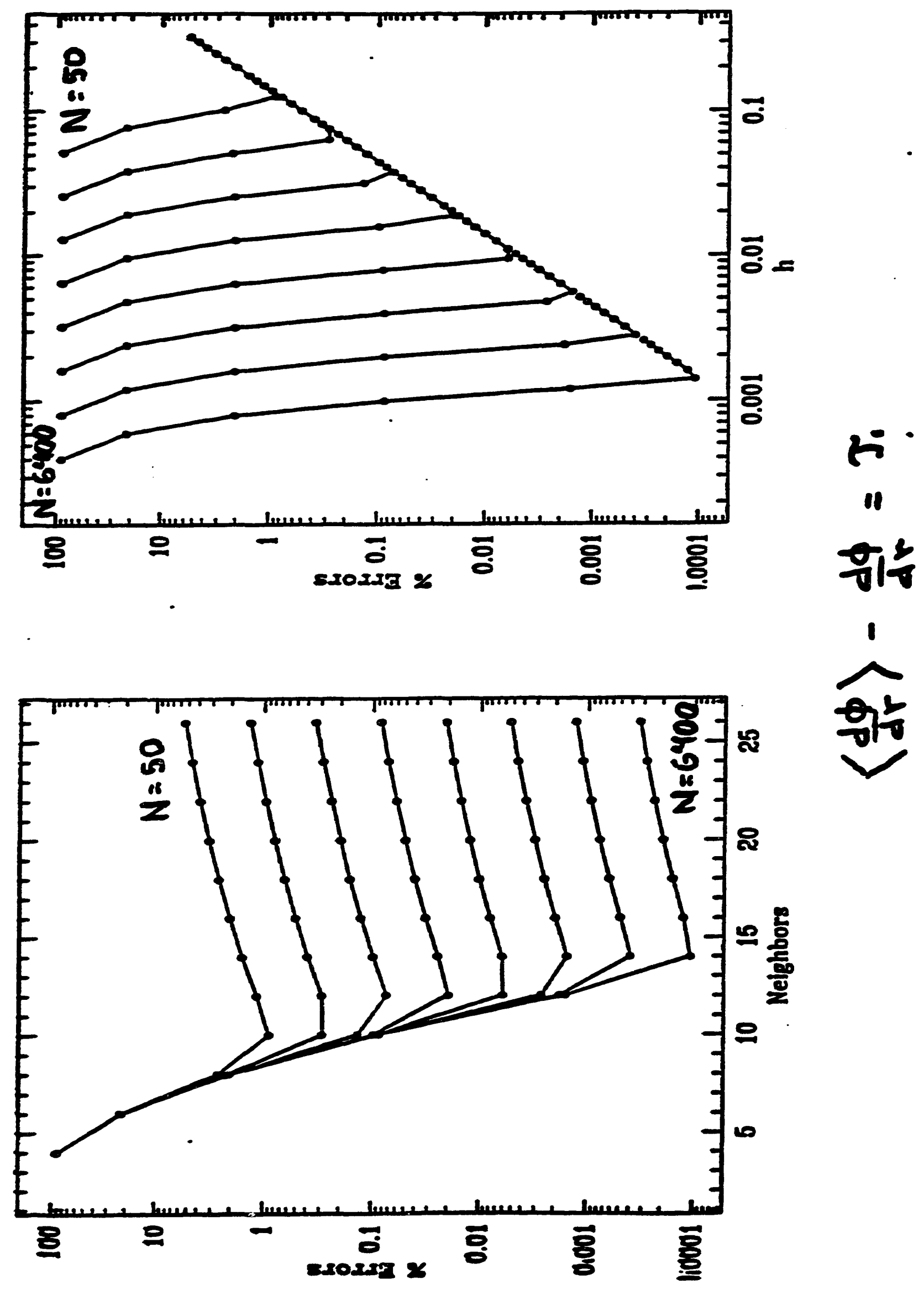

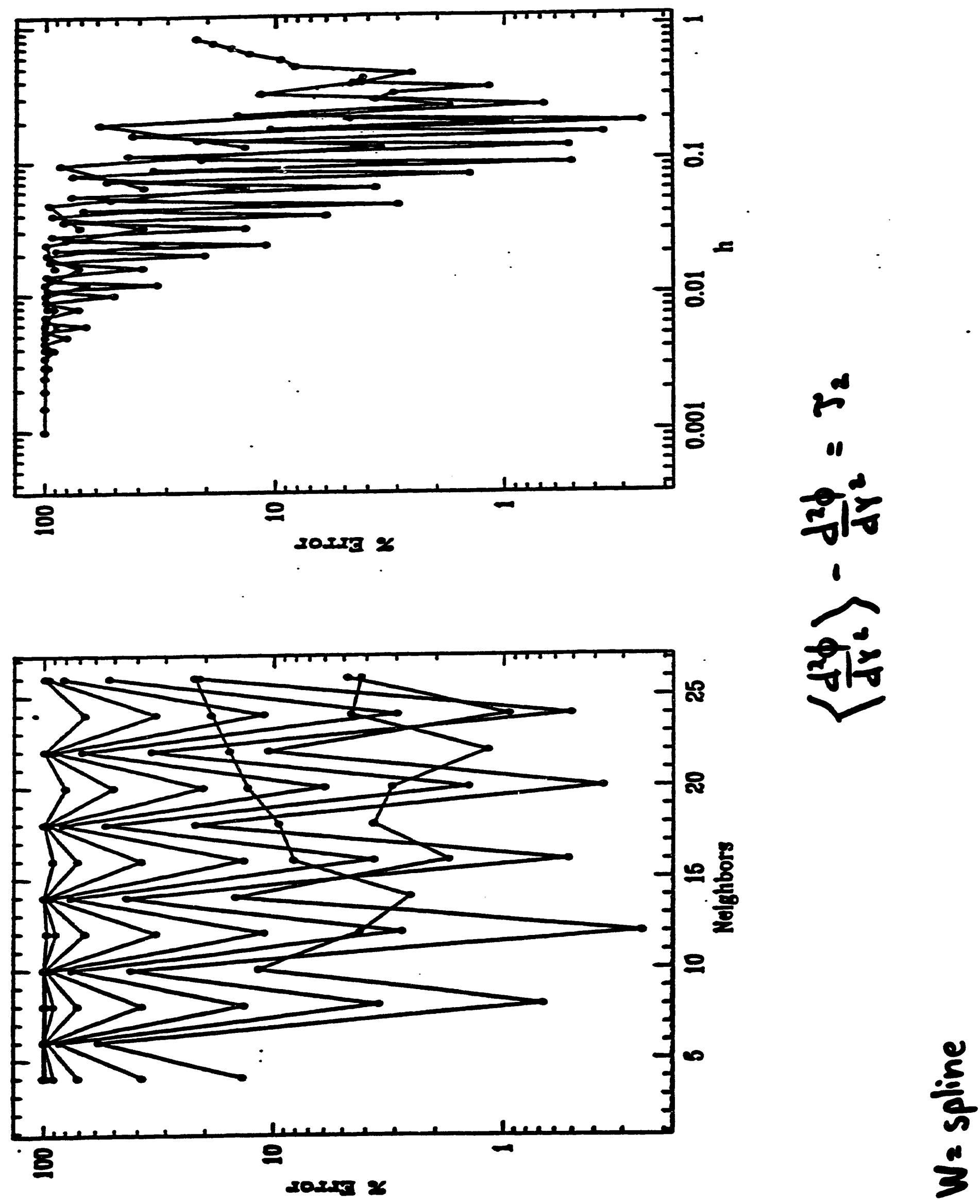

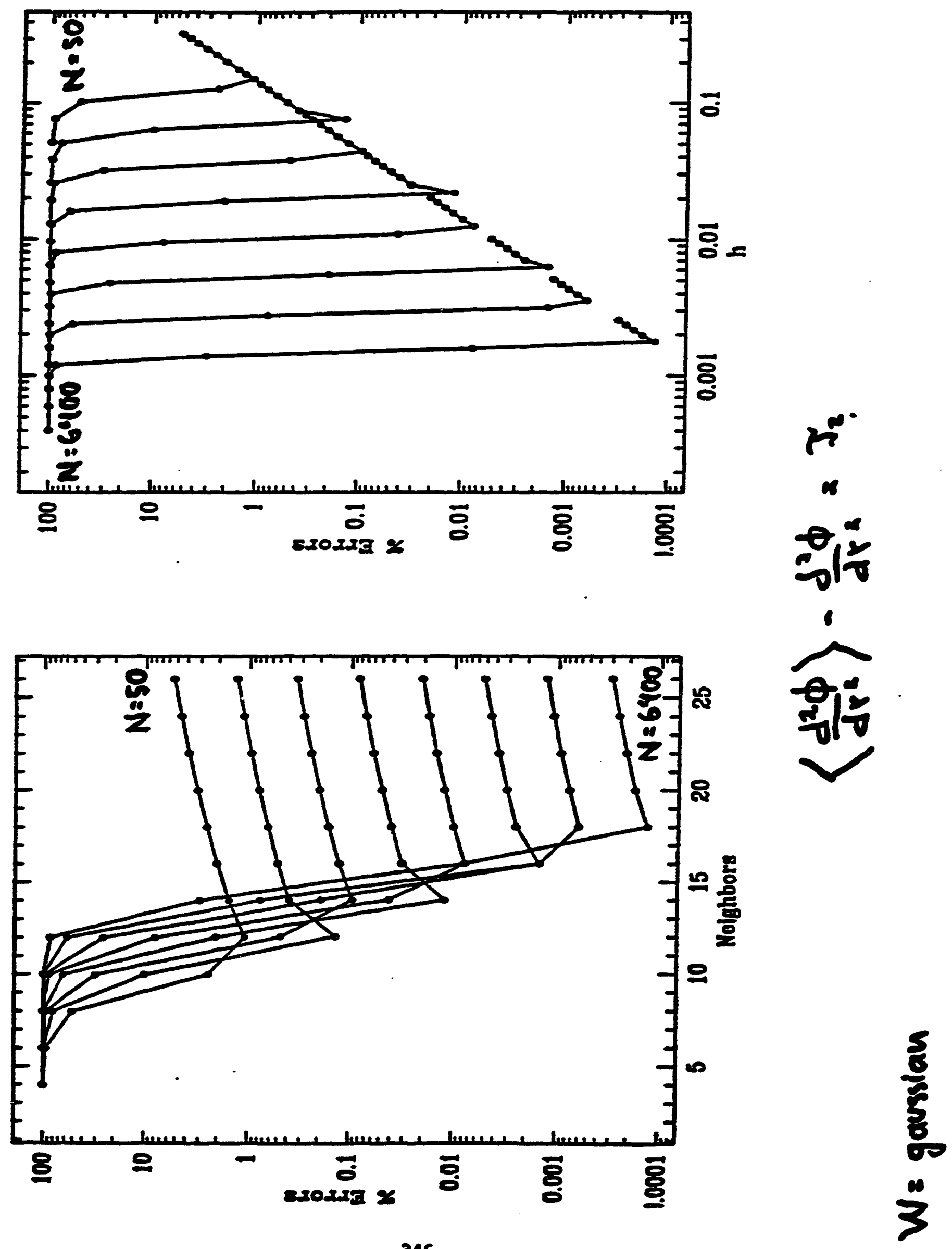
WAVE EQUATION $\quad \frac{\partial^{2} \phi}{\partial t^{2}}-c^{2} \Delta \phi=0$

Let $\Pi=\frac{\partial \phi}{\partial t}$, then $\left\{\begin{aligned}+\frac{\partial \phi}{\partial t} & =\pi \\ +\frac{\partial \pi}{\partial t} & =c^{2} \Delta \phi\end{aligned}\right.$

Applying SPI one gets

Eulenian form

$$
\begin{aligned}
& \frac{\partial \phi_{i}}{\partial t}=\pi_{i} \\
& \frac{\partial \Pi_{i}}{\partial t}=c^{2} \sum_{j} \frac{\phi_{j}}{n_{j}} \Delta_{i} W_{i j}
\end{aligned}
$$

Time integration using staggered leapfrog.

* Counant Condition: $v \Delta t \leqslant h$

${ }_{\text {not }}^{\uparrow} \Delta r$

$-347-$ 
Weve Equation and Moving Panticles

$$
\left.\begin{array}{l}
\partial_{t} \phi=\Pi \\
\partial_{t} \Pi=c^{2} \Delta \phi
\end{array}\right\} \begin{aligned}
& \text { Fixed Panticles, in genenal } \\
& \text { non-unifonum spatial distributiou }
\end{aligned}
$$

If particles move

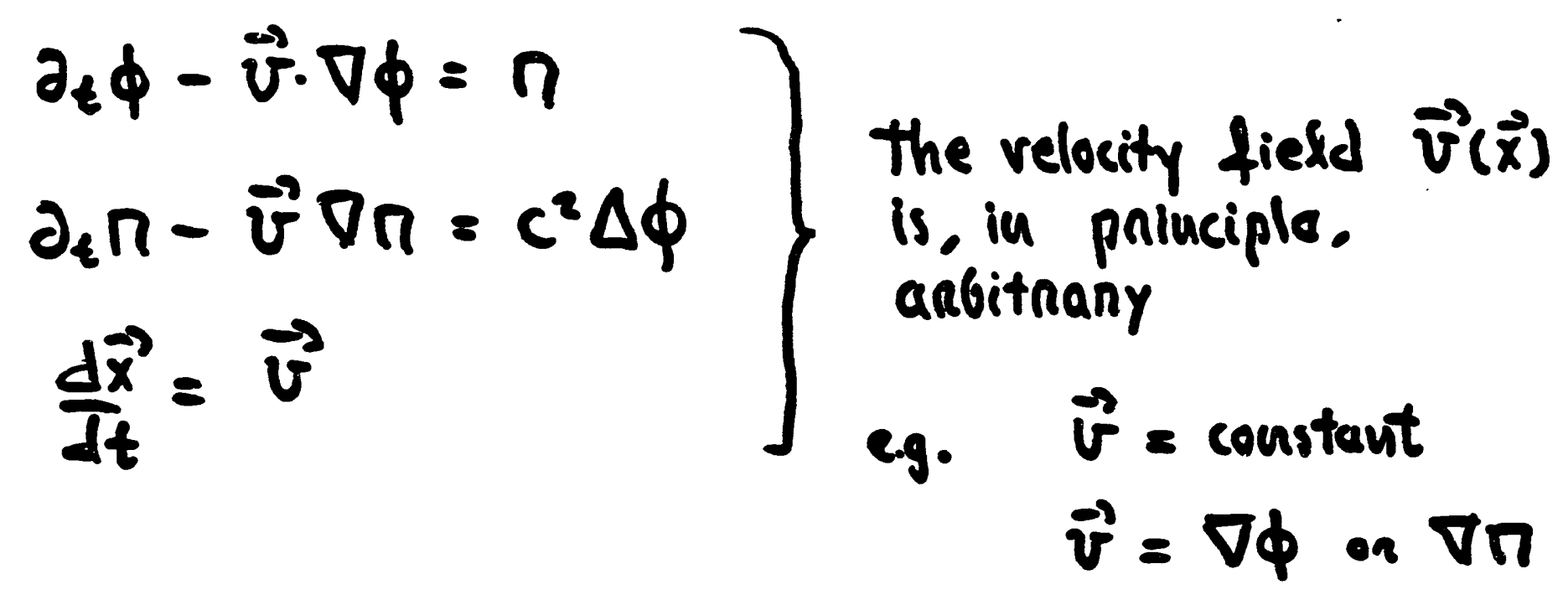

$-348-$ 

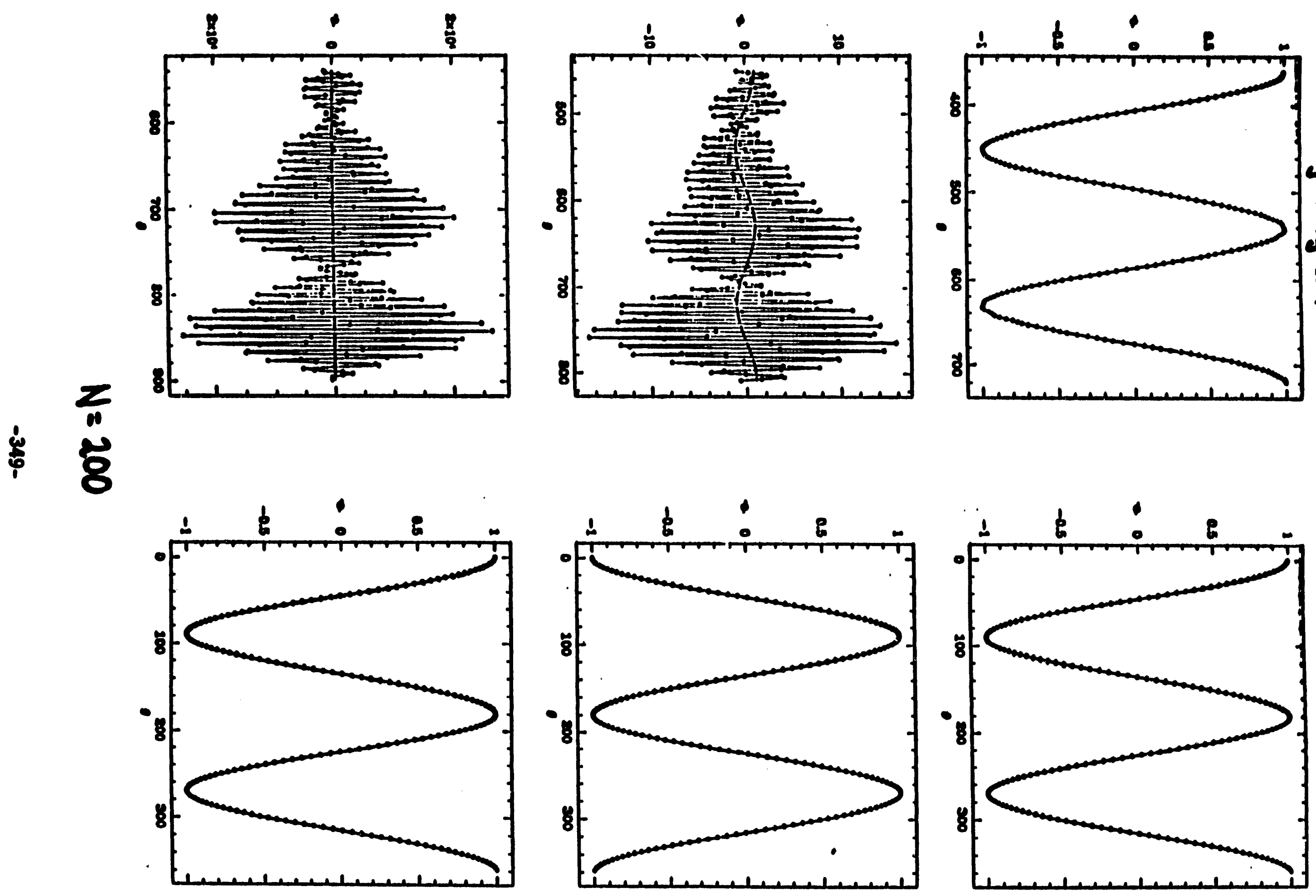
ADVECTION:

$$
\frac{\partial \phi}{\partial t}+v \frac{\partial \phi}{\partial r}=0
$$

Finite Difference

$$
\left(\phi_{j}^{n+1}-\phi_{j}^{n}\right)+v\left(\frac{\partial \phi}{\partial t}\right)_{j}^{n+y_{2}}=0
$$

We need

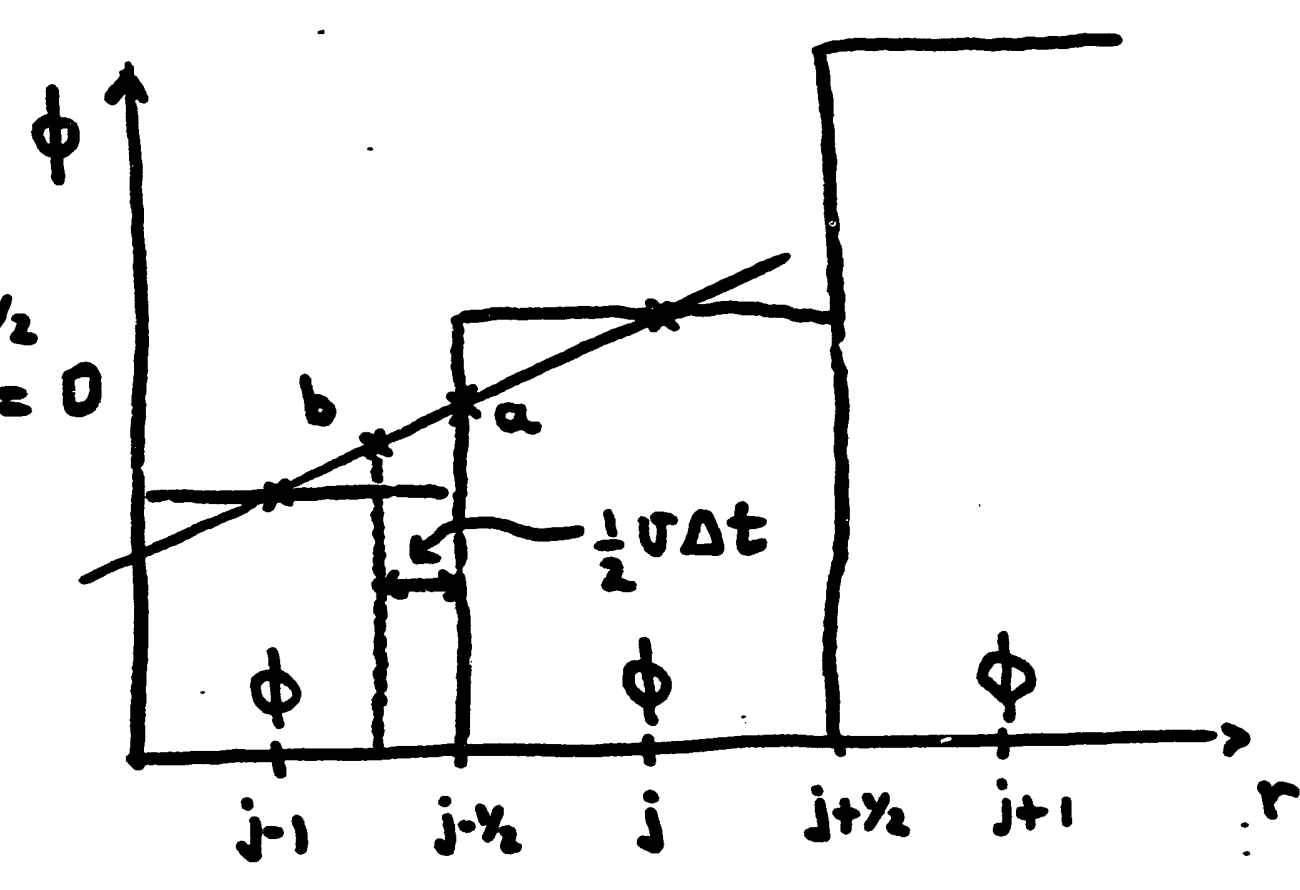

$$
\left(\frac{\partial \phi}{\partial r}\right)_{j}^{n+1 / 2}=\frac{\left(\phi_{j+y_{2}}^{n+y_{2}}-\phi_{j-1 / 2}^{n+1 / 2}\right)}{\Delta x} \quad \text { but } \phi \text { is }\left\{\begin{array}{l}
n \\
j
\end{array}\right\}
$$

a.. Let $\phi_{j-1 / 2}^{n+1 / 2}=\frac{1}{2}\left(\phi_{j}^{n}+\phi_{j-1}^{n}\right) \Rightarrow$ unstable

boo. Let $\phi_{j-y_{2}}^{n+y_{2}}=\frac{1}{2}\left(\phi_{j}^{n}+\phi_{j-1}^{n}\right)-\underbrace{\frac{1}{2}\left(\frac{\Delta t v}{\Delta x}\right)}_{c}\left(\phi_{j}^{n}-\phi_{j-1}^{n}\right)$

$-350-$ 
then

$$
\frac{\phi_{j}^{n+1}-\phi_{j}^{n}}{\Delta t}+\frac{v\left(\phi_{j+1}^{n}-\phi_{j}^{n}\right)}{2 \Delta x}-\frac{1}{2} \Delta t v\left(\frac{\phi_{j+1}^{n}+\phi_{j-1}^{n}-2 \phi_{j}^{n}}{\Delta x^{2}}\right)=c
$$

which is the finite difference representation of

$$
\frac{\partial \phi}{\partial t}+v \frac{\partial \phi}{\partial r}-\underbrace{\frac{\Delta t v}{2} \frac{\partial^{2} \phi}{\partial r^{2}}}_{\text {Diffusion }}=0
$$

In SPH one can then:

A.. Add diffusion teams to the equations.

B. Move particles a "distance" $\frac{v \Delta t}{2}$.

But, is the intrimsle numerical dissipation of SPH enough to cone the instability?

$-351-$ 


$$
\phi_{j}^{n+1} \frac{-}{\Delta x} \phi_{j}^{n}+v \sum_{k=j-N}^{j+N} \phi_{k}^{n} \frac{\partial}{n_{k}} \frac{\partial W_{j k}}{\partial r}=0
$$

fon a uniform distribution of panticles

$$
\phi_{j}^{n+1} \frac{-\phi_{j}^{n}}{\Delta x}+v \sum_{k=1}^{N} \frac{\left(\phi_{j+k}^{n}-\phi_{j-k}^{n}\right)}{n_{j}} \frac{\partial}{\partial r} W_{j j+k}=0
$$

UNSTABLE!

Futune Wonk

- Random distnibution of panticles

- Nou-unifonm panticle deusity

$-352-$ 
$=--^{-}$

?0
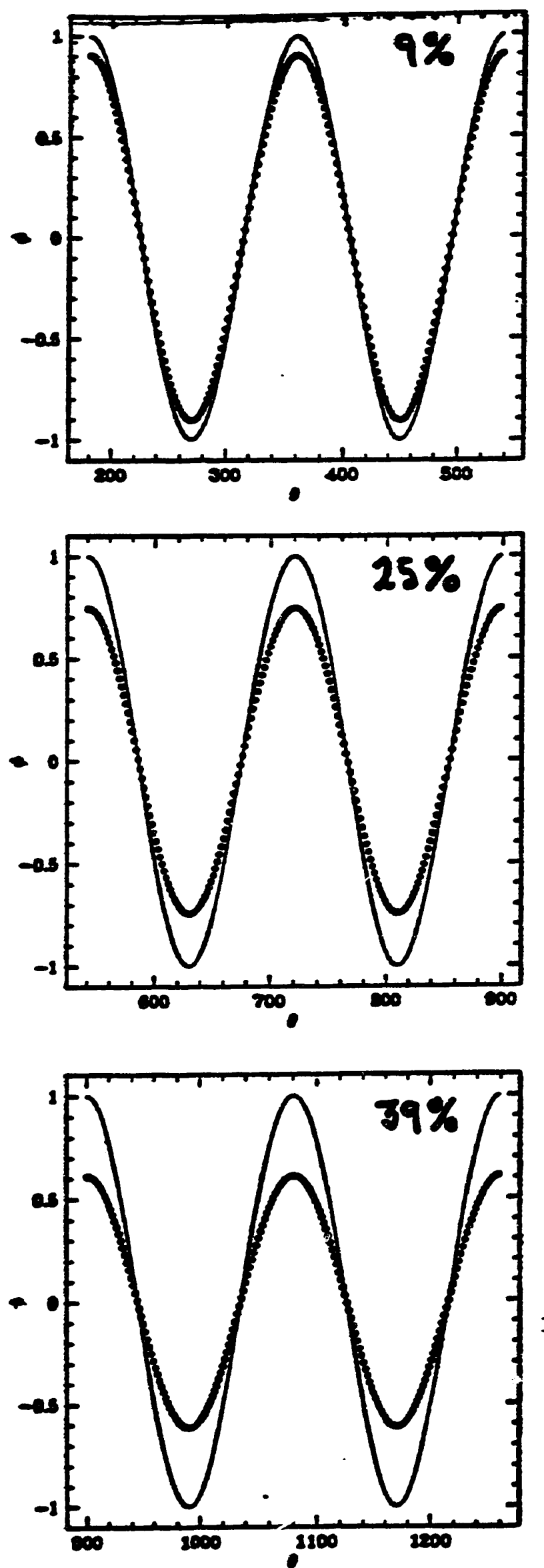
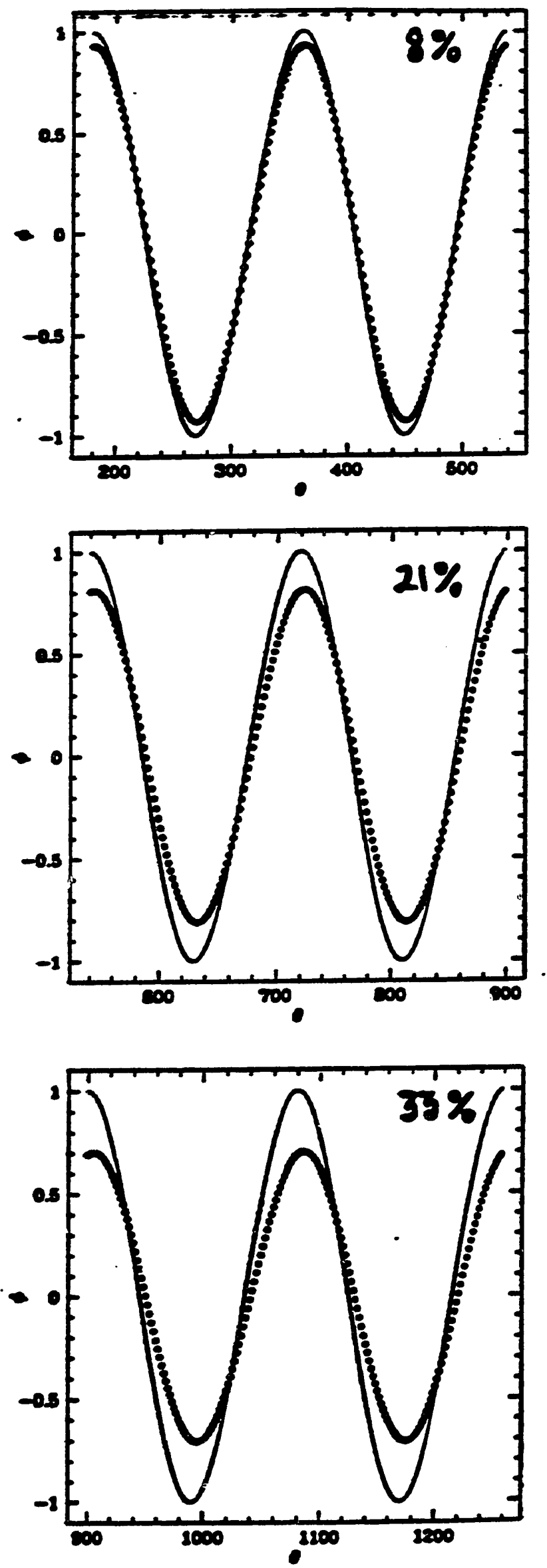

$N=200$

$\therefore \div \quad \therefore \quad \therefore \% \therefore \quad$-353- 
CONCLUSIONS:

- Higher denivatives seem more expeusive (Neighbons > few) unden SPI.

- Adrection tenms represent the main challenge

+ Modify the coutinuum eqs.

+Wonk with panticle "Iluxes"

FUTURE WORK:

- Vaniable $h$

- Highen dimeusions

- Mone genenal velocity fields $\vec{v}=\vec{v}(\vec{x})$

$-354-$ 
APPLICATION: Numenical Relativity

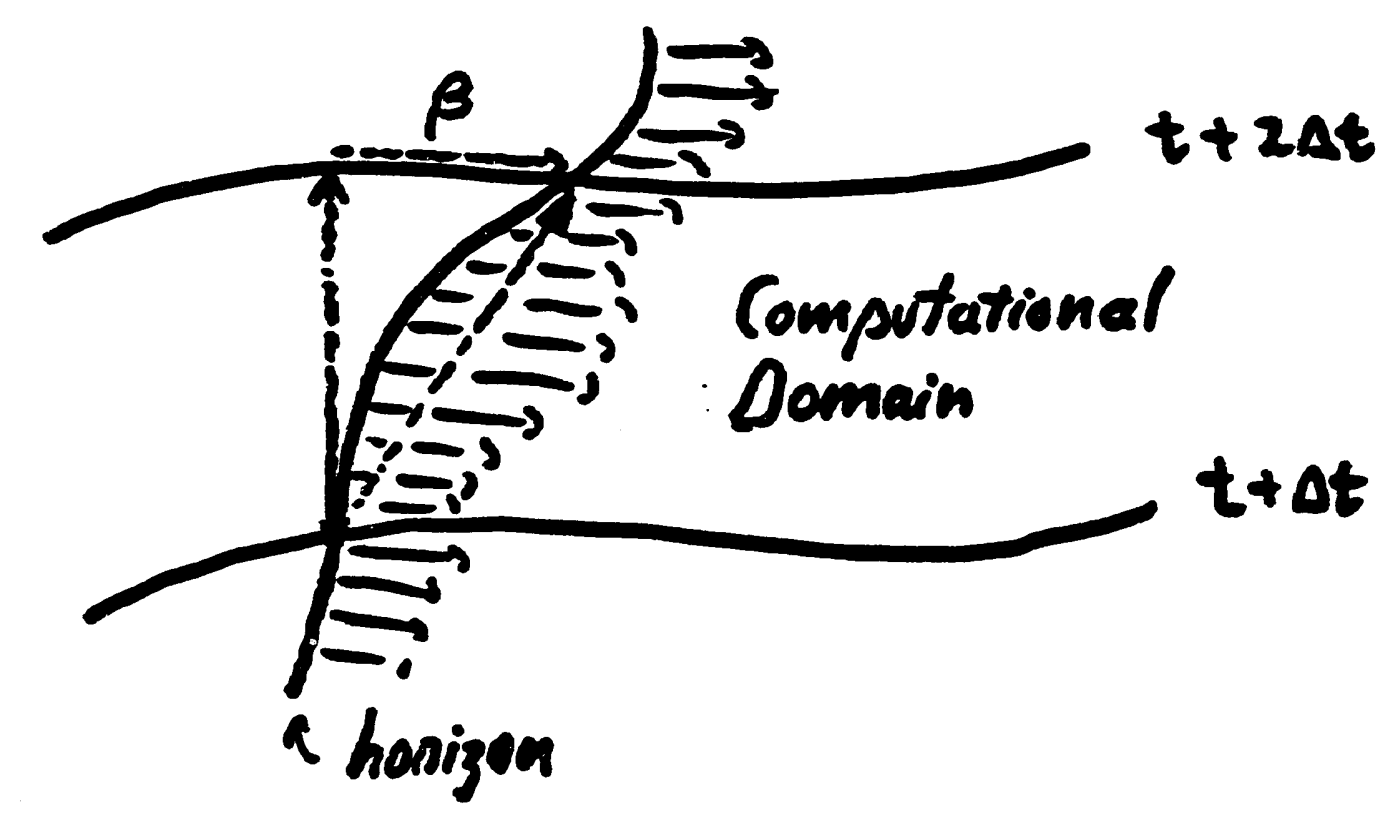

Einstein Eqs.

$$
\frac{\partial \phi}{\partial t}-\underbrace{\vec{\beta} \cdot \nabla \phi}_{\text {adrection }}=\ldots \ldots . .
$$

60AL: Use the shift vecton (coond. velocity) to lock the honizon (boundany cond.)

$-355-$ 
$\dot{4}$ 


\section{RELATTVISTIC SPH}

\section{AVOIDANCE OF VELOCITY-BIASED KERNELS}

Arkady Kheyfets, Warner A. Miller and Wojciech H. Zurek

Our contention is that any truly relativistic application of SPH should reflect the following three requrements:

1. The kinematics of the smoothed particles should be relativistic;

2. The state equation for the fluid should be relativistic; and

3. The interactions between the smoothed particles should be treated in a Lorentz-covariant fashion.

The Lorentz covariant treatment of interactions between the smoothed particles can be achieved if one assumes that the relativistic hydrodynamical contact interactions are mediated by kernels whose supports reside in the local frame comoving with the fluid. 


\section{SEMI-RELATIVISTIC SPH}

It appears to us to be common practice to satisfy only the first requirement and to neglect the other two.

- The first requirement is ordinarily taken care of automatically via applying the SPH discretization technique to the Lorentz covariant hydrodynamic equations (e.g. the Wilson equations).

- The equation of state is typically assumed to be either non-relativistic or vitrarelativistic. This should be more or less reasonable excluding problems involving variations in the temperature from non-relativistic to relativistic (collision of two clouds of cold gas moving with respect to each other at ultrarelativistic speeds). At mildly relativistic temperatures this could lead to quantitative errors, but probably, one could get away with this qualitatively. This is not a serious problem since these assumptions are made explicitly and can be easily corrected.

- The short range forces responsible for hydrodynamic interactions are ordinarily represented by spherical kernels in the proper space of an observer, or in an arbitrary spacelike section of spacetime. This procedure is obviously not relativistically invariant, as it depends on the choice of observer. It does not allow one to make any sound judgments concerning the scale of the kernels and does not allow one to draw any conclusions concerning the applicability of the SPH technique for any particular case. 


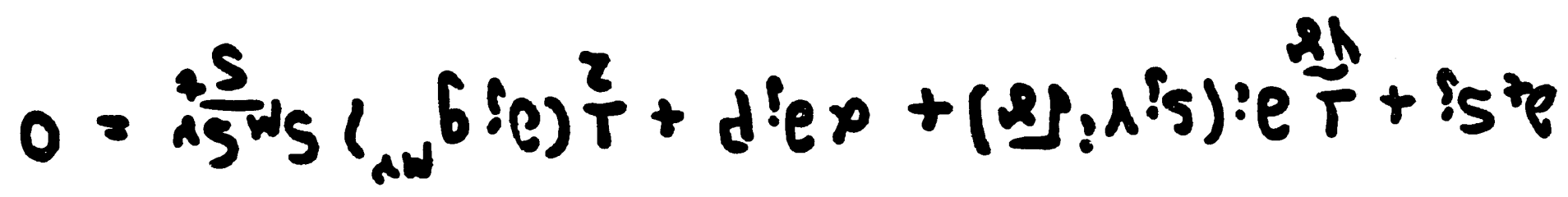
:no.unnusing mninamol.

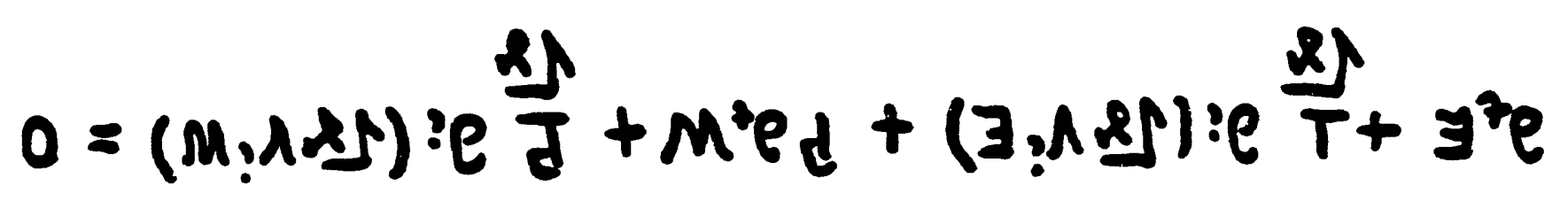
: mo!tbavasinos Abuauy

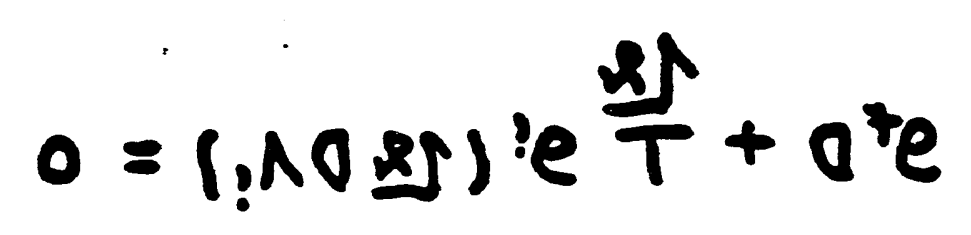

:usypunuasuog ushurg

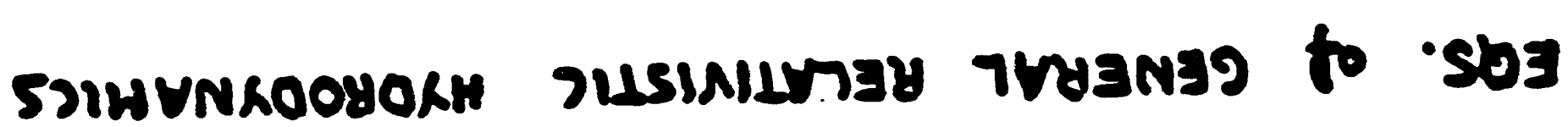




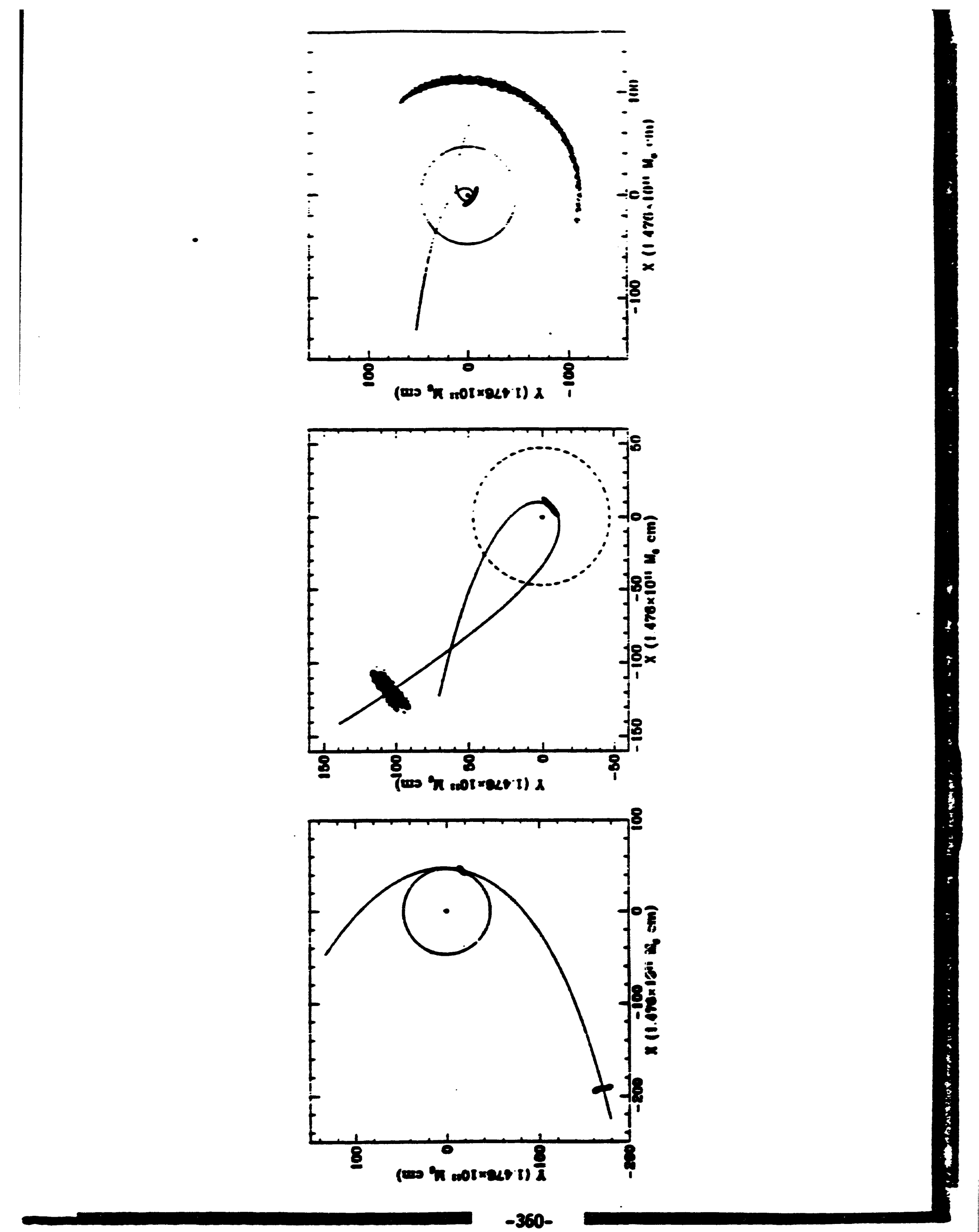




\section{RELATIVISTIC SPH}

The SPH representation of contact (hydrodynamical) forces can be made relativistically invariant if one one assumes that the relativistic hydrodynamical contact interactions are mediated by kernels whose supports reside in the local frame comoving with the fluid.

The discretized SPH equations for a 1-dimensional relativistic isentropic fluid

$$
\begin{aligned}
& n_{k} L\left(m \xi_{k}\right)=\text { const } \\
& c^{-2} \frac{d u_{l}}{d \tau}=-\frac{1}{m n_{l} G\left(m \xi_{l}\right)} \sum_{k} \frac{\alpha_{k}}{\xi_{k}} \nabla_{l} w_{l k} \\
& c^{-2} p_{k}=\frac{n_{k}}{\xi_{k}} \\
& \rho_{k}+c^{-2} p_{k}=m n_{k} G\left(m \xi_{k}\right) \\
& n_{k} V_{k}=\alpha_{k}=\text { const } \\
& u_{l} \cdot u_{l}=-c^{2}
\end{aligned}
$$

where

$$
\begin{aligned}
& G(m \xi)=\frac{K_{3}(\xi)}{K_{2}(\xi)} \\
& L(m \xi)=\frac{m \xi}{K_{2}(m \xi)} \exp \left[-\frac{m \xi K_{3}(m \xi)}{K_{2}(m \xi)}\right] \\
& \xi=\frac{c^{2}}{k T}
\end{aligned}
$$

and $K_{n}(m \xi)$ is the $n$-th hyperbolic Bessel function. 


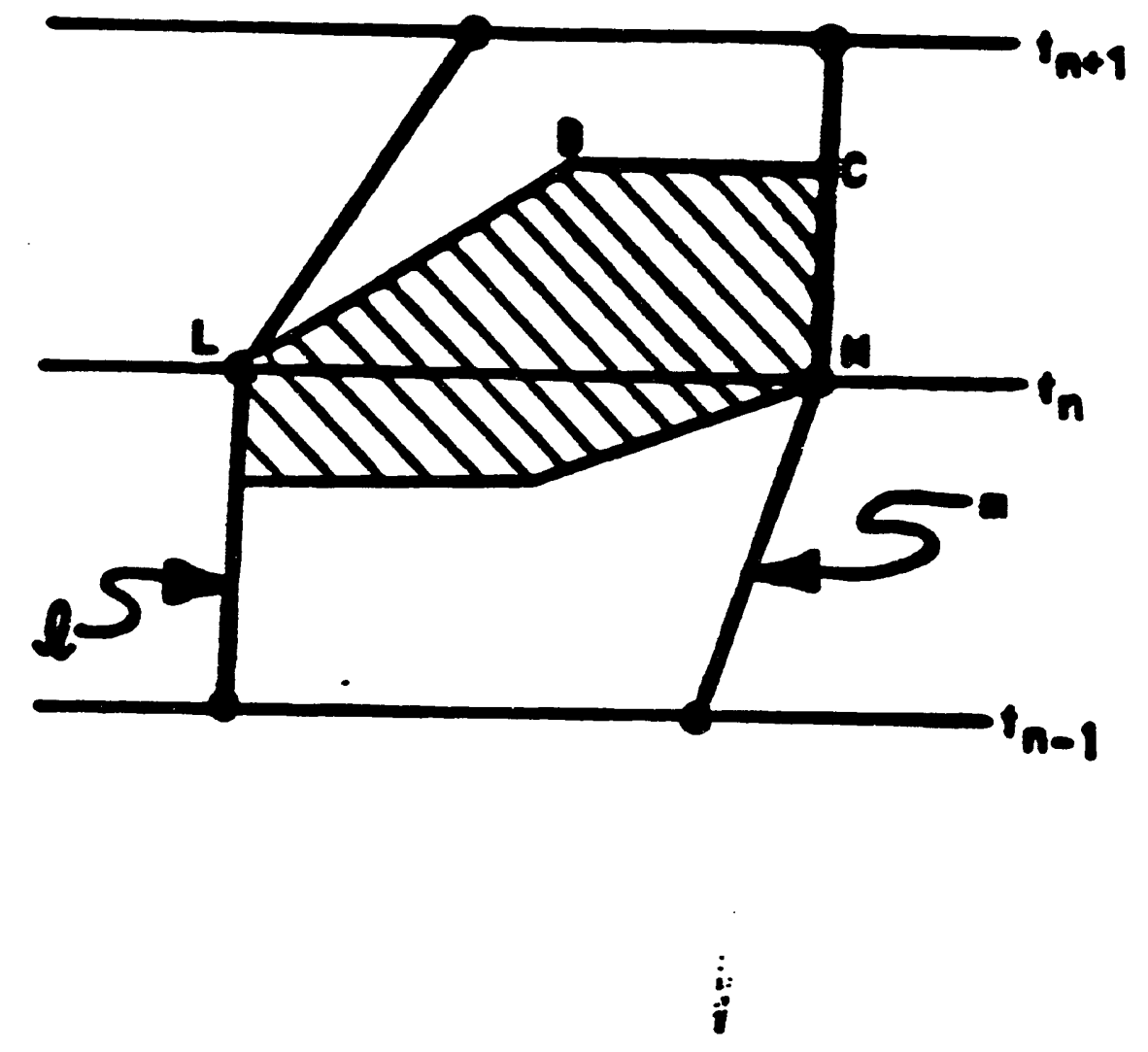

$-362-$ 

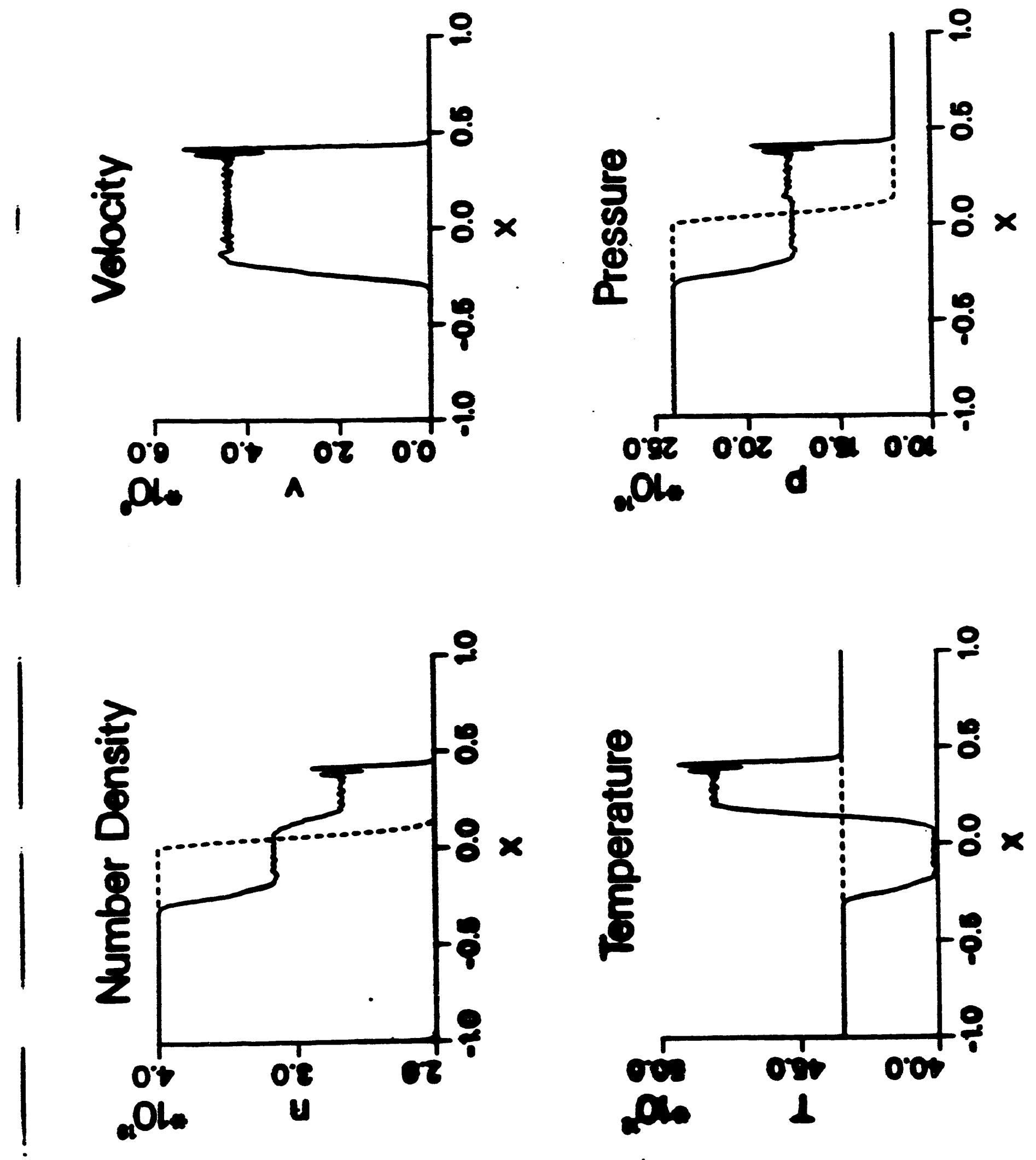


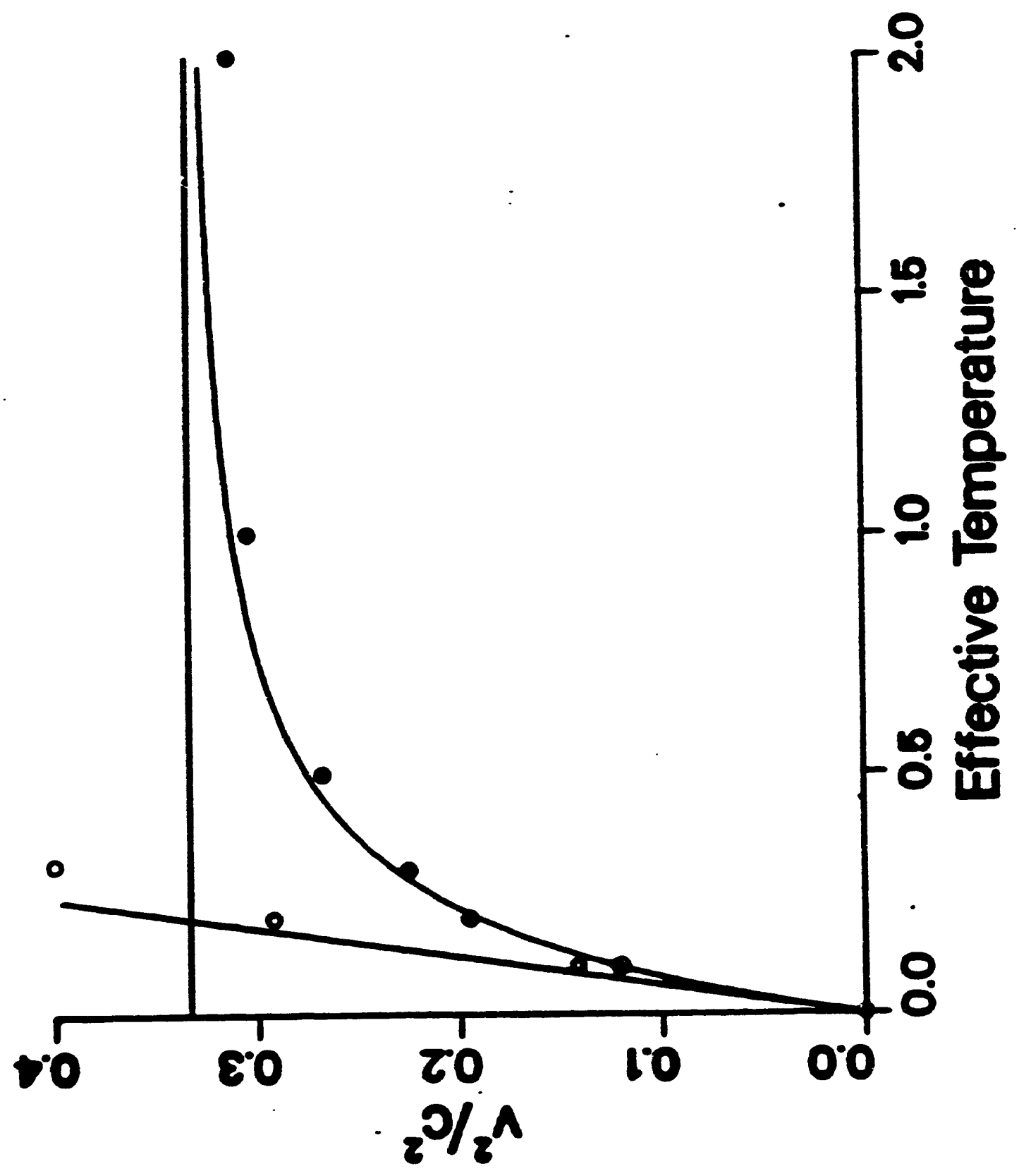




\section{DISCUSSION}

The main problem :

The kernels depend on the choice of observer. Their supports, if projected on the comoving frame of the fluid, become ellipsoidal. The ellipticity depends on the choice of the observer.

\section{Statement.}

For any choice of the smoothing length for the kernel (spherical kernels) in the observer's proper space, the observer can be picked in such a way that the smoothed particle approximation brakes down.

For any given scale, one can pick an observer such that in the direction of his motion the smoothed particles will become decoupled. The suggestion to start from an observer and to choose the scale such that there will be a sufficient number of particles in the direction of the observer's motion will ordinarily lead to silly situations (the scale of the smoothed particle becomes smaller than the baryon size, etc).

A suggestion to pick a "reasonable observer" is sometimes forwarded. However, a "reasonable observer" in this context means the one that does not move too fast (relativistically) with respect to fluid.

\section{Statement.}

The most "reasonable" observer is the one that does not move with respect to the fluid, i. e. the observer of the comoving frame. 


\section{CONCLUSION}

- Truly relativistic SPH is necessary for the problems that involve interactions of parts of fluid moving relativistically with respect to each other (no "reasonable" global observer exists).

- Truly relativistic SPH contains intrinsic criteria of its applicability. Such a critesion is totally absent in semirelativistic SPH.

- When using semi-relativistic SPH one should employ the fully relativistic SPH theory when picking a "reasonable" observer. When parts of the fluid move at relativistic speeds with respect to each other this should be done locally. 


\title{
Tidal compression and disruption of stars near a supermassive rotating black hole
}

\author{
Hanno Sponholz* \\ Intitut fur Theoretieche Actrophyrik \\ der Univeritht Beidelbers \\ In Nevenbeimer Feld 561 \\ D-69120 Eeidelbers
}

\begin{abstract}
Abetract
Capture and tidal break-np of atars by a supermasive black hole (SBH) and the subsequent accretion of the diopersed matter onto the central object may provide a crucial mechaniam for the fuelling of active galactic nuclei (AGN). It is generally asumed that very bright $A G N$ contrin a central rotating SBH. Becanse relativistic effects become important for the Roche-process for SBH $\geq 10^{6} M_{\odot}$ we present a first hydrodynamical compration of the tidal encounter of a main-eequence-star with a rotating black hole.

To consider qualitatively new effects, eppecially to include additional effects by a Kerr black hole, we apply the Smoothed Particle Hydrodynamics (SPH) technigue for a fixed background metric. Within the framework of SPH, the star is modelled as a polytrope - the Kerr metric of the rotating black hole is taken into account by nxing components of the Biemann-Tensor in a special chosen parallel-propagated tetrad-frame along the centre-of-mass trajectory of the star.

Bexide tests and stability investigations of the code, a comparion between the Newtonian and the relativistic investigntion is presented, as well as detailed calculations of the efficiency of tidal disruptions and their de pendence on the angular momentum of the SBH for a moderate rotating 5.107 $\mathrm{M}_{0}$ SBH. The tidal diruption of a 1. Mo polytrope by an extremely rotating $1 \cdot 10^{\circ} \mathrm{M}_{0} \mathrm{SBH}$ is considered and the fate of the resulting debris of the star is shortly discussed. Some results of the relativistic generalization of the problem of tidal equeesing of a star closely enconntering a Kers black hole are aketched. Finally the range of vaidity of these relativitic SPE-applications for tidal simplations around a SBH is derived in terms of the radius of curvature and the inhomogenity male of the external metric.
\end{abstract}

- E-mail: eponhols Cepikus sitanni-beidelberz.de 


\section{Tidal Processes around Black Holes - The scenario}

In addition to free gas accretion, tidal interaction between a compact object and a star (Rees, 1988) may play an important role in the process of fuelling active galaxies - concerning this fundamental process there exist the following lines of research: On one side there is the application of the theory of tides to incompressible bodies in both the relativistic (e.g. Fishbone, 1973, Mashhoon, 1975) and the classical context. On the other side we have the sophisticated works with the compressible affine star model (e.g. Iuminet and Carter, 1986) where the star is assumed to keep always an ellipsoidal density-shape, even during a very cloce encounter. Later, this application has been extended to a relativistic veraion (Lnminet and Marck, 1985) in the environment of a Schwarzschild-BH ${ }^{1}$, using an earlier formulation for relativistic tidal forces (Marck, 1983).

A third treatment of such 3D-problems with a reasonable computational effort has become more and more common - even if they are not that accurate as conventional hydrodynamic codes, mostly applied to restricted problems with less dimensions: The recently introduced particle methods (Lacy, 1977, Monaghan, 1992) are very helpful to understand gross effects of this intrinsic 3D-process.

Although we have principle difficulties to treat celf-gravitating systems in General Relativity properly, it seems to me nseful to include the formulation for tidal forces (Marck, 1983) in such a particle program. An older short description of simple SPH-nimulations for tidal actions of Schwarzechild- and Kert-Black-Holes on polytrope stars (Sponhol,, 1991) is here presented as more extended and elaborated paper. An actual application in the vicinity of a Schwarzschild-BH is described in Laguna et al., 1993

\section{Tidal Processes around Black Holes - moti- vation}

Earier it has been argued (Hills, 1975) that for the fuelling of AGNs, tidal disruption may be only effective if $M_{B H}<10^{3} M_{O}$ (if the mase $M_{B H}$ of the central Schwarzschild black hole exceeds $3.2 \times 10^{8} \mathrm{MO}_{\mathrm{O}}$, the radius of the innermont stable orbit becomes larger than the tidal radivs and the star will be captured wholly, withont emitting radiation). However, tidal dirruption of infinitesimal, incompressible fiuid bodies is not excluded for a more maxive Kerr BH with $M_{B A}<2 \times 10^{\circ} \mathrm{M}$, becanse for co-rotating orbits the Roche limit increnses while the radius of the last stable orbit decreases with increasing angular momentum of the Kerr black hole. Thus, of principle interest are the fraction of the star's mass which falls directly

\footnotetext{
${ }^{1}$ Ore of the reavits: a multiple comprosion of the etar inatead of the single one in the former Newtonian care of a very close encounter.
} 
into the hole, the fraction which is ejected into onter regions or at times, before the disk has fully evolved [CA90], and the fraction, which remains in the disk.

We consider as primary body a massive black hole (BH) with a mass of $10^{6} \ldots 10^{9} \mathrm{M}_{0}$, optionally with angular momentum. To model the secondary star as a polytrope, we divide the star into a number of fluid elements and simulate an encounter of the secondary star with the black hole; this means, that we colve the equations of motion in the combined field of (i) the black hole and (ii) the star itself.

\section{The Method: Smoothed Particle Hydrody- namics}

A recently introduced numerical method (Lucy, 1977, Bens and Hills, 1987, Evans and Kochanek, 1989) - Smoothed Particle Hydrodynamics (SPH) - uses a Lagrangian description to simulate the evolution of gaseons systems - the medium is modelled without a numerical grid as a collection of N gaseons parcels, elements, moving interpolation points or particles. To solve the exact real system one has to nee an infinite number of such elements. For a computational method with a finite number of elements, we have to perform local averaging over finite valumes, consequently, we have to introduce a procedure for smoothing out local fiuctuations in the particle number.

a) If the value of a physical field $f\left(x^{\prime}\right)$ is bnown at the radins vector $x_{0}^{\prime}$, the mean value at $r \mathrm{can}$ be obtained by averaging over a kernel $W_{h}\left(r-r^{\prime}\right)$ :

$$
\langle f(\mathbf{r})\rangle=\int f\left(\mathbf{r}^{\prime}\right) W_{h}\left(\mathbf{r}-\mathbf{r}^{\prime}\right) d \mathbf{r}^{\prime}
$$

b) The derivatives of the function $f$ are obtained by smoothing with the derived kernel:

$$
\left\langle\frac{\partial f(x)}{\partial x}\right\rangle=\int \frac{\partial f\left(x^{\prime}\right)}{\partial x^{\prime}} W_{h}\left(x-x^{\prime}\right) d r^{\prime}
$$

(after integration by parts:)

$$
\left\langle\frac{\partial f(x)}{\partial x}\right\rangle=\int f\left(x^{\prime}\right) \frac{\partial}{\partial x} W_{h}\left(x-x^{\prime}\right) d x^{\prime}
$$

c) If we choose the mase density $\&$ as amoothed function $W_{h}(r-y)$ bnown at a finite number of discrete points 5 , include the Poisson equation and an equation of state ${ }^{2}$ then we may write down a system of ODE's, approwimating the equations of hydrodynamics, including the lorentzimvariant modifications (cp. Mann, 1991).

To model the cecondary atar as a polytrope, we divide the atar into nomber of alid elements or "smoothed particles" and simulate an encounter of the secondary star with the black hole.

\footnotetext{
${ }^{2}$ For now, we take the polytropic equation of etate: $p=K_{p} p^{1+\frac{1}{t}}$.
} 


\section{Geodesics around a Black Hole and tidal ef- ficiency}

If that the star is much smaller ${ }^{3}$ than the BH, it is reasonable assumption that the star moves along a geodesic around the BH. The trajectory for a small main-sequence star orbiting in the equatorial plane $(\theta=\pi / 2$ and $\dot{\theta}=0$ ) around a massive BH is given by Carter's integrals of motion:

$$
\begin{aligned}
& i=\frac{(A E-2 M r a L)}{\Delta r^{2}}, \\
& \dot{r}^{2}=\frac{\left[E\left(r^{2}+a^{2}\right)-a L\right]^{2}-\Delta\left(\mu^{2} r^{2}+K\right)}{r^{4}}, \\
& \dot{\phi}=\left[\frac{2 M}{s} a E+\left(1-\frac{2 M}{r}\right) L\right] \frac{1}{\Delta} .
\end{aligned}
$$

where $\left(x^{0}, x^{1}, x^{2}, x^{3}\right) \equiv\left(x^{t}, x^{r}, x^{0}, x^{\phi}\right) \Rightarrow(t, r, \theta, \phi)$ denote the BoyerLindquist-coordinates and for the mass of the $B H$ we have $M_{B H}=M$. For the constants we nse the convention $G=c=1 . \mu^{2}, E, L$ are the rest mass, energy, and angular momentum about the axis of symmetry per unit mass, respectively; $K$ is Carter's third integral: $K=(a E-L)^{2}$. The dot denotes the differentiation with respect to the proper time $\tau_{\text {rel }}$. Without restriction we set $\mu=1$. Physically motivated - the star is assumed at rest in infinity distance - we use a "parabolic" orbit: $E=1$.

The geodesics may be obtained either as the formal solution or by numerical integration of the eqs. of motion (4) - (6). The numerical integration - here we use a Runge-Kutta-scheme seems to be reasonable sta. ble even close to nnstable orbits. (For Schwarzschildgeodesic an orbit close to the marginal bound orbit at $r_{m b}=4=I_{m b}$ is shown in Boyer-Lindquistcoordinates (Fig 1).) Already from the shape of geodesics we may discuss the infivence of relativistic effects and of the Black Hole's rotation on the tidal problem: The following three effects appear and $r_{p}=4.01$ )

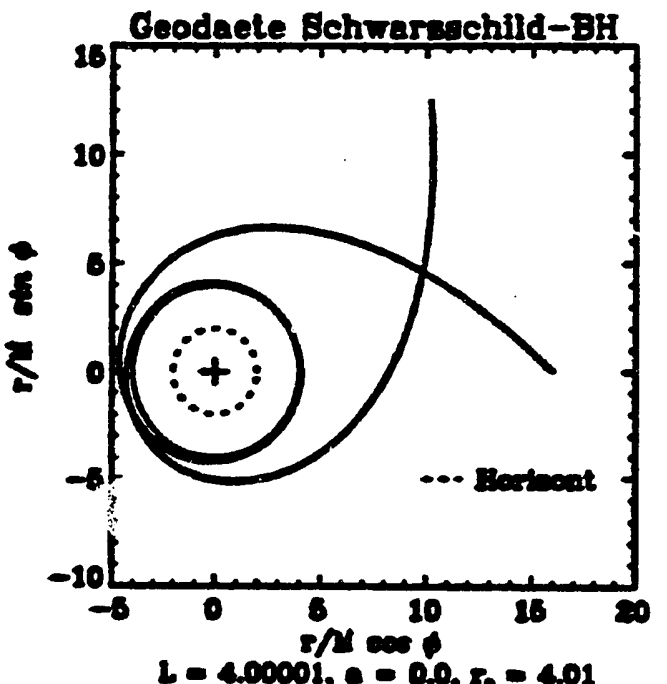

$L=400001,-0.0, x_{0}=4.01$

Figare 1: Schwarachild-geodecic (periation

may discussed even by conviderations about the geoderics:

\footnotetext{
For applieable conditions ree reet. 6
} 
(1) The geodesics of test-particles change by the infivence of the curvature effects of the space-time as well as by the angular momentum of the BH. In Boyer-Lindquist-coordinates e.g. all the typical radius of prograde test-particle-orbits goes to one Schwarzschild-radius for an extreme $(a \approx 1)$ rotating $B H$.

Increasing angular momentum of the BH decreases for prograde orbits the manginal bound rodius $r_{m b}$ : This enables a maller periastron-distance

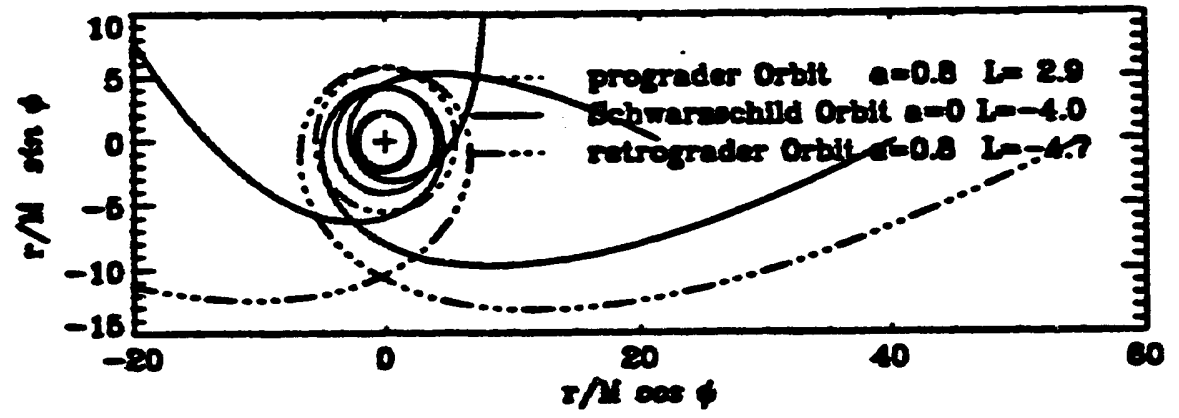

Figare 2: Critical unbound geodesics \& different epio-parameters.

$r_{p}$ between star and BH (Fig. 2). Potentially tidal effects on extended bodies increase roughly with decreasing distance from the compact object according to $r_{p}^{-3}$.

(2) Fixed periastron-distance:

- Relativistic orbits: the geodecic tends to wind around the BH (Fig. 1).

* Dependent from the spin-parameter of the BH; for retrograde orbit and chosen distance from the compact object, this tendency increases with the spin of the BH (Fig. 3).

- The clocer the itar reaches the marginal bound radius the longer is - the time it opends near the $B B$. In-

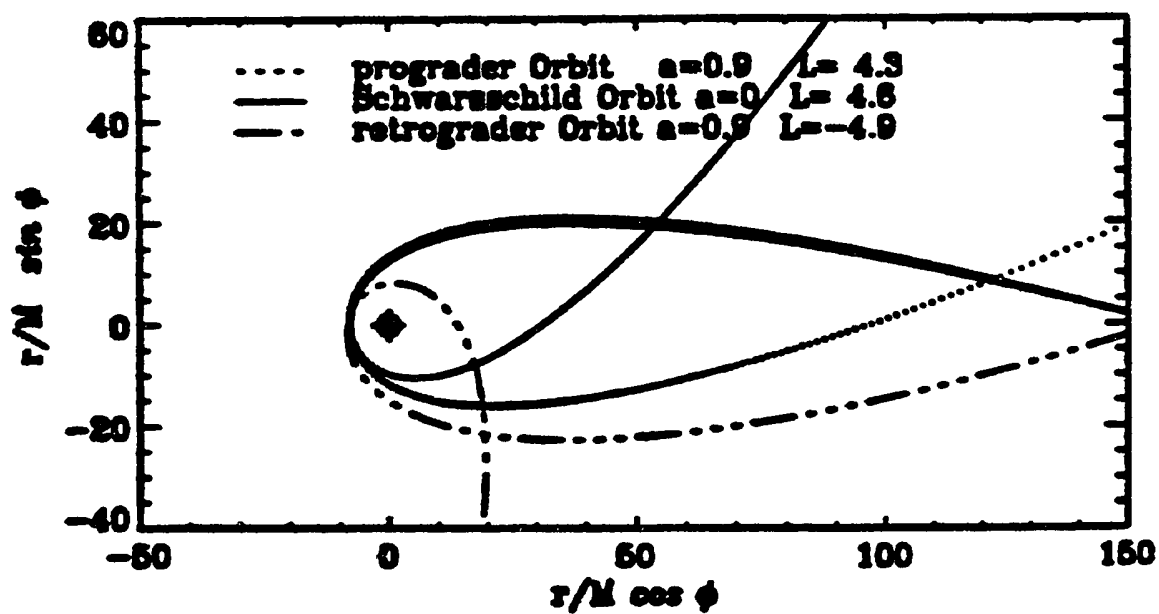

Fipure 8: Geodecice $r_{p}=7.9$ creases time-efificiency of tidal effects. 
(3) A non-vanishing spin-momentum $a$ of the BH results in the known "Frame-Dragging" effect; the borizon shifts inwards and on the former

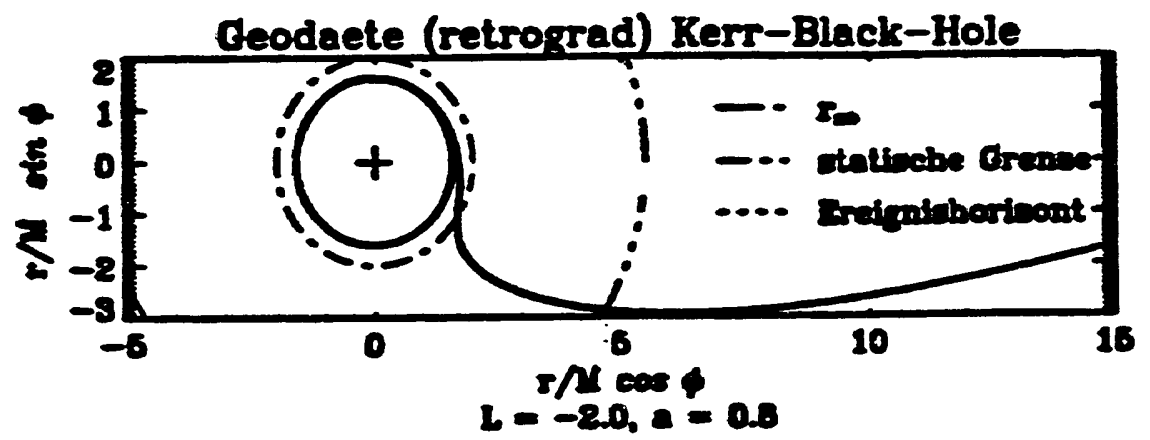

Figure 4: Kerr-geoderic (retrograde)

radius $2 M$ boates the "static limit", the "border" of the ergoophere. The Boyer-Iindguist-coordinate " $t$ " changes from a time-like to a space-like coordinate. This reflects the strong conpling of the individual orbits to the given space-iime of the BH and results in a stronger tidal force for the retrograde orbits compared to Schwarzschild ones and in all cases stronger than for a comparable (the same penetration factor $\beta$ ) newtonian constellation.

\section{The tidal field of the SBH Kerr-space-time}

The tidal problem is treated in a test-particle approximation - we assume that the space-time of and around the compact object is negligible disturbed by the secondary star. This is the condition to consider the orbit of the star as a geodesic given by Carter's formulae (4) - (6). With other words: the condition to include the self-gravitation of the star in a local flat way leads to the demand for a amall extension and apatial scales of the star against the radins of curvature of the primary metric (sect. 6).

The relative (tidal) acceleration of a hydrodynamical element of the star may be obtained by the equation of geodesic deviation. An orthonormal tetrad, locally defined and parallel-propagated along the centre-of-mass trajectory allows to derive a tidal teneor in terms of the Biemann tensor from the equation of geodesic deviation. The ceparability properties of a Kerr Black Hole make it poasible to construct such a tetrad analytically (Marck, 1983):

$$
\begin{aligned}
& \lambda_{[0]}^{(\mu)}=\frac{1}{r \sqrt{\Delta}}\left[E\left(r^{2}+a^{2}\right)-a L\right] \delta_{0}^{\mu}+\frac{r \dot{r}}{\sqrt{\Delta}} \delta_{1}^{\mu}+\frac{a E-L}{r} \delta_{3}^{\mu \mu}, \\
& \lambda_{[1]}^{(\mu)}=e_{[1]}^{(\mu)} \cos 1-e_{[H]}^{(\mu)} \sin \theta \text {, } \\
& \lambda_{[2]}^{(\mu)}=-\frac{a E-L}{\sqrt{K}} \delta_{2}^{\mu}, \\
& \lambda_{[3]}^{(\mu)}=e_{[1]}^{(\mu)} \sin 2+e_{[y]}^{(\mu)} \cos s \text {. }
\end{aligned}
$$


Tidal compression and disruption of stars near a rotating black bole

$$
\begin{aligned}
& e_{[1]}^{(\mu)}=\frac{r^{2} ;}{\sqrt{\Delta\left(r^{2}+K\right)}} \delta_{0}^{\mu}+\frac{E\left(r^{2}+a^{2}\right)-a L}{\sqrt{\Delta\left(r^{2}+R\right)}} \delta_{i}^{\mu}, \\
& e_{[\xi]}^{(\mu)}=\frac{\sqrt{K}\left[E\left(r^{2}+a^{2}\right)-a L\right]}{r \sqrt{\Delta\left(r^{2}+K\right)}} \delta_{0}^{\mu}+\frac{\sqrt{K} r i}{\sqrt{\Delta\left(r^{2}+K^{\prime}\right)}} \delta_{1}^{\mu}+\frac{\sqrt{\left(r^{2}+K\right)}}{r} \frac{a E-L}{\sqrt{K}} \delta_{3}^{*}
\end{aligned}
$$

For the angular-velocity of the coordinate-system we have:

$$
\dot{\varphi}=\frac{E \sqrt{K}-a \operatorname{sign}(a E-L)}{. r^{2}+K}
$$

For a rather high velocities in the beal tetrad system it is appropriate to nse a further extended equation of geoderic deviation (Hodghinson, 1972, Mashhoon, 1977), which gives the following equations for tidal acceleration of hydrodynamical elements with respect to the bocl tetrad system:

$$
\frac{d^{2} X^{i}}{d r^{2}}+C_{j}^{i} X^{j}=0
$$

The tidal tensor

$$
\begin{gathered}
C_{i j}=\bar{C}_{i 0 j 0}+2\left(\bar{C}_{i k j 0} \dot{X}^{k}+\bar{C}_{\left.0 k j 0 \dot{X}^{i} \dot{X}^{k}\right)}\right. \\
+\frac{2}{3}\left(\bar{C}_{i k j l} \dot{X}^{k} \dot{X}^{l}+\bar{C}_{0 k j l} \dot{X}^{i} \dot{X}^{k} \dot{X}^{\prime}\right)
\end{gathered}
$$

is derived in terms of the Riemann tensor with respect to the parallet propagated tetrad:

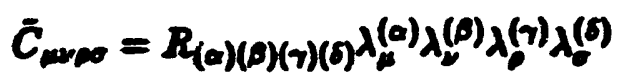

Explicitly, some non-vanishing independent components of the tidal tensor are given by the following

$$
\begin{aligned}
& C_{1010}=\left[1-3 \frac{\left(r^{2}+K\right)}{r^{2}} \cos ^{2} t\right] \frac{\mu}{r^{3}}, \\
& C_{2000}=\left[1+3 \frac{K}{r^{2}}\right] \frac{M}{r^{3}} \text {, } \\
& \left.C_{5000}=\left[1-3 \frac{\left(r^{2}+K\right)}{r^{2}} \sin ^{2}\right)\right] \frac{M}{r^{3}} \text {. } \\
& \bar{C}_{1000}=\bar{C}_{2010}=-8\left(r^{2}+K\right) M / r^{8} \cos \theta \sin \varphi \text {, }
\end{aligned}
$$

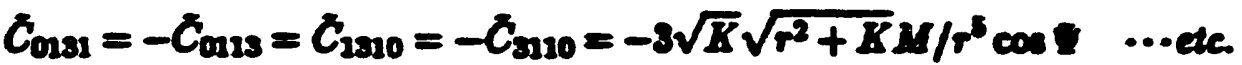




\section{Range of Application}

The noneract treatment of general relativity especially the flat (Newtonian) treatment of self-gravitation of the star refiects in the following conditions for range of application:

- The object moves on a teat-particle trajectory around the BH and

- Self-gravitation can be treated in a boclly flat way and

- Production of gravitational and electromagnetic radiation is sefigible

only if: the length-acale of the star $R_{(}\left(>M_{6}\right)$ is mall compared to the length scale of the metric. The external metric has a vacuum Biemann teneor characterized by the following three length scales:

- $R=$ Radius of curvature: $R^{2} \sim \frac{\lambda}{R_{\text {gaw }}}$.

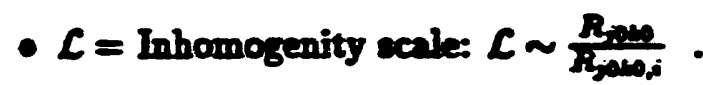

- $T$ = Time scales $T \sim \frac{R_{\text {nan }}}{R_{\text {sans }}}$. In terms of the Kerr-metric this conditions can be easily derived from the components $((13)-(16))$ of the Biemann-teneor with respect to the nsed tetrad aystem:

$$
R \sim r \sqrt{r / M B H}, C \sim r / 3, T \sim \infty
$$

The condition for the star's length scale $\boldsymbol{R}$

$$
R<R, R<L \text {. }
$$

is a real restriction for very close encornters, became the length-ecale of the deformed star increaces by ordess of magnitude especially during a vary close encounter.

The following table relates the typical stellar dates to curvature-radii of SBI with corresponding mans. 
Tidal compresion and distuption of stars near a rotating black hole

RANGE OF APPLCATION FOR TIDAL TENSORS - EXAMPLES

\begin{tabular}{|c|c|c|c|c|c|}
\hline Example" & $M_{0}\left(M_{0}\right)$ & $R\left(R_{\theta}\right)$ & $M_{\pi}\left(M_{\theta}\right)^{\prime}$ & Amae ${ }^{2}$ & $M_{B E}\left(M_{\Theta}\right)^{d}$ \\
\hline $\begin{array}{l}\text { BO V } \\
\text { AO V } \\
\text { GO V } \\
\text { MO V } \\
\text { FO III } \\
\text { KO III } \\
\text { YO III } \\
\text { AO I } \\
\text { FO I } \\
\text { RGinet (CAlor) }\end{array}$ & $\begin{array}{l}18 . \\
3.2 \\
1.1 \\
0.5 \\
2.5 \\
3.5 \\
5.0 \\
16 . \\
12 . \\
16 .\end{array}$ & $\begin{array}{l}7.5 \\
2.6 \\
1.1 \\
0.6 \\
5 . \\
16 . \\
40 . \\
40 . \\
60 . \\
245 .\end{array}$ & $\begin{array}{l}3.5 \cdot 10^{6} \\
1.2 \cdot 10^{6} \\
0.3 \cdot 10^{6} \\
0.3 \cdot 10^{6} \\
2.3 \cdot 10^{6} \\
7.5 \cdot 10^{6} \\
1.8 \cdot 10^{7} \\
1.8 \cdot 10^{7} \\
2.8 \cdot 10^{8} \\
1.2 \cdot 10^{8}\end{array}$ & $\begin{array}{l}1.22 \\
0.75 \\
0.46 \\
0.28 \\
1.58 \\
4.51 \\
10.0 \\
6.80 \\
11.2 \\
41.7\end{array}$ & $\begin{array}{l}1.6 \cdot 10^{9} \\
7.5 \cdot 10^{2} \\
3.6 \cdot 10^{6} \\
2.1 \cdot 10^{3} \\
2.3 \cdot 10^{0} \\
1.1 \cdot 10^{20} \\
3.7 \cdot 10^{10} \\
2.0 \cdot 10^{10} \\
4.3 \cdot 10^{10} \\
3.1 \cdot 10^{11}\end{array}$ \\
\hline
\end{tabular}

- Mes of a black bole with curvature-radive $R \sim T^{2 / 2} . R$.

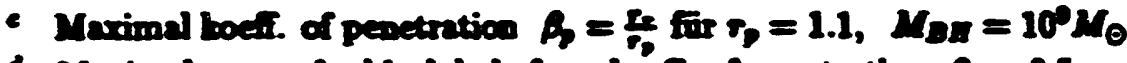

1 Maximal mas of a blact bole for a boeff. of pesetration. $A_{p}=0.5, r_{p}=2.0$

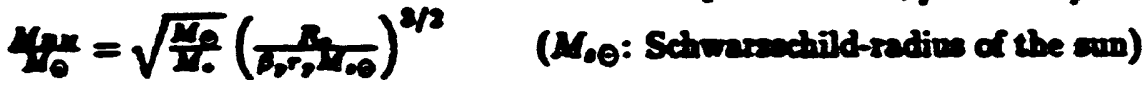

6.1 Classical treatment, non-relativistic case vs. Geodesic, Riemann-tensor

As simple test let's compare the chasionl treatment with the complete newtonian tidal forces to a similar compntation wing the integrated geodevics and the Riemann-tensor.

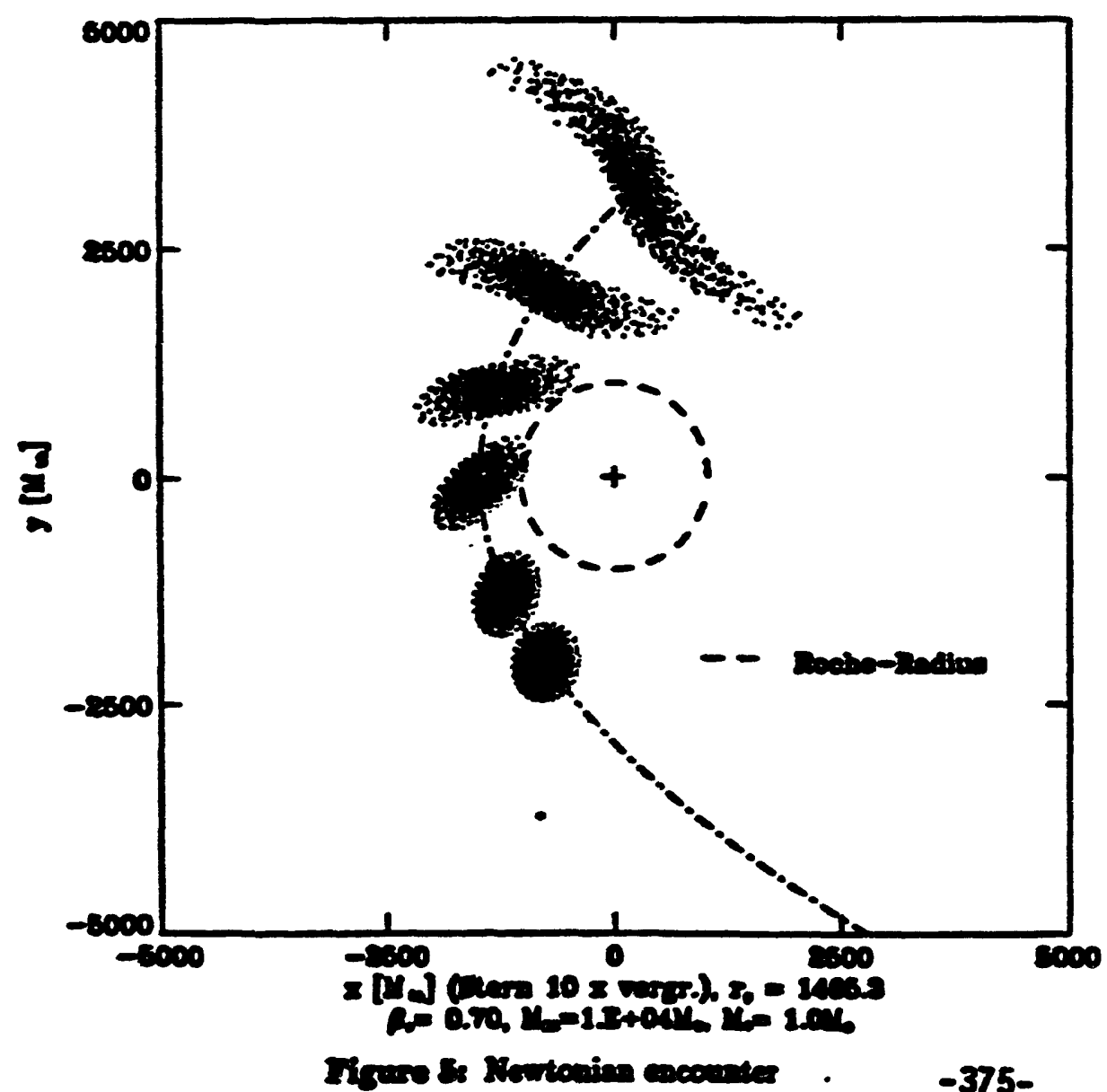


Both computations should show the rame results in a chasical, binear case: The linear sonrelativistic encounter happens, if $\left(M_{B H}<R<r_{P}\right)$. In Fig. 5 the formal Keplerian Orbit is shown; the compratation wes the exact (son-linear) Newtonian tidal forces.

This is a computation with 1000 particles; the SPH-width 2 h corresponds $\cong$ to the length of the ticks at the axis - all the length scale wits are in wnits of the Schwarechild radivs $\mathbf{M}_{B E ;}$, the projection of the interpolation-points cato the equatoriah-layer is enlarged by the factor of 10.

The simulation has to be compared with Fiz. 6.

Fig. 6 wes the geodacic equations; the tidal forces are veed as components of the Biemann tensor for the anme configuration (clasical, Finear cane) as Fis. 5. Both computations show casentially the anme particle distribution.

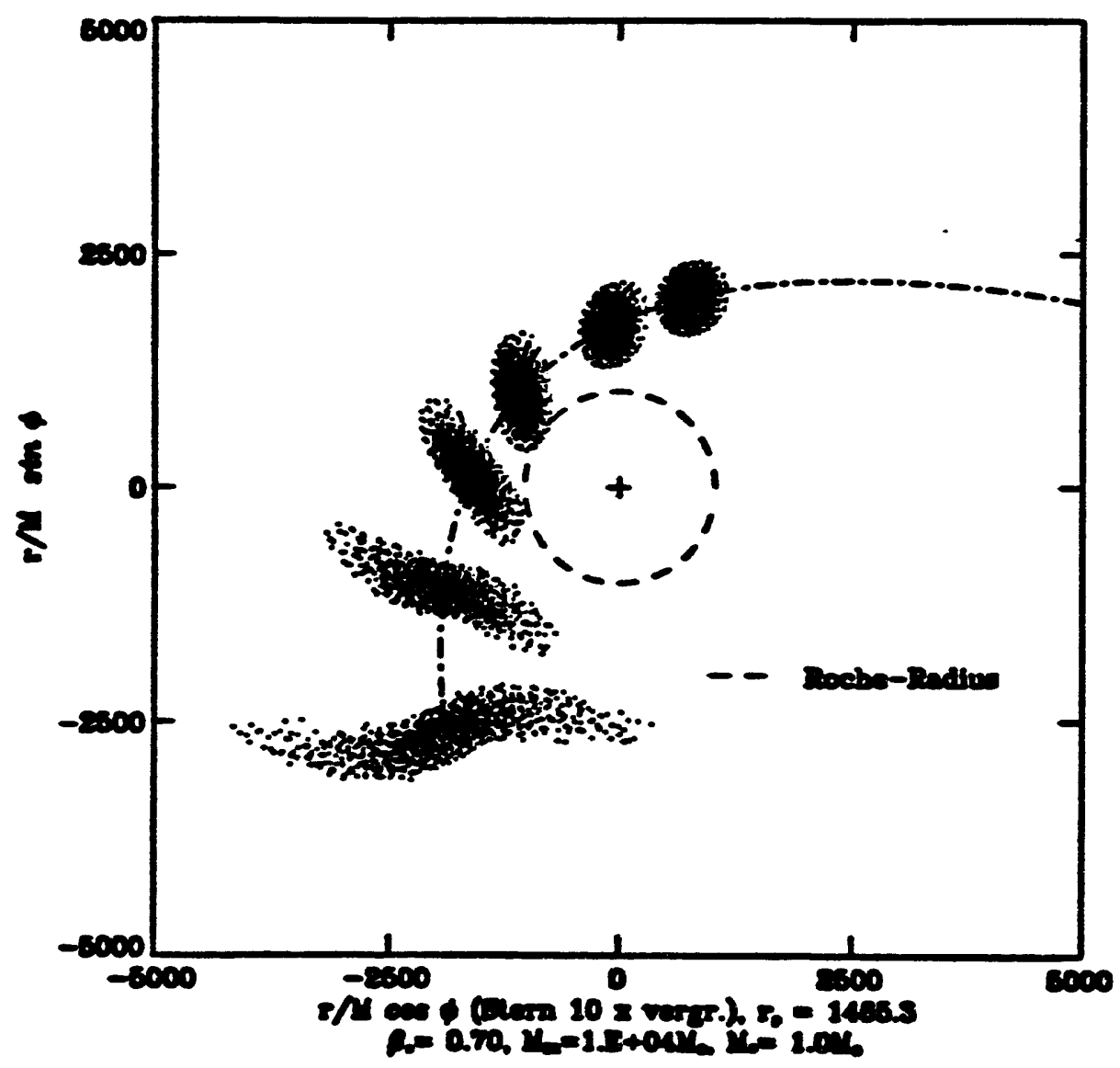

Figure Ge Generd relativitic enocunter in the Newtonin Iimit

Although the number of particlos (1000) is rather rextricted, the timviations gave a single teat for the correctines of the detivation of the (relativistic) tidal forces and the weed time and length-ecales. 
Tidal compression and disruption of stars near a rotating black hole

\section{Nonlinear Newtonian encounter}

- Very deep penetration of the star into the Roche bobe were discused in connection with

- tidd indeced neclear procesing of the star's matter (Ninclear

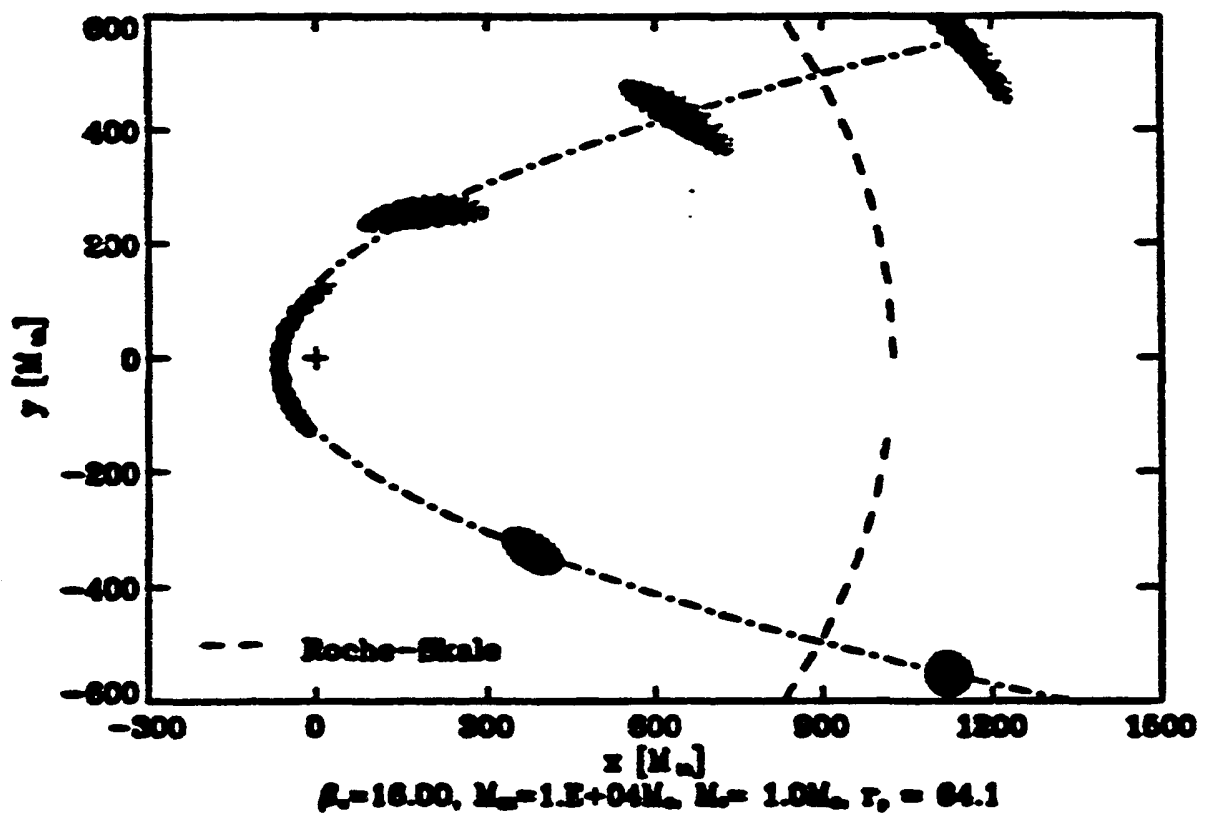

Figure 78 Nonlisear Newtonian encounter

runaway"-scenario (Lrminet and Carter, 1986]) and

- q-bursts (Carter, 1992).

- It is asomed, that the regime of the tidal internction is cocon-

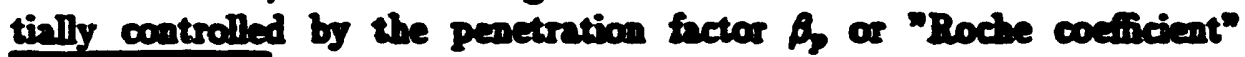

$$
\beta_{p}=\frac{R_{R}}{r_{p}}
$$

with the typical length coale in the tidal fiedd:

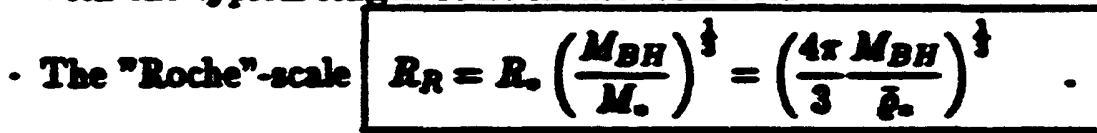

- Ior vay chere ( $\left.\beta_{2}>1\right)$ and sonimar $\left(r_{p} \simeq D_{0}\right)$ cocontes the polytrope is stretcled aloas the oxbit and cervely comprosead in the vertienl dircetion. This relocts in an barease for the centarl donity fat of ter the clowest apposech, before being div coived.

- The particles are bread buto bulividal Keglarian orbits and to crose a conall aree, which bads to the compresioncrot.

- Partides eo rather a "age of a ser

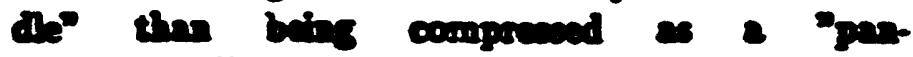
crib". (Die 8 sheme a vien cato the

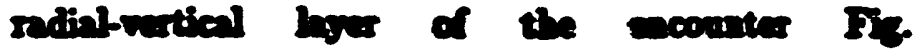
7.)

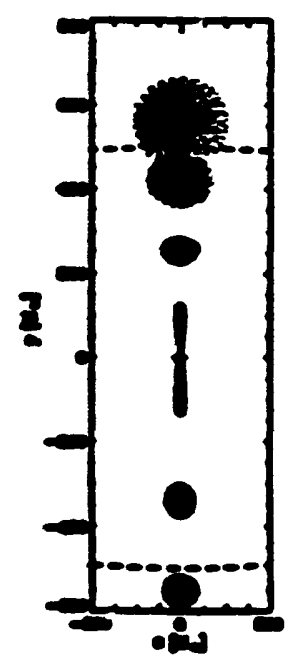

Itane 8 


\section{Tidal compression - relativistic case}

For the very dose encounters in the vicinity of a BH, this transition of the ceveral hydrodynamical elements through the equatorial layer occur repented. Thus, the corresponding size of the vertical principal axis will have ceveral minimums (Fis. 10). The coordinatetime $t$ of sech event (Fis. 9) is moctly very similar to the proper time $r_{r d}$ - if such violent process appear it must be observed with the same timescale of ceveral 5. $\boldsymbol{M}_{6}$ (where $\boldsymbol{M}_{6}$ is the mase of the $\mathrm{BH}$ in $\boldsymbol{M}_{0}$ ). A multiple peal in the density of the star conld not be recolved, probably due to the low recolution of the simulation.
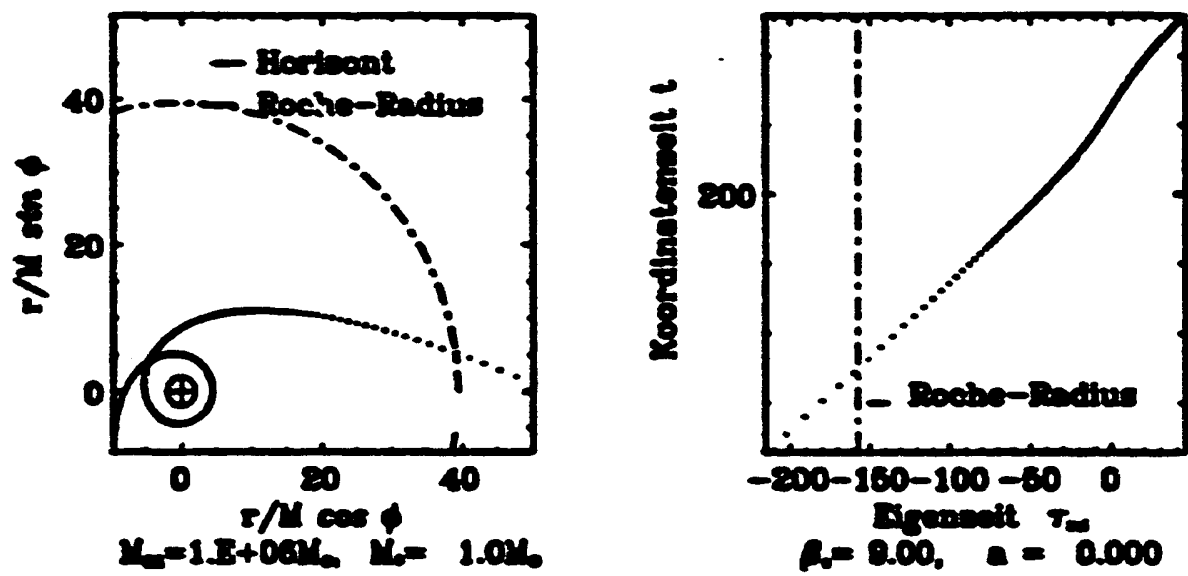

Figure 9: Geodesic for very close encounter. $\beta_{p}=R_{R} / r_{7}=9$. Proper-time remains neariy unchanged.
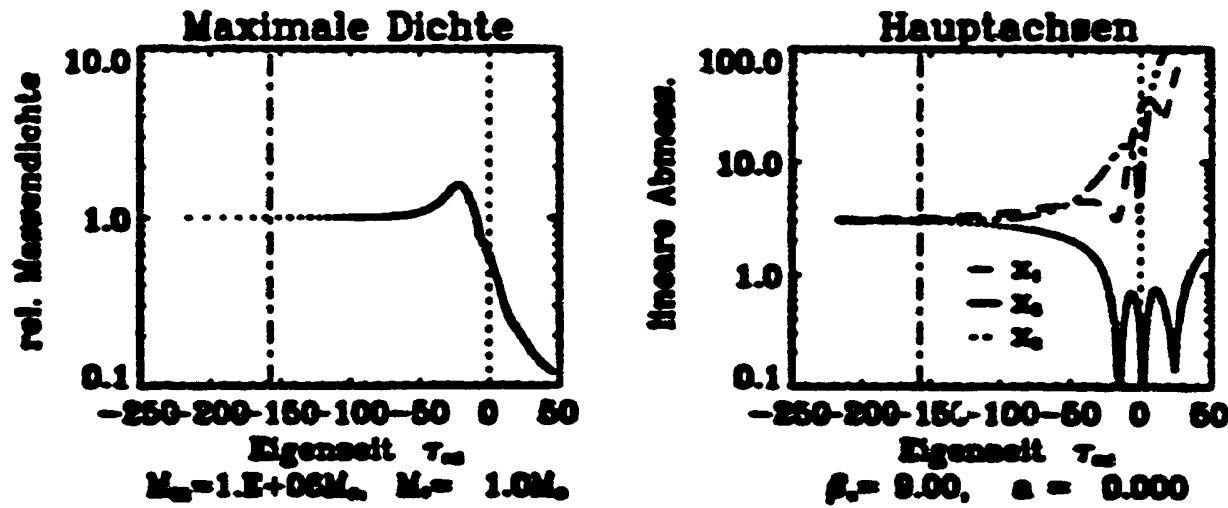

Figure 10: Time dependence of the central density and the length of axis for very clove encounter. (Fig. 9). The clower the marginal bound arbit is reached, the more tidal vertical compresions occur. ( 1000 Particlea) 


\section{Influence of BH-Rotation on Tidal compres- sion}

For the compreasion of the star there are intrences already by ite rotation-modified orbit of the star: For retrograde orbits, the orbit of the ctar is clower to the margial bound orbit for a given perited-distance compared to a sonrotating or a co-sotating BH. The phenomenon of ceveral compreations are therefore reached more easely.
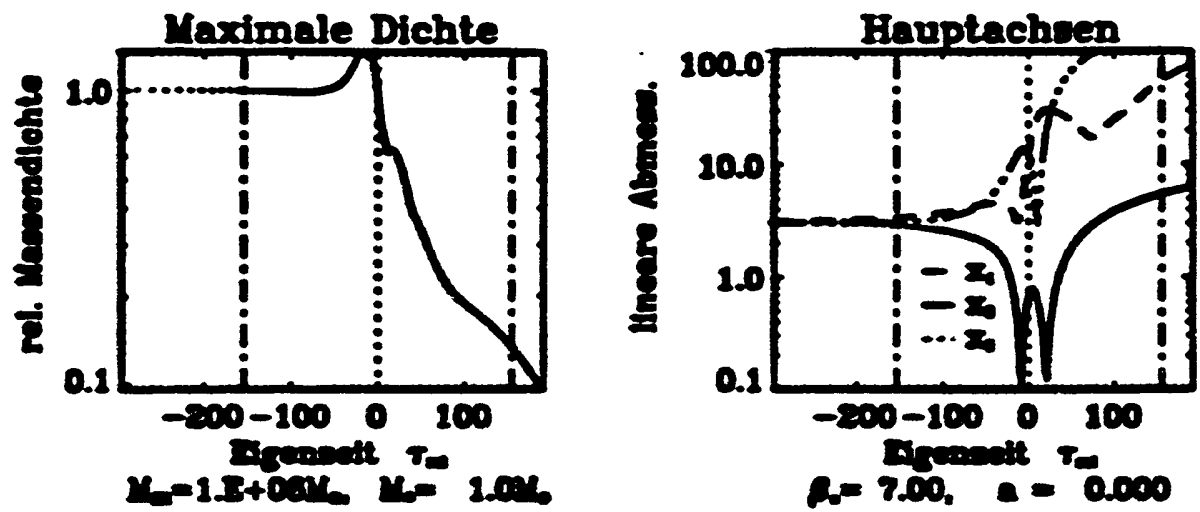

Figure 11: Dearity ve. time and relative magnitudss of the principal axes of the star for the eocounter with the Schwarachild BH (Cp. Fif. 12. $\beta_{p}=R_{R} / r_{p}=7$. 1000 Particles.)
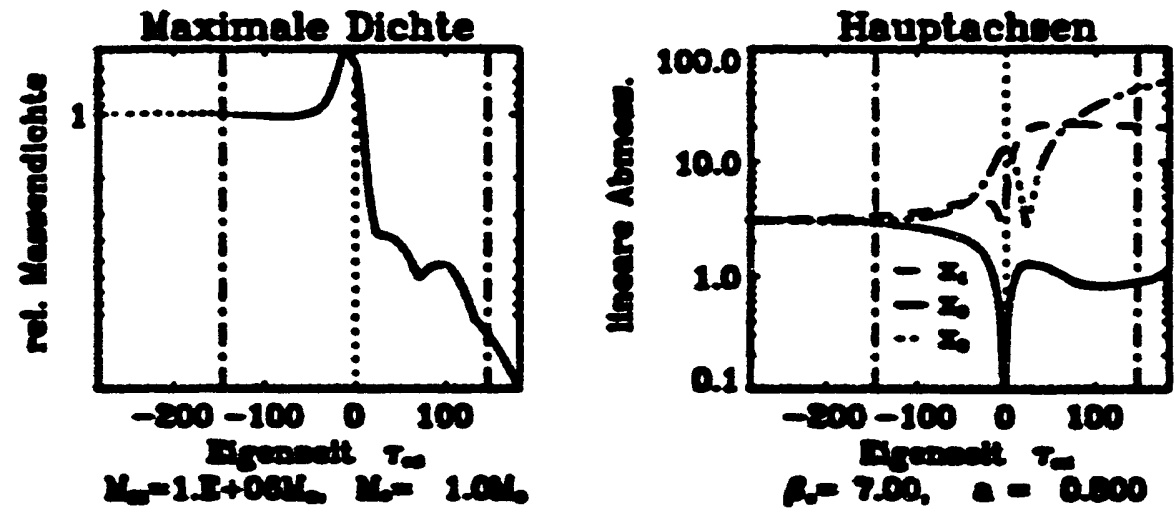

Figure 12: Deneity va time and selative magnitudes of the principal ares of the Atar for the prograde encounter with the Kerr BH (Cp. Fif. 11). $\beta_{p}=R_{R} / r_{p}=7$.

For the very dowe encounter we obtin dee to the son-linere - in the

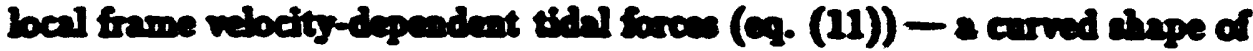

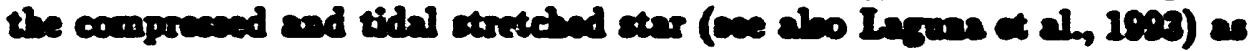
abown $b$ the figare 13. Howner we bave to be carefilly about the renge

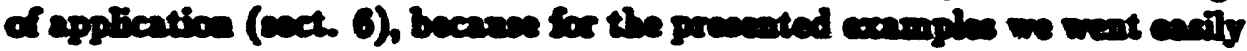


I

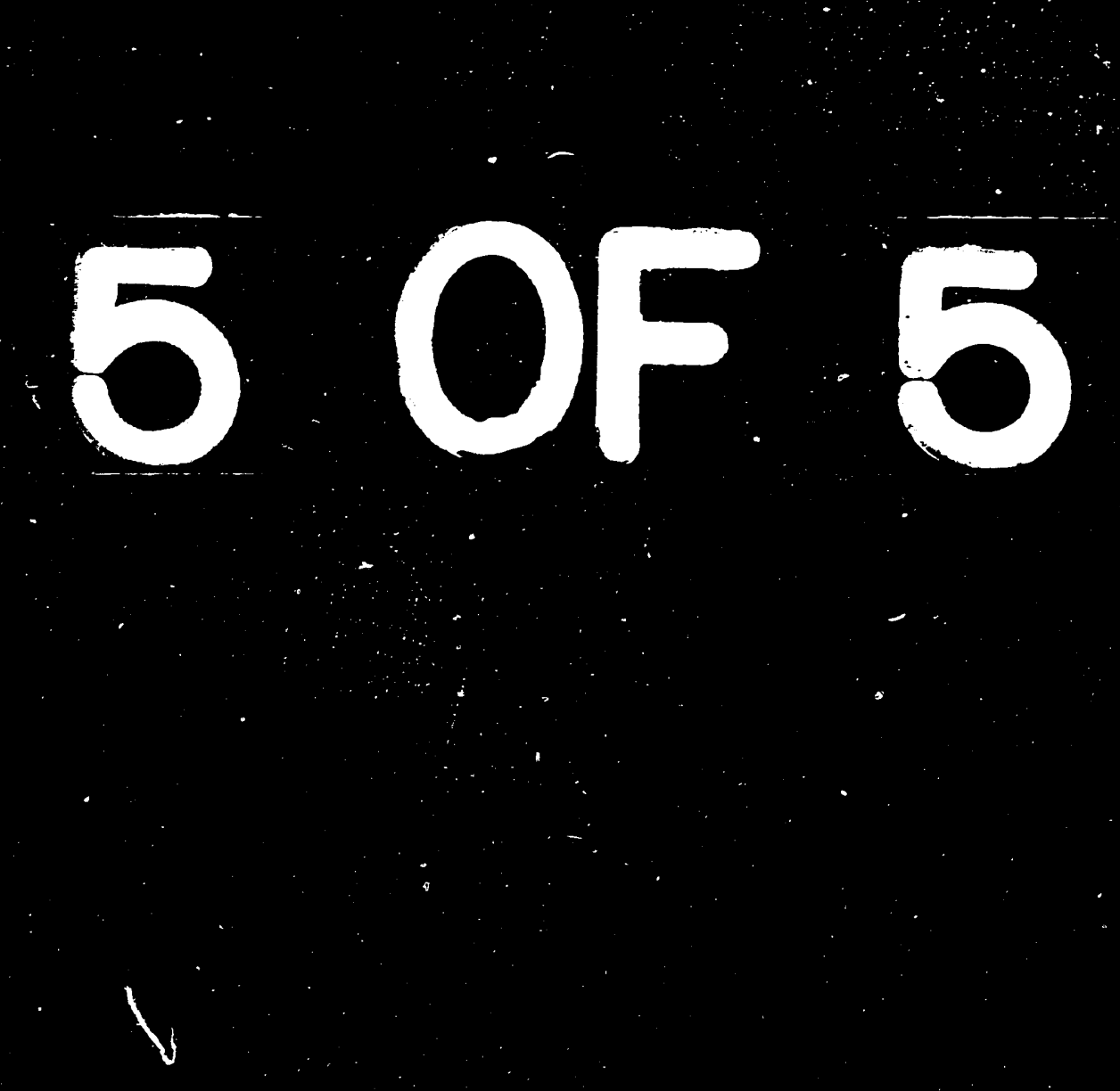

8

4 


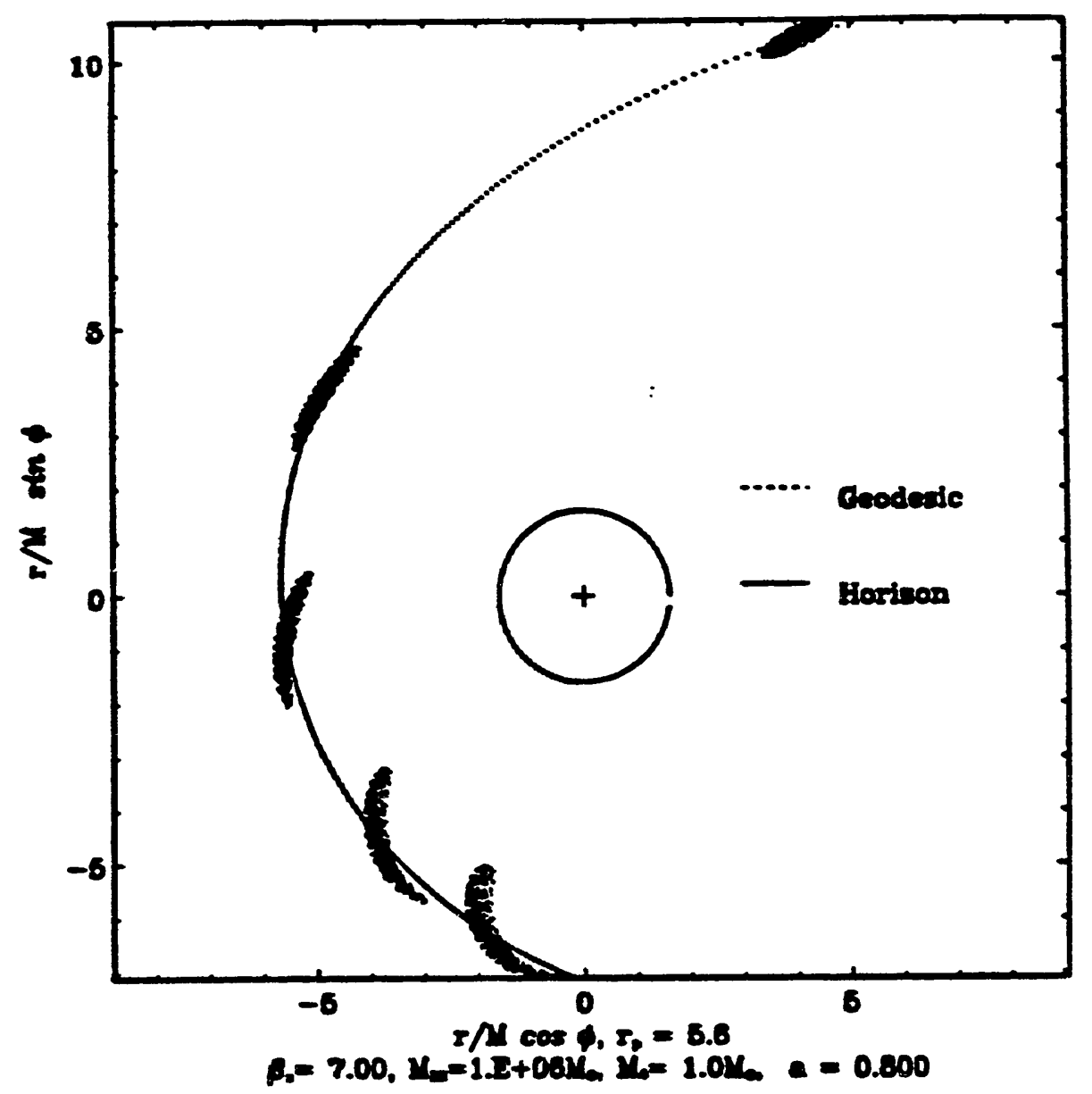

Figure 13: Non-linear treatment for Kerr-BH $a=0.8, \beta_{p}=R_{R} / r_{p}=7$.

into the dangerous region, where the length scale of the star has same order of magnitude as the inhomogenity scale, radius of curvature, respectively.

\section{Tidal disruptions}

To estimate Boche-limits the linear treatment $(R<R, R<\mathcal{L})$ is appiicable for computations of tidal disruptions $\left(\beta_{p} \simeq 1\right)$ of Main-SequenceStars and remains in the range of application. The influence of relativistic trajectories on tidal disruptions has been discused already in rect. 4:

- Minimal distance of the periastron is effected by opin of the SBH.

- Orbits near the marginally bound became nearly "circular" and the tidal time-fificiency encreaces.

- Tidal forces increase with respict to the Newtonian case.

- Tidal forces increase with spin of the SBH for retrograde trajectories (Frame-dragging). 
- Examples:

1) $\mathrm{SBH} \mathrm{MBH}_{\mathrm{BH}}=50 \cdot 10^{6} \mathrm{M}_{\odot}$.

- Simulations with the same $\beta$, but different angular momentum of the SBH (figs. 14-19).

2) Extreme Kerr-SBH $M_{B H}=10^{\circ} M_{\odot}$ (figs. 20-22).

A rough impreasion for the angular momentum-infinence of the SBH onto the tidal process may be obtained from the figs. 14-19. We identify the event of a successful disruption of the star as the increase of the sotal energy $E_{\text {tot }}$ above zero (figs. $\left.15,17,19\right)$ and the decrease of central stellar mass-density a well as a run-away of the principal axis of the atar (figs. 14,16,18).
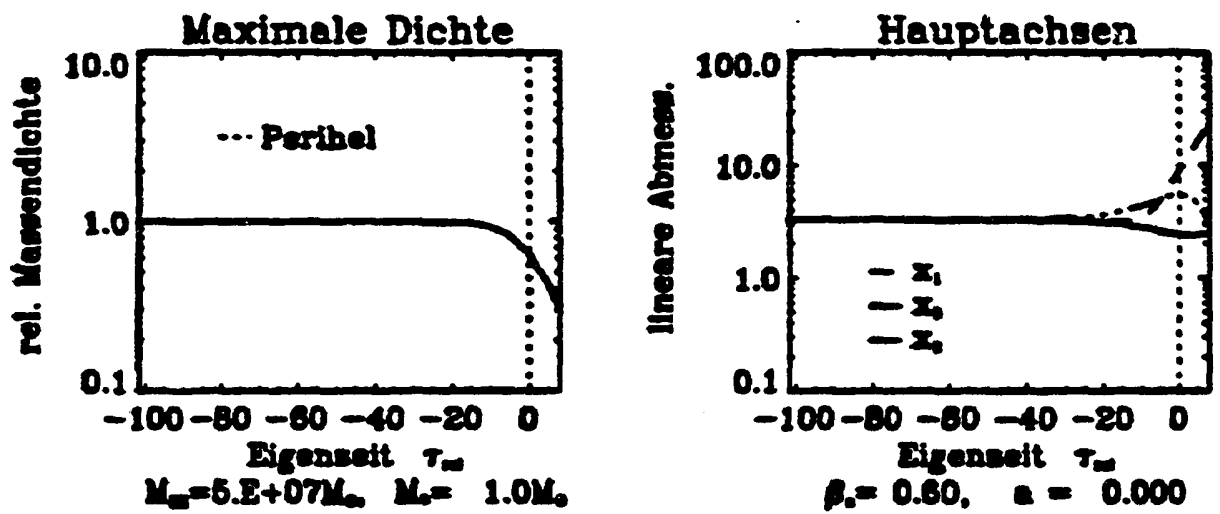

Figure 14: Density vs. time and relative magnitudes of the principal axes of the star for the encounter with the Schwarzschild BH. Transition of perihel at $\tau=0$.

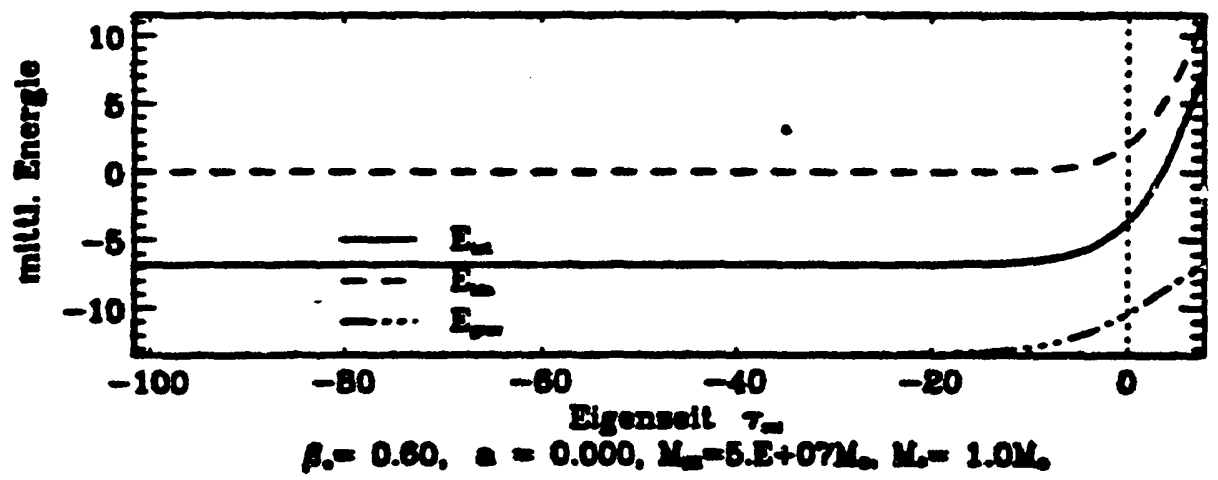

Figure 15: Kinetic, gravitational and total energy vo. time. Encounter with the Schwarzschild BH. Roche-limit if $E_{\text {tot }} \geq 0$ shortly after transition of perihel. (5000 Particles.)

Indeed, this disruption-event oceurs for a Schwarzschild-hole (figs. 14, 15) a moment after the transition of the star through the periantron. With 
the same Roche-coeff. $\beta$ (the came perihel-distance), but for a prograde orbit (figs. 16, 17), the tidal disruption-event happens a considerable time later (after the periastron-transition) for an encouriter with a moderately rotating Kerr-BH. On a related ratrograde orbit (figs. 18, 19), disruption occurs a reasonable time prior to the transition through the periastron (in all the computations, we interrupt the simulation, if the star is disrupted.
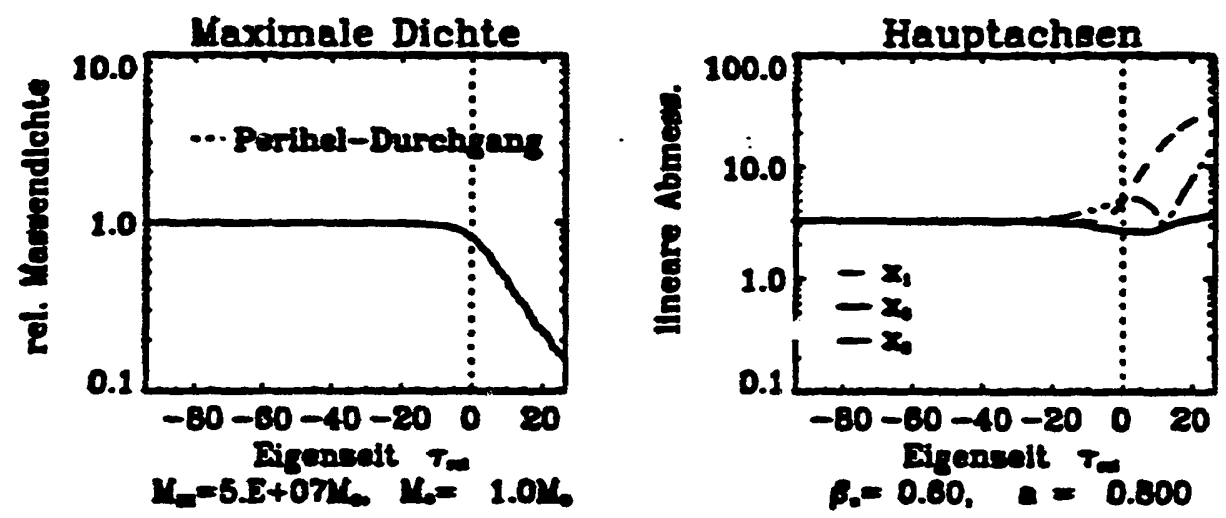

Figure 16: Density vs. time and relative magnitudes of the principal axes of the star for a prograde encounter with the Kerr BH. Transition of perihel at $\tau=0$.

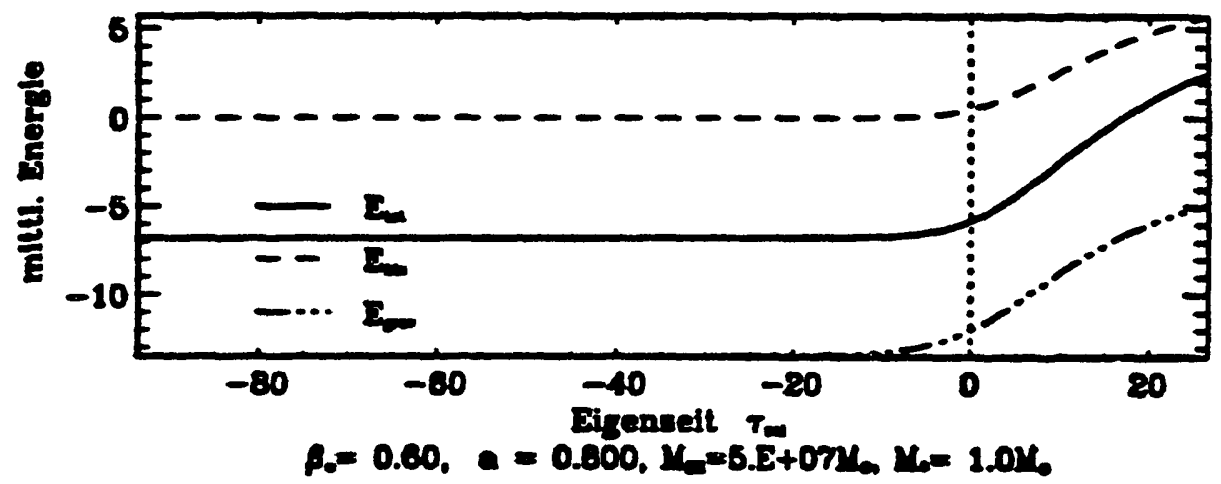

Figure 17: Kinetic, gravitational and total energy vs. time. Prograde encounter with the Kerr BH. Rochelimit if $E_{\text {zor }} \geq 0$ longer time after transition of perihel. (5000 Particles.)

\section{Tidal disruptions by a $10^{9} M_{\odot}$ Kerr-BH}

The encounter of a $1 M_{0}$ main-sequencestar encounter with a $10^{\circ} M_{0}$ extreme Kerr-BH (figs. 20 - 22) results in a clear tidal disruption-event. (Positive total energy, decreasing dencity of the star, increasing principle axis.) In connection with the fuelling of AGN we assume, that the tidal disuption events of atars are important as fuelling process aleo for the 

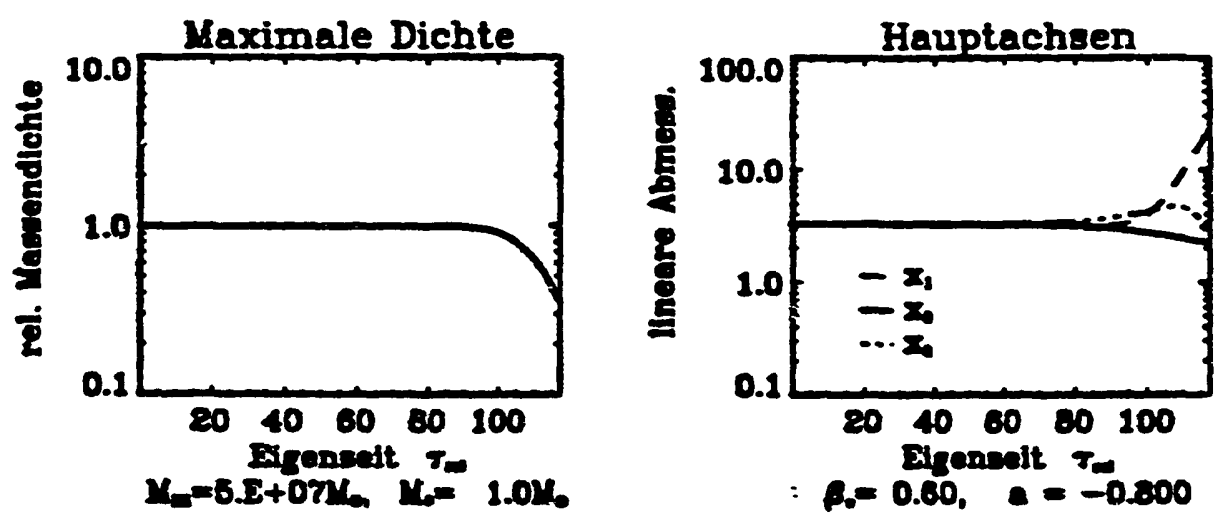

Figure 18: Density vs. time and relative magnitudes of the principal axes of the star for a retrograde encounter with the Kerr BH. Star being dissolved before reaching the periastron.

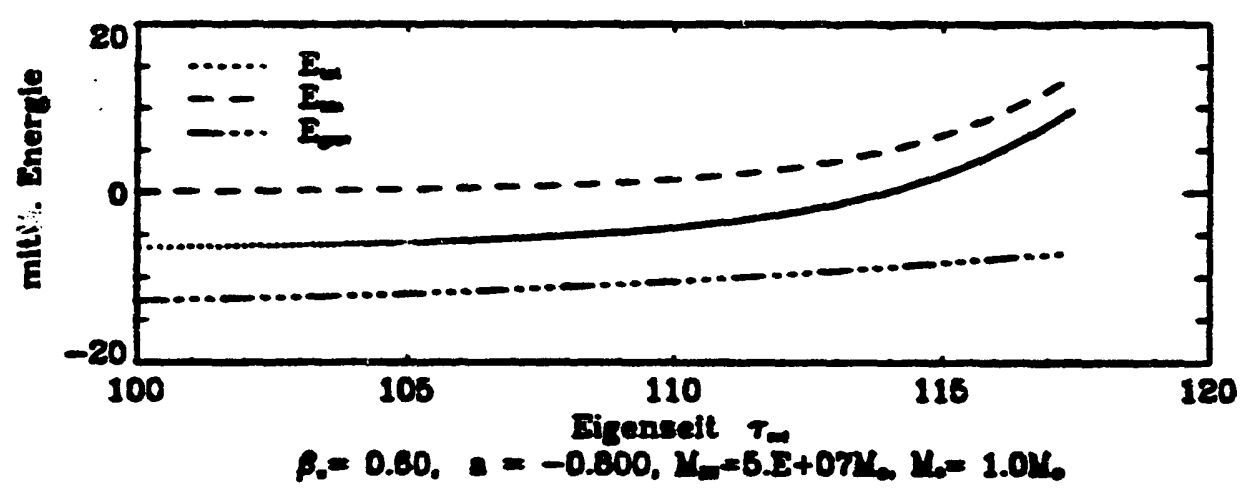

Figure 19: Kinetic, gravitational and total energy vs. time. Retrograde encounter with the Kerr $B H\left(a=-0.8,5000\right.$ particles) Roche-limit $E_{\text {tot }} \geq 0$ reached before transition of perihel. (5000 Particles.)

brightest AGN's (if the mass of the corresponding SBH is assumed for such BH's to be about $10^{\circ} \mathrm{M}_{0}$ and if it is rapidly rotating.) For such assumed SBH's, the mass-range for the tidal disruption event (due to the arguments given in Hills, 1975) will be extended in one order of magnitude.

The process may go to even higher masses of the BH, if one takes instead of a main-sequence-star a leas dense star, e.g. a red giant.

For tidal-events, the energy-distribution $E=-p_{t}$ and the orbital angular momentum-distribution $L_{z}=\eta_{\phi}$ of the debris may be calculated by the back-transformation by $p_{\mu}=\lambda_{\mu}^{[a]} \dot{X}_{\alpha}$. For events cloce to the Roche-limit, the envgy-distribution of the debris of the star is nearly the same as the energy of the former star: No dramatic changes occur for snch limiting case. The fate of the stellar debris is essentially the same as it would have been for the star itself; if the otar were on a geodesic, that is swallowed completely into the hole, than the fate of the debris will be the same and 
vice versa. For the above example eg. the distribution in energy and

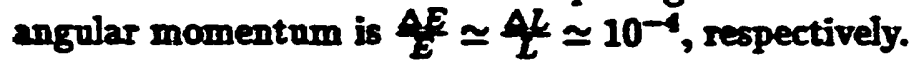

For the example described in (figs. 20-22), we expect as result of the tidal process that the debris leave the cloes environment of the BH on similar geodesic as the former star ((fig. 20). The resulting highly elongated fragment of an accretion-disk may be later very fast transformed into a circular accretion disk fragment due to perihel-shift-effects and viscosity (Syer et al, 1991).
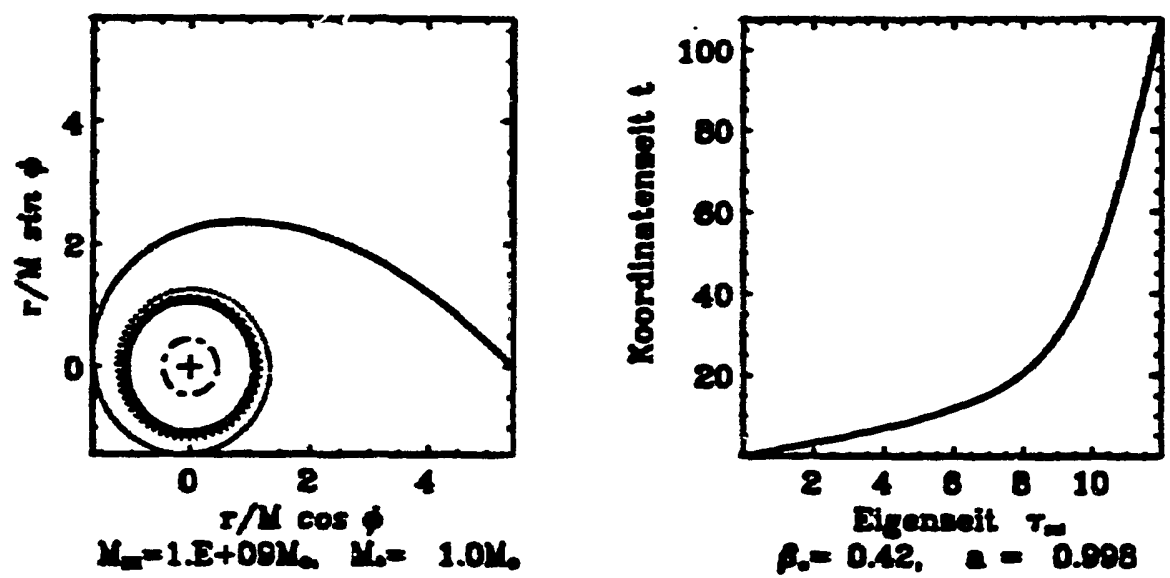

Figure 20: Trajectory of the centre of mass of the star. Position of the Schwarzschild-BH and its Schwarzchild radius. maseereichen Schwarzen Lochs. Roche-coeff. $\beta_{p}=R_{R} / r_{p}=0.42$, "canonical" Spin of the Kerr-SBH $a=0.998$. $r_{p}=r_{m b}+0.1$
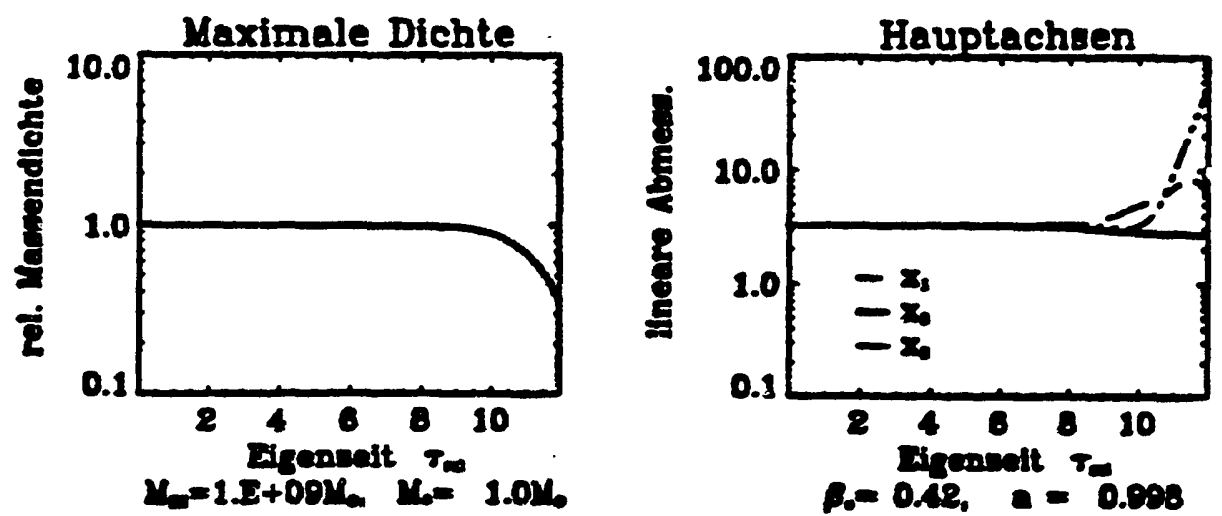

Figure 21: Kinetic, gravitational and total enerzy vs. time. Prograde encounter with the Kerr BH. Roche-limit if $E_{\text {ret }} \geq 0$. Trajectory (Fig. 20, 5000 Particles) 


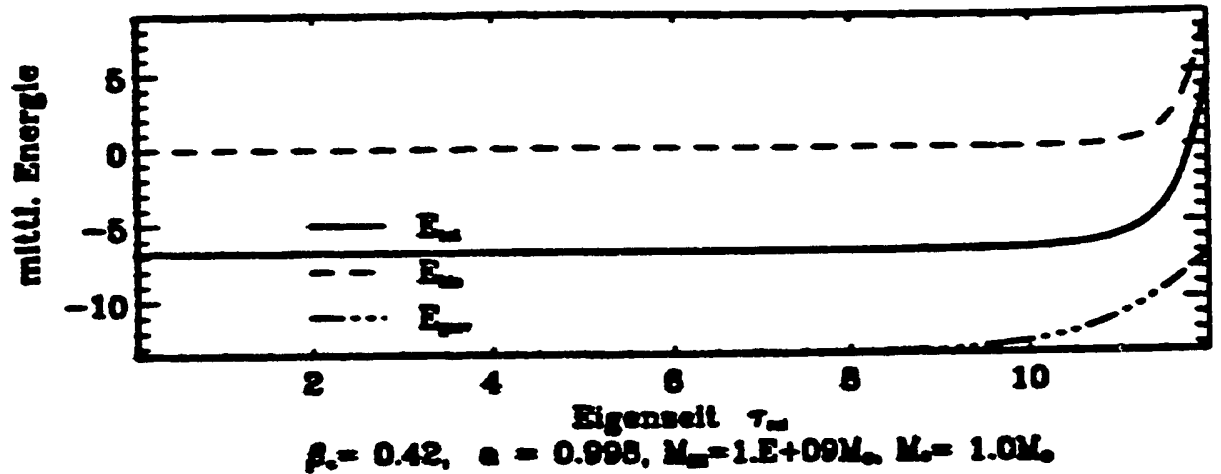

Figure 22: Kinetic, gravitational and total energy vs. time. Prograde encounter with the Kerr BH (Fig.20). Roche-limit if $E_{x \alpha 0} \geq 0$. (5000 Particles.)

\section{References}

Bens, W. and Bills, J. (1987). The Astrophysical Journal, 323:614.

Carter, B. (1992). The Astrophysical Journal, 391:L67.

Evans, C. and Kochanek, S. (1989). The Astrophyoical Journal, s48:I13.

Fishbone, L. (1973). The Aatrophysical Journal, 185:43.

Hills, J. (1975). Nature, 234:295.

Hodghinson, D. E. (1972). Gen. Rel. and Grov., 3:351.

Laguns, P., Miller, W. A., Zurek, W. H., and Davies, M. (1993). The Astrophysical Journah, 410:L83.

Lvcy, L. (1977). The Actronomical Journal, 82:1013.

Luminet, J.-P. and Carter, B. (1986). The Astrophysical Journal Suppl. Series., 61:219.

Luminet, J.-P. and Marck, J.-A. (1985). M.N.R.A.S., 212:57.

Mann, P. (1991). Comput. Phys. Commun., 67(2) :245-260.

Marck, J.-A. (1983). Proc R. Soc. London, A 385:431.

Mashhoon, B. (1975). The Astrophysical Journal, 107:705.

Mashhoon, B. (1977). The Astrophysical Journal, $216: 591$.

Monaghan, J. (1992). Ann. Rev. Astron. Astrophys., 30:543.

Rees, M. (1988). Nature, 333:523.

Spontolz, H. (1991). Gas release through tidal disruption of stars. In Duschl, W. and Wagner, S., editors, Physice of Active Galactic Nuclei, page 244. Berlin/Heidelberg/New York.

Syer, D., Clarke, C., and Rees, M. (1991). M.N.R.A.S., 250:505. 


\section{$-386-$}


Relativistic SPH

Viscosity and Energy

J.J. Monaghan Monash University jjm@vaxc.cc. monash. eder.au

Leo Brewin, Anthony Lun Monash. University 
PROGRAM

- basic equations

SPH EQUIVALENTS

- VISCOSITY, BARYON-BARYON SCATTERING

- munerical

- regge calculus 
SPH Relativistic Simulations

- N. Lahy MSc.Thesis. Monash Univ.

- P. Mann Comp.Phys. Comm 67, 245, (1991)

- P. Lugano, W. Moller, W. Zurek Ap.J. 404, 678, (1993)

- J.J. Monaghan Comp. Phajs. Rep.

Ann. Rev. Astr.Ap. 1992

P.I.C. Sinulations

Clare and Stiotimean Phys.Rep.141, 177, (1986)

Nise and Stretfman. LA-UR-81-3452

Amsden, Harlow, Nhe Phys.Rew. C 15, 2059, (1477)

$-389-$ 


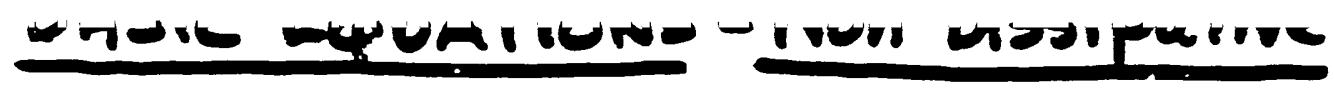

Landau, Lifshity Fluid Mechanics I Wilson.

$$
\begin{aligned}
& T^{\mu \nu}=\left(n m_{0} c^{2}+n \hat{\varepsilon}+P\right) u^{\mu} U^{\nu}+P g^{\mu \nu} \\
& g_{00}=-1, g_{1}=g_{22}=g_{33}=1 \\
& u^{0}=\gamma, v^{i}=\gamma v^{i} / c, \gamma=\frac{1}{\sqrt{1-v^{2} / c^{2}}} \\
& U_{2} U^{\nu}=-1
\end{aligned}
$$

From $\frac{\partial T^{\mu \nu}}{\partial x^{\nu}}=0$.

Get

$$
\begin{array}{ll}
\frac{d \vec{q}}{d t}=-\frac{1}{N} \nabla P & \text { Momentum } \\
\frac{d}{d t}=-\frac{1}{N} \nabla_{0}(P \vec{J}) & \text { Energy }
\end{array}
$$

Where

$$
\begin{aligned}
& \vec{q}=\frac{\vec{v} \gamma^{2}\left(P+n \hat{\varepsilon}+n m_{0} c^{2}\right)}{c^{2} N} \\
& \varepsilon=\frac{\gamma^{2}\left(P+n \hat{e}+n m_{0} c^{2}\right)-P}{N}
\end{aligned}
$$

and

$$
\frac{d}{d t} N=-N \nabla \cdot(U) \quad \text { Continuity }
$$

$-390-$ 
EQUATION OF STATE

$$
P=(r-1) n \hat{e} \quad \geqslant 0
$$

- S. Chandrasekhar (Intro .To Stellar Structure) For Relativistic Gas.

- Amsden, Hor low, Nix, STrottman for a nuclear equ. of state.

-391- 
- fran.ti mory asour un watidon ra mos voifonba Fbizng ayL

$.9,201$ arod Has m. Suofrug 8, on

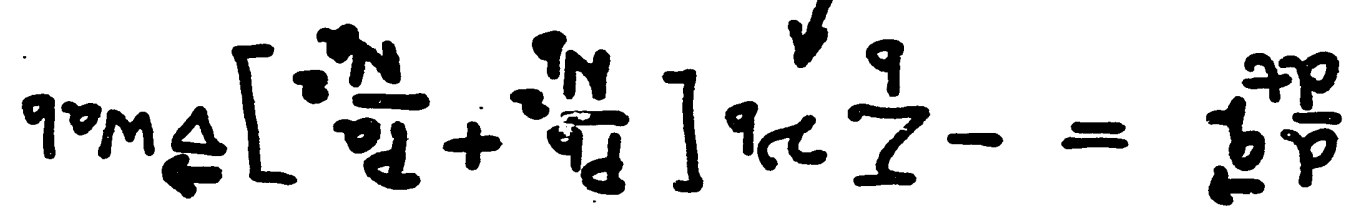

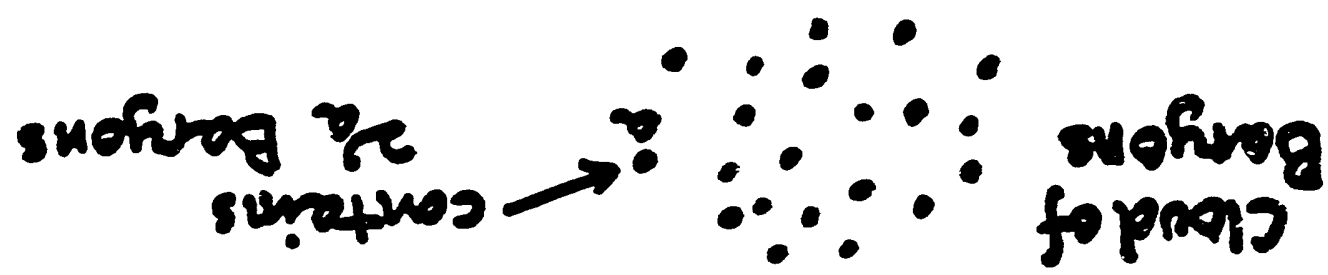
suo!tronb3 HdS 
TOTAL ENERGY

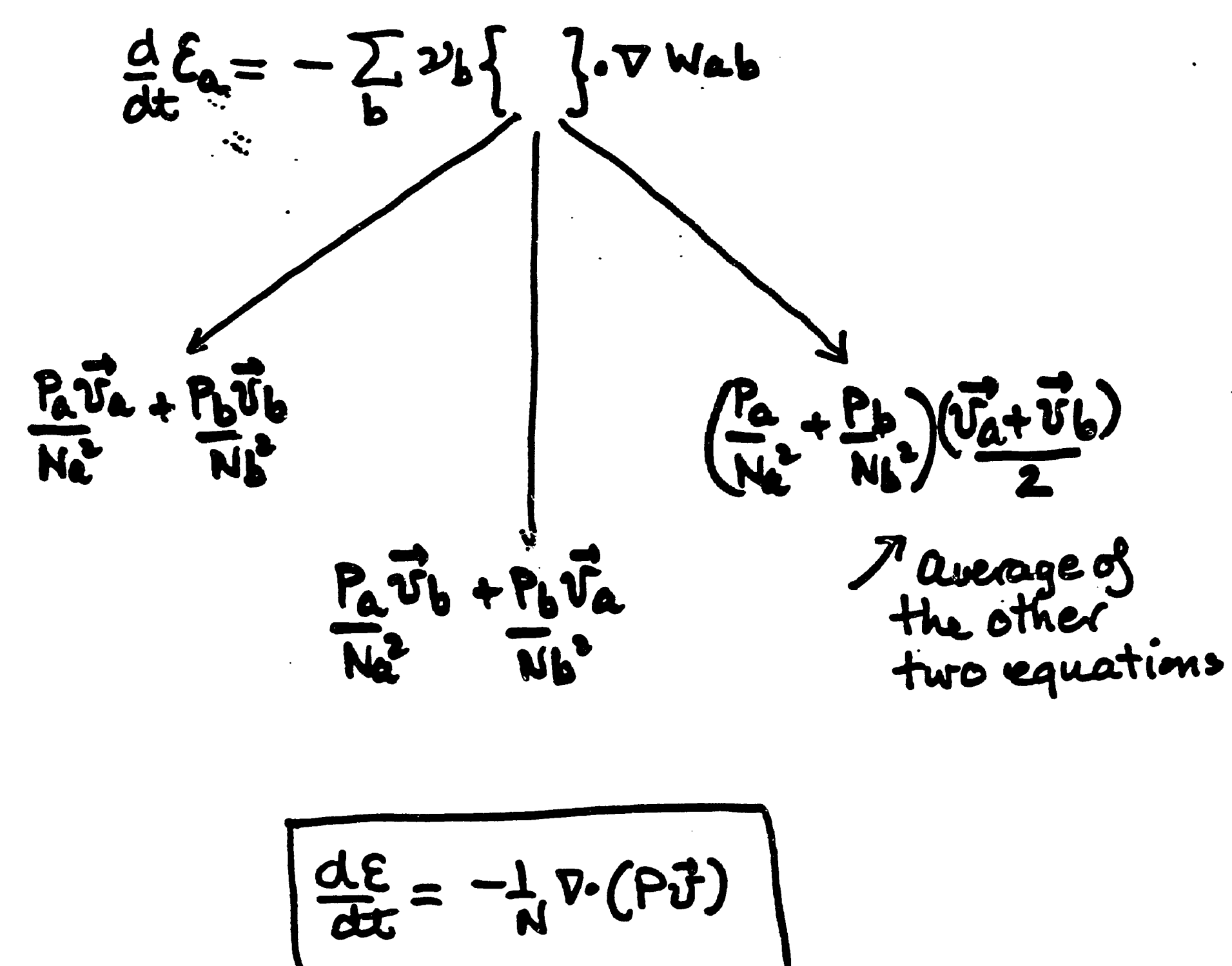

$-393-$ 
Thermal Energy

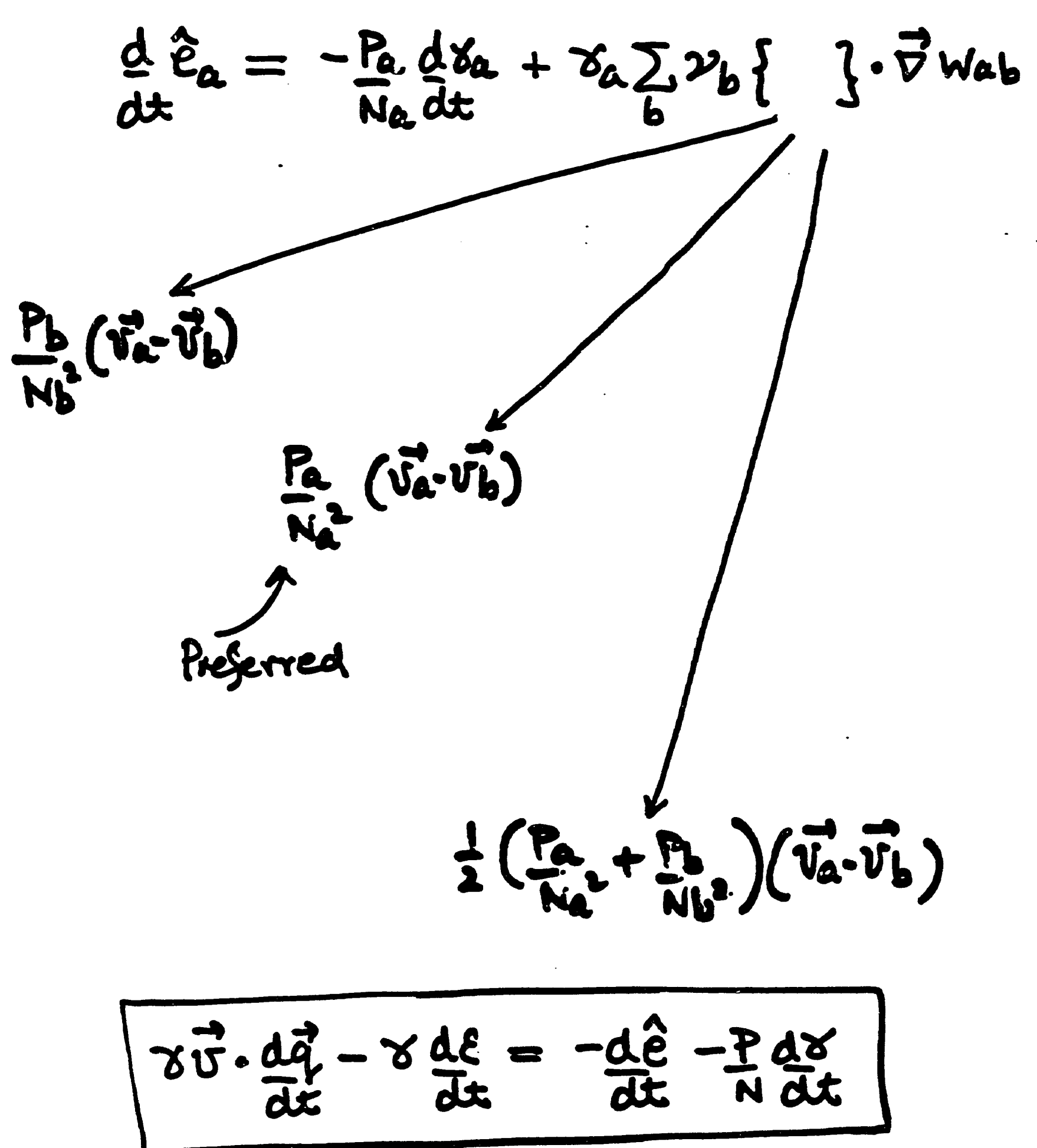

$-394-$ 
VISCOSITY

$\left.\begin{array}{l}\text { Laudau, Lifshit3 } \\ \text { Eckart } \\ \text { Weimberg, Carter }\end{array}\right\}$ Besed on Elegance

$$
\begin{aligned}
t^{\mu \nu}=\left(n m_{\alpha} c^{2}+\right. & n \hat{e}+P) u^{\mu} v^{\nu} \\
& +(P-\xi \theta) g^{\mu \nu} \\
\therefore \quad & \\
& \frac{1}{c} \frac{d \gamma}{d t}+\frac{\gamma}{c} \nabla . v
\end{aligned}
$$

All Unstable

Hiseoek, Lindblom Phys.Rew $D$

$$
\begin{aligned}
& \text { olson. Hiscoek } \\
& \text { 3], 725,(1985) } \\
& t \sim 10^{-3} y \text {. } \\
& \text { 41 ,3687, (1990) }
\end{aligned}
$$

Numerical

Hawteij, Smarr, Wilson Ap.J. Supp

$$
P_{\text {ant }}=\gamma l^{2}(D \cdot v)^{2}\left[n m_{0} c^{2}+n \hat{e}+P\right]
$$

Laguna, Moller, Zurek; Lahy (n.sc)

$$
\begin{gathered}
P a r t=N\left\{a l c|0 . v|+\ddot{\beta} l^{2}|\nabla . v|^{2}\right\} \\
\text { if } 0 . v<0 \\
\text { ottereme sero }
\end{gathered}
$$

These work okay, for $v \leqslant 0.8 \mathrm{C}$.

P.I.C. dissipation

$-395-$ 


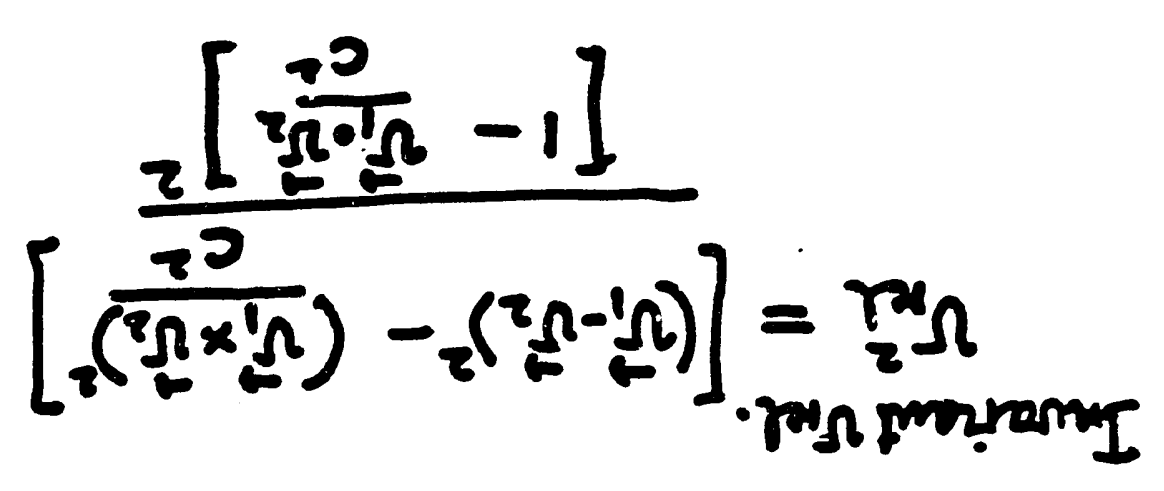

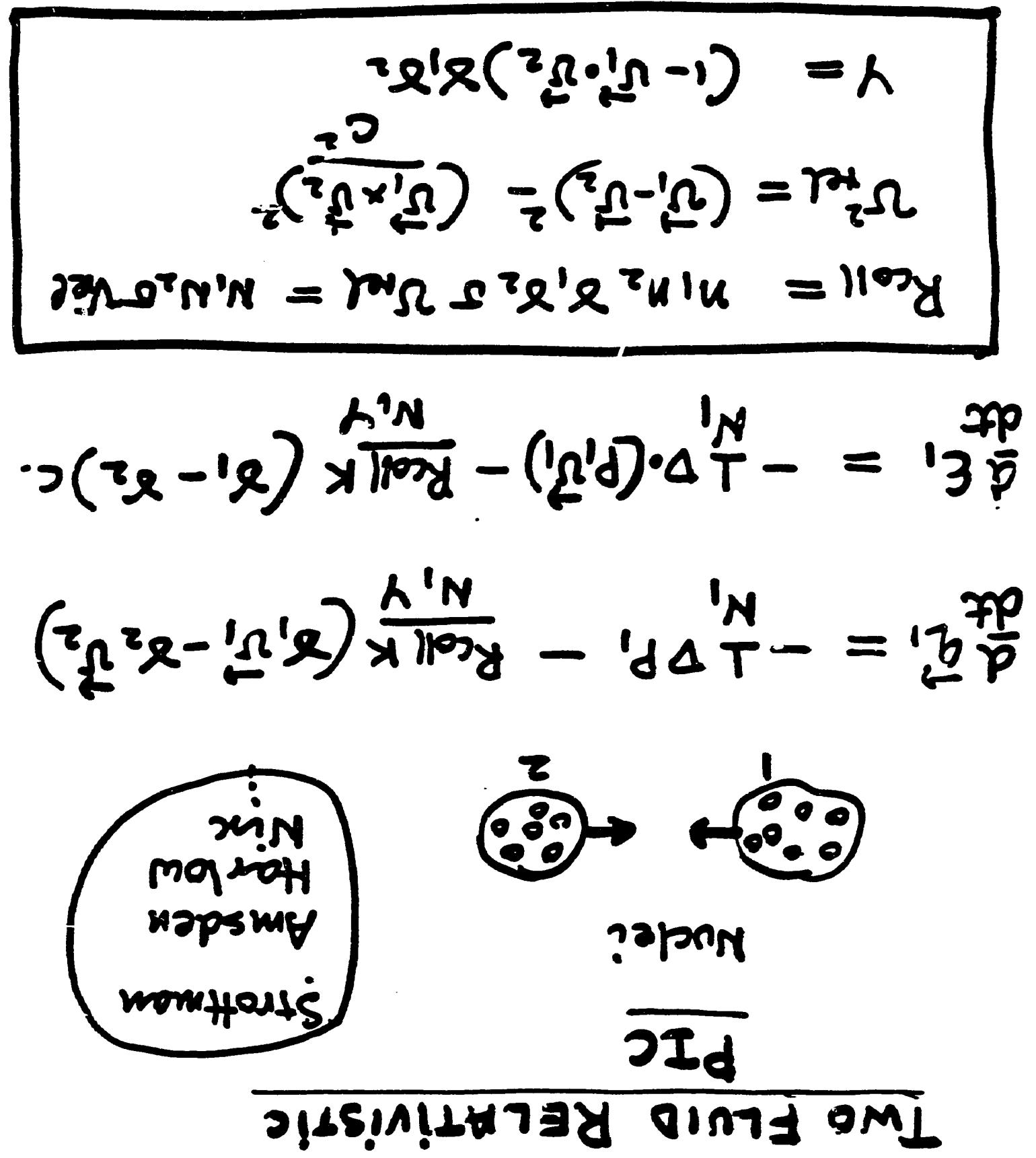


SPH FoRM of Baryon Drag

$$
\begin{aligned}
& \text { Use } \\
& \Pi_{a b}=-\frac{\mu_{a b}}{\bar{N}_{a b}} \bar{\hbar}_{a b}\left\{\bar{c}_{a b}+\left|\mu_{a b}\right|\right\} \\
& \mu_{a b}=\frac{\left(\gamma_{a} \vec{v}_{a}-\gamma_{b} \vec{v}_{b}\right) \cdot\left(\vec{v}_{a}-\vec{\gamma}_{b}\right)}{\left(r_{a}-r_{b}\right)^{2}+\eta^{2}}
\end{aligned}
$$

Same form ass Non Relativistic Viscous Term.

$-397-$ 
Equations

$$
\begin{aligned}
\frac{d}{d t} \vec{q}_{a}= & -\sum_{b} \nu_{b}\left\{\frac{P_{a}}{N_{a}^{2}}+\frac{P_{b}}{N_{b}^{2}}+\Pi_{a b}\right\} \vec{\nabla}_{c} w_{a b} \\
\frac{d}{d t} N_{a}= & +\sum_{b} \nu_{b}\left(\vec{v}_{a}-\vec{v}_{b}\right) \cdot \nabla w_{a b} \\
\frac{d}{d t} \hat{e}_{a}= & -\frac{P_{a}}{N_{a}} \frac{d}{d t} \gamma_{a} \\
& +\frac{\gamma_{a} P_{a}}{N_{a}^{2}} \sum_{b} \nu_{b}\left(v_{a}^{-}-\vec{v}_{b}\right) \cdot \nabla w_{a b} \\
& +\gamma_{a} \sum_{b} \nu_{b} \prod_{a b} \frac{1}{2}\left(\vec{v}_{a}-\vec{u}_{b}\right) \cdot \nabla W_{a b}
\end{aligned}
$$

- Spline Kernel

- Iteration on $\hat{e}$ and $d \gamma / d t$

- $h_{a b}=\frac{1}{2}\left(h_{a}+h_{b}\right)$

$$
\text { or } h_{a b}=\frac{2 h_{a} f_{b}}{\hbar_{a}+\hbar_{b}}
$$

or

$-398-$ 
Experiments

- Sound Wave. $\quad\left(D=1, \hat{e}=0.2, \Gamma=\frac{5}{3}\right.$

$$
\Delta v=0.05 \exp \left(-\frac{x^{2}}{l^{2}}\right)
$$

- Shock Tube

- Colliding Cold STreams

Smoothing

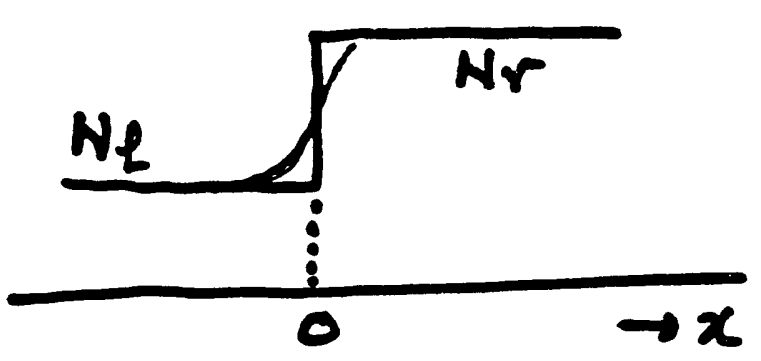

$$
N(x)=\frac{\Gamma N e+N r \operatorname{cxp}(y / t) I}{1+\exp (x / t)}
$$

$-399-$ 


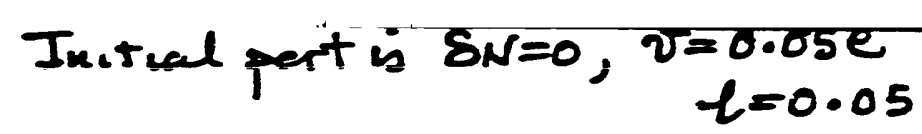
madel $=120 n=201 \quad n=0.010 \quad \lim \theta=4.5135 E-01 \quad \begin{aligned} & \alpha=1 \\ & \beta=2\end{aligned}$

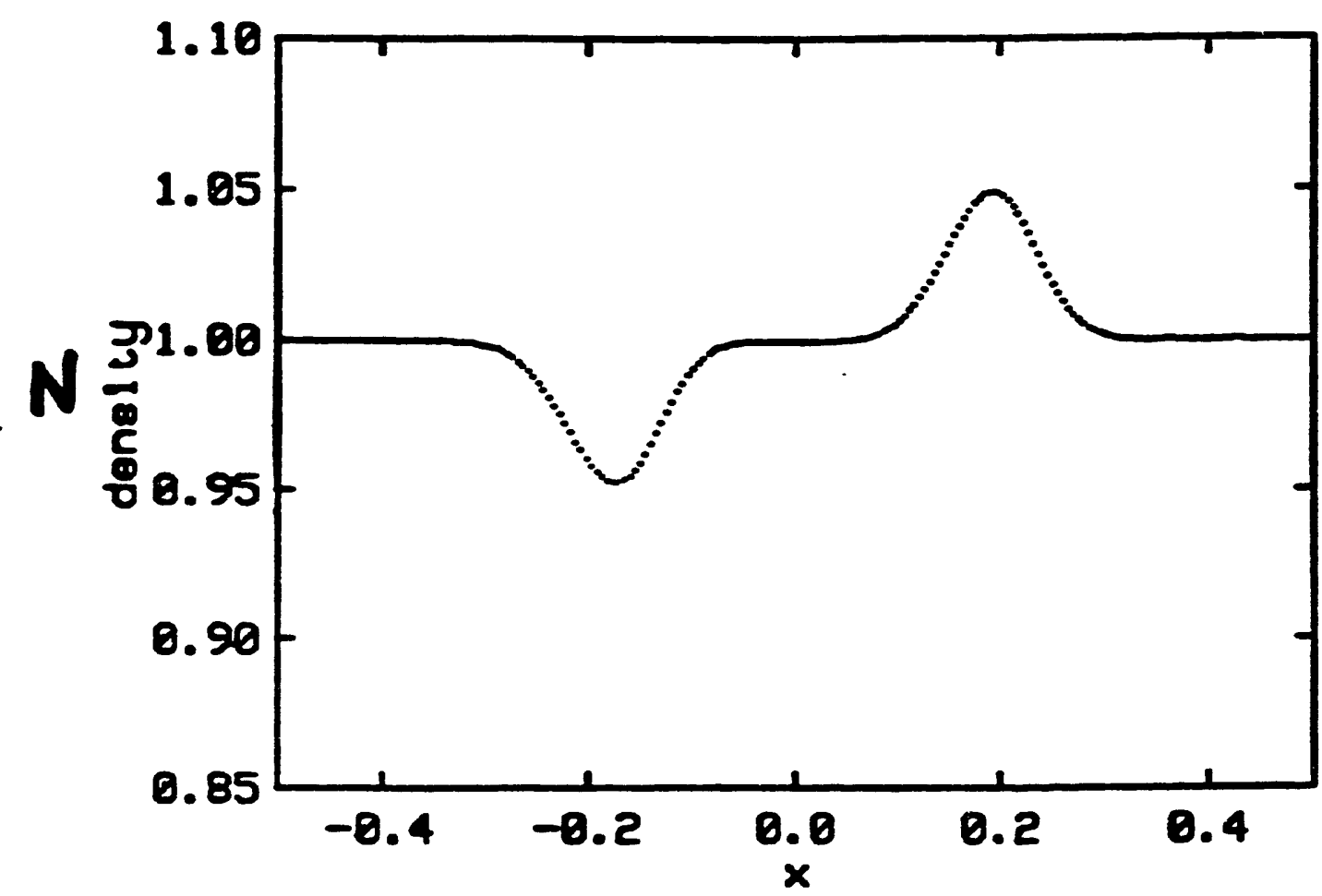

Propagation of SoUND WAVE IN A RELATIUISTLC GAS.

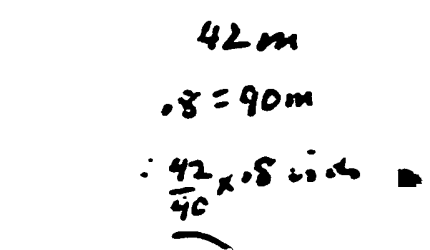

26-4uc-93

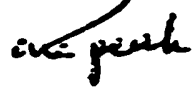
$42+\cdot 4$ 1t.5

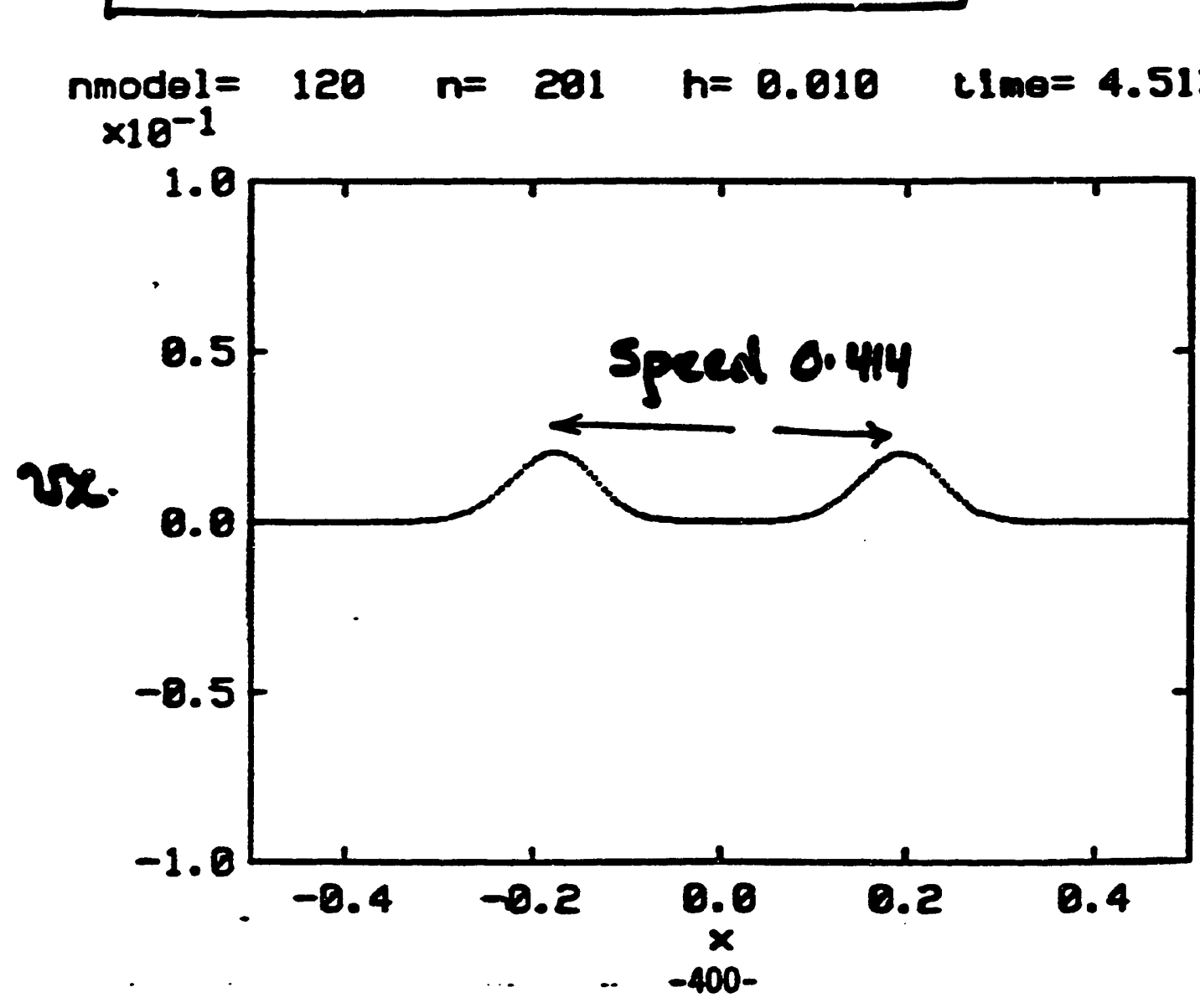




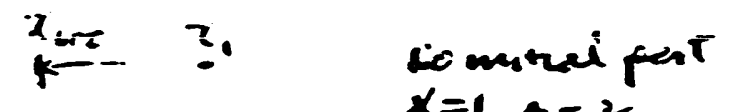

$$
\begin{array}{ll}
x=1, \beta=2 & r=073, \hat{e}=0.2
\end{array}
$$

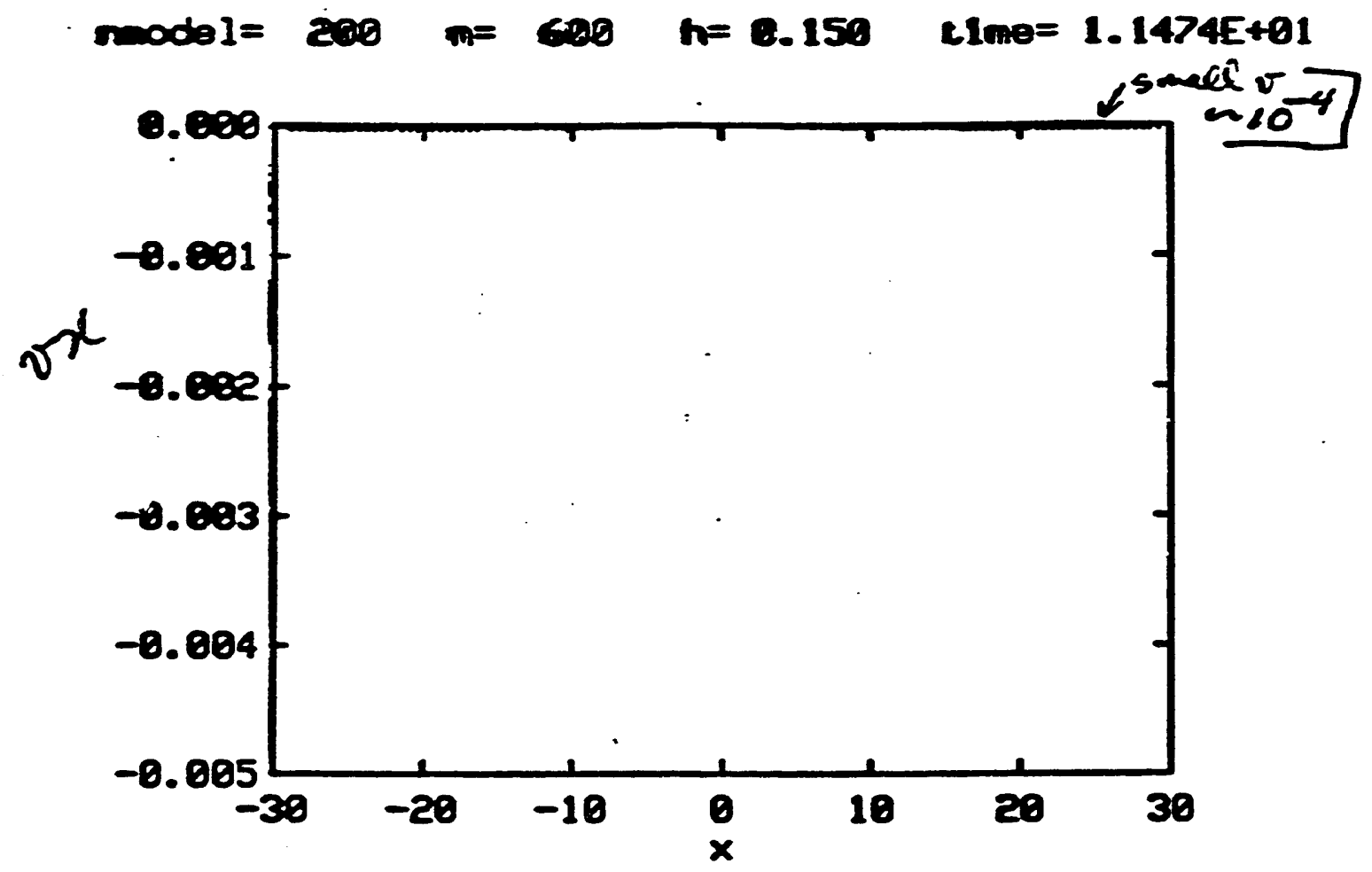

$25-\operatorname{archas}$

Why are the eros
small for $x=0$ ?

$$
\text { node }=280 \quad n=680 \quad h=0.158 \quad \text { lino }=1.1474 E+01
$$

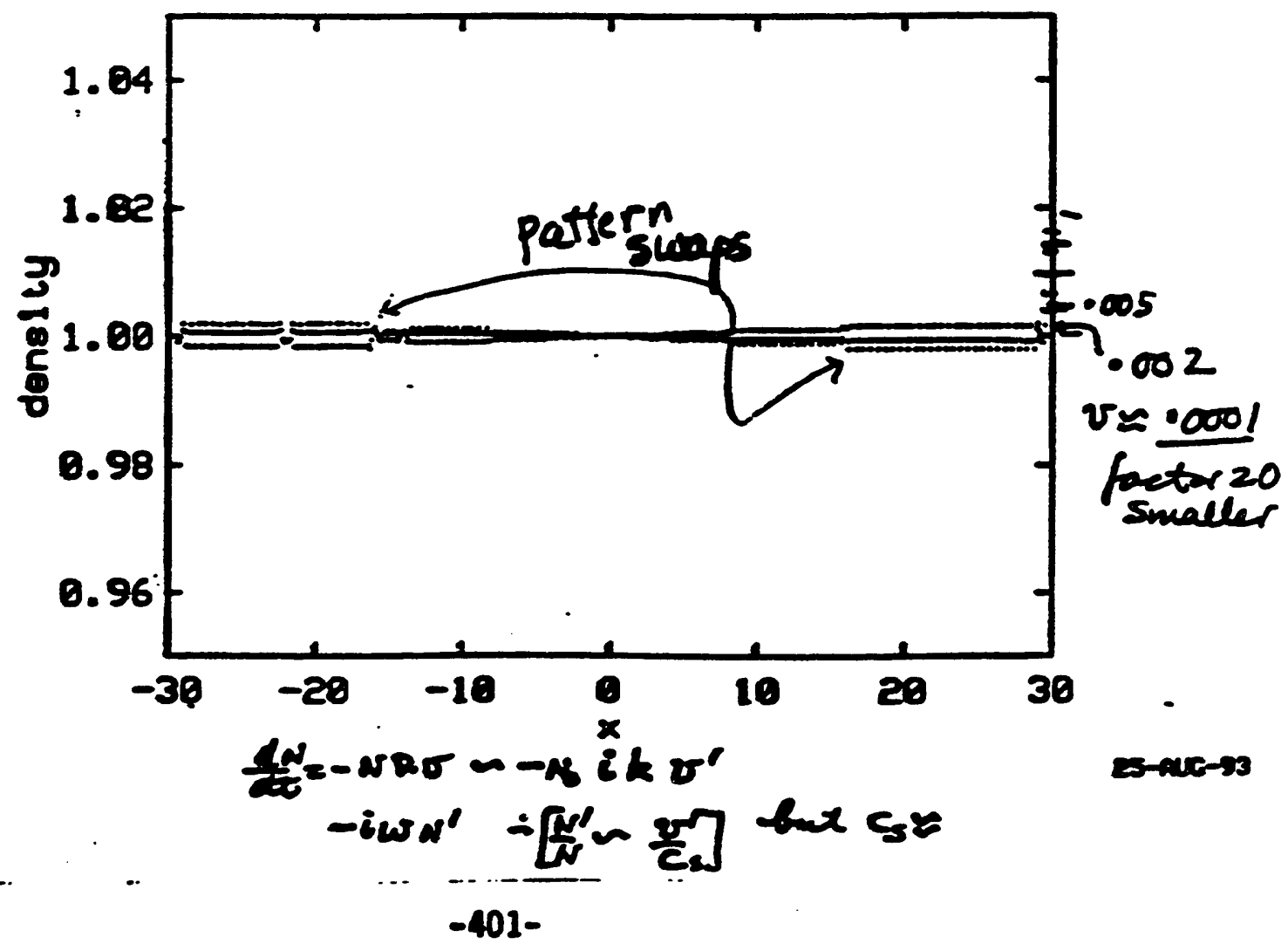




\section{Note: The pattern chanaes trom ine}

raodel $=200 h=600 \quad h=0.150$ Lime $=1.1471 E+\theta 1$

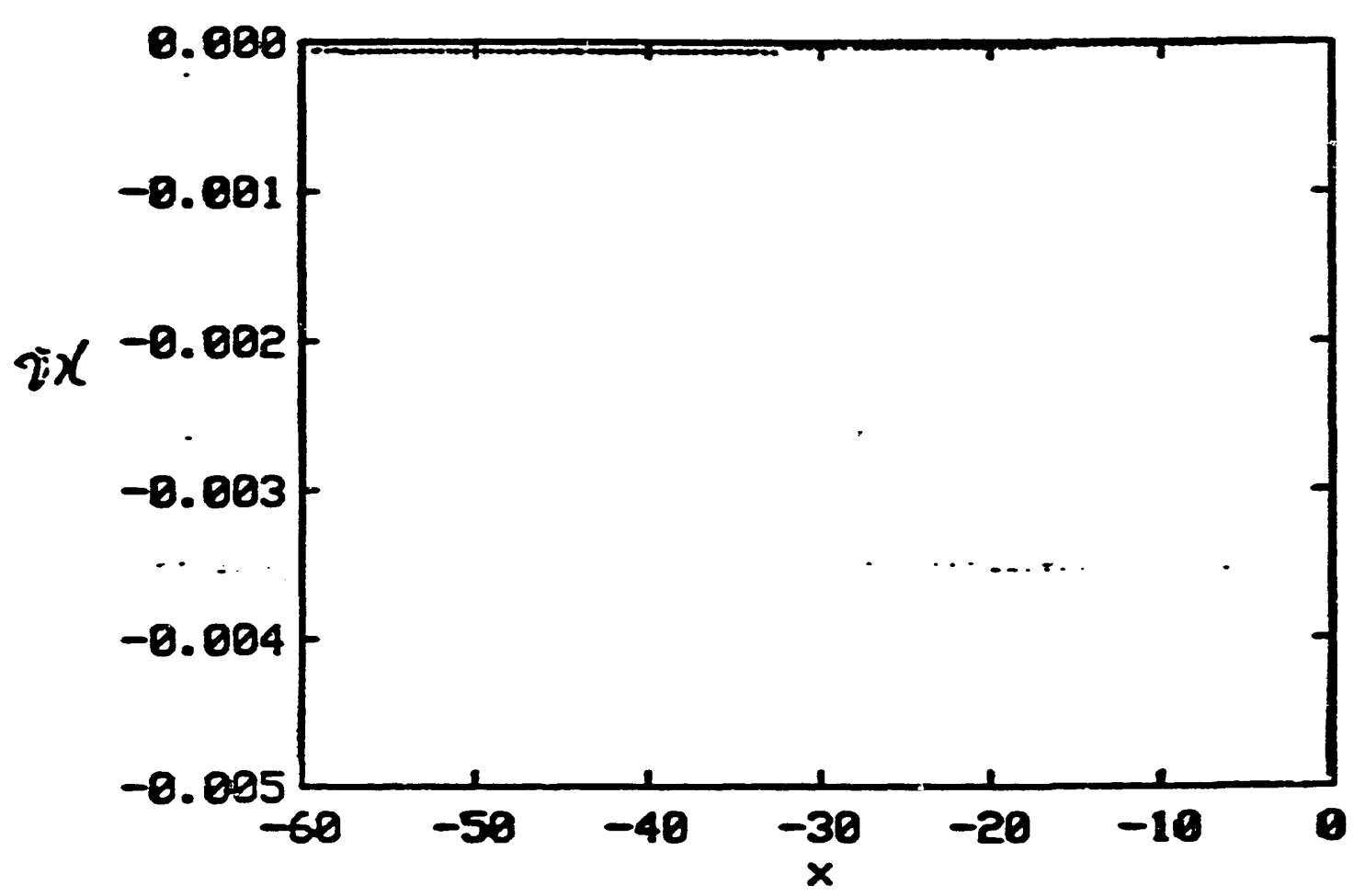

\section{No initial perturbations!}

es-anc-93

nmodel $=200 \quad n=680 \quad h=0.158 \quad$ time $=1.1471 E+01$

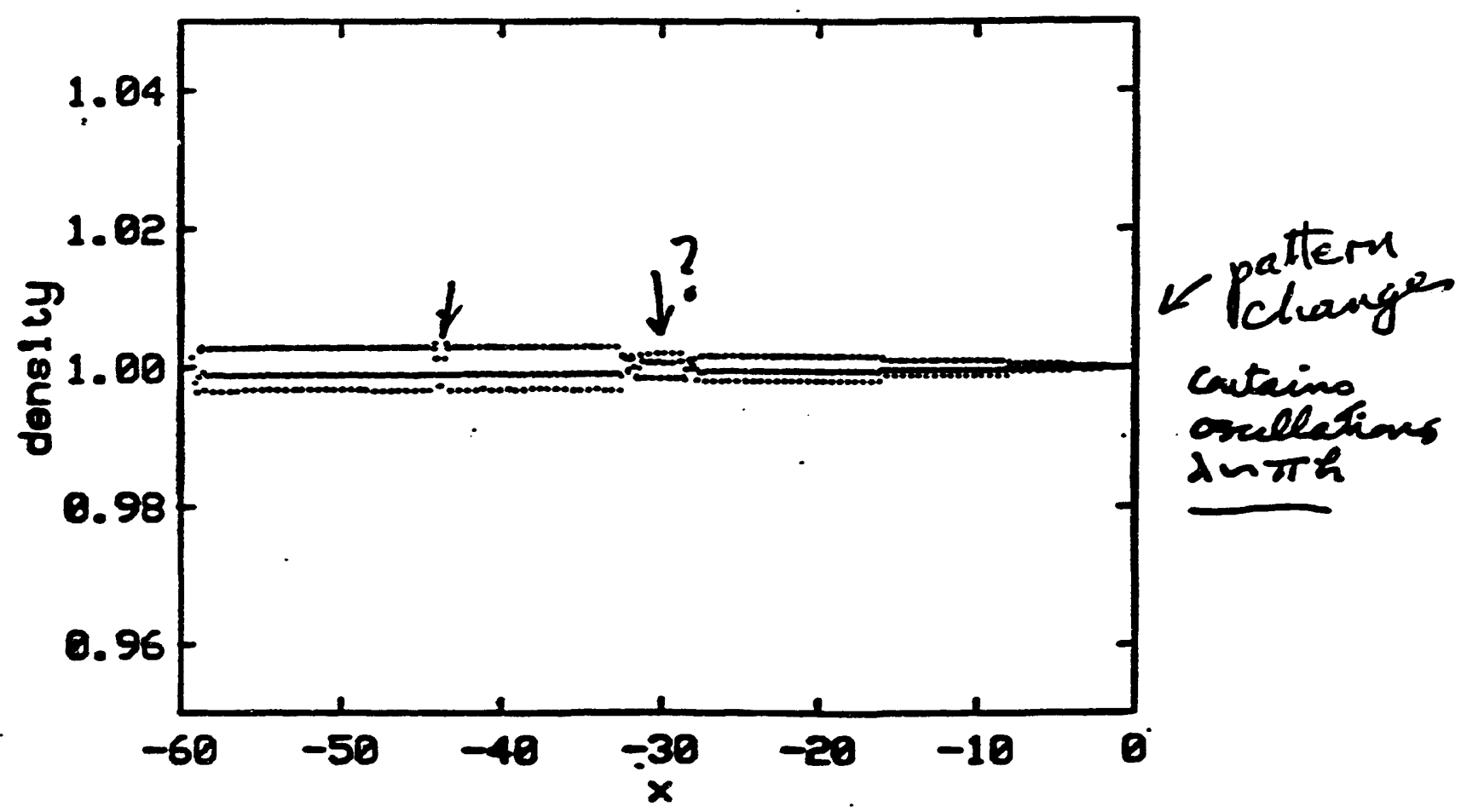


nnocto

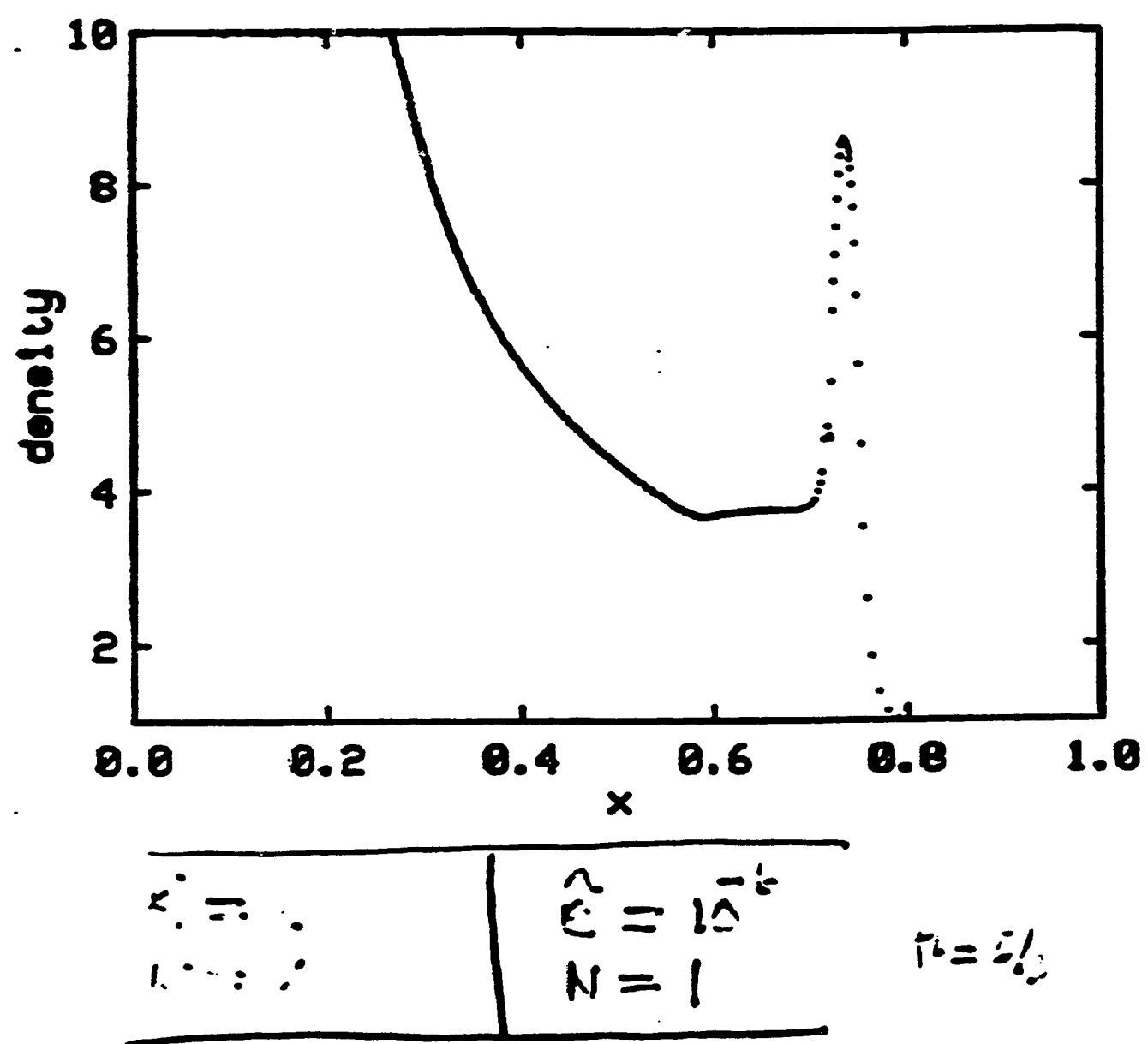

10-รयా-93

nmodel $=800 \quad m=551 \quad h=0.020 \quad$ LIme= $3.0983 E-01$

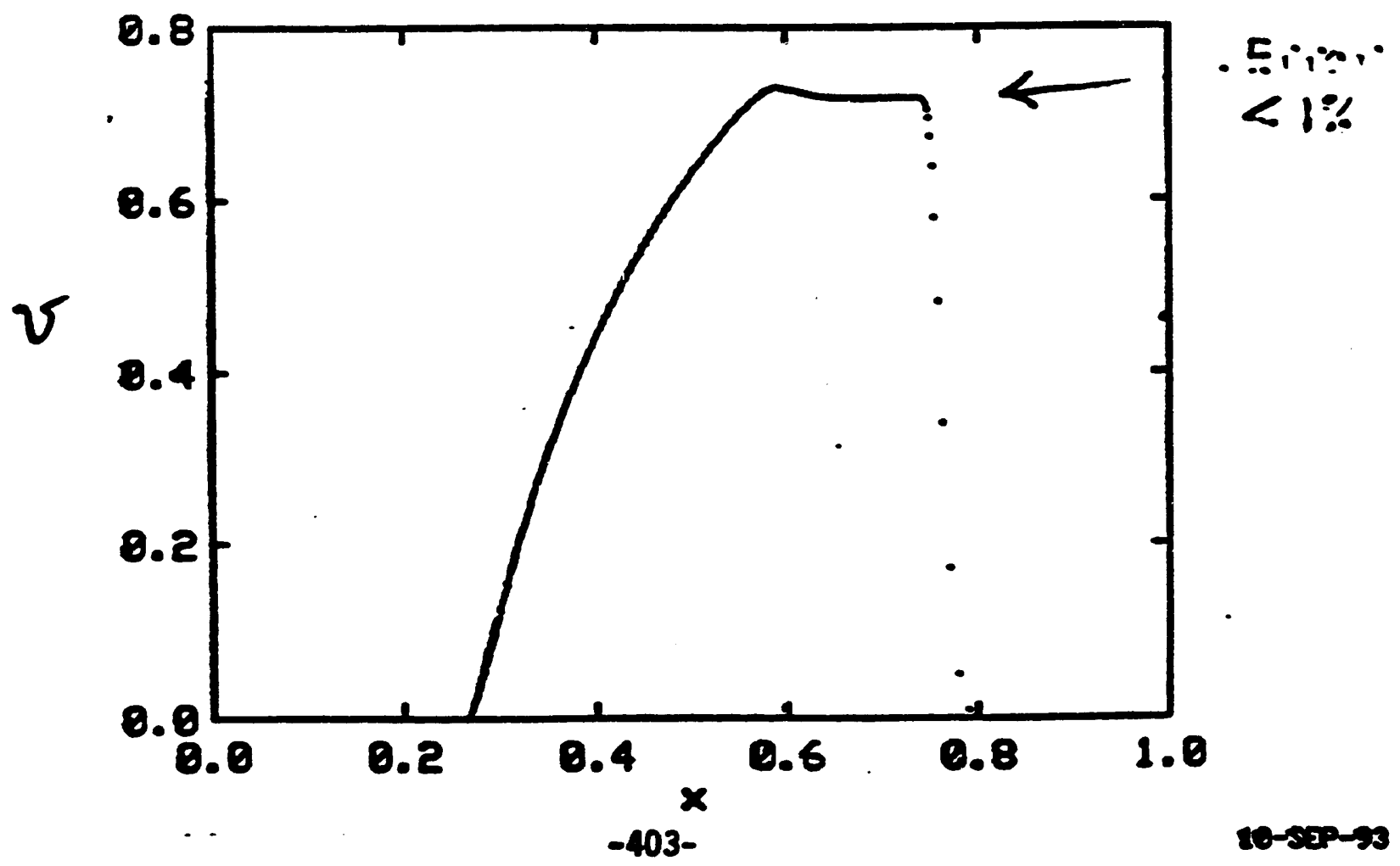


rmodel $=800 h=551 \quad h=0.020 \quad$ ime $=3.0983 E-01$

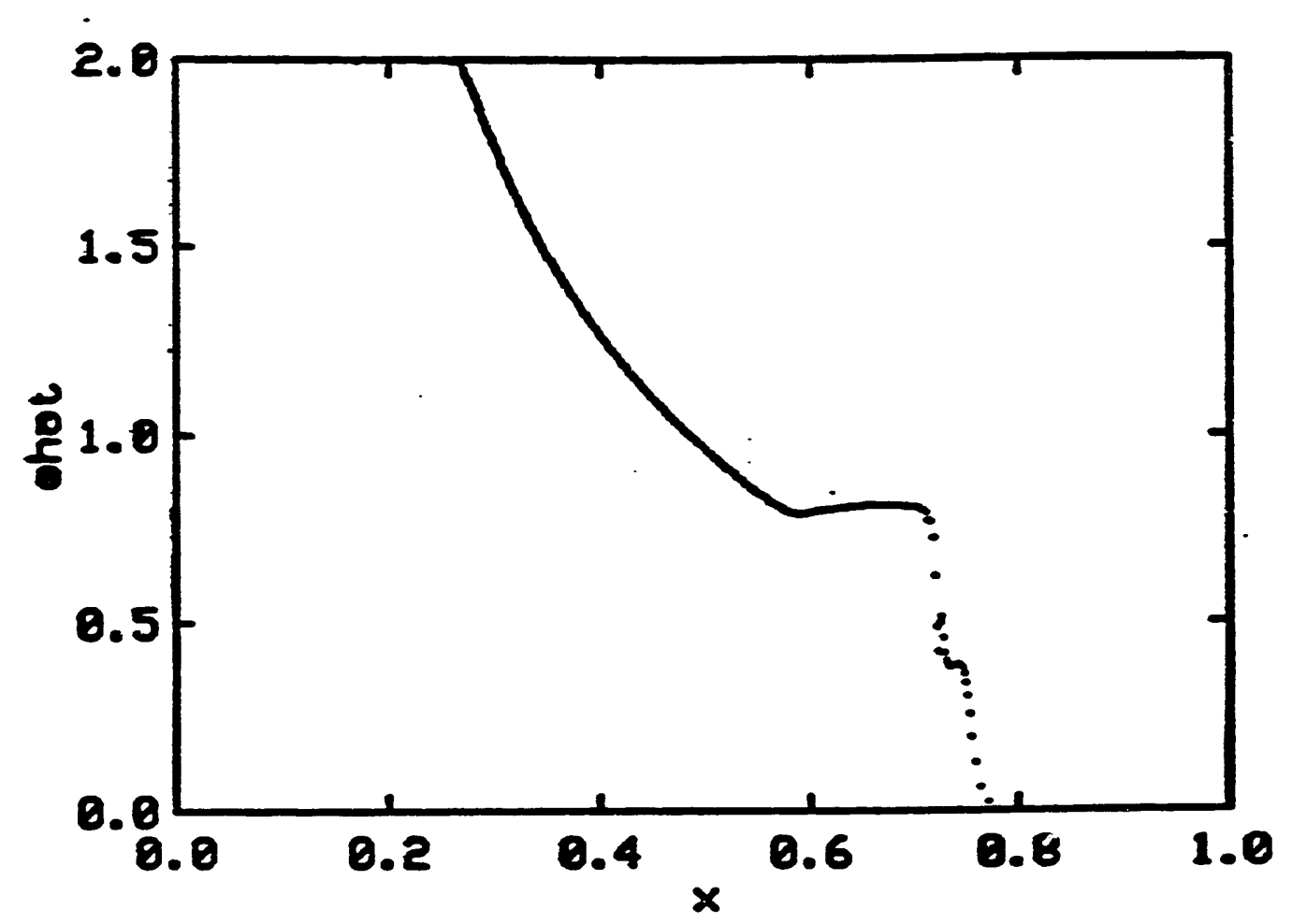

$10-2 x \operatorname{sen}-93$

$$
h_{a b}=\frac{1}{2}\left(h_{a}+h_{b}\right)
$$

nmodel $=800 \quad n=551 \quad h=0.020 \quad$ time $=3.0983 E-01$

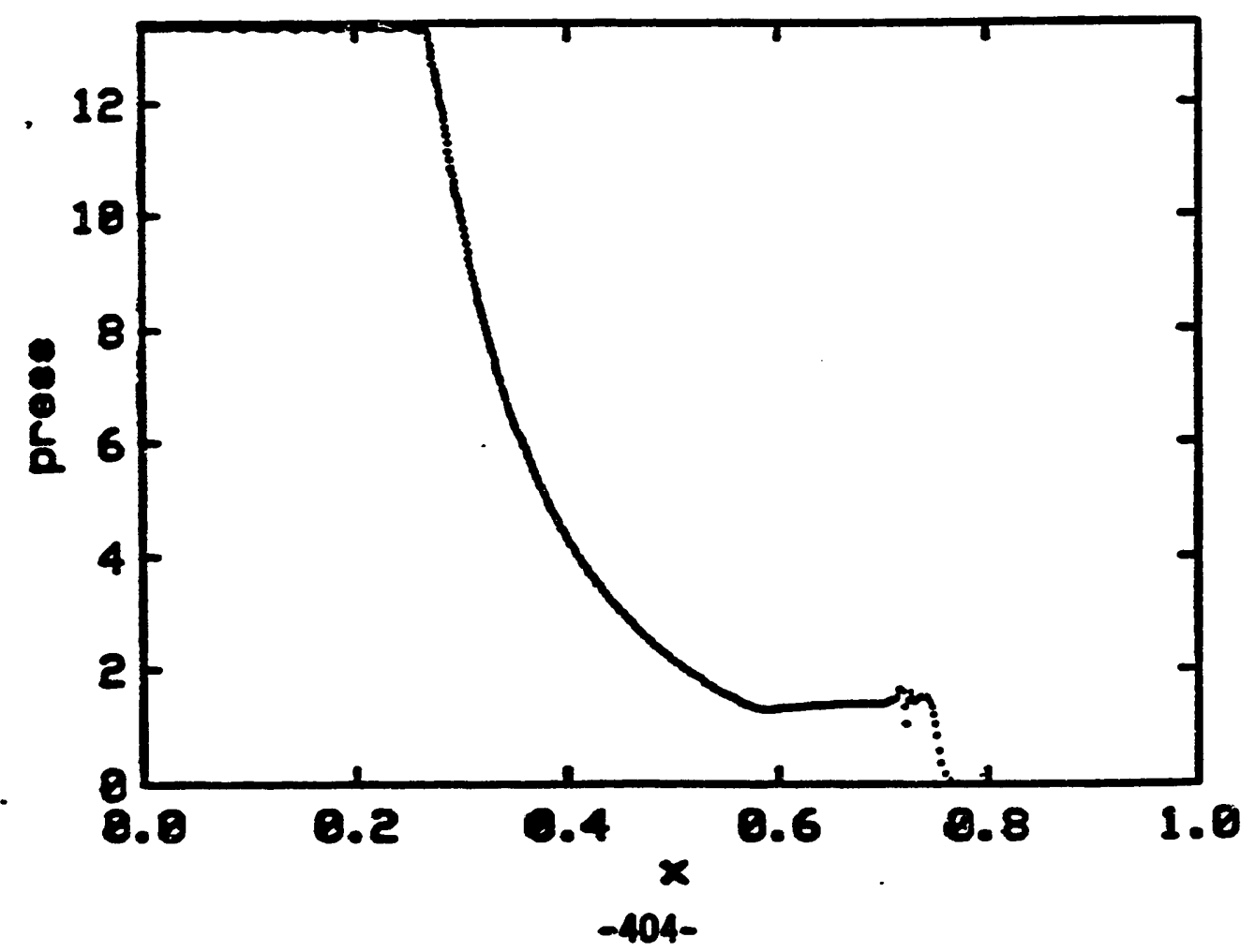




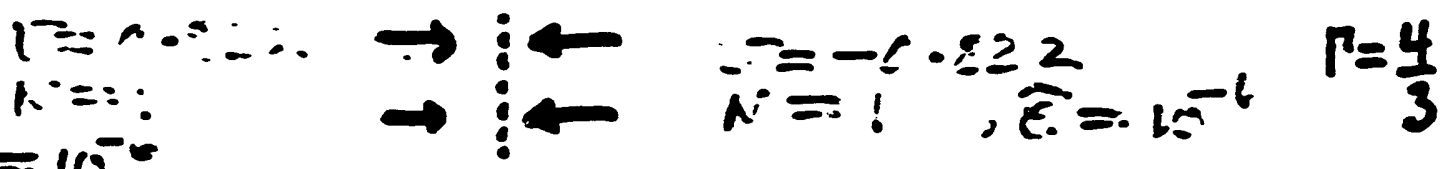

$$
\begin{aligned}
& \hat{\varepsilon}=w^{-\infty} \\
& \text { model }=400 \quad n=101 \quad h=0.815 \quad \text { tIme }=3.6499 E-01
\end{aligned}
$$

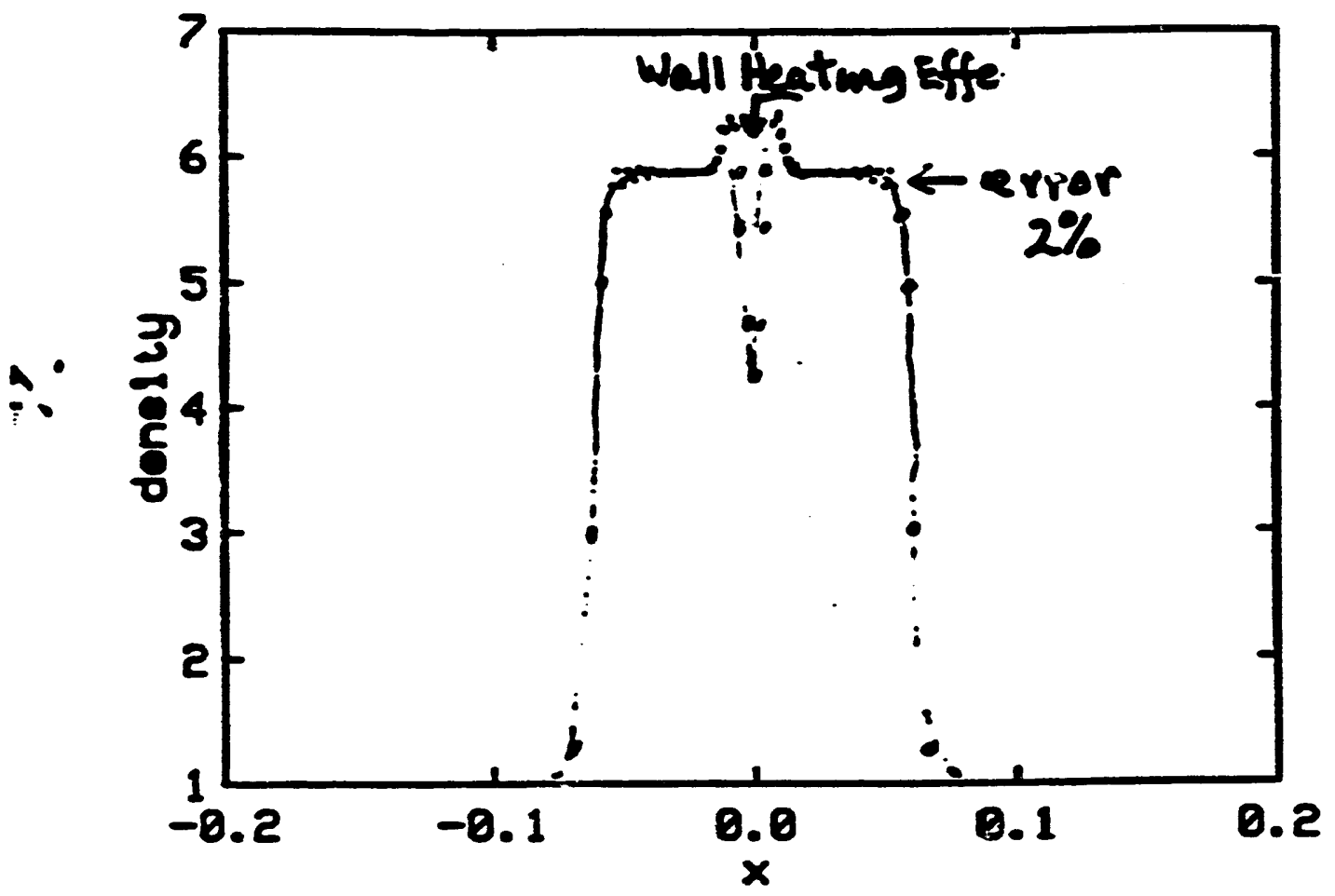

$h_{a b}=\frac{2 h_{a} h_{b}}{h_{a}+h_{b}} \quad \begin{aligned} & \text { Howler et al. } \\ & \text { get langer errors }\end{aligned}$

$$
\text { model }=400 \quad n=101 \quad h=0.015 \quad t \operatorname{lm} \theta=3.6499 E-01
$$

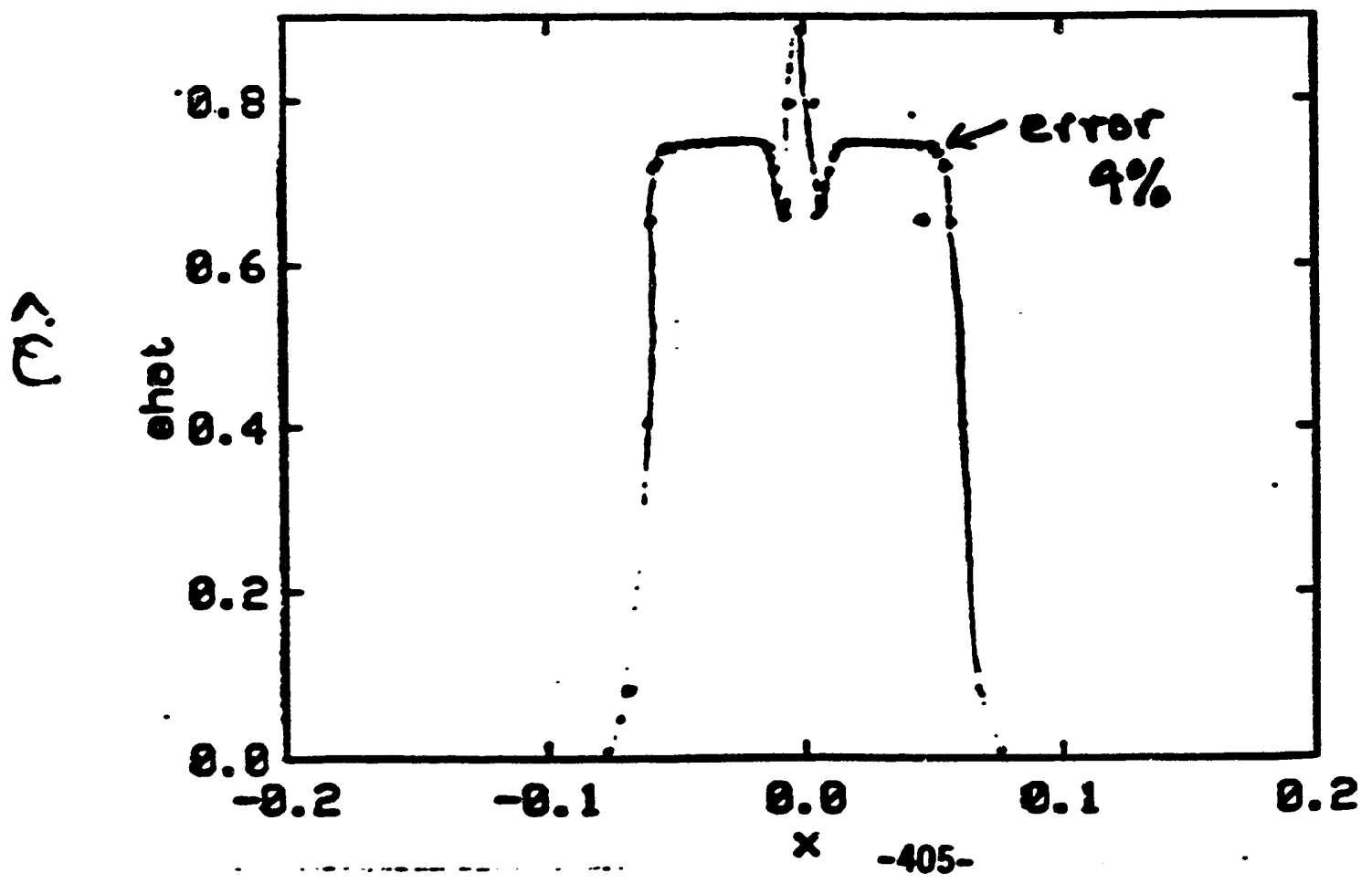


CONCLUSIONS

- The Baryon- Baryon Viscosity is known to work with $v \leqslant 0.95 \mathrm{c}$

- The results are comparable to those of Haw ley et. al. - often better!

- We need to decide best rule for choosing hab

- We definitely need a good heat conduction - for any method

- The calculations shew integrating the continuity equation works $0 . k$. for strong shocks.

$-406-$ 
Recent work on the Kegge Calculus at Monas Uni.

1) Constructing particle paths for Schwargschild + FR W spacetime

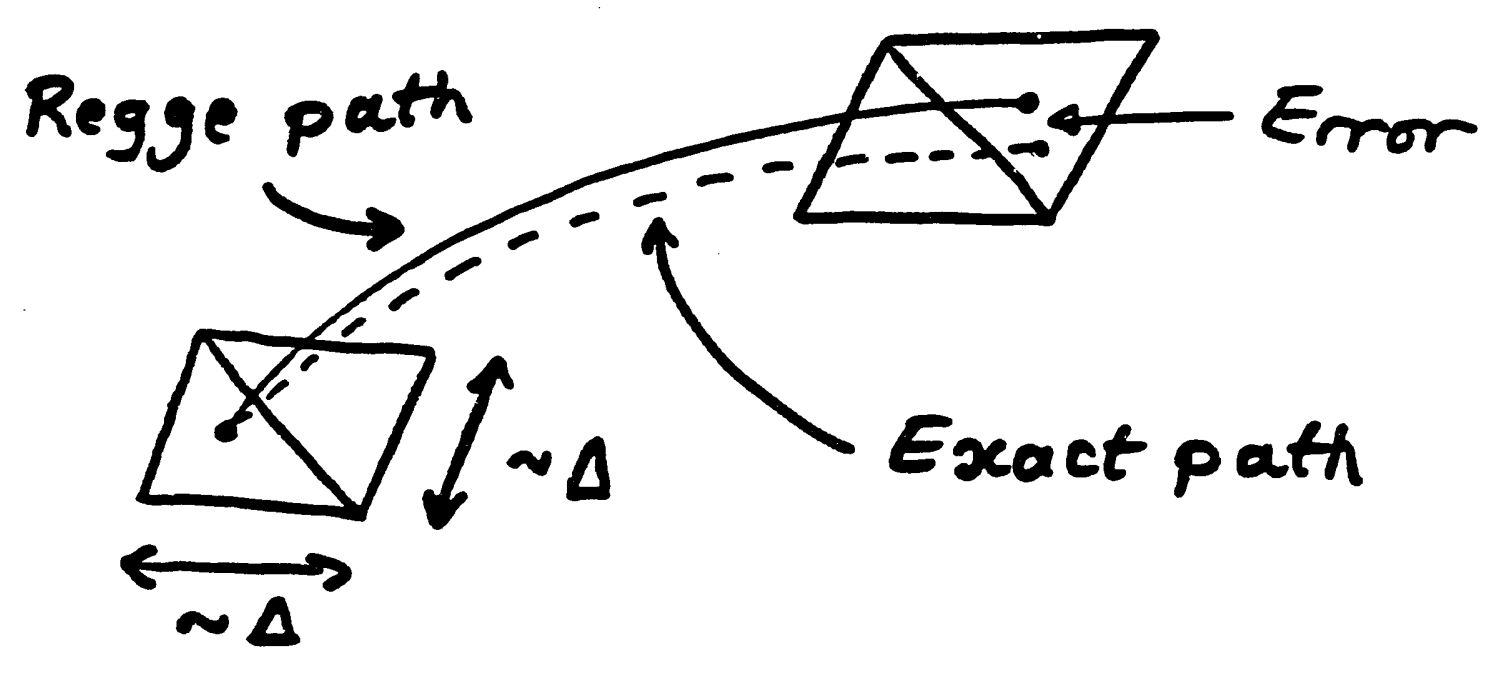

We showed that the global error $O(A)$ The Rage equations can be modified to give a global error $\sim O\left(\Delta^{2}\right)$

2) Boundary conditions Is there a systematic say to translate

$$
\frac{\partial \phi}{\partial r}+\frac{\phi-1}{r}=0
$$

into some $B C$ on the $L_{i j}$ is?

How can $r$ and $L_{-407-i}$ be related? 
5) Programming

We have a program that evaluates, for any 4-D simplicial space

i) all areas, volumes and defects

ii) all the field equations and

iii) all the derivatives of the field equations - required for NewtonRaphson.

The program takes about $6 \times 10^{-4} \mathrm{~S}$ per equation (+ derivatives).

Next step - port it to a CM 5.

- apply it to $2 \mathrm{BH}$ problem.

$-408-$ 
$\frac{1}{5}$ 
Caltech

130-33

Pasadena, CA 91125

Cray Research, Inc.

Suite 830

6565 Americas Parkway, N.E.

Albuquerque, NM 87110

Dagonet Software

2904 La Veta Dr. NE

Albuquerque, NM $87110-3110$

Kachina Technologies

1420 Carlisle Blvd. NE

Suite 202

Albuquerque, NM 87110

Kaman Sciences Corporation 1500 Garden of the Gods Rd.

P. O. Box 7463

Colorado Springs, CO 80933
Melvyn B. Davies

mbd@tapir.caltech.edu

Phil Campbell

505-883-8700

pmc@crayalb.cray.com

Dave Shirley

505-883-8700

dshirley@crayalb.cray.com

Lou Baker

505-883-0381

505-846-2018

baker@jake.plk.af.mil

Tony Giancola

505-268-8982

tony@objectsci.com

Laboratoire de Physique de la Matiere Condensee

Universite de Nice Sophia-Antipolis

Parc Valrose

Christian Vanneste $\quad 3393529972$

06108 NICE Cedex 2 France

Nasit Ari

Sheldon Jones

Joe Secary
719-599-1637

719-599-1911

719-599-1647
Lawrence Livermore National Laboratory PO B0X 808

Livermore, CA 94550
Leigh Brookshaw

Bill Hoover

Alan Spero

Joseph S. Oliveira

$510-423-2999$

vanneste@calypso.unice.fr
MS L-413

MS L-794

Los Alamos National Laboratory

Los Alamos NM 87545

\begin{tabular}{lrl} 
Stirling Colgate & $505-665-5254$ & MS B275 \\
Necia Cooper & $505-667-1447$ & MS M708 \\
Rich Davidson & $505-667-7438$ & MS K557 \\
\multicolumn{1}{r}{ rich @lanl.gov } & \\
Mike Garcia & $505-667-7965$ & MS C931 \\
Jack Hills & $505-667-9152$ & MS B288 \\
-412- & &
\end{tabular}




\section{LIST OF ATTENDEES AND OTHERS}

This list inlcudes people who attended the SPH workshop and people who were interested in the workshop but could not attend. This also constitutes the distribution list. This list is organized by company or institution. Following this list is a list organized by name.

Advanced Sciences Incorporated 6739 Academy Rd., NE

Albuquerque, NM 87109

Air Force Institute of Technology 2950 P Street

Wright-Patterson Air Force Base

Dayton, Ohio 45433-7765

Alliant Tech Systems Inc. (MN 11-2925)

600 2nd St NE

Hopkins, MN 55343

Alme and Associates

2 Stevens Forest Professional Center

9650 Santiago Road

Columbia, MD 21045

Applied Research Associates

4300 San Mateo Blvd. NE

Suite A-220

Albuquerque, NM 87110

Battelle

505 King Avenue

Columbus, OH 43201-2693
Mehdi Eliassi

\section{Michael Stoecker $\quad$ 513-255-3636 ext 4514 \\ Department of Mathematics and Statistics mstoecke afit.af.mil}

Dave Fulk

AFIT/ENC

dfulk@afitaf.mil

Gordon Johnson

612-931-5905

Marv Alme

$410-740-1118$

Ted Camey

$505-883-3636$

Mike Fisher

614-424-3620

fisher (tomahawk.dst.battelle.org

Doug Everhart

614-424-3214

doug@dragon.dst.battelle.org

Chuck Hargraves

614-424-4953 


\begin{tabular}{|c|c|c|}
\hline Brad Holian & $505-667-9237$ & MS B268 \\
\hline Kathy Holian & $505-665-4616$ & MS B295 \\
\hline Norman Johnson & 505-667-9094 & MS B216 \\
\hline Mike Jones & $505-667-7760$ & MS F645 \\
\hline Bob Karpp & & MS F663 \\
\hline Jerry Kerrisk & $505-665-8709$ & MS F663 \\
\hline Ron Kirkpatrick & $505-667-7208$ & MS F645 \\
\hline Raymond Lefiamme & $505-667-3394$ & MS B288 \\
\hline Peter Leonard & $404-667-1472$ & MS B288 \\
\hline Larry Luck & $505-667-3563$ & MS K557 \\
\hline Dave Mandell & $505-667-7145$ & MS F663 \\
\hline dam@lan & & \\
\hline Wamer Miller & $505-667-3747$ & MS B288 \\
\hline B. Nadiga & $\begin{array}{l}\text { 3.lanl.gov } \\
\text { S05-667-9466 }\end{array}$ & MS B213 \\
\hline Joe Repa & $505-667-4494$ & MS K574 \\
\hline Larry Schwalbe & $505-667-0325$ & MS F663 \\
\hline Barry Shafer & $505-667-1074$ & MS K574 \\
\hline Charles Snell & $505-667-9841$ & MS F665 \\
\hline cms@l & anl.gov & \\
\hline Warren Sparks & $505-667-4922$ & MS F663 \\
\hline wms@lan & & \\
\hline $\begin{array}{l}\text { Bob Stellingwerf } \\
\text { ffs@lanl. }\end{array}$ & $505-667-8905$ & MS F645 \\
\hline Robert Swift & $\begin{array}{l}505-665-7871 \\
\text { lanl }\end{array}$ & MS F665 \\
\hline ase & $505-667-8062$ & MS F663 \\
\hline Mire Women & & \\
\hline $\begin{array}{l}\text { Mike Warten } \\
\text { Doug Wilson }\end{array}$ & $\begin{array}{l}505-665-5023 \\
505-667-4370\end{array}$ & $\begin{array}{l}\text { MS B288 } \\
\text { MS F645 }\end{array}$ \\
\hline dew & & \\
\hline $\begin{array}{l}\text { ingate } \\
\text { caw@. }\end{array}$ & $\begin{array}{l}505-667-8954 \\
\text { ov }\end{array}$ & MS F645 \\
\hline Zurek & $505-667-683$ & (CDS \\
\hline
\end{tabular}

Louisiana State University Dept. of Mechanical Engineering Baton Rouge, LA 70803

Michigan Tech. University Math Deparment Houghton, Michigan 49931
Robert Courter

504-388-5891
505-667-9237$$
\text { 505-667-9094 }
$$

$505-667-7760$

505-665-8709

505-667-7208

404-667-1472

505-667-3563

ov

lanl.gov

505-667-0325

505-667-1074

$-667-984$

505-667-4922

sos

505-665-7871

505-667-8062

505-665-5023

505-667-4370

sos-

$505-667-6837$

MS B288
Darrell L. Hicks liebrock@es.mtu.edu 
Monash University

Mathematics Department

Clayton, Vic. 3168

Australia

NASA Ames Research Center

Moffett Field, CA 94035

NM Institute of Mining and Technology

Center for Explosives Technology Research

Socorro, NM 87801

Northwestem University

Dept of Mechanical Engineering

2145 Sheridan Rd.

Evanston, Il 60208-3111

Ohio State University

Dept. of Astronomy

174 W 18th Avenue

Columbus, $\mathrm{OH} 43210$

Phillips Lab

PLWSSD

Kirtland Air Force Base

Albuquerque, NM 87117

Purdue University

Dept. of Civil Engineering

West Lafayette, IN 47907
Joe Monaghan

035653867

jim@vaxc.cc.monash.edu.au

Erik Asphang

415-604-0786 MS 245-3

asphaug@cosmic.arc.nasa.gov

Larry Libersky

505-835-5941

larry@illusion.nmt.edu

Ted Beletchko

708-491-7270

Wing Kam Liu

Yun Yun Lu

708-491-5164

yunlu@tam4.mech.nwu.edu

Mike Owen

614-292-7881

Jens Villumsen jens@payne.mps.ohio-state.edu

Firooz Allahdadi $\quad$ 505-846-2016

Dave Amdahl 505-846-2017

amdahl@jake.plk.af.mil

Charles Luehr

505-846-2017

David Medina

luehr@jake.plk.af.mil

James Ninter

Brad Smith

505-846-2016

bsmith @jake.plk.af.mil

Patrick Fox

317-494-0697

pfox@ecn.purdue.edu

Hank Alme

713-665-0133

almehj@owhetrice.edu

Baker College

6320 South Main St.

Houston, TX 77005 
Sandia National Laboratory

P. O. Box 5800

Albuquerque, NM 87185

Tetra Corporation

3701 Hawkins St. NE

Albuquerque, NM $87109-4512$

University of Arizonia

Steward Observatory

Tucson, Arizona 85721

University of Heidelberg

Institut fur Theoretishche Astrospysik

Im Neuenheimer Feld 561

D - 69120 Heidelberg

Germany

University of New Mexico

Dept. of Chemical/Nuclear Engineering

Farris Engineering Center

Albuquerque, NM 87131-1341

University of Pennsylvannia

Dept. of Astronomy and Astrophysics

525 Davey Laboratory

University Park, PA 16802

University of Texas

Dept of Mechanical Engineering

ETC 5.160

Austin, TX 78712

University of Westem Ontario

Deparment of Applied Mathematics

London, Ontario, Canada, N6A 3K7

\begin{tabular}{|c|c|c|}
\hline Steve Attaway & $505-844-9288$ & Dept. 1425 \\
\hline swa & andiagov & U. \\
\hline $\begin{array}{l}\text { Jeff Swegle } \\
\text { iws }\end{array}$ & $\begin{array}{l}\text { 505-844-9369 } \\
\text { andia.gov }\end{array}$ & DepL 1 \\
\hline
\end{tabular}

Gary Hess

$505-345-8623$

Willy Benz

602-621-6530

wbenz@ptolemy.as.arizona.edu

Hanno Sponholz $\quad 49$ (6221) 562974

sponholz@epikurita.uni-heidelberg.de

Rita Smith

505-821-0416

rsmitheaquarius.unm.edu

A. Sharif Heger

heger@hydra.unm.edu

Pablo Laguna

814-863-8470

pablo@astro.psu.edu

Eric Fahrenthold 512-471-3064

meed522Qhermes.chpc.utexas.edu

Patrick Mann

pjom emonty.apinaths.uwo.ca 
i 


\section{LIST OF ATTENDEES AND OTHERS}

This list is the same as the previous list but organized by individual name.

Firooz Allahdadi

Hank Alme

Marv Alme

Dave Amdahl

Nasit Ari

Erik Asphang

Steve Altaway

Lon Baker

Ted Beletchko

Willy Benz

Leigh Brookshaw

Phil Campbell

Ted Carney

Stirling Colgate

Necia Cooper

Robert Courter

Rich Davidson

Melvyn B. Davies

Mehdi Eliassi

Doug Everhart

Eric Fahrenthold

Mike Fisher

Patrick Fox

Dave Fulk

Mike Garcia

Tony Giancola

Chuck Hargraves

A. Sharif Heger

Gary R. Hess

Darren L. Hicks

Jack Fills

Brad Holian

Rathy Holian

Bill Hoover

Tom Hughes

Gordon Johnson

Narman Johnson

Mike Jones

Sheidon Jones

Bob Kepp
Phillips Lab

Rice University

Alme and Associates

Prillips Lab

Raman Sciences Corporation

NASA Ames Research Center

Sandia National Laboratory

Dagonet Software

Northwestern University

University of Arizonia

Lawrence Livermore National Laboratory

Cray Research, Inc.

Applied Research Associates

Los Alamos National Laboratory

Los Alamos National Laboratory

Lonisiana State University

Los Alamos National Laboratory

Caltech

Advanced Sciences Incorporated

Battelle

University of Texas

Battelle

Purdue University

Air Force Institute of Technology

Los Alamos National Laboratory

Kachina Technologies

Battelle

University of New Mexico

Tetra Corporation

Michigan Tech. University

Los Alamos National Laboratory

Los Alamos National Laboratory

Los Alamos National Laboratory

Lawrence Livermore National Laboratory

Mission Research Corporation

Alliant Tech Systems Inc.

Los Alamos National Laboratory

Los Alamos National Laboratory

Kaman Sciences Corporation

Ios Alamos National Laboratory 
Jany Renisk

Ron Kirtpatrick

Pablo Laguna

Raymond Leflamme

Peter Leonard

Larry Libersky

Wing Kam Liu

Yun Yun Lu

Larry Lock

Charles Luehr

Dave Mandell

Patrick Mann

David Medina

Warner Miller

Joe Monaghan

B. Nadiga

James Ninter

Joseph S. Oliveira

Mike Owen

Joe Repa

Lany Schwalbe

Joe Secary

Barry Shafer

Dave Shirley

Brad Smith

Rita Smith

Charles Snell

Warren Sparks

Alan Spero

Hanno Sponholz

Bob Stellingwerf

Michael Stoecker

Jefi Swegle

Robert Swift

Harold Trease

Christian Vanneste

Jens Villumsen

Mike Warren

Doug Wilson

Chuck Wingate

Wojciech Zurek
Los Alamos National Laboratory

Los Alamos National Laboratory

University of Pennsylvannia

Los Alamos National Laboratory

Los Alamos National Laboratory

NM Institute of Mining and Technology

Northwestern University

Northwestem University

Los Alamos National Laboratory

Phillips Lab

Los Alamos National Laboratory

University of Western Ontario

Phillips Lab

Los Alamos National Laboratory

Monash University

Los Alamos National Laboratory

Phillips Lab

Lawrence Livermore National Laboratory

Ohio State University

Los Alamos National Laboratory

Los Alamos National Laboratory

Raman Sciences Corporation

Los Alamos National Laboratory

Cray Research, Inc.

Phillips Lab

University of New Mexico

Los Alamos National Laboratory

Los Alamos National Laboratory

Lawrence Livermore National Laboratory

University of Heidelberg

Los Alamos National Laboratory

Air Force Institute of Technology

Sandia National Laboratory

Los Alamos National Laboratory

Los Alamos National Laboratory

Laboratoire de Physique de la Matiere Condensee

Ohio State University

Los Alamos National Laboratory

Los Alamos National Laboratory

Los Alamos National Laboratory

Los Alamos National Laboratory 

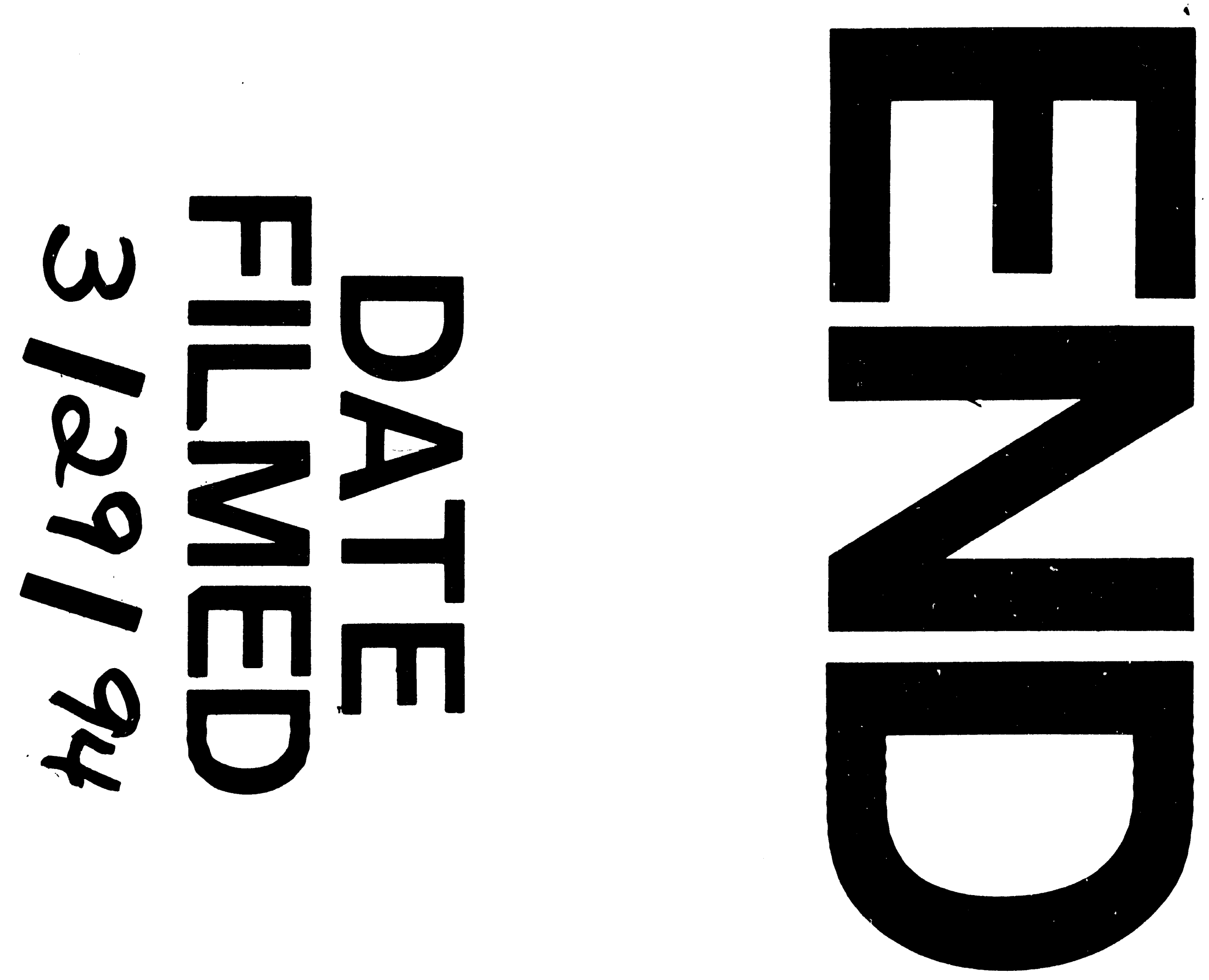